\title{
Development of a cardiovascular and lymphatic network model during human pregnancy
}

\author{
Carson, Jason M.
}

How to cite:

Carson, Jason M. (2019) Development of a cardiovascular and lymphatic network model during human pregnancy. Doctoral thesis, Swansea University.

http://cronfa.swan.ac.uk/Record/cronfa50058

Use policy:

This item is brought to you by Swansea University. Any person downloading material is agreeing to abide by the terms of the repository licence: copies of full text items may be used or reproduced in any format or medium, without prior permission for personal research or study, educational or non-commercial purposes only. The copyright for any work remains with the original author unless otherwise specified. The full-text must not be sold in any format or medium without the formal permission of the copyright holder. Permission for multiple reproductions should be obtained from the original author.

Authors are personally responsible for adhering to copyright and publisher restrictions when uploading content to the repository.

Please link to the metadata record in the Swansea University repository, Cronfa (link given in the citation reference above.)

http://www.swansea.ac.uk/library/researchsupport/ris-support/ 


\title{
Development of a Cardiovascular and Lymphatic Network Model During Human Pregnancy
}

\author{
Jason Matthew Carson
}

Submitted to Swansea University in fulfilment of the requirements for the Degree of Doctor of Philosophy.

Swansea University

2019 


\section{Summary}

The human physiology undergoes significant adaptation during pregnancy, particularly within the cardiovascular system. Insufficient cardiovascular adaptation can lead to several serious pathologies which can affect the growth of the foetus, such as hypertension, hypotension, pre-eclampsia, and placental insufficiency. Peripheral oedema occurs in the majority of woman over the course of a pregnancy, which is caused when the lymphatic system is unable to drain the excess fluid that has gathered in the interstitia.

In order to provide a platform for modelling these pathologies, a comprehensive closed-loop 1D-0D cardiovascular network model of pregnancy is developed and presented in this thesis. The computational framework allows in-vivo measurement data, including pressures, cardiac output, and gestational week, to be integrated into the cardiovascular model. New numerical schemes are presented for reducedorder modelling of the cardiovascular system and the lymphatic system with a view to providing a platform for a coupled cardiovascular and lymphatic model.

An automated parameter estimation technique is presented, which allows the integration of patient measurement data into the model through the iterative adaptation of haemodynamic parameters, and could be utilised in a wide variety of cardiovascular pathology modelling.

The pregnancy model is implemented using patient specific measurements and is extended to cover all gestational weeks for an idealised healthy pregnancy. The model solutions have shown good agreement with values from the literature for: the pulsatility index; pulse wave velocity; and flow rate waveforms in the uterine arteries, which includes the presence of a notch that is used in the clinic to detect pathologies. A novel aspect of the model is in predicting the blood supply to the uterus via the uterine and utero-ovarian communicating arteries, which could be useful in a clinical setting. The model is expected to provide a platform for modelling various pathologies that can develop during pregnancy. 


\section{Declarations}

This work has not previously been accepted in substance for any degree and is not being concurrently submitted in candidature for any degree.

Signed: Jason Carson Date: 18/01/2019

This work is the result of my own independent study/investigation, except where otherwise stated. Other sources are acknowledged by footnotes giving explicit references. A bibliography is appended.

\section{Signed: Jason Carson Date: 18/01/2019}

I hereby give my consent for my work, if relevant and accepted, to be available for photocopying and for inter-library loan, and for the title and summary to be made available to outside organisations.

Signed: Jason Carson Date: 18/01/2019 


\section{Acknowledgements}

Firstly I would like to thank my better half Rhiannon for her unwavering support, encouragement, and patience (not to be underestimated) during the many stressful times throughout the doctorate degree.

To my supervisors Dr. Raoul Van Loon and Prof. Michael Lewis, whose expertise, guidance and continued support throughout my doctorate has been exceptionally valuable. In addition I thank Dr. Dareyoush Rassi for his support and insight. I thank Daniel Watson for his expertise and ideas, particularly for his help in development of the lymphatic system mathematical model formulation.

Last but not least, I would like to think my family for their encouragement and support throughout my education (even though my education has taken an awful lot longer than they expected). 


\section{Contents}

Summary

Declarations $\quad$ ii

Acknowledgements iii

Peer-reviewed papers published during PhD candidature ix

Conference presentations during the PhD Candidature $\quad \mathrm{x}$

Common Symbols and Abbreviations xix

1 Introduction $\quad 1$

1.1 An Overview of Human Pregnancy . . . . . . . . . . . . . . . . 1

1.2 The Cardiovascular System . . . . . . . . . . . . . . . . 3

1.3 The Maternal Cardiovascular System . . . . . . . . . . . . . . . . 4

1.4 Utero-Ovarian Circulation . . . . . . . . . . . . . . . . . . . 6

1.5 Cardiovascular Models of Pregnancy . . . . . . . . . . . . . 8

1.6 The Lymphatic System . . . . . . . . . . . . . . . . . . . . . . 9

1.7 Outline of Thesis and Objectives . . . . . . . . . . . . . 11

2 The Cardiovascular System $\quad 14$

2.1 Introduction . . . . . . . . . . . . . . . . . . . . . 14

2.2 Modelling Human Haemodynamics . . . . . . . . . . . . . . . . . . 14

2.2.1 Deriving the Governing Equations of One-Dimensional Haemodynamics . . . . . . . . . . . . . . . . 16

2.2 .2 1D Formulation Variations . . . . . . . . . . . . . 20

2.2.3 Velocity Profiles . . . . . . . . . . . . . . . . . . . 21

2.2 .4 Constitutive Law . . . . . . . . . . . . . . . . . . . . . . . . . 22

2.2.5 External Pressures . . . . . . . . . . . . . . . 25

2.3 Zero-Dimensional Modelling . . . . . . . . . . . . . . . 26

2.3.1 Vascular Bed Models . . . . . . . . . . . . . . . 27

2.3.2 Heart Model . . . . . . . . . . . . . . . . . . . . . . . . . 29

2.3.3 Valve Model . . . . . . . . . . . . . . . . . . . . 32 
2.4 Mathematical Analysis of the One Dimensional Haemodynamics Equations . . . . . . . . . . . . . . . . . . . 34

2.4.1 Characteristic System . . . . . . . . . . . . . . 34

2.4.2 Wave Intensity Analysis Versus Fourier Analysis . . . . . . . . 37

2.4.3 Wave Reflection and Transmission . . . . . . . . . . . . . . . 41

2.5 Concluding Remarks . . . . . . . . . . . . . . . . . . . . . 43

3 Numerical Schemes $\quad 44$

3.1 1D Schemes . . . . . . . . . . . . . . . . . . . 44

3.2 Enhanced Trapezoidal Rule Method . . . . . . . . . . . . . . . . . 45

3.2 .1 Linearisation . . . . . . . . . . . . . . . . . . 46

3.2 .2 Discretisation . . . . . . . . . . . . . . . 47

3.2.3 Example of a 1D-1D Element Connectivity . . . . . . . . . . . 50

3.2.4 Lumped Models . . . . . . . . . . . . . . . . . . . 52

Vascular Beds . . . . . . . . . . . . . . . . . 53

Heart Model . . . . . . . . . . . . . . . . . . 56

Valve Model . . . . . . . . . . . . . . . . . . . . 58

3.2.5 Vessel to Vessel Interface Conditions . . . . . . . . . . . . . . 60

3.2.6 Physical Interpretation of the Lagrange Multipliers . . . . . . 62

3.2.7 Treatment of Duplicate 1D Vessels or Lumped Elements . . . 64

3.3 Concluding Remarks . . . . . . . . . . . . . . . . . . 66

4 Benchmark Problems and Methodology Testing 67

4.1 Introduction . . . . . . . . . . . . . . . . . . . . . 67

4.2 Benchmarked Problems . . . . . . . . . . . . . . . . . . . 68

4.2.1 Single Pulse in a Long Reflection-Free Vessel . . . . . . . . . . 69

4.2 .2 Common Carotid Artery . . . . . . . . . . . . . . . 71

4.2 .3 Upper Thoracic Aorta . . . . . . . . . . . . . . . . . 71

4.2.4 Aortic Bifurcation . . . . . . . . . . . . . . 75

4.2.5 Arterial Network Containing 37 Vessels . . . . . . . . . . . . . 77

4.2 .6 ADAN56 Arterial Network . . . . . . . . . . . . . . 81

4.3 Numerical Comparisons For Problems With Theoretical Solutions . . 89

4.3.1 Shockwave within a Vessel . . . . . . . . . . . . . . . 91

4.3.2 Aneurysm and Stenosis with Steady Volumetric Flow Rate . . 92 
4.3.3 Discontinuous Material Properties and Geometry . . . . . . . 95

4.3.4 Reflections at Junctions . . . . . . . . . . . . . . . . . . . 101

4.3.5 Single Pulse in bifurcation and unification case. . . . . . . . . 104

4.4 Convergence Behaviour of the ETM Scheme . . . . . . . . . . . . . 108

4.4.1 Mass Conservation in an Arterial Network . . . . . . . . . . 108

4.4 .2 Numerical Convergence Study . . . . . . . . . . . . . . . . . . 110

4.5 Conclusions . . . . . . . . . . . . . . . . . . . . . . 112

5 Investigation of Non-Linearities in a 1D-0D Closed-Loop Cardio$\begin{array}{lr}\text { vascular System } & 113\end{array}$

5.1 Introduction . . . . . . . . . . . . . . . . . . . . . 113

5.2 Methodology . . . . . . . . . . . . . . . . . . . 114

5.2 .1 Isolated Vessel Junctions . . . . . . . . . . . . . . . . . . . 115

5.3 Results . . . . . . . . . . . . . . . . . . . . . 116

5.3.1 Effects on waveforms . . . . . . . . . . . 117

5.3 .2 Error analysis . . . . . . . . . . . . . . . 118

5.3.3 Overview of the main systemic vessels . . . . . . . . . 120

5.3.4 Additional results from the systemic arteries . . . . . . . . . . 122

5.3.5 Additional results from the systemic veins . . . . . . . . . . 124

5.3.6 Results from the pulmonary system . . . . . . . . . . . . 126

5.3.7 Bifurcation Test . . . . . . . . . . . . . . 126

5.3.8 Confluence Test . . . . . . . . . . . . . . . . . . . 128

5.3 .9 Trifurcation Test . . . . . . . . . . . . . . . . . . . 130

5.4 Discussion . . . . . . . . . . . . . . . . . . . . . . . . 132

5.5 Conclusion . . . . . . . . . . . . . . . . . 136

6 Parameter Estimation and Measurement Integration 152

6.1 Model Parameters From Literature . . . . . . . . . . . . . . . . . . . 154

6.1.1 Heart Model . . . . . . . . . . . . . . . . . . 155

6.1.2 Valve Model . . . . . . . . . . . . . . . . . . . . . 155

6.1.3 1D Vascular Networks . . . . . . . . . . . . . . . . . 155

Pulmonary System . . . . . . . . . . . . . 156

Systemic System . . . . . . . . . . . . . . . 156

Coronary Network and Coronary Vascular Bed Model . . . . . 158 
6.1.4 Utero-Ovarian Circulation . . . . . . . . . . . . . . . . 159

6.2 Measured Data . . . . . . . . . . . . . . . . . . . . . . 160

6.3 Initial Parameter Estimation . . . . . . . . . . . . . . . . . . . 162

6.3.1 Total Peripheral Resistance . . . . . . . . . . . . . 163

6.3.2 Estimation of Total Systemic Compliance . . . . . . . . . . . . 164

6.4 Iterative Parameter Estimation . . . . . . . . . . . . . . . . 165

6.4 .1 Blood Volume . . . . . . . . . . . . . . . . 166

6.4.2 Total Peripheral Resistance . . . . . . . . . . . . . . 167

6.4.3 Systemic Arterial Compliance . . . . . . . . . . . . . . . . . 168

6.5 Concluding Remarks . . . . . . . . . . . . . . . . . . . 170

7 Computational Model of Pregnancy $\quad 175$

7.1 Physiological Adaptation . . . . . . . . . . . . . . . . . . . . . . . 175

7.2 The Uterus and The Placenta . . . . . . . . . . . . . . . . . . . . . . 177

7.3 Doppler Studies of Pregnancy . . . . . . . . . . . . . . . . . . . 179

7.4 Computational Modelling Of Pregnancy . . . . . . . . . . . . . . . . 179

7.5 Computational Algorithm . . . . . . . . . . . . . . . . . . 181

7.6 Pulse Wave Velocity and Pulsatility Index . . . . . . . . . . . . . . 182

7.7 Patient Specific Pregnancy Modelling . . . . . . . . . . . . . . . . 185

7.7 .1 Results Overview . . . . . . . . . . . . . . . . 185

7.7.2 Three Participant Comparison . . . . . . . . . . . . . . . 188

7.8 An Idealised Pregnancy Model For All Weeks . . . . . . . . . . . . 196

7.8.1 Simulation Results . . . . . . . . . . . . . . . . . 197

7.8.2 Changes in Vessel Adaptation . . . . . . . . . . . . . . . . . . 201

7.8.3 Sensitivity of Parameters . . . . . . . . . . . . . . . . 203

7.9 Limitations . . . . . . . . . . . . . . . . . . . . 206

7.10 Conclusions . . . . . . . . . . . . . . . . . . 207

8 The Lymphatic System 210

8.1 Introduction . . . . . . . . . . . . . . . . . . . 210

8.1 .1 Lymph . . . . . . . . . . . . . . . . . . 211

8.1 .2 Lymph Vessels . . . . . . . . . . . . . . . . . . . . . 211

8.2 Mathematical Model of Lymphatics . . . . . . . . . . . . . . . . . 214

8.2.1 Modelling Lymph Vessels, Lymphangions . . . . . . . . . . . . 216 
8.2.2 Constitutive Law and Contraction Model . . . . . . . . . . . . 218

8.2 .3 Valve Model . . . . . . . . . . . . . . . . . . . . . . . . . 220

8.2.4 Linearisation and Methodology . . . . . . . . . . . . 221

8.2.5 Example of an Assembled Matrix for a Lymphangion . . . . . 223

8.3 Lymphatic Simulation Results . . . . . . . . . . . . . . . . . . . 224

8.3 .1 Single Lymphangion . . . . . . . . . . . . . . . . . . . 224

8.3.2 Chain of Four Lymphangions . . . . . . . . . . . . . . 224

8.4 Limitations . . . . . . . . . . . . . . . . . . 226

8.5 Conclusions . . . . . . . . . . . . . . . . . . . 227

9 Conclusions $\quad 231$

9.1 Summary of Thesis Achievements . . . . . . . . . . . . . . . . 231

9.2 Additional Achievements . . . . . . . . . . . . . . . . . . . 235

9.3 Main Limitations . . . . . . . . . . . . . . . . . . . . . . . . . . . 235

9.4 Future Work . . . . . . . . . . . . . . . . . . 236

9.4.1 Pregnancy Modelling and Clinical Applications . . . . . . . 236

9.4.2 Other Modelling and Clinical Applications . . . . . . . . . . 237

9.5 Concluding Remarks . . . . . . . . . . . . . . . . . . . . . . 239

$\begin{array}{ll}\text { References } & 241\end{array}$ 


\section{Peer-reviewed papers published dur- ing PhD candidature}

J. Carson, R. Van Loon. An implicit solver for 1D arterial network models. Int $J$ Num Meth Biomed Eng, 33(7), 2017. doi: 10.1002/cnm.2837.

J. Carson, M. Lewis, D. Rassi, R. Van Loon. A data-driven model to study uteroovarian blood flow physiology during pregnancy. Biomech Model Mechanobiol, 2019. doi: 10.1007/s10237-019-01135-3.

I. Sazonov, A.W. Khir, W.S. Hacham, E. Boileau, J.M. Carson, R. Van Loon, C. Ferguson, P. Nithiarasu. A novel method for non-invasively detecting the severity and location of aortic aneurysms. Biomech Model Mechanobiol. 16(4): 1225-1242, 2017. doi: 10.1007/s10237-017-0884-8.

A. Coccarelli, H. M. Hasan, J. Carson, D. Parthimos, P. Nithiarasu. Influence of ageing on human body blood flow and heat transfer: A detailed computational modelling study. Int J Num Meth Biomed Eng, ePub, e3120, 2018. doi: 10.1002/cnm.3120. 


\section{Conference presentations during the PhD Candidature}

J. Carson, M. Lewis, D. Rassi, R. Van Loon. Development of a 1D cardiovascular model during pregnancy. 24th UK Conference of the Association for Computational Mechanics in Engineering, 31 March - 01 April 2016, Cardiff University, Cardiff, $\mathrm{UK}$.

J.M. Carson, M.J. Lewis, D. Rassi, R. Van Loon. Development of a 1D-0D cardiovascular model of pregnancy in humans. 25th UKACM Conference on Computational Mechanics, 12 - 13 April 2017, University of Birmingham, Birmingham, UK.

J. Carson, M. Lewis, D. Rassi, S. Emery, R. Van Loon. Development of a cardiovascular network model during pregnancy in humans. MPEC MEIbioeng 13th 14th September 2017. Sandown Park Racecourse, Esher, Surrey, UK.

J. Carson, S. Pant, P. Nithiarasu. The use of reduced order models to determine fractional flow reserve using non-invasive techniques. MPEC MEIbioeng 13th - 14th September 2017. Sandown Park Racecourse, Esher, Surrey, UK.

J. Carson, M. Lewis, D. Rassi, R. Van Loon. A multi-scale model of the maternal human cardiovascular system during pregnancy. 5th International Conference on Computational and Mathematical Biomedical Engineering, 1012 April 2017, University of Pittsburgh, USA.

J. Carson, S. Pant, C. Roobottom, P. Nithiarasu. Non-invasive assessment of fractional flow reserve using reduced-order models. 6th European Conference on Computational Mechanics (ECCM 6) and the 7th European Conference on Computational Fluid Dynamics (ECFD 7), 11-15 June 2018, Glasgow, UK.

Pg. $\mathrm{x} / 284$ 


\section{List of Figures}

1.1 General overview of the cardiovascular system. Showing the right atrium (RA), right ventricle (RV), left atrium (LA), and the left ventricle (LV); along with the aortic valve (AV), mitral valve (MV), pulmonary valve $(\mathrm{PV})$, and the tricuspid valve $(\mathrm{TV}) \ldots \ldots . . . .5$

1.2 Diagram of the utero-ovarian circulation. Showing an example of a one-sided connection, with the right side representing the arterial circulation and the left side representing the venous circulation . . . . 7

1.3 Vascular bed arrangement for non-pregnant and pregnant humans . . 8

1.4 Example uterine artery waveform examples for non-pregnant, healthy pregnant, and unhealthy pregnant conditions . . . . . . . . . 8

1.5 Overview of fluid leakage from cardiovascular capillaries (red vessel) to interstitia, and fluid uptake by the lymphatic capillaries (green vessel) . . . . . . . . . . . . . . . . . . 11

2.1 Overview of flow in a vessel . . . . . . . . . . . . . . . . . 17

2.2 Comparison of various velocity profiles . . . . . . . . . . . . 22

2.3 Womersley velocity profiles development for different Womersley num-

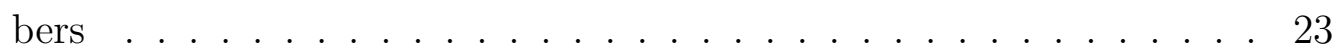

2.4 Comparison of a linear elastic, a non-linear elastic, a non-linear elastic power law, and a collapsible tube model . . . . . . . . . . . . . 24

2.5 Comparison of the four common Windkessel models. Art and Vein are connections to artery/arteries and vein/veins, respectively. . . . . 29

2.6 Fluid storage in a control volume . . . . . . . . . . . . . . . 30

2.7 Elastance curves and volumes of left ventricle and left atria . . . . . . 30

2.8 Interactions of the heart . . . . . . . . . . . . . . . . . . 31

2.9 Heart model (one side) . . . . . . . . . . . . . . . . . 31

2.10 Comparison of left ventricular pressure and aortic root pressure . . . 33 
2.11 Frequency domain analysis, forward (F WI) and backward (B WI) wave intensity, and pressure and velocity waveforms in the ascending aorta and left common carotid artery. The main forward and backward waves are abbreviated as follows: FCW - the forward compression wave, FEW - forward expansion wave, BCW - backward compression wave . . . . . . . . . . . . . . . . . . . . . . . 39

2.12 Overview of reflected and transmitted wave from an incident wave at a bifurcation. . . . . . . . . . . . . . . . . . . . 42

3.1 Generic vascular bed . . . . . . . . . . . . . . . . 53

3.2 Liver vascular bed model . . . . . . . . . . . . . . . . . . . . . . 54

3.3 Coronary vascular bed model . . . . . . . . . . . . . . . . . . . 55

3.4 Heart model (one side) . . . . . . . . . . . . . . . . . . . 56

3.5 Valve model . . . . . . . . . . . . . . . . . . . . . . 59

3.6 An example of the ETM schemes use of Lagrange multipliers at a bifurcation. Arrows indicate assumption of flow direction, hence the parent vessel (reference vessel) is on the left with pressure nodes $P_{1}$ and $P_{2}$. However, this is for indication of the reference vessel only, and is capable of handling flow in any direction. Lagrange multiplier $\lambda_{1}$ connects pressure nodes 2 and 3 , while $\lambda_{2}$ connects pressure nodes 2 and $5 \ldots \ldots \ldots \ldots \ldots \ldots \ldots \ldots$

4.1 Decrease in the pressure wave peak along a vessel length for the single pulse case. The pressure of inviscid case is shown by the black line, while the pressure decrease due to viscous effects is shown by the blue line. The theoretical decrease of pressure for viscous case is given by the red line. . . . . . . . . . . . . . . . . . . . . . . 71

4.2 Pressure and volumetric flow rate waveforms in the common carotid artery test comparing the ETM, DCG, LCG and 3D data. . . . . . . 73

4.3 Pressure and volumetric flow rate waveforms in the upper thoracic aorta test comparing the ETM, DCG, LCG and 3D data. . . . . . . . 73

4.4 Pressure and flow rates for the aortic bifurcation case. A comparison between the ETM, STM and LCG schemes with 3D data. . . . . . . . 77 
4.5 Pressure and flow waveforms in the 37-arterial tree network, comparison between ETM, DCG, LCG, and experimental measurements. . . 81

4.6 Pressure and flow waveforms in the 37-arterial tree network, comparison between ETM, DCG, LCG, and experimental measurements. . . 82

4.7 Pressure and flow waveforms in the 37-arterial tree network, comparison between ETM, DCG, LCG, and experimental measurements. . . 83

4.8 Pressure and flow rate waveforms in the aortic arch I, thoracic aorta III, and abdominal aorta V. Results are shown for the ETM, STM, DCG, and LCG methods. ETMa is the ETM scheme with $\Delta t=1 \mathrm{~ms}$ and $\Delta x=1 \mathrm{~cm}$, ETMb is the ETM scheme with $\Delta t=2 \mathrm{~ms}$ and $\Delta x=2 \mathrm{~cm}$, ETMc is the ETM scheme with $\Delta t=5 \mathrm{~ms}$ and $\Delta x=5 \mathrm{~cm} .85$

4.9 Pressure and flow rate waveforms in right common carotid artery, right renal artery, and right common iliac artery. Results are shown for the ETM, STM, DCG, and LCG methods. ETMa is the ETM scheme with $\Delta t=1 \mathrm{~ms}$ and $\Delta x=1 \mathrm{~cm}, \mathrm{ETMb}$ is the ETM scheme with $\Delta t=2 \mathrm{~ms}$ and $\Delta x=2 \mathrm{~cm}$, ETMc is the ETM scheme with $\Delta t=5 \mathrm{~ms}$ and $\Delta x=5 \mathrm{~cm} . \ldots \ldots \ldots 6$

4.10 Pressure and flow rate waveforms in right internal carotid artery, right radial artery, and right internal iliac artery. Results are shown for the ETM, STM, DCG, and LCG methods. ETMa is the ETM scheme with $\Delta t=1 \mathrm{~ms}$ and $\Delta x=1 \mathrm{~cm}, \mathrm{ETMb}$ is the ETM scheme with $\Delta t=2 \mathrm{~ms}$ and $\Delta x=2 \mathrm{~cm}$, ETMc is the ETM scheme with $\Delta t=5 \mathrm{~ms}$ and $\Delta x=5 \mathrm{~cm}$.

4.11 Pressure and flow rate waveforms in right posterior interosseous, right femoral artery, and right anterior tibial. Results are shown for the ETM, STM, DCG, and LCG methods. ETMa is the ETM scheme with $\Delta t=1 \mathrm{~ms}$ and $\Delta x=1 \mathrm{~cm}$, ETMb is the ETM scheme with $\Delta t=2 \mathrm{~ms}$ and $\Delta x=2 \mathrm{~cm}$, ETMc is the ETM scheme with $\Delta t=5 \mathrm{~ms}$ and $\Delta x=5 \mathrm{~cm} . \quad \ldots \ldots \ldots \ldots \ldots$

4.12 Waveforms for vessels with maximum peak errors in pressure and flow rates for the ETMc, in comparison with the ETMa. . . . . . . . . . . 90

4.13 Pressure and flow rate waveforms for shock test with and without artificial diffusion at different time instances (seconds). . . . . . . . 93

4.14 Waveforms for the aneurysm case. . . . . . . . . . . . . . . 95 
4.15 Waveforms for the stenosis case. . . . . . . . . . . . . . . . 96

4.16 Waveforms for a discontinuous area decrease. . . . . . . . . . . . . . 100

4.17 Waveforms for a discontinuous area increase. . . . . . . . . . . . . . . 101

4.18 Waveforms for a discontinuous decrease in material properties. . . . . 102

4.19 Waveforms for a discontinuous increase in material properties. . . . . 103

4.20 Reflections at a single bifurcation . . . . . . . . . . . . . . 105

4.21 Configuration for bifurcation and unification test . . . . . . . . 106

4.22 A comparison of outflow and inflow at junctions with Lagrange multipliers. . . . . . . . . . . . . . . . . 107

4.23 Inflow flow rate for mass conservation tests . . . . . . . . . . . . . . . 109

4.24 Numerical convergence curves of the ETM scheme for the common carotid problem. Investigating the choice of discretisation of the nonlinear convection term, and the number of Newton iterations performed.111

5.1 Comparison of NL-TP, NL-SP, L-TP, L-SP, L0-SP formulations for various vessels in the systemic arterial system . . . . . . . . . . 119

5.2 Comparison of NL-TP, NL-SP, L-TP, L-SP, LO-SP formulations for various vessels in the systemic venous system . . . . . . . . . . 120

5.3 RMSE (relative to the NL-TP formulation) of pressure, flow rate, and wave intensities in the systemic arterial and venous systems (including hepatic veins, cerebral and coronary circulation), and pulmonary arterial and venous systems . . . . . . . . . . . . 137

5.4 Percentage errors (relative to the NL-TP formulation) of peak pressures, flow rates, and wave intensities in the systemic arterial and venous systems (including hepatic veins, cerebral and coronary circulation), and pulmonary arterial and venous systems . . . . . . . 138

5.5 Deviation of formulations for the systemic arterial system (cerebral and coronary vessels omitted for clarity), compared to NL-TP form . 139

5.6 Deviation of formulations in the systemic venous system (cerebral and coronary vessels omitted for clarity), compared to NL-TP form. As shown in the figure the veins on the left side (arms and legs) are deep veins, while on the right side are superficial veins . . . . . . . . . 140 
5.7 Deviation of L-SP for the systemic arterial and venous systems (cerebral and coronary vessels omitted for clarity), compared to NL-TP form. For the veins, the left side (arms and legs) are deep veins, while on the right side are superficial veins . . . . . . . . . . . . . . 141

5.8 Comparison of NL-TP, NL-SP, L-TP, L-SP, L0-SP formulations for various vessels in the arterial system . . . . . . . . . . . . . . . . 142

5.9 Comparison of NL-TP, NL-SP, L-TP, L-SP, L0-SP formulations for various vessels in the arterial system . . . . . . . . . . . . . 143

5.10 Comparison of NL-TP, NL-SP, L-TP, L-SP, L0-SP formulations for various vessels in the venous system . . . . . . . . . . . . . . . . . 144

5.11 Comparison of NL-TP, NL-SP, L-TP, L-SP, L0-SP formulations for various vessels in the venous system . . . . . . . . . . . . . 145

5.12 Comparison of NL-TP, NL-SP, L-TP, L-SP, L0-SP formulations for various vessels in the pulmonary system . . . . . . . . . . . . 146

5.13 Comparison of NL-TP, NL-SP, L-TP, L-SP, L0-SP formulations for various vessels in the pulmonary system . . . . . . . . . . . . . 147

5.14 Configuration of the bifurcation case where $A_{P}$ is the area of the parent vessel, $A_{d 1}$ and $A_{d 2}$ are the areas of the first and second daughter vessels, respectively . . . . . . . . . . . . . . . . . . . . . . . 148

5.15 Configuration of the trifurcation case where $A_{P}$ is the area of the parent vessel, $A_{d 1}, A_{d 2}$, and $A_{d 3}$ are the areas of the first, second, and third daughter vessels, respectively . . . . . . . . . . . . . . 148

5.16 Percentage error of static pressure compared with total pressure for bifurcation and confluence cases for parent (Par) and daughter (Dau) vessels . . . . . . . . . . . . . . . . . . . . . . . . . . . . . . . . . 149

5.17 Percentage error of static pressure compared with total pressure for trifurcation case for parent (Par) and daughter (Dau) vessels . . . . . 150

5.18 Percentage error of static pressure compared with total pressure for the trifurcation case for parent (Par) and daughter (Dau) vessels . . . 151

6.1 1D pulmonary artery and venous vascular networks. . . . . . . . . 156

6.2 1D systemic system network . . . . . . . . . . . . . . 157

$6.31 \mathrm{D}$ cerebral artery and venous vascular networks. . . . . . . . . . 158 
6.4 1D coronary artery and venous vascular networks, and hepatic portal system. . . . . . . . . . . . . . . . . . . . . . . . 159

6.5 Utero-Ovarian System . . . . . . . . . . . . . . . . 160

6.6 Flowchart of the implementation of the initial and iterative parameter estimation techniques . . . . . . . . . . . . . . . 166

7.1 Comparison of diagram of the non-pregnant and pregnant human uterus, including the development and vasculature of the placenta. . . 178

7.2 Typical digitised Doppler waveforms for a non-pregnant woman and for the first trimester . . . . . . . . . . . . . . . . . . . . 180

7.3 Estimated increase of problem parameters. . . . . . . . . . . . . 181

7.4 The pulsatility index and percentage of total cardiac output through the uterine arteries and utero-ovarian communicating arteries; and pulse wave velocity using Aortic PWV from the model and an estimate of brachial-ankle PWV, for all patients. Circles represent patient simulated values, and the dashed red line is the $95 \%$ confidence interval of the simulated data. . . . . . . . . . . . . . 187

7.5 Right uterine artery flow rate waveforms for three patients over the course of pregnancy, and post-partum, compared with in-vivo waveforms from literature. The black line is for participant one, the blue line is participant two, and the red line is participant three. Waveforms are normalised in time for comparison purposes. Digitised waveforms are scaled by physiologically realistic areas, as they are originally velocity waveforms for Doppler studies. . . . . . . . . . . . 192

7.6 Right uterine vein flow rate waveforms for three patients over the course of pregnancy, and post-partum. The black line is for participant one, the blue line is participant two, and the red line is participant three. . . . . . . . . . . . . . . . . . . . . 193

7.7 Ascending aorta flow rate waveforms for three patients over the course of pregnancy, and post-partum. The black line is for participant one, the blue line is participant two, and the red line is participant three. . 194 
7.8 Left brachial artery pressure waveforms for three patients over the course of pregnancy, and post-partum. The black line is for participant one, the blue line is participant two, and the red line is participant three. . . . . . . . . . . . . . . . . . 195

7.9 Comparison of model outputs with idealised data. The aimed parameters which the parameter estimation technique converges towards are shown by the red dashed line, where applicable. . . . . . . . . . . . 198

7.10 Comparison of model outputs with idealised data, and convergence behaviour. . . . . . . . . . . . . . . . . . . 199

7.11 Number of cardiac cycles required to achieve convergence to the idealised data input and periodic convergence . . . . . . . . . . . . 200

7.12 Volumetric flow rates in vessels toward the uterus for the following cases: the utero-ovarian communicating artery has the same area as the ascending uterine - $\mathrm{A}$, has half the original area - $\mathrm{A} / 2$, and has a

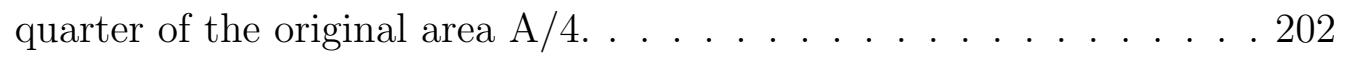

7.13 Comparison of flow rate waveforms in vessels carrying blood to the uterus for symmetric (case 1 and 3) and asymmetric (case 2 and 4)adaptation . . . . . . . . . . . . . . . . . . . 203

7.14 Mean absolute percentage error in flow rates through the utero-ovarian communicating and uterine arteries, and pressures in the brachial artery. Subscripts $p$ and $m$ in the figures indicate whether the parameter is perturbed by an increase, or decrease respectively. . . . . . 205

7.15 Comparison of flow rate waveforms in the utero-ovarian communicating arteries and uterine arteries, and pressure waveforms in the brachial artery . . . . . . . . . . . . . . . 206

8.1 Overview of lymphatic system in relation to the cardiovascular system 212

8.2 A lymph vessel containing a chain of 5 lymphangions . . . . . . . . . 213

8.3 Lumped representation of a lymphangion . . . . . . . . . . . . . 217

8.4 Comparison of passive constitutive relationships and contraction functions . . . . . . . . . . . . . . . . . . . . . 220

8.5 Lumped representation of lymphangion chain . . . . . . . . . . . 221

8.6 Comparison of lymph valve models. Resistance and pressure are normalised by maximum resistance and maximum pressure respectively . 222 
8.7 Results of a single lymphangion test comparing the valve model of Bertram [29], the proposed valve model modified from [226], and the new contraction model. . . . . . . . . . . . . . . . . . . . 229

8.8 Results for a chain of four lymphangions _ . . . . . . . . . 230 


\section{Common Symbols and Abbreviations}

\begin{tabular}{|c|c|c|c|}
\hline $\begin{array}{l}\text { Parameter } \\
\text { Name }\end{array}$ & Symbol & Value & Units \\
\hline viscosity & $\mu$ & 0.035 & poise \\
\hline density & $\rho$ & 1.06 & $\mathrm{~g} / \mathrm{cm}^{3}$ \\
\hline $\begin{array}{l}\text { cross- } \\
\text { sectional } \\
\text { area }\end{array}$ & $A$ & -- & $\mathrm{cm}^{2}$ \\
\hline pressure & $P$ & -- & $\mathrm{mmHg}$ \\
\hline flow rate & $Q$ & -- & $\mathrm{ml} / \mathrm{s}$ \\
\hline $\begin{array}{l}\text { temporal co- } \\
\text { ordinate }\end{array}$ & $t$ & -- & $\mathrm{s}$ \\
\hline $\begin{array}{l}\text { axial coordi- } \\
\text { nate }\end{array}$ & $x$ & -- & $\mathrm{cm}$ \\
\hline $\begin{array}{l}\text { viscous fric- } \\
\text { tion constant }\end{array}$ & $\xi$ & -22 & $\mathrm{~cm}^{3} / \mathrm{g}$ \\
\hline $\begin{array}{l}\text { reference } \\
\text { pressure }\end{array}$ & $P_{0}$ & -- & $\mathrm{mmHg}$ \\
\hline $\begin{array}{l}\text { external pres- } \\
\text { sure }\end{array}$ & $P_{e x t}$ & 0 & $\mathrm{mmHg}$ \\
\hline $\begin{array}{l}\text { reference } \\
\text { cross- } \\
\text { sectional } \\
\text { area }\end{array}$ & $A_{0}$ & -- & $\mathrm{cm}^{2}$ \\
\hline $\begin{array}{l}\text { wall viscosity } \\
\text { coefficient }\end{array}$ & $\Gamma$ & -- & $\mathrm{g} / \mathrm{s}$ \\
\hline $\begin{array}{l}\text { collapse pres- } \\
\text { sure }\end{array}$ & $P_{\text {collapse }}$ & -10 & $\mathrm{mmHg}$ \\
\hline $\begin{array}{l}\text { reference } \\
\text { wave speed }\end{array}$ & $c_{0}$ & -- & $\mathrm{cm} / \mathrm{s}$ \\
\hline resistance & $R$ & -- & $\mathrm{mmHg} \min / \mathrm{l}$ \\
\hline compliance & $C$ & -- & $\mathrm{ml} / \mathrm{mmHg}$ \\
\hline inductance & $L$ & -- & $\mathrm{mmHg} \mathrm{s}^{2} / \mathrm{ml}$ \\
\hline
\end{tabular}

Table 1: Glossary of terms for blood flow equations. Includes values where applicable. 


\section{Chapter 1}

\section{Introduction}

In this thesis a closed-loop 1D-0D computational model of the human cardiovascular system during pregnancy is developed. The model utilises participant data from in-vivo measurements, using an automated parameter estimation technique. This chapter provides an overview of: human pregnancy, including physiological adaptation and the development of pathologies; the role and function of the cardiovascular system; the adaptation of the cardiovascular system during pregnancy; and the role of the lymphatic system. At the end of this chapter the outline and objectives of this thesis are presented.

\subsection{An Overview of Human Pregnancy}

Approximately five days after fertilisation of the egg, the collection of cells begins to organise into a structure called a blastocyte before implantation (adherence to the uterine wall). The blastocyte structure has three main sections: an inner cell mass, referred to as the embryoblast; a blastocoel, which is a fluid-filled cavity; and trophoblasts, which are located on the outer wall of the blastocyte. The blastocyte embeds itself into the innermost layer of the uterus (endometrium), and develops to become the embryo, and then eventually the foetus.

Pregnancy causes many significant changes to the physiology of the female human, which includes mechanical and chemical adaptation. The following are some of the changes that occur in the blood:

- an increase in plasma volume by up to $50 \%$ and a smaller increase in red blood cell mass creates a decrease in haemoglobin concentration [320];

- a small decrease in platelet count, though normally still within a healthy range [320];

- changes in blood coagulation leads to an increased risk of blood clotting and venous thrombosis [320]. 
Additional adaptations of the blood and cardiovascular system are described in section 1.3.

The respiratory system is also affected by pregnancy. There is an increased demand for oxygen during pregnancy, which is primarily caused by a $15 \%$ increase in the rate of metabolism and a $20 \%$ increase in oxygen consumption [320]. There are changes in the renal vasculature, anatomy, and function. These include the vasodilation of renal blood vessels, increased renal plasma flow, and an increase in protein excretion.

With all the adaptation that needs to occur for a healthy pregnancy, there are also many pathologies that can develop. Many of these pathologies are caused by a mixture of poor cardiovascular adaptation and chemical imbalances. Pregnant woman are at an increased risk of developing: depression and anxiety disorders [14] due to the chemical and hormonal changes that occur; peripheral oedema, due to the lymphatic system being unable to drain the increased amount of fluid that gathers within the interstitia; and pulmonary oedema, due to a decrease in the colloid osmotic pressure by $10-15 \%$ [320]. There are also several serious cardiovascular related pathologies which may develop during pregnancy and can impact foetal development, such as pre-eclampsia, placental insufficiency, hypertension, and hypotension, which are discussed in section 1.3.

Due to the interaction of the cardiovascular and lymphatic systems, it would be advantageous to create a coupled cardiovascular and lymphatic network model. This could lead to a greater understanding of how several pathologies develop, and potentially aid clinicians in diagnosis and treating these pathologies. Thus in this thesis a framework is developed for cardiovascular and lymphatic system models. Cardiovascular models are quite well-developed and are currently being used to answer clinical questions. However, the lymphatic system is still poorly understood and currently no full body lymphatic vessel network has been developed, nor is there enough data from literature to create one. Therefore no coupling between the lymphatic and cardiovascular system is performed at this stage in the development of the framework. 


\subsection{The Cardiovascular System}

The human circulatory system is comprised of the cardiovascular system and the lymphatic system, with the former being investigated and understood much more thoroughly. From an outside perspective, the cardiovascular system is comprised of the heart, blood vessels, and approximately 5 litres of blood. However, it serves many important roles which includes: the transport of oxygen, carbon dioxide and other waste products to and from body cells; transport of hormones and blood cells; is a critical component in regulating body temperature and $\mathrm{pH}$ levels; and aids the healing process from injuries contains cells to fight disease causing pathogens.

The cardiovascular system is a closed double-loop system comprised of the systemic loop and the pulmonary loop. In the systemic circulation oxygenated blood is pumped into the aorta from the left ventricle of the heart. The blood transports oxygen and nutrients to organs, tissues, and body cells, before returning de-oxygenated blood and carbon dioxide to the right atria of the heart. The pulmonary loop brings the de-oxygenated, carbon dioxide rich blood from the right ventricle of the heart to the lungs where gas exchange takes place. The function of the lungs removes carbon dioxide and other waste products from the body and binds oxygen to the haemoglobin protein, then returns the oxygenated blood to the left atria of the heart.

Both systemic and pulmonary system loops have similar structures and processes, although the systemic system has more auto-regulation mechanisms. The arterial systems are comprised from arteries transporting blood to the micro-circulation. The micro-circulation can be separated into three main components: the arterioles, which are small muscular arteries that branch from the larger arteries, and can contract/relax to change downstream resistance and resistance distribution; the capillaries branch from the arterioles and have thin walls which aids in their function for nutrient exchange; the capillaries confluence into the venules, which are small veins with smooth muscle present in their walls allowing vasoconstriction and vasodilation to take place. Finally the venules join to become larger veins which bring blood back to a heart atria. Figure 1.1 shows the general overview of the cardiovascular circulation.

The systemic arterial system transports nutrients and minerals to the majority 
of organs in the body which includes the brain via the cerebral arteries, the kidney via the renal arteries, the liver via the hepatic artery and hepatic portal vein, the heart via the coronary arteries, the large and small intestines via the inferior and superior mesenteric arteries, and the pancreas, spleen, and stomach via the celiac artery. The circulation of the liver is both interesting and unusual as it not only receives oxygen-rich blood from the hepatic artery, but also receives low oxygenated, nutrient-rich blood from the hepatic portal vein. The hepatic portal vein receives blood from the spleen, and both large and small intestines which have absorbed nutrients.

There are many auto-regulation mechanisms present in the cardiovascular system, particularly in the systemic circulation. These mechanisms aid in controlling/maintaining blood pressures and/or blood flow rates. There are baroreceptors located in the carotid sinus and aortic arch. The receptors are pressure sensitive and/or stretch sensitive nerve endings that continuously sends signals to the automatic nervous system, the brain uses this information to regulate blood pressure and flow rates by adapting the heart rate and heart contractility, vascular compliances, and vascular resistances.

There are many varieties of cardiovascular disease (CVD) ranging from problems with the heart such as cardiac arrhythmia (irregular heart beats), blood vessel stenosis (narrowing) or aneurysms, hypertension (high blood pressure), and hypotension (low blood pressure). With the many types of pathologies effecting the cardiovascular system, cardiovascular disease is responsible for the majority of deaths worldwide with $31 \%$ of all global deaths attributed to CVDs according to the world health organisation [247].

\subsection{The Maternal Cardiovascular System}

During a healthy human pregnancy the cardiovascular system undergoes significant adaptation; with an increase in cardiac output by $30-50 \%[209,320]$, which is due to an increase in both stroke volume and heart rate; up to a $50 \%$ increase in blood volume [161]; significant increases to vascular compliance [26, 87, 150, 218]; and significant decreases in total peripheral resistance [150]. With these physiological changes, the mean arterial pressure remains relatively stable during a healthy preg-

Pg. 4 / 284 


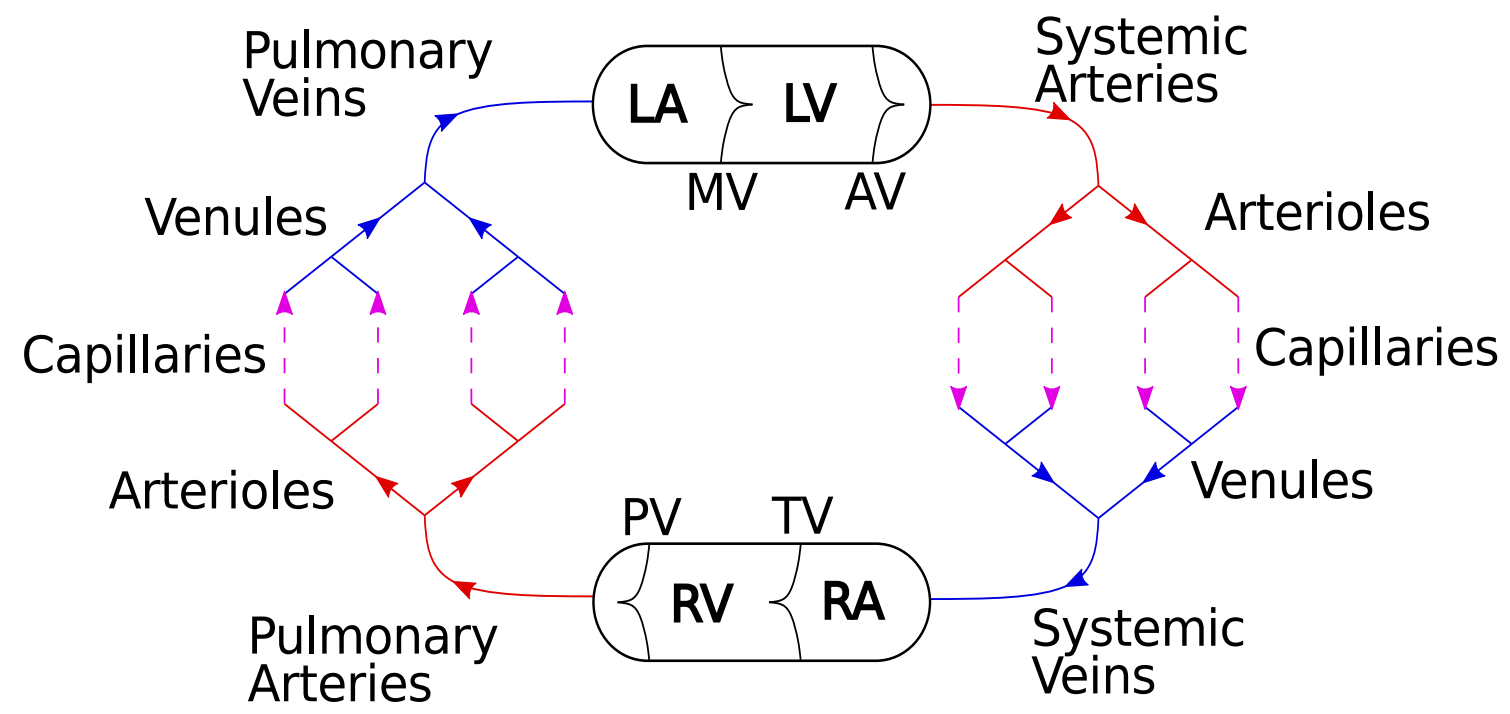

Figure 1.1: General overview of the cardiovascular system. Showing the right atrium (RA), right ventricle (RV), left atrium (LA), and the left ventricle (LV); along with the aortic valve $(\mathrm{AV})$, mitral valve $(\mathrm{MV})$, pulmonary valve $(\mathrm{PV})$, and the tricuspid valve $(\mathrm{TV})$.

nancy, often decreasing slightly for the first to second trimesters and then increasing towards term [150]. If cardiovascular adaptation does not occur sufficiently, a number of pathologies may result. For example, an insufficient decrease in peripheral resistance relative to the increase in cardiac output will result in high blood pressure (hypertension). While an insufficient increase in blood volume relative to the amount of vascular remodelling could result in low blood pressure (hypotension). More serious pathologies such as placental insufficiency and pre-eclampsia are associated with hypertension, often caused by significantly elevated resistance of the placenta/uterine system; additionally in pre-eclampsia, elevated levels of protein are present in urine (proteinuria) [343, 263, 20, 131], while placental insufficiency can lead to reduced foetal growth $[127,232,181]$.

The maternal system is required to undergo a number of significant physiological adaptations, including the remodelling of various components in the cardiovascular system. There is significant heart remodelling [150], with an increase in wall thickness and left ventricular mass [145]; there is also significant remodelling of the vascular network, such as increased vessel area, increased vessel compliance, and the creation and development of a new specialised organ to facilitate nutrient exchange between maternal and foetal systems called the placenta. The utero-ovarian system 
undergoes the most significant changes $[53,66,200]$. The spiral arteries, which are the vessels that deliver blood to the uterine micro-circulation and placenta, undergo the most drastic change, with a complete change in their micro-structure, whereby their endothelial and smooth muscle cells are replaced by trophoblast cells from the foetus. These trophoblast cells originate in the foetus, and propagate through the maternal uterine vessels and tissues, beginning with entering the walls of the spiral arteries before migrating through the entire inner third of the myometrium (which is the outer layer of the uterus) [53]. Remodelling of the uterine vessels occurs by increasing the unstressed vessel lumen diameter, and also significantly increases the vessel wall compliance, which lowers resistance of the entire uterine region, and encourages and increase of blood flow to the placenta [270, 367, 153].

The most significant regional increases of blood flow are to the uterus, ovaries, kidneys, and skin. The uterus/placenta receives the largest increase of blood flow, with significant increases to flow in the uterine artery, which supplies the lower and mid portions of the uterus, being observed [98, 137]. The utero-ovarian communicating artery, which supplies the fundus of the uterus is also expected to increase its blood flow rate significantly, although direct measurements of this flow have so far been unsuccessful due to the complex flow behaviour in this region [262, 47]. However, it has been mentioned that if the utero-ovarian communicating artery reaches the same diameter as the ascending uterine artery, then the communicating artery is capable of supplying the uterus with the majority of its blood supply needs [52].

\subsection{Utero-Ovarian Circulation}

The utero-ovarian circulation contains a complex arrangement of blood vessels. Although there are numerous anatomical variations possible; the most common anatomical configuration in the systemic arteries contains a loop which is shown in figure 1.2, whereby the ovarian artery anastomoses with the uterine artery. This creates complex flow behaviour in the uterine region [52, 47, 96], as blood can travel from the uterus via the ovarian artery, which branches from either the descending aorta or renal artery, and also reach the uterus via the left and right uterine arteries, which is one of the branches from the internal iliac artery. In addition, the placenta and uterine vascular beds are in parallel, significantly lowering the overall resistance 


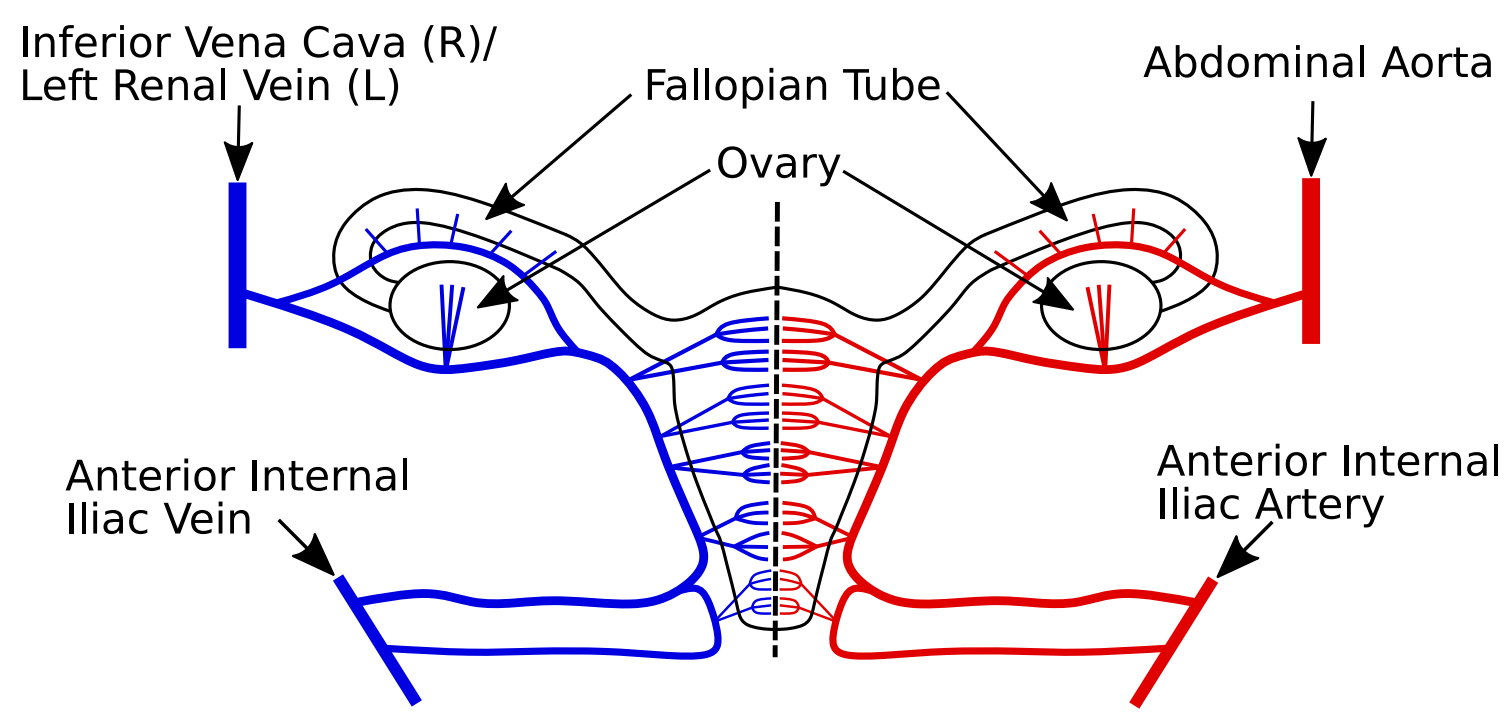

Figure 1.2: Diagram of the utero-ovarian circulation. Showing an example of a onesided connection, with the right side representing the arterial circulation and the left side representing the venous circulation

of the uterine region during healthy pregnancy. The vascular bed configuration before and after the development of the placenta are shown in figure 1.3.

The uterine artery generally supplies the largest percentage of blood to the uterus, although for a non-pregnant woman each uterine artery typically receives less than $0.5 \%$ of cardiac output [52]. There are numerous other named (and unnamed) vessels which may also carry blood to the uterus, such as the lumbar arteries and vaginal arteries. As in the majority of the cardiovascular system, the systemic veins and systemic arteries are essentially symmetric [52].

During pregnancy the utero-ovarian circulation undergoes considerable adaptation, including the development of the placenta, structural changes to many vessels, and the enlargement of the uterus itself. The shape and characteristics of the uterine artery velocity waveform have been studied extensively via Doppler ultrasound, with examples of uterine artery waveform shapes shown in 1.4. The presence of a notch in early diastole in the uterine artery waveform has been linked to several pathologies, and has been investigated as an indicator of increased risk to a patient developing a pathology later in pregnancy $[98,137]$. The notch in the waveform is typically seen in non-pregnant individuals (Figure 1.4a), however during a healthy pregnancy the notch in the waveform is dampened out as pregnancy progresses, which is a direct 


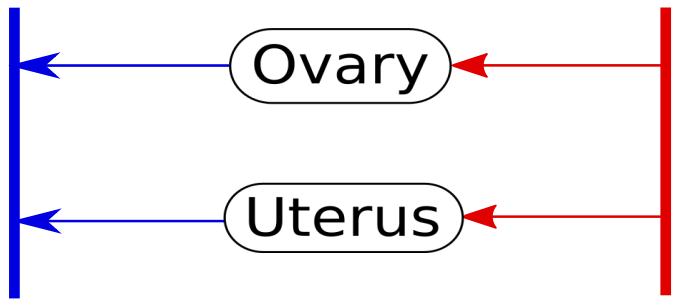

(a) Diagram of vascular bed configuration in the non-pregnant uterus.

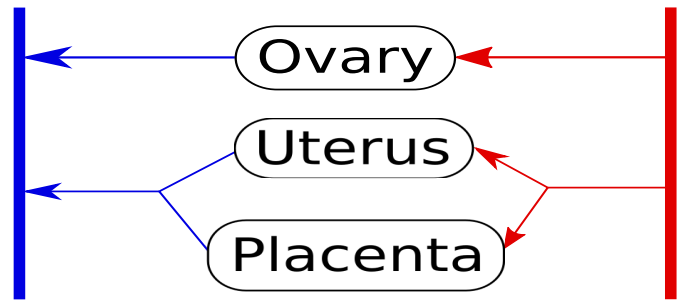

(b) Diagram of vascular bed configuration in the pregnant uterus.

Figure 1.3: Vascular bed arrangement for non-pregnant and pregnant humans

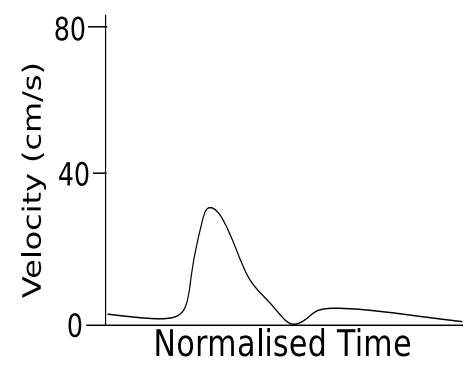

(a) uterine artery waveform non-pregnant.

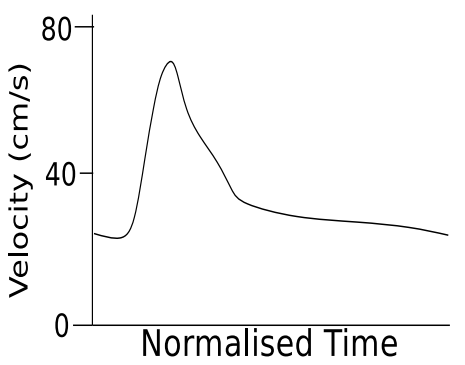

(b) healthy uterine artery waveform mid pregnancy.

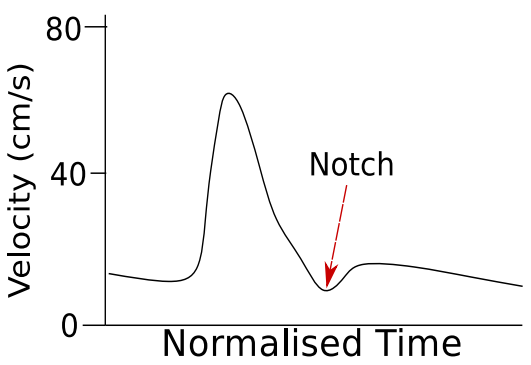

(c) unhealthy uterine artery waveform mid pregnancy.

Figure 1.4: Example uterine artery waveform examples for non-pregnant, healthy pregnant, and unhealthy pregnant conditions

result of the increased vessel compliance, and typically the notch should be absent in most patients by the week 20-22 (middle of second trimester), which is shown in Figure 1.4b. The presence of the notch in the uterine artery waveform (Figure 1.4c) has been linked to the development of pre-eclampsia in later pregnancy, and to poor foetal growth [109]. Flow rates in the uterine arteries are routinely measured to ensure the foetus is receiving enough blood for growth, however, flow rates from the utero-ovarian communicating artery are currently unknown and attempts to measure this have currently been unsuccessful $[262,47]$ due to the complex anatomy and flow behaviour in the pelvic region.

\subsection{Cardiovascular Models of Pregnancy}

Current models of pregnancy mainly exclude the maternal system; instead focusing on the foetal cardiovascular system [266, 130, 227], and flow in the umbilical cord 
$[273,311]$. Although recently, a lumped parameter model of the maternal system was proposed [89]. However, it is well known that lumped parameter models are unable to account for wave-propagation phenomena, and therefore would have a limited range of applicability, as the clinical detection of many pathologies require waveform measurements and estimations of arterial stiffness, which are dependant on wave-propagation and wave-reflection phenomena. Moreover, blood vessels in the utero-ovarian system form a loop, such that the uterus can be supplied with blood from the ovarian arteries (which originate from the descending aorta), and the uterine arteries (which originate from the internal iliac arteries). Thus, there is no current model of the maternal circulation which can account for various wavepropagation phenomena, and that considers the various pathways of blood supply to the uterus, from the heart.

\subsection{The Lymphatic System}

The primary role of the lymphatic system is to drain fluid from body tissues and transport the fluid, now called lymph, back to the systemic venous system. The lymphatic system also has an important function in the immune system, being one of the first lines of defence, and recently has been shown to play a greater role in fighting disease than first thought [280]. Hydrostatic pressure from the arterial side of the capillaries causes fluid to leak into the interstitia, becoming interstitial fluid. Colloid osmotic pressure causes the majority of this interstitial fluid to be reabsorbed into the cardiovascular system via the venous side of the blood capillaries, while the remaining fluid is absorbed and carried by the lymphatic system. There is a wide range of estimates for the amount of blood which is re-absorbed, with 1 to 12 litres per day being claimed [308, 305].

When there is insufficient uptake of fluid from the interstitia to the lymphatic system, the fluid will gather in the interstitia and body tissues, causing swelling that is called oedema. Oedema can occur in many location in the body, but the most common type is peripheral oedema, which occurs in the feet, ankles, legs, and hands. There are many causes of peripheral oedema, such as hypertension, where high blood pressure forces more fluid from the capillary blood vessels and into the interstitia; congestive heart failure, where the heart is unable to pump sufficient blood around 
the body; trauma, where a region of tissue has been damaged; and renal failure, where the kidneys fail the regulate fluid volume in the body correctly. A common cause of oedema is from the damage or removal of lymph nodes as a result of cancer treatment. Edema which is caused by the damage (or removal) of lymph vessels or nodes is commonly referred to as lymphedema. This type of pathology can arise in cancer patients whose lymph nodes have been removed or damaged by surgery or radiation therapy. Currently there exists no method of predicting whether a patient will suffer from edema after the removal of these lymph nodes [168].

The lymphatics system has not been investigated as thoroughly as the cardiovascular system, and many aspects and functions of the lymphatic system are not fully understood [79, 280]. Due to the small size of lymph vessels a full description of the lymphatic vessel network is currently impossible as current in-vivo imaging techniques are insufficient at capturing this small scale. The lymphatic system is comprised of: initial lymphatics, which are thin-walled capillaries that absorb fluid from the interstitia with valve like structures in their wall [44]; pre-collecting and collecting lymphatics, which contain one-way valves and smooth muscle in the vessel walls which contract; there are also lymph ducts and trunks which are the largest lymph vessels. The lymph ducts drain directly into the venous system near one of the subclavian veins. There are various organs that are considered part of the lymphatic system including: lymph nodes, bone marrow, thymus gland, tonsils, spleen, and Peyer's patches in the ileum. Figure 1.5 shows an example of a lymphatic capillary vessel and its valve like structure, which allows fluid uptake and prevents fluid leakage back into the interstitia.

During pregnancy significant lymphangiogenesis (creation of lymphatic vessels) occurs in the region near the placenta [282]. This may be an indication that the lymphatic system plays an important role at the maternal-foetal interface, however this function is currently not known. The lymphatic system also needs to increase its workload during pregnancy as there is an increased amount of blood plasma that leaks into the interstitia. This can cause peripheral oedema, which occurs at some stage in $80 \%$ of pregnancies [94]. Due to the significant increase in plasma volume, pregnant individuals are also at a greater risk of developing other types of oedema, such as pulmonary oedema, where the amount of fluid that leaks into the interstitia around the lungs exceeds the drainage capacity of the lymphatic vessels in the region. 


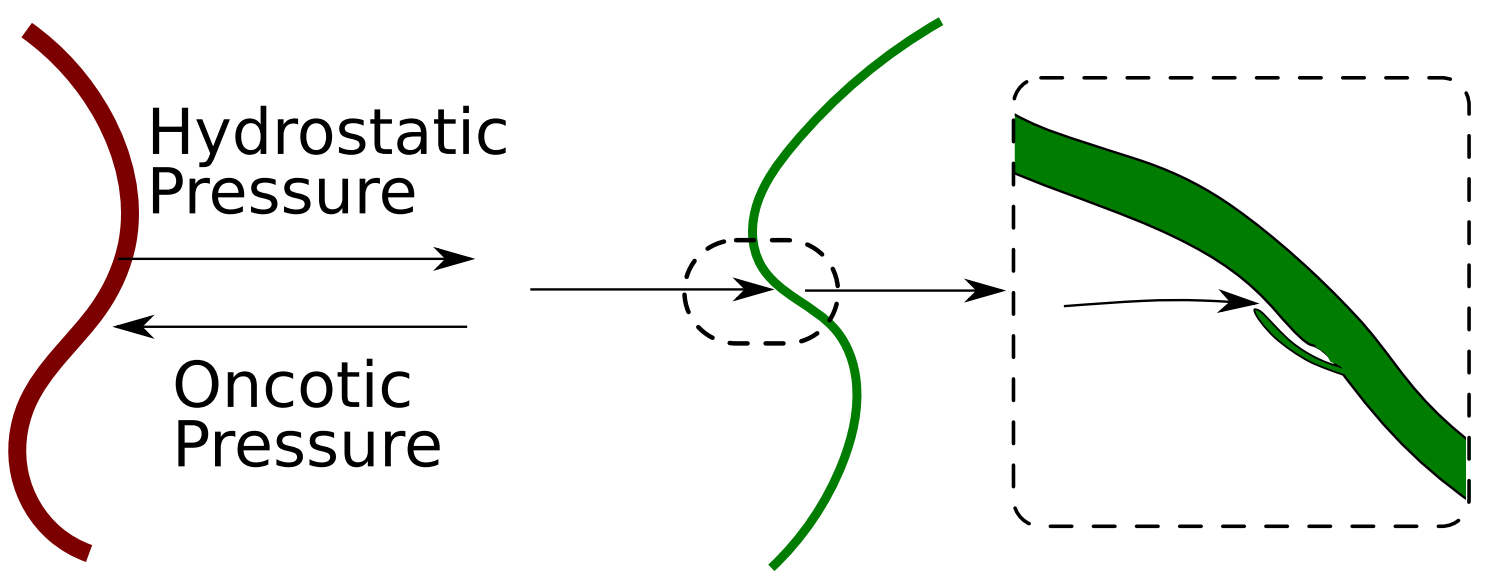

Figure 1.5: Overview of fluid leakage from cardiovascular capillaries (red vessel) to interstitia, and fluid uptake by the lymphatic capillaries (green vessel)

A coupled cardiovascular and lymphatic modelling approach could lead to improved diagnosis and treatment of edema, whether in aiding a clinicians decision making when removing lymph nodes in cancer treatment, or to help understand why oedema occurs in the majority of pregnancies. However, modelling of the lymphatic system is still in its infancy and many improvements are required before clinically relevant lymphatic models can be developed.

\subsection{Outline of Thesis and Objectives}

The aim of this thesis is to develop a computer modelling framework of the cardiovascular and lymphatic systems during pregnancy, that is capable of integrating patient measurement data to aid parameter estimation techniques.

Chapter 2 In chapter 2 background information about the cardiovascular system is presented. The chapter begins by giving an overview of literature in cardiovascular modelling. The derivation of the one-dimensional blood flow equations is described, along with the various components used to construct a closed-loop 1D-0D model. The end of the chapter introduces the concepts used to analyse the model through mathematical means, including: the separation of waves into forward and backward components, wave intensity analysis, frequency domain analysis, and the basis of transmission line theory, which comes from the linearisation of the one-dimensional 
blood flow equations.

Chapter 3 This chapter begins by giving background information on the numerical schemes which are typically used to solve the 1D-0D haemodynamic equations. The Enhanced Trapezoidal Rule Method (ETM), which is the numerical scheme used in this thesis, is presented in detail: including the linearisation of equations for all modelling components; the construction of elemental matrices; and the various coupling procedures used.

Chapter 4 In this chapter the ETM scheme is rigorously tested through a series of carefully chosen problems. The scheme is tested on published benchmark problems, and cases with analytical solutions, which includes shock-wave problems, aneurysm, and stenosis problems. The chapter finishes with a convergence study.

Chapter 5 In this chapter further numerical simulations are performed to investigate the effects of non-linearities in the system of equations. This includes a comparison of the non-linear set of equations, the linearised set of equations, and the lumped model equations, in a closed-loop 1D-0D cardiovascular network model. In addition the impact of the choice of boundary conditions at vessel junctions is investigated, with a comparison of using either continuity of static pressure, or continuity of total pressure.

Chapter 6 In this chapter a framework for estimating model parameters from invivo participant measurements is presented. The chapter begins by describing the various parameters which are estimated from literature. Finally the initial estimation of parameters and an automated parameter adaptation technique are described.

Chapter 7 In this chapter the closed-loop 1D-0D model of the maternal cardiovascular system during pregnancy is presented. The chapter begins by providing background information on the adaptation of the maternal cardiovascular system. The model is then implemented for patient-specific measurement data in the three trimesters and post-partum. Three of the patient waveforms are then compared with digitised Doppler waveforms for pregnant and non-pregnant conditions. The model is extended to an idealised pregnancy, which is performed for all gestational weeks. The simulations results are validated through comparisons with several clin- 
ical indices such as pulse wave velocity, and the pulsatility index. The chapter ends with a sensitivity analysis, showing the dependence on several model parameters such as: cardiac parameters, initial conditions, and haemodynamic quantities.

Chapter 8 In this chapter a computational model of the lymphatic system is presented. The chapter presents a new numerical scheme for the collecting lymphatic vessels, which allows the frequency of lymphangion contraction to be determined automatically based on the pressure in the lymphangion.

Chapter 9 The final chapter provides a discussion on the strengths and limitations of the modelling frameworks. Finally an overview of the main findings of the thesis is given, and possible directions and applications for future work are presented. 


\section{Chapter 2}

\section{The Cardiovascular System}

\section{$2.1 \quad$ Introduction}

In this chapter, an introductory description of human cardiovascular physiology is presented, along with the theory used to construct the modelling components of the human cardiovascular system. Derivations of the governing equations used in the model is given, using a reduction from the three-dimensional system to derive the one-dimensional blood flow equations, and in turn the linearisation and reduction of the one-dimensional system to determine the zero-dimensional lumped model equations. Lumped parameter models used to describe vascular beds, the heart, and valves are formulated and described. The chapter ends with a mathematical analysis of the one-dimensional blood flow equations, which describes how the governing equations can be written in terms of forward-propagating and backward propagating characteristics. Finally, several commonly used analytical techniques are presented, including: wave intensity analysis, which is performed in the time domain; Fourier analysis, which is performed in the frequency domain; and an approach that predicts the transmission and reflection of an incoming wave in the presence of changes in vessel properties, and at vessel junctions. Parts of this chapter have been published in $[65]$.

\subsection{Modelling Human Haemodynamics}

Three-dimensional fluid-structure interaction (FSI) models of haemodynamics are recognised as the most accurate models, and several highly complex FSI models exist [346]. However, these models are very computationally expensive, and hence are generally only applied to a small number of vessels, such as the aortic arch [215], cerebral arteries [346, 351, 335], or coronary arteries [345, 99, 77]. Furthermore, these FSI models require large amounts of input data ranging from: vessel geometry, which is often extracted using 3D or 4D radiographic imaging techniques, such as rotational angiography [351, 335], computed tomography (CT) [346, 345, 
99] or magnetic resonance imaging (MRI) [215]; haemodynamic quantities such as volumetric flow rate, which often require an assumption of the velocity profile at the inlet and of laminar flow; and pressure measurements, which would generally require the invasive surgical technique of catheterisation.

Material properties of the vessel wall would also be required in order to accurately estimate vessel wall motion (compliance). However this is not feasible or ethical, as it would require a sample of a patients artery. In addition a sample taken outside of the body would exhibit significantly different conditions than the environment in-vivo. The mechanical properties may be estimated from a sample from a cadaver, which will also be under significantly different conditions than in-vivo. Due to lack of experimental testing, the majority of FSI models assume uniform material properties in a vessel and consider the wall to have isotropic properties [110, 345, 215], when significant anisotropy has been observed experimentally [380]; or from expensive four-dimensional imaging data such as 4D flow MRI, which can track the three dimensional wall motion and/or blood velocity [195, 133]. This wall motion can be used to either estimate the elastic moduli of the material, or to define wall motion of the vessels during the simulation, essentially avoiding the fluid-structure interface problem as the boundary/interface of the fluid and vessel wall is defined, allowing the vessel wall (solid) model to be neglected. Vessel compliance can also be estimated from the wave speed by finding the time difference between two measurable waveforms (normally pressure) for specific wave characteristics, such as the initial rise of pressure at the beginning of diastole; however such a method can only be performed non-invasively for a small selection of vessels, and is typically used to find the overall/average wave speed in the cardiovascular network, rather than the wave speed in one specific vessel $[251,236]$. The requirement to know these material properties can be overcome by assuming a rigid wall, however these rigid wall 3D computational fluid dynamic (CFD) models are un-physiological, as significant enlargement of vessel areas occur from pressure changes between diastole to systole, and to a much lesser extend vessel axial and circumferential elongation.

Early contributions to the development of 1D haemodynamic models include Barnard et al. [23], Hughes and Lubliner [160], Rooz et al. [293], Stergiopoulos [324], Stergiopoulos et al. [325]. Recently, the field of one-dimensional blood flow modelling has received more interest with further development of the one-dimensional model via the use of different numerical methods such as finite differences [156] and 
[188], finite volume methods [216], and finite element methods [313, 117, 224]; there also exists a semi-implicit scheme which allows a 1D or 2D model to be implemented within the same framework [69]. One-dimensional blood flow models have also been used to model the calf muscle pump [169], cerebral artery flow regulation [10], foetal veins [188], neonatal pulmonary atresia [225], coronary circulation [224, 228, 229], heat transfer in the systemic system in [85], and the entire human cardiovascular system [220, 221, 230].

Validation of these one-dimensional models can generally be collected into three main methodologies: in-vivo which includes outlet boundary conditions [244], flow in bypass grafts in porcine subjects[322], patient specific arterial network [288], and coronary artery flow [228]; in-vitro using experimental set-ups [295, 205, 31, 12]; in-silico studies comparing 3D and 1D models [142, 372, 42], or several 1D models $[41,357]$.

The use of one-dimensional blood flow models has become more common as they have gained recognition as a powerful tool for analysis of haemodynamic waveforms in the cardiovascular system. One-dimensional modelling provides a computationally inexpensive alternative to the 3D CFD and/or fluid-structure interaction models. This allows 1D models to consider the entire circulation and take considerably less time to run compared 3D models, which can only be performed on relatively small networks due to computational expense. The main drawback with 1D modelling is that they are unable to capture local flow fields, as they consider flow only in the axial direction. This has led to several studies coupling 1D and 3D models, enabling the 1D model to act as boundary conditions for the 3D model, while the 3D domain covers the specific region of interest [116, 35, 253, 234, 199, 34].

\subsubsection{Deriving the Governing Equations of One-Dimensional Haemodynamics}

There are (at least) three ways to derive the one-dimensional blood flow equations [272]:

- an asymptotic analysis can be performed on the three-dimensional NavierStokes equations with an assumption that the ratio vessel radius is small. This reduces the dimensionality of the system of equations, and allows higher-order terms to be neglected [23]; 


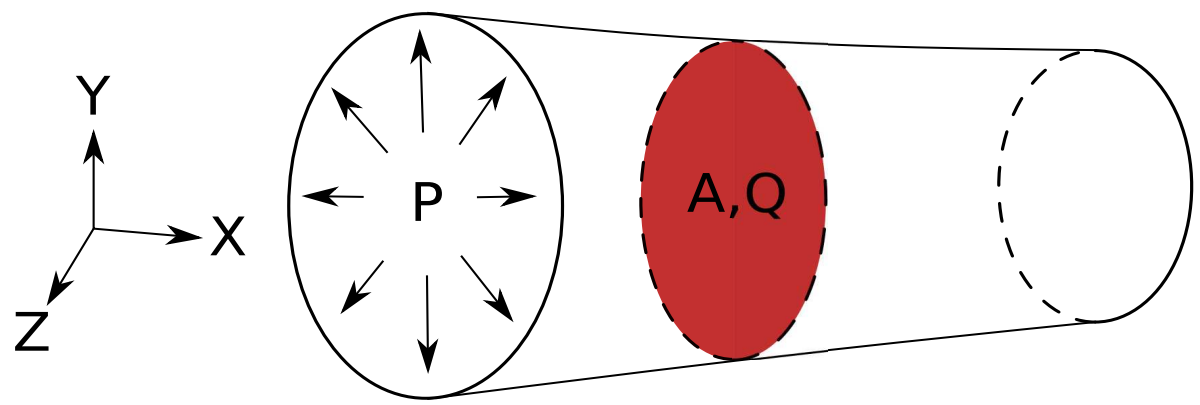

Figure 2.1: Overview of flow in a vessel

- the one-dimensional blood flow equations governing blood flow can be derived directly from first principals on a control volume [160];

- the three-dimensional system can be reduced to the one-dimensional system by making the several assumptions, which includes assuming that vessels are axially-symmetric, and that the flow in the axial direction is much larger than the flow in the radial and circumferential directions (which is the approach described below).

The three-dimensional incompressible Navier-Stokes equations describe the flow of viscous fluid with a constant density. The system is governed by the continuity equation

$$
\nabla \cdot \mathbf{u}=\frac{\partial u_{x}}{\partial x}+\frac{\partial u_{y}}{\partial y}+\frac{\partial u_{z}}{\partial z}=0
$$

where $\mathbf{u}=\left[u_{x}, u_{y}, u_{z}\right]$ is the fluid velocity in the $x, y$, and $z$ directions, respectively; the momentum equations of the three directions, which are expressed as

$$
\frac{\partial u_{x}}{\partial t}+\left(u_{x} \frac{\partial u_{x}}{\partial x}+u_{x} \frac{\partial u_{y}}{\partial y}+u_{x} \frac{\partial u_{z}}{\partial z}\right)+\frac{1}{\rho}\left(\frac{\partial P}{\partial x}\right)-\left(\frac{\partial \tau_{x x}}{\partial x}+\frac{\partial \tau_{x y}}{\partial y}+\frac{\partial \tau_{x z}}{\partial z}\right)=f_{x},
$$

in the $x$ direction,

$$
\frac{\partial u_{y}}{\partial t}+\left(u_{y} \frac{\partial u_{x}}{\partial x}+u_{y} \frac{\partial u_{y}}{\partial y}+u_{y} \frac{\partial u_{z}}{\partial z}\right)+\frac{1}{\rho}\left(\frac{\partial P}{\partial y}\right)-\left(\frac{\partial \tau_{y x}}{\partial x}+\frac{\partial \tau_{y y}}{\partial y}+\frac{\partial \tau_{y z}}{\partial z}\right)=f_{y}
$$

in the $y$ direction, and

$$
\frac{\partial u_{z}}{\partial t}+\left(u_{z} \frac{\partial u_{x}}{\partial x}+u_{z} \frac{\partial u_{y}}{\partial y}+u_{z} \frac{\partial u_{z}}{\partial z}\right)+\frac{1}{\rho}\left(\frac{\partial P}{\partial z}\right)-\left(\frac{\partial \tau_{z x}}{\partial x}+\frac{\partial \tau_{z y}}{\partial y}+\frac{\partial \tau_{z z}}{\partial z}\right)=f_{z}
$$


in the $z$ direction. Where $f_{x}, f_{y}$, and $f_{z}$ are external source/sink terms that may arise depending on the problem of interest, such as from the inclusion of gravitational forces. $\tau$ is the Cauchy stress tensor, and $P$ is the hydrostatic pressure. The NavierStokes equations are closed by the conservation of energy and an additional equation, normally referred to as an equation of state (relating pressure, temperature and density). However, neither the energy conservation equation or equation of state are required for the purposes of this thesis as these are not required by the onedimensional formulation, and are not presented or described further.

In order to reduce the three-dimensional system into a one-dimensional system, the three dimensional incompressible Navier-Stokes equations are first transformed into cylindrical coordinates by applying

$$
\begin{aligned}
(x, y, z) & \rightarrow(x, r, \theta), \\
x=x, \quad y=r \sin (\theta), \quad z & =r \cos (\theta) .
\end{aligned}
$$

Making an assumption of a Newtonian fluid, the 3D continuity equation becomes

$$
\frac{1}{r} \frac{\partial\left(r u_{r}\right)}{\partial r}+\frac{1}{r} \frac{\partial u_{\theta}}{\partial \theta}+\frac{\partial u_{x}}{\partial x}=0
$$

While the radial component of momentum becomes

$$
\begin{gathered}
\rho\left[\frac{\partial u_{r}}{\partial t}+u_{r} \frac{\partial u_{r}}{\partial r}+\frac{u_{\theta}}{r} \frac{\partial u_{r}}{\partial \theta}-\frac{u_{\theta}^{2}}{r}+u_{x} \frac{\partial u_{r}}{\partial x}\right]+\frac{\partial P}{\partial r} \\
-\mu\left[\frac{1}{r} \frac{\partial}{\partial r}\left(r \frac{\partial u_{r}}{\partial r}\right)-\frac{u_{r}}{r^{2}}+\frac{1}{r^{2}} \frac{\partial^{2} u_{r}}{\partial \theta^{2}}-\frac{2}{r^{2}} \frac{\partial u_{\theta}}{\partial \theta}+\frac{\partial^{2} u_{r}}{\partial x^{2}}\right]=\rho f_{r},
\end{gathered}
$$

the circumferential component becomes

$$
\begin{gathered}
\rho\left[\frac{\partial u_{\theta}}{\partial t}+u_{r} \frac{\partial u_{\theta}}{\partial r}+\frac{u_{\theta}}{r} \frac{\partial u_{\theta}}{\partial \theta}-\frac{u_{r} u_{\theta}}{r}+u_{x} \frac{\partial u_{\theta}}{\partial x}\right]+\frac{\partial P}{\partial \theta} \\
-\mu\left[\frac{1}{r} \frac{\partial}{\partial r}\left(r \frac{\partial u_{\theta}}{\partial r}\right)-\frac{u_{\theta}}{r^{2}}+\frac{1}{r^{2}} \frac{\partial^{2} u_{\theta}}{\partial \theta^{2}}-\frac{2}{r^{2}} \frac{\partial u_{r}}{\partial \theta}+\frac{\partial^{2} u_{\theta}}{\partial x^{2}}\right]=\rho f_{\theta},
\end{gathered}
$$


and the axial component becomes

$$
\begin{array}{r}
\rho\left[\frac{\partial u_{x}}{\partial t}+u_{r} \frac{\partial u_{x}}{\partial r}+\frac{u_{\theta}}{r} \frac{\partial u_{x}}{\partial \theta}+u_{x} \frac{\partial u_{x}}{\partial x}\right]+\frac{\partial P}{\partial x} \\
-\mu\left[\frac{1}{r} \frac{\partial}{\partial r}\left(r \frac{\partial u_{x}}{\partial r}\right)+\frac{1}{r^{2}} \frac{\partial^{2} u_{x}}{\partial \theta^{2}}+\frac{\partial^{2} u_{x}}{\partial x^{2}}\right]=\rho f_{x} .
\end{array}
$$

Finally, the three-dimensional system in cylindrical coordinates can be reduced to one dimension by the following assumptions:

- Axial symmetry. Indicates all quantities including velocity will be independent of the angle $\theta$.

- Radial displacement. The vessel wall displaces in the radial direction only (no circumferential or axial displacement occurs).

- The pressure is assumed constant in each cross-section. Hence no pressure differentials are considered in the radial or circumferential directions.

- Axial velocity is considered much larger than both radial and circumferential velocities, allowing radial and circumferential components of velocity to be neglected.

The momentum equation then becomes

$$
\frac{\partial u_{x}}{\partial t}+u_{x} \frac{\partial u_{x}}{\partial x}+\frac{1}{\rho} \frac{\partial P}{\partial x}-\frac{\mu}{\rho}\left[\frac{1}{r} \frac{\partial}{\partial r}\left(r \frac{\partial u_{x}}{\partial r}\right)+\frac{\partial^{2} u_{x}}{\partial x^{2}}\right]=f_{x}
$$

Now integrating over a section gives momentum equation as

$$
\frac{\partial Q}{\partial t}+\frac{\partial\left(\alpha \frac{Q^{2}}{A}\right)}{\partial x}+\frac{A}{\rho} \frac{\partial P}{\partial x}-\int_{C S} \frac{\mu}{\rho}\left(\frac{1}{r} \frac{\partial}{\partial r}\left(r \frac{\partial u_{x}}{\partial r}\right)\right) d \sigma+\frac{\partial^{2} Q}{\partial x^{2}}=A f_{x}
$$

where $Q=A u$ is the volumetric flow rate in a cross section, $u$ is the average crosssectional velocity in the axial direction, $r$ is the coordinate in the radial direction, and $x$ is the coordinate in the axial direction. The term $\alpha$ is a momentum flux correction factor given as

$$
\alpha=\frac{\int_{C S} u_{x}^{2} d \sigma}{A u^{2}}
$$


which accounts for the non-uniform velocity distribution (called the Boussinesq coefficient), and depends on the choice of velocity profile (for a uniform profile $\alpha=1$ ) [314]. The continuity equation becomes

$$
\frac{\partial A}{\partial t}+\frac{\partial Q}{\partial x}=\Phi
$$

where $A$ is the cross-sectional area, and $\Phi$ accounts for any source/sink terms.

There are three unknowns in this system, which are the area $A$, flow rate $Q$ (or velocity $u$ ), and hydrostatic pressure $P$ which are shown in figure 2.1. Thus in order to close the system of equations, an additional relation is required. The additional equation is the constitutive law, which links the transmural pressure to the crosssectional area. The various constitutive laws used in one-dimensional blood flow models are discussed in section 2.2.4. Furthermore, in order to estimate the friction term, a velocity profile needs to be chosen. The choice and implications of the velocity profile is discussed in section 2.2.3.

\subsubsection{D Formulation Variations}

Through various manipulations of equations (2.11) and (2.13), various formulations of the $1 \mathrm{D}$ blood flow equations can be found. The three most common variants are the $A U, A Q$, and $P Q$ formulations. The AU system of equations have been implemented in $[314,224]$ and can be expressed as

$$
\begin{cases}\frac{\partial A}{\partial t}+\frac{\partial A u}{\partial x}=0 & \text { continuity } \\ \frac{\partial u}{\partial t}+(2 \alpha-1) u \frac{\partial u}{\partial x}+(1-\alpha) \frac{u^{2}}{A} \frac{\partial A}{\partial x}+\frac{1}{\rho} \frac{\partial P}{\partial x}-\frac{f}{\rho}=0, & \text { momentum. }\end{cases}
$$

The $A Q$ system used by $[220,117,349]$ can be written as

$$
\begin{cases}\frac{\partial A}{\partial t}+\frac{\partial Q}{\partial x}=0, & \text { continuity } \\ \frac{\rho}{A} \frac{\partial Q}{\partial t}+\frac{\rho}{A} \frac{\partial\left(\alpha \frac{Q^{2}}{A}\right)}{\partial x}+\frac{\partial P}{\partial x}-\frac{f}{A}=0, & \text { momentum }\end{cases}
$$

The $P Q$ system of equations is implemented in [30, 182]. The $P Q$ system can be derived by utilising the chain rule on the continuity equation (2.15), which gives 
$\frac{\partial A}{\partial t}=\frac{\partial A}{\partial P} \frac{\partial P}{\partial t}=C \frac{\partial P}{\partial t}$.

$$
\begin{cases}\frac{\partial A}{\partial P} \frac{\partial P}{\partial t}+\frac{\partial Q}{\partial x}=0, & \text { continuity } \\ \frac{\rho}{A} \frac{\partial Q}{\partial t}+\frac{\rho}{A} \frac{\partial\left(\alpha \frac{Q^{2}}{A}\right)}{\partial x}+\frac{\partial P}{\partial x}-\frac{f}{A}=0, & \text { momentum. }\end{cases}
$$

There are also more intricacies involved when it actually comes to solving these equations. For example in the $A U$ formulation, [314] chooses to keep the equations in conservation form, while [224] performs the chain rule on the term $\frac{\partial P}{\partial x}$. This will have an impact on not only implementation, but also may potentially causes numerical issues if the geometry is not smooth (which is the case in patient-specific geometric simulations).

\subsubsection{Velocity Profiles}

The choice of velocity profile effects both the momentum flux correction factor $\alpha$ in equation 2.12, and the friction term in the momentum equation $\int_{C S} \frac{\mu}{\rho}\left(\frac{1}{r} \frac{\partial}{\partial r}\left(r \frac{\partial u_{x}}{\partial r}\right)\right) d \sigma$. The commonly used velocity profile $[11,41]$ is implemented throughout this thesis, which satisfies the no slip condition $\left(\left.u_{x}\right|_{r=R}=0\right)$, is given by

$$
u(x, r, t)=U \frac{\zeta+2}{\zeta}\left[1-\left(\frac{r}{R}\right)^{\zeta}\right]
$$

where the radius of the vessel lumen is $R, r$ is the radial coordinate, and $\zeta=$ $\frac{2-\alpha}{\alpha-1}$. Integrating the velocity profile in equation (2.17) for a cross-section causes the friction term in the momentum equation to become

$$
f=\int_{C S} \frac{\mu}{\rho}\left(\frac{1}{r} \frac{\partial}{\partial r}\left(r \frac{\partial u_{x}}{\partial r}\right)\right) d \sigma=\frac{-2(\zeta+2) \mu \pi Q}{A}
$$

For the velocity profile given in (2.17), a flat profile can be obtained for $\alpha=1$, however as $\lim _{\alpha \rightarrow 1} \zeta(\alpha)=\infty$, a flat profile is only chosen for the non-linear term in (2.11) as the friction term would tend to infinity. The velocity profile chosen is almost always considered to be fully developed. Furthermore, it is relatively common to assume a different velocity profile for the non-linear convection term and the friction term. In most models $\alpha$ is chosen to be a constant $[118,318,314]$ for the momentum flux correction factor with either $\alpha=1$ (flat profile) [314], or 
$\alpha=4 / 3$ (parabolic profile) [118], while the friction term is often considered to be either $\zeta=2$ (for a parabolic profile) $[119,224,194]$; or $\zeta=9[318,230,205]$, which produces a profile that is mostly flat but with a boundary layer.

In [30] the velocity profile is constructed by splitting the profile into inertia dominant and viscous dominant regions; While a velocity profile using Witzig-Womersley pulsatile theory is presented in [287]. Figure 2.2a shows velocity profiles using (2.17), figure $2.2 \mathrm{~b}$ shows fully developed Womersley velocity profiles for a variety of Womersley numbers, and figure 2.3 shows Womersley profiles during different stages of development for various Womersley numbers.

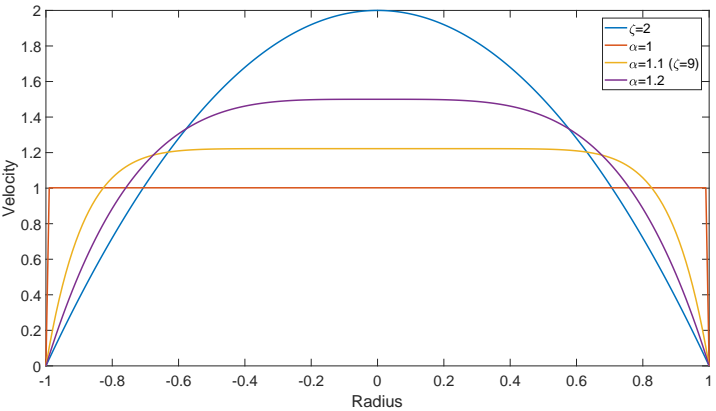

(a) Velocity profiles generated from equation (2.17)

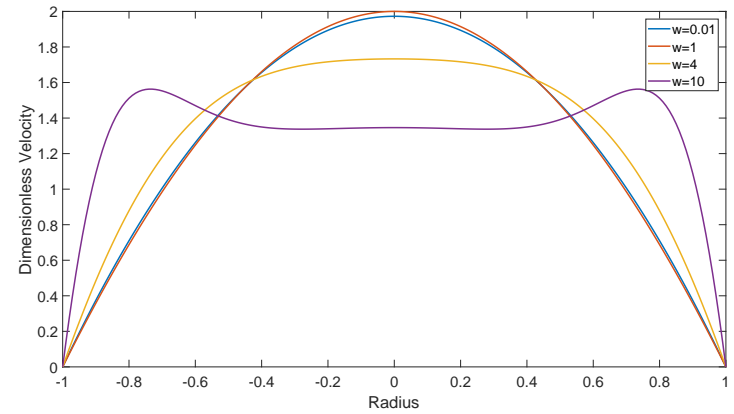

(b) Velocity profiles generated from equation (2.17)

Figure 2.2: Comparison of various velocity profiles

\subsubsection{Constitutive Law}

A constitutive law is required to solve the system in equation (2.16). The constitutive law provides a relation between a sections cross-sectional area, and the transmural pressure $P_{t m}(A)=P-P_{\text {ext }}$ where $P_{\text {ext }}$ is the external pressure. There are numerous constitutive laws in use for the one-dimensional model, these may be classified as elastic (linear and non-linear variants), collapsible vessel models, or visco-elastic wall models. Table 2.1 shows a selection of the main constitutive laws used in onedimensional haemodynamic models and figure 2.4 compares the elastic constitutive laws from the table. The meaning of terms in the equations in 2.1 are as follows: $P_{0}$ is a reference pressure, $c_{0}^{2}$ and $A_{0}$ are the wave speed and area at the reference pressure, $P_{\text {collapse }}$ is the pressure at which the vessel is fully collapsed, and $K$ is the 

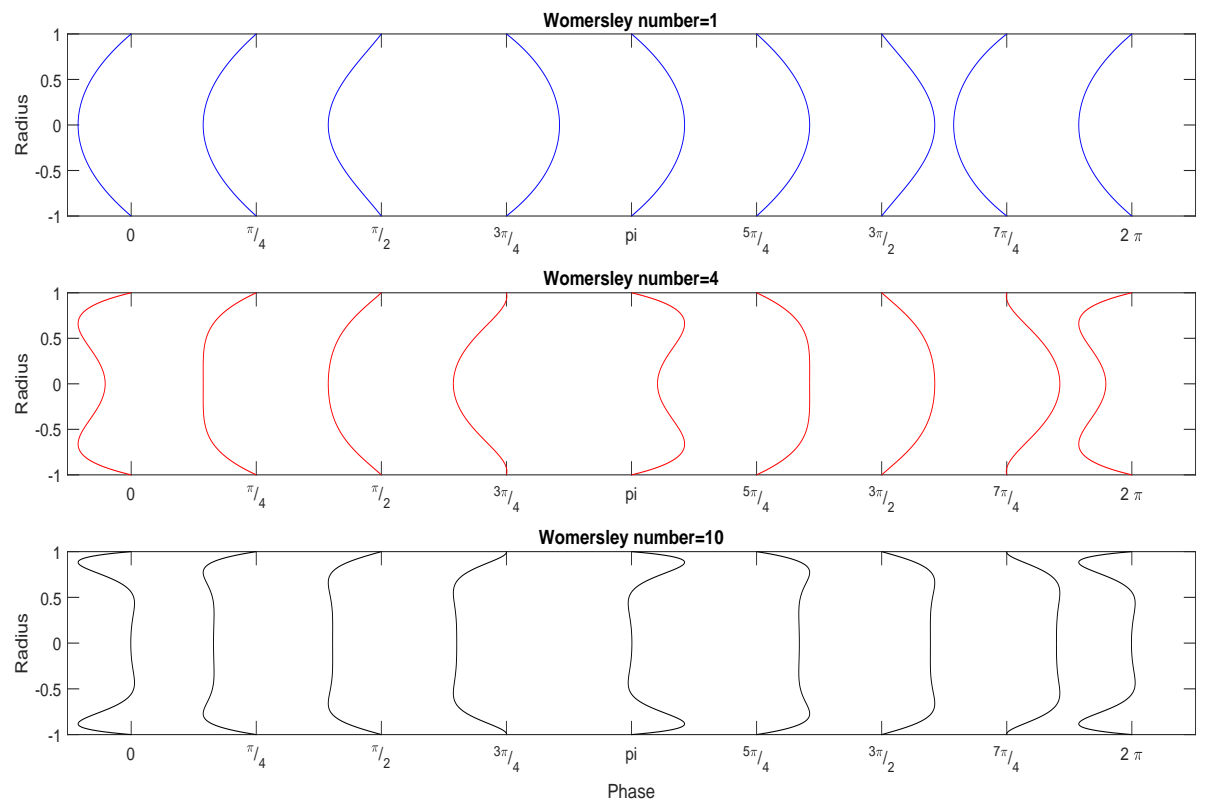

Figure 2.3: Womersley velocity profiles development for different Womersley numbers

stiffness which is often expressed as

$$
K=\frac{E}{12 \sqrt{1-\sigma^{2}}}\left(\frac{h}{R}\right)^{3}
$$

where $E$ is the elastic modulus, $\sigma=0.5$ is Poisson's ratio, $h$ is the wall thickness, and $R$ the vessel radius.

In order to account for the visco-elastic properties of vessel walls, an additional term can be added to the original constitutive law, such as in $[117,12,230]$ given by

$$
\frac{\Gamma}{A_{0} \sqrt{A}} \frac{\partial A}{\partial t}
$$

with comparisons between different visco-elastic models being performed by [276, 135]. [117] also included more complex wall behaviour, such as wall inertia and a longitudinal elastic term in the constitutive law, although these terms tend to have a minor effect on the solution.

From the constitutive law, one can extract the compliance of the vessel for a given pressure. The compliance describes the relationship between the change of area, with respect to the change of pressure. For the non-linear elastic constitutive 
2.2. MODELLING HUMAN HAEMODYNAMICS

\begin{tabular}{|c|c|c|}
\hline Type & Equation & References \\
\hline $\begin{array}{l}\text { linear elas- } \\
\text { tic }\end{array}$ & $P_{t m}=\rho c_{0}^{2}\left(\frac{A}{A_{0}}-1\right)+P_{0}$ & {$[277,278]$} \\
\hline $\begin{array}{l}\text { non-linear } \\
\text { elastic }\end{array}$ & $\begin{array}{l}P_{t m}=2 \rho c_{0}^{2}\left(\frac{\sqrt{A}}{\sqrt{A_{0}}}-1\right)+P_{0}= \\
\frac{\beta}{A_{0}}\left(\sqrt{A}-\sqrt{A_{0}}\right)+P_{0}\end{array}$ & $\begin{array}{l}{[117,314,313,} \\
224]\end{array}$ \\
\hline $\begin{array}{l}\text { non-linear } \\
\text { elastic } \\
\text { (power } \\
\text { law) }\end{array}$ & $\begin{array}{l}P_{t m}=\frac{2 \rho c_{0}^{2}}{b}\left(\left(\frac{A}{A_{0}}\right)^{b / 2}-1\right)+P_{0}, \quad b= \\
\frac{2 \rho c_{0}^{2}}{P_{0}-P_{\text {collapse }}}\end{array}$ & {$[230,318,55]$} \\
\hline $\begin{array}{l}\text { collapsible } \\
\text { elastic }\end{array}$ & $P-P_{e x t}=P_{0}+K\left(\left(\frac{A}{A_{0}}\right)^{m}-\left(\frac{A}{A_{0}}\right)^{n}\right)$ & {$[45,220]$} \\
\hline
\end{tabular}

Table 2.1: Example of each constitutive law type

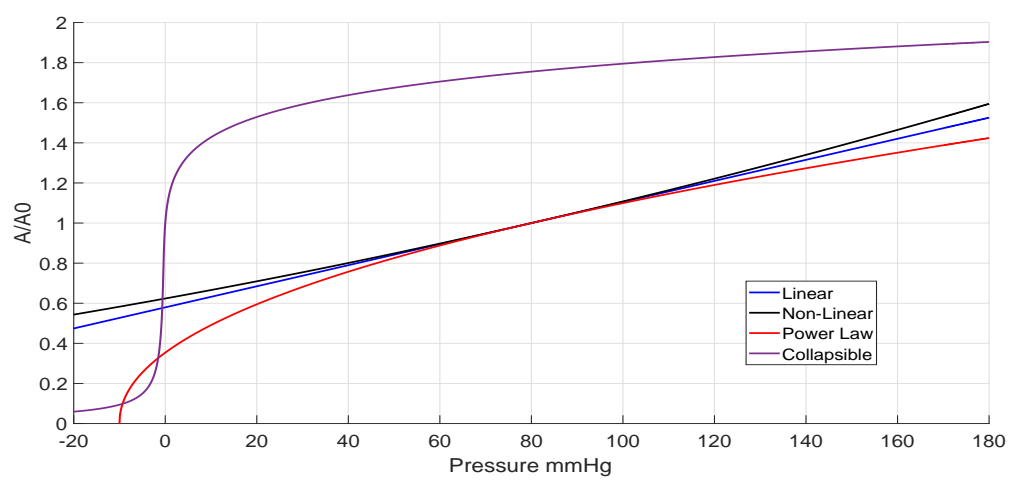

Figure 2.4: Comparison of a linear elastic, a non-linear elastic, a non-linear elastic power law, and a collapsible tube model

law, the compliance can be calculated as

$$
\frac{\partial A}{\partial P}=\left(\frac{2 A_{d}\left(P-P_{e x t}-P_{d}\right)}{\beta}+2 \sqrt{A_{d}}\right) \frac{A_{d}}{\beta} .
$$

In general the constitutive law is written in the form $P=y(A)$, and due to the nonlinear relation of pressure and area (for the non-linear constitutive laws), it may not be possible (or trivial) to rearrange the function to be in the form $A=y^{-1}(P)$, and hence the derivative $\frac{\partial A}{\partial P}$, may not be straightforward to calculate. However, from the inverse function theorem: for a continuously differentiable function $y$ with a non-zero derivative at a point in its domain, then the function $y$ is invertible in 
a neighbourhood of this point, and it's inverse $y^{-1}$ is continuously differentiable; furthermore the derivative of the inverse function in this neighbourhood is the reciprocal of the derivative of $y$. Which means the derivative of $P=y(A)$ with respect to A, can be written as:

$$
\frac{\partial P}{\partial A}=\frac{1}{\frac{\partial A}{\partial P}}
$$

When calculating the compliance for a visco-elastic constitutive law, only the elastic part of the constitutive law is considered as the viscous term is much smaller in magnitude.

\subsubsection{External Pressures}

In the majority of works the external pressure in the one-dimensional model is neglected, and hence $P_{e x t}=0$. However, external pressures are known to significantly influence haemodynamics, for example many deep veins and arteries traverse between muscles and will experience external pressures when these muscles contract. One of the many auto-regulation mechanisms, termed the calf muscle pump has been presented in [169]. Although the network in [169] only considered one artery and two veins (one deep, one superficial), it was shown that the addition of an external pressure on the deep vein improved venous return by partially overcoming the force produced from gravity.

The vessels in the coronary system experience external pressure from the surrounding heart muscle. [229] presented a complex network of coronary vessels that experience external pressure from the ventricles of the heart model. The model was developed in order to study the influence of heart dominance on flow rate patterns. In a right dominant coronary system, the right coronary arteries supply the septum and parts of the left ventricle with blood; while in left dominance, the circumflex artery supplies the septum with blood (co-dominance also exists, although was not studied).

There remains a number of additional areas where external pressure plays a significant role, and to-date have not been studied in sufficient detail to understand the implications of these external pressures. These include both: healthy physiological conditions, such as the effect of the muscle pump on either a venous or arterial network (ankle, calf, and thigh), the effect of the respiratory pump, which acts on 
vessels in the thorax during respiration, and the effect of respiration and thus the external pressure from the lungs, on pulmonary vessels; and also during pathological conditions such as the effect of external pressures on stenosis (such as in the coronary vessels), or on aneurysms. Of course 1D modelling has its limitations for modelling these situations, due to being unable to account for local fluid dynamics, as only fluid flow in the axial direction is accounted for.

\subsection{Zero-Dimensional Modelling}

In many situations, the fluid flow at a specific location may not be of interest, instead the more global aspects of the cardiovascular system may be of interest, such as heart rate changes due to postural change [198]. In these case, a 1D model may be more complex than needed, and thus a 0D lumped parameter model may more appropriate. Typically, there are two variants of lumped models: single compartment models, which represent the entire circulation as a single lumped model; or multicompartment models, which may treat each section of the cardiovascular system as separate lumped models [191, 2, 271]. Another common use of lumped models is for boundary conditions for more complex methodologies, such as outflow boundary conditions for 1D [324, 116, 314, 230, 205, 220, 221, 41], 2D [69], or 3D [175, 372, 185] models. For the one-dimensional models, this may be to represent a specific vascular bed, such as the coronary vascular bed [228] or lungs [275]; or may be to represent the remainder of the circulation [103, 41].

The lumped parameter model equations can be derived using first principles, or can be found by linearising the $1 \mathrm{D}$ system in a $\mathrm{PQ}$ formulation (similarly shown in [11]), as in equation (2.16), and then averaging physical properties over the domain length. In order to reduce the $1 \mathrm{D}$ system of equations to $0 \mathrm{D}$, the $1 \mathrm{D}$ system is linearised around its reference state, which means the non-linear convection term $\frac{\partial\left(\frac{Q^{2}}{A}\right)}{\partial x}$ is first neglected. This leads to the system of equations

$$
\begin{cases}C_{1 D, L} \frac{\partial P_{t m}}{\partial t}+\frac{\partial Q}{\partial x}=0, & \text { continuity } \\ L_{1 D, L} \frac{\partial Q}{\partial t}+\frac{\partial P}{\partial x}+R_{1 D, L} Q=0, & \text { momentum } \\ C_{1 D, L}=\frac{\partial A_{0}}{\partial P_{t m, 0}}, \quad L_{1 D, L}=\frac{\rho}{A_{0}}, & R_{1 D, L}=\frac{\xi \mu \pi}{A_{0}^{2}}\end{cases}
$$


averaging properties in a segment and integration over its length, leads to the final form of the zero-dimensional system of equations

$$
\begin{cases}C_{0 D} \frac{\partial P_{t m}}{\partial t}+\Delta Q=0, & \text { continuity } \\ L_{0 D} \frac{\partial Q}{\partial t}+\Delta P+R_{0 D} Q=0, & \text { momentum } \\ C_{0 D}=\frac{\partial A_{0}}{\partial P_{t m, 0}} \Delta x, \quad L_{0 D}=\frac{\rho}{A_{0}} \Delta x, & R_{0 D}=\frac{8 \mu \pi}{A_{0}^{2}} \Delta x\end{cases}
$$

where the capacitance (compliance) of a volume is $C_{0 D}$, the fluid inertia in a volume is $L_{0 D}$, and the resistance from viscous forces in a volume is $R_{0 D}$. Furthermore, the system of equations (2.24) can be de-constructed into the following three basic elements: a resistance element, an inductance and a compliance (capacitance) element.

$$
\begin{aligned}
& \Delta P=R Q \\
& \Delta P=L \frac{\partial Q}{\partial t}, \\
& C \frac{\partial P_{t m}}{\partial t}=Q_{n e t}=Q_{\text {in }}-Q_{\text {out }} .
\end{aligned}
$$

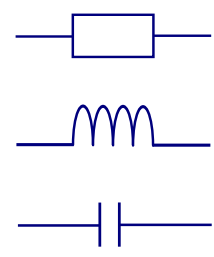

The three basic elements shown in equation (2.25) can be used to construct any of the lumped models used in haemodynamic modelling, albeit may need to be edited slightly, such as in the case of the heart model as will be discussed in subsection 2.3.2. Additional information, such as the implementation of the lumped models used in this thesis, is discussed further in subsection 3.2.4 of chapter 3 .

\subsubsection{Vascular Bed Models}

In this subsection, the main types of lumped parameter models used for vascular beds will be presented and discussed. Early implementations of these vascular bed (often called Windkessel) models [120], used the two-element Windkessel model to describe the effect of downstream vasculature on upstream haemodynamics. These two-element Windkessel models are comprised of a single resistance and a single compliance in parallel, as shown in figure 2.5a. However, it was found that while these models typically represented compliance quite well (when used in conjunction 
with the pulse pressure method) [364], the model poorly captured the overall waveshape and inadequately estimated the impedance at high frequencies.

In order to improve on the shortcomings of the two-element Windkessel models, an additional resistance element was introduced, creating the commonly used three-element Windkessel model. The three-element Windkessel model considers a resistance in series, with a resistance and compliance in parallel, as shown in figure $2.5 \mathrm{~b}$. The first resistance $Z_{\text {art }}$, was found to represent the characteristic impedance of the connecting vessel [365], and can be considered as an intermediary between a lumped model (which cannot account for wave propagating phenomena) and the wave propagating aspects/phenomena in the vascular systems. Moreover, it has been observed [327, 364] that treating the characteristic impedance as a resistor leads to errors in the lower frequency range. However, these errors are generally small due to the characteristic impedance being significantly smaller than the peripheral resistance. The three-element Windkessel model is generally treated using constant resistances and compliances, however attempts have been made to implement pressure dependant resistances [230] (as used in this thesis for the closed loop system), and pressure dependant compliances [114].

To reduce the errors at low frequencies, caused by the introduction of the characteristic impedance, an additional element (inertia) was introduced into the threeelement Windkessel model. This four-element Windkessel model has two variations: the traditional four-element model $[50,329]$ considers the inertial element and the characteristic impedance to be in parallel, as shown in figure 2.5c; while $[50,51]$ implemented an inertial element in series with the characteristic impedance, shown in figure $2.5 \mathrm{~d}$. In practice it is very difficult to find an accurate estimate of the inertance, and hence the three-element Windkessel model is often chosen for this reason.

Further extensions to these lumped models have occurred, such as: the ability to connect multiple arteries and/or veins to the same vascular bed; and more complex vascular beds for the coronaries [228], which includes external pressure from heart chambers and volume dependant resistances; and the vascular bed for the liver [230], which connects to three systems, the systemic arteries, hepatic portal veins, and systemic veins. Further description of the vascular bed models used in this thesis, including their implementation, are discussed in section 3.2.4. 


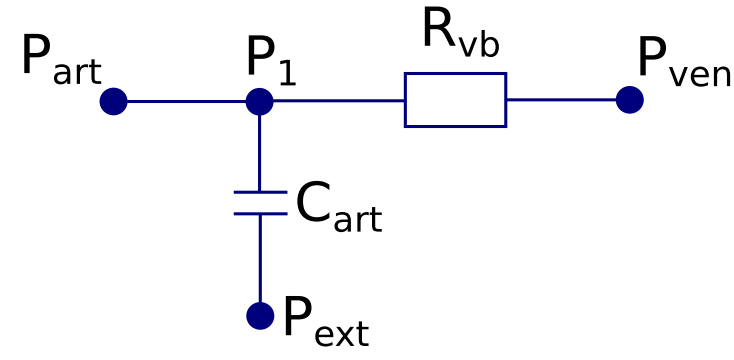

(a) Two-element Windkessel model

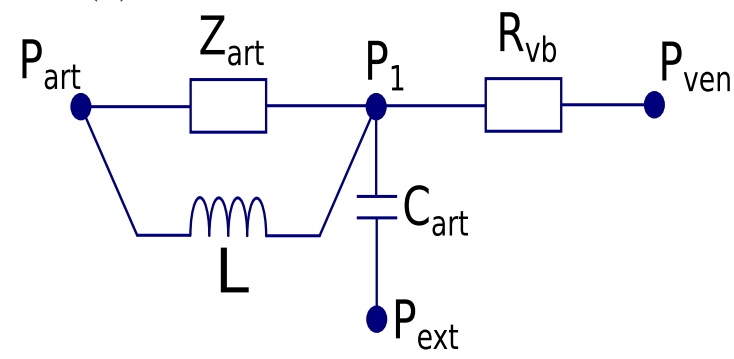

(c) Four-element Windkessel model with inertia (inductance) in parallel

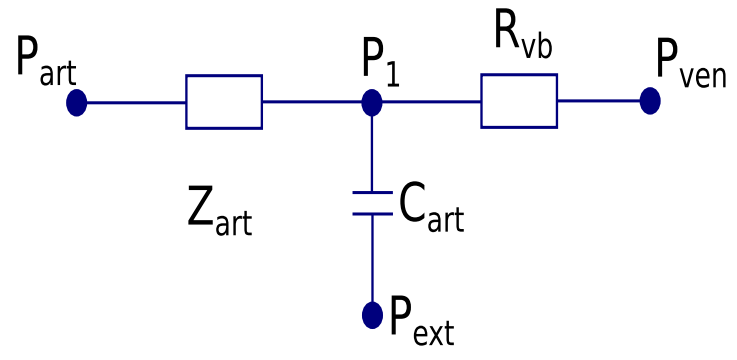

(b) Three-element Windkessel model

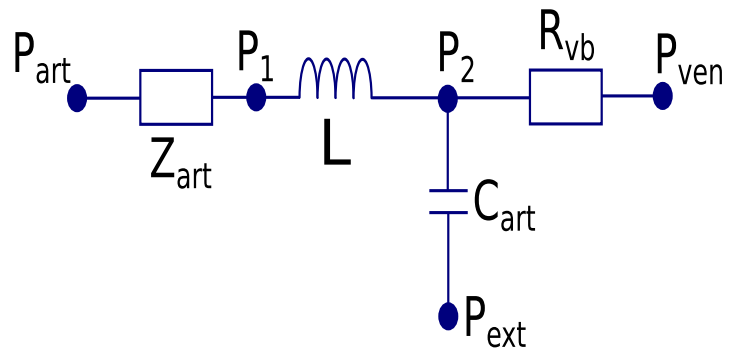

(d) Four-element Windkessel model with inertia (inductance) in series

Figure 2.5: Comparison of the four common Windkessel models. Art and Vein are connections to artery/arteries and vein/veins, respectively.

\subsubsection{Heart Model}

In some studies, an upstream boundary condition of volumetric inflow rate or pressure is prescribed $[324,116,314,224,41]$; however several studies [119, 226, 230, $220,221]$ have instead attached the inlet of the $1 \mathrm{D}$ vessel domain with a lumped parameter heart model. The contractility of the heart model is generally pre-defined through an elastance curve such as in figure $2.7 \mathrm{a}$, while the closed-loop system from section 7.8 (for the non-pregnant case) produced the physiologically representative volume curves for the left atria and ventricle, and the right atria and ventricle, which are shown in $2.7 \mathrm{~b}$.

Before introducing the equations of the heart model, it is useful to note the following relation between volume, volumetric flow rate and pressure, which is outlined by figure 2.6

$$
\begin{aligned}
\frac{\partial V}{\partial t} & =\frac{\partial V}{\partial P} \frac{\partial P}{\partial t}=Q_{\text {in }}-Q_{\text {out }}, \\
V & =A_{\text {avg }} L, \quad \frac{\partial V}{\partial P}=\frac{1}{E},
\end{aligned}
$$


where $A_{\text {avg }}$ is the average area in a segment of length $L$, and $E$ is the elastance of the heart chamber. The basic equations which describe the heart model can be written in the form [119]

$$
P=E\left(V-V_{0}\right)-R_{s} Q, \quad R_{s}=K_{s} E\left(V-V_{0}\right),
$$

where $P$ is the pressure in the heart chamber, $Q$ is the net flow rate in the heart chamber which is defined to be positive in the direction of flow $Q=Q_{\text {out }}-Q_{\text {in }}, R_{s}$ is a source resistance, and $K_{s}$ is a constant. There have been several implementations of the heart model with a single left ventricle model used to generate flow and pressure waveforms for inflow to the aorta implemented in [119], while other studies $[230,220]$ have used the heart model within a closed-loop framework.

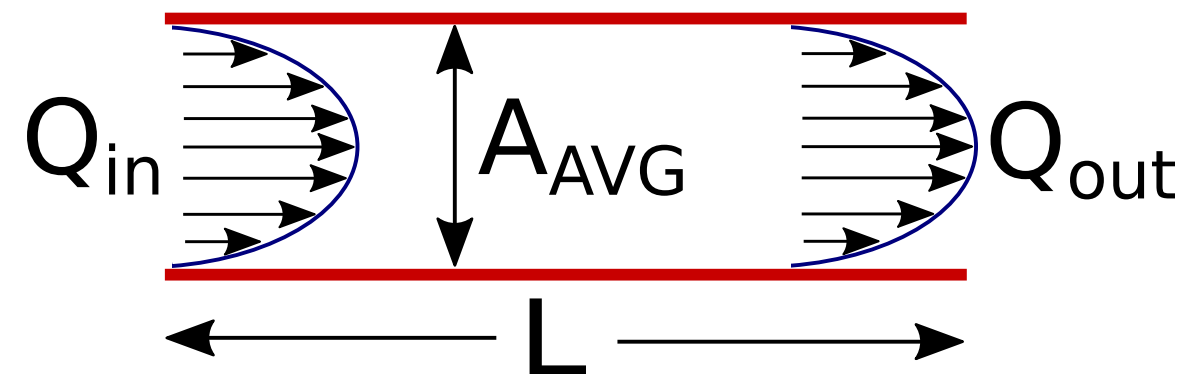

Figure 2.6: Fluid storage in a control volume

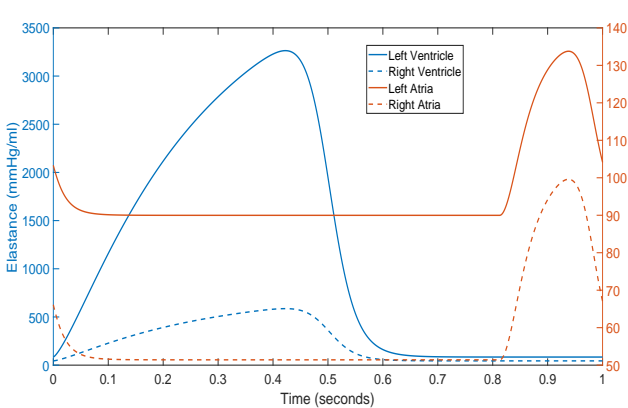

(a) Elastance curves for one cardiac cycle

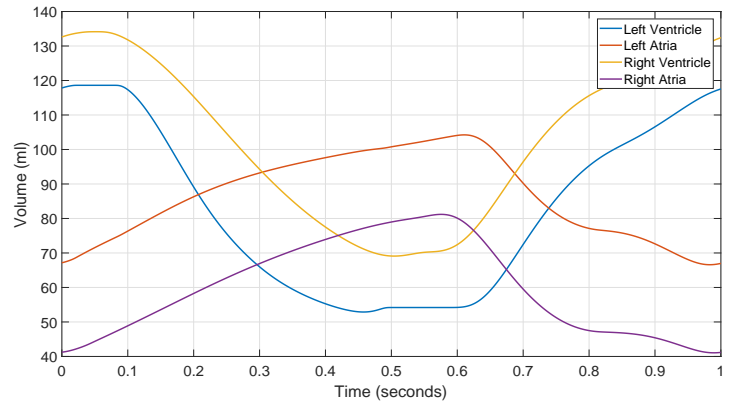

(b) Volume curves for one cardiac cycle

Figure 2.7: Elastance curves and volumes of left ventricle and left atria

In this thesis, the heart model by [230] is implemented for the closed-loop system. A lumped element diagram of this heart model can be seen in figure 2.9. This complex heart model considers three types of interaction: 
1. External pressures acting between contra-lateral chambers (interaction between the left and right side of the heart interaction), shown in figure 2.8a.

2. During ventricular contraction, a piston like effect causes a suction like effect on the atria, aiding atrial filling, shown in figure $2.8 \mathrm{~b}$.

3. External pressures are exerted from the pericardia (where the heart is situated) which acts on all chambers equally, shown in figure $2.8 \mathrm{c}$.

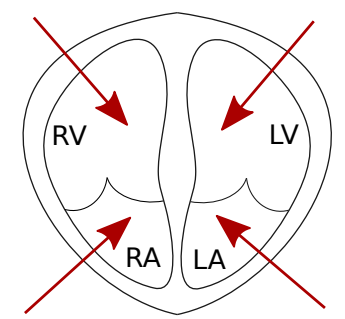

(a) Pericardial cavity pressure

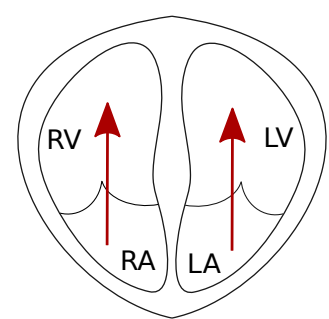

(b) Piston effect on atria

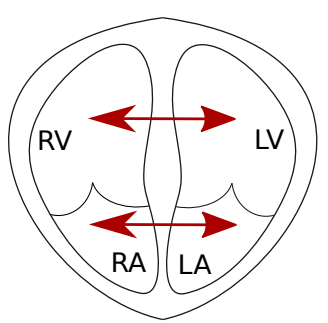

(c) Left and right interaction

Figure 2.8: Interactions of the heart

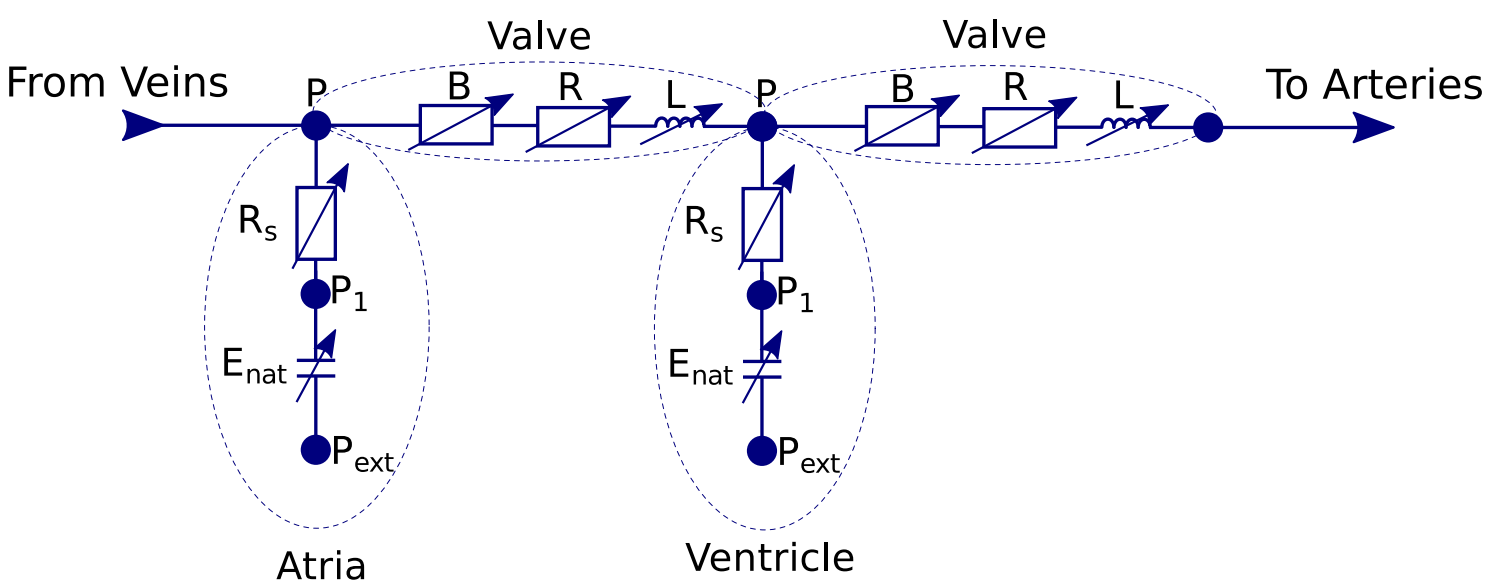

Figure 2.9: Heart model (one side)

Full details of the discretisation and implementation of the heart model used in this thesis is provided in subsection 3.2.4. 


\subsubsection{Valve Model}

Valves are located in several locations in the human body. In the cardiovascular system valves are located in the heart and in the systemic venous system. There are also valves located in the lymphatic system, separating lymphangions. Valves in the cardiovascular system are present to prevent blood from flowing in the opposite direction. A healthy valve is one that opens easily in the presence of a positive pressure gradient across the valve (opens for forward flow), and closes completely to prevent back flow during a negative pressure gradient. A valve may: become damaged as a result of excessive opening and closing pressures/forces and may lose its effectiveness to fully close, allowing blood flow backwards through the valve, which is called valve regurgitation [189]; or a heart valve may be affected by calcification, which leads to valve stenosis where the cross-sectional area of the valve decreases causing a greater resistance to blood flow, requiring the heart to pump with more force [57].

Early models of valves treated the valve state like a simple diode, either being fully open for a positive pressure gradient, or fully closed for negative flow rate [119, 377, 125]. More complex lumped models have been developed, such as describing valve opening and closing behaviour based on balancing forces [180], or by determining valve opening and closing behaviour from the pressure gradient across the valve [226]. The model from [226] was extended by [252] to include and improve modelling of valve regurgitation. Under healthy conditions viscous losses are generally small through the valve, however these will become more significant in the case of valve stenosis. [169] added this viscous term into the valve model for systemic venous valves.

In this thesis the valve model implemented is that of [226]; however, for completeness the viscous term will remain in the formulation in order to show the full set of equations. A modification to [226] is given by

$$
\Delta P=B|Q| Q+R Q+L \frac{d Q}{d t}
$$

where the Bernoulli resistance $B$, the viscous resistance $R$, and the inertance (in- 
ductance) $L$ are given by

$$
B=\frac{\rho}{2 A_{e f f}^{2}}, \quad L=\frac{\rho l_{e f f}}{A_{e f f}}, \quad R=\frac{2(\zeta+2) \pi \mu l_{e f f}}{A_{e f f}^{2}},
$$

where $l_{\text {eff }}$ is an effective length, and $A_{\text {eff }}$ is the current orifice area, bounded between a minimum $A_{e f f, \min }$ and maximum $A_{e f f, \max }$ orifice area, given by

$$
A_{e f f}(t)=\left(A_{e f f, \max }-A_{e f f, \min }\right) \xi(t)+A_{e f f, \min } .
$$

The coefficient $\xi$ is the valve state, which is bounded between zero and one, and can be calculated by one of the following ordinary differential equations

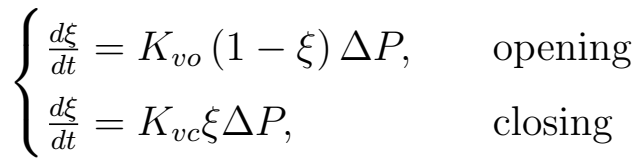

where $K_{v o}$ and $K_{v c}$ are coefficients which affect the sensitivity of the opening and closing behaviour respectively, due to the trans-valvular pressure $\Delta P$.

Figure 2.10 compares the pressure in the left ventricle with the pressure in the aortic root, while the effect of valve closure can be seen with the presence of the dicrotic notch.

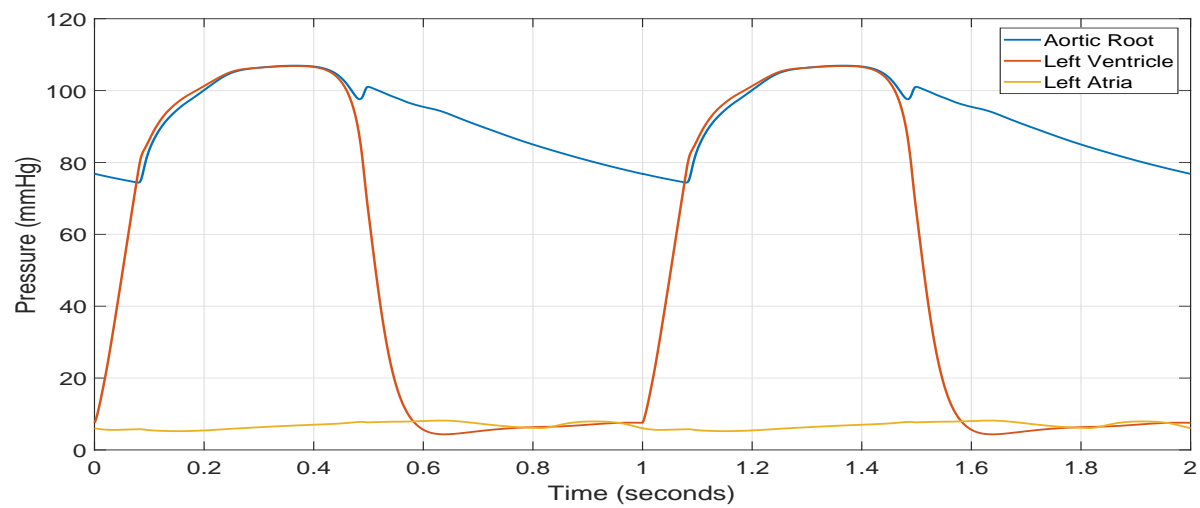

Figure 2.10: Comparison of left ventricular pressure and aortic root pressure 


\subsection{Mathematical Analysis of the One Dimensional Haemodynamics Equations}

In this section an overview of the typical approaches used in the analysis of the 1D system is described. The section will begin by studying the characteristic structure of the 1D blood flow equations, which allows the equations to be re-written in terms of forward and backward propagating characteristic variables. The section will continue by presenting the two main types of analysis for the one-dimensional blood flow equations: wave intensity analysis, which is performed in the time domain; and Fourier analysis methods, which are performed in the frequency domain. Finally, an analytical description of wave reflections is presented, which is valid for the linearised system of equations.

\subsubsection{Characteristic System}

In order to derive the characteristic system, the chain rule is often used on the pressure term in the momentum equation. For example, consider the 1D system written as

$$
\begin{gathered}
\frac{\partial A}{\partial t}+\frac{\partial Q}{\partial x}=0 \\
\frac{\partial Q}{\partial t}+\frac{\partial\left(\frac{Q^{2}}{A}\right)}{\partial x}+\frac{A}{\rho} \frac{\partial P}{\partial x}=-\frac{\zeta \pi \mu Q}{\rho A}
\end{gathered}
$$

together with the constitutive law $P-P_{\text {ext }}-P_{0}=\frac{\beta}{A_{0}}\left(\sqrt{A}-\sqrt{A_{0}}\right)$. Performing the chain rule on the spatial derivative for pressure gives (assuming $P_{0}$ is space independent)

$$
\frac{\partial P}{\partial x}=\frac{\partial P}{\partial A} \frac{\partial A}{\partial x}+\frac{\partial P}{\partial \beta} \frac{\partial \beta}{\partial x}+\frac{\partial P}{\partial A_{0}} \frac{\partial A_{0}}{\partial x}+\frac{\partial P}{\partial P_{e x t}} \frac{\partial P_{e x t}}{\partial x}
$$

This allows the system of equations to be written in quasi-linear form as

$$
\frac{\partial \boldsymbol{U}}{\partial t}+\boldsymbol{H} \frac{\partial \boldsymbol{U}}{\partial x}=\boldsymbol{S}
$$


Where

$$
\boldsymbol{U}=\left[\begin{array}{l}
A \\
Q
\end{array}\right], \quad \boldsymbol{H}=\left[\begin{array}{cc}
0 & 1 \\
\frac{\beta \sqrt{A}}{2 \rho A_{0}}-\frac{Q^{2}}{A^{2}} & 2 \frac{Q}{A}
\end{array}\right]
$$

and

$$
\boldsymbol{S}=\left[\begin{array}{c}
0 \\
-\frac{\zeta \pi \mu Q}{\rho A}-\frac{A}{\rho} \frac{\partial P}{\partial P_{e x t}} \frac{\partial P_{e x t}}{\partial x}-\frac{A}{\rho} \frac{\partial P}{\partial A_{0}} \frac{\partial A_{0}}{\partial x}-\frac{A}{\rho} \frac{\partial P}{\partial \beta} \frac{\partial \beta}{\partial x}
\end{array}\right] .
$$

The eigenvalues $\boldsymbol{\Lambda}$ are found by $\operatorname{det}(\boldsymbol{\Lambda I}-\boldsymbol{H})$, and are calculated as

$$
\boldsymbol{\Lambda}=\left[\begin{array}{l}
\lambda_{+} \\
\lambda_{-}
\end{array}\right]=\left[\begin{array}{l}
\frac{Q}{A}+\sqrt{\frac{\beta \sqrt{A}}{2 \rho A_{0}}} \\
\frac{Q}{A}-\sqrt{\frac{\beta \sqrt{A}}{2 \rho A_{0}}}
\end{array}\right]=\left[\begin{array}{l}
u+c \\
u-c
\end{array}\right],
$$

where $\lambda_{+}$and $\lambda_{-}$are the propagation speeds in the forward and backward direction respectively and $c=\sqrt{\frac{\beta \sqrt{A}}{2 \rho A_{0}}}$ is the wave speed. Solving $\boldsymbol{l}_{i} \boldsymbol{H}=\lambda_{i} \boldsymbol{l}_{i}$ gives the left eigenmatrix as

$$
\boldsymbol{L}=\left[\begin{array}{l}
\boldsymbol{l}_{1}^{T} \\
\boldsymbol{l}_{2}^{T}
\end{array}\right]=\left[\begin{array}{cc}
-\frac{Q}{A}+c, & 1 \\
-\left(\frac{Q}{A}+c\right), & 1
\end{array}\right] .
$$

Note that $\boldsymbol{H}$ can be written as

$$
\boldsymbol{H}=\boldsymbol{R} \bar{\Lambda} \boldsymbol{L}
$$

Where $\boldsymbol{R}$ is the right eigenvector, $\boldsymbol{L}$ is the left eigenvector and

$$
\bar{\Lambda}=\left[\begin{array}{cc}
\lambda_{+} & 0 \\
0 & \lambda_{-}
\end{array}\right]
$$

Noting that $\boldsymbol{L} \boldsymbol{R}=\boldsymbol{I}$, the identity matrix. System (2.35) can be written in an equivalent form

$$
\boldsymbol{L} \frac{\partial U}{\partial t}+\bar{\Lambda} \boldsymbol{L} \frac{\partial U}{\partial x}-\boldsymbol{L} \boldsymbol{S}=0
$$

If quantities $w_{+}$and $w_{-}$exist as

$$
\frac{\partial w_{+}}{\partial U}=\boldsymbol{l}_{1}, \quad \frac{\partial w_{-}}{\partial U}=\boldsymbol{l}_{2}
$$


then they are referred to as characteristic variables. Defining $\boldsymbol{W}=\left[\begin{array}{ll}w_{+}, & w_{-}\end{array}\right]$, system (2.42) can be written as

$$
\frac{\partial \boldsymbol{W}}{\partial t}+\bar{\lambda} \frac{\partial \boldsymbol{W}}{\partial t}+\boldsymbol{T}=\mathbf{0}
$$

with

$$
\boldsymbol{T}=-\boldsymbol{L} \boldsymbol{S}-\frac{\partial \boldsymbol{W}}{\partial A_{0}} \frac{\partial A_{0}}{\partial x}-\frac{\partial \boldsymbol{W}}{\partial \beta} \frac{\partial \beta}{\partial x} .
$$

An assumption is often made to simplify the problem, where it is assumed $A_{0}$ and $\beta$ are both constant, and the viscous resistance term is small enough to be neglected. Which results in $\boldsymbol{T} \approx 0$. The characteristic variables $w_{+}, w_{-}$can be calculated from

$$
w_{ \pm}=u-u_{0}+\int_{A_{0}}^{A} \frac{c}{A} d A=u-u_{0}+\int_{P_{0}}^{P} \frac{1}{\rho c} d P
$$

where $A_{0}, u_{0}$, and $P_{0}$ are reference values. The characteristic variables are determined to be

$$
w_{ \pm}=u-u_{0} \pm 4\left(c-c_{0}\right)
$$

or considering $u_{0}$ to be zero, and using the constitutive law $P-P_{\text {ext }}-P_{0}=$ $\frac{\beta}{A_{0}}\left(\sqrt{A}-\sqrt{A_{0}}\right)$, leads to

$$
w_{+}=\frac{Q}{A}+4\left(\sqrt{\frac{\beta \sqrt{A}}{2 \rho A_{0}}}-\sqrt{\frac{\beta}{2 \rho \sqrt{A_{0}}}}\right), \quad w_{-}=\frac{Q}{A}-4\left(\sqrt{\frac{\beta \sqrt{A}}{2 \rho A_{0}}}-\sqrt{\frac{\beta}{2 \rho \sqrt{A_{0}}}}\right) .
$$

It is also possible to calculate the primitive variables via manipulation of the characteristic variables which gives

$$
A=\left(\frac{w_{+}-w_{-}}{8}\right)^{4}\left(\frac{2 \rho A_{0}}{\beta}\right)^{2}, \quad u=\frac{w_{+}+w_{-}}{2},
$$

while the volumetric flow rate can be calculated using the relation $Q=A u$ and the pressure can be found from substituting $A$ into the constitutive law.

The forward propagating wave is governed by the forward travelling characteristic $w_{+}$, and is generated by ventricular contraction pumping blood into the arterial system (in the arteries); while the backward propagating wave is governed by the 
backward travelling characteristic $w_{-}$, and is caused by wave reflections which occur as a result of junctions and/or changes in vessel properties (material properties and/or geometry). Furthermore, these reflected waves are also re-reflected etc. and hence at any point in the vessel network, the pressure and flow rate waves can be considered as a combination of these forward and backward propagating waves.

\subsubsection{Wave Intensity Analysis Versus Fourier Analysis}

There are two main approaches to modelling wave propagation. Traditionally, Fourier analysis has been used in signal processing, which includes area such as audio signals, seismology and various wave propagation phenomena, including wave which occur in biological systems. Fourier analysis requires the signal to be converted into the frequency domain. This is performed by describing a complex waveform using a series of sinusoidal functions, whose frequencies form a harmonic series. For the purposes of studying haemodynamcs, this has the advantage of being capable of detecting small changes in frequency or the presence of additional frequencies, which may be due to changes in vascular stiffness, or changes in wave reflections due to pathological conditions such as stenosis or aneurysms. However, there also exist some shortcomings of working in the frequency domain, primarily:

- the circulatory system is not in steady state oscillation [259]. It is constantly adapting to the environment with changes in heart rate, pressures, flow rates, resistances, and compliances.

- Analysis in the frequency domain considers the cardiovascular system as a linear system, which is certainly not the case as significant non-linearities exist, with regards to both flow behaviour and vessel wall behaviour. This is particularly true for the venous system, where vessels are capable of collapsing, and many valves are present to encourage and support blood return to the heart.

- It is generally not possible to relate a specific harmonic to an event in time, as an entire heart beat or multiple heart beats are used to calculate the spectrum of frequencies.

In order to overcome these problems, [260, 258] proposed a method which can allow the separation and study of the forward and backward propagating waves. The 
method eventually became known as wave intensity analysis (WIA) [259], and although it is more mathematically intensive than Fourier analysis, WIA overcomes some of its shortcomings. WIA is performed in the temporal domain, allowing specific events to be studied in time, which may lead to an improved understanding of the underlying mechanisms involved in creating this specific event. WIA uses the method of characteristics, which is described in section 2.4.1, as a basis for the analysis. In addition, separation of waves can be performed linearly [259], or non-linearly [106].

Derivation of the WIA requires the method of characteristics, with equation (2.46) being used as a basis. Expressing the characteristic variable in terms of the pressure $P$ instead of using the cross-sectional area $A$, finding the differences between two points in the space-time plane for characteristic variables which intersect, gives the following the difference equation

$$
d w_{ \pm}=d U \pm \frac{d P}{\rho c}
$$

where $w_{ \pm}, d P$ and $d U$ are the differences in the characteristic variables, pressures and velocities over the time interval $\Delta t$. The change in pressure and change in velocity can then be written in terms of the change in characteristic variables (by adding and subtracting both equations) to give

$$
d P=\frac{\rho c}{2}\left(d w_{+}-d w_{-}\right), \quad d U=\frac{1}{2}\left(d w_{+}+d w_{-}\right) .
$$

The product of $d P$ and $d U$ is called the wave intensity $d I$, and is defined as

$$
d I=d P d U=\frac{\rho c}{4}\left(d w_{+}^{2}-d w_{-}^{2}\right)
$$

which has a positive contribution to wave intensity for forward travelling waves, and a negative contribution for backward travelling waves.

The waves can also be separated (linearly) into forward and backward propagating waves by defining the overall change in pressure and velocity are from the addition of forward and backward components

$$
d P=d P_{+}+d P_{-}, \quad d U=d U_{+}+d U_{-} .
$$




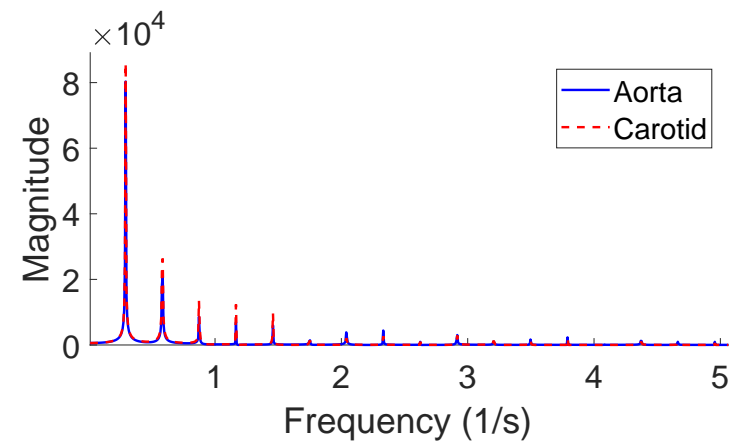

(a) Frequency domain analysis of pressure

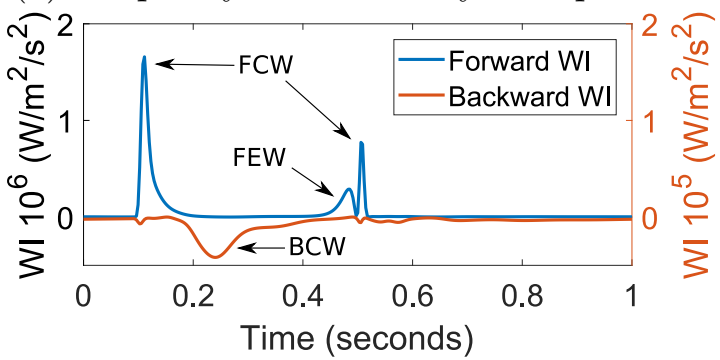

(c) Wave intensity in ascending aorta

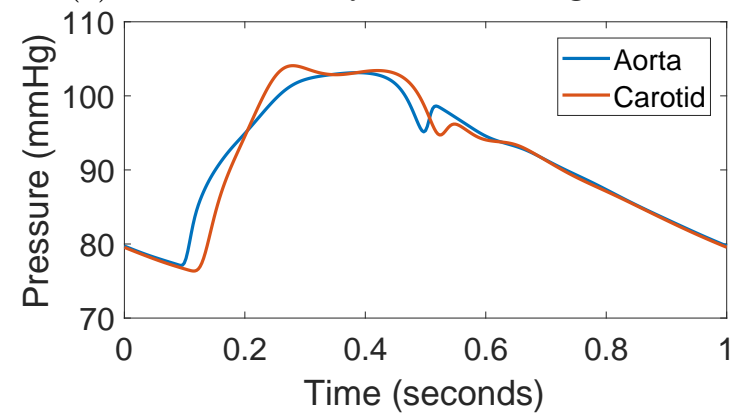

(e) Pressure waveform

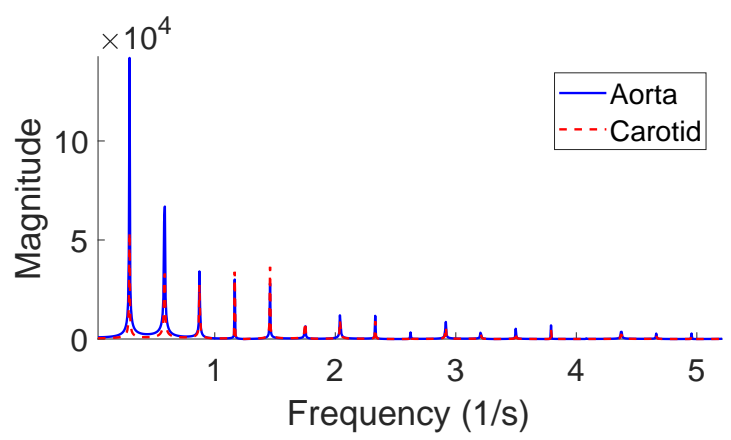

(b) Frequency domain analysis of velocity

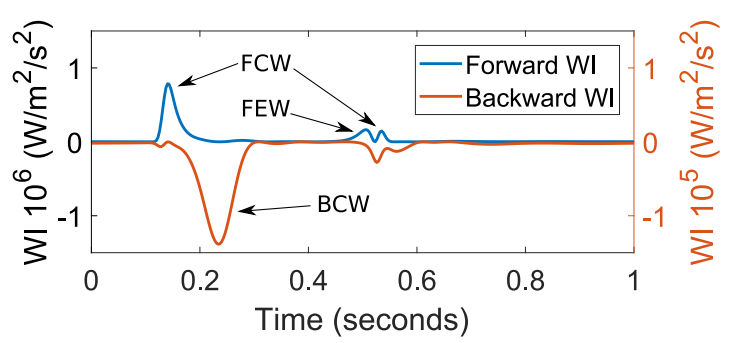

(d) Wave intensity in left carotid

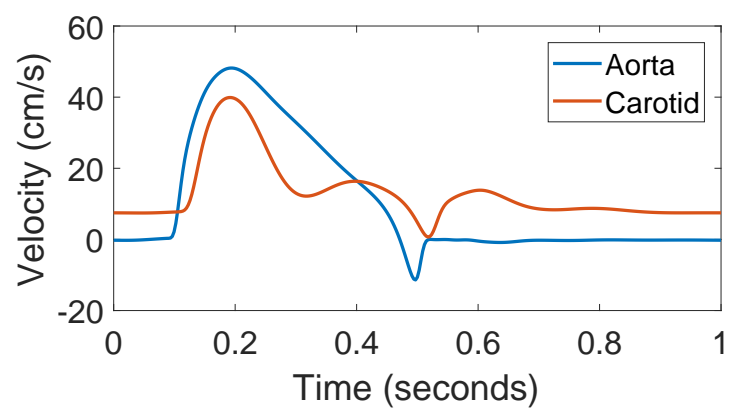

(f) Velocity Waveform

Figure 2.11: Frequency domain analysis, forward (F WI) and backward (B WI) wave intensity, and pressure and velocity waveforms in the ascending aorta and left common carotid artery. The main forward and backward waves are abbreviated as follows: FCW - the forward compression wave, FEW - forward expansion wave, $\mathrm{BCW}$ - backward compression wave

By considering forward components for forward pressure and velocity, and backward components for backward pressure and velocity from equation 2.51, the following is found

$$
\begin{array}{cc}
d P_{+}=\frac{\rho c}{2} d w_{+}, & d P_{-}=-\frac{\rho c}{2} d w_{-} \\
d U_{+}=\frac{1}{2} d w_{+}, & d U_{-}=\frac{1}{2} d w_{-} .
\end{array}
$$




\subsection{MATHEMATICAL ANALYSIS OF THE ONE DIMENSIONAL HAEMODYNAMICS EQUATIONS}

Or writing in terms of the overall pressure and velocity change, the forward and backward pressure and velocity waves can be written as

$$
d P_{ \pm}=\frac{1}{2}(d P \pm \rho c d U), \quad d U_{ \pm}=\frac{1}{2}\left(d U \pm \frac{d P}{\rho c}\right)
$$

Thus calculation of the forward and backward wave intensity can be described as

$$
d I_{ \pm}=d P_{ \pm} d U_{ \pm}=\frac{ \pm 1}{4 \rho c}(d P \pm \rho c d U)^{2}
$$

A time corrected version of wave intensity was proposed in [279]. The main reason for this time correction was due to the majority of signals used in analysis being at discrete points, thus the magnitude of wave intensity was also dependant on the sample rate of the signal. The time corrected version is found using

$$
w i=\frac{d p}{d t} \frac{d u}{d t}
$$

The time corrected forward and backward wave intensity, when performing linear wave separation is

$$
w i_{ \pm}=\frac{ \pm 1}{4 \rho c}\left(\frac{d P}{d t} \pm \rho c \frac{d U}{d t}\right)^{2} .
$$

A non-linear wave separation can be performed by considering the wave speed to also vary in the forward and backward direction, which means the wave intensity is

$$
d w_{ \pm}=d U \pm \frac{1}{\rho c_{ \pm}} d P
$$

Using this equation and following the previous steps to find the time corrected forward and backward wave intensity [227] gives

$$
w i_{ \pm}=\frac{ \pm \rho c}{4}\left(\frac{d w_{ \pm}}{d t}\right)^{2}
$$

The wave intensity used in combination with $d P_{ \pm}$and $d U_{ \pm}$can be used to determine the type of wave.

- Forward expansion wave (FEW) - where $d P d U>0$ with a deceleration $d U<0$ and an expansion $d P<0$ wave. 
- Forward compression wave (FCW) - where $d P d U>0$ with an acceleration $d U>0$ and a compression $d P>0$ wave.

- Forward expansion wave $(\mathrm{BEW})$ - where $d P d U<0$ with an acceleration $d U>$ 0 and an expansion $d P<0$ wave.

- Forward compression wave $(\mathrm{BCW})$ - where $d P d U<0$ with a deceleration $d U<0$ and a compression $d P>0$ wave

A fast Fourier transform (fft) can be performed on the pressure or velocity waveforms to convert them from the time domain into the frequency domain. Figure 2.11 compares the frequency domain analysis for pressure and velocity, the time-corrected forward and backward wave intensities, and the pressure and velocity waveforms in the ascending aorta and carotid artery. The wave intensity analysis can be used to detect specific events and reflections such as the first forward compression wave being caused by the heart; while the frequency domain combines the waves from the entire cardiac cycle and detects changes in frequency due to reflections and transmissions, making it more difficult to make conclusions on a specific event.

\subsubsection{Wave Reflection and Transmission}

An analytical approach can determine the magnitude of wave reflections and transmissions caused by changes in vessel properties or at vessel junctions [365, 8]. The analysis relies on the linearised system from equation (2.23), and the admittance of the vessel. The admittance $Y$ is the inverse of the characteristic impedance $Z$ and is given by

$$
Y=\frac{1}{Z}=\frac{A_{r e f}}{\rho c_{r e f}}
$$

for a reference area $A_{r e f}$ and wave speed $c_{r e f}$, and the fluid density $\rho$.

For a change in material properties and/or geometry, an incoming wave will be partially reflected. The reflection and transmitted coefficients can be determined as

$$
R_{r e f}=\frac{Y_{u}-Y_{d}}{Y_{u}+Y_{d}}, \quad R_{t r a n}=\frac{Y_{d}-Y_{u}}{Y_{u}+Y_{d}}
$$

where subscript $u$ and $d$ are the upstream and downstream position with respect to the change in vessel properties. For any perturbation in the forward travelling 


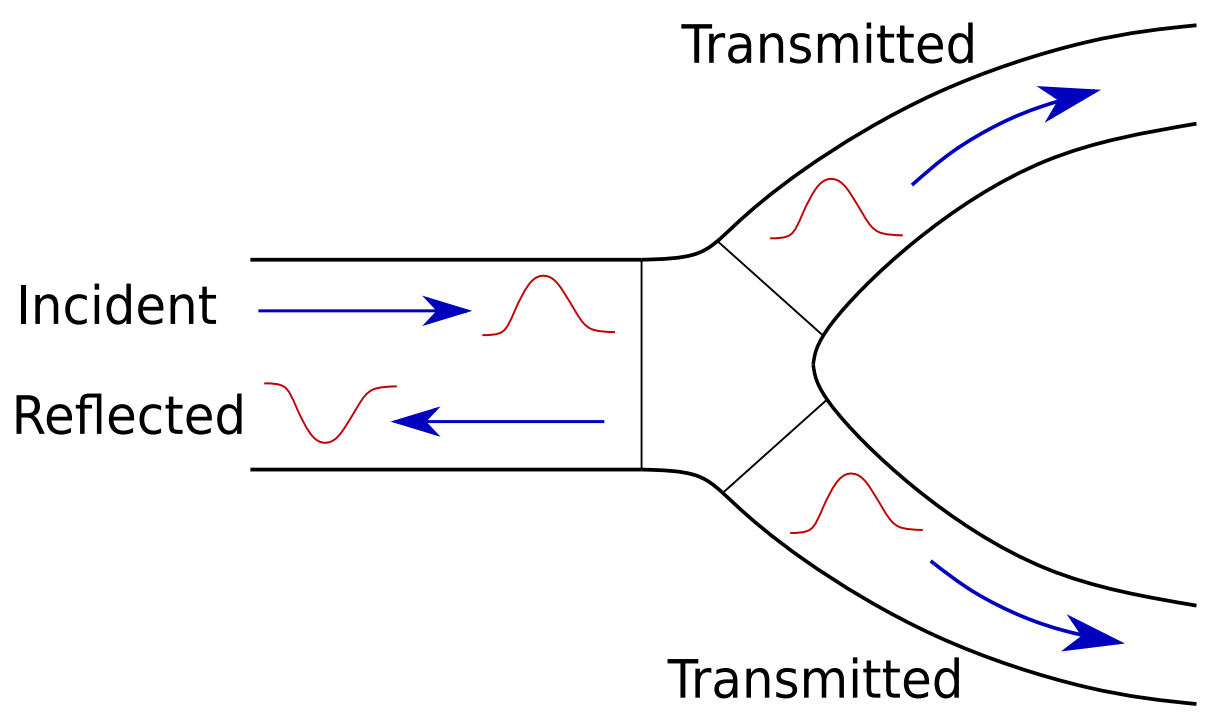

Figure 2.12: Overview of reflected and transmitted wave from an incident wave at a bifurcation.

incident pressure wave the reflected pressure can be determined as

$$
\delta P_{\text {ref }}=\delta P_{\text {inc }} R_{\text {ref }}
$$

The transmitted pressure wave can be found from

$$
\delta P_{\text {tran }}=\delta P_{\text {inc }}+\delta P_{\text {ref }}=\left(1+R_{\text {ref }}\right) \delta P_{\text {inc }} .
$$

The transmitted flow rate can be found by considering the conservation of mass at a point in the domain

$$
\delta Q_{i n c}=\delta Q_{t r a n}-\delta Q_{r e f}
$$

where the reflected flow rate can be determined from

$$
\delta Q_{\text {ref }}=-\delta Q_{\text {inc }} R_{\text {ref }}
$$

This process can be extended to determine the reflection of waves at vessel junctions. For example, at a bifurcation as shown in figure 2.12, the reflection coefficients can be determined as

$$
R_{r e f}=\frac{Y_{p}-Y_{d 1}-Y_{d 2}}{Y_{p}+Y_{d 1}+Y_{d 2}}, \quad R_{d 1}=\frac{Y_{d 1}-Y_{d 2}-Y_{p}}{Y_{d 1}+Y_{d 2}+Y_{p}}, \quad R_{d 2}=\frac{Y_{d 2}-Y_{d 1}-Y_{p}}{Y_{d 1}+Y_{d 2}+Y_{p}}
$$


where subscripts p, d1, and d2 represent the parent vessel, and the first and second daughter vessels, respectively. A process that is similar to the one used for a single vessel can be performed to find the reflected and transmitted pressure and flow waves from a given incident wave.

\subsection{Concluding Remarks}

This chapter presented the mathematical formulation of reduced order modelling, and the modelling components utilised in this thesis. The chapter began by describing the reduction of dimensionality of the three-dimensional incompressible NavierStokes equations, which leads to the derivation of the one-dimensional blood flow equations. The components of the one-dimensional blood flow model were described, which included a description of the different velocity profiles, and constitutive laws that are implemented in the literature. The zero-dimensional model, often referred to as a lumped parameter model, is then derived from the one-dimensional system of equations by performing a linearisation around the reference state, averaging the properties of a vessel segment, and then integrating the equations in space. The various types of lumped parameter models used in reduced-order cardiovascular modelling were then presented, such as models of: the vascular beds, which included specialised models of the liver and coronary vascular beds; the heart, which included all four heart chambers; the heart valves and systemic venous valves. Finally, the different analytical techniques that are commonly utilised for the analysis of the one-dimensional blood flow equations were described. The analytical methods included a characteristic analysis of the one-dimensional system of equations, wave intensity analysis, and wave reflection and transmission theory. 


\section{Chapter 3}

\section{Numerical Schemes}

In this chapter, details of the most common numerical schemes used to solve the one-dimensional blood flow equations are presented. The numerical scheme used in this thesis, including the linearisation and discretisation of the blood flow equations, are described in detail for all model components. These components include: 1D vessels and 1D vessel junctions, 0D models representing vascular beds, the heart and valves, and connectivity between 1D and 0D models. Parameter estimation techniques which are used in conjunction with the numerical scheme are described in chapter 6 .

\subsection{D Schemes}

There have been many important contributions to the development of 1D cardiovascular models, with early works by Hughes and Lubliner [160], Stergiopoulos et al. [324, 325] and Brook et al. [45]. The field of 1D cardiovascular modelling received renewed interest in the early 2000s with Olufsen et al. [244], Formaggia et al. [116, 117], Sherwin et al. [314, 313]. This was followed by further developments to the field: proposing new numerical methods and coupling procedures such as Mynard and Nithiarasu [224], Müller and Toro [216, 220], Blanco and Malossi et al. [35, 199], and Formaggia [118, 119]; new arterial and venous networks [194, 37, 36, 216, 359, 220, 230]; model validation and comparisons [205, 31, 244, 287, 288, 12, 228, 41, 42, 349, 357]; auto-regulation [10, 169]; clinical related topics [225, 156, 188]; model components such as velocity profile [30], heart and valve models [119, 226, 180, 252]; and closed loop models [34, 230, 216, 220].

Several studies have compared different numerical schemes. Wang et al. [357] compared the local nodal discontinuous Galerkin, MacCormack, Taylor-Galerkin, and the finite volume scheme, monotonic upwind scheme for conservation laws (MUSCL). Comparisons were performed on a single vessel, a bifurcation, and a 55 vessel arterial network. The MacCormack scheme performed well when nonlinearities were small, but struggled for cases which included larger gradients; the MUSCL scheme performed best if shock-like phenomena were present; the Taylor- 
Galerkin scheme struggled with shock-like phenomena, and was the slowest of the schemes; the discontinuous Galerkin scheme performed well for systems with small physical diffusion. A benchmark study by Boileau et al. [41] compared six numerical schemes for a range of problems, including comparisons with problems that had analytic solutions, and also compared the $1 \mathrm{D}$ schemes with 3D solutions, and in-vitro solutions. The six schemes were: the locally conservative Galerkin [224], a modal discontinuous Galerkin [314], a Galerkin least-squares finite element method [34], finite volume method [216], a MacCormack scheme [188], and simplified trapezoidal rule method (STM) [182]. Results were generally consistent among the schemes, although the STM scheme deviated from the other schemes for an arterial network consisting of 55 arteries. The reason why the STM scheme deviated from the other schemes in this case was due to the restriction of using conservation of static pressure as a boundary condition at vessel junctions, while the other numerical schemes in [41] implemented conservation of total pressure at junctions. This essentially means that rather than any problems with accuracy, the STM scheme was actually solving a different system of equations compared to the other schemes in [41]. The scheme proposed in this thesis generalises the STM scheme, and overcomes the restriction at vessel junctions by allowing any type of pressure conservation (including methods involving estimates for pressure/energy loses) to be imposed as a boundary condition at vessel junctions.

\subsection{Enhanced Trapezoidal Rule Method}

In this thesis the enhanced trapezoidal rule method is proposed. Some parts of this section have been published in [64], however the description of the numerical model in this thesis is much more thorough and complete compared with that published in [64] as additional modelling components are introduced. The enhanced trapezoidal rule method (ETM) scheme is essentially an extension to the method proposed by Kroon et al. [182]. The ETM scheme can be considered to be a sub-domain collocations method, which is technically on the boundary of finite element (FEM) and finite volume methods, as it is part of a class of weighted residual methods (FEM), yet can also be viewed as the generalisation and basis of a FVM.

Consider the following PQ formulation from equation (2.16) using the velocity 
profile given by equation (2.17) and a visco-elastic constitutive law equation. The continuity equation is

$$
\frac{\partial A}{\partial P} \frac{\partial P_{t m}}{\partial t}+\frac{\partial Q}{\partial x}=\Phi
$$

the momentum equation is given by

$$
\frac{\rho}{A} \frac{\partial Q}{\partial t}+\frac{\rho}{A} \frac{\partial\left(\alpha \frac{Q^{2}}{A}\right)}{\partial x}+\frac{\partial P}{\partial x}-\frac{\zeta \mu \pi Q}{A^{2}}=0
$$

and the visco-elastic constitutive law is

$$
P-P_{0}-P_{e x t}=\frac{2 \rho c_{0}^{2}}{b}\left(\left(\frac{A}{A_{0}}\right)^{b / 2}-1\right)+\frac{\Gamma}{A_{0} \sqrt{A}} \frac{\partial A}{\partial P} \frac{\partial P}{\partial t} .
$$

The scheme can be derived using a second order backward difference discretisation in time and the trapezoidal rule in space. Firstly the formulation is modified slightly by moving the viscous term in the constitutive law to the momentum equation. Thus the momentum equation becomes

$$
\frac{\rho}{A} \frac{\partial Q}{\partial t}+\frac{\rho}{A} \frac{\partial\left(\alpha \frac{Q^{2}}{A}\right)}{\partial x}+\frac{\partial P}{\partial x}+\frac{\partial}{\partial x}\left(\frac{\Gamma}{A_{0} \sqrt{A}} \frac{\partial A}{\partial P} \frac{\partial P}{\partial t}\right)-\frac{\zeta \mu \pi Q}{A^{2}}=0
$$

Moreover, the compliance calculation only considers the elastic part of the constitutive law as the viscous term is much smaller in magnitude, which can be written as

$$
\frac{\partial A}{\partial P}=C_{A}=\frac{A_{0}}{\rho c_{0}^{2}}\left(\left(P-P_{0}-P_{e x t}\right) \frac{b}{2 \rho c_{0}^{2}}+1\right)^{\left(\frac{2}{b}-1\right)} .
$$

\subsubsection{Linearisation}

In order to discretise the non-linear system of equations, the terms are linearised in the following way

$$
\begin{gathered}
C_{A}^{n+1} \approx C_{A}^{n+1, k}, \quad Q^{n+1} \approx Q^{n+1, k+1}, \quad P^{n+1} \approx P^{n+1, k+1}, \\
{\frac{Q^{2}}{A}}^{n+1}{\frac{Q^{2}}{A}}^{n+1, k}, \quad \frac{\rho}{A}^{n+1} \approx \frac{\rho}{A}^{n+1, k}, \quad\left(\frac{8 \mu \pi Q}{A^{2}}\right)^{n+1} \approx\left(\frac{8 \mu \pi Q}{A^{2}}\right)^{n+1, k},
\end{gathered}
$$




$$
\left(\frac{\Gamma}{A_{0} \sqrt{A}} C_{A}\right)^{n+1} \approx\left(\frac{\Gamma}{A_{0} \sqrt{A}} C_{A}\right)^{n+1, k},
$$

where superscript $k$ is the iteration level, and as an initial guess $k=1 \approx n$. However, in this thesis only the first iteration is performed for the majority of cases, which means $(n+1, k) \approx(n)$ and $(n+1, k+1) \approx(n+1)$. The choice of performing only one iteration, and the convergence of the iterative scheme, will be demonstrated and discussed in Chapter 4. The linearised system then has the form

$$
\begin{gathered}
C_{A}^{n} \frac{\partial P}{\partial t}+\frac{\partial Q^{n+1}}{\partial x}=0 \\
\frac{\rho}{A^{n}} \frac{\partial Q}{\partial t}+\frac{\partial P^{n+1}}{\partial x}+\frac{\partial}{\partial x}\left(\left(\frac{C_{A} \Gamma}{A_{0} \sqrt{A}}\right)^{n} \frac{\partial P}{\partial t}\right)=\left(-\frac{8 \mu \pi Q}{A^{2}}-\frac{\rho}{A} \frac{\partial\left(\frac{Q^{2}}{A}\right)}{\partial x}\right)^{n}
\end{gathered}
$$

where equations (3.7) and (3.8) are the continuity equation and momentum equation respectively.

\subsubsection{Discretisation}

Integration within the domain is performed by partitioning each vessel into smaller intervals and integrating each of these intervals using the trapezoidal rule. Thus integration over the length of a vessel can be considered as the composite trapezoidal rule (second-order accurate), and creates two nodes per element. Integrating each term individually in the continuity equation for an element gives

$$
\begin{gathered}
\int_{e}\left(C_{A}^{n} \frac{\partial P}{\partial t}\right) d x=\frac{\Delta x}{2}\left(C_{A, 1}^{n} \frac{\partial P_{1}}{\partial t}+C_{A, 2}^{n} \frac{\partial P_{2}}{\partial t}\right), \\
\int_{e}\left(\frac{\partial Q^{n+1}}{\partial x}\right) d x=\left(Q_{2}^{n+1}-Q_{1}^{n+1}\right) .
\end{gathered}
$$

Integrating the momentum equation gives

$$
\int_{e}\left(\frac{\rho}{A^{n}} \frac{\partial Q}{\partial t}\right) d x=\frac{\Delta x}{2}\left(\frac{\rho}{A_{1}^{n}} \frac{\partial Q_{1}}{\partial t}+\frac{\rho}{A_{2}^{n}} \frac{\partial Q_{2}}{\partial t}\right) .
$$


The second term of the momentum equation is

$$
\int_{e}\left(\frac{\partial P^{n+1}}{\partial x}\right) d x=\left(P_{2}^{n+1}-P_{1}^{n+1}\right)
$$

The third term is integrated as

$$
\int_{e} \frac{\partial}{\partial x}\left(\left(\frac{C_{A} \Gamma}{A_{0} \sqrt{A}}\right)^{n} \frac{\partial P}{\partial t}\right) d x=\left(\left(\frac{C_{A} \Gamma}{A_{0} \sqrt{A}}\right)^{n} \frac{\partial P}{\partial t}\right)_{2}-\left(\left(\frac{C_{A} \Gamma}{A_{0} \sqrt{A}}\right)^{n} \frac{\partial P}{\partial t}\right)_{1}
$$

Letting the right side of Equation (3.8) be

$$
h=\left(\frac{-8 \pi \mu Q}{A^{2}}-\frac{\rho}{A} \frac{\partial\left(\frac{Q^{2}}{A}\right)}{\partial x}\right)^{n} .
$$

Integrating gives

$$
\int_{e}(h)^{n} d x=\frac{\Delta x}{2}\left(h_{1}+h_{2}\right)^{n} .
$$

This indicates that the mass and momentum is conserved at the element centre, while the variables are defined on the nodes. Using the following second-order backward difference scheme for the time derivatives $\left(\frac{\partial U}{\partial t} \approx \frac{3}{2 \Delta t} U^{n+1}-\frac{2}{\Delta t} U^{n}+\frac{1}{2 \Delta t} U^{n-1}\right)$ gives the system in the form

$$
\begin{aligned}
& {\left[\begin{array}{cc}
\frac{3}{2 \Delta t} \frac{\Delta x}{2} C_{A, 1}^{n}, & \frac{3}{2 \Delta t} \frac{\Delta x}{2} C_{A, 2}^{n} \\
-1-\left(\left(\frac{C_{A} \Gamma}{A_{0} \sqrt{A}}\right)^{n} \frac{3}{2 \Delta t}\right)_{1}, & 1+\left(\left(\frac{C_{A} \Gamma}{A_{0} \sqrt{A}}\right)^{n} \frac{3}{2 \Delta t}\right)_{2}
\end{array}\right]\left[\begin{array}{l}
P_{1} \\
P_{2}
\end{array}\right]_{e}^{n+1}} \\
& +\left[\begin{array}{cc}
-1, & 1 \\
\frac{3}{2 \Delta t} \frac{\Delta x}{2} \frac{\rho}{A_{1}^{n}}, & \frac{3}{2 \Delta t} \frac{\Delta x}{2} \frac{\rho}{A_{2}^{n}}
\end{array}\right]\left[\begin{array}{l}
Q_{1} \\
Q_{2}
\end{array}\right]_{e}^{n+1}=\left[\begin{array}{c}
0 \\
\frac{\Delta x}{2}\left(h_{1}^{n}+h_{2}^{n}\right)
\end{array}\right]_{e} \\
& +\left[\begin{array}{l}
\frac{\Delta x}{2}\left(C_{A}^{n} \frac{2}{\Delta t} P^{n}-C_{A}^{n} \frac{1}{2 \Delta t} P^{n-1}\right)_{1} \\
\frac{\Delta x}{2}\left(\frac{\rho}{A^{n}} \frac{2}{\Delta t} Q^{n}-\frac{\rho}{A^{n}} \frac{1}{2 \Delta t} Q^{n-1}\right)_{1}
\end{array}\right]_{e}+\left[\begin{array}{l}
\frac{\Delta x}{2}\left(C_{A}^{n} \frac{2}{\Delta t} P^{n}-C_{A}^{n} \frac{1}{2 \Delta t} P^{n-1}\right)_{2} \\
\frac{\Delta x}{2}\left(\frac{\rho}{A^{n}} \frac{2}{\Delta t} Q^{n}-\frac{\rho}{A^{n}} \frac{1}{2 \Delta t} Q^{n-1}\right)_{2}
\end{array}\right]_{e} \\
& -\left[\left(\left(\frac{C_{A} \Gamma}{A_{0} \sqrt{A}}\right)^{n}\left(\frac{2}{\Delta t} P^{n}-\frac{1}{2 \Delta t} P^{n-1}\right)\right)_{1}\right]_{e}+\left[\left(\left(\frac{C_{A} \Gamma}{A_{0} \sqrt{A}}\right)^{n}\left(\frac{2}{\Delta t} P^{n}-\frac{1}{2 \Delta t} P^{n-1}\right)\right)_{2}\right]_{e} \text {. }
\end{aligned}
$$


Which can be generalised to

$$
\boldsymbol{F}_{e} \boldsymbol{P}_{e}^{n+1}+\boldsymbol{G}_{e} \boldsymbol{Q}_{e}^{n+1}=\boldsymbol{h}_{e}^{n},
$$

where subscript $e$ represents the elemental system of equations, and

$$
\begin{gathered}
\boldsymbol{F}_{e}=\left[\begin{array}{cc}
\frac{3}{2 \Delta t} \frac{\Delta x}{2} C_{A, 1}^{n}, & \frac{3}{2 \Delta t} \frac{\Delta x}{2} C_{A, 2}^{n} \\
-1-\left(\left(\frac{C_{A} \Gamma}{A_{0} \sqrt{A}}\right)^{n} \frac{3}{2 \Delta t}\right)_{1}, & 1+\left(\left(\frac{C_{A} \Gamma}{A_{0} \sqrt{A}}\right)^{n} \frac{3}{2 \Delta t}\right)_{2}
\end{array}\right], \\
\boldsymbol{P}_{e}^{n+1}=\left[\begin{array}{c}
P_{1} \\
P_{2}
\end{array}\right]_{e}^{n+1}, \quad \boldsymbol{G}_{e}=\left[\begin{array}{cc}
-1, & 1 \\
\frac{3}{2 \Delta t} \frac{\Delta x}{2} \frac{\rho}{A_{1}^{n}}, & \frac{3}{2 \Delta t} \frac{\Delta x}{2} \frac{\rho}{A_{2}^{n}}
\end{array}\right], \quad \boldsymbol{Q}_{e}^{n+1}=\left[\begin{array}{l}
Q_{1} \\
Q_{2}
\end{array}\right]_{e}^{n+1},
\end{gathered}
$$

and $\boldsymbol{h}_{e}^{n}$ is the right-hand side of Equation (3.16).

The second term on the right side of Equation (3.14) uses the following first-order upwind discretisation for node $i$ for an elastic constitutive law, while a central difference discretisation is performed if a visco-elastic constitutive law is implemented. For node $i$ the upwind scheme can be written as

$$
\frac{\partial\left(\frac{Q^{2}}{A}\right)^{n}}{\partial x} \approx\left\{\begin{array}{l}
{\left[\left(\frac{Q^{2}}{A}\right)_{i}-\left(\frac{Q^{2}}{A}\right)_{i-1}\right] \frac{1}{\Delta x} \quad \text { if } \quad Q_{i}^{n}>0} \\
{\left[\left(\frac{Q^{2}}{A}\right)_{i+1}-\left(\frac{Q^{2}}{A}\right)_{i}\right] \frac{1}{\Delta x} \quad \text { if } \quad Q_{i}^{n}<0}
\end{array}\right.
$$

and a ghost node is used at the vessel boundaries when needed.

A modification at the elemental is performed by enforcing flows to be directed inwards (defined to point towards element centre). This causes a change of the sign (to a negative) of the second row of $\boldsymbol{Q}_{e}$ (Q at the second node) and changing the sign of the second column of the matrix $\boldsymbol{G}_{e}$.

$$
\boldsymbol{G}_{e}^{c}=\left[\begin{array}{ll}
G_{11} & G_{12} \\
G_{21} & G_{22}
\end{array}\right], \quad \text { becomes } \boldsymbol{G}_{\boldsymbol{e}}=\left[\begin{array}{ll}
G_{11} & -G_{12} \\
G_{21} & -G_{22}
\end{array}\right]
$$

While flows $Q_{e}=\left[Q_{1}, Q_{2}\right]^{T}$ become $Q_{e}=\left[Q_{1},-Q_{2}\right]^{T}$. This means that the system equations will still be the same as before when multiplied out. However this will significantly change the meaning of the flow rate column at the global level. Matrix 
$\boldsymbol{G}_{\boldsymbol{e}}$ is then inverted so the final elemental system of equations is given by

$$
\left[-\boldsymbol{G}_{\boldsymbol{e}}^{-1} \boldsymbol{F}_{e}\right] \boldsymbol{P}_{e}^{n+1}=\left[-\boldsymbol{G}_{e}{ }^{-1} \boldsymbol{h}_{e}^{n}\right]+\boldsymbol{Q}_{e}^{n+1}
$$

Once these elemental systems are assembled into the global system matrix it can be seen that the flow column $\boldsymbol{Q}_{g}^{n+1}$ becomes the conservation of mass between connected elements and is zero for all internal nodes. In essence the flow rates at the local element level become a column of mass change between elements at the global assembled level. This global mass change (flow) vector only has non-zero values on the boundaries, which represent external flows, and are accounted for through the boundary conditions. Thus the global mass change vector looks like

$\boldsymbol{Q}_{g}^{n+1}=\left[Q_{\text {inflow }}, 0, \ldots, 0, Q_{\text {outflow }}\right]$. This can also have major implications on the implementation of vessel junction conditions, such as for bifurcations, as the continuity of mass is automatically satisfied and when the same pressure node is used (last node in parent and first nodes in daughters), the conservation of static pressure is also satisfied (as performed in the STM scheme [182]).

However, this limits the STM method as only conservation of static pressure can be imposed (it is not possible to use total pressure, or any pressure loss terms). Therefore the ETM method extends the existing STM method to allow conservation of total pressure (or any pressure loss method) to be applied between vessel segments, the ETM uses Lagrange multipliers to hold conservation of total pressure as a system constraint. This difference only occurs at vessel junctions as will be discussed in section 3.2.5. Once the global matrix is assembled the implicit system is solved for pressures and Lagrange multipliers, then the flows are updated using the elemental system (3.21).

\subsubsection{Example of a 1D-1D Element Connectivity}

Consider two neighbouring elements within the same vessel with

$$
\left[-\boldsymbol{G}_{\boldsymbol{e}}^{-1} \boldsymbol{F}_{e}\right]=\boldsymbol{K}_{e}, \quad \text { and } \quad\left[-\boldsymbol{G}_{\boldsymbol{e}}^{-1} \boldsymbol{h}_{e}^{n}\right]=\boldsymbol{f}_{e}
$$

which means Equation (3.21) will take the form

$$
\boldsymbol{K}_{e} \boldsymbol{P}_{e}^{n+1}=\boldsymbol{f}_{e}^{n}+\boldsymbol{Q}_{e}^{n+1}
$$


where

$$
\boldsymbol{K}_{e}=\left[\begin{array}{cc}
K_{11}^{e} & K_{12}^{e} \\
K_{21}^{e} & K_{22}^{e}
\end{array}\right], \quad \boldsymbol{f}_{e}^{n}=\left[\begin{array}{l}
f_{1} \\
f_{2}
\end{array}\right],
$$

and $\boldsymbol{P}_{e}^{n+1}=\left[\begin{array}{ll}P_{1} & P_{2}\end{array}\right]^{T}, \boldsymbol{Q}_{e}^{n+1}=\left[\begin{array}{ll}Q_{1} & -Q_{2}\end{array}\right]^{T}$. Element one has pressure nodes $P_{1}$ and $P_{2}$ and flows $Q_{1}$ and $Q_{2}$, and element two has pressure nodes $P_{2}$ and $P_{3}$ and flows $Q_{3}$ and $Q_{4}$ respectively. Assembling these two elemental system leads to the system,

$$
\left[\begin{array}{ccc}
K_{11}^{1} & K_{12}^{1} & 0 \\
K_{21}^{1} & K_{22}^{1}+K_{11}^{2} & K_{12}^{2} \\
0 & K_{21}^{2} & K_{22}^{2}
\end{array}\right]\left[\begin{array}{c}
P_{1} \\
P_{2} \\
P_{3}
\end{array}\right]^{n+1}=\left[\begin{array}{c}
f_{1}^{1} \\
f_{1}^{2}+f_{2}^{1} \\
f_{2}^{2}
\end{array}\right]+\left[\begin{array}{c}
Q_{1} \\
-Q_{2}+Q_{3} \\
-Q_{4}
\end{array}\right]^{n+1}
$$

where the superscripts on $K$ and $f$ represent which element they belong to. The conservation of mass between element one and two requires the outflow of element one $\left(Q_{2}\right)$ to be equal to inflow of element two $\left(Q_{3}\right)$, hence $Q_{2}=Q_{3}$ or rearranged gives $0=Q_{3}-Q_{2}$. This is exactly the middle term of the flow vector (last term on right) in Equation (3.25). Thus only inflow $Q_{1}$ and outflow $Q_{4}$ (boundary conditions) of this column vector are potentially non-zero, where either pressure or flow rate would be prescribed. This implicit use of conservation of mass occurs when: 1D elements connect with other 1D elements; when 1D elements connect to one, or multiple lumped (0D) elements; and when any number of lumped elements connects to any number of other $0 \mathrm{D}$ elements.

The inlet boundary condition for the benchmark problems is given as a flow rate, which is easily implemented in the ETM scheme using the external flow column vector $\boldsymbol{Q}_{g}^{n+1}$. If the pressure is required as a boundary condition then this can be imposed as a Dirichlet boundary condition. At vessel junctions Lagrange multipliers are used to constrain conservation of total pressure which will be discussed in section 3.2 .5 .

The outlet of the 1D system is often connected to a lumped parameter model, whether it is a heart model, valve model, or vascular bed model. As an example to show the connectivity of a $1 \mathrm{D}$ element with a $0 \mathrm{D}$ element, consider the 3-element Windkessel model, which is constructed from a resistor $R_{1}$ (often characteristic impedance) in series with a resistor $R_{2}$ (peripheral resistance) and capacitor C (compliance) in parallel. 
The Windkessel model can be treated using a combination of the three basic elemental equations, as described in equation (2.25). The 3-elements, each described by two pressure nodes and two flow rate nodes, which can be expressed as

$$
\begin{gathered}
\frac{1}{R_{1}}\left(P_{1}^{n+1}-P_{2}^{n+1}\right)=q_{1}^{n+1}=-q_{2}^{n+1}, \\
\frac{1}{R_{2}}\left(P_{2}^{n+1}-P_{3}^{n+1}\right)=q_{3}^{n+1}=-q_{4}^{n+1}, \\
C\left(\frac{\partial P_{2}}{\partial t}-\frac{\partial P_{4}}{\partial t}\right)=q_{5}^{n+1}=-q_{6}^{n+1} .
\end{gathered}
$$

Note that the flow rates for node two of each element $\left(q_{2}, q_{4}\right.$ and $\left.q_{5}\right)$ are negative due to the requiring flow rates to be defined towards the element centre. The temporal term in Equation (3.28) is once again discretised by a second order backward difference scheme. The elemental lumped equations have the same form as the 1D blood flow equations, which can be expressed as

$$
\boldsymbol{K}_{e}^{0 D} \boldsymbol{P}_{e}^{n+1}=\boldsymbol{f}_{e}+\boldsymbol{Q}_{e}^{n+1}
$$

Similarly to the 1D equations, the local flow rate column once again becomes a global mass change vector after assembly, with conservation of mass for internal nodes, with only external flows being non-zero (boundary condition). This also allows lumped model elements to be easily connected to the 1D elements by sharing a pressure node, where once again the conservation of mass appears in the flow rate column. The outlet boundary condition for the Windkessel model is typically a defined pressure, which is applied to the system matrix directly. The flow rate could be given in which case the boundary condition would simply be added to the flow rate vector, $\boldsymbol{Q}^{n+1}$.

\subsubsection{Lumped Models}

As discussed in the subsection 2.3, all lumped models can be constructed using a combination of three basic elemental equations: a resistance element, an inductance (inertial) element, and a capacitance (compliant) element. The most complex versions of these models, which are used implemented in chapter 7 , and their discretisations are presented in this section. 


\section{Vascular Beds}

Vascular beds are defined by three different models, which are all identical to that from [230], and thus only an overview of the models are presented here.

The majority of vascular beds are treated using the model configuration shown in figure 3.1. The generic vascular bed model allows multiple arteries and/or veins to connect to the same vascular bed, which is more physiologically realistic than treating each terminal vessel with a separate vascular bed. The generic vascular bed includes: characteristic impedances from the arteries $Z_{\text {art }}$ and veins $Z_{\text {ven }}$, a compliance element representing the arteriole and arterial side of the capillaries $C_{a r t}$, a pressure dependant resistance from the capillaries $R_{v b}$, and a compliance element representing the venous side of the capillaries and venules $C_{v e n}$. The pressure dependant resistance varies as

$$
R_{v b}=\left\{\begin{array}{l}
R_{0}\left(\frac{P_{t m, 0}-P_{z f}}{P_{t m}-P_{z f}}\right), \quad P_{t m}>P_{z f} \\
\text { inf }, \quad P_{t m} \leq P_{z f}
\end{array}\right.
$$

where $R_{0}$ is the resistance at the reference pressure $P_{t m, 0}$, and $P_{z f}$ is the transural pressure when flow becomes zero $\left(5 \mathrm{mmHg}\right.$ as in [230]). The value of $P_{t m, 0}$ is set to equal $P_{t m}=P_{1}-P_{e x t, 1}$ in figure 3.1 when the pressure $P_{\text {art }}$ is equal to the aimed diastolic pressure in the arteries, and $P_{v e n}$ is the reference pressure of the veins.

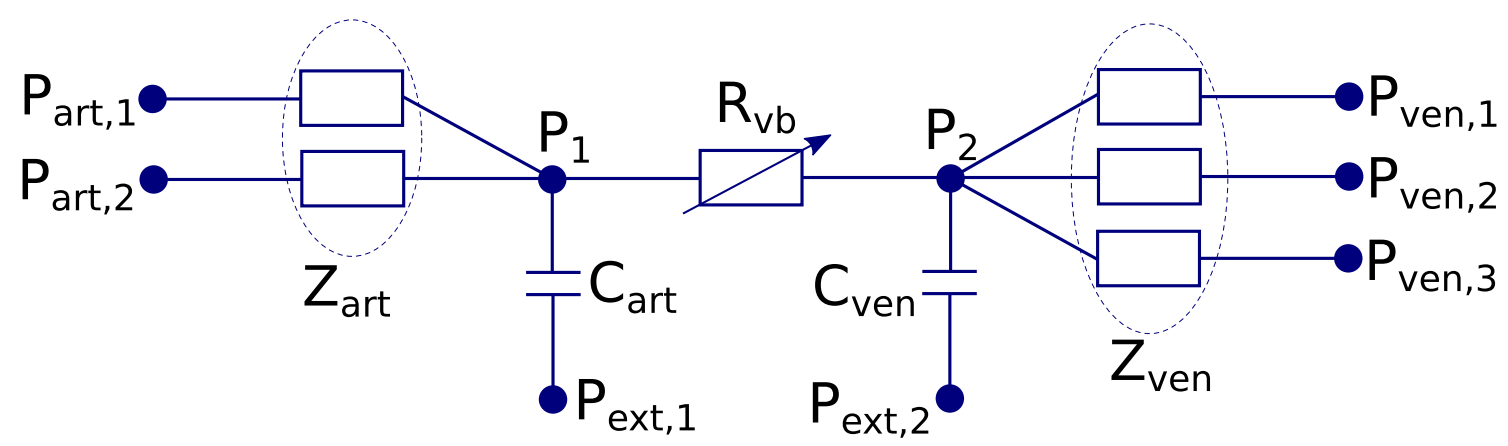

Figure 3.1: Generic vascular bed

The liver vascular bed is shown in figure 3.2, and connects the hepatic artery (art) and hepatic portal (hpv) vein, before reaching the variable resistance $R_{v b}$ which represents the liver. All other aspects are the same as the generic vascular bed, with the resistance being dependant on the transmural pressure $P_{t m}=P_{2}-P_{e x t, 2}$ from 
figure 3.2.

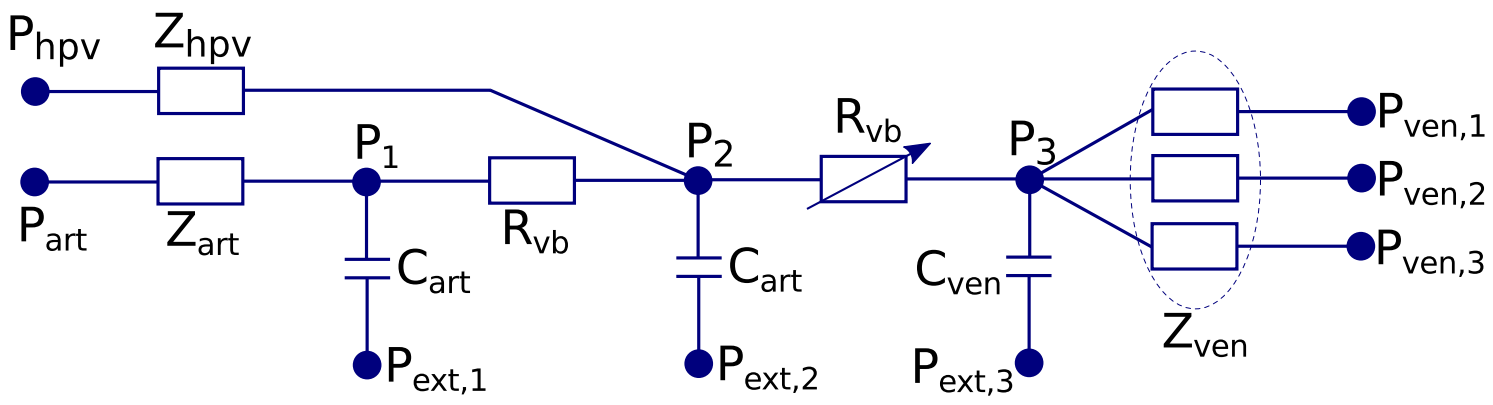

Figure 3.2: Liver vascular bed model

The coronary model is shown in figure 3.3 and is more complex than the other vascular beds. The coronary vascular bed essentially including four layers: the epicardium, subepicardium, midwall, and subendocardium. The model contains nine variable resistances, which depend on the volume of blood in the vascular bed compartment, rather than the pressure. In addition, the external pressures are defined to be a proportion of heart chamber pressures, which leads to the physiologically representative waveforms for coronary arteries, which have low systolic flow, and larger diastolic flows.

For completeness the model equations presented in [230] are repeated here. Each compliance element $C$ is associated with a compartment volume $V$, which is updated using

$$
V(t)=V_{0}+\int_{0}^{t} C \frac{d p_{t m}}{d t} d t
$$

where $V_{0}$ is the initial compartment volume. This volume is used to update the bed resistances as

$$
R_{i}=R_{0, i} \frac{V_{0, i}^{2}}{V_{i}^{2}}
$$

for subscripts $R_{1, i}$ and $R_{2, i}$ from figure 3.3, and

$$
R_{v b}=R_{0, v b}\left(0.75 \frac{V_{0,1}^{2}}{V_{1}^{2}}+0.25 \frac{V_{0,2}^{2}}{V_{2}^{2}}\right)
$$

The external pressures are split between the subepicardium, midwall, and subendo- 


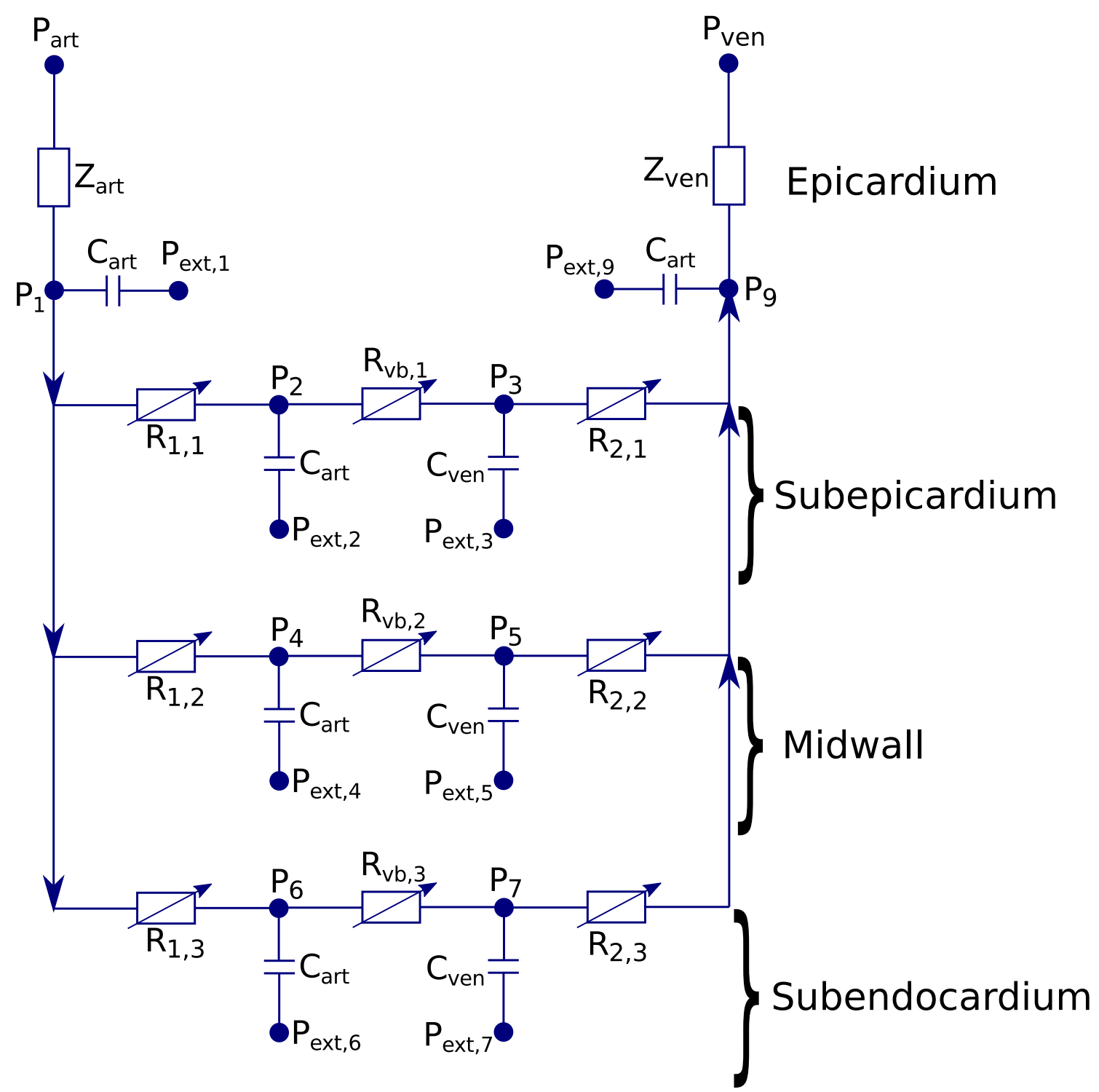

Figure 3.3: Coronary vascular bed model

cardium respectively as

$$
\begin{aligned}
& 1 / 6 P_{c h}+\alpha \frac{P_{c h}}{\left(V_{c h}-V_{0, c h}\right)} \\
& 1 / 2 P_{c h}+\alpha \frac{P_{c h}}{\left(V_{c h}-V_{0, c h}\right)} \\
& 5 / 6 P_{c h}+\alpha \frac{P_{c h}}{\left(V_{c h}-V_{0, c h}\right)},
\end{aligned}
$$


where $P_{c h}$ is the pressure in a heart chamber, and $V_{c h}$ is the volume of the heart chamber. For more details of the model see [230].

\section{Heart Model}

The heart model is identical to that of [230]. For completeness, all equations that describe the heart model are presented. The lumped representation of the heart model is shown in figure 3.4. The pressure in a heart chamber is described by

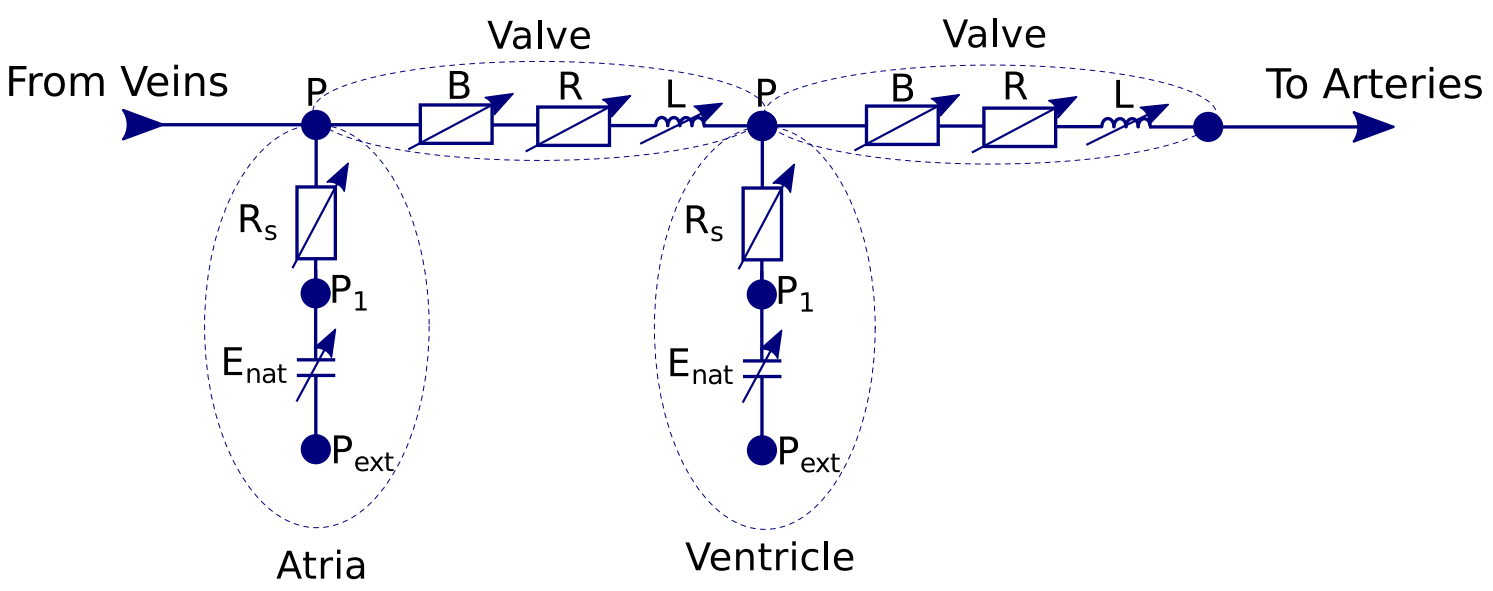

Figure 3.4: Heart model (one side)

$$
P=P_{p c}+E_{n a t}\left(V-V_{0}\right)-R_{s} Q+\frac{E_{n a t}}{E_{s e p}} P^{*}
$$

where the source resistance is

$$
R_{s}=K_{s} E_{n a t}\left(V-V_{0}\right),
$$

for a constant $K_{s}$, and current $V$ and unstressed $V_{0}$ chamber volume. The pericardial pressure is calculated using

$$
P_{p c}=K_{p c} \exp \left[\frac{V_{p c}-V_{0, p c}}{\Phi}\right]
$$

where $K_{p c}, V_{0, p c}$, and $\Phi$ are constants for the pericardium, and $V_{p c}$ is the current volume of the pericardium, which includes heart chamber volumes, intramycardial volume, and pericardial fluid volume. The effective heart chamber elastance curves 
(native elastances) are constructed using a relation between the free-wall elastance (local elastance) and septal elastance (a linear combination of the free-wall elastances of right and left heart chambers). The native elastance of a heart chamber is described by

$$
E_{n a t}=\frac{E_{f w} E_{s e p}}{E_{f w}+E_{s e p}}-\mu_{A V} Q_{V}
$$

for an atrio-ventricular piston constant $\mu A V$ and the net volumetric flow rate in the ventricle. The septal elastance is

$$
E_{\text {sep }}=\kappa_{L} E_{f w, L}+\kappa_{R} E_{f w, R}
$$

for chamber constants $\kappa$, and the free wall elastance is

$$
E_{f w}=k_{c h}\left[\frac{g_{1}}{1+g_{1}}\right]\left[\frac{1}{1+g_{2}}\right]+E_{m i n}
$$

The free-wall elastance is constructed from the following three functions

$$
g_{1}=\left(\frac{t-t_{\text {onset }}}{\tau_{1}}\right)^{m_{1}}, \quad g_{2}=\left(\frac{t-t_{\text {onset }}}{\tau_{2}}\right)^{m_{2}}
$$

and

$$
k_{c h}=\left(E_{f w}^{\max }-E_{f w}^{\min }\right) / \max \left[\left(\frac{g_{1}}{1+g_{1}}\right),\left(\frac{1}{1+g_{2}}\right)\right] .
$$

From a lumped element perspective, each heart chamber can be constructed from one modified capacitance (compliance) element and one resistive element. Before describing the discretisation of the equations, it is important to understand the physical meaning of the terms in the equation. In particular there are two external pressures acting in equation (3.35). There is an external pressure acting on all heart chambers from the pericardium via $P_{p c}$ (as shown in figure 2.8a), and an external pressure from the contra-lateral heart chamber (i.e. left to right sided chamber interaction) $\frac{E_{\text {nat }}}{E_{\text {sep }}} P^{*}$ (shown in figure 2.8c). Thus defining the total external pressure on a chamber as as $P_{c h, e x t}=P_{p c}+\frac{E_{n a t}}{E_{s e p}} P^{*}$, which is to be used to describe the discrete form of the equations. 
The discretisation of equation (3.35) (assuming iterations are performed) is

$$
\begin{aligned}
P^{n+1, k+1} & =\left(\frac{E_{\text {nat }}}{E_{\text {sep }}}\right)^{n+1, k+1} P^{*, n+1, k}+P_{p c}^{n+1, k} \\
& +E_{\text {nat }}^{n+1, k+1}\left(V^{n+1, k+1}-V_{0}\right)-R_{s}^{n+1, k+1} Q^{n+1, k+1} .
\end{aligned}
$$

In order to split this equation into two elements, consider a heart chamber to have three pressure nodes: an external pressure $P_{c h, e x t}$, a node connecting elastance element and resistance element $P_{1}$, and the chamber pressure $P$. The following relation is used to update the volume in every heart chamber

$$
\frac{d V}{d t}=Q_{n e t}=Q_{\text {in }}-Q_{\text {out }}
$$

where $Q_{n e t}$ is the difference between flow going into the chamber $Q_{i n}$ and flow going out of the chamber $Q_{\text {out }}$. Performing a Crank-Nicolson discretisation allows the volume update to the current time step to be

$$
V^{n+1, k+1}-V^{n}=\frac{\Delta t}{2}\left(Q_{n e t}^{n+1, k+1}+Q_{n e t}^{n}\right)
$$

This allows the elastance element (modified compliant element) to be described by

$$
\frac{1}{E_{n a t}^{n+1, k+1}}\left(P_{1}^{n+1, k+1}-P_{c h, e x t}^{n+1, k+1}\right)=V^{n}+\frac{\Delta t}{2}\left(Q_{n e t}^{n+1, k+1}+Q_{n e t}^{n}\right)-V_{0},
$$

which can be written as a linear system consistent with equation (3.17). The source resistance element can be written as

$$
\frac{1}{R_{s}^{n+1, k+1} E_{n a t}^{n+1, k+1}\left(V^{n}+\frac{\Delta t}{2}\left(Q_{n e t}^{n+1, k}+Q_{n e t}^{n}\right)-V_{0}\right)}\left(P^{n+1, k+1}-P_{1}^{n+1, k+1}\right)=Q_{n e t},
$$

which is in the required form to be used in the ETM scheme.

\section{Valve Model}

Valves can be constructed using three elements: a Bernoulli resistance element, a viscous resistance, and an inertial element. The valve model is shown in figure 3.5. For a change in pressure across the valve $\Delta P$, the equation which describes valve 


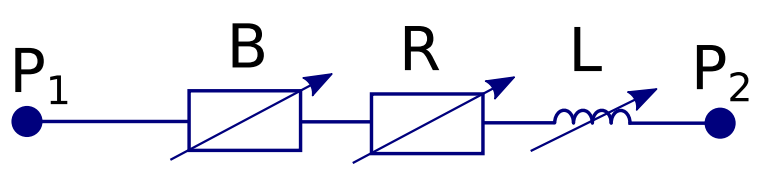

Figure 3.5: Valve model

dynamics is

$$
\Delta P=B Q|Q|+R Q+L \frac{\partial Q}{\partial t},
$$

where $B, R$, and $L$ are the Bernoulli resistance, viscous resistance, and the inertance respectively and are given by

$$
B=\frac{\rho}{2 A_{\text {eff }}^{2}}, \quad R=\frac{\zeta \pi \mu l_{\text {eff }}}{A_{\text {eff }}^{2}}, \quad L=\frac{\rho l_{\text {eff }}}{A_{\text {eff }}},
$$

with an effective orifice area of

$$
A_{e f f}(t)=\left(A_{e f f, \max }-A_{e f f, \min }\right) \zeta(t)+A_{e f f, \min } .
$$

The valve state $0 \leq \zeta \leq 1$ from equation (2.32), can be updated by applying the second order backward differences for the time derivatives and a semi-implicit discretisation

$$
\frac{3}{2 \Delta t} \zeta^{n+1}-\frac{2}{\Delta t} \zeta^{n}+\frac{1}{2 \Delta t} \zeta^{n-1}=K_{v o}\left(1-\zeta^{n+1, k+1}\right) \Delta P^{n+1, k}
$$

for valve opening and

$$
\frac{3}{2 \Delta t} \zeta^{n+1}-\frac{2}{\Delta t} \zeta^{n}+\frac{1}{2 \Delta t} \zeta^{n-1}=K_{v c} \zeta^{n+1, k+1} \Delta P^{n+1, k}
$$

for valve closing. The discretisation of (3.48) can be found by using a second order backward difference scheme for the time derivative which gives

$$
\begin{aligned}
\Delta P^{n+1, k+1} & =B^{n+1, k}\left|Q^{n+1, k}\right| Q^{n+1, k+1}+R^{n+1, k} Q^{n+1, k+1} \\
& +L^{n+1, k}\left(\frac{3}{2 \Delta t} Q^{n+1}-\frac{2}{\Delta t} Q^{n}+\frac{1}{2 \Delta t} Q^{n-1}\right),
\end{aligned}
$$

which is consistent with the required form from equation (3.17) to be used in the ETM scheme. 


\subsubsection{Vessel to Vessel Interface Conditions}

In the majority of works $[224,12,188,220]$ extrapolation of characteristic variables is used in conjunction with conservation of mass and conservation of total pressure at vessel junctions. A Newton-Raphson method is required to update variables to the next time step which are then used as boundary conditions. This needs to be performed at every vessel junction and could become computationally expensive depending on the number of vessels at a junction, and how many junctions are considered in the network.

In the ETM scheme, Lagrange multipliers are used as system constraints to impose a conservation of pressure (in this case total pressure). In order to implement Lagrange multipliers, first consider a system of equations (such as those representing 1D blood flow) written in the form $\boldsymbol{A} \boldsymbol{x}=\boldsymbol{b}$, which are subject to constraints at an interface (vessel junction). For $N$ number of vessels at a junction, a reference vessel is chosen (normally a parent vessel), then the system of equations needs to be supplemented with $N-1$ constraints $g_{i} i=1, \ldots, N-1$, which relates the pressure in the reference vessel to that of the daughter vessels (or non-reference parent vessels). The constraint chosen in this example is the conservation of total pressure, although static pressure or the inclusion of pressure loses can be easily introduced. The constraint is written in the form

$$
g_{i}=\frac{\rho}{2} \frac{Q_{1}^{2}}{A_{1}^{2}}+P_{1}-\frac{\rho}{2} \frac{Q_{i}^{2}}{A_{i}^{2}}-P_{i}=0, \quad i=1, \ldots, N-1,
$$

where subscript 1 is the chosen reference vessel. These constraints need to be linearised and are imposed for the time level $n+1$. After linearisation, the constraints for all junctions can be combined into the system $\boldsymbol{B} \boldsymbol{x}=\boldsymbol{c}$, which is the same form as the original equations (blood flow equations).

At each junction there are $N-1$ equations which need to be satisfied at the interface between the parent and $N-1$ daughter vessels, implying that $N-1$ Lagrange multipliers will be needed to impose these pressure constraints at each vessel junction. For the global system, the Lagrange multipliers for all vessel junctions are written as $\lambda_{1, \ldots, M}$. Where $M$ is the total number of Lagrange multipliers needed in the entire system in order to constrain conservation of total pressure at all vessel junctions. In order to constrain the system, first let $\boldsymbol{\Lambda}$ be a vector containing all 
$\lambda_{1, \ldots, M}$, and

$$
\boldsymbol{\Lambda}^{T}(\boldsymbol{B} \boldsymbol{x}-\boldsymbol{c})=0 .
$$

Defining an energy functional to be

$$
\delta I=(\boldsymbol{A x}-\boldsymbol{b})^{T} \delta x=\delta\left(\boldsymbol{x}^{T} \boldsymbol{A} \boldsymbol{x}-\boldsymbol{x}^{T} \boldsymbol{b}\right),
$$

where $\boldsymbol{A} \boldsymbol{x}-\boldsymbol{b}$ are the original system of equations which describe the 1D blood flow model. The stationary condition that satisfies this is

$$
I=\left(\boldsymbol{x}^{T} \boldsymbol{A} \boldsymbol{x}-\boldsymbol{x}^{T} \boldsymbol{b}\right) .
$$

A functional can be created by adding Equations (3.57) and (3.55), which gives

$$
J=\boldsymbol{x}^{T} \boldsymbol{A} \boldsymbol{x}-\boldsymbol{x}^{T} \boldsymbol{b}+\boldsymbol{\Lambda}^{T}(\boldsymbol{B} \boldsymbol{x}-\boldsymbol{c}) .
$$

By requiring that the total variation of this functional $\delta J=0$,

$$
\delta J=\frac{\partial J}{\partial x_{i}} \delta x_{i}+\frac{\partial J}{\partial \lambda_{i}} \delta \lambda_{i}=0 .
$$

It is easily deduced that

$$
\frac{\partial J}{\partial x_{i}}=0, \quad \text { and } \quad \frac{\partial J}{\partial \lambda_{i}}=0,
$$

as $\delta x_{i}$ and $\delta \lambda_{i}$ are arbitrary. Thus the full linear system to be solved can be written as

$$
\left[\begin{array}{cc}
\boldsymbol{A} & \boldsymbol{B}^{T} \\
\boldsymbol{B} & 0
\end{array}\right]\left[\begin{array}{l}
\boldsymbol{x} \\
\Lambda
\end{array}\right]=\left[\begin{array}{l}
\boldsymbol{b} \\
\boldsymbol{c}
\end{array}\right]
$$

Interestingly when conservation of total pressure is constrained via Lagrange multipliers, mass conservation is automatically satisfied and hence does not need to be explicitly constrained, this will be described and become clear in subsection 3.2.6. The ETM scheme requires the constraint system of equations $\boldsymbol{B} \boldsymbol{x}=\boldsymbol{c}$ to be in the same form as equation (3.23). As a result, the junction constraints (including both pressure and flow) need to follow the same format. The linearisation of the 


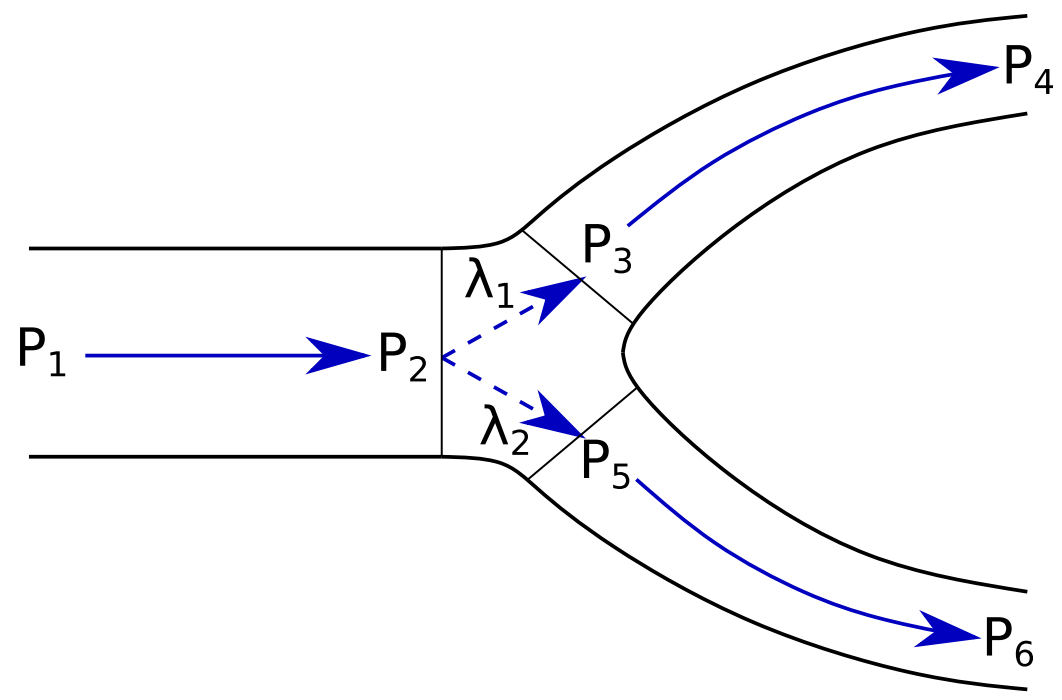

Figure 3.6: An example of the ETM schemes use of Lagrange multipliers at a bifurcation. Arrows indicate assumption of flow direction, hence the parent vessel (reference vessel) is on the left with pressure nodes $P_{1}$ and $P_{2}$. However, this is for indication of the reference vessel only, and is capable of handling flow in any direction. Lagrange multiplier $\lambda_{1}$ connects pressure nodes 2 and 3 , while $\lambda_{2}$ connects pressure nodes 2 and 5 .

conservation of total pressure terms are as follows

$$
P^{n+1} \approx P^{n+1, k+1}, \quad \frac{\rho}{2}\left(\frac{Q^{2}}{A^{2}}\right)^{n+1} \approx \frac{\rho}{2}\left(\frac{Q^{2}}{A^{2}}\right)^{n+1, k}
$$

where $k$ is the iteration level. As mentioned before only one solve is performed per time step (no iterations), hence, the iteration levels would be approximated by $(n+1, k) \approx(n)$ and $(n+1, k+1) \approx(n+1)$.

The Lagrange multipliers can be used to impose continuity of static pressure, continuity of total pressure, and can also implement methods which estimate energy/pressure losses at junctions, such as that of [231].

\subsubsection{Physical Interpretation of the Lagrange Multipliers}

In order to determine a physical interpretation of the Lagrange multipliers it is advantageous to consider one parent vessel connecting to one daughter vessel, which can then be easily extended to multiple parent or daughter vessels. Consider the 
last element in the parent vessel $p_{1}$ and the first element in its daughter vessel $d_{1}$. The system of equations representing this system is given by

$$
\left[\begin{array}{cccc}
k e_{11}^{p_{1}} & k e_{12}^{p_{1}} & 0 & 0 \\
k e_{21}^{p_{1}} & k e_{22}^{p_{1}} & 0 & 0 \\
0 & 0 & k e_{11}^{d_{1}} & k e_{12}^{d_{1}} \\
0 & 0 & k e_{21}^{d_{1}} & k e_{22}^{d_{1}}
\end{array}\right]\left[\begin{array}{c}
P_{1}^{p_{1}} \\
P_{2}^{p_{1}} \\
P_{1}^{d_{1}} \\
P_{2}^{d_{1}}
\end{array}\right]^{n+1}=\left[\begin{array}{c}
f_{1}^{p_{1}} \\
f_{2}^{p_{1}} \\
f_{1}^{d_{1}} \\
f_{2}^{d_{1}}
\end{array}\right]^{n}+\left[\begin{array}{c}
Q_{1}^{p_{1}} \\
-Q_{2}^{p_{1}} \\
Q_{1}^{d_{1}} \\
-Q_{2}^{d_{1}}
\end{array}\right]^{n+1}
$$

Noting that the final column on the right hand side of Equation (3.63) represents the flow rate column. The first flow of the system $Q_{1}^{p_{1}}$ and the last flow of the system $Q_{2}^{d_{1}}$ will either: become the conservation of mass when connected another element within the vessel as shown in Equation (3.25) (becomes zero); or be a specified boundary condition, which could be either prescribed flow or pressure. After taking this into account the only flows left in this column are the outflow of the parent vessel $Q_{2}^{p_{1}}$, and the inflow of the daughter vessel $Q_{1}^{d_{1}}$. However, from mass conservation the outflow of the parent vessel must equal the inflow of the daughter vessel. Defining $\lambda_{1}=Q_{2}^{p_{1}}=Q_{1}^{d_{1}}$ and adding it as a variable to the left hand side of system (3.63), and adding the conservation of total pressure equation (3.54) between one parent vessel and one daughter vessel gives

$$
\left[\begin{array}{ccccc}
k e_{11}^{p_{1}} & k e_{12}^{p_{1}} & 0 & 0 & 0 \\
k e_{21}^{p_{1}} & k e_{22}^{p_{1}} & 0 & 0 & 1 \\
0 & 0 & k e_{11}^{d_{1}} & k e_{12}^{d_{1}} & -1 \\
0 & 0 & k e_{21}^{d_{1}} & k e_{22}^{d_{1}} & 0 \\
0 & 1 & -1 & 0 & 0
\end{array}\right]\left[\begin{array}{c}
P_{1}^{p_{1}} \\
P_{2}^{p_{1}} \\
P_{1}^{d_{1}} \\
P_{2}^{d_{1}} \\
\lambda_{1}
\end{array}\right]^{n+1}=\left[\begin{array}{c}
f_{1}^{p_{1}} \\
f_{2}^{p_{1}} \\
f_{1}^{d_{1}} \\
f_{2}^{d_{1}} \\
f_{\lambda_{1}}
\end{array}\right]^{n}
$$

This implies that the Lagrange multiplier is in fact the flow between parent vessel and daughter vessel. Writing the equations corresponding to the last node of the parent and the first node of the daughter gives

$$
\begin{aligned}
& k e_{21}^{p_{1}} P_{1}^{p_{1}}+k e_{22}^{p_{1}} P_{2}^{p_{1}}+\lambda_{1}=f_{2}^{p_{1}}, \\
& k e_{11}^{d_{1}} P_{1}^{d_{1}}+k e_{12}^{d_{1}} P_{2}^{d_{1}}-\lambda_{1}=f_{2}^{d_{1}} .
\end{aligned}
$$

A dimensional analysis can be done on either of these equations to find that $\lambda_{1}$ has the same units of flow rate. 
This can be extended to any number of parent vessels and/or daughter vessels. For example, at a bifurcation, the equations of the last node of the parent vessel and the first node of the two daughter vessels are

$$
\begin{aligned}
k e_{21}^{p_{1}} P_{1}^{p_{1}}+k e_{22}^{p_{1}} P_{2}^{p_{1}}+\lambda_{1}+\lambda_{2} & =f_{2}^{p_{1}}, \\
k e_{11}^{d_{1}} P_{1}^{d_{1}}+k e_{12}^{d_{1}} P_{2}^{d_{1}}-\lambda_{1} & =f_{2}^{d_{1}}, \\
k e_{11}^{d_{2}} P_{1}^{d_{2}}+k e_{12}^{d_{2}} P_{2}^{d_{2}}-\lambda_{2} & =f_{2}^{d_{2}} .
\end{aligned}
$$

It is easily seen that $\lambda_{1}$ is the inlet flow of the first daughter vessel and $\lambda_{2}$ is the inlet flow of the second daughter vessel and hence $\lambda_{1}+\lambda_{2}$ is the total flow leaving (or entering) the parent vessel.

In the case of two parent vessels to one daughter vessel the equations of the last node of the two parents and first node of the daughter become

$$
\begin{aligned}
k e_{21}^{p_{1}} P_{1}^{p_{1}}+k e_{22}^{p_{1}} P_{2}^{p_{1}}+\lambda_{1}+\lambda_{2} & =f_{2}^{p_{1}}, \\
k e_{21}^{p_{2}} P_{1}^{p_{2}}+k e_{22}^{p_{2}} P_{2}^{p_{2}}-\lambda_{1} & =f_{2}^{p_{2}}, \\
k e_{11}^{d_{1}} P_{1}^{d_{1}}+k e_{12}^{d_{1}} P_{2}^{d_{1}}-\lambda_{2} & =f_{2}^{d_{1}} .
\end{aligned}
$$

The Lagrange multiplier $\lambda_{1}=-Q_{2}^{p_{2}}$ (negative the outlet flow of the second parent vessel), while $\lambda_{2}$ is the inlet flow of the daughter vessel, hence, $\lambda_{1}+\lambda_{2}$ will be the outlet flow of the first parent (reference) vessel. This can be extended to any number of parent and/or daughter vessels.

Thus the use of Lagrange multipliers to constrain conservation of total pressure (or static pressure) also finds the external flows of the vessels, meaning that conservation of mass is also automatically satisfied for the connected vessels.

\subsubsection{Treatment of Duplicate 1D Vessels or Lumped Ele- ments}

There are a large number of variations in a cardiovascular network between individuals. Moreover, in the model it is assumed no fluid leaks radially from the vessels. It is also unrealistic to account for all minor vessels which branch from major vessels (such as the inter-costal arteries from the descending aorta). In order to account for the blood that is being removed by such arteries, it can be assumed that several 
vessels branch from the same point of the major vessel, and connect to the same vascular bed. Thus creating several vessels with exact same properties and inlet/outlet boundary conditions on both sides. This allows a trick to be applied at the vessel junction, which takes into account that multiple vessels are connected to a junction and vascular bed, but only calculates the fluid within one of these vessels.

There are two different treatments for the different occurrences of these duplicate vessels. These are from: a 1D-1D vessels junctions, where the trick needs to be taken into account through the Lagrange multipliers at the junction, as described in section 3.2.5; and a connection of 1D-0D, or 0D-0D models.

First consider the case for a connection of 1D-1D vessels. For simplicity consider a bifurcation, where the two child vessels have the same material properties (such as length and area), and have the same inlet boundary conditions (branch from the same point of the parent vessel). For this the corresponding constraint system matrix (only considering the nodes at the junction), which would normally be written as equation (3.66), could be written as:

$$
\begin{gathered}
k e_{21}^{p_{1}} P_{1}^{p_{1}}+k e_{22}^{p_{1}} P_{2}^{p_{1}}+\lambda_{1}=f_{2}^{p_{1}}, \\
2 k e_{11}^{d_{1}} P_{1}^{d_{1}}+2 k e_{12}^{d_{1}} P_{2}^{d_{1}}-\lambda_{1}=2 f_{2}^{d_{1}} .
\end{gathered}
$$

This essentially tricks the code to consider the effect of duplicate child vessels at a junction, and can be extended to any number of child vessels, and can also extend to include any number of parent vessels. It is important to note that the Lagrange multiplier will now be the total flow leaving the parent vessels, which is twice the flow going into each child vessel.

A similar trick can be performed at junctions where pressure nodes are shared by neighbouring elements, such as 1D-0D or 0D-0D connectivities. Considering two identical 1D vessels connecting to a $0 \mathrm{D}$ vascular bed, the original system (at the shared pressure node) can be expressed as

$$
\begin{aligned}
& k e_{21}^{1 D, 1} P^{1 D, 1}+k e_{22}^{1 D, 1} P_{2}^{1 D, 1, S}+k e_{21}^{1 D, 2} P^{1 D, 2}+k e_{22}^{1 D, 2} P_{2}^{1 D, 2, S}+k e_{21}^{0 D} P^{0 D, S}+k e_{22}^{0 D} P_{2}^{0 D} \\
= & f_{2}^{1 D, 1}+f_{2}^{1 D, 2}+f_{1}^{0 D}
\end{aligned}
$$

or in a linear system when considering the shared nodes $P^{\text {Shared }}=P_{2}^{1 D, 1, S}=$ 


$$
\begin{aligned}
& P_{2}^{1 D, 2, S}=P_{2}^{0 D, 1, S} \text { is } \\
& {\left[\begin{array}{llll}
k e_{21}^{1 D, 1} & k e_{22}^{1 D, 1}+k e_{22}^{1 D, 2}+k e_{21}^{0 D} & k e_{21}^{1 D, 2} & k e_{22}^{0 D}
\end{array}\right]\left[\begin{array}{c}
P^{1 D, 1} \\
P^{\text {Shared }} \\
P_{2}^{1 D, 1} \\
P^{0 D, 2}
\end{array}\right]=f_{2}^{1 D, 1}+f_{2}^{1 D, 2}+f_{1}^{0 D} .}
\end{aligned}
$$

This can be manipulated and re-written as

$$
\left[\begin{array}{lll}
2 k e_{21}^{1 D, 1} & 2 k e_{22}^{1 D, 1}+k e_{21}^{0 D} & k e_{22}^{0 D}
\end{array}\right]\left[\begin{array}{c}
P^{1 D, 1} \\
P^{\text {Shared }} \\
P^{0 D, 1, S}
\end{array}\right]=2 f_{2}^{1 D, 1}+f_{1}^{0 D}
$$

It is also important to remember that these tricks are performed on the global assembled matrix (not the elemental systems), as the local flow updates are performed at the elemental level.

\subsection{Concluding Remarks}

This chapter presented the formulation and implementation of the numerical schemes used to solve the one-dimensional and zero-dimensional modelling components. The chapter began by comparing the common types of schemes that are implemented for the one-dimensional system of equations. This was followed by the description of the ETM scheme utilised in this thesis. The ETM scheme is an implicit sub-domain collocation scheme, which uses a second-order backward difference discretisation for the temporal domain, and the composite trapezoidal rule for spatial integration. The scheme involves a novel technique for the treatment of vessel junctions, which involves the use of Lagrange multipliers to constrain conservation of mass, and a continuity of pressure (static, total, or inclusion of pressure loss estimation methods). The treatment of other boundary condition types for the one-dimensional model was also described, including the coupling to the lumped parameter models of the heart, valves, and vascular beds. 


\section{Chapter 4}

\section{Benchmark Problems and Method- ology Testing}

\subsection{Introduction}

In this chapter the Enhanced Trapezoidal Rule Method (ETM) is rigorously tested through a series of comparisons with published benchmark problems [41] which include comparisons with analytical solutions, in-vitro data, and in-silico data. The scheme is further tested in order to determine its convergence behaviour, and is compared to additional problems for which a theoretical solution exists.

This chapter is primarily split into three parts: section 4.2 compares the ETM scheme with benchmark problems published in [41]; section 4.3 compares the ETM with several additional cases with theoretical solutions; finally, section 4.4 tests the spatial and temporal convergence behaviour of the scheme and the effect on the choice of linearisation implemented.

Unless otherwise stated, the governing equations used for each test case in this chapter are the following continuity and momentum equations

$$
\left\{\begin{array}{l}
C_{A} \frac{\partial P}{\partial t}+\frac{\partial Q}{\partial x}=0 \\
\frac{\rho}{A} \frac{\partial Q}{\partial t}+\frac{\rho}{A} \frac{\partial}{\partial x}\left(\frac{Q^{2}}{A}\right)+\frac{\partial P}{\partial x}-\frac{f}{A}=0
\end{array}\right.
$$

While the constitutive law is the non-linear elastic constitutive law described by

$$
P=P_{e x t}+P_{d}+\frac{\beta}{A_{d}}\left(\sqrt{A}-\sqrt{A_{d}}\right), \quad \beta(x)=4 / 3 \sqrt{\pi} E h,
$$

for which the vessel compliance can be determined by

$$
C_{A}=\frac{\partial A}{\partial P}=\left(\frac{2 A_{d}\left(P-P_{e x t}-P_{d}\right)}{\beta}+2 \sqrt{A_{d}}\right) \frac{A_{d}}{\beta} .
$$


The velocity profile chosen is given by

$$
u(x, \xi, t)=U(x, t) \frac{\xi+2}{\xi}\left[1-\left(\frac{\xi}{r}\right)^{\zeta}\right]
$$

which causes the friction term due to viscous forces to become

$$
f=-2(\zeta+2) \mu \pi \frac{Q}{A}
$$

The outlet boundary conditions will differ between each problem tested and range between reflection-free outlet conditions, a single resistance outlet model, and a three-element Windkessel model. For completeness, the details of each problem tested, including the network and boundary conditions, will be described in each section. At vessel junctions conservation of total pressure, and conservation of mass, are held as constraints between vessels, unless stated otherwise.

\subsection{Benchmarked Problems}

In this section the ETM scheme is compared with six published benchmark problems [41], which includes comparisons with analytical solutions (single pulse in a long vessel), in-vitro experimental measurements (37-artery network), in-silico solutions from a 3D model (common carotid artery, upper thoracic aorta, and aortic bifurcation), and an in-silico comparison between several one-dimensional numerical schemes (56-artery network). Table 4.1 contains the numerical parameters used for all benchmarked cases. For the benchmark cases the ETM scheme which is at least first-order accuracy in space is implemented (due to the non-linear convection discretisation), however the scheme can be easily adapted to second-order accuracy in space, which is tested in section 4.4.2.

The following error metrics are used to aid the comparison between the ETM scheme and 3D or in-vitro measurements for the benchmark problems:

$$
\epsilon_{P}^{R M S}=\sqrt{\frac{1}{n} \sum_{i=1}^{n}\left(\frac{P_{i}^{1 D}-\mathcal{P}_{i}}{\mathcal{P}_{i}}\right)^{2}}, \quad \epsilon_{Q}^{R M S}=\sqrt{\frac{1}{n} \sum_{i=1}^{n}\left(\frac{Q_{i}^{1 D}-\mathcal{Q}_{i}}{\max _{j} \mathcal{Q}_{i}}\right)^{2}},
$$




\begin{tabular}{|c|c|c|c|c|}
\hline Case & $\Delta t(\mathrm{~ms})$ & $\Delta x(\mathrm{~cm})$ & Space (order) & Time (order) \\
\hline Single pulse & 0.1 & 0.1 & $1 \mathrm{st}$ & $2 \mathrm{nd}$ \\
Carotid & 1.0 & 1.0 & $1 \mathrm{st}$ & $2 \mathrm{nd}$ \\
Aorta & 1.0 & 1.0 & $1 \mathrm{st}$ & $2 \mathrm{nd}$ \\
Aortic bifurcation & 1.0 & 1.0 & $1 \mathrm{st}$ & $2 \mathrm{nd}$ \\
37-artery & 1.0 & 1.0 & $1 \mathrm{st}$ & $2 \mathrm{nd}$ \\
ADAN56 & 1.0 & 1.0 & $1 \mathrm{st}$ & $2 \mathrm{nd}$ \\
\hline
\end{tabular}

Table 4.1: Numerical parameters used for the benchmark cases

$$
\begin{array}{cl}
\epsilon_{P}^{M a x}=\max _{j}\left|\frac{P_{i}^{1 D}-\mathcal{P}_{i}}{\mathcal{P}_{i}}\right|, & \epsilon_{Q}^{\text {Max }}=\max _{j}\left|\frac{Q_{i}^{1 D}-\mathcal{Q}_{i}}{\max _{j} \mathcal{Q}_{i}}\right|, \\
\epsilon_{P}^{\text {Sys }}=\frac{\max \left(P^{1 D}\right)-\max (\mathcal{P})}{\max (\mathcal{P})}, & \epsilon_{Q}^{\text {Sys }}=\frac{\max \left(Q^{1 D}\right)-\max (\mathcal{Q})}{\max (\mathcal{Q})}, \\
\epsilon_{P}^{\text {Dias }}=\frac{\min \left(P^{1 D}\right)-\min (\mathcal{P})}{\min (\mathcal{P})}, & \epsilon_{Q}^{\text {Dias }}=\frac{\min \left(Q^{1 D}\right)-\min (\mathcal{Q})}{\max (\mathcal{Q})},
\end{array}
$$

where $P^{1 D}$ and $Q^{1 D}$ are the solutions of the model at the spatial location and time point $i(i=1, \ldots, n)$, while $\mathcal{P}$ and $\mathcal{Q}$ are the $3 \mathrm{D}$ solutions (or in-vitro measurement). The error measures are used for the pressure $(P)$ and flow rates $(Q)$ in the vessel (middle of vessel unless otherwise stated), the pressure difference between the inlet and outlet of the vessel $(\Delta P)$, and the difference in vessel radius from diastole $(\Delta r)$.

\subsubsection{Single Pulse in a Long Reflection-Free Vessel}

The first test is a single, non-physiological pulse in a long reflection-free vessel. Numerically, this is challenging for any scheme due to the sharp gradient of the pulse. As mentioned, this is a non-physiological test as a physiological wave has a wavelength in metres, while in this problem the defined pulse has a wavelength in centimetres. Furthermore, an analytical solution can be found for the linearised system, hence only the linearised system, shown in equation (2.23) are implemented, which involves the removal of the non-linear convection term from the momentum equation.

The vessel considered has uniform properties along its length. At the inlet the volumetric inflow rate is a Gaussian-shaped wave with a peak flow rate of $1 \mathrm{ml} \mathrm{s}^{-1}$ 


\begin{tabular}{|c|c||c|}
\hline Property & Units & Vessel 1 \\
\hline Length, $L$ & $\mathrm{~cm}^{2}$ & 1000 \\
Area, $A_{d}$ & $\mathrm{~cm}^{2}$ & $\pi$ \\
Initial Area, $A(x, 0)$ & $\mathrm{cm}^{2}$ & $A_{d}$ \\
Initial flow, $Q(x, 0)$ & $\mathrm{ml} \mathrm{s}^{-1}$ & 0 \\
Wall thickness, $h$ & $\mathrm{~cm}^{-3}$ & 0.15 \\
Blood density, $\rho$ & $\mathrm{kg} \mathrm{m}^{-3}$ & 1050 \\
Blood viscosity, $\mu$ & $\mathrm{mPas}$ & 4 or 0 \\
Velocity profile order $\zeta$ & - & 9 \\
Elastic modulus, $E$ & $\mathrm{kPa}$ & 400.0 \\
Diastolic pressure, $P_{d}$ & $\mathrm{kPa}$ & 0 \\
External pressure, $P_{\text {ext }}$ & $\mathrm{Pa}$ & 0 \\
Initial pressure, $P(x, 0)$ & $\mathrm{kPa}$ & 0 \\
Outflow pressure, $P_{\text {out }}$ & $\mathrm{Pa}$ & 0 \\
\hline
\end{tabular}

Table 4.2: Properties for a single pulse in a single vessel

given by

$$
Q_{\text {inflow }}(t)=\exp \left(-10000(t-0.05)^{2}\right) \quad \mathrm{ml} \mathrm{s}^{-1},
$$

while a reflection-free outflow boundary condition is chosen (although the simulation terminates before the pulse reaches the end of the computational domain). A theoretical solution is constructed for the linearised system, with a theoretical decrease of peak pressure [11] in the viscid case given by

$$
\text { Ppeak }, \operatorname{viscid}(x)=\text { Ppeak, inviscid } \exp \left(-\frac{(\zeta+2) \pi \mu x}{\rho c_{0} A_{d}}\right),
$$

which is applicable when the fluid velocity is small. Parameters used in this case are given in Table 4.2. For more details on this problem, see the original description in [11], which is repeated in [41]. The time step used for this case is $\Delta t=0.1 \mathrm{~ms}$ and the element size is $\Delta x=0.1 \mathrm{~cm}$.

Figure 4.1 compares the ETM scheme with the theoretical solutions for both the viscous and inviscid cases. The ETM scheme preserves the peak in the inviscid case, and correctly predicts the pressure peak reduction along the vessel length for the viscous case. The solution indicates that the ETM scheme can correctly capture high frequencies. 


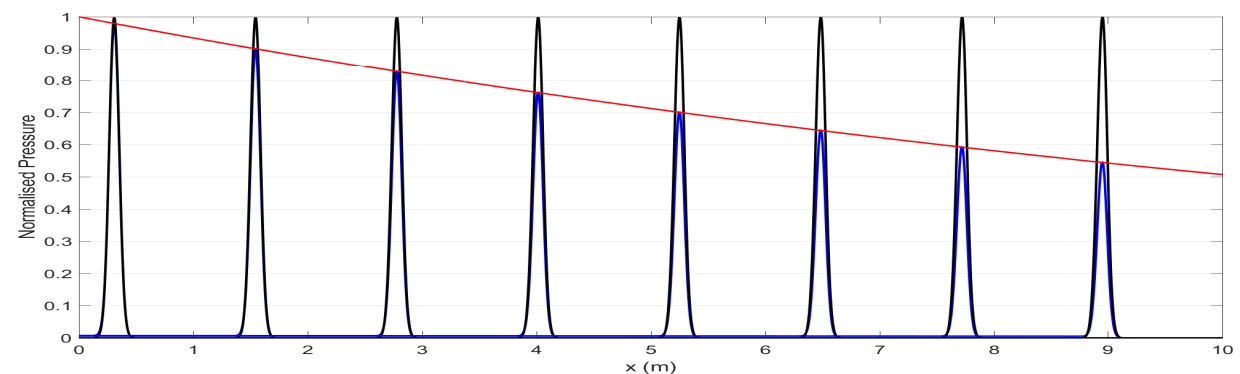

Figure 4.1: Decrease in the pressure wave peak along a vessel length for the single pulse case. The pressure of inviscid case is shown by the black line, while the pressure decrease due to viscous effects is shown by the blue line. The theoretical decrease of pressure for viscous case is given by the red line.

\subsubsection{Common Carotid Artery}

The common carotid arteries are the largest arteries in the neck and supply the majority of blood to the head and brain. In this test case a single vessel, representing a common carotid artery, is considered to have uniform material properties (cross-sectional area, wall thickness, and elastic modulus). The outlet of the vessel is connected to a three-element lumped parameter model which represents the downstream vessel network properties. The volumetric inflow rate $Q_{\text {inlet }}$ was measured in-vivo and was published in [372] with the problem description. The inflow rate is also given in supplementary material from [41]. Table 4.3 contains information on various parameters of the problem.

For this problem, the spatial mesh (element size) used is $\Delta x=1 \mathrm{~cm}$, with a temporal mesh (time step) of $\Delta t=1 \mathrm{~ms}$. The pressure and flow rate waveforms of the ETM scheme, shown in figures $4.2 \mathrm{a}$ and $4.2 \mathrm{~b}$ respectively, are in good agreement with the 3D solution and are consistent with the published solutions of the other 1D schemes. Relative errors are shown in Table 4.4.

\subsubsection{Upper Thoracic Aorta}

The thoracic aorta is the first section of the descending aorta, located distal to the aortic arch. The thoracic aorta test considered here, is a single 1D vessel with uniform properties. The inlet boundary condition is a defined volumetric inflow rate $Q_{\text {inlet }}$, while the 1D outlet boundary is coupled with a three element Windkessel 


\begin{tabular}{|c|c||c|}
\hline Property & Units & Common Carotid \\
\hline Length, $L$ & $\mathrm{~cm}^{2}$ & 12.6 \\
Area at diastolic pressure, $A_{d}$ & $\mathrm{~cm}^{2}$ & 0.28274 \\
Initial Area, $A(x, 0)$ & $\mathrm{cm}^{2}$ & 0.22038 \\
Initial flow, $Q(x, 0)$ & $\mathrm{ml} \mathrm{s}^{-1}$ & 0 \\
Wall thickness, $h$ & $\mathrm{~cm}^{-3}$ & 0.03 \\
Blood density, $\rho$ & $\mathrm{kg} \mathrm{m}^{-3}$ & 1060 \\
Blood viscosity, $\mu$ & $\mathrm{mPas}$ & 4 \\
Velocity profile order $\zeta$ & - & 2 \\
Elastic modulus, $E$ & $\mathrm{kPa}$ & 700.0 \\
Diastolic pressure, $P_{d}$ & $\mathrm{kPa}$ & 10.933 \\
External pressure, $P_{\text {ext }}$ & $\mathrm{Pa}$ & 0 \\
Initial pressure, $P(x, 0)$ & $\mathrm{kPa}$ & 0 \\
Outflow pressure, $P_{\text {out }}$ & $\mathrm{Pa}$ & 0 \\
Windkessel resistance, $R 1$ & $\mathrm{Pas} \mathrm{m}^{-3}$ & $2.4875 \times 10^{8}$ \\
Windkessel resistance, $R 2$ & $\mathrm{~Pa} \mathrm{~s} \mathrm{~m}^{-3}$ & $1.8697 \times 10^{9}$ \\
Windkessel compliance, $C$ & $\mathrm{Pas} \mathrm{m}^{-3}$ & $1.7529 \times 10^{-10}$ \\
\hline
\end{tabular}

Table 4.3: Properties for the common carotid artery case.

\begin{tabular}{|c|c|c|c|c|}
\hline Error (\%) & ETM & DCG & LCG & STM \\
\hline$\epsilon_{P}^{R M S}$ & 0.31 & 0.33 & 0.32 & 0.32 \\
$\epsilon_{Q}^{R M S}$ & 0.35 & 0.34 & 0.30 & 0.35 \\
$\epsilon_{\Delta r}^{R M S}$ & 1.11 & 1.15 & 1.11 & 1.10 \\
$\epsilon_{\Delta P}^{R M S}$ & 4.16 & 4.15 & 4.24 & 4.35 \\
$\epsilon_{P}^{M a x}$ & 0.75 & 0.81 & 0.76 & 0.77 \\
$\epsilon_{Q}^{\text {Max }}$ & 1.30 & 1.26 & 1.19 & 1.31 \\
$\epsilon_{\Delta r}^{M a x}$ & 2.48 & 2.63 & 2.48 & 2.54 \\
$\epsilon_{\Delta P}^{M a x}$ & 15.48 & 15.43 & 15.77 & 16.47 \\
$\epsilon_{P}^{\text {Sys }}$ & -0.26 & -0.27 & -0.27 & -0.26 \\
$\epsilon_{Q}^{\text {Sys }}$ & -0.54 & -0.55 & -0.46 & -0.55 \\
$\epsilon_{\Delta r}^{S y s}$ & -1.61 & -1.65 & -1.63 & -1.63 \\
$\epsilon_{\Delta P}^{S y s}$ & -15.11 & -15.05 & -15.37 & -15.76 \\
$\epsilon_{P}^{D i a}$ & 0.27 & 0.28 & 0.28 & 0.27 \\
$\epsilon_{Q}^{D i a}$ & 0.26 & 0.25 & 0.22 & 0.27 \\
$\epsilon_{\Delta r}^{D i a}$ & 0.07 & 0.08 & 0.11 & 0.8 \\
$\epsilon_{\Delta P}^{D i a}$ & 4.99 & 4.99 & 4.76 & 4.85 \\
\hline
\end{tabular}

Table 4.4: Comparison of relative errors from the 3D solution of the ETM, DCG, LCG, and STM, for the common carotid artery case 


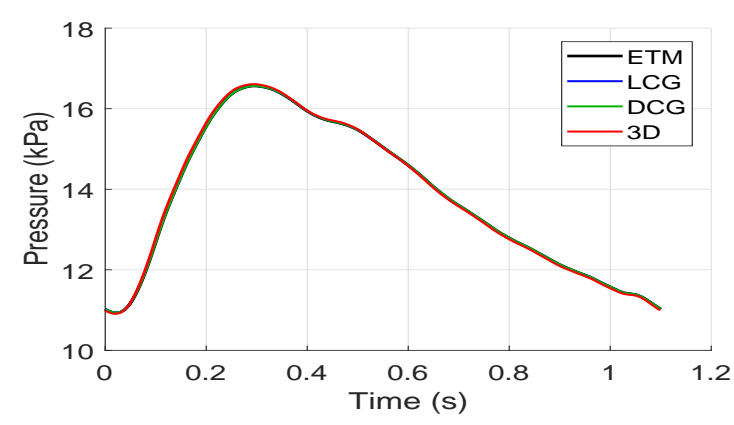

(a) Pressure in common carotid artery test

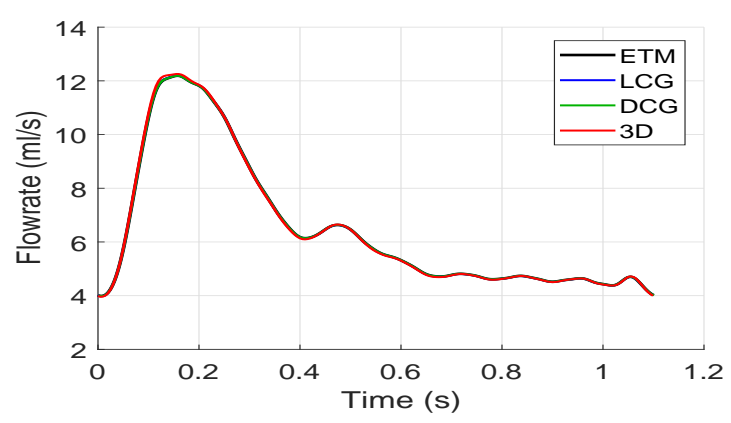

(b) Flow rate in common carotid artery test

Figure 4.2: Pressure and volumetric flow rate waveforms in the common carotid artery test comparing the ETM, DCG, LCG and 3D data.

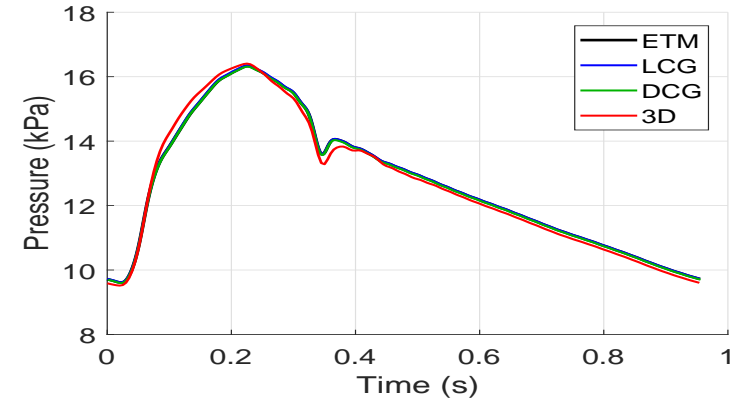

(a) Pressure in upper thoracic aorta test

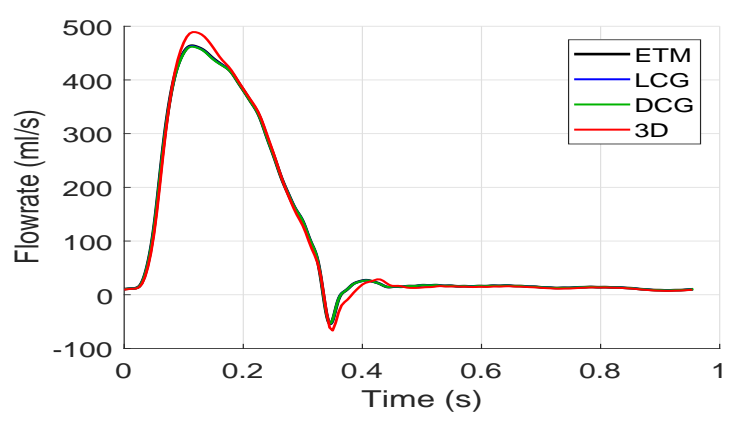

(b) Flow rate in upper thoracic aorta test

Figure 4.3: Pressure and volumetric flow rate waveforms in the upper thoracic aorta test comparing the ETM, DCG, LCG and 3D data.

model which simulates the effect of downstream vasculature. The original test case was published in [372], while [41] also describes the problem along with supplying additional material, which includes the volumetric inflow rate and both $1 \mathrm{D}$ and $3 \mathrm{D}$ model solutions.

The time step used for the ETM scheme is $\Delta t=1 \mathrm{~ms}$ and element size is $\Delta x=$ $1 \mathrm{~cm}$. Figures $4.3 \mathrm{a}$ and $4.3 \mathrm{~b}$ compare the pressure and flow waveforms for the ETM, DCG and LCG schemes, along with a 3D solution. The ETM scheme solution is consistent with the other published 1D method solutions published in [41], with noticeable discrepancies with the 3D data, particularly during systole. Table 4.6 shows the relative errors of the ETM, STM, DCG, and LCG schemes. 


\begin{tabular}{|c|c||c|}
\hline Property & Units & Thoracic Aorta \\
\hline Length, $L$ & $\mathrm{~cm}^{2}$ & 24.137 \\
Area at diastolic pressure, $A_{d}$ & $\mathrm{~cm}^{2}$ & 4.5239 \\
Initial Area, $A(x, 0)$ & $\mathrm{cm}^{2}$ & 3.0605 \\
Initial flow, $Q(x, 0)$ & $\mathrm{ml} \mathrm{s}^{-1}$ & 0 \\
Wall thickness, $h$ & $\mathrm{~cm}^{-3}$ & 0.12 \\
Blood density, $\rho$ & $\mathrm{kg} \mathrm{m}^{-3}$ & 1060 \\
Blood viscosity, $\mu$ & $\mathrm{mPas}$ & 4 \\
Velocity profile order $\zeta$ & - & 2 \\
Elastic modulus, $E$ & $\mathrm{kPa}$ & 700.0 \\
Diastolic pressure, $P_{d}$ & $\mathrm{kPa}$ & $9.4 \dot{6}$ \\
External pressure, $P_{\text {ext }}$ & $\mathrm{Pa}$ & 0 \\
Initial pressure, $P(x, 0)$ & $\mathrm{kPa}$ & 0 \\
Outflow pressure, $P_{\text {out }}$ & $\mathrm{Pa}$ & 0 \\
Windkessel resistance, $R 1$ & $\mathrm{~Pa} \mathrm{~s} \mathrm{~m}^{-3}$ & $1.1752 \times 10^{7}$ \\
Windkessel resistance, $R 2$ & $\mathrm{~Pa} \mathrm{~s} \mathrm{~m}^{-3}$ & $1.1167 \times 10^{8}$ \\
Windkessel compliance, $C$ & $\mathrm{~Pa} \mathrm{~s} \mathrm{~m}^{-3}$ & $1.0163 \times 10^{-8}$ \\
\hline
\end{tabular}

Table 4.5: Properties for upper thoracic aorta case.

\begin{tabular}{|c|c|c|c|c|}
\hline Error (\%) & ETM & $\overline{\mathrm{DCG}}$ & LCG & STM \\
\hline$\epsilon_{P}^{R M S}$ & 1.30 & 1.20 & 1.31 & 1.23 \\
\hline$\epsilon_{Q}^{R M S}$ & 1.72 & 1.78 & 1.74 & 1.82 \\
\hline$\epsilon_{\Delta r}^{R M S}$ & 2.63 & 2.56 & 2.61 & 3.08 \\
\hline$\epsilon_{\Delta P}^{\vec{R} M S}$ & 6.48 & 6.46 & 6.43 & 6.71 \\
\hline$\epsilon_{P}^{M a x}$ & 2.94 & 3.05 & 2.99 & 3.23 \\
\hline$\epsilon_{Q}^{M a x}$ & 5.60 & 5.83 & 5.68 & 5.94 \\
\hline$\epsilon_{\Delta r}^{\operatorname{Max}}$ & 6.67 & 6.74 & 6.75 & 8.94 \\
\hline$\epsilon_{\Delta P}^{\bar{M} a x}$ & 25.51 & 24.16 & 24.16 & 26.07 \\
\hline$\epsilon_{P}^{\text {Sys }}$ & -0.42 & -0.57 & -0.32 & -0.59 \\
\hline$\epsilon_{Q}^{\text {Sys }}$ & -5.17 & -5.56 & -5.36 & -5.56 \\
\hline$\epsilon_{\Delta r}^{\text {Sys }}$ & -2.18 & -2.54 & -1.93 & -4.48 \\
\hline$\epsilon_{\Delta P}^{\prime S y s}$ & -9.42 & -8.91 & -8.89 & -9.70 \\
\hline$\epsilon_{P}^{\bar{D} i a}$ & 1.08 & 0.85 & 1.22 & 0.85 \\
\hline$\epsilon_{Q}^{D i a}$ & 2.55 & 2.75 & 2.67 & 2.95 \\
\hline$\epsilon_{\Delta r}^{D i a}$ & 2.17 & 1.85 & 2.24 & 0.00 \\
\hline$\epsilon_{\Delta P}^{D i a}$ & 6.95 & 7.32 & 7.24 & 7.47 \\
\hline
\end{tabular}

Table 4.6: Comparison of relative errors from the 3D solution of the ETM, DCG, LCG, and STM, for the upper thoracic aorta case 


\begin{tabular}{|c|c||c|c|}
\hline Property & Units & Aorta & Iliac \\
\hline Length, $L$ & $\mathrm{~cm}^{2}$ & 8.6 & 8.5 \\
Area at diastolic pressure, $A_{d}$ & $\mathrm{~cm}^{2}$ & 2.3235 & 1.1310 \\
Initial Area, $A(x, 0)$ & $\mathrm{cm}^{2}$ & 1.8062 & 0.94787 \\
Initial flow, $Q(x, 0)$ & $\mathrm{ml} \mathrm{s}^{-1}$ & 0 & 0 \\
Wall thickness, $h$ & $\mathrm{~cm}$ & 0.1032 & 0.072 \\
Blood density, $\rho$ & $\mathrm{kg} \mathrm{m}^{-3}$ & 1060 & - \\
Blood viscosity, $\mu$ & $\mathrm{mPa} \mathrm{s}$ & 4 & - \\
Velocity profile order $\zeta$ & - & 9 & - \\
Elastic modulus, $E$ & $\mathrm{kPa}$ & 500.0 & 700.0 \\
Diastolic pressure, $P_{d}$ & $\mathrm{kPa}$ & 9.46 & 9.46 \\
External pressure, $P_{\text {ext }}$ & $\mathrm{Pa}$ & 0 & 0 \\
Initial pressure, $P(x, 0)$ & $\mathrm{kPa}^{\prime}$ & 0 & 0 \\
Outflow pressure, $P_{\text {out }}$ & $\mathrm{Pa}$ & 0 & 0 \\
Windkessel resistance, $R 1$ & $\mathrm{~Pa} \mathrm{~s} \mathrm{~m}^{-3}$ & - & $6.8123 \times 10^{7}$ \\
Windkessel resistance, $R 2$ & $\mathrm{~Pa} \mathrm{~s} \mathrm{~m}^{-3}$ & - & $3.1013 \times 10^{9}$ \\
Windkessel compliance, $C$ & $\mathrm{~Pa} \mathrm{~s} \mathrm{~m}^{-3}$ & - & $3.6664 \times 10^{-10}$ \\
\hline
\end{tabular}

Table 4.7: Properties for aortic bifurcation case, which is a symmetric configuration and includes the abdominal aorta and two common iliac arteries.

\subsubsection{Aortic Bifurcation}

The section of the descending aorta contains a bifurcation to the left and right common iliac arteries, which distributes blood towards the pelvic region and the legs. This location is often called the aortic bifurcation and the geometric configuration of this region can lead to an abdominal aortic aneurysm (AAA), particularly in older individuals with more tortuous vessels [304]. The bifurcation model is symmetric, i.e. both iliac arteries are identical and the vessels considered have uniform properties. The volumetric inflow rate is defined for the inlet boundary condition, while the outflow is coupled to a three-element Windkessel model. The original problem is described in [372], while supplementary material from [41] contains volumetric inflow rate, and the solutions of various models, including 1D and 3D methodologies.

The time step used in this case $\Delta t=1 \mathrm{~ms}$ and the element size is $\Delta x=1 \mathrm{~cm}$. Figure 4.4 compares the ETM, DCG, and LCG methods with the 3D model solutions. Good agreement is observed for pressure and flow waveforms between the ETM scheme and 3D data for the abdominal aorta and common iliac arteries. Table 4.8 shows the relative errors for the ETM, DCG, LCG, and STM schemes. 


\begin{tabular}{|c|c|c|c|c|c|}
\hline & Error (\%) & $\overline{\text { ETM }}$ & DCG & LCG & STM \\
\hline \multirow{12}{*}{ Midpoint aorta } & $\epsilon_{P}^{R M S}$ & 0.43 & 0.45 & 0.45 & 0.44 \\
\hline & $\epsilon_{Q}^{R M S}$ & 0.93 & 0.92 & 0.83 & 0.93 \\
\hline & $\epsilon_{\Delta r}^{R M S}$ & 2.50 & 2.52 & 2.45 & 2.48 \\
\hline & $\epsilon_{P}^{M a x}$ & 0.73 & 0.77 & 0.75 & 0.74 \\
\hline & $\epsilon_{O}^{\operatorname{Max}}$ & 2.79 & 2.74 & 2.49 & 2.76 \\
\hline & $\epsilon_{\Delta r}^{\operatorname{Max}}$ & 4.07 & 4.18 & 4.04 & 4.11 \\
\hline & $\epsilon_{P}^{\vec{S} y s}$ & -0.52 & -0.53 & -0.54 & -0.51 \\
\hline & $\epsilon_{Q}^{\text {Sys }}$ & -2.51 & -2.47 & -2.23 & -2.52 \\
\hline & $\epsilon_{\Delta r}^{\text {Sys }}$ & -3.92 & -3.96 & -3.86 & -3.93 \\
\hline & $\epsilon_{P}^{\overrightarrow{D i a}}$ & 0.48 & 0.50 & 0.51 & 0.48 \\
\hline & $\epsilon_{Q}^{D i a}$ & 1.16 & 1.16 & 1.03 & 1.17 \\
\hline & $\epsilon_{\Delta r}^{D i a}$ & -1.41 & -1.39 & -1.37 & -1.41 \\
\hline \multirow{12}{*}{ End aorta } & $\overline{\epsilon_{P} M S}$ & 0.46 & 0.48 & 0.47 & 0.47 \\
\hline & $\epsilon_{Q}^{R M S}$ & 1.18 & 1.16 & 1.19 & 0.40 \\
\hline & $\epsilon_{\Delta r}^{R M S}$ & 4.08 & 4.09 & 4.03 & 4.06 \\
\hline & $\epsilon_{P}^{M a x}$ & 0.84 & 0.89 & 0.86 & 0.85 \\
\hline & $\epsilon_{Q}^{\operatorname{Max}}$ & 3.63 & 3.55 & 3.64 & 1.68 \\
\hline & $\epsilon_{\Delta r}^{\operatorname{Max}}$ & 6.94 & 7.02 & 6.88 & 7.08 \\
\hline & $\epsilon_{P}^{S ?}$ & -0.69 & -0.71 & -0.70 & -0.69 \\
\hline & $\epsilon_{Q}^{S}$ & -3.52 & -3.47 & -3.53 & -1.08 \\
\hline & $\epsilon_{\Delta}^{S}$ & -6.80 & -6.84 & -6.73 & -6.90 \\
\hline & $\epsilon_{P}^{\vec{D} i a}$ & 0.53 & 0.54 & 0.55 & 0.53 \\
\hline & $\epsilon_{Q}^{D i a}$ & 1.77 & 1.76 & 1.78 & 0.69 \\
\hline & $\epsilon_{\Delta r}^{D i a}$ & -1.95 & -1.93 & -1.92 & -1.88 \\
\hline \multirow{12}{*}{ Midpoint iliac } & $\epsilon_{P}^{R M S}$ & 0.49 & 0.50 & 0.50 & 0.48 \\
\hline & $\epsilon_{Q}^{R M S}$ & 0.59 & 0.56 & 0.60 & 0.59 \\
\hline & $\epsilon_{\Delta r}^{R N}$ & 4.31 & 4.31 & 4.31 & 4.27 \\
\hline & $\epsilon_{P}^{M a x}$ & 0.94 & 0.99 & 0.96 & 0.94 \\
\hline & $\epsilon_{Q}^{\operatorname{Max}}$ & 2.16 & 2.05 & 2.16 & 2.18 \\
\hline & $\epsilon_{\Delta r}^{\operatorname{Max}}$ & 7.45 & 7.50 & 7.48 & 7.45 \\
\hline & $\epsilon_{P}^{S y}$ & -0.82 & -0.83 & -0.83 & -0.81 \\
\hline & $\epsilon_{Q}^{S y}$ & -1.80 & -1.68 & -1.84 & -1.79 \\
\hline & $\epsilon_{\Delta r}^{\text {Sys }}$ & -7.23 & -7.25 & -7.26 & -7.22 \\
\hline & $\epsilon_{P}^{\overline{D i a}}$ & 0.53 & 0.55 & 0.55 & 0.54 \\
\hline & $\epsilon_{Q}^{D i a}$ & 1.18 & 1.17 & 1.19 & 1.18 \\
\hline & $\epsilon_{\Delta r}^{D i a}$ & -2.39 & -2.36 & -2.37 & -2.41 \\
\hline
\end{tabular}

Table 4.8: Comparison of relative errors from the 3D solution of the ETM, DCG, LCG, and STM, for the aortic bifurcation case 


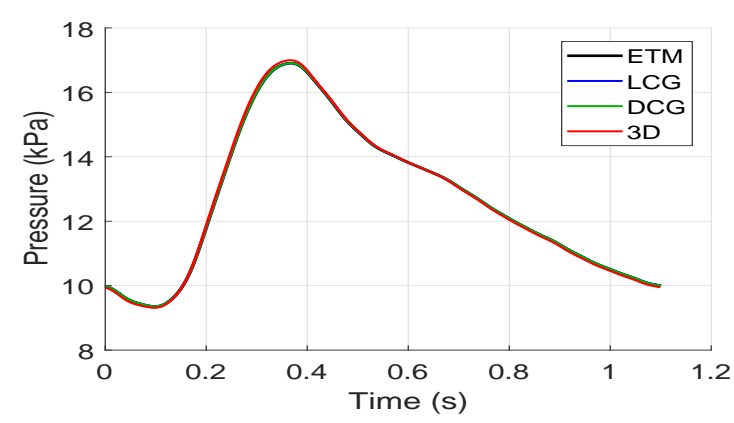

(a) Pressure in abdominal aorta

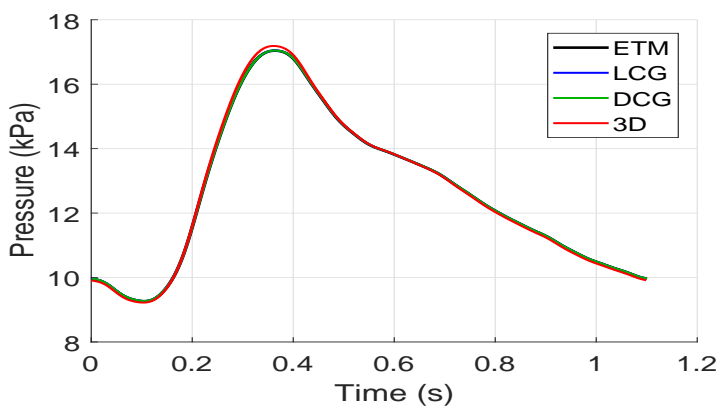

(c) Pressure in iliac arteries

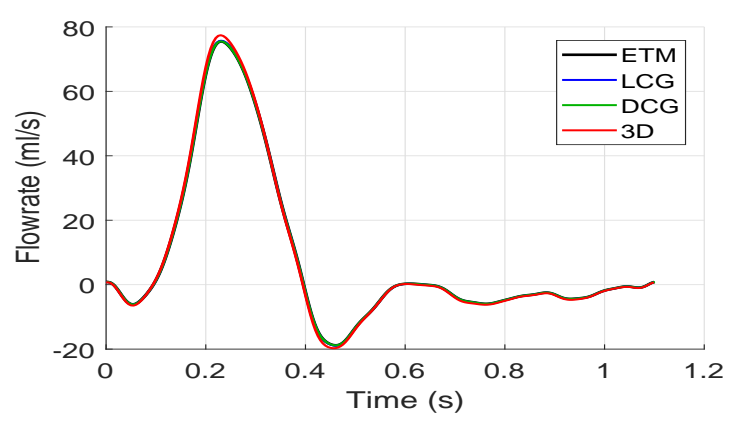

(b) Flow rate in abdominal aorta

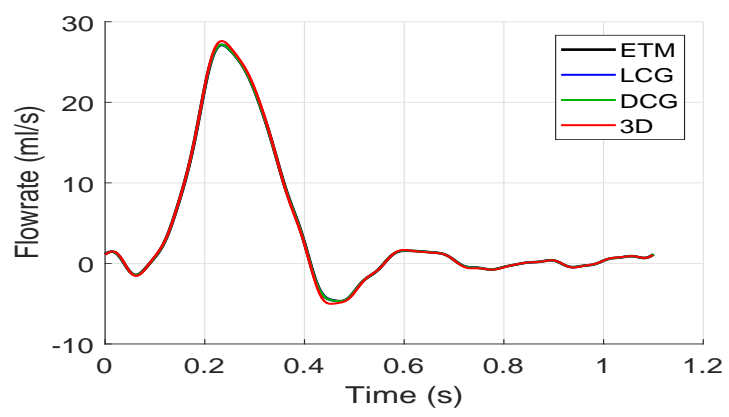

(d) Flow Rate in iliac arteries

Figure 4.4: Pressure and flow rates for the aortic bifurcation case. A comparison between the ETM, STM and LCG schemes with 3D data.

\subsubsection{Arterial Network Containing 37 Vessels}

The 37-arterial network was first presented in [205], who compared a 1D haemodynamics model of purely elastic vessels with in-vitro measurements from an identical network of silicone tubing. An extension, which included a visco-elastic wall model for the 1D methodology, was compared with the same experimental set-up [12]. The inlet boundary condition for the model uses volumetric inflow rate from the in-vitro measurements, while the outflow boundaries are attached to lumped resistance elements. In order to be consistent with the benchmark paper by [41], the case considered in this section is for the elastic wall model. All parameters in the model are given as supplementary material in [41]. Table 4.9 shows the haemodynamic parameters used in the simulation.

Figures 4.5, 4.6, and 4.7 compare pressure and volumetric flow rates from in-vitro measurements with the ETM, DCG, and LCG methods for various vessels throughout the network. The vessels considered are the: aortic arch II, which connects the 


\begin{tabular}{|c|c||c|}
\hline Property & Units & Parameter Value \\
\hline Blood density, $\rho$ & $\mathrm{kg} \mathrm{m}^{-3}$ & 1050 \\
Blood viscosity, $\mu$ & $\mathrm{mPas}$ & 2.5 \\
Velocity profile order $\zeta$ & - & 9 \\
Elastic modulus, $E$ & $\mathrm{kPa}$ & 1200.0 \\
Diastolic pressure, $P_{d}$ & $\mathrm{kPa}$ & 0 \\
External pressure, $P_{\text {ext }}$ & $\mathrm{Pa}$ & 0 \\
Initial pressure, $P(x, 0)$ & $\mathrm{kPa}$ & 0 \\
Outflow pressure, $P_{\text {out }}$ & $\mathrm{Pa}$ & 0 \\
\hline
\end{tabular}

Table 4.9: Properties for thirty-seven arterial network case.

ascending and descending parts of the aorta; thoracic aorta II, which is towards the top of the descending aorta; left subclavian artery I, which is primarily responsible for carrying blood to the arms (and to the vertebral arteries which supply a minority of blood to the head and brain); right iliac femoral, which although is not an artery in the artery by itself, it represents the combination of the right common and external iliac arteries and right femoral artery, which physiologically begins in the pelvic region (iliac) and carries the majority of blood to the leg (via femoral); left ulnar and right ulnar arteries, which are located in each forearm; splenic artery, which is an artery that splits from the celiac branch (a branch that supplies blood to organs which eventually brings blood to the liver) and carries blood the the spleen; and the right anterior tibial artery, which is located in the lower right leg.

The ETM scheme gives solutions which are consistent with the other 1D modelling methodologies. Comparing with the in-vitro measurements, the numerical methods over-predict the presence and magnitude of high frequency oscillations for both pressure and flows, particularly in the second and third vessel generations. The ETM scheme uses a time step of $\Delta t=1 \mathrm{~ms}$ and an element size of $\Delta x=1 \mathrm{~cm}$, which gives a maximum CFL number of $C F L=2.3517$. Table 4.10 shows the relative errors of the ETM, LCG, DCG, and STM schemes compared to the measurements. 


\begin{tabular}{|c|c|c|c|c|c|c|c|c|c|c|c|c|c|c|c|c|c|c|c|}
\hline & 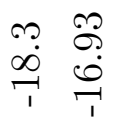 & 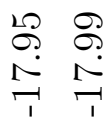 & $\stackrel{\infty}{\infty}$ & $\begin{array}{l}2 \\
0 \\
0 \\
+1 \\
1\end{array}$ & 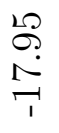 & 疍 & $\begin{array}{l}\infty \\
\stackrel{\infty}{1}\end{array}$ & $\begin{array}{l}m \\
0 \\
0 \\
1 \\
1\end{array}$ & 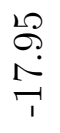 & $\begin{array}{l}\text { S } \\
\text { N } \\
+1\end{array}$ & $\begin{array}{l}\infty \\
\infty \\
+1\end{array}$ & $\begin{array}{l}2 \\
0 \\
0 \\
1 \\
1\end{array}$ & $\begin{array}{l}\stackrel{2}{2} \\
\stackrel{2}{N} \\
\stackrel{1}{1}\end{array}$ & $\begin{array}{l}\text { S } \\
\text { N } \\
+1\end{array}$ & $\begin{array}{l}\infty \\
\infty \\
+1\end{array}$ & $\begin{array}{l}\infty \\
0 \\
0 \\
11\end{array}$ & $\stackrel{0 ?}{r}$ & & \\
\hline & $\begin{array}{ll}\vec{H} & \stackrel{0}{0} \\
\infty & \stackrel{0}{0}\end{array}$ & $\begin{array}{ll}\infty & 12 \\
1 & \stackrel{1}{1} \\
\infty & \infty\end{array}$ & $\begin{array}{l}\vec{H} \\
\infty \\
\infty\end{array}$ & $\begin{array}{l}\stackrel{o}{0} \\
\stackrel{0}{0}\end{array}$ & $\frac{\infty}{1}$ & & $\begin{array}{l}\vec{H} \\
\infty \\
\infty\end{array}$ & $\underset{\stackrel{O}{0}}{\stackrel{0}{0}}$ & $\frac{\infty}{1}$ & & $\begin{array}{l}\vec{H} \\
\infty \\
\infty\end{array}$ & $\begin{array}{l}\stackrel{1}{0} \\
\stackrel{0}{0}\end{array}$ & $\begin{array}{l}\infty \\
\infty \\
\infty\end{array}$ & $\begin{array}{l}\frac{10}{10} \\
\infty\end{array}$ & $\begin{array}{l}\vec{H} \\
\dot{\infty} \\
\infty\end{array}$ & $\begin{array}{l}\stackrel{O}{0} \\
\stackrel{0}{0}\end{array}$ & & & \\
\hline & 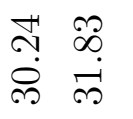 & 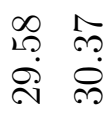 & $\begin{array}{l}\stackrel{H}{N} \\
\text { S. } \\
\text { So }\end{array}$ & $\begin{array}{c}\infty \\
\infty \\
\dot{\infty}\end{array}$ & 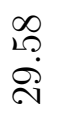 & & 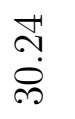 & $\begin{array}{c}\infty \\
\infty \\
\infty \\
\infty\end{array}$ & $\begin{array}{l}\infty \\
2 \mathfrak{1} \\
\stackrel{1}{ }\end{array}$ & & $\begin{array}{l}\stackrel{H}{N} \\
\stackrel{0}{\infty}\end{array}$ & $\begin{array}{c}\infty \\
\dot{\infty} \\
\infty\end{array}$ & 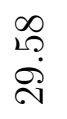 & م⿱⺈ & 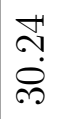 & $\begin{array}{l}\infty \\
\infty \\
\infty \\
\infty\end{array}$ & $\stackrel{N}{N}$ & $\begin{array}{l}\text { no } \\
\stackrel{0}{\infty}\end{array}$ & \\
\hline & 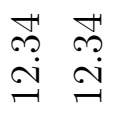 & 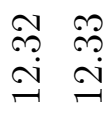 & 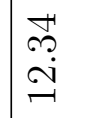 & $\begin{array}{l}\stackrel{+}{\infty} \\
\stackrel{2}{1} \\
\stackrel{-1}{*}\end{array}$ & \begin{tabular}{l} 
ஸै \\
$\stackrel{\sim}{*}$ \\
\multirow{-}{*}{}
\end{tabular} & & $\begin{array}{l}\vec{H} \\
\stackrel{+}{7} \\
\stackrel{7}{1}\end{array}$ & $\begin{array}{l}\vec{H} \\
\stackrel{\leftrightarrow}{+} \\
\stackrel{-}{ت}\end{array}$ & 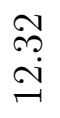 & $\stackrel{\sim}{-}$ & $\begin{array}{l}\stackrel{H}{\infty} \\
\stackrel{+}{\sigma}\end{array}$ & $\begin{array}{l}\stackrel{H}{H} \\
\stackrel{\leftrightarrow}{-}\end{array}$ & $\begin{array}{l}\stackrel{\text { Oे }}{2} \\
\stackrel{\sim}{\sim}\end{array}$ & 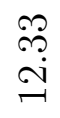 & 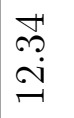 & $\begin{array}{l}\stackrel{+}{W} \\
\stackrel{n}{-}\end{array}$ & $\stackrel{\sim}{-1}$ & $\Re$ & \\
\hline & $\begin{array}{ll}\stackrel{20}{P} & 20 \\
\stackrel{0}{0} & 0 \\
& 0\end{array}$ & 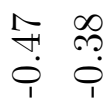 & 点 & $\begin{array}{l}\stackrel{2}{2} \\
0 \\
0 \\
1\end{array}$ & $\stackrel{n}{\stackrel{N}{+}}$ & $\infty$ & 畩 & $\begin{array}{l}\stackrel{2}{2} \\
\stackrel{0}{0} \\
1\end{array}$ & $\stackrel{r}{\stackrel{n}{0}}$ & & 点 & $\begin{array}{l}\stackrel{2}{2} \\
\stackrel{0}{0} \\
\end{array}$ & $\begin{array}{l}\text { fr } \\
\stackrel{0}{0}\end{array}$ & $\begin{array}{l}\infty \\
? \\
? \\
0 \\
1\end{array}$ & $\stackrel{109}{\stackrel{10}{0}}$ & $\begin{array}{l}\stackrel{20}{Q} \\
\stackrel{?}{0} \\
1\end{array}$ & $P_{1}$ & $\begin{array}{l}\infty \\
\stackrel{2}{?} \\
0 \\
\end{array}$ & \\
\hline & 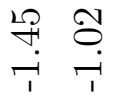 & ت. & 点 & 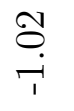 & $\stackrel{+4}{i}$ & קִ & 皇 & $\underset{\text { Oे }}{\stackrel{\text { Tे }}{1}}$ & $\stackrel{\vec{H}}{\vec{H}}$ & קִ & $\frac{\stackrel{20}{7}}{\stackrel{7}{+}}$ & $\underset{\substack{0\\
}}{+}$ & $\stackrel{\leftrightarrow}{\rightarrow}$ & קִ & 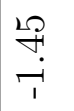 & 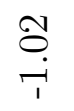 & 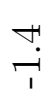 & קి & \\
\hline$\sum_{0}^{8}$ & $\begin{array}{ll}\mathscr{0} & \vec{\sim} \\
\dot{\oplus} & \ddot{s}\end{array}$ & $\begin{array}{ll}\stackrel{N}{i c} & \stackrel{1}{1} \\
\leftrightarrow & \leftrightarrow\end{array}$ & $\begin{array}{l}\mathscr{O} \\
\dot{0} \\
\infty\end{array}$ & $\vec{\sim}$ & $\begin{array}{l}\text { के } \\
\text { के }\end{array}$ & $\stackrel{N}{i}$ & $\begin{array}{l}\tilde{b} \\
\dot{\infty}\end{array}$ & $\begin{array}{l}\vec{N} \\
\ddot{n}\end{array}$ & $\begin{array}{l}8 \\
\text { is } \\
\text { is }\end{array}$ & $\stackrel{i}{i}$ & $\begin{array}{l}\mathscr{\bigcup} \\
\oplus \dot{0}\end{array}$ & $\vec{N}$ & $\begin{array}{l}\text { 今. } \\
\text { is }\end{array}$ & $\stackrel{N}{i}$ & $\begin{array}{l}\tilde{0} \\
\dot{\infty}\end{array}$ & $\overrightarrow{\stackrel{N}{N}}$ & $\begin{array}{l}\text { शे } \\
\text { ते }\end{array}$ & i & \\
\hline$\sum_{0}^{a}$ & 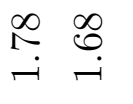 & $\stackrel{\substack{\infty \\
亡}}{\sim}$ & $\stackrel{\infty}{\stackrel{\infty}{L}}$ & 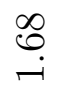 & 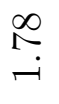 & 닫 & 足 & $\stackrel{\infty}{0}$ & $\stackrel{\infty}{\stackrel{\infty}{L}}$ & $\stackrel{N}{\stackrel{N}{r}}$ & 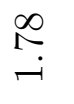 & 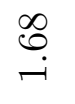 & 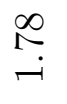 & $\stackrel{N}{\stackrel{N}{N}}$ & $\stackrel{\infty}{\substack{1 \\
-i}}$ & 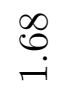 & $\stackrel{\infty}{\substack{1 \\
-i}}$ & 卡 & 0 \\
\hline 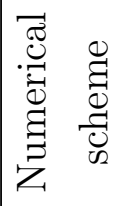 & 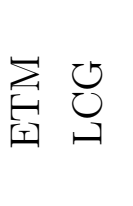 & 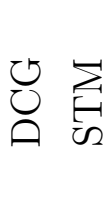 & $\underset{\text { 至 }}{\sum_{1}}$ & $\begin{array}{l}\circlearrowright \\
己 \\
\smile\end{array}$ & Uِ & $\sum$ & $\underset{\text { 田 }}{\sum_{1}}$ & 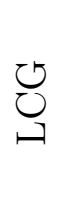 & Uִ & is & $\underset{\text { 至 }}{\sum_{1}}$ & ঠુ & Uِ & $\sum_{i=1}$ & $\underset{\text { 至 }}{\sum_{1}}$ & U్త & & $\sum_{\xi}$ & \\
\hline 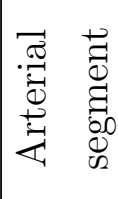 & 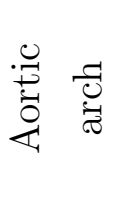 & $\Xi$ & 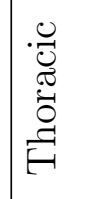 & 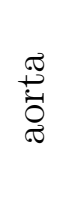 & છ & & 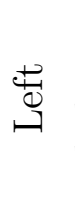 & 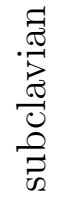 & 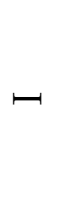 & & $\begin{array}{l}\boxminus \\
\overrightarrow{ \pm} \\
.00 \\
\overrightarrow{01}\end{array}$ & 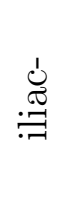 & 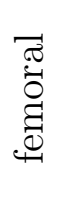 & & 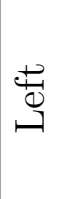 & $\underset{\Xi}{\Xi}$ & & & \\
\hline
\end{tabular}


$\stackrel{9}{+}$

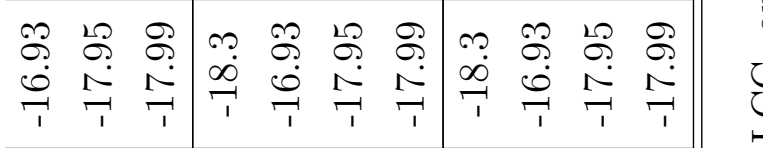

.

$\sum_{i}$

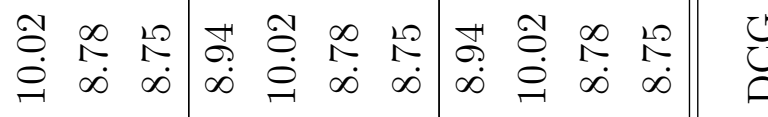

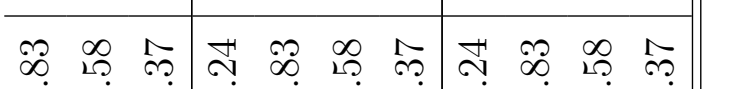

m ते की

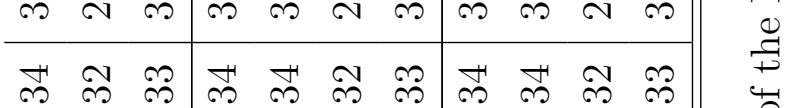

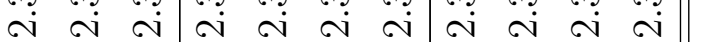

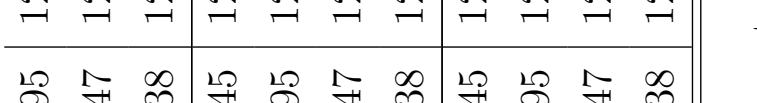

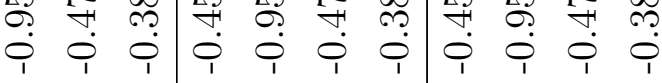

จิ

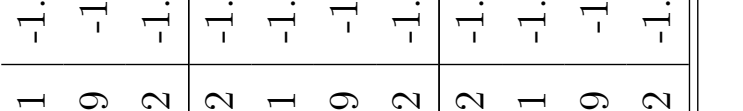

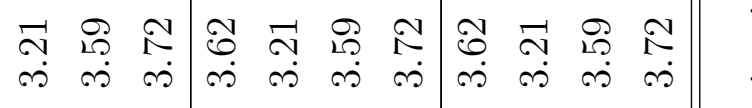

$\infty$ 我

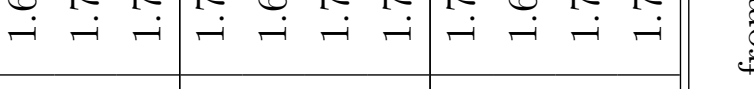

导

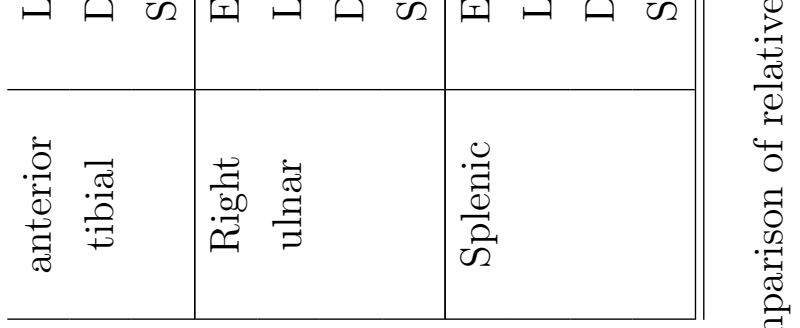

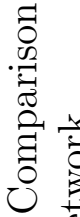

$\ddot{0}$

궁

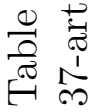

Pg. $80 / 284$ 


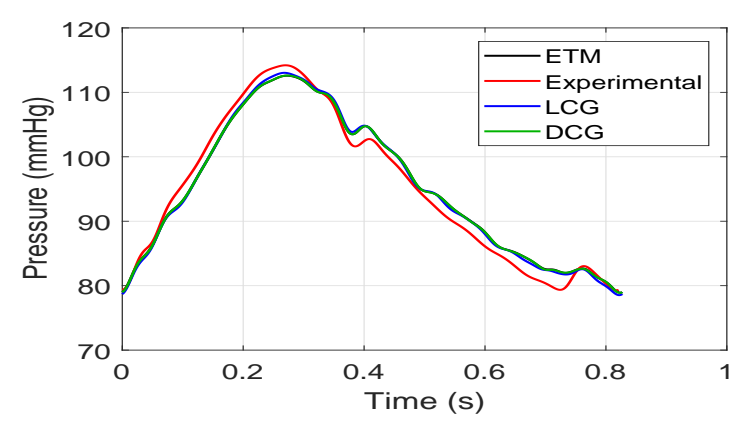

(a) Pressure in aortic arch II

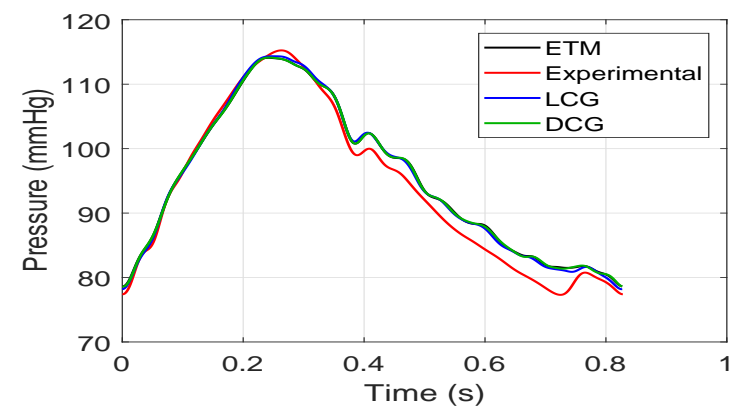

(c) Pressure in thoracic aorta II

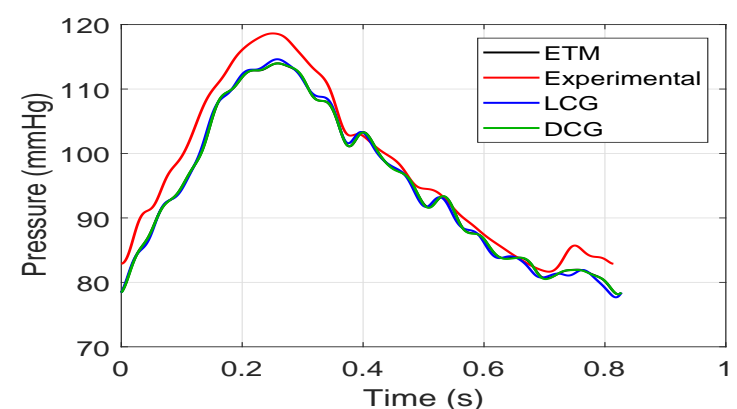

(e) Pressure in left subclavian I

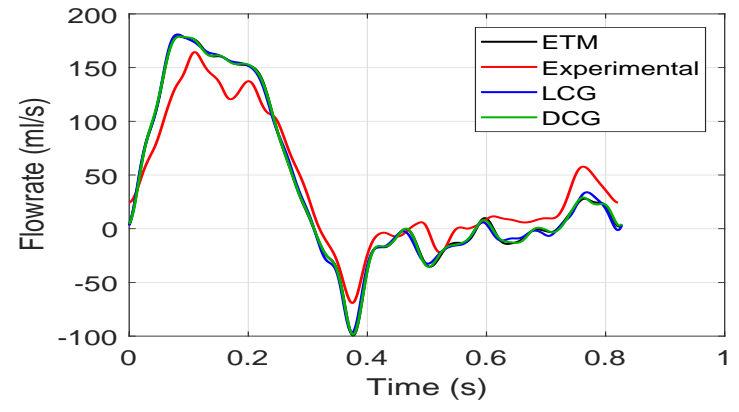

(b) Flow rate in aortic arch II

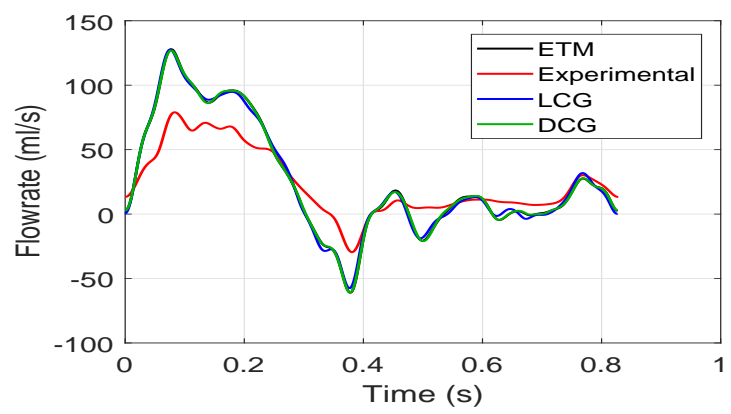

(d) Flow rate in thoracic aorta II

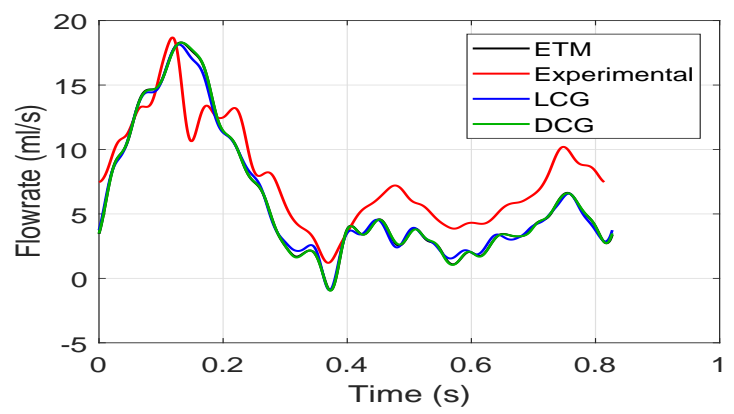

(f) Flow rate in left subclavian I

Figure 4.5: Pressure and flow waveforms in the 37-arterial tree network, comparison between ETM, DCG, LCG, and experimental measurements.

\subsubsection{ADAN56 Arterial Network}

The largest network tested in the benchmark paper [41] is a network consisting of 56 of the major systemic arteries, which is a reduced model of an arterial network originally proposed in $[37,36]$. Table 4.11 shows the main haemodynamic parameters used for this simulation. For more details on parameters, including volumetric inflow rate and the arterial network description, see [41]. There exists no in-vitro 


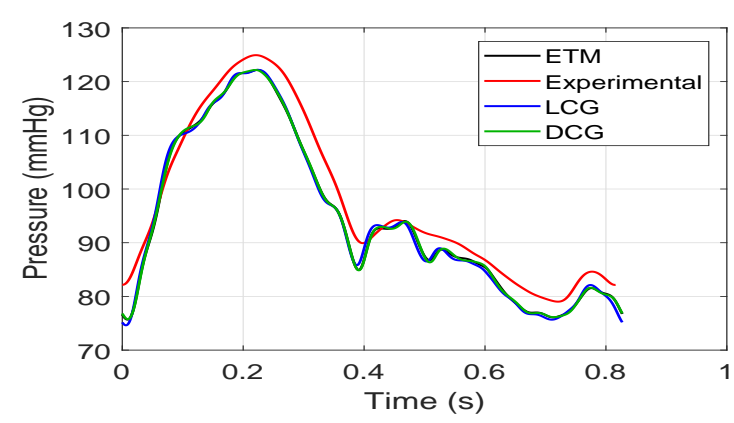

(a) Pressure in right iliac femoral II

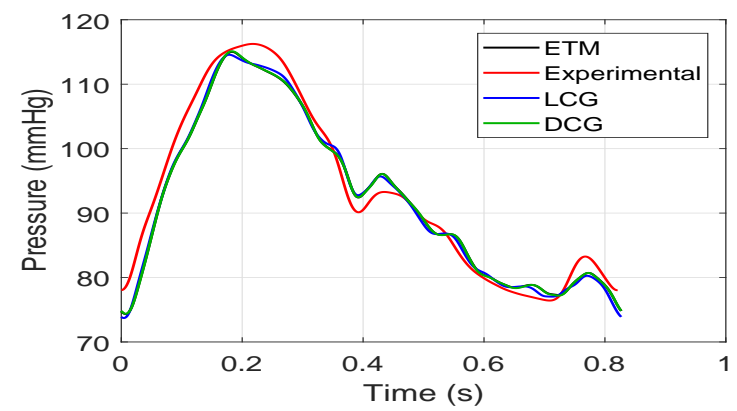

(c) Pressure in left ulnar

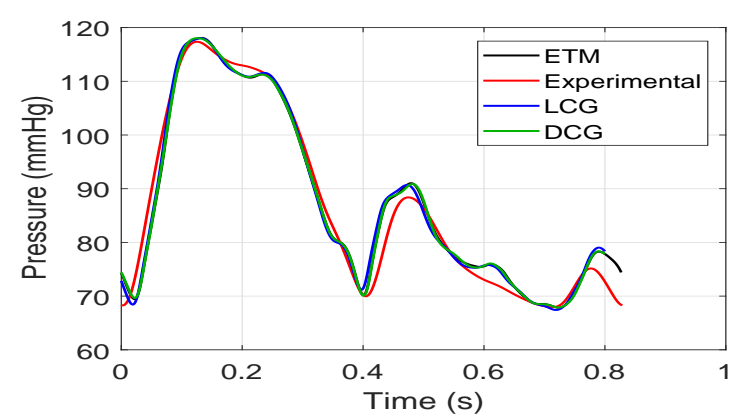

(e) Pressure in right anterior tibial

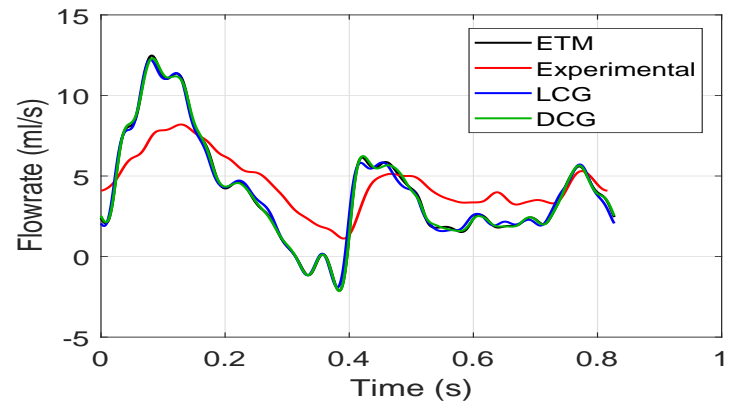

(b) Flow rate in right iliac femoral II

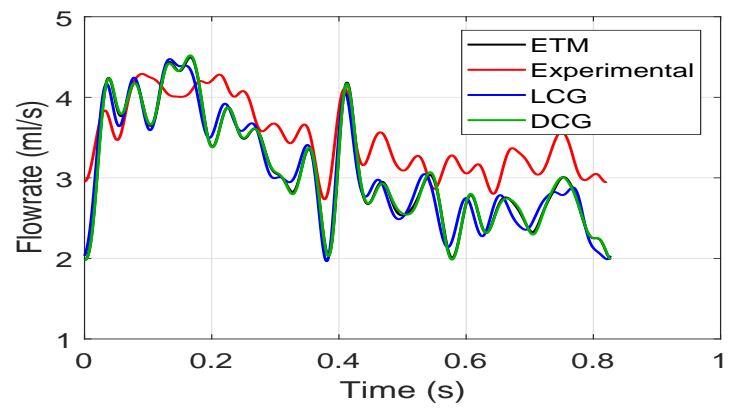

(d) Flow rate in left ulnar

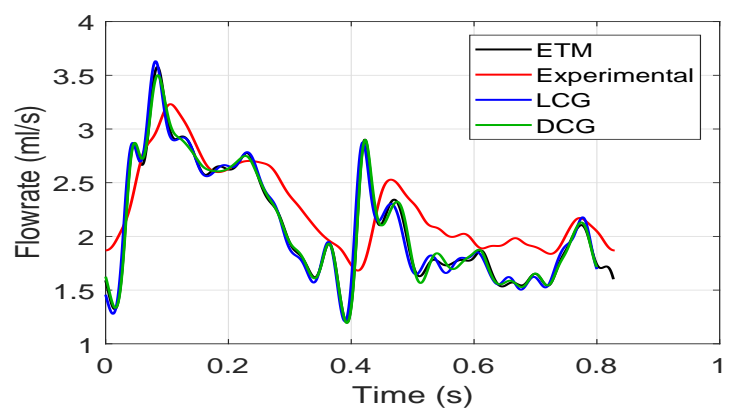

(f) Flow rate in right anterior tibial

Figure 4.6: Pressure and flow waveforms in the 37-arterial tree network, comparison between ETM, DCG, LCG, and experimental measurements.

data, or in-silico data from 3D models for this network, and hence the solutions of six 1D models were compared and published in [41]. Thus in this subsection, the ETM scheme is compared with three of the solutions from the benchmark paper. Furthermore, while the majority of current schemes are explicit and must adhere to a CFL number restriction for stability purposes, the ETM scheme is implicit and hence is not restricted by the CFL number. Thus the ETM scheme is simulated for several combinations of element size $\Delta x$ and time step size $\Delta t$, in order to investi- 


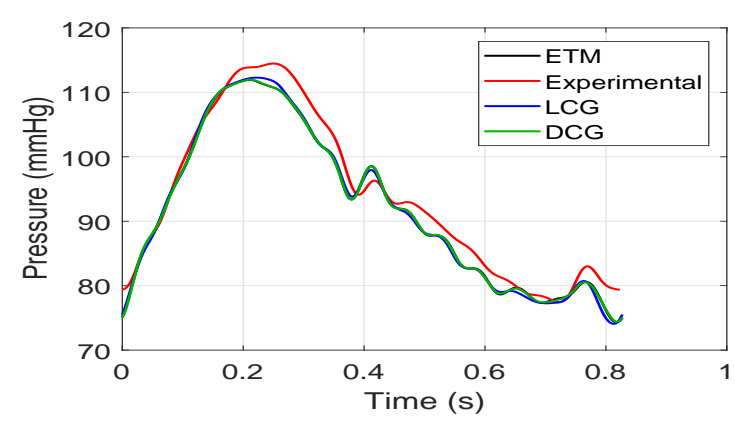

(a) Pressure in right ulnar

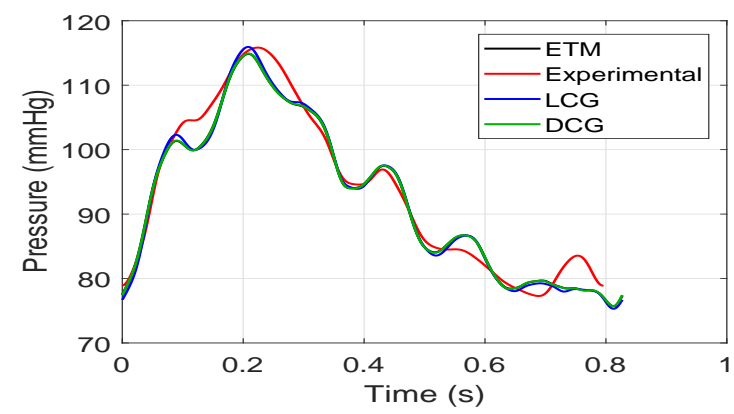

(c) Pressure in splenic

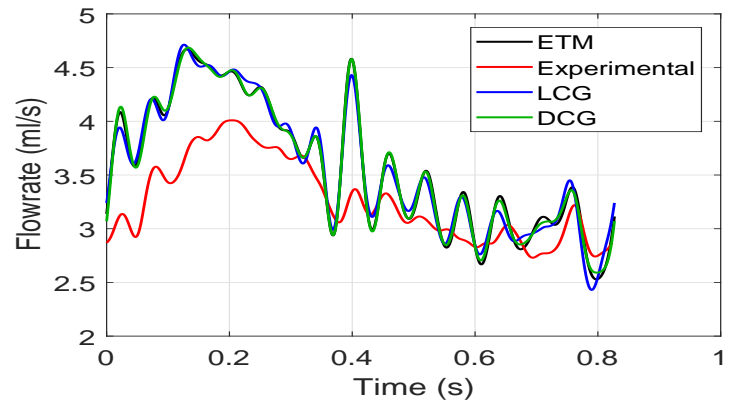

(b) Flow rate in right ulnar

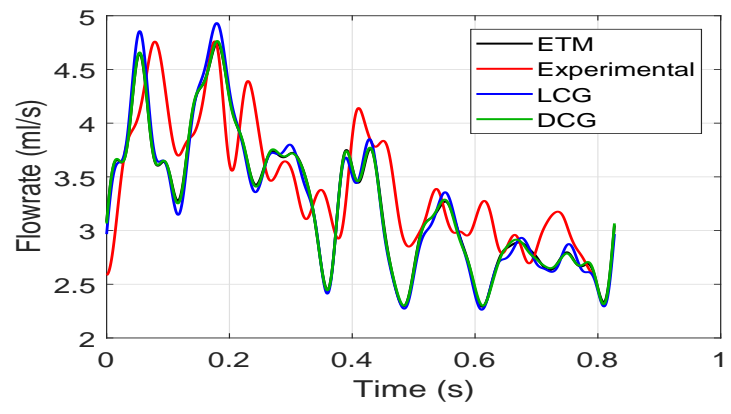

(d) Flow rate in splenic

Figure 4.7: Pressure and flow waveforms in the 37-arterial tree network, comparison between ETM, DCG, LCG, and experimental measurements.

gate whether using higher CFL numbers would still give acceptable solutions. As mentioned in section 3.2 the ETM is an extension of the STM scheme by [182]. The STM scheme performed poorly when compared with the other schemes published in [41] for the ADAN network, however the STM gave results that were consistent with other schemes for all other cases in the benchmark paper. The differences in solution of the STM scheme for the ADAN model were attributed to the STM's inability to hold conservation of total pressure at vessel junctions as the STM was restricted to use conservation of static pressure by sharing a pressure node between parent and child vessels. This explanation of the choice of junction condition was not originally investigated or confirmed, as all other numerical methods in [41] implemented conservation of total pressure, and conservation of static pressure was not tested for any other scheme. Thus implementing the ETM scheme for this problem also confirms the hypothesis from [41] that the ADAN model is more sensitive to the choice of the pressure conservation condition at vessel junctions. The effect of junction conditions and non-linearities in the system are investigated for a closed 


\begin{tabular}{|c|c||c|}
\hline Property & Units & Parameter Value \\
\hline Blood density, $\rho$ & $\mathrm{kg} \mathrm{m}^{-3}$ & 1040 \\
Blood viscosity, $\mu$ & $\mathrm{mPas}$ & 4 \\
Velocity profile order $\zeta$ & - & 2 \\
Elastic modulus, $E$ & $\mathrm{kPa}$ & 225.0 \\
Diastolic pressure, $P_{d}$ & $\mathrm{kPa}$ & 10 \\
External pressure, $P_{\text {ext }}$ & $\mathrm{Pa}$ & 0 \\
Initial pressure, $P(x, 0)$ & $\mathrm{kPa}$ & 0 \\
Outflow pressure, $P_{\text {out }}$ & $\mathrm{Pa}$ & 0 \\
\hline
\end{tabular}

Table 4.11: Properties for ADAN network case.

loop system in section 5 .

Figures 4.8, 4.9, 4.10, and 4.11 compare pressure and volumetric flow rate wave forms of the ETM (for three different combinations of element size and time step), STM, LCG, and DCG schemes for several vessel through the systemic arterial system. The solutions are compared in the following vessels: aortic arch I, which physiologically is located just after the coronary arteries in the ascending aorta; thoracic aorta III, which is located close to the midpoint in the descending part of the aorta; abdominal aorta $\mathrm{V}$, which is located at the very bottom of the descending aorta; the right common and internal carotid arteries, which are located in the neck; the right renal artery, which brings blood to the right kidney and branches from the abdominal aorta; right common and internal iliac arteries, which are located in the pelvic region; the right radial artery and right posterior interosseous, which are located in the forearm; the right femoral and right anterior tibial artery, which are located in the upper and lower leg, respectively.

The solutions show that the ETM scheme gives solutions consistent with the LCG, and DCG schemes. The STM scheme can be seen to deviate from the other numerical schemes for all vessels, which even includes pressure in the aortic arch (which is the inlet for the model). The ETM scheme has been tested with conservation of static pressure, and then gives the same solution as the STM, implying that the choice of pressure conservation law at vessel junctions is responsible for the deviation of the solution for the STM scheme.

In addition, three different combinations of element size and time step size are 


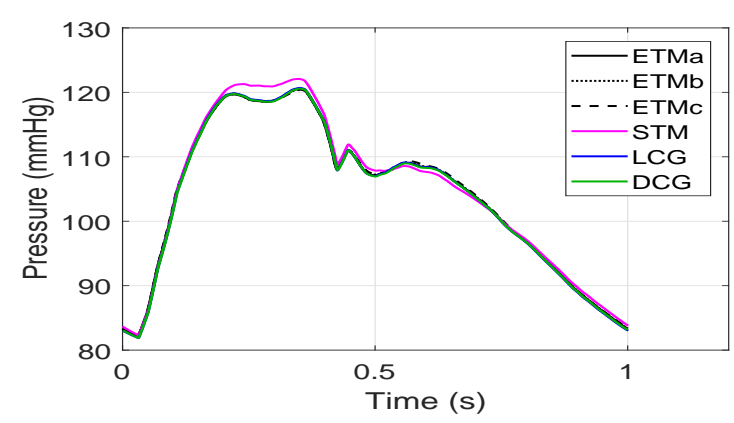

(a) Pressure in aortic arch I

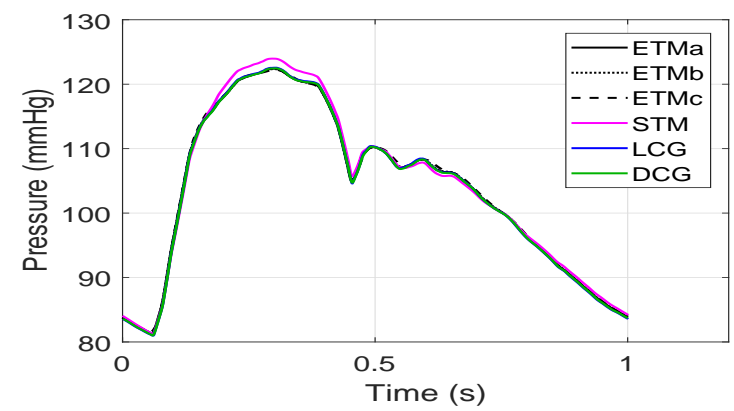

(c) Pressure in thoracic aorta III

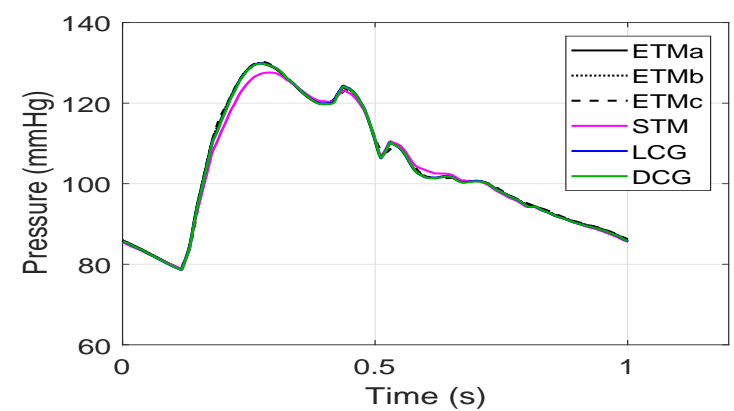

(e) Pressure in abdominal aorta V

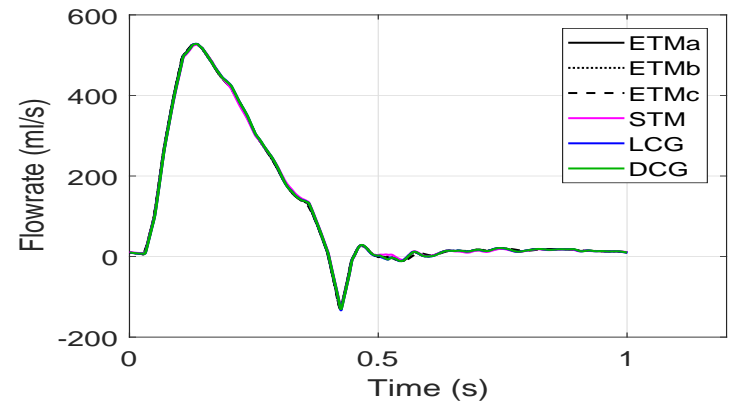

(b) Flow rate in aortic arch I

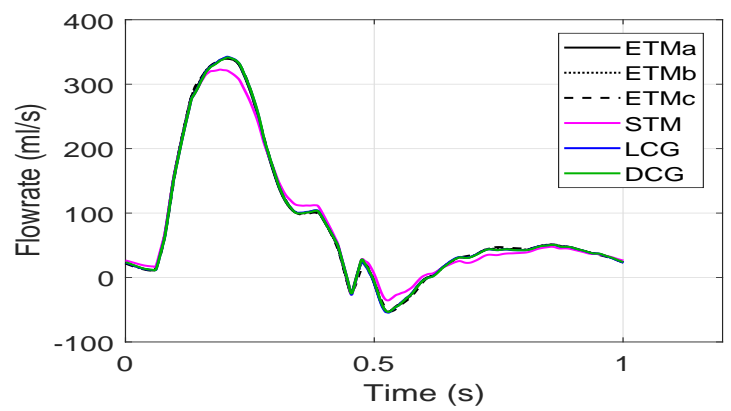

(d) Flow rate in thoracic aorta III

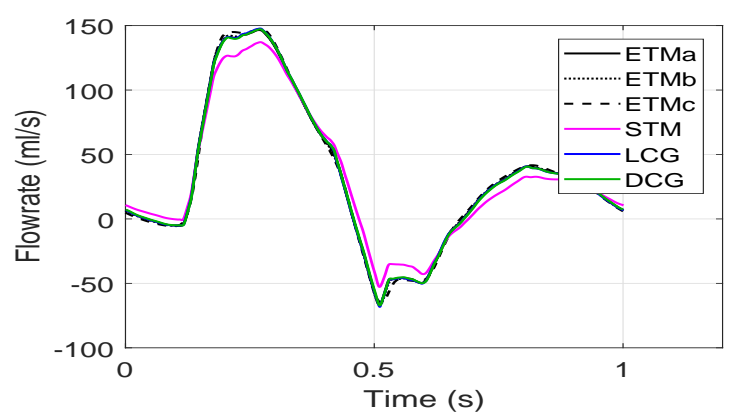

(f) Flow rate in abdominal aorta $\mathrm{V}$

Figure 4.8: Pressure and flow rate waveforms in the aortic arch I, thoracic aorta III, and abdominal aorta V. Results are shown for the ETM, STM, DCG, and LCG methods. ETMa is the ETM scheme with $\Delta t=1 \mathrm{~ms}$ and $\Delta x=1 \mathrm{~cm}, \mathrm{ETMb}$ is the ETM scheme with $\Delta t=2 \mathrm{~ms}$ and $\Delta x=2 \mathrm{~cm}$, ETMc is the ETM scheme with $\Delta t=5 \mathrm{~ms}$ and $\Delta x=5 \mathrm{~cm}$.

considered by the ETM scheme. The time step for the ETMa simulation is $\Delta t=1 \mathrm{~ms}$ while the element size is $\Delta x=1 \mathrm{~cm}$; the ETMb uses a time step of $\Delta t=2 \mathrm{~ms}$ and $\Delta x=2 \mathrm{~cm}$ for the element size; the ETMc uses $\Delta t=5 \mathrm{~ms}$ for the time step and an element size of $\Delta x=5 \mathrm{~cm}$. Table 4.12 shows the maximum CFL number 


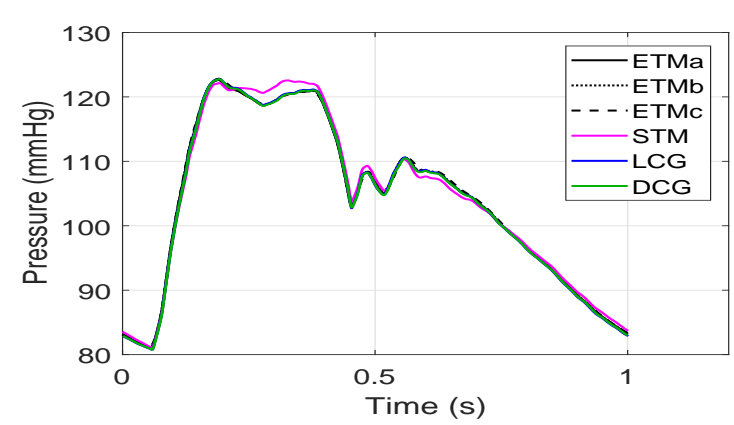

(a) Pressure in right common carotid artery

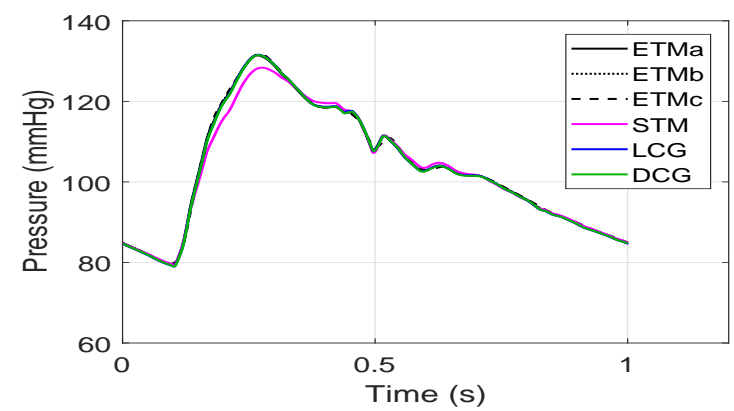

(c) Pressure in right renal artery

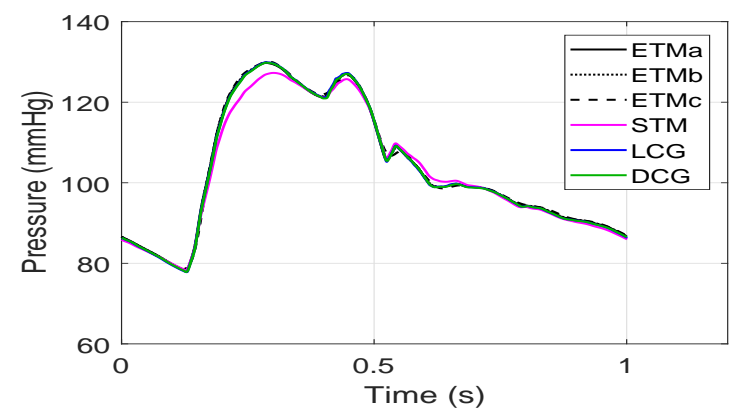

(e) Pressure in right common iliac artery

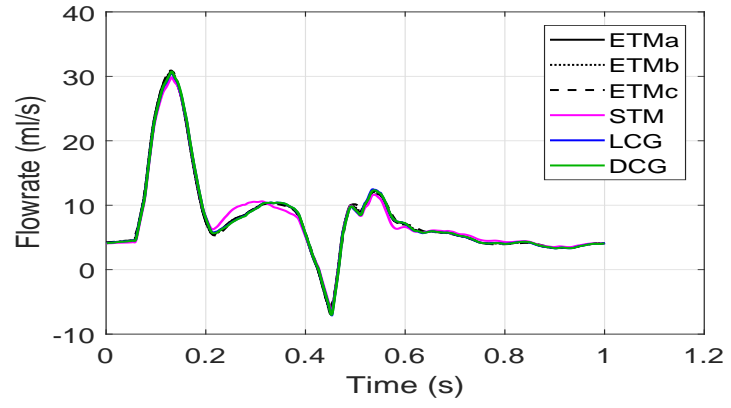

(b) Flow rate in right common carotid artery

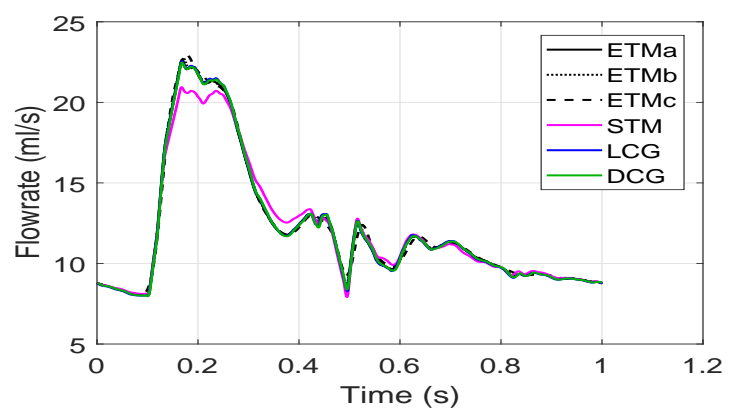

(d) Flow rate in right renal artery

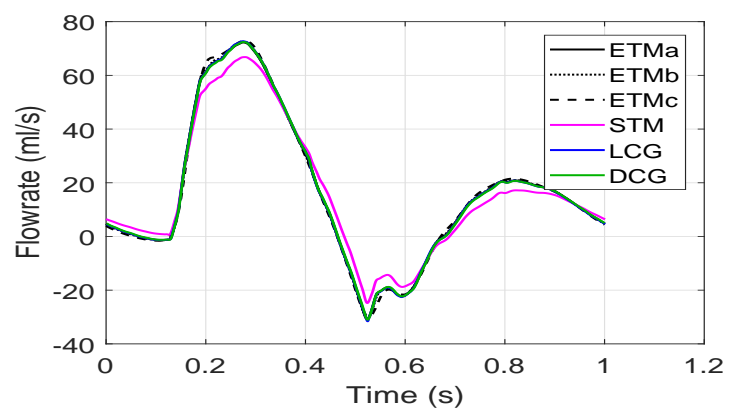

(f) Flow rate in right common iliac artery

Figure 4.9: Pressure and flow rate waveforms in right common carotid artery, right renal artery, and right common iliac artery. Results are shown for the ETM, STM, DCG, and LCG methods. ETMa is the ETM scheme with $\Delta t=1 \mathrm{~ms}$ and $\Delta x=$ $1 \mathrm{~cm}$, ETMb is the ETM scheme with $\Delta t=2 \mathrm{~ms}$ and $\Delta x=2 \mathrm{~cm}$, ETMc is the ETM scheme with $\Delta t=5 \mathrm{~ms}$ and $\Delta x=5 \mathrm{~cm}$.

(Courant-Friedrichs-Lewy condition) and CPU time taken per cardiac cycle for each simulation. The lowest CFL number gives results almost identical to the LCG and DCG schemes from [41], however explicit schemes would be unstable using this time step and element size combination. It is important to note that a lower limit of two 


\begin{tabular}{|c|c|c|c|c|}
\hline ETM & Time Step $(\mathrm{ms})$ & Max Element Size $(\mathrm{cm})$ & Max CFL & Time Taken $(\mathrm{s})$ \\
\hline $\mathrm{a}$ & 1 & 1 & 3.6789 & 2.31 \\
$\mathrm{~b}$ & 2 & 2 & 7.3631 & 0.83 \\
$\mathrm{c}$ & 5 & 5 & 18.4272 & 0.24 \\
\hline
\end{tabular}

Table 4.12: ETM scheme simulation for three different combinations of time step and element size.

elements per vessel is chosen for the simulation, and as a result of the shortest vessel being defined by two elements for all simulation cases (ETMa, ETMc, and ETMc), the maximum CFL number for each case only scales with the time step as the ratio of wave speed to element size does not change for the vessel with the highest CFL number.

Furthermore, only small discrepancies are seen for the ETM scheme when using the coarser temporal and spatial meshes. Table 4.13 shows the maximum peak and mean percentage errors for pressures and flow rates (for the vessels where these errors are largest), with the waveforms for these vessels shown in figure 4.12 for the ETMc and ETMa simulations. The maximum errors for P (and Q) at the centre of each vessel were calculated as follows

$$
\operatorname{Max} P_{i}=\left|\left(\frac{\operatorname{Max}\left(P_{i, \text { fine }}\right)-\operatorname{Max}\left(P_{i, \text { coarse }}\right)}{\operatorname{Max}\left(P_{i, \text { fine }}\right)}\right)\right|,
$$

while mean errors were calculated via

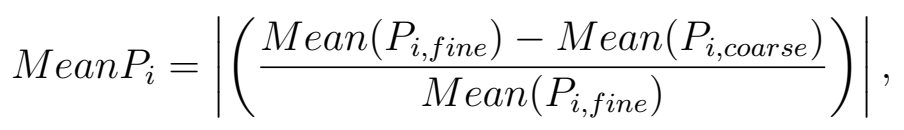

for $i=1: N$, where $N$ is the number of vessels. The largest $\operatorname{Max}_{i}, \operatorname{Mean} P_{i}$, $\operatorname{Max} Q_{i}$, and $M e a n Q_{i}$ were chosen for table 4.13. Although the peak values have errors, the waveforms of the ETMc simulations still capture the main trends in the waveforms quite well. Hence it may be of value to quickly estimate the solution (in close to real time) using a coarser mesh if only mean values of pressure and flow are required, or a coarse mesh could be used for several cardiac cycles to quickly converge, with a refined mesh used for the final one or two cardiac cycles. 


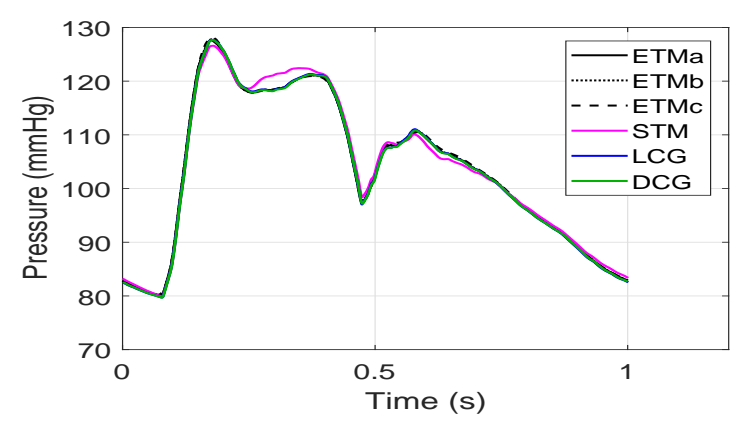

(a) Pressure in right internal carotid artery

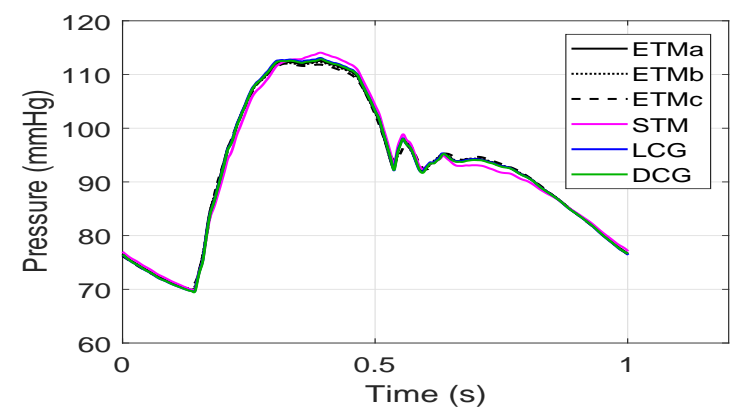

(c) Pressure in right radial artery

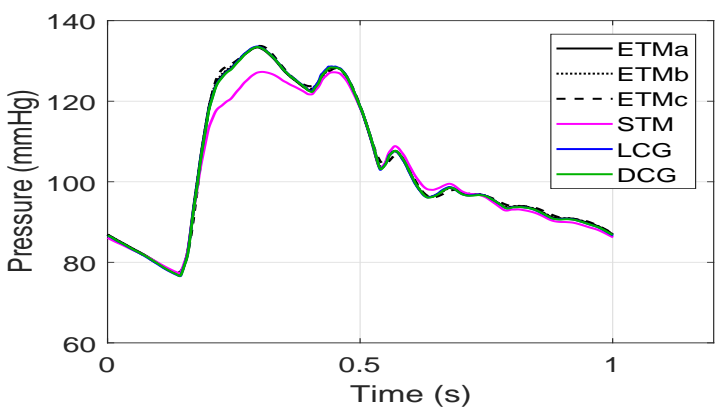

(e) Pressure in right internal iliac artery

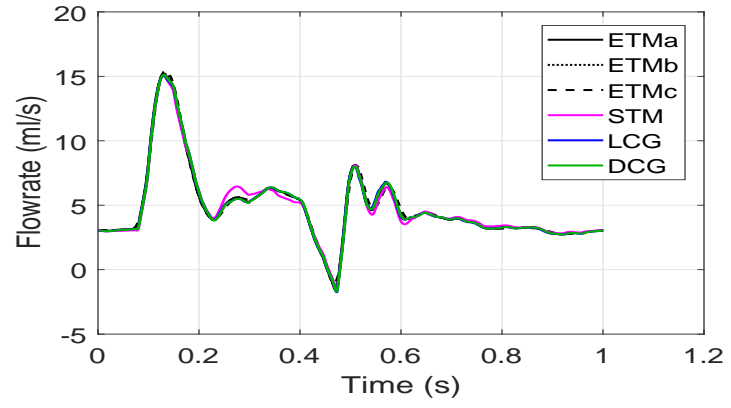

(b) Flow rate in right internal carotid artery

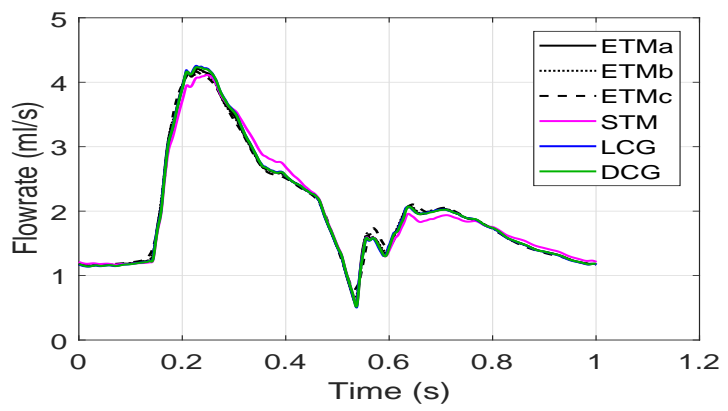

(d) Flow rate in right radial artery

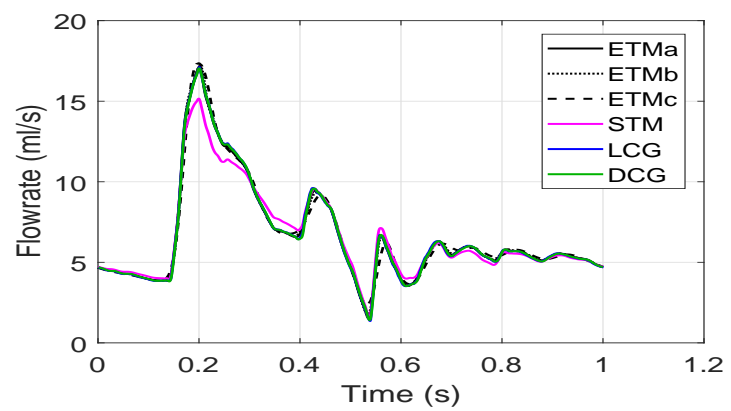

(f) Flow rate in right internal iliac qrtery

Figure 4.10: Pressure and flow rate waveforms in right internal carotid artery, right radial artery, and right internal iliac artery. Results are shown for the ETM, STM, DCG, and LCG methods. ETMa is the ETM scheme with $\Delta t=1 \mathrm{~ms}$ and $\Delta x=$ $1 \mathrm{~cm}$, ETMb is the ETM scheme with $\Delta t=2 \mathrm{~ms}$ and $\Delta x=2 \mathrm{~cm}$, ETMc is the ETM scheme with $\Delta t=5 \mathrm{~ms}$ and $\Delta x=5 \mathrm{~cm}$. 


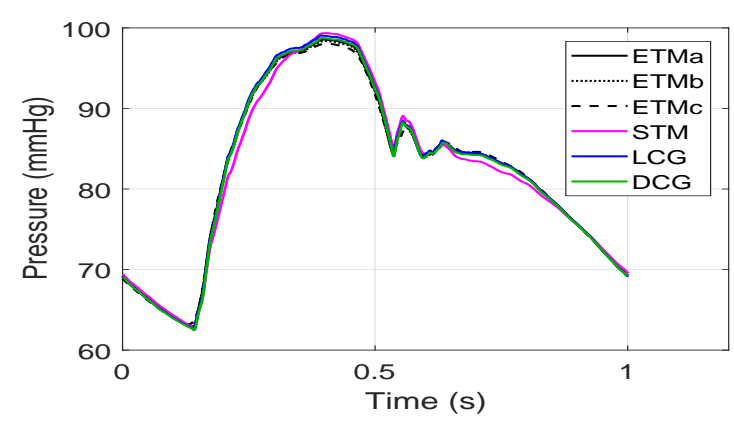

(a) Pressure in right posterior interosseous

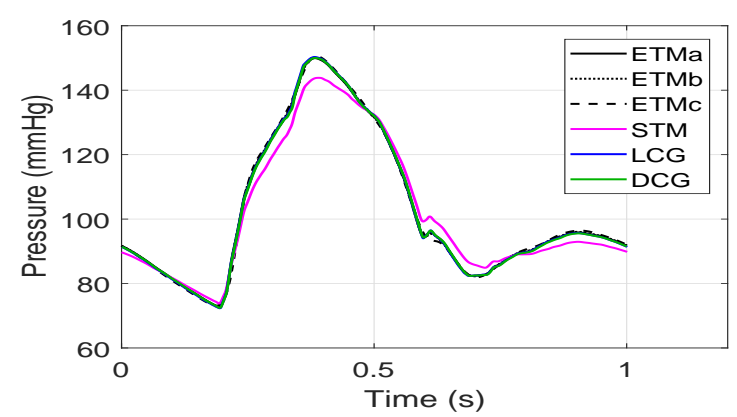

(c) Pressure in right femoral II artery

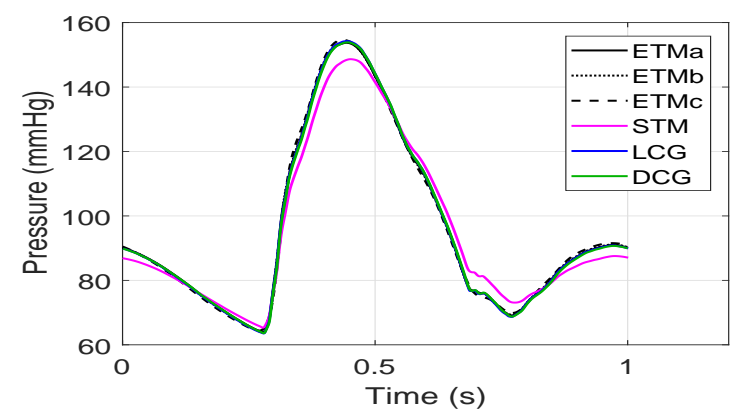

(e) Pressure in right anterior tibial artery

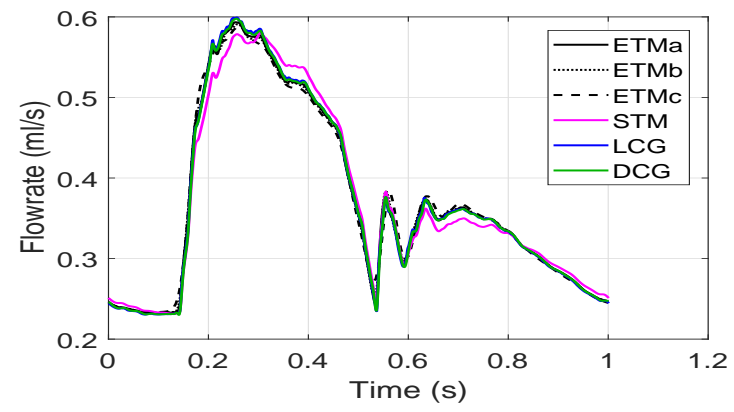

(b) Flow rate in right posterior interosseous

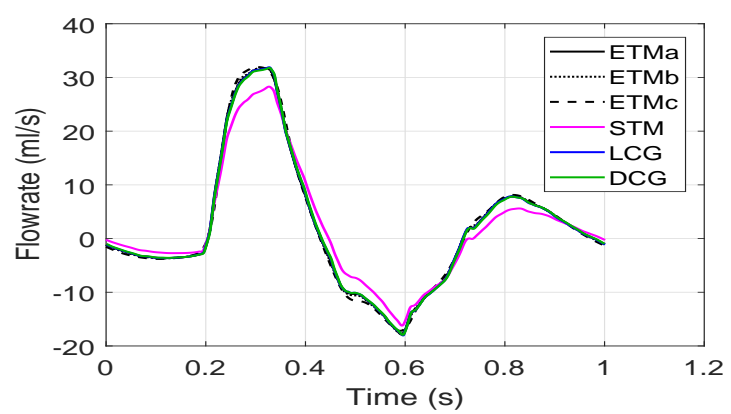

(d) Flow rate in right femoral II artery

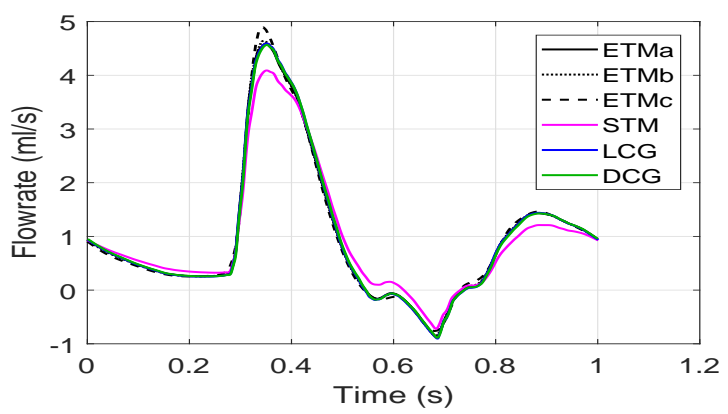

(f) Flow rate in right anterior tibial artery

Figure 4.11: Pressure and flow rate waveforms in right posterior interosseous, right femoral artery, and right anterior tibial. Results are shown for the ETM, STM, DCG, and LCG methods. ETMa is the ETM scheme with $\Delta t=1 \mathrm{~ms}$ and $\Delta x=1 \mathrm{~cm}$, $\mathrm{ETMb}$ is the ETM scheme with $\Delta t=2 \mathrm{~ms}$ and $\Delta x=2 \mathrm{~cm}$, ETMc is the ETM scheme with $\Delta t=5 \mathrm{~ms}$ and $\Delta x=5 \mathrm{~cm}$.

\subsection{Numerical Comparisons For Problems With Theoretical Solutions}

In this section additional cases are chosen to test the ability of the ETM scheme to handle various challenging problems. These will test a number of important concepts 


\begin{tabular}{|c|c|c|c|c|}
\hline ETM & Max P & Mean P & Max Q & Mean Q \\
\hline b & $1.776 \%$ & $0.041 \%$ & $3.231 \%$ & $0.238 \%$ \\
c & $6.782 \%$ & $0.129 \%$ & $11.259 \%$ & $0.998 \%$ \\
\hline
\end{tabular}

Table 4.13: Error of ETM scheme for coarser meshes ETMb and ETMc, in comparison with the ETMa simulation

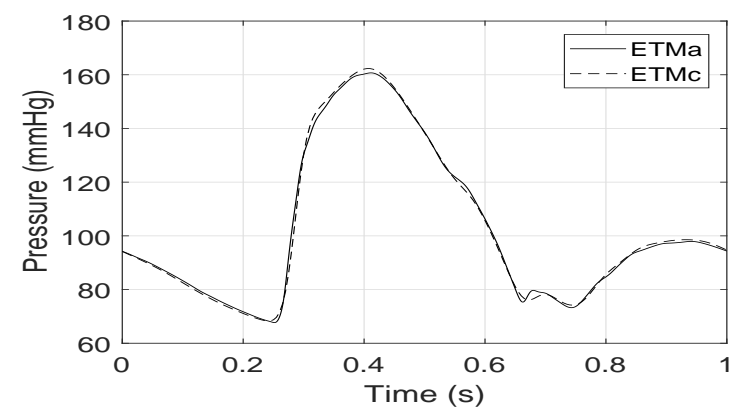

(a) Pressure in right popliteal artery II

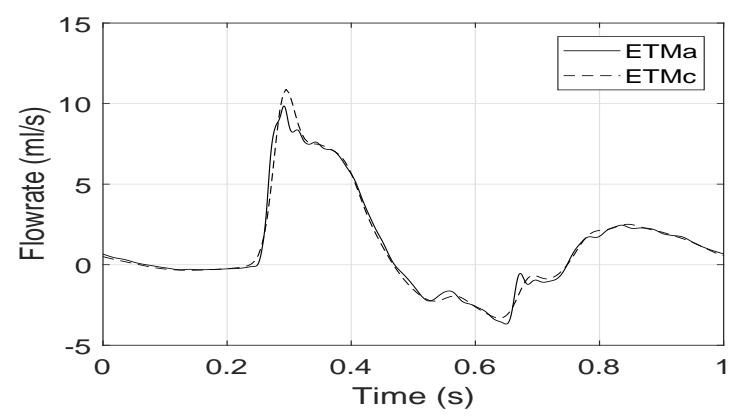

(b) Flow Rate in right tibiofibular trunk

Figure 4.12: Waveforms for vessels with maximum peak errors in pressure and flow rates for the ETMc, in comparison with the ETMa.

and situations in order to improve understanding of the numerical scheme, and to investigate any limitations that may be present, including:

- Investigating whether the scheme is well-balanced. In the computational community, well-balanced refers to the ability of a numerical scheme to correctly handle source terms, which is particularly important for stiff source terms, which is the case in the governing equations of 1D haemodynamics, particularly in the presence of a stenosis (narrowing) of a vessel.

- Conservation properties of the scheme (in particular mass conservation). Theoretically the scheme for the 1D system is mass conservative, as the equations are formulated in such a way as to solve for pressures and net flow rates (mass change, which includes Lagrange multipliers) in the global system. However, as with any scheme inaccuracies can occur due to order of accuracy (spatial and/or temporal order of the scheme). For example, it would be expected that the scheme would perform worse with respect to mass conservation for larger time steps or lower order of accuracy. Furthermore for an implicit method, conservation can also depend on the choice of convergence criteria, lineari- 
sation, and also on the condition number of the global system matrix and machine precision.

- Numerical order of accuracy, stability, convergence (spatial and temporal mesh, and periodic convergence) of the scheme and its components (1D and 0D).

- If the scheme can handle un-smooth changes in vessel geometry and/or material properties, which may even include discontinuous jumps.

- The ability to capture sharp gradients of pressure or flow rates, including shock waves, which could potentially occur due to external pressures, or from inclusion of gravity due to a sudden change in posture.

There are various cases that can be constructed in order to test the effectiveness, and correctness, of the numerical scheme. Due to the complex, non-linear behaviour of the system, there exists no analytical solution for the full non-linear set of equations. Therefore, in order to construct problems with analytical solutions, additional assumptions need to be made, which reduces the complexity of the system. These assumptions involve: reducing the system to a linear set of equations with similar properties, which can be used to investigate wave propagating phenomena in the vessel and at junctions; employing a steady state solution for a rigid tube for an inviscid fluid, which allows verification that the non-linear convection term is treated correctly by the scheme; and considering discontinuous material properties and geometry, which allows any jump in pressure or flow rate to be analytically calculated.

\subsubsection{Shockwave within a Vessel}

In order to ensure the numerical scheme correctly handles sharp gradients which may occur due to external pressure on a a vessel, a case similar to a shock tube problem is investigated. For this problem the inviscid set of equations are implemented $(\mu=0)$, with vessel properties and the parameters of the test given in Table 4.14. Reflectionfree boundary conditions are implemented at both the vessel inlet and outlet. The 
initial discontinuous pressure chosen to be quite severe and is given by

$$
P_{\text {initial }}(x)= \begin{cases}16.3995 \mathrm{kPa} & \text { if } \quad x<\frac{L}{2}, \\ 10.9333 \mathrm{kPa} & \text { if } \quad x \geq \frac{L}{2} .\end{cases}
$$

For this problem the time step is chosen to be $\Delta t=0.5 \mathrm{~ms}$, while the element size is $\Delta x=1 \mathrm{~cm}$.

The ETM scheme is compared with the theoretical solution (see [97] for details of the theoretical solution) in figure 4.13. A small amount of artificial diffusion is added to the momentum equation in order to suppress numerical oscillations that would occur due to the sharp gradient. The artificial diffusion term is chosen to have the same form as the visco-elasticity term from equation (2.20), which is written in the format

$$
\frac{\partial}{\partial x}\left(\frac{\Gamma C_{A}}{A_{0} \sqrt{A_{0}}} \frac{\partial P}{\partial t}\right)
$$

and is linearised and written in discrete form as described in section 3.13. For this problem the artificial diffusion $\Gamma=200$ was applied over the entire vessel, as the main reason was to investigate whether the scheme correctly predicts the wave speed at sharp gradients, although artificial diffusion could be added only in the region of the shock if required.

It can be seen from figures $4.13 \mathrm{~b}$ and $4.13 \mathrm{~d}$, that without artificial diffusion, oscillations develop. However, the introduction of a relatively low amount artificial diffusion $\Gamma=200$ eliminates these oscillations. Moreover, the results indicate the ETM scheme correctly handles and predicts the wave speed for the shock travelling in the forward (right) direction, and a rarefaction travelling in the backward (left) direction.

\subsubsection{Aneurysm and Stenosis with Steady Volumetric Flow Rate}

Thus far problems have been implemented to test the schemes ability to handle source terms correctly, and to correctly capture the wave speed of the system. How- 


\subsection{NUMERICAL COMPARISONS FOR PROBLEMS WITH THEORETICAL SOLUTIONS}

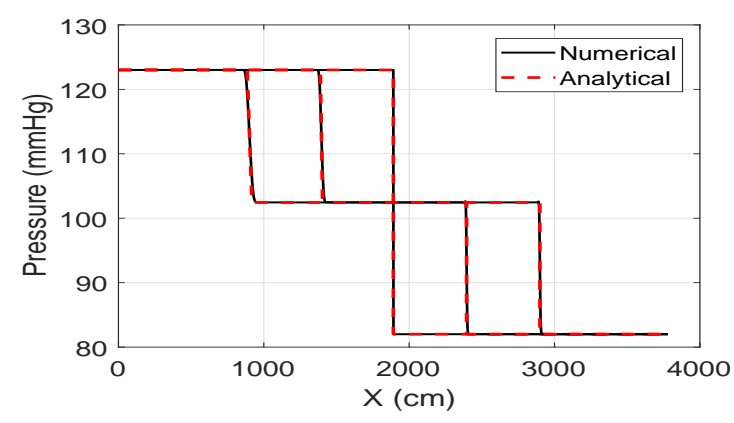

(a) Pressure with artificial diffusion

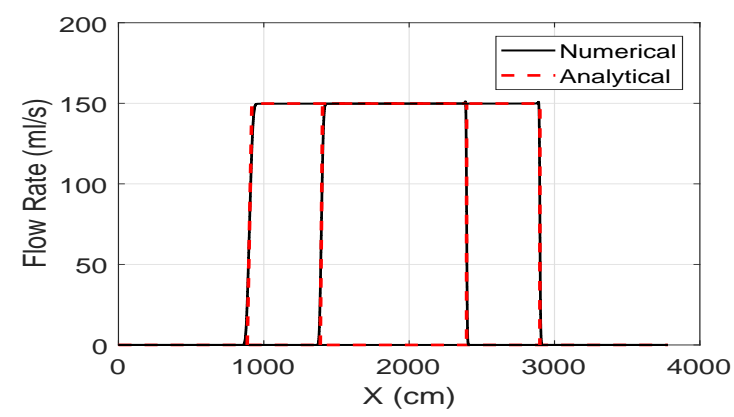

(c) Flow rate with artificial diffusion

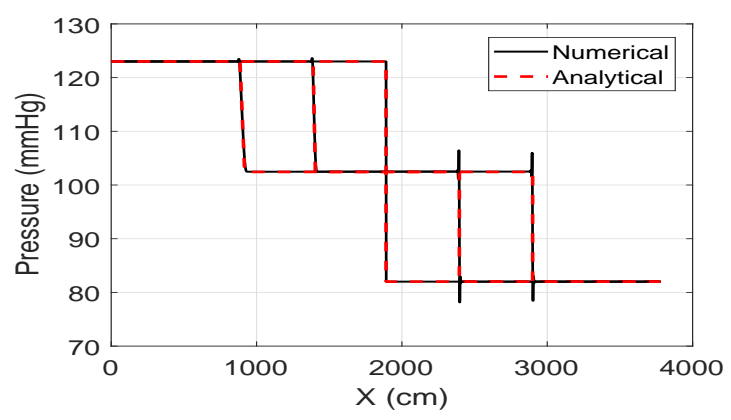

(b) Pressure without artificial diffusion

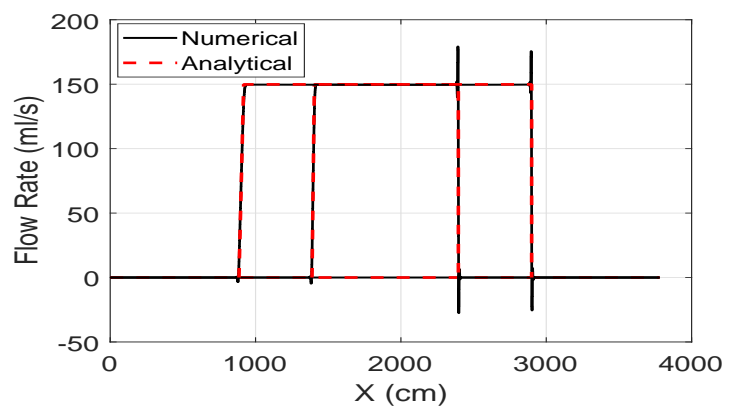

(d) Flow rate without artificial diffusion

Figure 4.13: Pressure and flow rate waveforms for shock test with and without artificial diffusion at different time instances (seconds).

ever, the ETM scheme has not been tested to determine whether the non-linear convection terms are correctly handled. In order to do this, two cases are considered with the inviscid formulation $\mu=0$ and a rigid wall model for the constitutive law, where the vessel wall is made rigid by choosing $\beta=\infty$, which causes the compliance to become $C_{A}=0$ everywhere in the domain. This technique would cause problems for explicit schemes due to the CFL condition, however the implicit ETM scheme is unconditionally stable for any CFL. The two cases considered are an aneurysm case and a stenosis case (both with severe changes in area), with the problem parameters being defined in table 4.15. The outlet condition is a single characteristic impedance. The system is considered to have a steady flow rate of $100 \mathrm{ml} \mathrm{s}^{-1}$.

For both aneurysm and stenosis cases, the pressure before and after the aneurysm or stenosis will be equal in magnitude. Within a stenosis the pressure should decrease as the velocity increases until the location of the minimum area is reached, the pressure will then increase until the healthy area is reached while the velocity de- 


\begin{tabular}{|c|c||c|}
\hline Property & Units & Vessel 1 \\
\hline Length, $L$ & $\mathrm{~cm}^{2}$ & 3780 \\
Area, $A_{d}$ & $\mathrm{~cm}^{2}$ & 5.6549 \\
Initial flow, $Q(x, 0)$ & $\mathrm{ml} \mathrm{s}^{-1}$ & 0 \\
Wall thickness, $h$ & $\mathrm{~cm}^{-3}$ & 0.3 \\
Blood density, $\rho$ & $\mathrm{kg} \mathrm{m}^{-3}$ & 1060 \\
Blood viscosity, $\mu$ & $\mathrm{mPas}$ & 0 \\
Velocity profile order $\zeta$ & - & 9 \\
Young's modulus, $E$ & $\mathrm{kPa}$ & 700.0 \\
Diastolic pressure, $P_{d}$ & $\mathrm{mmHg}$ & 82 \\
External pressure, $P_{\text {ext }}$ & $\mathrm{Pa}$ & 0 \\
Inflow pressure, $P_{\text {In }}$ & $\mathrm{mmHg}$ & 123 \\
Outflow pressure, $P_{\text {out }}$ & $\mathrm{mmHg}$ & 82 \\
Viscous wall coefficient, $\Gamma$ & $\mathrm{g} \mathrm{s}^{-1}$ & 200 \\
\hline \multicolumn{2}{|c|}{}
\end{tabular}

Table 4.14: Properties for shock wave test

creases to return to the same magnitude as before the stenosis. Within an aneurysm the opposite occurs, where the pressure increases as the velocity decreases until the location of the maximum area is reached, and then the pressure will decrease until the healthy area is reached while the velocity increases to return to the same magnitude as before the aneurysm.

The aneurysm or stenosis is chosen to be at the centre of the domain and has a length $L_{A}=10 \mathrm{~cm}$ defined in terms of its diameter by

$$
D(x)=D_{0}+\frac{D_{A}-D_{0}}{2}\left(1-\cos \left(\frac{x}{L_{A} 2 \pi}\right)\right)
$$

where $D_{0}$ is the healthy diameter, $D_{A}$ is the diseased diameter, and $x \in\left[0, L_{A}\right]$ is the spatial coordinate.

Figure 4.14 and figure 4.15 show the pressure, flow rate, area, and velocity for the aneurysm and stenosis cases respectively. The pressure solutions obtained for both cases indicate the ETM scheme treat the non-linear term correctly, as the pressure before and after the stenosis are equal in magnitude, while the behaviour of pressure and velocity within the aneurysm and stenosis show the expected behaviour. 


\begin{tabular}{|c|c||c||c|}
\hline Property & Units & Aneurysm & Stenosis \\
\hline Length, $L$ & $\mathrm{~cm}$ & 100 & 100 \\
Area (healthy), $A_{d}$ & $\mathrm{~cm}^{2}$ & $\pi$ & $\pi$ \\
Diameter (unhealthy), $D_{A}$ & $\mathrm{~cm}^{2}$ & $2 D_{0}$ & $D_{0} / 2$ \\
flow rate, $Q(x, 0)$ & $\mathrm{ml} \mathrm{s}^{-1}$ & 100 & 100 \\
Blood density, $\rho$ & $\mathrm{kg} \mathrm{m}^{-3}$ & 1050 & 1050 \\
Blood viscosity, $\mu$ & $\mathrm{mPas}$ & 0 & 0 \\
Velocity profile order $\zeta$ & - & 9 & 9 \\
Young's modulus, $E$ & $\mathrm{kPa}$ & $\infty$ & $\infty$ \\
Diastolic pressure, $P_{d}$ & $\mathrm{mmHg}$ & 0 & 0 \\
External pressure, $P_{\text {ext }}$ & $\mathrm{Pa}$ & 0 & 0 \\
Outflow pressure, $P_{\text {out }}$ & $\mathrm{mmHg}$ & 0 & 0 \\
\hline
\end{tabular}

Table 4.15: Properties for aneurysm and stenosis tests

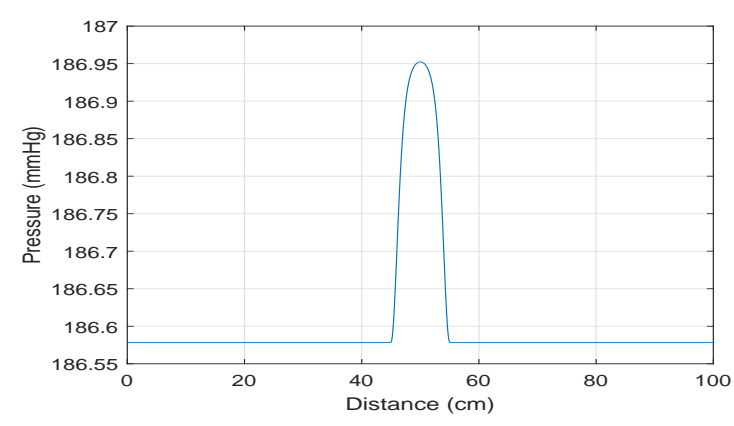

(a) Pressure

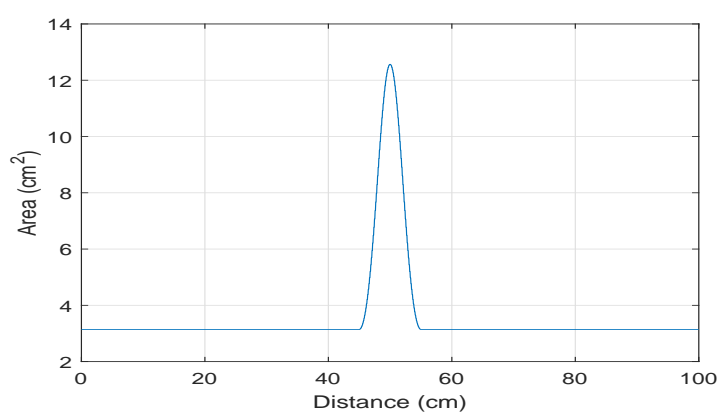

(c) Area

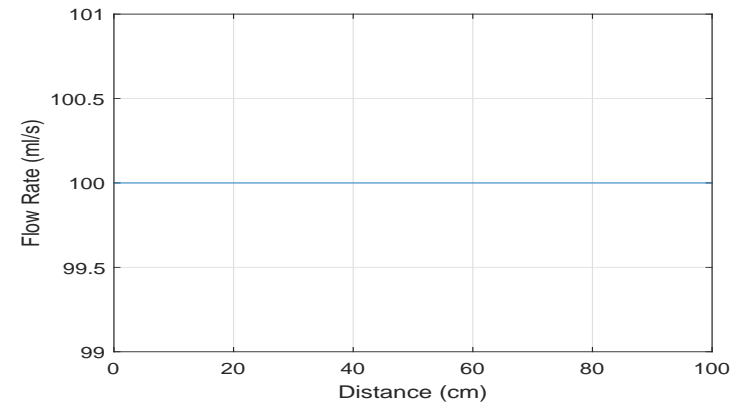

(b) Flow rate

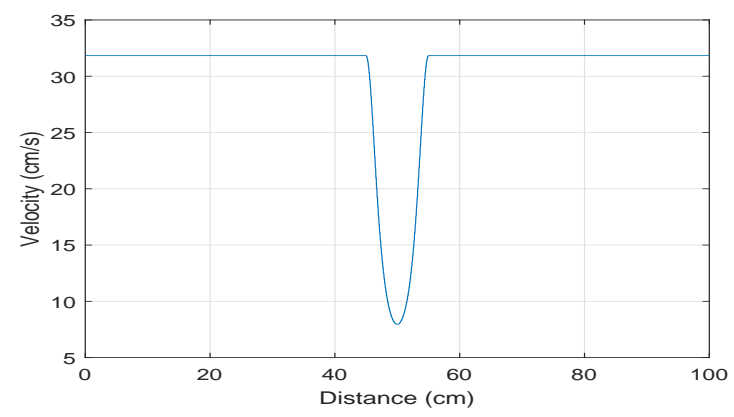

(d) Velocity

Figure 4.14: Waveforms for the aneurysm case.

\subsubsection{Discontinuous Material Properties and Geometry}

Wave reflections will occur in the system for any changes in material properties and geometry, hence it is important for the numerical scheme to correctly predict 


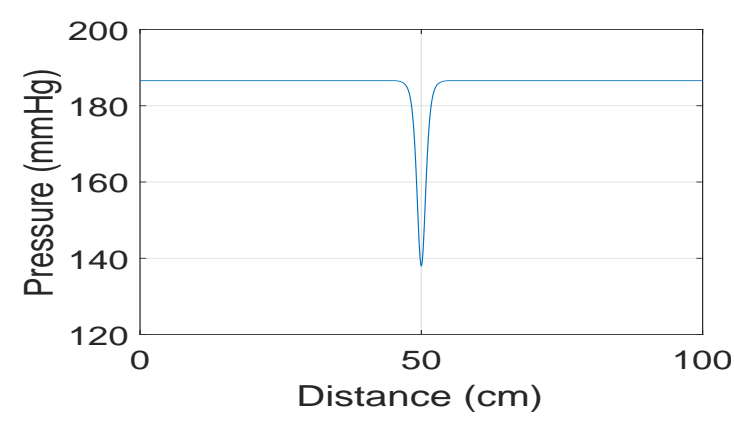

(a) Pressure

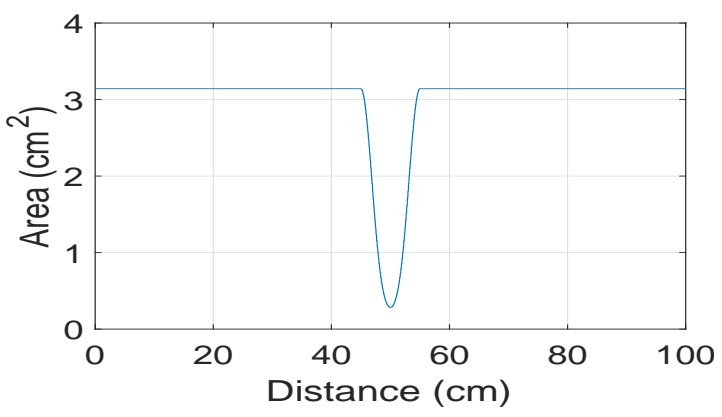

(c) Area

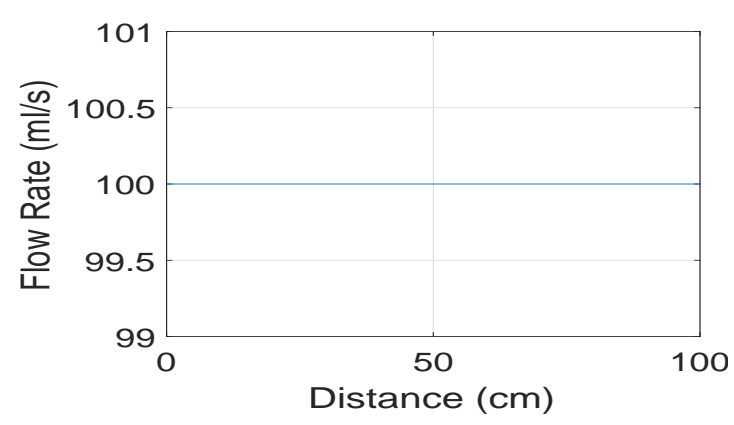

(b) Flow rate

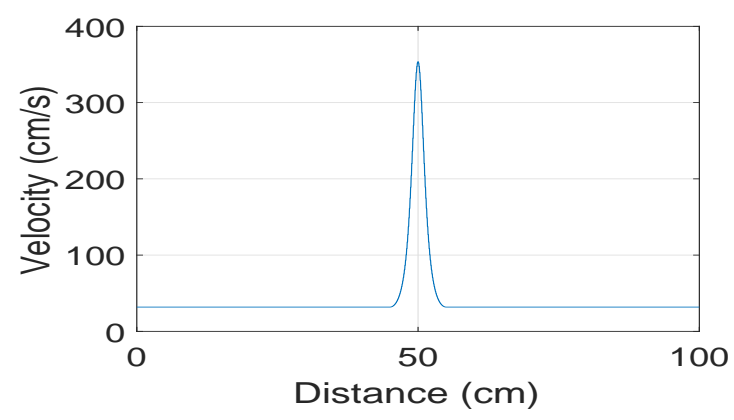

(d) Velocity

Figure 4.15: Waveforms for the stenosis case.

any wave reflections which may occur due to geometrical aspects such as tapering. In this subsection, the schemes ability to handle static discontinuities is tested, which includes discontinuous material properties and geometries in a single vessel. The inviscid set of equations are chosen for these test cases. The non-linear terms are considered, however by considering the flow rate to be quite small, the nonlinearities are also small, it allows the use of an analytical approach to determine the magnitude of wave reflections and transmissions caused by the discontinuity. This is determined with the use of transmission line theory which is performed on the linear system $[365,8]$. In order to determine these wave reflections, it is first advantageous to define the initial admittance $Y$ (inverse of characteristic impedance $Z)$, which can be written as

$$
Y=\frac{1}{Z}=\frac{A_{d}}{\rho c_{0}}
$$

where $A_{d}$ is the initial cross-sectional area, $\rho$ is the density, and $c_{0}$ is the initial wave speed. The reflection coefficient $R_{f}$ will depend on the change of admittance in a vessel. The linear formulation is used to find the analytical solution. At the point 


\begin{tabular}{|c|c|c|}
\hline Property & Units & Value \\
\hline Length, $L$ & $\mathrm{~cm}^{-3}$ & 100 \\
Blood density, $\rho$ & $\mathrm{kg} \mathrm{m}^{-3}$ & 1050 \\
Blood viscosity, $\mu$ & $\mathrm{mPa} \mathrm{s}$ & 0 \\
Diastolic pressure, $P_{d}$ & $\mathrm{mmHg}$ & 0 \\
External pressure, $P_{\text {ext }}$ & $\mathrm{Pa}$ & 0 \\
Initial pressure, $P(x, t=0)$ & $\mathrm{mmHg}$ & 0 \\
Wall thickness, $h$ & $\mathrm{~cm}$ & 0.15 \\
Elastic modulus, $E$ & $\mathrm{kPa}$ & 200.0, or 400.0 or 800.0 \\
Reference area, $A_{d}$ & $\mathrm{~cm}^{2}$ & $0.5 \pi, \pi$ or $2 \pi$ \\
Cardiac period, $T$ & $\mathrm{~s}$ & 1.5 \\
\hline
\end{tabular}

Table 4.16: Consistent properties across all four simulations for discontinuous material property $\beta$, and discontinuous reference area $A_{0}$.

of any change in material properties or geometry the following equations must hold

$$
R_{f, r e f}=\frac{Y_{r e f}-Y_{\text {tran }}}{Y_{\text {ref }}+Y_{\text {tran }}}, \quad R_{f, \text { tran }}=\frac{Y_{\text {tran }}-Y_{\text {ref }}}{Y_{\text {ref }}+Y_{\text {tran }}},
$$

where subscript ref is for the backwards propagating reflected wave and tran is for the forward propagating transmitted wave. The reflection coefficient has limiting values of 1 for a complete positive reflection, and - 1 for a complete negative reflection, while a value of 0 corresponds with a zero reflection of the wave. Furthermore, the a perturbation in the reflected pressure wave $P_{\text {ref }}$ can be determined from the perturbation of the forward travelling incident pressure wave $P_{i n c}$ as

$$
\delta P_{\text {ref }}=\delta P_{\text {inc }} R_{f, r e f}
$$

The transmitted pressure can be determined from the linear separation of pressure into reflected pressure and transmitted pressure $P_{\text {tran }}$, in a similar way to section 2.4 .2 to give

$$
\delta P_{\text {tran }}=\delta P_{i n c}+\delta P_{\text {ref }}=\left(1+R_{f, r e f}\right) \delta P_{i n c} .
$$

The flow rate is related by the conservation of mass at the point, that is the flow entering must equal all flow leaving (whilst taking into account that the reflected 
flow wave is in the negative direction)

$$
\delta Q_{i n c}=\delta Q_{\text {tran }}-\delta Q_{\text {ref }}
$$

where the reflected flow rate wave can be determined as

$$
\delta Q_{\text {ref }}=-\delta Q_{\text {inc }} R_{f, r e f}
$$

Thus from the initial wave speed, initial area, and a known incident flow rate and pressure wave, both reflected and transmitted flow rates and pressures can be estimated given the assumption of small system non-linearities.

The main haemodynamic parameters, which are consistent across all four test cases implemented, are given in table 4.16, where the base values for $A_{d}$ and $\beta$ are before any increases are imposed. These cases include

- A discontinuous decrease in reference area $A_{d}$ with a uniform $\beta$.

- A discontinuous increase in reference area $A_{d}$ with a uniform $\beta$.

- A uniform reference area $A_{d}$ with a discontinuous decrease in $\beta$.

- A uniform reference area $A_{d}$ with a discontinuous increase in $\beta$.

The same Gaussian shaped inflow rate is used for all simulations and is given by

$$
Q_{\text {inflow }}(t)=\exp \left(-10000(t-0.05)^{2}\right) \quad \mathrm{ml} \mathrm{s}^{-1}
$$

which has a maximum flow rate of $1 \mathrm{ml} \mathrm{s}^{-1}$, while non-reflecting boundary conditions are imposed.

Figure 4.16 shows the case where $A_{d}$ has a step decrease from $\pi$ to $\pi / 2$ at $50 \mathrm{~cm}$ into the domain. It can be seen from the figure that the numerical solution correctly predicts the reflected and transmitted waves from the incident wave. For this case the admittance is determine as follows: given the value of $E=400 \mathrm{kPa}$, and $\beta$ calculated from equation (4.2), and the values of $A_{0}$ and $\rho$, means that the first half of the vessel domain has a wave speed of $c_{0, s}=617.2134 \mathrm{~cm} \mathrm{~s}^{-1}$, while the second have of the vessel domain is $c_{0, e}=733.9946 \mathrm{~cm} \mathrm{~s}^{-1}$. Thus the admittance is given 
by

$$
Y_{s}=\frac{A_{0, s}}{\rho c_{0, s}} \approx 0.0048, \quad Y_{e}=\frac{A_{0, e}}{\rho c_{0, e}} \approx 0.0020
$$

which means the reflection coefficient can be determined to be

$$
R_{f, r e f}=\frac{Y_{s}-Y_{e}}{Y_{s}+Y_{e}}=0.4080
$$

Therefore for this problem the reflected peak flow rate can be found analytically as (when considering $\delta Q=Q_{i n c}-Q_{\text {init }}=Q_{i n c}$, as the initial flow rate $Q_{\text {init }}=0$ )

$$
Q_{\text {ref }}=-Q_{i n c} R_{f, r e f}=-0.4080 \mathrm{ml} \mathrm{s}^{-1}
$$

and the transmitted flow rate is $Q_{\text {tran }}=Q_{\text {inc }}+Q_{\text {ref }}=1-0.4080=0.5920 \mathrm{ml} \mathrm{s}^{-1}$. Similarly for pressure, the incident pressure is $0.1547 \mathrm{mmHg}$, and hence the reflected pressure is analytically determined to be

$$
P_{\text {ref }}=P_{\text {inc }} R_{f, r e f}=0.0631 \mathrm{mmHg}
$$

The transmitted pressure can be calculated as $P_{\text {tran }}=P_{\text {inc }}\left(1+R_{f, r e f}\right)=0.2178 \mathrm{mmHg}$.

The second problem involves a step increase in the reference diameter $A_{d}$ from $\pi$ to $2 \pi$ at $50 \mathrm{~cm}$ into the domain. The elastic modulus for this problem is chosen to be $400 \mathrm{kPa}$. This causes the initial wave speed to be calculated as $c_{0, s}=617.2134 \mathrm{~cm} \mathrm{~s}^{-1}$ for the first half of the domain, and $c_{0, e}=519.0125 \mathrm{~cm} \mathrm{~s}^{-1}$, which leads to a negative reflection coefficient of $R_{f, r e f}=-0.4080$. This causes the reflected pressure to become negative, while the reflected flow is positive. Figure 4.17 indicates that the numerical scheme correctly predicts the wave reflections from a step increase in area.

The third problem investigates a severe step decrease in $\beta$ caused by a decrease in elastic modulus from $400 \mathrm{kPa}$ in the first half of the domain, to $200 \mathrm{kPa}$ in the second half. The area is defined to be uniform with $A_{d}=\pi$. For this problem the wave speed can be calculated to be $c_{0, s}=617.2134 \mathrm{~cm} \mathrm{~s}^{-1}$ for the start of the domain, and $c_{0, s}=436.4358 \mathrm{~cm} \mathrm{~s}^{-1}$ in the second half. This leads to a small negative reflection coefficient of $R_{f, r e f}=-0.1716$, which leads to a negative reflected pressure and a positive reflected flow rate. Figure 4.18 shows the numerical scheme correctly predicts the magnitude of wave reflections and transmissions at the discontinuity.

The final problem tests a case with a a severe step increase in $\beta$ caused by a 


\subsection{NUMERICAL COMPARISONS FOR PROBLEMS WITH THEORETICAL SOLUTIONS}

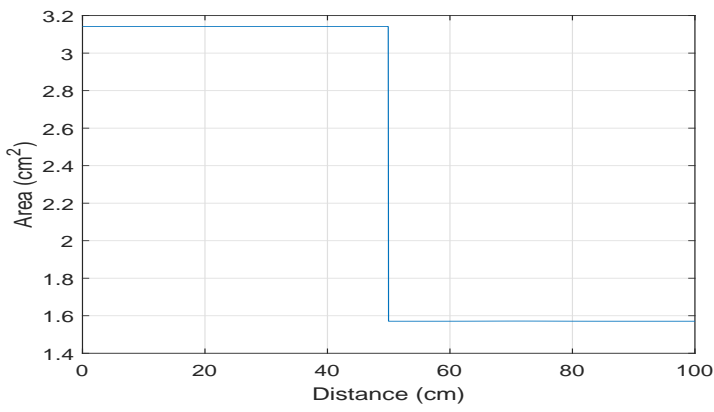

(a) Initial area

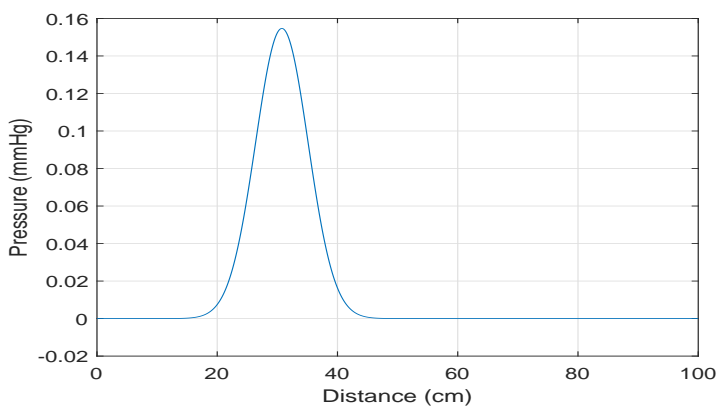

(c) Incident pressure wave

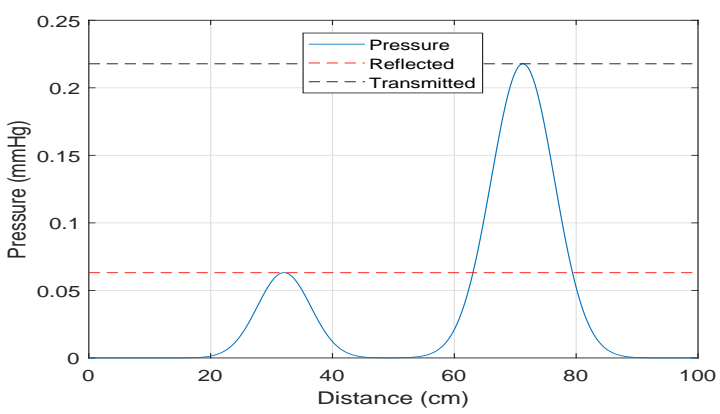

(e) reflected and transmitted pressure waves

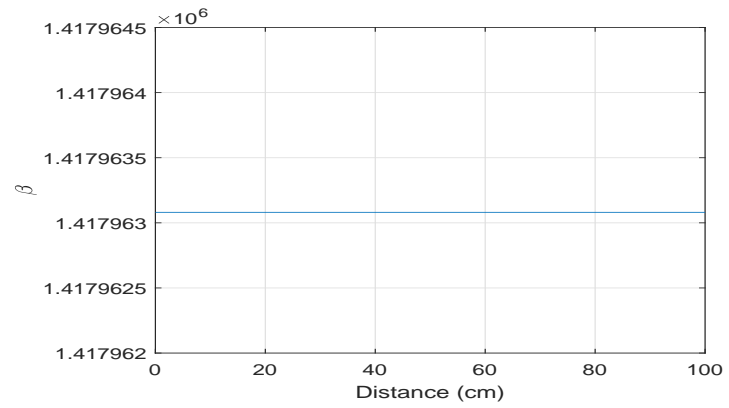

(b) Initial material property

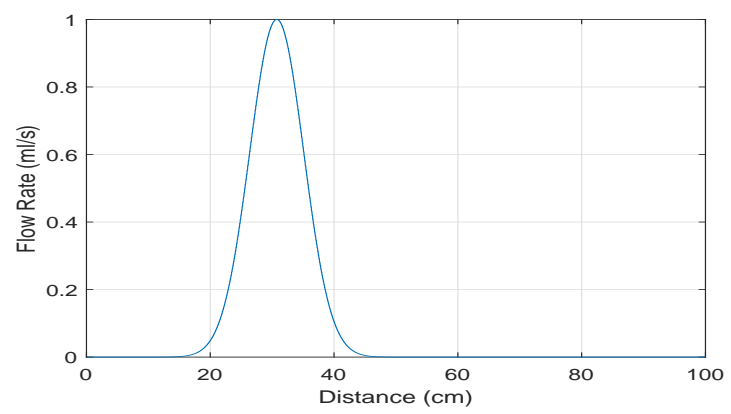

(d) Incident flow rate wave

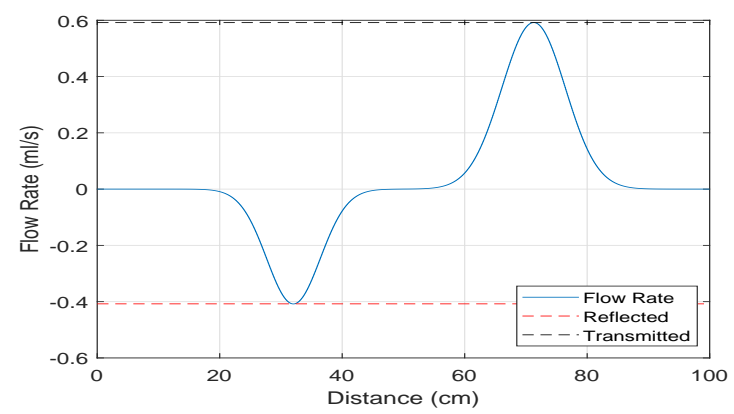

(f) reflected and transmitted flow rate waves

Figure 4.16: Waveforms for a discontinuous area decrease.

increase in elastic modulus from $400 \mathrm{kPa}$ in the first half of the domain, to $800 \mathrm{kPa}$ in the second half. The area is uniform across the length of the vessel $A_{d}=\pi$. For this problem the wave speed can be calculated to be $c_{0, s}=617.2134 \mathrm{~cm} \mathrm{~s}^{-1}$ in the first half of the vessel, and $c_{0, s}=872.8716 \mathrm{~cm} \mathrm{~s}^{-1}$ in the second half. This leads to a small positive reflection coefficient of $R_{f, r e f}=0.1716$, which leads to a positive reflected pressure and a negative reflected flow rate. Figure 4.19 indicates that the magnitude of both reflected and transmitted waves are correctly predicted by the 


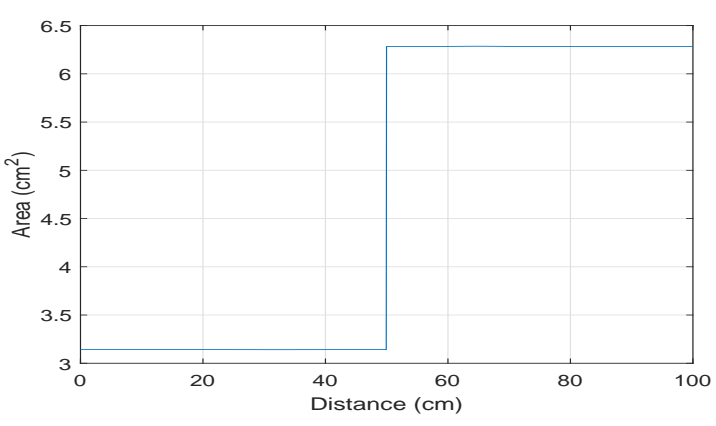

(a) Initial Area

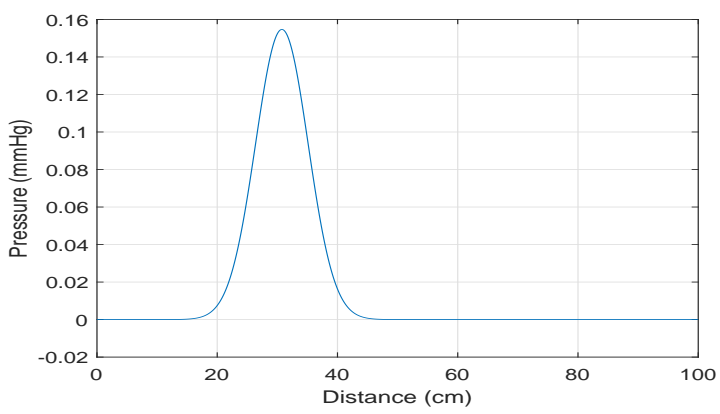

(c) Incident Pressure Wave

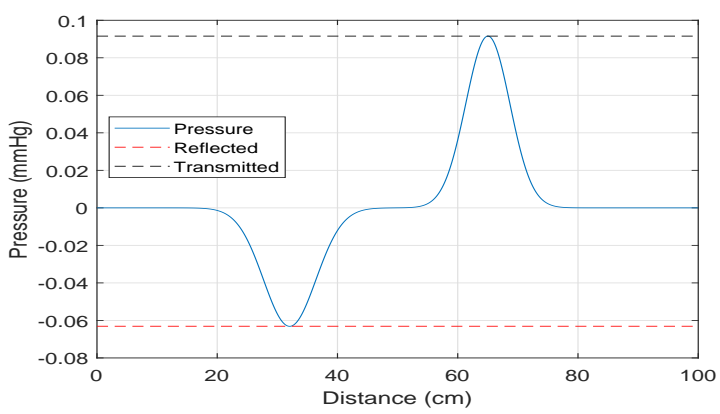

(e) Reflected and Transmitted Pressure Waves

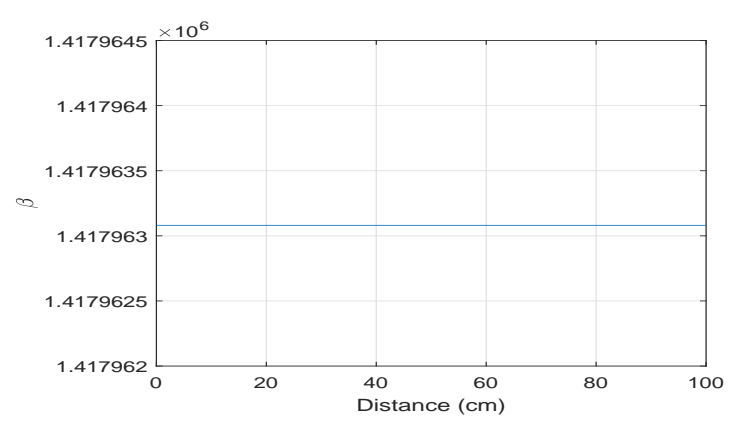

(b) Initial material property

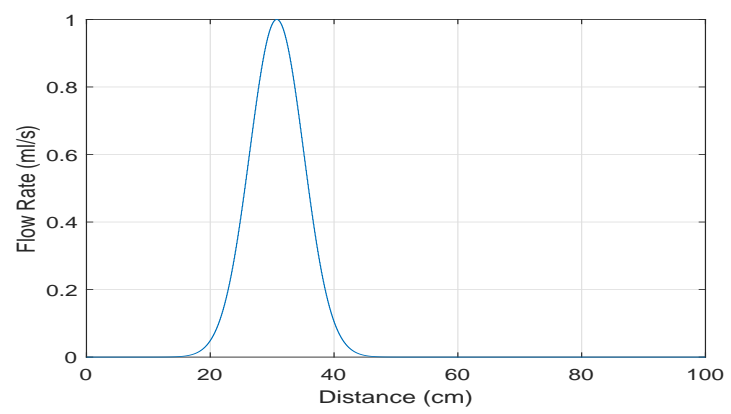

(d) Incident Flow Rate Wave

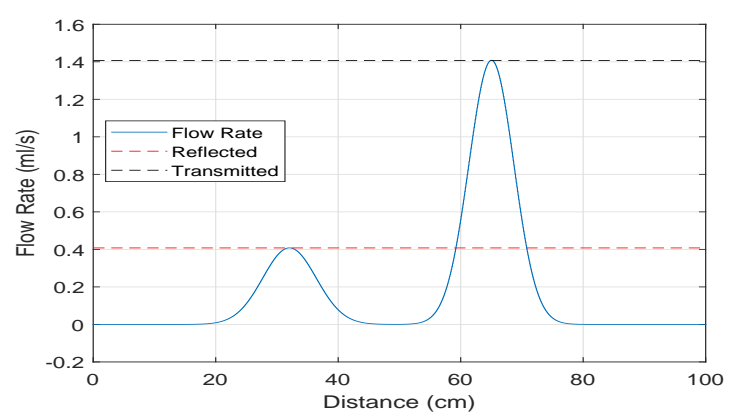

(f) Reflected and Transmitted Flow Rate Waves

Figure 4.17: Waveforms for a discontinuous area increase.

numerical scheme.

\subsubsection{Reflections at Junctions}

In the previous subsection, the numerical scheme was tested to determine if wave reflections were correctly predicted in the presence of discontinuities in vessel stiffness and geometry. This section makes use of transmission line theory in the prediction of 


\subsection{NUMERICAL COMPARISONS FOR PROBLEMS WITH THEORETICAL SOLUTIONS}

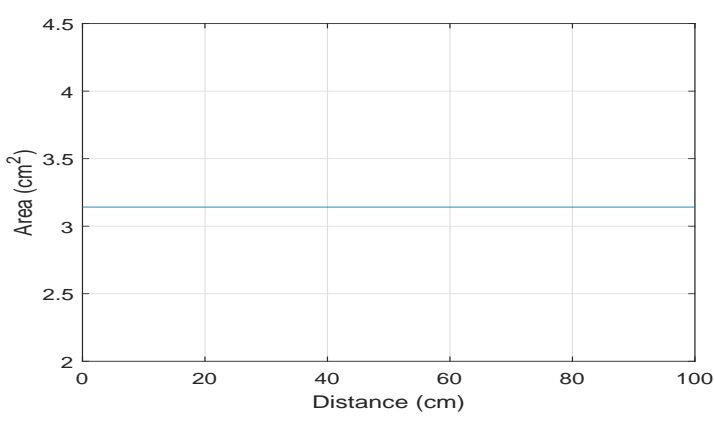

(a) Initial Area

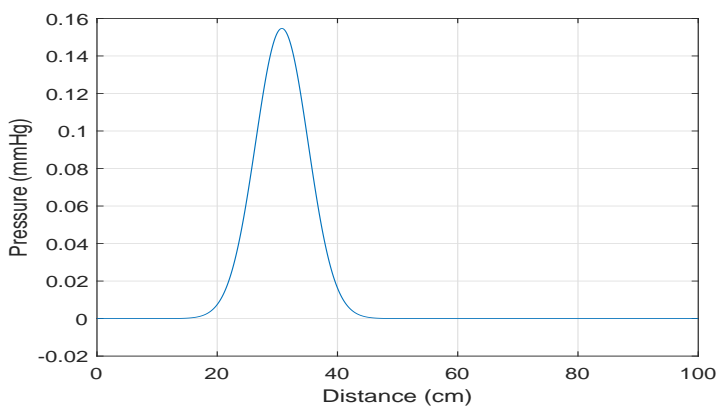

(c) Incident Pressure Wave

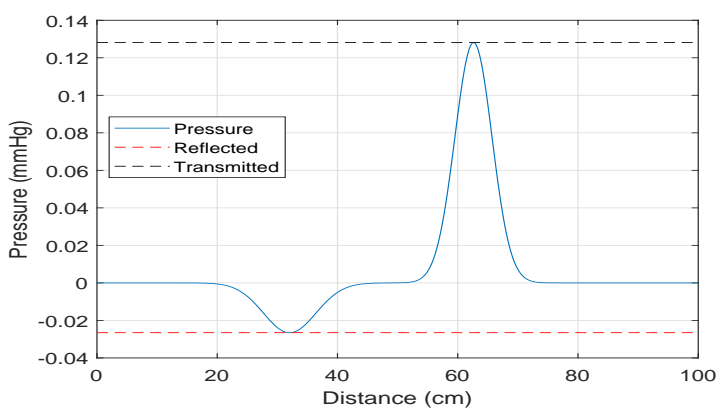

(e) Reflected and Transmitted Pressure Waves

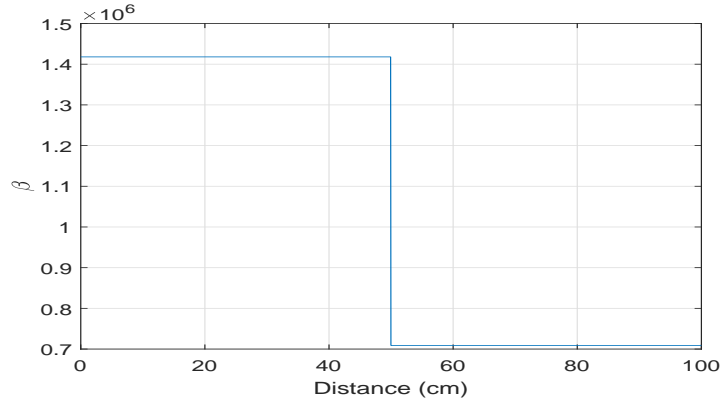

(b) Initial material property

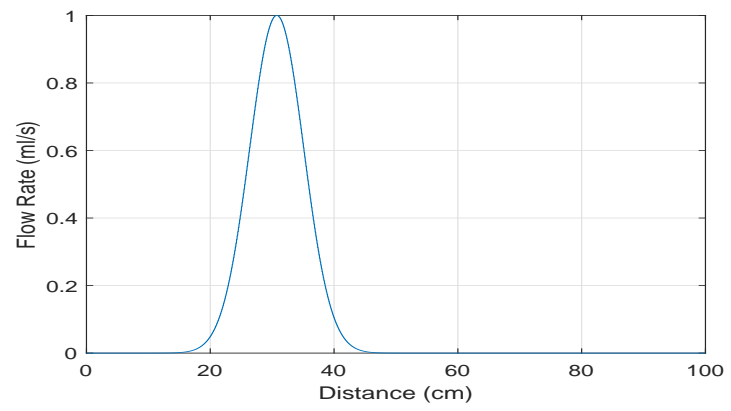

(d) Incident Flow Rate Wave

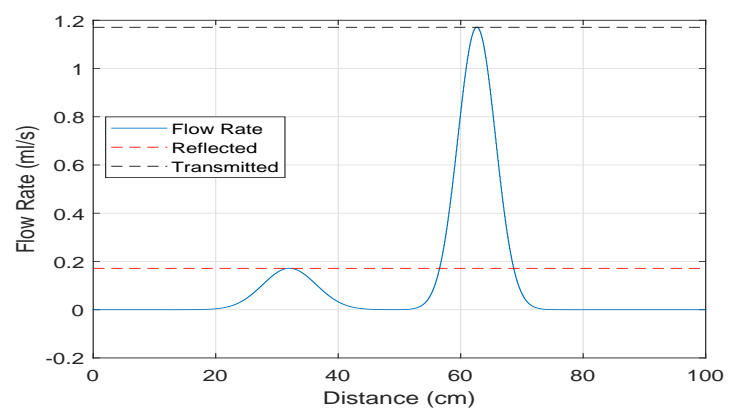

(f) Reflected and Transmitted Flow Rate Waves

Figure 4.18: Waveforms for a discontinuous decrease in material properties.

wave reflections and transmissions at vessel junctions. Table 4.17 shows the parameters and vessel information of a bifurcation problem. The inviscid set of equations are implemented once again in order to prevent a decrease in peak values for pressure and flow rate waveforms as the pulse propagates. Furthermore, conservation of mass and conservation of hydrostatic pressure are chosen as interface conditions at the junction. A single Gaussian shaped wave, given by equation (4.23), is used as the inlet flow rate. 


\subsection{NUMERICAL COMPARISONS FOR PROBLEMS WITH THEORETICAL SOLUTIONS}

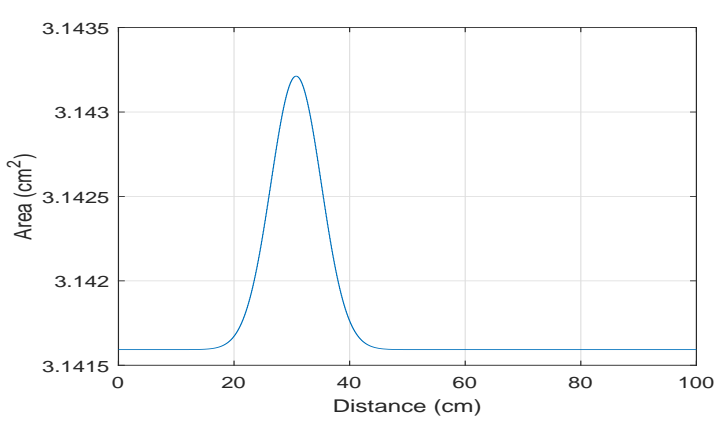

(a) Initial Area

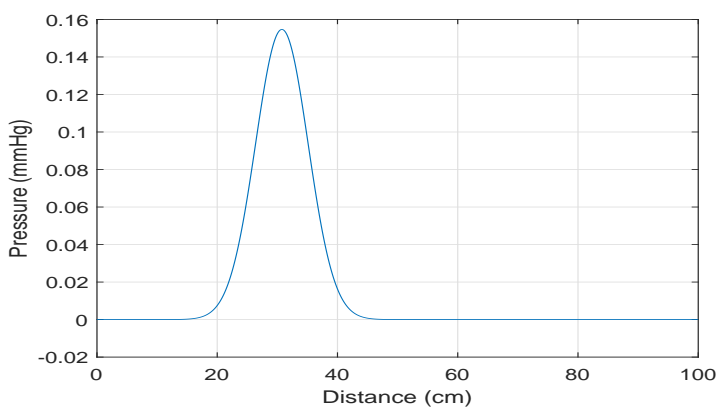

(c) Incident Pressure Wave

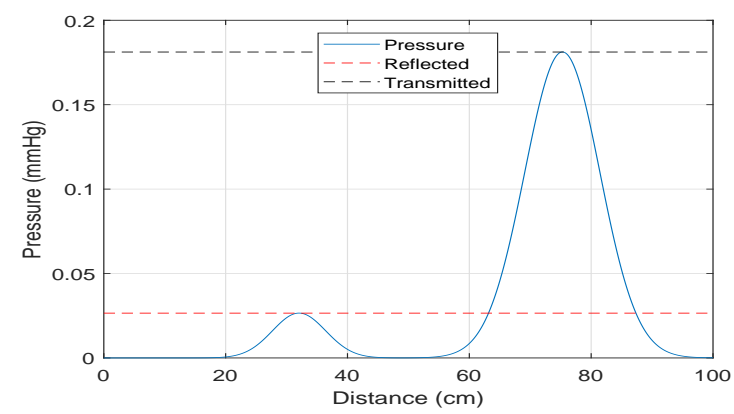

(e) Reflected and Transmitted Pressure Waves

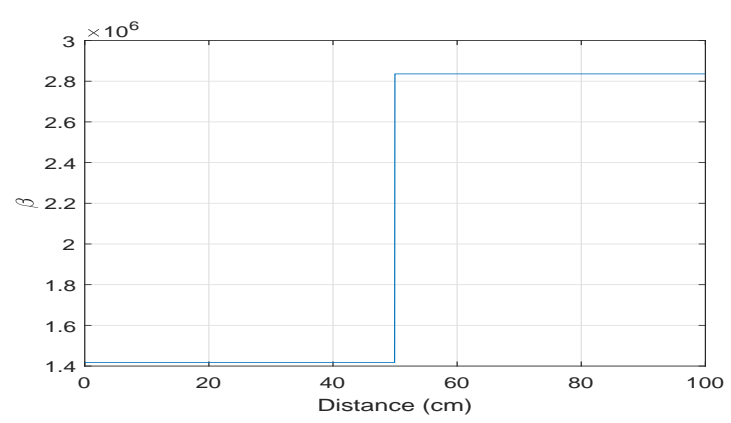

(b) Initial material property

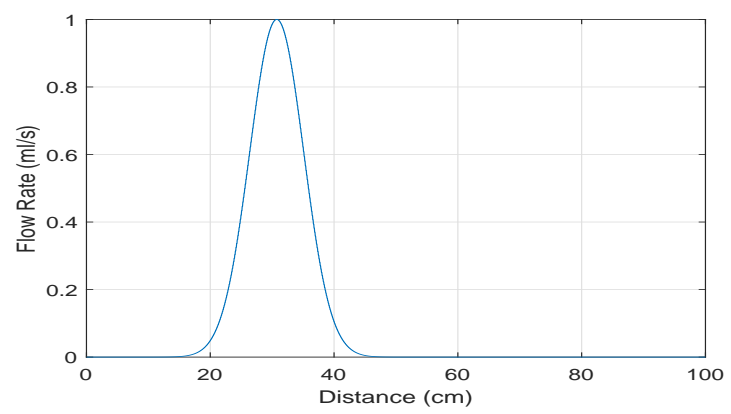

(d) Incident Flow Rate Wave

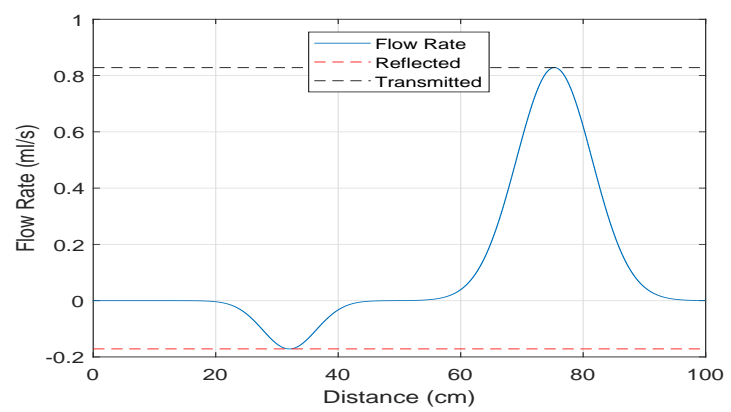

(f) Reflected and Transmitted Flow Rate Waves

Figure 4.19: Waveforms for a discontinuous increase in material properties.

The analytical reflection coefficient can be determined from linear analysis of the system equations, and is calculated from the admittances $Y$ of all vessels at the junction as

$$
R_{p, r e f}=\frac{Y_{p}-Y_{d 1}-Y_{d 2}}{Y_{p}+Y_{d 1}+Y_{d 2}}=0.2522,
$$

where the admittances are calculated from equation (4.17), and the initial wave 


\begin{tabular}{|c|c|c|c|c|}
\hline Property & Units & Vessel 1 & Vessel 2 & Vessel 3 \\
\hline Length, $L$ & $\mathrm{~cm}^{-3}$ & 50 & 50 & 50 \\
Blood density, $\rho$ & $\mathrm{kg} \mathrm{m}^{-3}$ & 1050 & 1050 & 1050 \\
Blood viscosity, $\mu$ & $\mathrm{mPas}$ & 0 & 0 & 0 \\
Diastolic pressure, $P_{d}$ & $\mathrm{mmHg}$ & 0 & 0 & 0 \\
External pressure, $P_{\text {ext }}$ & $\mathrm{Pa}$ & 0 & 0 & 0 \\
Initial pressure, $P(x, t=0)$ & $\mathrm{mmHg}$ & 0 & 0 & 0 \\
Wall thickness, $h$ & $\mathrm{~cm}$ & 0.15 & 0.15 & 0.15 \\
Elastic modulus, $E$ & $\mathrm{kPa}$ & 400.0 & 400.0 & 400.0 \\
Reference Area, $A_{d}$ & $\mathrm{~cm}^{2}$ & $\pi$ & $\pi / 2$ & $\pi / 4$ \\
Cardiac Period, $T$ & $\mathrm{~s}$ & 1.1 & - & - \\
\hline
\end{tabular}

Table 4.17: Haemodynamic parameters used in bifurcation case to investigate wave reflections

speeds of the parent $p$, and two daughter vessels $d 1$ and $d 2$, are $c_{0}=617.2134 \mathrm{~cm} \mathrm{~s}^{-1}$, $c_{0}=733.9946 \mathrm{~cm} \mathrm{~s}^{-1}$, and $c_{0}=872.8716 \mathrm{~cm} \mathrm{~s}^{-1}$.

The theoretical reflected peak pressure can be calculated once again from the peak incident pressure as $P_{r e f}=R_{p, r e f} P_{\text {inc }}=0.0390 \mathrm{mmHg}$, while the transmitted pressure for both daughter vessels can be calculated as $P_{\text {trans }}=\left(1+R_{p, r e f}\right) P_{\text {inc }}=$ $0.1937 \mathrm{mmHg}$.

Similarly the reflected peak flow rate can be calculated as $Q_{r e f}=-R_{p, r e f} Q_{i n c}=$ $-0.2522 \mathrm{ml} \mathrm{s}^{-1}$. The theoretical transmitted flow rates can be determined through the use of the admittances of both daughter vessels as

$$
Q_{t r a n, d 1}=\left(Q_{i n c}+Q_{r e f}\right) \frac{Y_{d 1}}{Y_{d 1}+Y_{d 2}}, \quad Q_{t r a n, d 2}=\left(Q_{i n c}+Q_{r e f}\right) \frac{Y_{d 2}}{Y_{d 1}+Y_{d 2}},
$$

where $\left(Q_{\text {inc }}+Q_{\text {ref }}\right)=0.7478 \mathrm{ml} \mathrm{s}^{-1}$ corresponds with the remaining flow that is transmitted into one of the two downstream vessels. Thus the theoretic peak flows are determined to be $Q_{t r a n, d 1}=0.5265 \mathrm{ml} \mathrm{s}^{-1}$ and $Q_{\text {tran }, d 2}=0.2214 \mathrm{ml} \mathrm{s}^{-1}$. Figure 4.20 indicates that the ETM scheme correctly predicts wave reflection and transmission behaviour for a given incident wave.

\subsubsection{Single Pulse in bifurcation and unification case.}

This test case is constructed to confirm the physical interpretation of the Lagrange multipliers in the ETM scheme, which are used at vessel junctions to constrain a 


\subsection{NUMERICAL COMPARISONS FOR PROBLEMS WITH THEORETICAL SOLUTIONS}

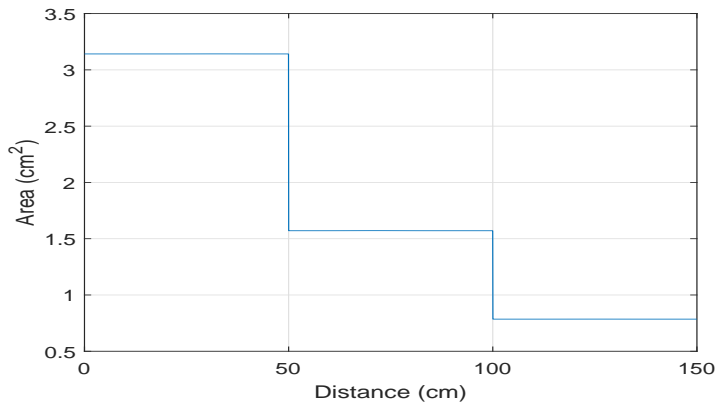

(a) Initial Area

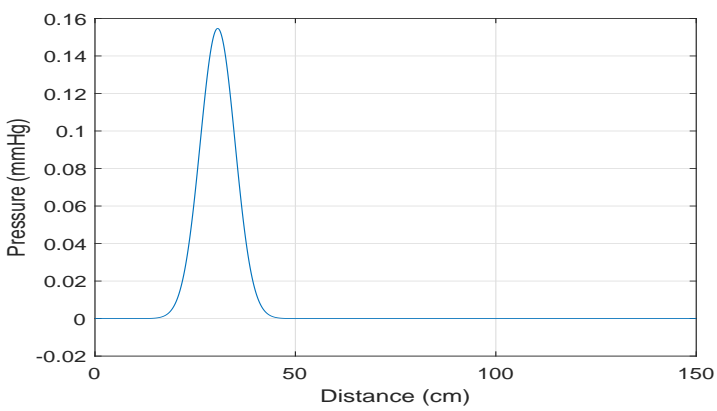

(c) Incident pressure waveform

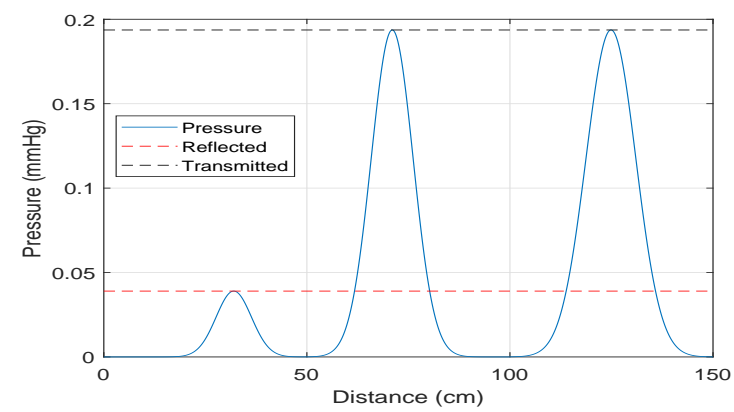

(e) Reflected and Transmitted Pressure Waved

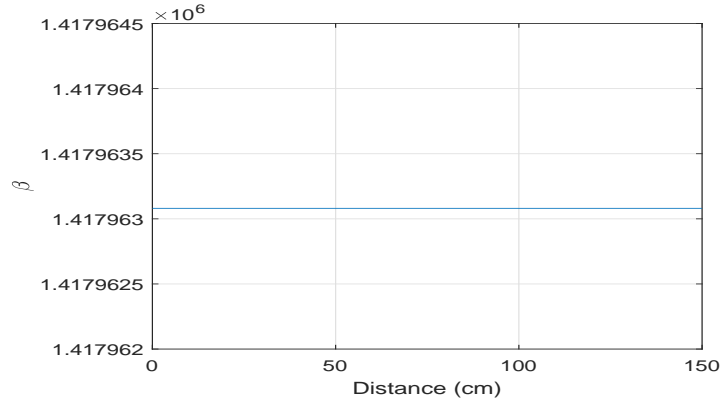

(b) Initial material property

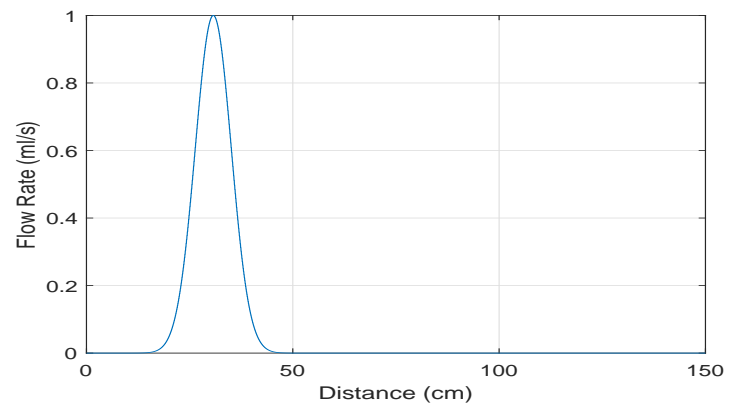

(d) Incident flow rate waveform

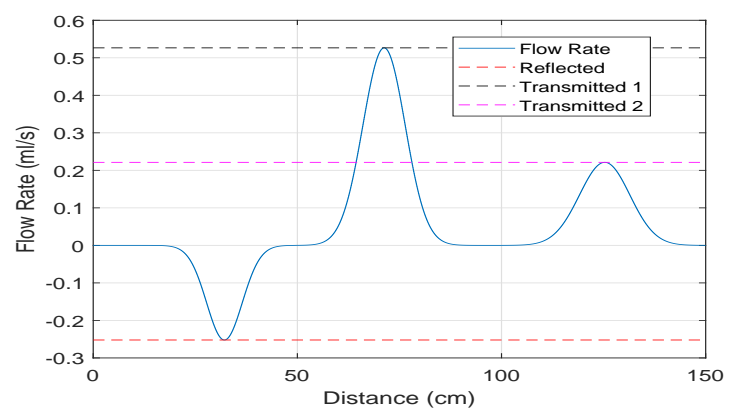

(f) Reflected and Transmitted flow rate waveforms

Figure 4.20: Reflections at a single bifurcation

conservation of pressure, actually correspond with flow rates of the non-reference vessels (at a junction one of the vessels is chosen as the reference vessel, to which all other vessels are constrained). The test is a configuration of four vessels, where the initial vessel bifurcates and then these two child vessels rejoin again. The inflow rate is a sinusoidal shaped wave, while the outlet condition is attached to a resistance element with a resistance of infinity, which causes a full reflection of the waves. The full non-linear set of equations are implemented in this problem for a viscous fluid. 


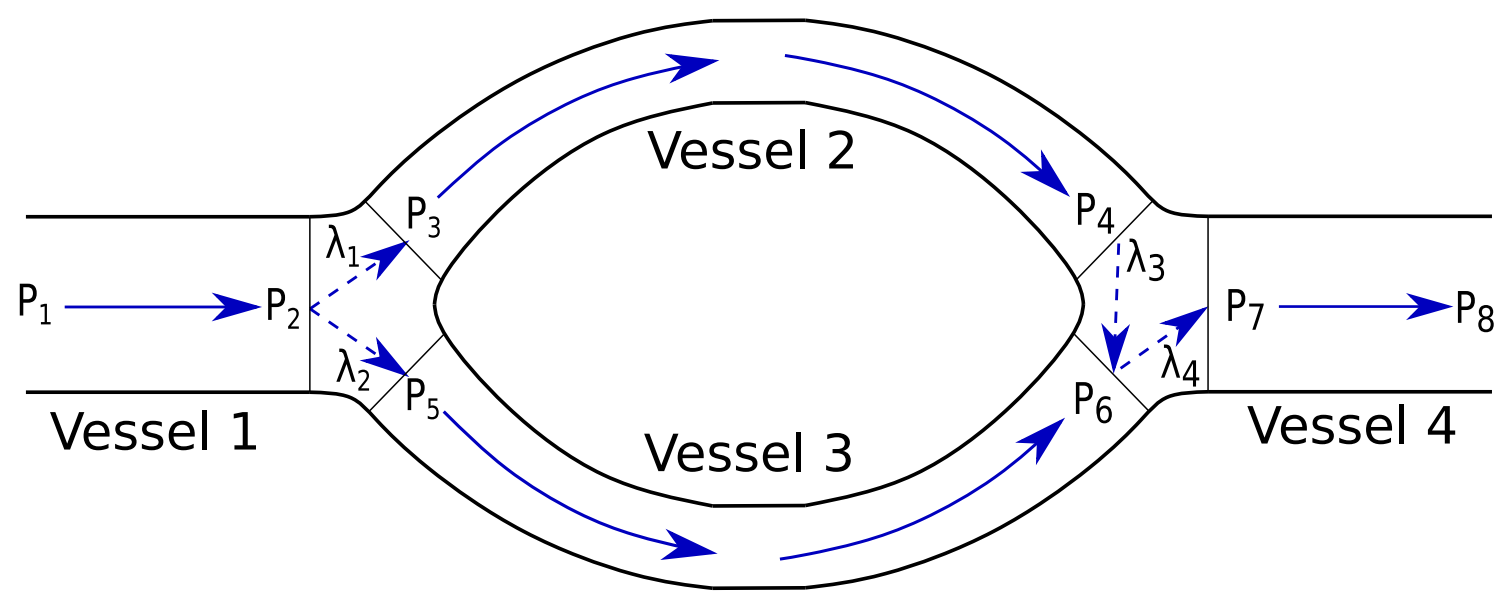

Figure 4.21: Configuration for bifurcation and unification test

The haemodynamic properties of the problem are given in Table 4.18, while the problem configuration is shown in Figure 4.21. As the non-linear set of equations are chosen, no theoretical solution exists for this problem. The element size chosen for this problem is $\Delta x=1 \mathrm{~cm}$, while the time step is $\Delta t=1 \mathrm{~ms}$.

The inflow rate is given by

$$
Q_{\text {inlet }}=\left\{\begin{array}{l}
40 \sin \left(\frac{\pi t}{0.3636 T}\right), \quad \text { for } \quad 0<t \leq 0.3636 T \\
0, \quad \text { for } \quad 0.3636 T<t \leq T
\end{array}\right.
$$

The cardiac period is $T=1.1 \mathrm{~s}$, while four cardiac cycles are required to achieve periodic convergence. The downstream resistance was set to infinity to enforce both positive and negative flow rates to occur at all the junctions.

The numerical predicted flow rates, as calculated in equation (3.21), are compared to the Lagrange multipliers at each junction in Figure 4.22. Figure 4.22a shows the bifurcation case where, as described in Section 3.2.6, the parent vessel flow rate is equal to the sum of the Lagrange multipliers $\left(\lambda_{1}\right.$ and $\left.\lambda_{2}\right)$, which correspond with the inflow rates of vessels 2 and 3 , respectively. The confluence to unification case is shown in figure $4.22 \mathrm{~b}$, and it can be seen that the flow rate in the reference parent vessel (vessel 2), is equal to the sum of $\lambda_{3}$ and $\lambda_{4}$. Furthermore, the outflow of parent vessel 4 is equal to the negative value of $\lambda_{3}$, while the inflow of vessel 4 is equal to the Lagrange multiplier $\lambda_{4}$. These results indicate that the 


\begin{tabular}{|c|c||c|c|c|c|}
\hline Property & Units & Vessel 1 & Vessel 2 & Vessel 3 & Vessel 4 \\
\hline Length, $L$ & $\mathrm{~cm}$ & 400 & 400 & 400 & 400 \\
Radius, $r_{d}$ & $\mathrm{~cm}$ & 1.3 & 0.8 & 1.1 & 1.3 \\
Area, $A_{d}$ & $\mathrm{~cm}^{2}$ & 5.3093 & 2.3093 & 3.8013 & 5.3093 \\
Initial area, $A(x, 0)$ & $\mathrm{cm}^{2}$ & 5.3093 & 2.3093 & 3.8013 & 5.3093 \\
Initial flow, $Q(x, 0)$ & $\mathrm{ml} \mathrm{s}^{-1}$ & 0 & 0 & 0 & 0 \\
Initial pressure, $P(x, 0)$ & $\mathrm{kPa}$ & 10.666 & 10.666 & 10.666 & 10.666 \\
Wall thickness, $h$ & $\mathrm{~cm}$ & 0.07 & 0.07 & 0.07 & 0.07 \\
Blood density, $\rho$ & $\mathrm{kg} \mathrm{m}^{-3}$ & 1060 & 1060 & 1060 & 1060 \\
Blood viscosity, $\mu$ & $\mathrm{mPa}$ & 4 & 4 & 4 & 4 \\
Velocity profile order $\zeta$ & - & 9 & 9 & 9 & 9 \\
Young's modulus, $E$ & $\mathrm{kPa}$ & 700.0 & 700.0 & 700.0 & 700.0 \\
Diastolic pressure, $P_{d}$ & $\mathrm{kPa}$ & 10.666 & 10.666 & 10.666 & 10.666 \\
External pressure, $P_{\text {ext }}$ & $\mathrm{Pa}$ & 0 & 0 & 0 & 0 \\
Outflow pressure, $P_{\text {out }}$ & $\mathrm{Pa}$ & 0 & 0 & 0 & 0 \\
Windkessel resistance, $R_{1}$ & $\mathrm{Pasm}$ & - & - & - & $\infty$ \\
Daughter vessel$(\mathrm{s})$, & - & $2 \& 3$ & 4 & 4 & - \\
Parent vessel(s), & - & - & 1 & 1 & $2 \& 3$ \\
\hline
\end{tabular}

Table 4.18: Properties for bifurcation to unification test.

Lagrange multipliers can be physically interpreted as flow rates in the non-reference vessels at each junction.

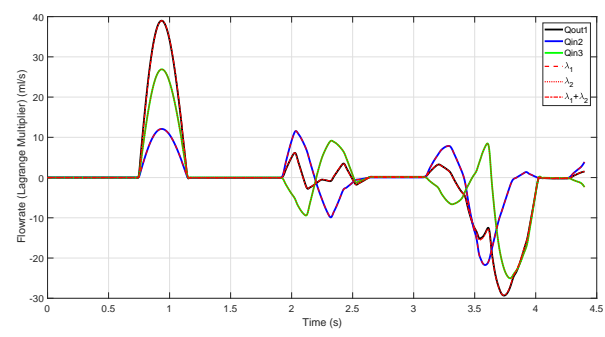

(a) Junction 1 (Bifurcation)

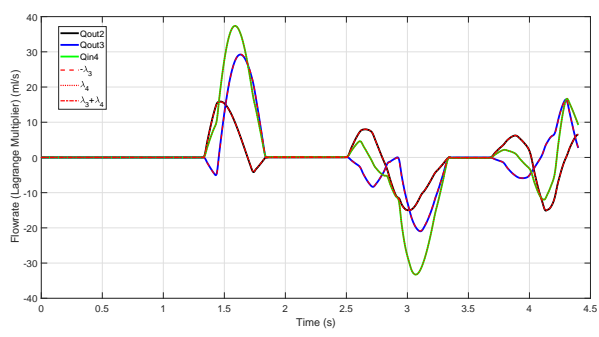

(b) Junction 2 (Unification)

Figure 4.22: A comparison of outflow and inflow at junctions with Lagrange multipliers. 


\subsection{Convergence Behaviour of the ETM Scheme}

The ETM scheme has been rigorously tested for a wide range of problems. However, the convergence behaviour of the scheme, and the effect of performing one iteration per time step, has not yet been investigated. Thus this section tests the convergence behaviour of the scheme, which includes comparing the choice to use either an upwind or central difference discretisation for the non-linear convection term, and comparing the choice of performing one Newton iteration per time step, or multiple iterations.

\subsubsection{Mass Conservation in an Arterial Network}

In this subsection, the ADAN arterial network that was implemented in section 4.2.6 is used. However, this time the interest is of mass conservation, rather than the pressure and flow waveforms. The inflow of the network is defined and has an average flow rate of $112.3899 \mathrm{ml} \mathrm{s}^{-1}$ and is shown in Figure 4.23, while the outflow is a three element windkessel model with constant resistances and compliances, and a defined outflow pressure for the windkessel model of $0 \mathrm{mmHg}$. In order to investigate mass conservation the following is true of the system:

- Due to the periodic inflow boundary condition and constant outflow conditions, the solution of the numerical problem will converge to a periodic solution.

- When a periodic solution is achieved, the inflow of the $1 \mathrm{D}$ system must be equal to the sum of outflows of the system over a cardiac cycle. Which can be described by stating, over a cardiac cycle the mass entering the system must equal the mass exiting the system.

Of course not all numerical schemes are mass conservative, and for schemes that do theoretically conserve mass, numerical errors will still cause deviations (albeit small) in the schemes mass conservation properties. The ETM scheme is theoretically mass conservative, in fact the scheme implicitly solves pressure and mass change in the system (not flow rates), per time step.

The first test is to investigate the impact of time step and element size of mass conservation in the system. In the ETM scheme it could be anticipated that the time step will have a greater impact on mass conservation as greater errors would be 


\begin{tabular}{|c||c|c|c|}
\hline $\begin{array}{c}\text { Newton } \\
\text { Iterations }\end{array}$ & $\begin{array}{c}\text { time step } \\
(\mathrm{ms})\end{array}$ & $\begin{array}{c}\text { element size } \\
(\mathrm{cm})\end{array}$ & $\begin{array}{c}\text { (inflow - outflow)/ } \\
\text { inflow }(\%)\end{array}$ \\
\hline 1 & 1.0 & 1.0 & -0.1180 \\
2 & 1.0 & 1.0 & 0.000198 \\
3 & 1.0 & 1.0 & 0.000157 \\
4 & 1.0 & 1.0 & 0.000157 \\
5 & 1.0 & 1.0 & 0.000157 \\
1 & 0.1 & 1.0 & -0.0397 \\
1 & 1.0 & 0.1 & -0.1443 \\
\hline
\end{tabular}

Table 4.19: Results for the mass conservation simulation tests on the ADAN model, where inflow minus outflow divided by inflow column corresponds with the percentage difference between inflow rate with outflow rate, which is normalised by the inflow rate (should be zero to exactly conserve mass).

expected for larger time steps, especially due to the result of the system linearisation, and choice of performing only one Newton iteration. The errors would be expected to decrease in magnitude when more Newton iterations are performed. For these tests, an upwind discretisation is chosen for the non-linear convection term.

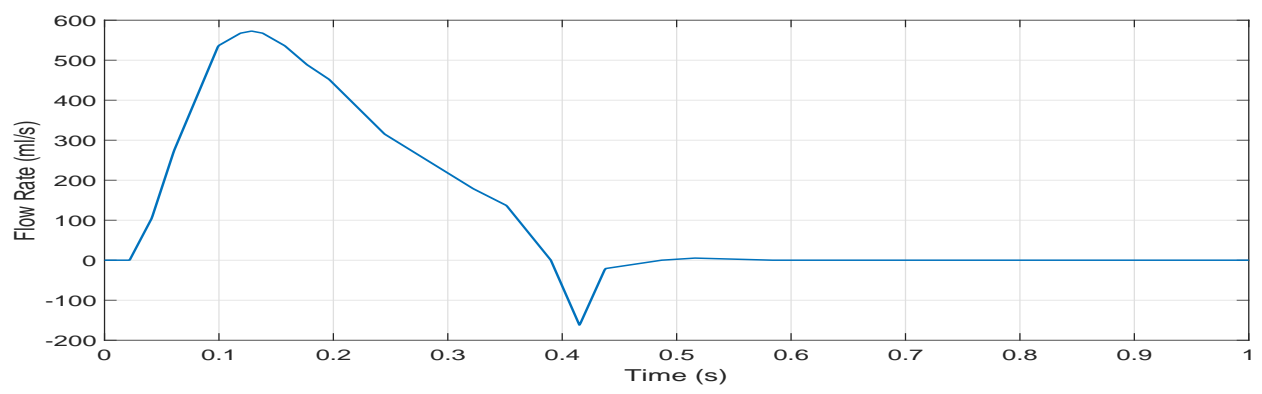

Figure 4.23: Inflow flow rate for mass conservation tests

Table 4.19 shows the results of the simulations several different configurations. The difference between inflow and outflow per cardiac cycle is shown to decrease from $-0.1180 \%$ to $-0.0397 \%$ with a decrease in time step by a factor of 10 . Furthermore, the error is also seen to decrease when more Newton iterations are performed, until a peak convergence is reached for 3 Newton iterations. When more than 3 Newton iterations per time step are performed, there is no change in performance, as the residual does not decrease any further (due to machine precision and the condition number of the system matrix). Although the waveforms show very little change with the use of more than 1 Newton iteration. 
Interestingly, the error in mass actually increases with a reduction of the maximum element size in the system. This is likely caused by an increase of the CFL number from $C F L=3.6789$ to $C F L=8.0868$. Although the CFL number does not have an impact on the stability of the scheme, it does have an impact on the accuracy, as the CFL number determines how far propagating information travels per time step. A CFL number of 1 would indicate information travelling in the forward direction will propagate from one node to the next node in exactly one time step.

Needless to say, the ETM scheme has been shown to successfully conserve mass in a network scale situation. The ability to conserve mass will be particularly important when implementing a closed loop system, as will be performed in chapter 7 .

\subsubsection{Numerical Convergence Study}

In this section numerical convergence tests are performed. The common carotid case from section 4.2.2 is used for this study. Four different cases are tested here:

- A first order upwind discretisation for the non-linear convection term with one Newton iteration used for time stepping.

- A second order central difference discretisation for the non-linear convection term with one Newton iteration used for time stepping.

- A first order upwind discretisation for the non-linear convection term with four Newton iteration used for time stepping.

- A second order central difference discretisation for the non-linear convection term with four Newton iteration used for time stepping.

Thus this test investigates and compares the space-time convergence behaviour of the different variations of the ETM scheme, and serves to improve understanding of the choice on performing one Newton iteration.

Figure 4.24 shows the spatial and temporal convergence for all cases studied. The solution of the smallest time step and element size is chosen to be the reference solution, where $\Delta t=0.1 \mathrm{~ms}$ and $\Delta x=0.1 \mathrm{~cm}$. When considering the choice of discretisation for the non-linear convection term, the first order upwind form provides a spatial convergence in the order of 1.6 , which increases to the order of 2.09 when 


\subsection{CONVERGENCE BEHAVIOUR OF THE ETM SCHEME}

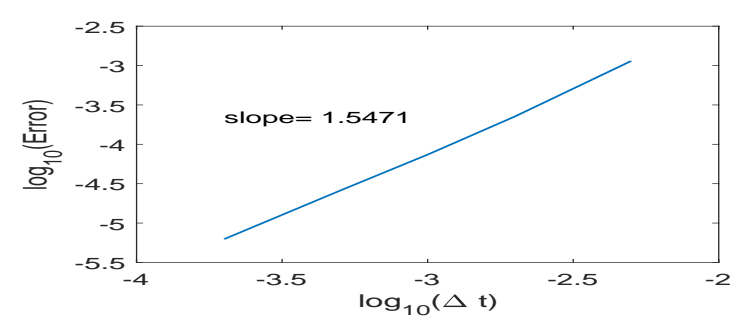

(a) Temporal convergence of upwind discretisation, with one Newton iteration

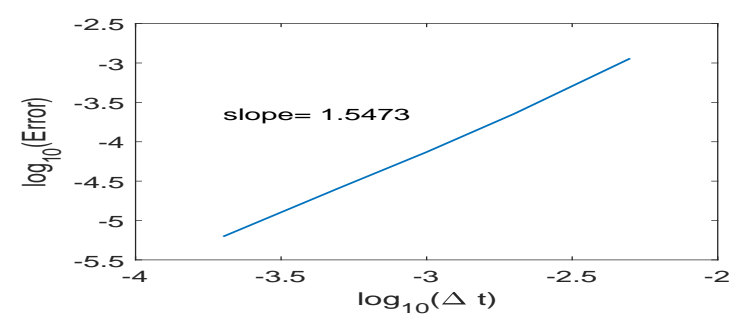

(c) Temporal convergence of central discretisation, with one Newton iteration

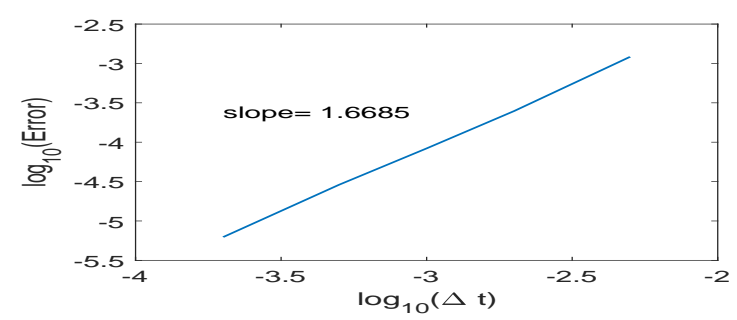

(e) Temporal convergence of upwind discretisation, with four Newton iterations

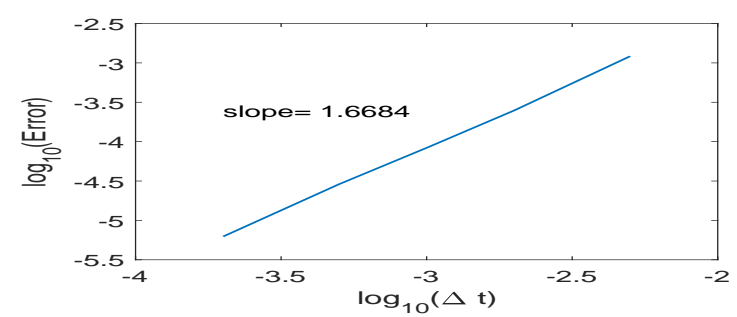

(g) Temporal convergence of central discretisation, with four Newton iterations

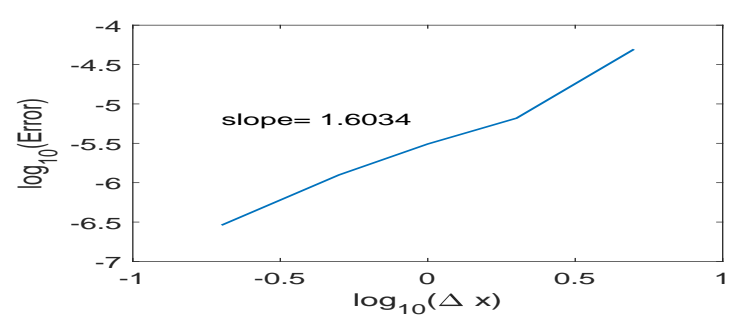

(b) Spatial convergence of upwind discretisation, with one Newton iteration

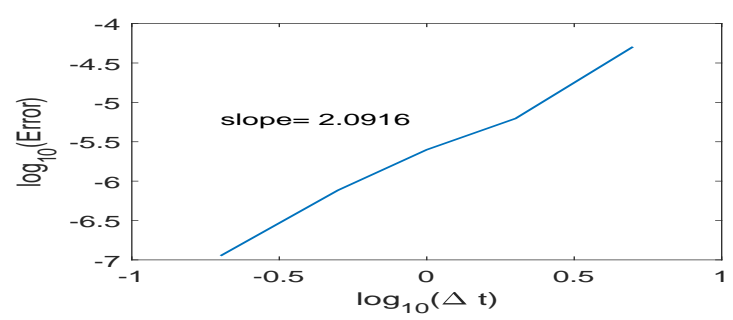

(d) Spatial convergence of central discretisation, with one Newton iteration

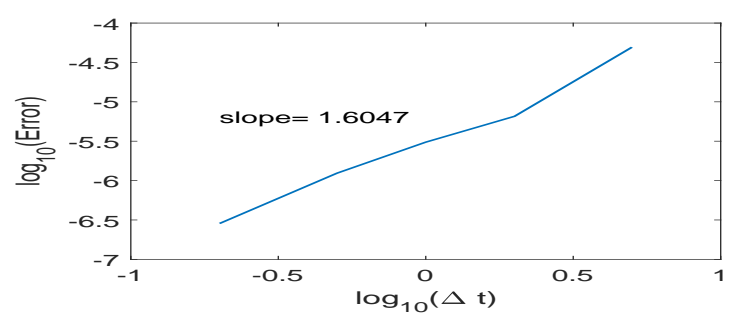

(f) Spatial convergence of upwind discretisation, with four Newton iterations

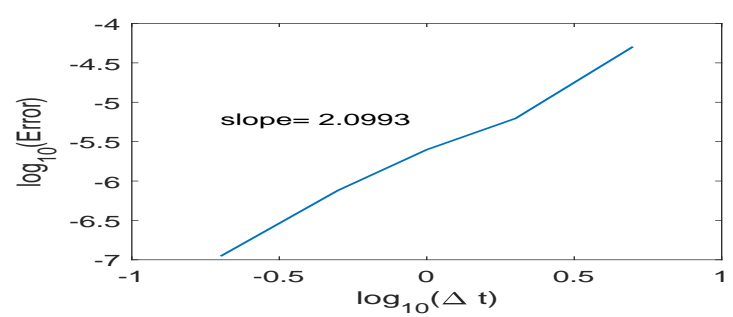

(h) Spatial convergence of central discretisation, with four Newton iterations

Figure 4.24: Numerical convergence curves of the ETM scheme for the common carotid problem. Investigating the choice of discretisation of the non-linear convection term, and the number of Newton iterations performed.

a second order central difference form is implemented. The spatial convergence rate remains relatively consistent whether one Newton iteration or four Newton itera- 
tions are performed per time step, with a small improvement in performing four iterations for both forms of convection discretisation. The temporal convergence is not affected by the choice of convection discretisation. However, an improvement of temporal convergence is seen when performing four Newton iterations as opposed to one iteration.

The scheme exhibits super-linear convergence in both time and space, with the highest order of temporal convergence of 1.6685 when using four Newton iterations, and a quadratic (order of 2.0993) spatial convergence when using a central difference discretisation for the non-linear convection term.

\subsection{Conclusions}

The ETM scheme has shown to give solutions which are consistent with other commonly used 1D numerical methods for the various benchmarked problems tested. When compared with a theoretical solution for a single pulse in a long, reflectionfree vessel, the ETM correctly predicted the drop in peak pressure across the length of a vessel for the viscid case, and correctly retained the pressure peak for the inviscid case. The scheme has also been compared with additional problems which have a theoretical solution and successfully captured: a shock wave and rarefaction in a shock tube problem; and wave reflections caused by discontinuous geometries and material properties; and wave reflections at vessel junctions. Furthermore, the ETM scheme has been shown to exhibit good mass conservation properties, while a numerical convergence study showed super-linear convergence in both time and space. This provides confidence in the numerical scheme. 


\section{Chapter 5}

\section{Investigation of Non-Linearities in a 1D-0D Closed-Loop Cardiovascu- lar System}

\subsection{Introduction}

In the field of 1D cardiovascular modelling, research groups tend to use conservation of mass and either continuity of static pressure, or of total pressure, at vessel junctions. However, it is important to realise that neither the use of total pressure conservation nor static pressure conservation fully describe the dynamics at the junction. The effect of pressure loss at junctions was investigated for various bifurcation configurations (both converging and diverging flows) by [231]. They compared the performance of their 1D model to a three-dimensional model, observing that the influence of the pressure conservation law chosen at the junction was inconsistent across different vessel configurations (bifurcations), which led to the development of their unified method, which estimates the pressure loss at junctions based on the angle between vessels at the junction. It was found that for diverging flow at a bifurcation where a small vessel branches from a larger vessel, that static pressure gave a loss coefficient closer to the 3D solution in the smaller side vessel, while total pressure gave a better representation for the larger straight vessel. Moreover, for a configuration similar to the abdominal aorta branching to the common iliac arteries, that static pressure overestimated the loss coefficient, while total pressure underestimated the loss coefficient by a similar amount. However, these comparisons were only performed for steady flow conditions, not pulsatile flows which are more common in blood flow. The effect of the non-linearities in blood flow have been studied for the arterial system by $[38,7]$, but not for the venous system.

The main aim of this section is therefore to investigate how cardiovascular waveforms are affected by

1. including or excluding non-linear components in the models, and 
2. using the conservation of either total pressure or static pressure to describe the transition at junctions in both the arterial and venous systems.

To investigated these, the following comparisons and investigations are performed:

1. four model formulations comprised of either non-linear or linear models, describing junction transitions using the conservation of either total or static pressure in each case,

2. a comparison of these models with the performance of a linear $0 \mathrm{D}$ formulation in which resistances, compliances and inductances were constant,

3. and commonly used configurations such as a non-linear formulation for either the major systemic vessels, or just the major systemic arteries, with a lumped formulation for the remainder of the circulation. Which are performed to investigate whether errors from the linear regimes propagated into other systems.

\subsection{Methodology}

The closed loop model for the non pregnant case is used as the basis, the parameters of the model are described in Chapter 6 . The formulations and simulation cases used are abbreviated as follows, the four cases with unified formulations for 1D vessels are:

- NL-TP includes the non linear convection term $\left(\alpha_{1}=1\right)$ within the vessel with conservation of total pressure at junctions.

- NL-SP includes the non linear convection term $\left(\alpha_{1}=1\right)$ within the vessel with conservation of static pressure at junctions.

- $\mathbf{L}-\mathbf{T P}$ excludes the non linear convection term $\left(\alpha_{1}=0\right)$ within the vessel with conservation of total pressure at junctions.

- L-SP excludes the non linear convection term $\left(\alpha_{1}=0\right)$ within the vessel with conservation of static pressure at junctions. 


\begin{tabular}{|l||c|r|}
\hline Parameter & Value & Units \\
\hline$\rho$ & 1.06 & $\mathrm{~g} / \mathrm{cm}^{3}$ \\
$\mu$ & 0.035 & poise \\
$\xi$ & 22 & $\mathrm{~cm}^{3} / \mathrm{g}$ \\
$g$ & 0 & $\mathrm{~cm} / \mathrm{s}^{2}$ \\
$\Delta t$ & 1 & $\mathrm{~ms}$ \\
$\Delta x$ & 1 & $\mathrm{~cm}$ \\
\hline
\end{tabular}

Table 5.1: Parameters used in this section

- L0-SP is the linear 0D formulation $\left(\alpha_{1}=0\right)$ within the vessel with conservation of static pressure at junctions.

- Mixed-A uses a non-linear (NL-TP) formulation for the systemic arteries and veins (including portal, cerebral and coronary systems), whilst using a linear 0D (L0-SP) formulation for the pulmonary system. This is a similar form to the model proposed by [220, 221].

- Mixed-B uses a non-linear (NL-TP) formulation for the systemic arteries (cerebral and coronary systems), whilst using a linear 0D (L0-SP) formulation for the systemic veins (including portal, cerebral and coronary systems) and the pulmonary system. This is a similar form to the model proposed by [34].

These abbreviations will be used throughout the results and discussion sections. It should be noted that the linear 0D model used in this section is a multi-compartment model, and contains the same number of elements (vessel segments) as the 1D model.

\subsubsection{Isolated Vessel Junctions}

Three additional cases are considered to better understand the differences caused by using either static pressure conservation (NL-SP) or total pressure conservation (NL-TP) at vessel junctions. These cases are:

- Bifurcation case, where one parent vessel bifurcates to two daughter vessels.

- Confluence case, where two parent vessels unify to one daughter vessel.

- Trifurcation case, where one parent vessel trifurcates to three daughter vessels. 
These cases were chosen as they are the most common type of junctions in the closed loop cardiovascular model.

In the bifurcation and trifurcation cases the area of the parent vessel is chosen, while in the confluence case the area of the daughter vessel is chosen. Murray's law [223] given by

$$
\sum_{p=1}^{N_{p}} r_{p}^{\kappa}=\sum_{d=1}^{N_{d}} r_{d}^{\kappa}, \quad \kappa=2.76,
$$

is used along with area ratios, to determine the initial radii of the daughter vessels for bifurcation and trifurcation cases (or parent vessels for the confluence case). Where subscript $p$ and $d$ denote the parent and daughter vessels respectively.

- The bifurcation case has one area ratio defined as $\gamma_{1}=\frac{A_{d 2}}{A_{d 1}}$.

- The confluence case has one area ratio defined as $\gamma_{1}=\frac{A_{p 2}}{A_{p 1}}$.

- In the trifurcation case there are three ratios defined as $\gamma_{1}=\frac{A_{d 2}}{A_{d 1}}, \gamma_{2}=\frac{A_{d 3}}{A_{d 1}}$, and $\gamma_{3}=\frac{\gamma_{1}}{\gamma_{2}}=\frac{A_{d 2}}{A_{d 3}}$.

The input for these three test cases use a half cosine wave for flow rate at the inlet given by

$$
Q_{\text {in }}= \begin{cases}\frac{Q_{\max }}{2}\left[1-\cos \left(\frac{2 \pi t 3}{T}\right)\right], & \text { for } 0 \leq t \leq T / 3 \\ 0, & \text { for } T / 3<t \leq T,\end{cases}
$$

where $Q_{i n}$ is the flow rate at the inlet, $Q_{\max }$ is the maximum flow rate, and $T=0.9$ $s$ is the period for one cardiac cycle. In the confluence there are two system inlets and as a result the system inflow is distributed based on reference area $A_{0}$ such that both inlets have approximately the same velocity $\frac{Q_{i n, p 1}}{A_{0, p 1}}=\frac{Q_{i n, p 2}}{A_{0, p 2}}$. A three element Windkessel model is used at the outlet of the system.

\subsection{Results}

A typical simulation took approximately $18.2 \mathrm{~s}$ per cardiac cycle (typically 15 cardiac cycles needed to achieve a periodic solution, when there is less than $1 \%$ change in peak pressure and flow rates). All waveforms shown are from the middle of each vessel. Names of the vessels correspond with names used in [230]. 
The error measures used in this section are as follows: Root-Mean-Square-Error (RMSE)

$$
\epsilon_{R M S E}=\sqrt{\frac{1}{n} \sum_{i=1}^{n}\left(X_{i}-\bar{X}_{i}\right)^{2}},
$$

where $X$ is a value of the NL-TP formulation, $\bar{X}$ is a value of one of the other formulations and $n=n_{v} \times n_{t}$ is the number of monitored points $\left(n_{v}\right)$ plus the number of time steps $\left(n_{t}\right)$; A scaled Mean-Absolute-Error (MAE) is needed for flow rates to avoid dividing by zero.

$$
\epsilon_{M A E}=\frac{100}{n} \sum_{v=1}^{n_{v}} \sum_{t=1}^{n_{t}}\left|\frac{X_{v, t}-\bar{X}_{v, t}}{\max (X)_{v}}\right|,
$$

where $\max (X)_{v}$ is the maximum value in each vessel; The Mean-Absolute-PercentageError (MAPE)

$$
\epsilon_{\text {MAPE }}=\frac{100}{n} \sum_{i=1}^{n}\left|\frac{X_{i}-\bar{X}_{i}}{X_{i}}\right|,
$$

is used to measure error of the peak pressures, flow rates, and wave intensities. It is important to note that although error is used to describe the differences between NL-TP and the other formulations (as error measures such as RMSE and MAE are used to compare the methods), it does not necessarily mean that the NL-TP formulation captures the physics most accurately for a given situation (although the non-linear form is often considered the most complete).

\subsubsection{Effects on waveforms}

The waveforms for pressure, flow rate, forward and backward components of wave intensity in the systemic arterial network are shown in figure 5.1. The solutions in the aortic root are shown in figures 5.1a, 5.1b, 5.1c, 5.1d and in the right ulnar artery II in figures 5.1e, 5.1f, 5.1g, 5.1h. Systemic venous system waveforms are shown in figure 5.1, for the inferior vena cava IV in figures $5.2 \mathrm{a}, 5.2 \mathrm{~b}, 5.2 \mathrm{c}, 5.2 \mathrm{~d}$ and ulnar vein II in figures $5.2 \mathrm{e}, 5.2 \mathrm{f}, 5.2 \mathrm{~g}, 5.2 \mathrm{~h}$. The aortic root and inferior vena cava were chosen as they are close the heart, while the ulnar artery and vein were chosen as they are both close to the vascular beds and are also a sufficient distance from the heart to view the effects of the non-linearities. 
Comparing the NL-TP and NL-SP formulations, the results indicate that the use of static pressure with non-linear effects captures the main characteristics of the waveforms quite well. The use of static pressure tends to induce a very slight shift of waveforms normally affecting the rate at which pressure and/or flow increases and decreases. These effects can also be seen when comparing the L-TP and L-SP waveforms.

Comparing the NL-TP and L-TP formulations shows the effect of the nonlinear convection term on waveforms. The linearised formulation tends to shift the waveform to the right in the systemic arterial system as seen in figure 5.1, and shifts waveforms to the left in the systemic venous system as seen in figure 5.1. This effect on waveforms is accentuated toward peripheries, although the main characteristics of the waveforms are generally still captured.

The linear formulation L0-SP typically shows the greatest deviation in waveforms when compared with the NL-TP formulation. In the systemic arterial and venous systems the waveforms are significantly shifted towards the right and fail to capture a number of characteristics in the waveforms such as those in figures 5.1e and 5.1f for the ulnar arteries, and figures 5.2e and 5.2f for the ulnar veins. Furthermore, figures $5.1 \mathrm{~g}$ and $5.1 \mathrm{~h}$ demonstrate that the wave intensities are poorly captured using the linear system of equations towards the periphery. In comparison, figure 5.1c shows that the forward wave intensity in the aortic root is relatively well captured for all methods. This is due to the forward wave intensity being primarily formed by the outflow of the lumped heart model (via aortic valve), which is the same model for all formulations. Similarly, figure 5.2d shows that the backward wave intensity in the inferior vena cava is relatively well captured for all methods, as the heart model creates the backward travelling waves from atria contraction. However, the linear system of equations performs poorly for the backward wave intensity $5.1 \mathrm{~d}$, which indicates the inability of the method to capture wave reflections. This can also be seen in figure 5.1a, where the pressure waveform fails to reach the same peak pressure as a result of poorly capturing wave reflections from downstream vessels.

\subsubsection{Error analysis}

The RMSE for waveforms in the systemic systems (including coronary and cerebral circulations) and pulmonary systems can be seen in Figure 5.3. The NL-SP 


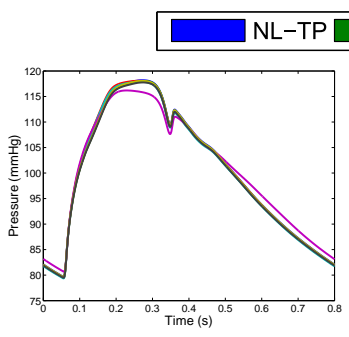

(a) Pressure in the aortic root

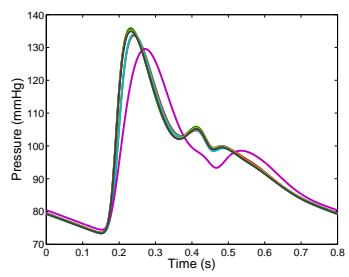

(e) Pressure in the right ulnar artery II

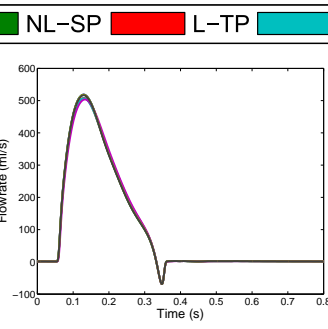

(b) Flow rate in the aortic root

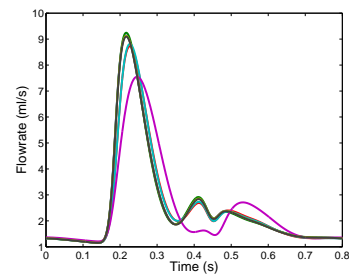

(f) Flow rate in the right ulnar artery II

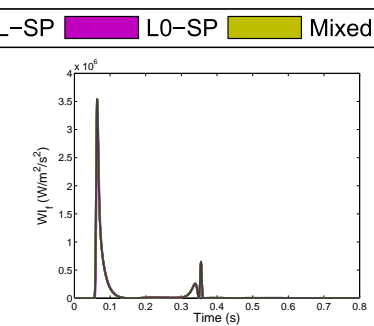

(c) Forward wave intensity in aortic root

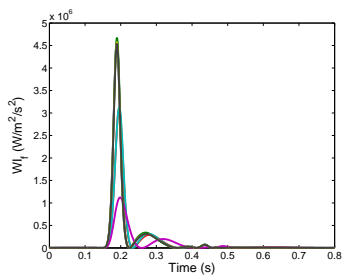

(g) Forward wave intensity in right ulnar artery II

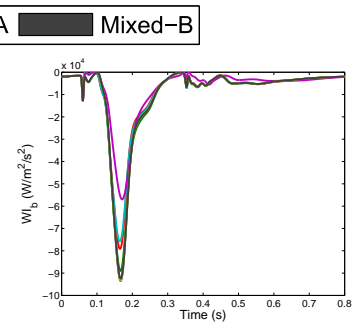

(d) Backward wave intensity in aortic root

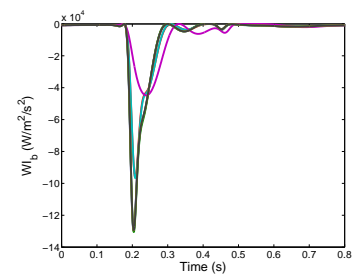

(h) Backward wave intensity in right ulnar artery II

Figure 5.1: Comparison of NL-TP, NL-SP, L-TP, L-SP, L0-SP formulations for various vessels in the systemic arterial system

formulation typically shows the least deviation (of the unified formulations) from the NL-TP formulation for pressure (figure 5.3a), flow rates (figure 5.3b) and wave intensities (figures 5.3c and 5.3d).

The linearised formulations L-TP and L-SP tended to perform similarly. The linear L0-SP method shows the greatest deviation of all the unified formulations, performing particularly poorly in the systemic arterial system. The Mixed-A configuration typically performed well for the systemic system, while the Mixed-B configuration performed quite well in the systemic arteries. The two mixed configurations performed poorly for the pulmonary system as both use the linear 0D formulation in those vessels.

The percentage error of the peak pressure, flow rate, forward wave intensity and backward wave intensity are shown in Figure 5.4. Typically the NL-SP formulation deviated least from the NL-TP formulation for peak wave intensities. However, this is not the case for pressure and flow rates, where in a number of systems (such as the pulmonary arterial system in figures $5.4 \mathrm{a}$ and $5.4 \mathrm{~b}$ ) the L-TP formulation deviated 


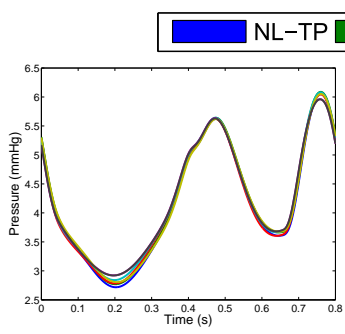

(a) Pressure in inferior vena cava IV

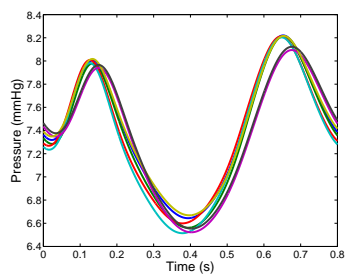

(e) Pressure in the right ulnar vein II

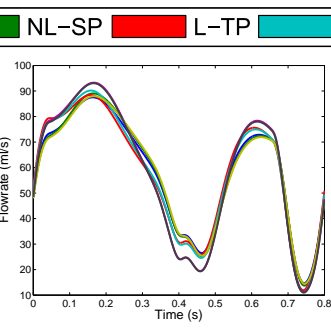

(b) Flow rate in inferior vena cava IV

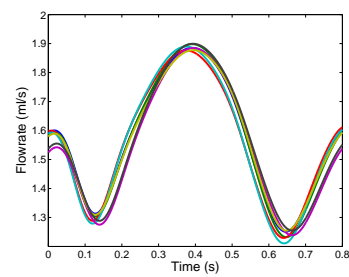

(f) Flow rate in the right ulnar vein II

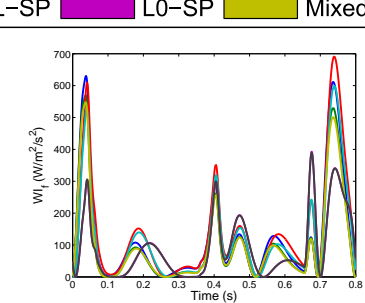

(c) Forward wave intensity inferior vena cava IV

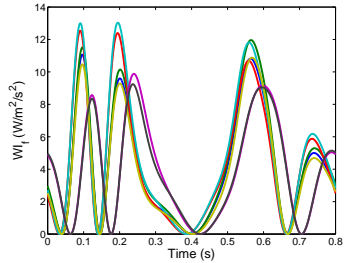

(g) Forward wave intensity in right ulnar vein II
Mixed-B

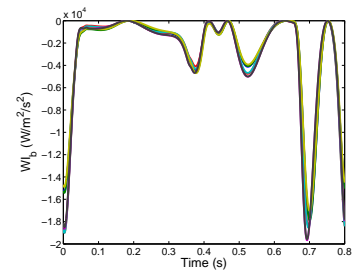

(d) Backward wave intensity inferior vena cava IV

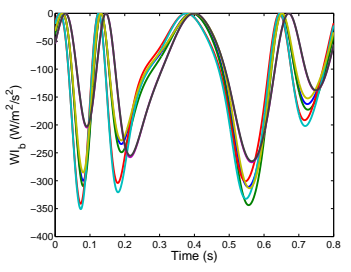

(h) Backward wave intensity in right ulnar vein II

Figure 5.2: Comparison of NL-TP, NL-SP, L-TP, L-SP, L0-SP formulations for various vessels in the systemic venous system

less.

Of the unified formulations the linear L0-SP method again deviated the most. Although this model showed only a $2 \%$ error for pressures, the method performs much worse for flow rates (up to $12 \%$ error) and wave intensities (just over $50 \%$ error).

\subsubsection{Overview of the main systemic vessels}

Figures 5.5 and 5.6 provide a visual representation of errors in the systemic arterial and venous systems respectively (excluding coronary and cerebral circulations for clarity). The pulmonary circulation is not shown as it is a smaller network and generally has much smaller errors. The L-SP form are omitted from the combined figures 5.5 and 5.6 for clarity, and also as the results are somewhat similar to the $\mathbf{L}$ TP formulation. However, the L-TP results for the systemic arteries and systemic veins are shown in figure 5.7. The two mixed configurations are also omitted as they are a mix of non-linear and linear formulations. Note that in the systemic venous 
trees the left side (of the network) shows only deep veins in the arm and leg, while the right side shows superficial veins.

Figure 5.5a shows the RMSE of the NL-SP formulation while figure 5.5b shows the scaled MAE of the NL-SP for flow rates in the major systemic arterial vessels. It can be seen that the largest errors in pressure tend to be in smaller peripheral vessels which have branched from much larger vessels, although a significant error can also be seen in the abdominal aorta between the celiac arterial branch and the superior mesenteric artery. This error in pressure also has a knock on effect on flow rates.

Figure 5.5c and 5.5d show the RMSE and scaled MAE of the L-TP formulation for pressure and flow rate, respectively. The errors for both pressure and flow rates tend to increase toward peripheries. The L-TP formulation has larger errors than the NL-SP formulation for both pressure and flow rates.

The RMSE and scaled MAE of the L0-SP formulation for pressure and flow rate are shown in Figures 5.5e and 5.5f. Significant differences can be seen between the left and right arm in 5.5f, this is due to the larger flow rates which the right arm experiences, meaning that the dynamic pressure term at junctions, and the non-linear convection effect has a greater impact in the right arm. The general distribution of errors is similar, albeit significantly larger, than the L-TP formulation.

The errors of the NL-SP formulation in the major systemic venous vessels for pressure and flow rates are shown in Figures 5.6a and 5.6b respectively. The largest errors in pressure occur in the inferior vena cava between the renal veins and the hepatic veins. Errors in flow rates are spread across the venous system, but tend to be larger close to the iliac veins and inferior vena cava.

Figures 5.6c and 5.6d show the errors for the L-TP formulation for pressure and flow rates. Errors in flow rates follow a similar pattern to the NL-SP form, although are in general larger. The errors in pressure tend to be larger around the junction of the iliac veins and the inferior vena cava. Although there is no sudden jump in pressure error around the renal veins to inferior vena cava (as seen when using static pressure conservation at junctions).

The errors of the linear L0-SP formulation are shown in Figures 5.6e and 5.6f for pressure and flow rates respectively. Again errors in flow rate have a similar pattern to the other formulations, albeit substantially larger in magnitude. The errors in pressure are more distributed when compared with other formulations. However, the 
largest error occurs in the inferior vena cava, between the renal and hepatic veins; which is the same place as the NL-SP formulation, implying that this deviation is due to the junction conditions of pressure conservation.

\subsubsection{Additional results from the systemic arteries}

Additional results from the systemic arterial network are shown in figure 5.8 and 5.9. The results are shown for pressure, volumetric flow rate, forward wave intensity, and backward wave intensity; for the right common carotid artery, the descending thoracic aorta, the right femoral artery, the septal artery in the coronary arteries, and the anterior communicating artery in the cerebral circulation. While table 5.2 shows results the number of vessels for each formulation which deviated from the reference solution, from most to least deviation; and also shows the average root-mean-square-error RMSE and peak errors for pressure, flow rate, forward and backward wave intensities. The Lumped formulation L0-SP deviated the most for all measures tested.

\begin{tabular}{|l||c|c|c|c|}
\hline & NL SP & L TP & L SP & L0 \\
\hline $\begin{array}{l}\text { RMSE } \\
\text { Ranking } P\end{array}$ & $86 / 15 / 8 / 0$ & $19 / 50 / 40 / 0$ & $4 / 44 / 61 / 0$ & $0 / 0 / 0 / 109$ \\
\hline $\begin{array}{l}\text { RMSE } \\
\text { Ranking } Q\end{array}$ & $99 / 9 / 1 / 0$ & $1 / 42 / 66 / 0$ & $9 / 58 / 42 / 0$ & $0 / 0 / 0 / 109$ \\
\hline $\begin{array}{l}\text { RMSE } \\
\text { Ranking } \\
W I_{f}\end{array}$ & $109 / 0 / 0 / 0$ & $0 / 69 / 40 / 0$ & $0 / 40 / 69 / 0$ & $0 / 0 / 0 / 109$ \\
\hline $\begin{array}{l}\text { RMSE } \\
\text { Ranking } \\
W I_{b}\end{array}$ & $103 / 6 / 0 / 0$ & $6 / 76 / 27 / 0$ & $0 / 27 / 82 / 0$ & $0 / 0 / 0 / 109$ \\
\hline $\begin{array}{l}\text { Average } \\
\text { RMSE } P\end{array}$ & 0.3985 & 0.8368 & 0.8989 & 2.4693 \\
\hline $\begin{array}{l}\text { Average } \\
\text { RMSE } Q\end{array}$ & 0.1341 & 0.6406 & 0.6106 & 1.7049 \\
\hline
\end{tabular}

Pg. $122 / 284$ 
5.3. RESULTS

\begin{tabular}{|c|c|c|c|c|}
\hline $\begin{array}{l}\text { Average } \\
\text { RMSE } \\
W I_{f}\end{array}$ & 13,725 & 66,090 & 67,686 & 114,690 \\
\hline $\begin{array}{l}\text { Average } \\
\text { RMSE } \\
W I_{b}\end{array}$ & 5,935 & 12,096 & 12,596 & 26,846 \\
\hline $\begin{array}{l}\text { Peak } \\
(\max ) \\
\text { Ranking } P\end{array}$ & $26 / 19 / 46 / 18$ & $52 / 20 / 20 / 17$ & $14 / 56 / 28 / 11$ & $17 / 14 / 15 / 63$ \\
\hline $\begin{array}{l}\text { Peak } \\
(\max ) \\
\text { Ranking } Q\end{array}$ & $50 / 21 / 36 / 2$ & $48 / 32 / 28 / 1$ & $9 / 55 / 43 / 2$ & $2 / 1 / 2 / 104$ \\
\hline $\begin{array}{l}\text { Peak } \\
(\max ) \\
\text { Ranking } \\
W I_{f} \\
\end{array}$ & $107 / 2 / 0 / 0$ & $2 / 77 / 30 / 0$ & $0 / 30 / 78 / 1$ & 0/0/1/108 \\
\hline $\begin{array}{l}\text { Peak (min) } \\
\text { Ranking } \\
W I_{b} \\
\end{array}$ & $91 / 16 / 2 / 0$ & $17 / 66 / 26 / 0$ & $1 / 27 / 81 / 0$ & 0/0/0/109 \\
\hline $\begin{array}{l}\text { Average } \\
\text { Peak } \\
(\max ) \quad \% \\
\text { Error } P\end{array}$ & 0.8156 & 0.5320 & 0.8179 & 1.4645 \\
\hline $\begin{array}{l}\text { Average } \\
\text { Peak } \\
(\max ) \quad \% \\
\text { Error } Q\end{array}$ & 2.6680 & 3.1645 & 3.5772 & 7.7570 \\
\hline $\begin{array}{l}\text { Average } \\
\text { Peak } \\
(\max ) \quad \% \\
\text { Error } W I_{f}\end{array}$ & 4.2053 & 20.4602 & 22.8622 & 38.005 \\
\hline
\end{tabular}




\begin{tabular}{|l||l|l|l|l|}
\hline Average & & & & \\
Peak (min) & 6.7846 & 16.6803 & 20.6903 & 41.8134 \\
$\% \quad$ Error & & & & \\
$W I_{b}$ & & & & \\
\hline \hline
\end{tabular}

Table 5.2: Arterial RMSE. Ranking numbers in the form $a / b / c / d$ indicate the number of vessel segments (out of 109) for each formulation which have $a$-least deviation, $b$-second least deviation, $c$-second most deviation, and $d$-most deviation from the solution of the NL-TP formulation. Average RMSE is the root-mean-square-error calculated for each vessel, then averaged over all vessels

\subsubsection{Additional results from the systemic veins}

Additional results from the systemic venous network is shown in figure 5.10 and 5.11. The results are shown for pressure, volumetric flow rate, forward wave intensity, and backward wave intensity; for the right internal jugular vein, superior vena cava, the right femoral vein, the septal vein and the superior sagittal sinus in the cerebral circulation. A significant shift in waveforms can be seen between non-linear NL formulations and linear/lumped formulations $\mathbf{L} / \mathbf{L} \mathbf{0}$. While table 5.3 shows results the number of vessels for each formulation which deviated from the reference solution, from most to least deviation; and also shows the average root-mean-square-error RMSE and peak errors for pressure, flow rate, forward and backward wave intensities. Once again the Lumped formulation L0-SP deviated the most for all measures tested.

\begin{tabular}{|l||c|c|c|c|}
\hline & NL SP & L TP & L SP & L0 \\
\hline $\begin{array}{l}\text { RMSE } \\
\text { Ranking } P\end{array}$ & $104 / 27 / 7 / 2$ & $28 / 87 / 23 / 2$ & $4 / 26 / 110 / 0$ & $4 / 0 / 0 / 136$ \\
\hline $\begin{array}{l}\text { RMSE } \\
\text { Ranking } Q\end{array}$ & $140 / 0 / 0 / 0$ & $0 / 91 / 49 / 0$ & $0 / 49 / 91 / 0$ & $0 / 0 / 0 / 140$ \\
\hline $\begin{array}{l}\text { RMSE } \\
\text { Ranking } \\
W I_{f}\end{array}$ & $137 / 3 / 0 / 0$ & $3 / 116 / 20 / 45$ & $0 / 11 / 119 / 10$ & $0 / 10 / 1 / 129$ \\
\hline
\end{tabular}

Pg. $124 / 284$ 
5.3. RESULTS

\begin{tabular}{|c|c|c|c|c|}
\hline $\begin{array}{l}\text { RMSE } \\
\text { Ranking } \\
W I_{b}\end{array}$ & $140 / 0 / 0 / 0$ & $0 / 105 / 35 / 0$ & $0 / 27 / 105 / 8$ & 0/8/0/132 \\
\hline $\begin{array}{l}\text { Average } \\
\text { RMSE } P\end{array}$ & 0.0834 & 0.1274 & 0.1474 & 0.2375 \\
\hline $\begin{array}{l}\text { Average } \\
\text { RMSE } Q\end{array}$ & 0.0842 & 0.2528 & 0.2492 & 0.3432 \\
\hline $\begin{array}{l}\text { Average } \\
\text { RMSE } \\
W I_{f}\end{array}$ & 6.5366 & 17.1062 & 20.2746 & 32.0045 \\
\hline $\begin{array}{l}\text { Average } \\
\text { RMSE } \\
W I_{b} \\
\end{array}$ & 48.1556 & 238.4696 & 250.9585 & 374.4662 \\
\hline $\begin{array}{l}\text { Peak } \\
(\max ) \\
\text { Ranking } P\end{array}$ & $43 / 37 / 53 / 7$ & $24 / 30 / 29 / 57$ & $57 / 31 / 32 / 20$ & $16 / 42 / 26 / 56$ \\
\hline $\begin{array}{l}\text { Peak } \\
(\max ) \\
\text { Ranking } Q\end{array}$ & $65 / 64 / 8 / 3$ & $71 / 63 / 6 / 0$ & $2 / 12 / 121 / 5$ & $2 / 1 / 5 / 132$ \\
\hline $\begin{array}{l}\text { Peak } \\
(\max ) \\
\text { Ranking } \\
W I_{f}\end{array}$ & $75 / 32 / 23 / 10$ & $41 / 71 / 24 / 4$ & $19 / 24 / 83 / 14$ & $5 / 13 / 10 / 112$ \\
\hline $\begin{array}{l}\text { Peak (min) } \\
\text { Ranking } \\
W I_{b}\end{array}$ & $72 / 32 / 28 / 8$ & $49 / 61 / 29 / 1$ & $10 / 41 / 77 / 12$ & $9 / 6 / 6 / 119$ \\
\hline $\begin{array}{l}\text { Average } \\
\text { Peak } \\
(\max ) \quad \% \\
\text { Error } P\end{array}$ & 0.5640 & 0.8461 & 0.6303 & 0.9142 \\
\hline
\end{tabular}




\begin{tabular}{|l||c|c|c|c|}
\hline $\begin{array}{l}\text { Average } \\
\text { Peak } \\
(\max ) \% \\
\text { Error } Q\end{array}$ & 2.0366 & 2.0582 & 3.9173 & 5.9333 \\
\hline $\begin{array}{l}\text { Average } \\
\text { Peak } \\
(\max \%\end{array}$ & 11.4586 & 19.6975 & 26.7254 & 49.3712 \\
Error $W I_{f}$ & & 14.2311 & 18.3907 & 37.2798 \\
\hline $\begin{array}{l}\text { Average } \\
\text { Peak (min) } \\
\% \quad \text { Error }\end{array}$ & 7.4202 & & \\
$W I_{b}$ & & & \\
\hline \hline
\end{tabular}

Table 5.3: Ranking numbers in the form $a / b / c / d$ indicate the number of vessel segments (out of 140) for each formulation which have $a$-least deviation, $b$-second least deviation, $c$-second most deviation, and $d$-most deviation from the solution of the NL-TP formulation. Average RMSE is the root-mean-square-error averaged over all vessels

\subsubsection{Results from the pulmonary system}

Figures 5.12 and 5.13 show the solutions for all formulations for a selection of vessels in the pulmonary circulation. In the pulmonary arteries, the main pulmonary artery and the vessel denoted as RSA0-2-54 are chosen as they are near the start and end of the pulmonary arteries respectively. In the pulmonary veins, the superior right pulmonary vein and the vessel denoted as RSV0-2-54 are chosen as they are near the end and start of the pulmonary veins respectively. There is generally less deviation in the pulmonary circulation than is seen in the systemic circulation.

\subsubsection{Bifurcation Test}

Due to significant deviations seen between the use of static pressure or total pressure at various junctions, such as the bifurcation of the abdominal aorta and celiac 
branch, additional cases are investigated, comparing only the NL-TP and NL-SP formulations. The bifurcation case uses a half sinusoidal wave for volumetric flow rate at the inlet of the parent vessel, and a three element Windkessel model at the outlets of both daughter vessels. The configuration is shown in figure 5.14. The test has three main variables that are defined: maximum flow rate at the inlet, area of the parent vessel, and the area ratio between the two daughter vessels $\gamma_{1}$. The cases investigated are:

- $A_{p 1}=1.584 \mathrm{~cm}^{2}, \gamma_{1} \in[1,50]$, $Q_{\text {in,max }}=[100,200,300] \mathrm{cm}^{3} / \mathrm{s}$.

- $A_{p 1}=2 \pi \mathrm{cm}^{2}, \gamma_{1} \in[1,50]$, $Q_{\text {in,max }}=[200,400,600] \mathrm{cm}^{3} / \mathrm{s}$.

- $A_{p 1}=\pi \mathrm{cm}^{2}, \gamma_{1} \in[1,50]$, $Q_{\text {in, } \max }=[200] \mathrm{cm}^{3} / \mathrm{s}$.

Figures 5.16a, 5.16b and 5.16c show the percentage error of maximum flow rate, maximum pressure and mean pressure for the bifurcation case, respectively. The test compares the effect of using either static or total pressure conservation at the junction. Thus the solutions are compared at the oulet of the parent vessel and the inlets of the daughter vessels (not at the centre of each vessel as in the full cardiovascular network). The case shown is using $A_{p 1}=1.584 \mathrm{~cm}^{2}$, with $\gamma_{1} \in[1,50]$, and a maximum inlet flow rate of $Q_{i n, \max }=200 \mathrm{~cm}^{3} / \mathrm{s}$. The area ratio of the celiac branch of the closed loop model is shown by the vertical line. In general the error graphs show the same behaviour for all the cases simulated. However, for the cases $A_{p 1}=2 \pi \mathrm{cm}^{2}$ and a maximum inlet flow rate of $Q_{i n, \max }=200 \mathrm{~cm}^{3} / \mathrm{s}$, a different maximum flow rate peak can be found in the parent vessel as a result of wave reflections at the junction depending on the value of $\gamma_{1}$.

The differences between solutions of the static and total pressure conservation junction condition tend to increase rapidly for an area ratio $\gamma_{1}$ from 1 to 5 at the inlet of the smallest daughter vessel $D a u_{1}$ for maximum flow rate, and both maximum and mean pressures. After this point the percentage error levels off to around $8 \%$ for maximum flow rate, $2 \%$ for maximum pressure, and $0.35 \%$ for mean pressures. The error of the largest daughter $D_{a} u_{2}$ and the parent vessel Par tends to zero as the area ratio increases for both pressures and flow rates. This is due to the area of 


\begin{tabular}{|l||c|c|c|}
\hline$Q_{\max } \mathrm{ml} / \mathrm{s}$ & 100 & 200 & 300 \\
\hline \% error Mean P & 0.1 & 0.35 & 0.65 \\
\% error Max P & 0.5 & 2 & 4 \\
\% error Max Q & 5 & 8 & 9.5 \\
\hline
\end{tabular}

Table 5.4: percentage error of mean and maximum pressure, and maximum flow rates for $D a u_{1}$ in the bifurcation case with $A_{p}=1.584 \mathrm{~cm}^{2}$

\begin{tabular}{|l||c|c|c|}
\hline & $A=1.584$ & $A=\pi$ & $A=2 \pi$ \\
\hline \% error Mean P & 0.35 & 0.1 & 0.025 \\
\% error Max P & 2 & 0.4 & 0.045 \\
\% error Max Q & 8 & 5 & - \\
\hline
\end{tabular}

Table 5.5: percentage error of mean and maximum pressure, and maximum flow rates for $D a u_{1}$ in the bifurcation case with $Q_{i n, \max }=200$. Empty entry - due to a different peak becoming the maximum as a result of wave reflections

largest daughter vessel tending towards the area of the parent vessel, while a large proportion of the flow travels from the parent to the largest daughter vessel, and hence the two dynamic pressure terms $\frac{\rho}{2} \frac{Q^{2}}{A^{2}}$ in total pressure conservation almost cancel. For an area ratio of $\gamma_{1}=1$ the difference in maximum flow between the static and total pressure cases is close to zero for all three vessels. However, for pressures only solutions of the daughter vessels are close to zero for $\gamma_{1}=1$, while in the parent vessel there is a negative error for the static pressure case.

The shape of the error waveforms generally remain consistent when changing the maximum flow rate or area of the parent. However, the error increases (decreases) with an increase (decrease) in flow rates as shown in table 5.4; while when increasing the parent and daughter vessel areas the error increases (decreases) as the area decreases (increases) as shown in table 5.5.

\subsubsection{Confluence Test}

The confluence case also has three main variables that are defined: maximum inlet flow rate, area of the daughter vessel, and the area ratio between the two parent vessels $\gamma_{1}$. The cases investigated are:

- $A_{d 1}=\pi \mathrm{cm}^{2}, \gamma_{1} \in[1,50]$, $Q_{\text {in, } \max }=[100,200,300] \mathrm{cm}^{3} / \mathrm{s}$.

Pg. $128 / 284$ 


\begin{tabular}{|l||c|c|c|}
\hline$Q_{\max } \mathrm{ml} / \mathrm{s}$ & 100 & 200 & 300 \\
\hline \% error Mean P & -0.014 & -0.05 & -0.1 \\
\% error Max P & -0.01 & -0.1 & -0.35 \\
$\%$ error Max Q & -0.047 & -0.12 & -0.18 \\
\hline
\end{tabular}

Table 5.6: percentage error of mean and maximum pressure, and maximum flow rates for $\mathrm{Par}_{1}$ in the confluence case with $A_{d}=\pi \mathrm{cm}^{2}$. Maximum inlet flow rates $Q_{\max }$ are distributed to achieve same velocity at both inlets

\begin{tabular}{|l||c|c|c|}
\hline & $A=\frac{\pi}{2}$ & $A=\pi$ & $A=2 \pi$ \\
\hline \% error Mean P & -0.2 & -0.05 & -0.001 \\
\% error Max P & -0.8 & -0.1 & -0.01 \\
\% error Max Q & -0.23 & -0.12 & -0.046 \\
\hline
\end{tabular}

Table 5.7: percentage error of mean and maximum pressure, and maximum flow rates for $P a r_{1}$ in the confluence case with $Q_{i n, \max }=200$. Empty entry - due to a different peak becoming the maximum as a result of wave reflections

- $A_{d 1}=2 \pi \mathrm{cm}^{2}, \gamma_{1} \in[1,50]$, $Q_{\text {in, } \max }=[200] \mathrm{cm}^{3} / \mathrm{s}$.

- $A_{d 1}=\frac{\pi}{2} \mathrm{~cm}^{2}, \gamma_{1} \in[1,50]$, $Q_{i n, \max }=[200] \mathrm{cm}^{3} / \mathrm{s}$.

Figure 5.16 shows the confluence test for $A_{d 1}=\pi$, which is a similar size to the inferior vena cava, and $Q_{i n, \max }=200$ which would be achieved during moderate exercise (although a physiological flow waveform in the inferior vena cava generally does not have zero flow). Figures 5.16d, 5.16e and 5.16f shows the percentage error between the static pressure and total pressure junction condition, of maximum flow rate, maximum pressure and mean pressure for the confluence case, respectively. Generally the error is largest for the smallest parent vessel $P a r_{1}$. For flow rates the error tends to decrease as the ratio $\gamma_{1}$ decreases. However, this is not the case for pressure as the error increases with increased $\gamma_{1}$ in parent vessel $\operatorname{Par}_{1}$.

The waveform has the same general shape when changing inlet flow rates or areas. The error tends to increase as the flow rate increases as shown in table 5.6, while the error tends to decrease as the areas increase as shown in table 5.7. 


\subsubsection{Trifurcation Test}

An additional trifurcation case is investigated, comparing the NL-TP and NLSP formulations, as significant deviations were seen at trifurcations such as at the junction of the internal thoracic artery, vertebral artery and subclavian artery II that branch from the subclavian artery I. The trifurcation case has four main variables that are defined: maximum inlet flow rate, area of the parent vessel, and two area ratios $\gamma_{1}$ and $\gamma_{2}$. An additional area ratio $\gamma_{3}$ has also been defined to aid analysis. The configuration of the trifucation case can be seen in figure 5.15. The cases investigated are:

- $A_{p 1}=\frac{\pi}{2} \mathrm{~cm}^{2}, \gamma_{1} \in[1,50], \gamma_{2} \in[1,50]$, $Q_{\text {in, } \max }=[100,200,300] \mathrm{cm}^{3} / \mathrm{s}$.

- $A_{p 1}=\pi \mathrm{cm}^{2}, \gamma_{1} \in[1,50], \gamma_{2} \in[1,50]$, $Q_{i n, \max }=[200] \mathrm{cm}^{3} / \mathrm{s}$.

- $A_{p 1}=2 \pi \mathrm{cm}^{2}, \gamma_{1} \in[1,50], \gamma_{2} \in[1,50]$, $Q_{i n, \max }=[200] \mathrm{cm}^{3} / \mathrm{s}$.

Figures 5.17a, 5.17b, 5.17c show the percentage difference (between static and total pressure conservation at junctions) of maximum pressure in the parent vessel $\mathrm{Par}_{1}$, and daughter vessels $D a u_{1}$ and $D a u_{2}$, respectively. Figures $5.17 \mathrm{~d}, 5.17 \mathrm{e}, 5.17 \mathrm{f}$ show the percentage difference of the maximum flow rate. The results shown are for the case $A_{p 1}=\frac{\pi}{2}$ and $Q_{i n, \max }=100$. The solutions for $D a u_{3}$ are shown in the supplementary file, as they show similar behaviour as $D a u_{2}$ (the same graphs would be produced by swapping the $\mathrm{x}-\mathrm{y}$ axes).

The main diagonal of these figures (bottom left to top right) correspond with $\gamma_{3}=1$. Moreover, the figures for the parent and daughter $\left(\operatorname{Dau}_{1}\right)$ vessels are symmetric along this diagonal.

There is a negative error for pressure in the parent vessel along the main diagonal $\gamma_{3}=1$, with the largest negative error occurring at $\gamma_{1}=\gamma_{2}=\gamma_{3}=1$. For flow rates there is a small positive error along the diagonal where $\gamma_{3}=1$. For the parent vessel $\operatorname{Par}_{1}$ :

- The largest errors for both maximum pressure and flow rate occurred in the interval $\gamma_{3}=[0.3,0.4]$ and $\gamma_{3}=[1 / 0.3,1 / 0.4]$. 
- The lowest errors for maximum pressure and flow rate occurred in the interval $\gamma_{3}=[0.57,0.6]$ and $\gamma_{3}=[1 / 0.57,1 / 0.6]$, at high values of $\gamma_{1}$ when $\gamma_{2}=1$, and at high values of $\gamma_{2}$ when $\gamma_{1}=1$.

For the daughter vessel $D a u_{1}$ :

- The lowest error for both maximum pressure and flow rate occurred at $\gamma_{1}=$ $\gamma_{2}=\gamma_{3}=1$.

- Large errors for maximum flow rate occurred for $\gamma_{1}>2$ and $\gamma_{2}>2$. The largest errors occurred along the diagonal where $\gamma_{3}=1$, at high values of $\gamma_{1}$ when $\gamma_{2}=1$, and at high values of $\gamma_{2}$ when $\gamma_{1}=1$.

- The largest errors for maximum pressure occurred at $\gamma_{3}=[0.3,0.4]$ and $\gamma_{3}=$ $[1 / 0.3,1 / 0.4]$.

- Medium errors occurred for maximum pressure along the diagonal $\gamma_{3}=1$ (i.e. $\left.\gamma_{1}=\gamma_{2}\right)$

For daughter vessel $\mathrm{Dau}_{2}$ :

- The lowest errors occurred along the diagonal $\gamma_{3}=1$; and for high values of $\gamma_{2}$ with low values of $\gamma_{1}$.

- The largest errors occurred along $\gamma_{3} \geq 1 / 0.4$.

For daughter vessel $\mathrm{Dau}_{3}$ :

- The lowest errors occurred along the diagonal $\gamma_{3}=1$; and for high values of $\gamma_{1}$ with low values of $\gamma_{2}$.

- The largest errors occurred along $\gamma_{3} \leq 0.4$.

Figure 5.17 shows the deviation between using static pressure and total pressure conservation at vessel junctions for the third daughter vessel $D a u_{3}$. The case show is for $A_{p 1}=\frac{\pi}{2}$ and $Q_{i n, \max }=100$. Figure 5.18 shows the deviation of the choice of pressure conservation condition at a trifurcation junction for the parent vessel, and all three daughter vessels. The case shown is for $A_{p 1}=\frac{\pi}{2}$ and $Q_{i n, \max }=200$. Indicating an increase in flow rate increases the magnitude of the errors, but follows a similar pattern for the area ratios $\gamma_{1}, \gamma_{2}$, and $\gamma_{3}$. 


\subsection{Discussion}

The performance of six 1D model configurations were compared (two employing non-linear convection terms and two without non-linear convection, each of which was formulated separately using conservation of either total pressure or static pressure); a 0D linear formulation in which resistances, compliances and inductances were constant; a configuration which uses a non-linear formulation for systemic arteries and veins, with a 0D linear formulation for the pulmonary system; and a configuration which uses a non-linear formulation for systemic arteries, with a 0D linear formulation for the systemic veins and pulmonary system. These configurations were compared with the reference solution (non-linear with total pressure conservation at junctions, NL-TP).

For systemic arterial networks we observed that:

- peak flow rate solutions were most sensitive to the choice of pressure conservation rule at junctions;

- the NL-SP simulation showed the smallest deviation from the reference solution in pressure, flow rate, and forward and backward wave intensities, but contained larger deviations at asymmetric vessel junctions particularly for the smallest daughter vessel;

- estimation of peak pressure was better with the L-TP formulation, but peak wave intensities were better estimated with the NL-SP formulation;

- the 0D linear formulation showed the greatest deviation for all measures investigated and poorly captured wave reflections;

- treating the systemic venous and/or pulmonary system as linear models, whilst treating the systemic arterial system as non-linear, does not have a significant impact on the solution in the systemic arterial system.

For systemic venous networks:

- the NL-SP simulation again deviated least overall, especially for flow rate estimation; 
- the NL-SP solution deviated significantly from the NL-TP solution when very asymmetric vessel junctions were considered;

- L0-SP (linear) formulation deviated the most.

For the pulmonary circulation:

- The influence of pressure conservation at junctions was greater than the nonlinear convection effect in pulmonary vessels;

- overall the L-TP formulation deviated the least for pressures in both arterial and venous systems;

- L-TP deviated the least for peak pressures in both arterial and venous networks, and for peak flows in arterial networks;

- L0-SP formulation performed the worst for all error measures tested (of the unified formulations).

The NL-SP simulation provided solutions for arterial configurations that were closer to the NL-TP (reference model) solution than any of the other three model formulations (being closer to the reference solution in 86 out of 109 vessels for pressure, in 99 out of these vessels for flow rate, in all 109 vessels for forward wave intensity, and in 103 vessels for backward wave intensity. However, the NL-SP solutions showed greater deviations from the reference solution in vessels that had very asymmetric junctions, this being particularly evident for peak systolic pressure and peak flow rates. The junction is considered to be asymmetric if there are large differences in cross-sectional area between the junctions daughter vessels. These asymmetric junctions tend to increase the error in estimates of flow rates during systole (i.e. peak flow rates) but not during diastole (i.e. low flow rates), when the total (dynamic) pressure term has a much reduced impact. In general, the more asymmetric the junction the larger the deviation of the NL-SP solution from the NL-TP reference solution, especially in the smaller daughter vessel. This influence of junction cross-sectional area mismatches could explain the observations of Boileau et al.[41], who described deviations in solutions for the Adan56 arterial network (compared with reference solutions) but not for the more symmetric 37-vessel arterial network, despite using static pressure conservation in both cases. 
To illustrate this point further the NL-SP model was also compared to the NL-TP model for isolated bifurcation and trifurcation cases. The results of the isolated junction cases supported the finding that larger differences between the solutions were present at more asymmetric junctions, mainly affecting the solution of the smallest daughter vessel. However, the isolated junction cases also showed that differences in pressure were found in the parent vessel at symmetric junctions (although this difference was relatively small). Moreover, it was found that these differences increased at higher flow rates. Figures 5.16a, 5.16b and 5.16c showed a configuration (both areas and flow rates) that is similar to the celiac branching from the descending aorta. There was approximately a $5 \%$ difference in maximum flow rate for what could be considered the celiac artery where the area ratio between the daughter vessels was $\gamma_{1}=2.575$. Interestingly [231] showed that static pressure better represented the pressure solution in a smaller daughter vessel (which branched from a much larger vessel), while dynamic pressure better represented the pressure solution in the larger daughter vessel (when compared to 3D simulations). In the systemic arterial network, significant differences were seen at the trifurcation of the internal thoracic artery, vertebral artery and subclavian artery II branching from the subclavian artery I. The isolated trifurcation test junction again supported this finding as significant differences between the static and total pressure conservation cases were observed for $\gamma_{3}=[0.3,0.4]$ (largest difference occurred around $\gamma_{3}=0.35$ ). At this trifurcation in the systemic arterial tree the value of $\gamma_{3}=0.39$. Moreover, the largest daughter vessel showed less differences in solutions (between the static pressure and total pressure conservation junction condition) than the other two much smaller daughter vessels. As one of the daughter vessels is much larger than the other/others, there is a much larger percentage of blood entering the larger daughter vessel (from the parent vessel), and as the area of the largest daughter vessel tends towards the area of the parent vessel, the dynamic pressure terms (in total pressure conservation) $\frac{\rho}{2} \frac{Q^{2}}{A^{2}}$ almost cancel.

It is important to consider the impact on waveforms of the non-linearity term and the use of either conservation of total pressure or static pressure at junctions throughout the network. In general, the non-linearity in the momentum equation causes the flow and pressure solutions to lean upstream towards the heart. The use of conservation of total pressure (which includes dynamic pressure term similar to the non-linear convection term) mainly influences the rate of change of pressure (and flow 
rate) waveforms, however the main characteristics of the waveforms are generally well captured albeit with different extrema. The linear 0D L0-SP formulation performed poorly, differences can even be seen in the aortic root for pressure in figure 5.1a, indicating the $0 \mathrm{D}$ form particularly struggles with capturing wave reflections. While in the ulnar artery shown in figures 5.1e and 5.1f many features of the pressure and flow waveforms are not captured.

Similarly, for venous network configurations the non-linear convection term tended to have a greater impact on model solutions than the dynamic/total pressure term. The NL-SP configuration again performed best compared to the reference (NLTP) solution, particularly for the flow rate solutions: the average flow rate solution was better for this model in all 140 vessels, whilst the wave intensity solution was better in 137 vessels for forward wave intensity and in all 140 vessels for backwards wave intensity. The pressure solutions in the NL-SP model again became less accurate when considering smaller vessels connected to very asymmetrical junctions (i.e. small veins joining a much larger vein). Once again the lumped formulation deviated the most from the reference solution for both average and peak values of pressure, flow rate and wave intensity.

The non-linear convection term in the momentum equation causes the estimated venous waveforms to lean downstream (towards the heart). As in the arterial system, the use conservation of total pressure mainly influences the rate of change of pressure (and flow rate) waveforms, which again captured the main characteristics of the waveforms albeit with different extrema. The L-TP solution was generally closer to the reference solution than the L-SP solution for pressures, flow rates and wave intensities. The direction of waveform lean in veins was found to be the opposite of that in the arterial system, in which the non-linear convection term leans the waveform upstream. This is most likely a result of the diverging flow and tapering of vessels in the flow direction for the arterial system, whilst the venous system displays converging flow and a widening of the vessels in the direction of flow.

For the pulmonary circulation the NL-SP formulation deviated the least from the reference solution for average flow rates and average wave intensities, however the $\mathbf{L}$-TP solution performed best for average and peak pressures as seen in figures 5.3a and 5.4a. The choice of pressure conservation rule at junctions tends to have a larger effect in the pulmonary system than the inclusion or exclusion of the non-linear convection term, as the non-linearity has less time/distance to affect the solution. 


\subsection{Conclusion}

The effects of non-linearities and the pressure conservation junction condition has been investigated across the vessels of an extensive closed-loop cardiovascular system. The greatest deviations of solutions, when comparing conservation of total pressure and conservation of static pressure, tended to occur at asymmetric junctions (i.e. junctions which have large differences in daughter vessel cross-sectional areas). If only the systemic system is of interest then it is reasonable to consider the pulmonary system as a lumped model. The non-linear convection effect, and the effect of variable inductances, compliances and resistances in 1D was shown to significantly affect the solutions of pressure, flow rates, and wave intensities and should be included in the systems of interest. The lumped formulation performed poorly, particularly with respect to wave reflections. Thus the lumped formulation is unsuitable for problems/investigations for which wave reflections are important, such as stenosis, aneurysms, and investigating arterial stiffness using the augmentation index. This indicates that lumped models, even multi-compartment models should only be used to close the loop, that is to ensure that flow returns to the heart. Furthermore, when using the ETM scheme there is no computational overhead of a 1D formulation, compared with a lumped model (when considering the same number of elements); that is the 1D and 0D approaches take the same amount of computational time. Therefore a 0D model should be lumped as much as possible if the aim is to reduce computational cost, otherwise the more versatile and more accurate 1D model should be implemented. The 1D linearised formulation which omits the non-linear convection term (though non-linearities are present through inductance, compliance and viscous effects) is an acceptable compromise, particularly for problems involving smaller vessels. If regions of interest include small vessels branching from larger branches such as investigating renal function and liver function, the choice of junction condition may be of importance. In order to investigate the effects of gravity in such a system, various auto-regulation effects such as muscle pumping and the baroreceptor reflex mechanism need to be integrated into the model. 


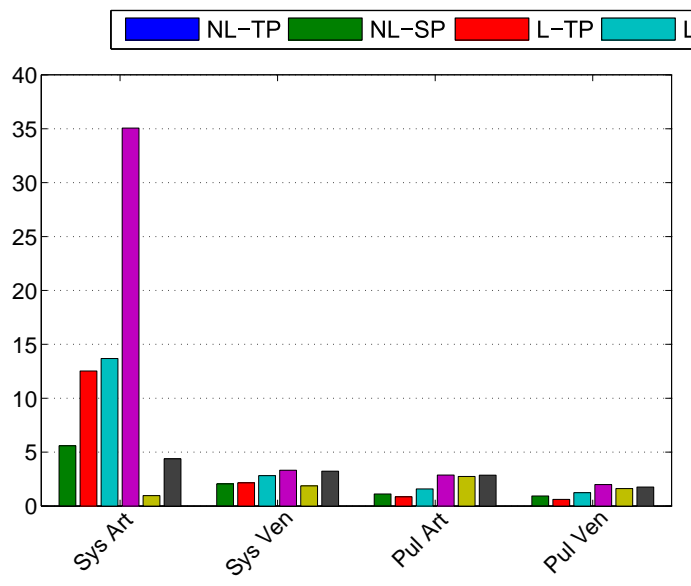

(a) Pressure

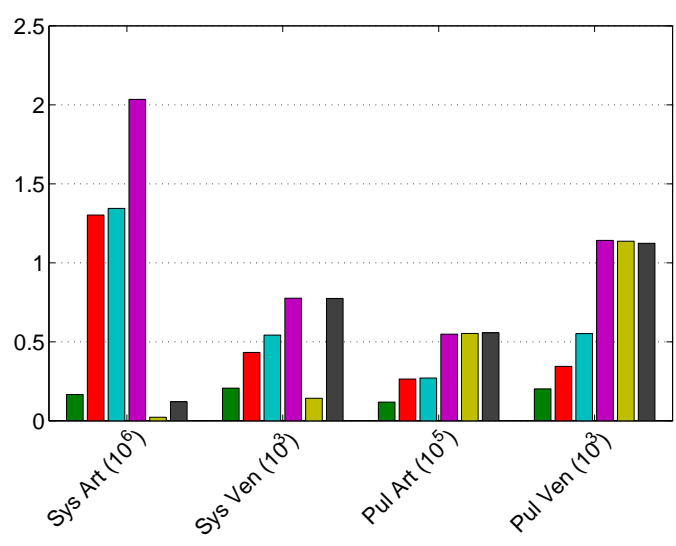

(c) Forward wave intensity (values in brackets are scaling factors)

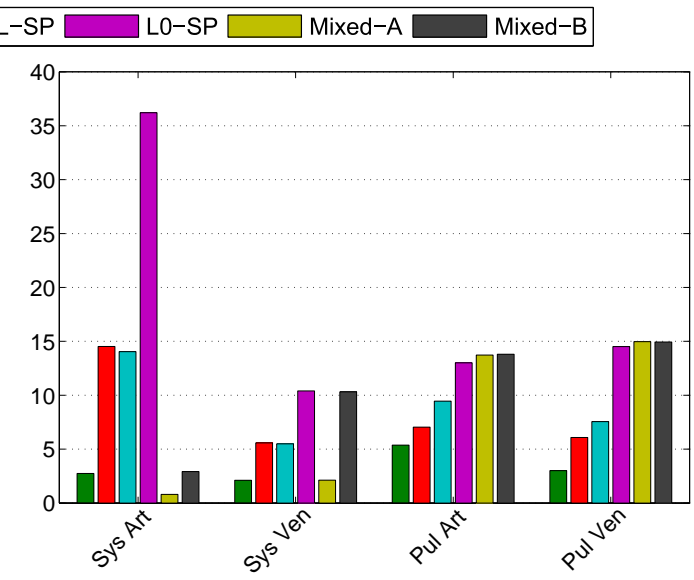

(b) Flow rates

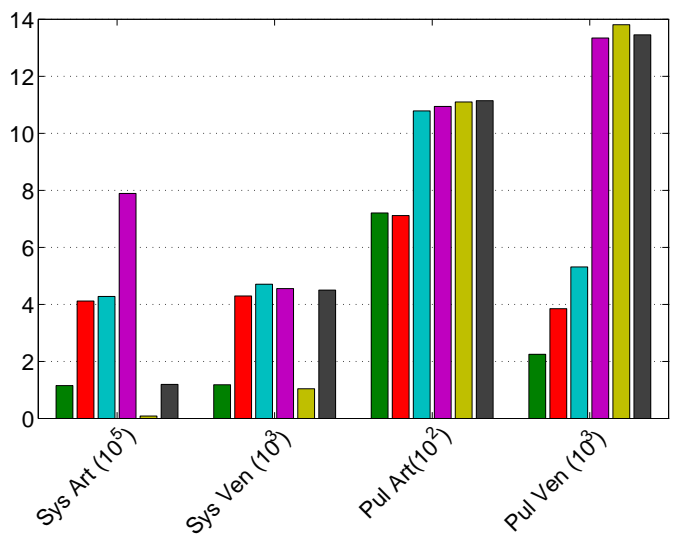

(d) Backward wave intensity (values in brackets are scaling factors)

Figure 5.3: RMSE (relative to the NL-TP formulation) of pressure, flow rate, and wave intensities in the systemic arterial and venous systems (including hepatic veins, cerebral and coronary circulation), and pulmonary arterial and venous systems 


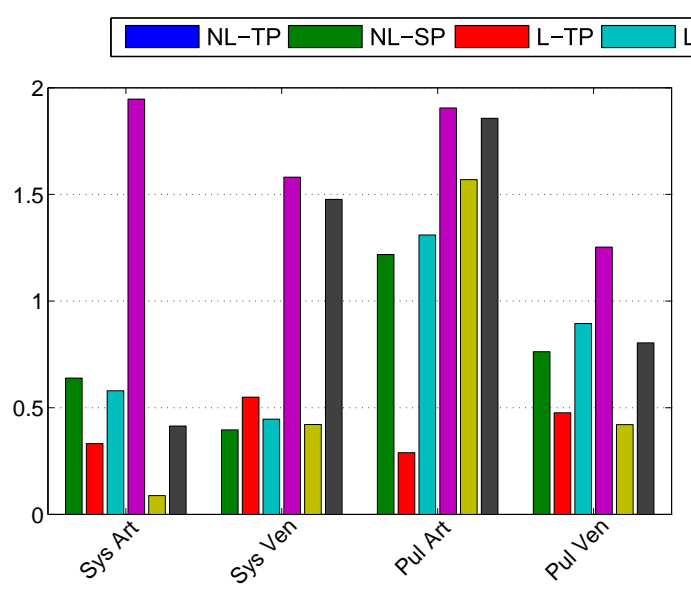

(a) Pressure

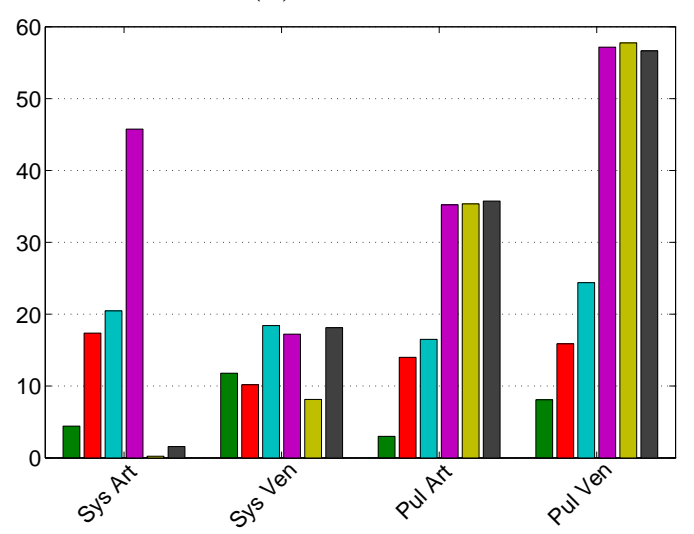

(c) Forward wave intensity

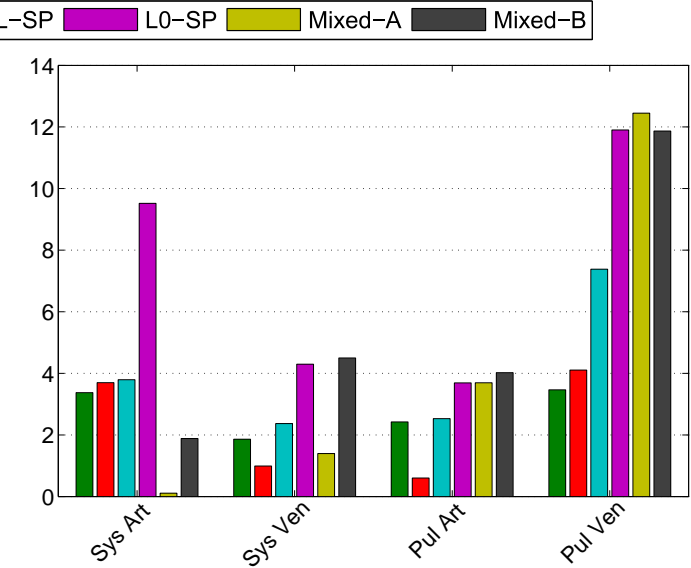

(b) Flow rates

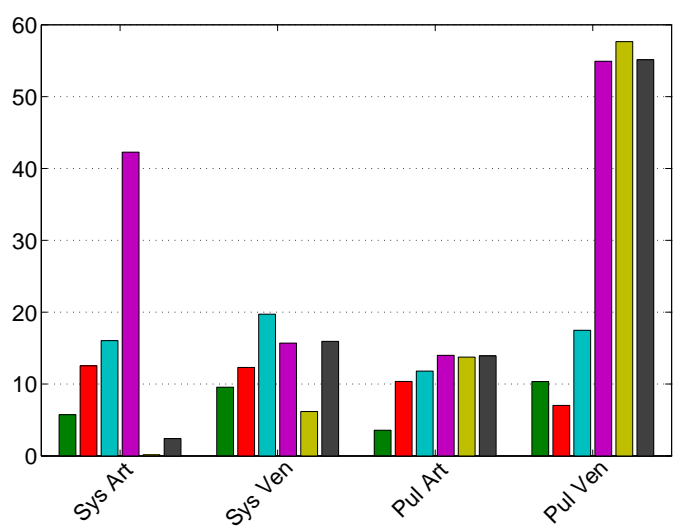

(d) Backward wave intensity

Figure 5.4: Percentage errors (relative to the NL-TP formulation) of peak pressures, flow rates, and wave intensities in the systemic arterial and venous systems (including hepatic veins, cerebral and coronary circulation), and pulmonary arterial and venous systems 

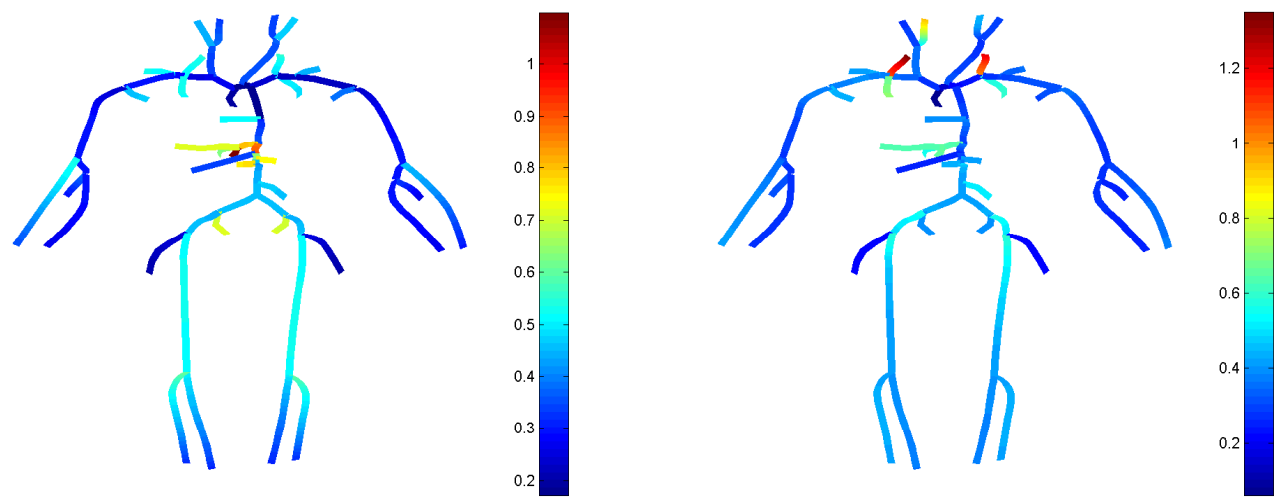

(a) RMSE of NL-SP for pressure

(b) Scaled MAE of NL-SP for flow rate
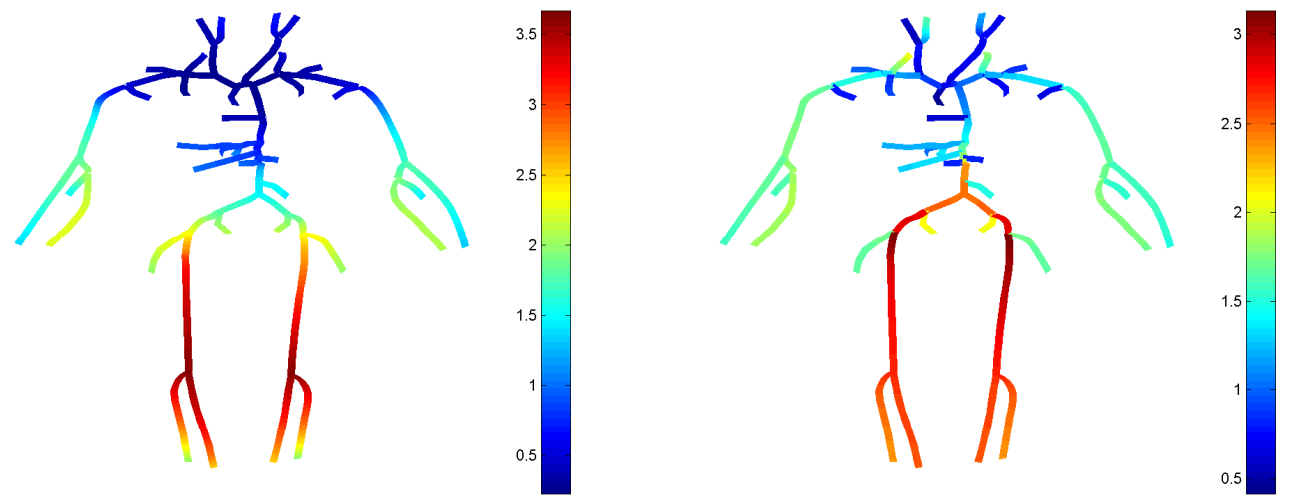

(c) RMSE of L-TP for pressure

(d) Scaled MAE of $\mathbf{L}-\mathbf{T P}$ for flow rate
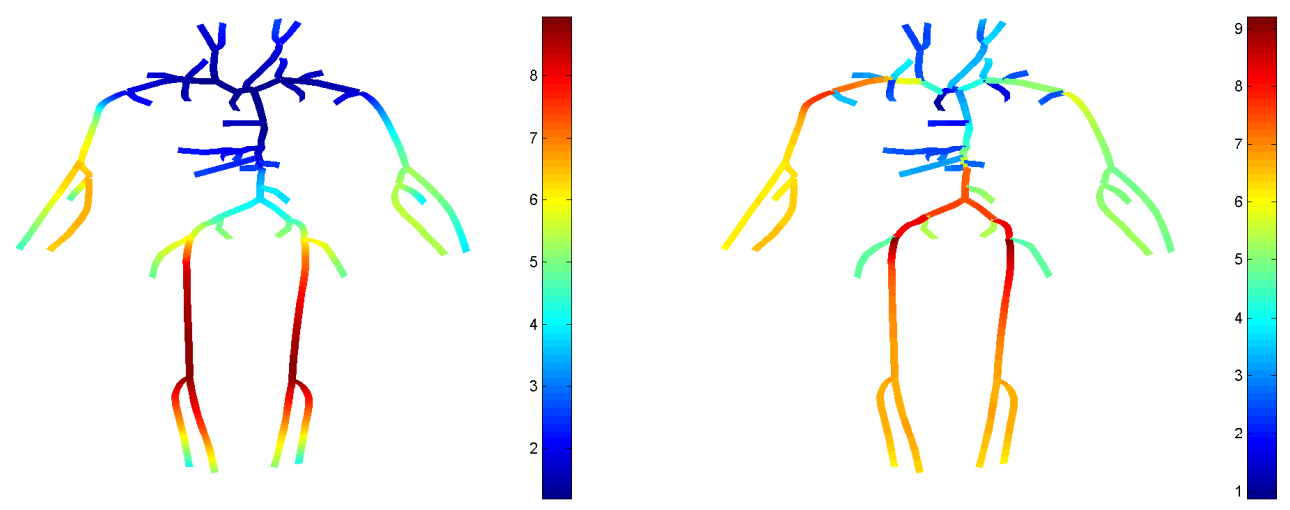

(e) RMSE of L0-SP for pressure

(f) Scaled MAE of L0-SP for flow rate

Figure 5.5: Deviation of formulations for the systemic arterial system (cerebral and coronary vessels omitted for clarity), compared to NL-TP form 

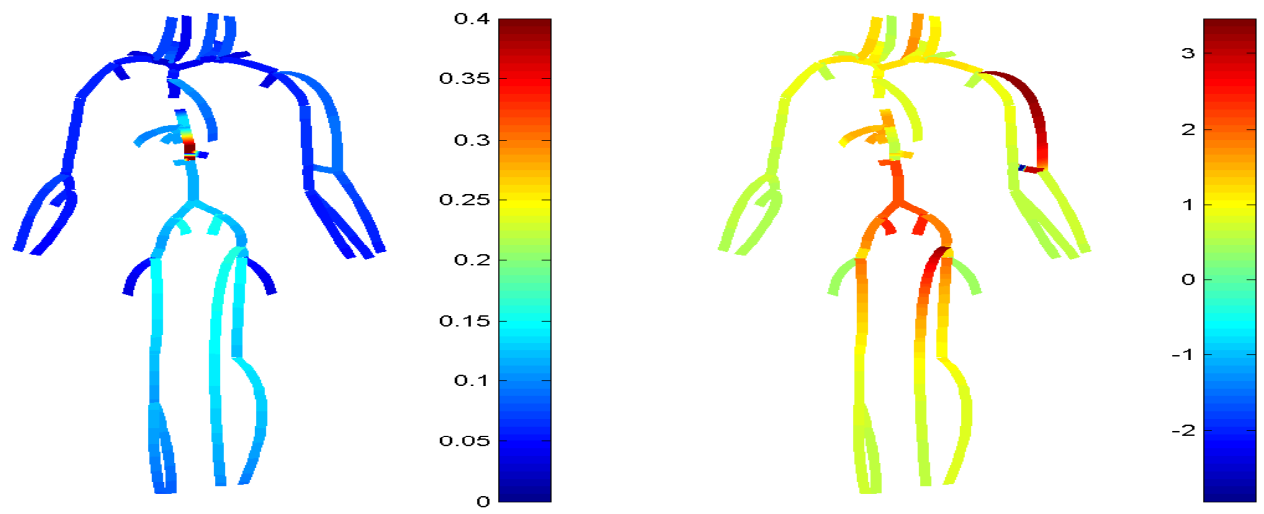

(a) RMSE of NL-SP for pressure

(b) Scaled MAE of NL-SP for flow rate
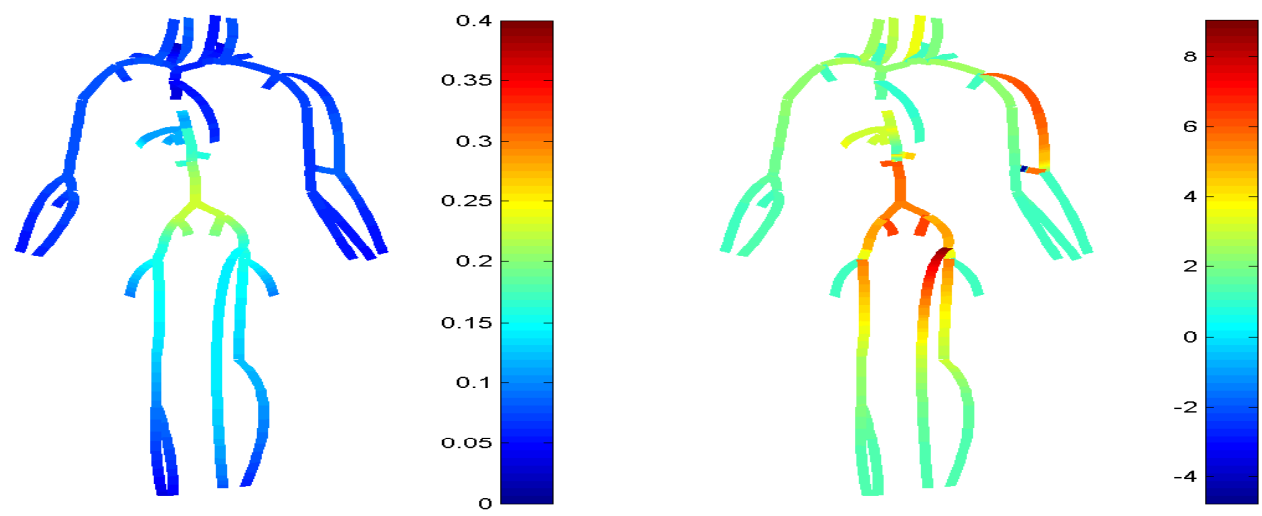

(c) RMSE of L-TP for pressure
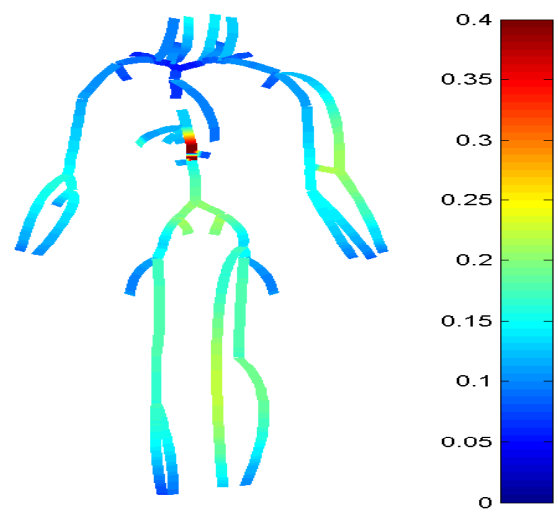

(d) Scaled MAE of L-TP for flow rate

(e) RMSE of L0-SP for pressure

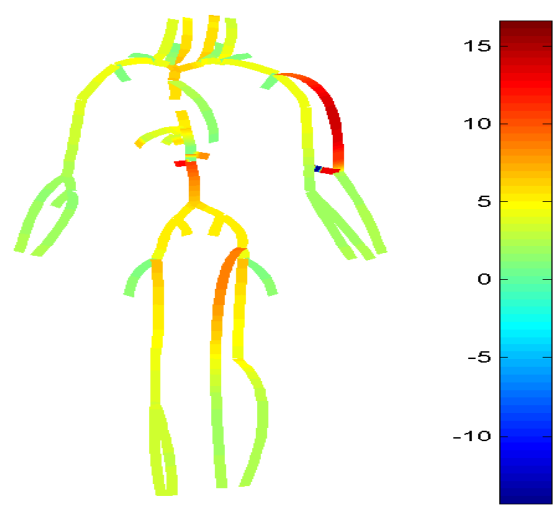

(f) Scaled MAE of L0-SP for flow rate

Figure 5.6: Deviation of formulations in the systemic venous system (cerebral and coronary vessels omitted for clarity), compared to NL-TP form. As shown in the figure the veins on the left side (arms and legs) are deep veins, while on the right side are superficial veins 

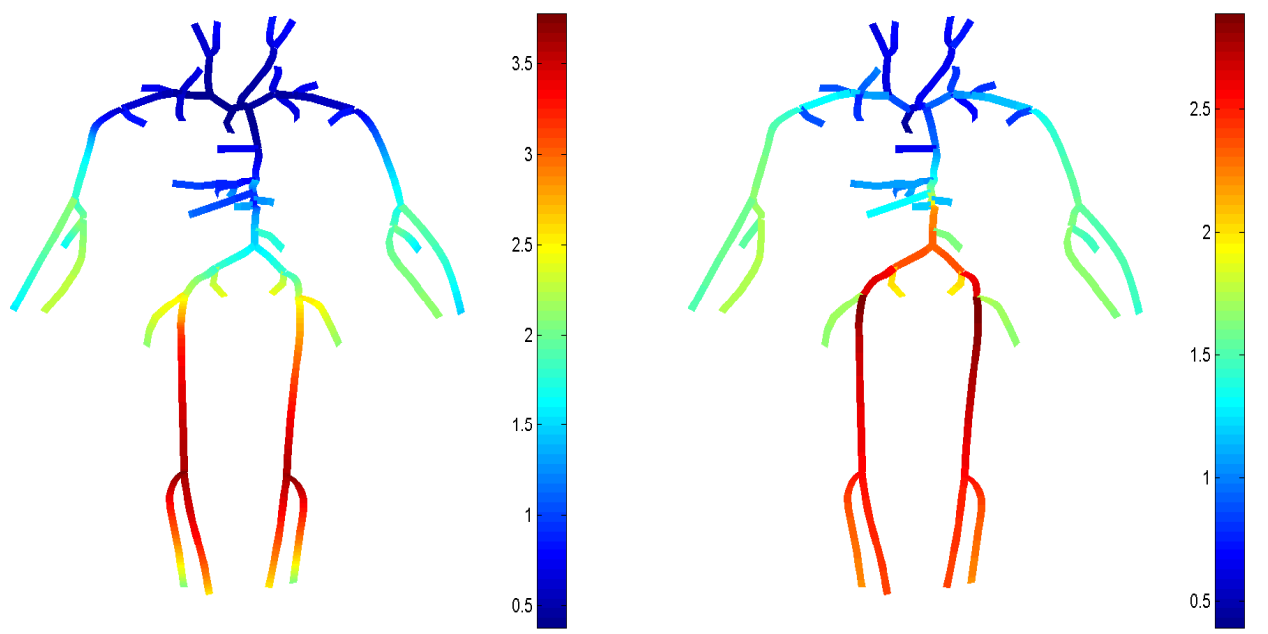

(a) RMSE of L-SP for pressure in arter-

(b) Scaled MAE of $\mathbf{L - S P}$ for flow rate in ies arteries
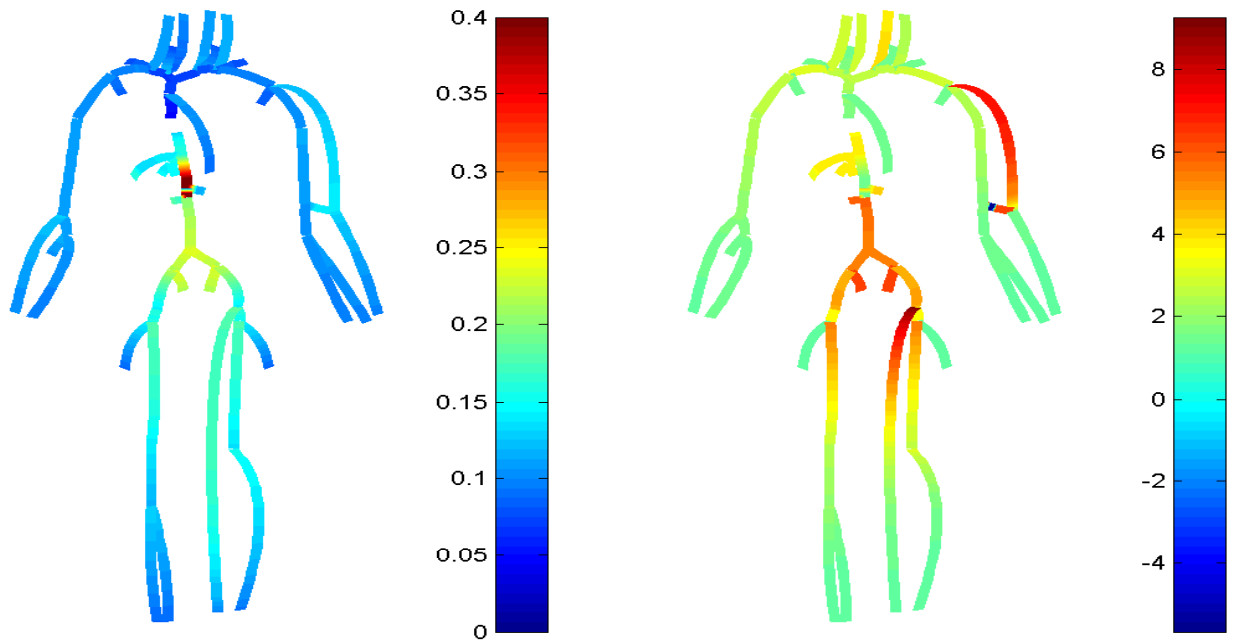

(c) RMSE of L-SP for pressure in veins (d) Scaled MAE of L-SP for flow rate in veins

Figure 5.7: Deviation of L-SP for the systemic arterial and venous systems (cerebral and coronary vessels omitted for clarity), compared to NL-TP form. For the veins, the left side (arms and legs) are deep veins, while on the right side are superficial veins 


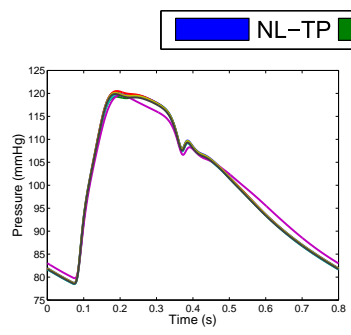

(a) Pressure in the right common carotid artery

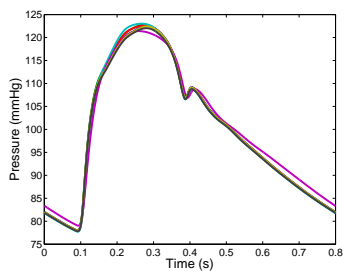

(e) Pressure in the descending thoracic aorta II

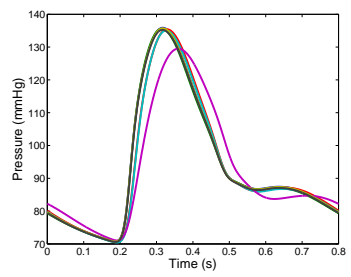

(i) Pressure in the right femoral artery
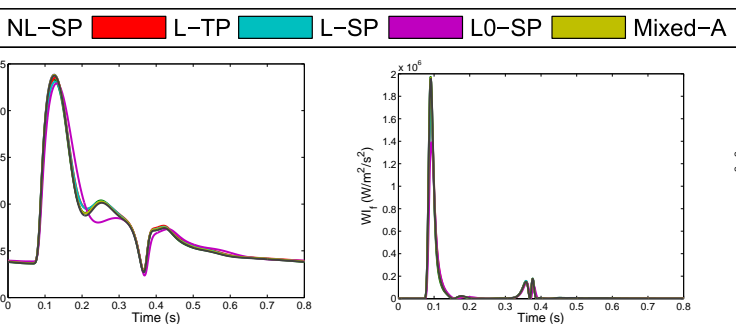

(c) Forward wave intensity right common carotid artery

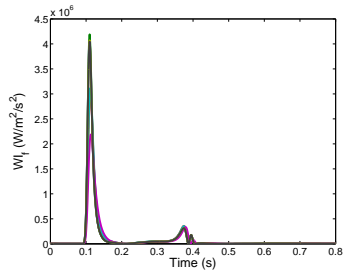

(g) Forward wave intensity in descending thoracic aorta II

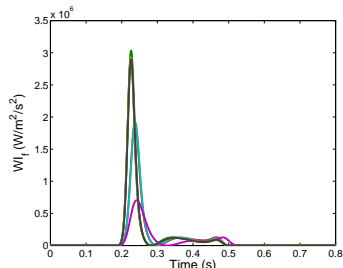

(k) Forward wave intensity in the right femoral artery

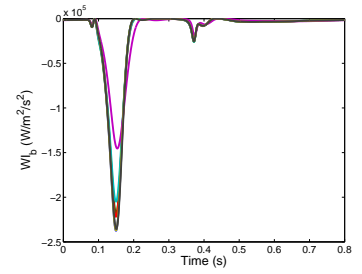

(d) Backward wave intensity right common carotid artery

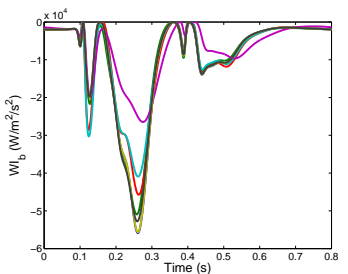

(h) Backward wave intensity in descending thoracic aorta II

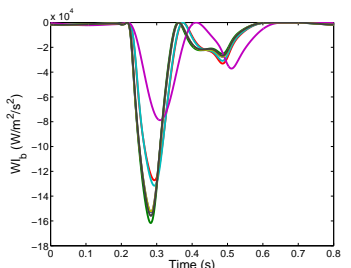

(1) Backward wave intensity in the right femoral artery

Figure 5.8: Comparison of NL-TP, NL-SP, L-TP, L-SP, L0-SP formulations for various vessels in the arterial system 


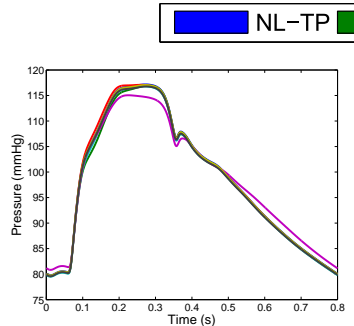

(a) Pressure in the septal artery

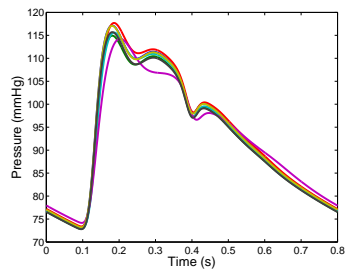

(e) Pressure in the anterior communicating artery

\section{$\mathrm{NL}-\mathrm{SP}$}

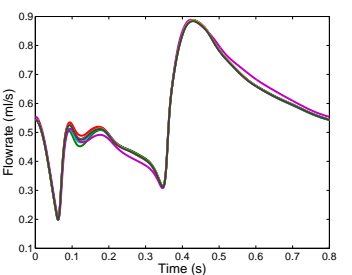

(b) Flow rate in the septal artery

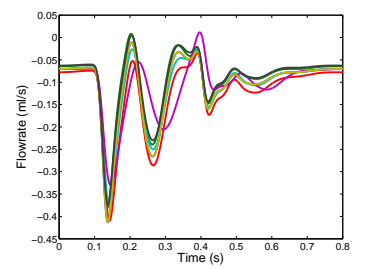

(f) Flow rate in the anterior communicating artery

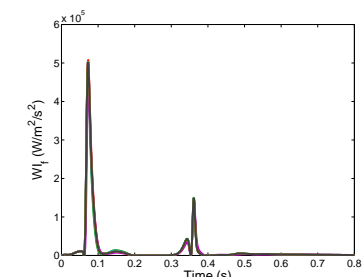

(c) Forward wave intensity in the septal artery

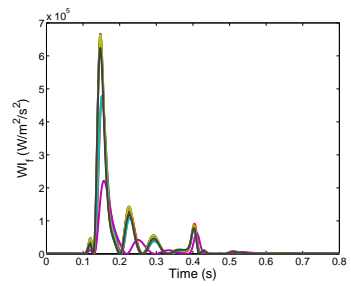

(g) Forward wave intensity anterior communicating artery

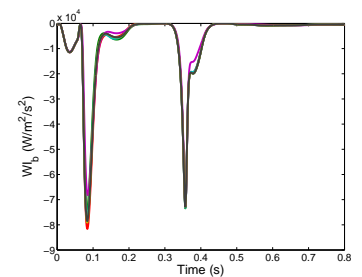

(d) Backward wave intensity in the septal artery

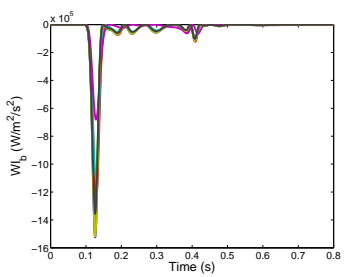

(h) Backward wave intensity anterior communicating artery

Figure 5.9: Comparison of NL-TP, NL-SP, L-TP, L-SP, L0-SP formulations for various vessels in the arterial system 


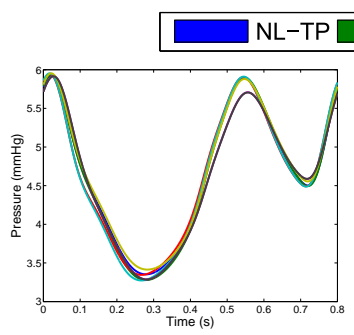

(a) Pressure in right internal jugular vein

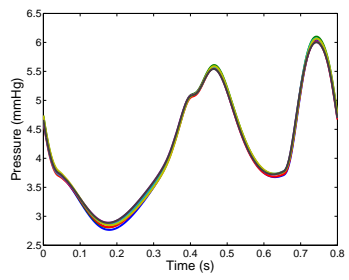

(e) Pressure in superior vena cava II

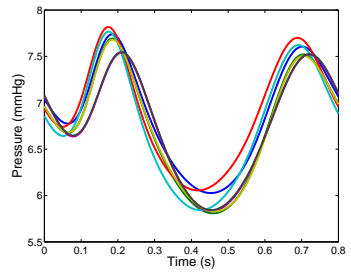

(i) Pressure in right femoral vein I

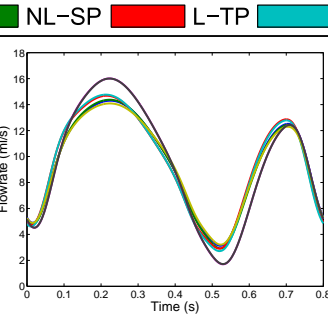

(b) Flow rate in right internal jugular vein

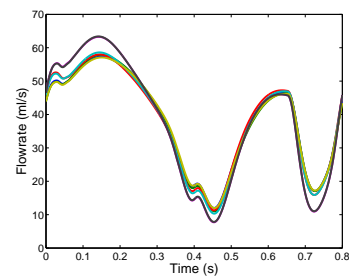

(f) Flow rate in superior vena cava II

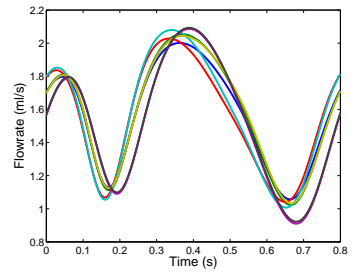

(j) Flow rate in right femoral vein I

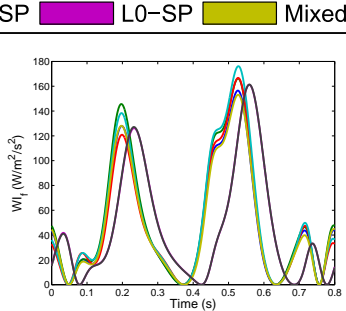

(c) Forward wave intensity in right internal jugular vein

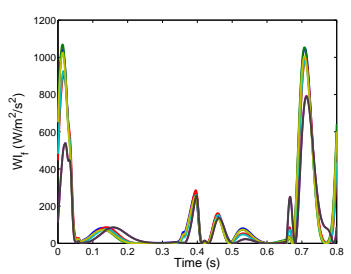

(g) Forward wave intensity in superior vena cava II

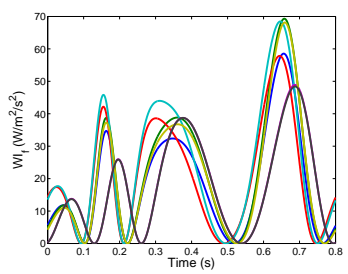

(k) Forward wave intensity in right femoral vein I

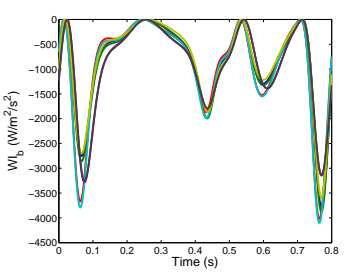

(d) Backward wave intensity in right internal jugular vein

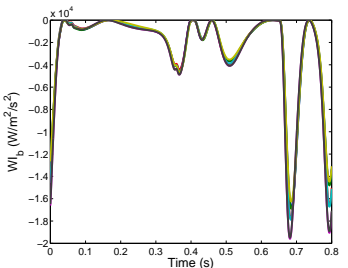

(h) Backward wave intensity in superior vena cava II

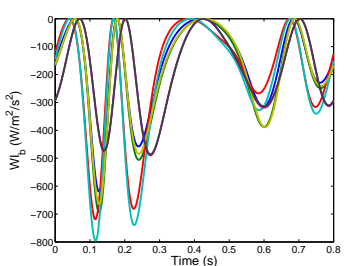

(l) Backward wave intensity in right femoral vein I

Figure 5.10: Comparison of NL-TP, NL-SP, L-TP, L-SP, L0-SP formulations for various vessels in the venous system 


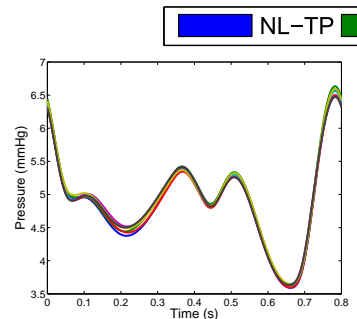

(a) Pressure in the septal vein

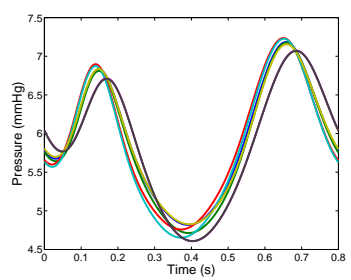

(e) Pressure in superior sagittal sinus III

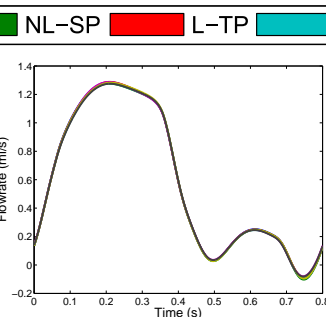

(b) Flow rate in the septal vein

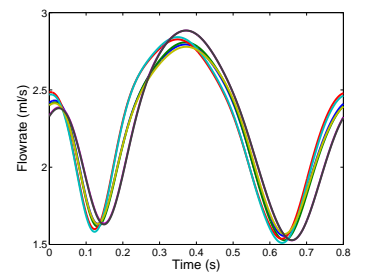

(f) Flow rate in superior sagittal sinus III

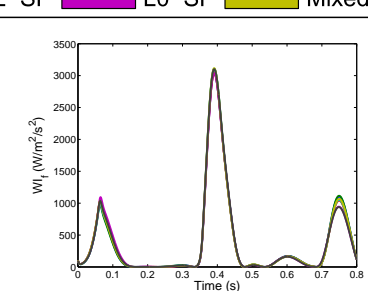

(c) Forward wave intensity in the septal vein

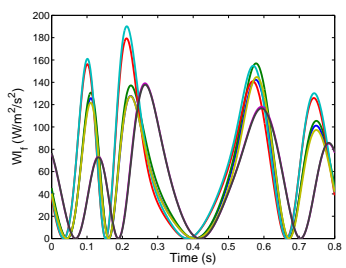

(g) Forward wave intensity superior sagittal sinus III

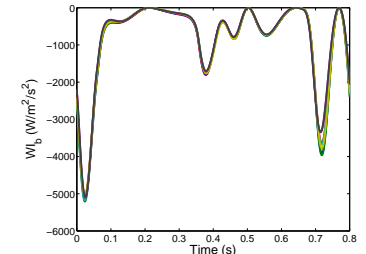

(d) Backward wave intensity in the septal vein

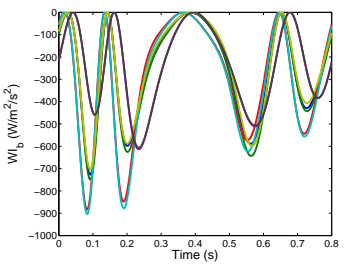

(h) Backward wave intensity superior sagittal sinus III

Figure 5.11: Comparison of NL-TP, NL-SP, L-TP, L-SP, L0-SP formulations for various vessels in the venous system 


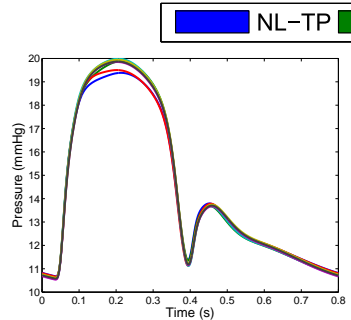

(a) Pressure in the main pulmonary artery

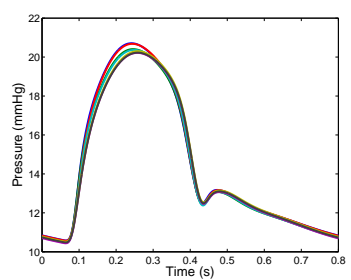

(e) Pressure in pulmonary artery (RSA0-2-54)

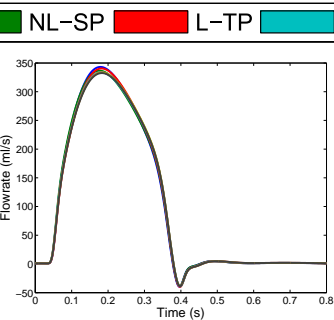

(b) Flow rate in the main pulmonary artery

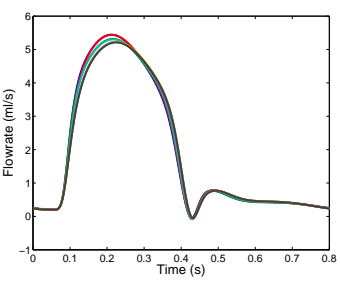

(f) Flow rate in pulmonary artery (RSA0-2-54)

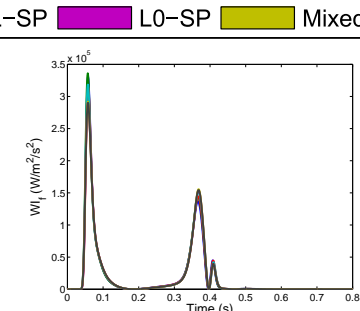

(c) Forward wave intensity in the main pulmonary artery

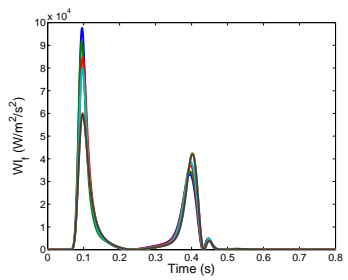

(g) Forward wave intensity in pulmonary artery (RSA0-2-54)

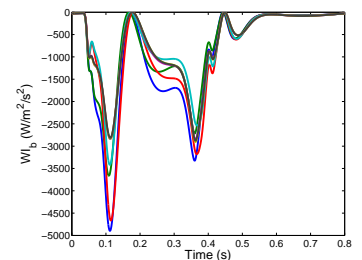

(d) Backward wave intensity in the main pulmonary artery

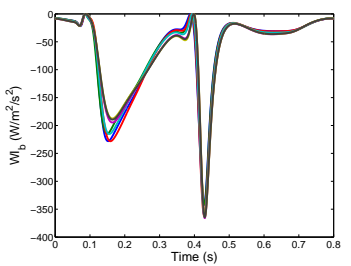

(h) Backward wave intensity in pulmonary artery (RSA0-2-54)

Figure 5.12: Comparison of NL-TP, NL-SP, L-TP, L-SP, L0-SP formulations for various vessels in the pulmonary system 


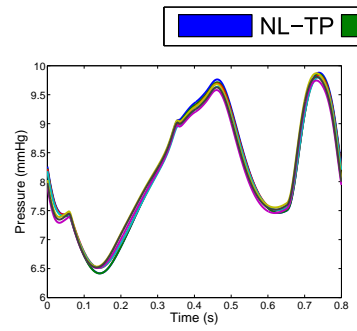

(a) Pressure in right superior pulmonary vein

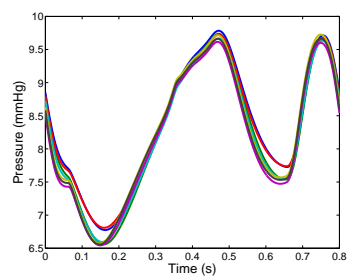

(e) Pressure in the pulmonary (RSV0-2-54)

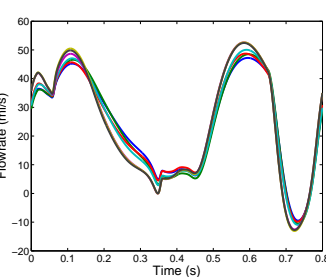

(b) Flow rate in right superior pulmonary vein

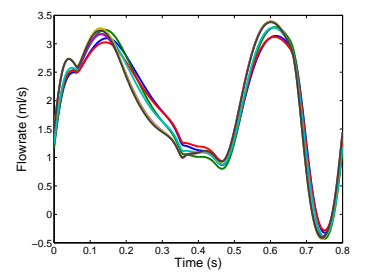

(f) Flow rate in the pulmonary vein (RSV0-2-54)

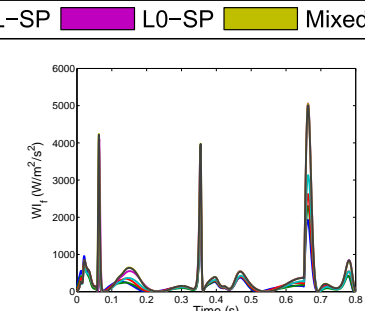

(c) Forward wave intensity in right superior pulmonary vein

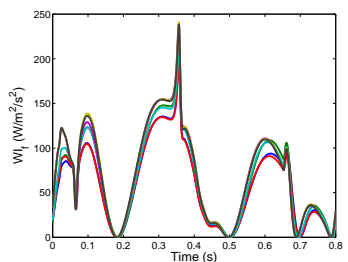

(g) Forward wave intensity in pulmonary vein (RSV0-2-54)

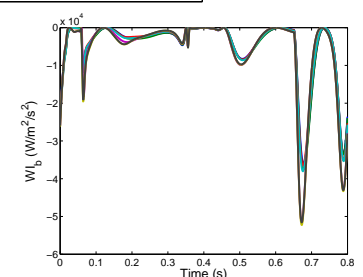

(d) Backward wave intensity in right superior pulmonary vein

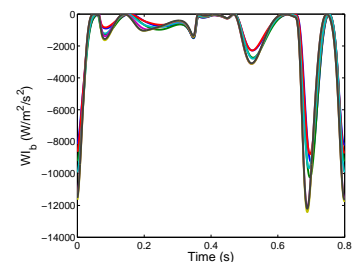

(h) Backward wave intensity in pulmonary vein (RSV0-2-54)

Figure 5.13: Comparison of NL-TP, NL-SP, L-TP, L-SP, L0-SP formulations for various vessels in the pulmonary system 


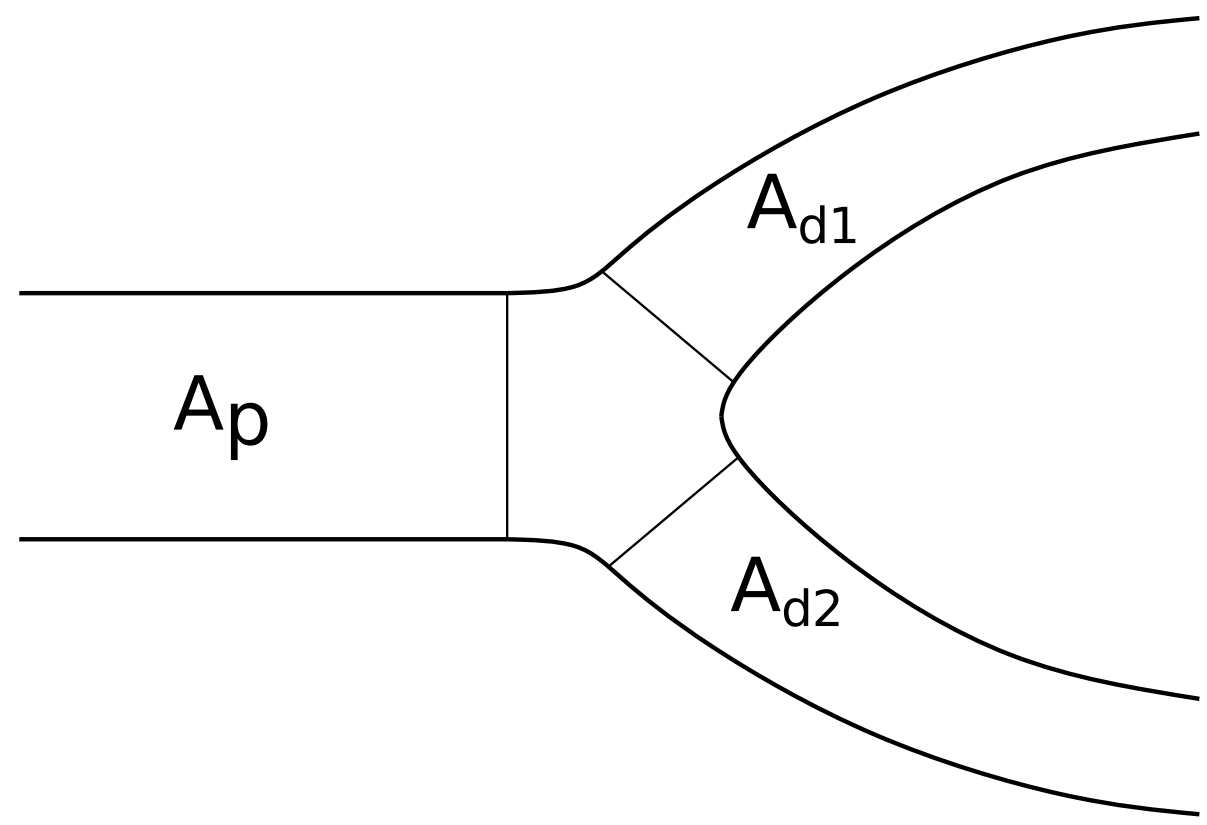

Figure 5.14: Configuration of the bifurcation case where $A_{P}$ is the area of the parent vessel, $A_{d 1}$ and $A_{d 2}$ are the areas of the first and second daughter vessels, respectively

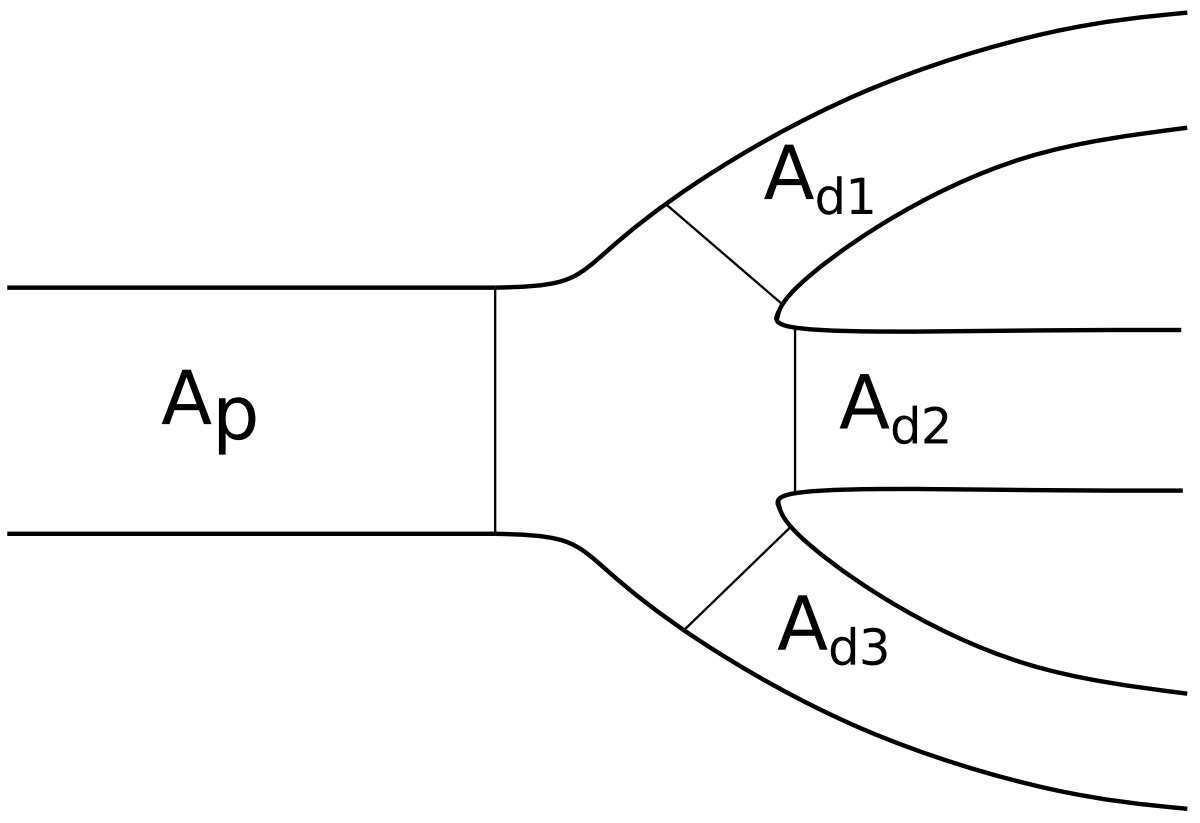

Figure 5.15: Configuration of the trifurcation case where $A_{P}$ is the area of the parent vessel, $A_{d 1}, A_{d 2}$, and $A_{d 3}$ are the areas of the first, second, and third daughter vessels, respectively 


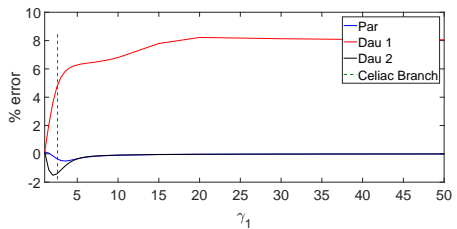

(a) $\%$ error of maximum flow rate in bifurcation case

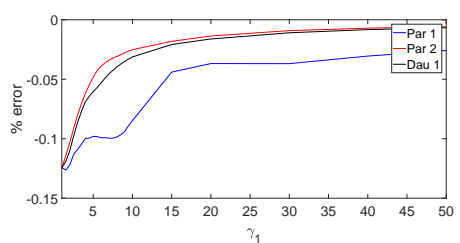

(d) $\%$ error of maximum flow rate in confluence case

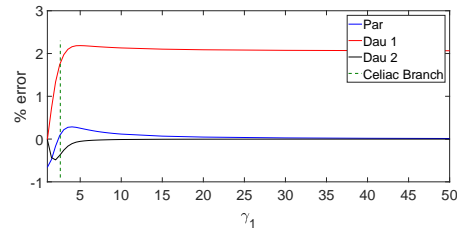

(b) $\%$ error of maximum pressure in bifurcation case

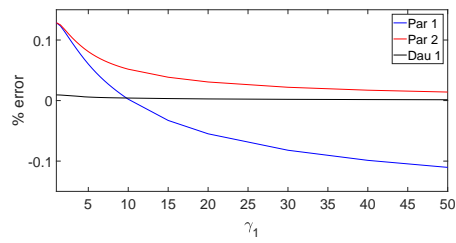

(e) $\%$ error of maximum pressure in confluence case

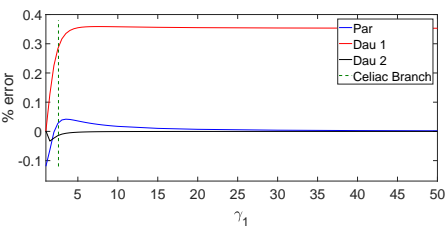

(c) $\%$ error of mean pressure in bifurcation case

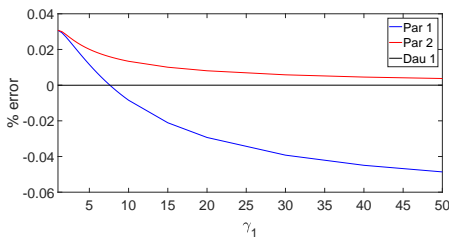

(f) $\%$ error of mean pressure in confluence case

Figure 5.16: Percentage error of static pressure compared with total pressure for bifurcation and confluence cases for parent (Par) and daughter (Dau) vessels 


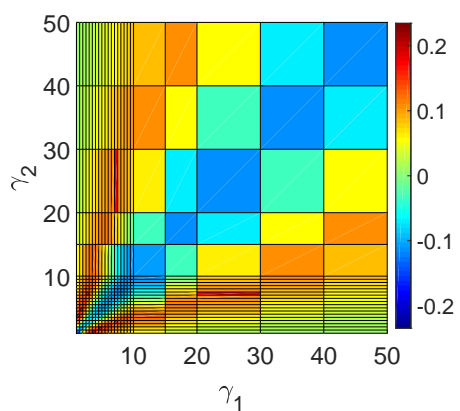

(a) $\%$ error of pressure for $\mathrm{Par}_{1}$ in trifurcation case

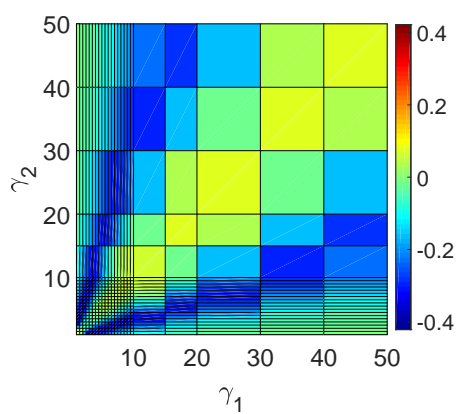

(d) $\%$ error of flow rate for $\mathrm{Par}_{1}$ in trifurcation case

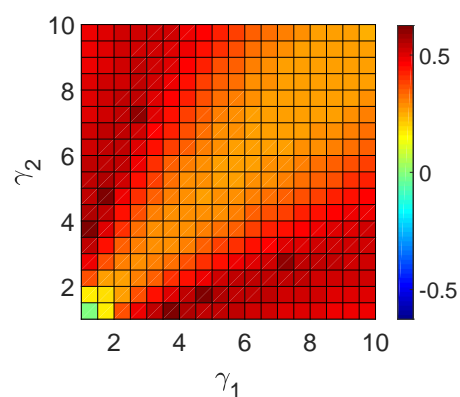

(b) \% error of pressure for $D a u_{1}$ in trifurcation case

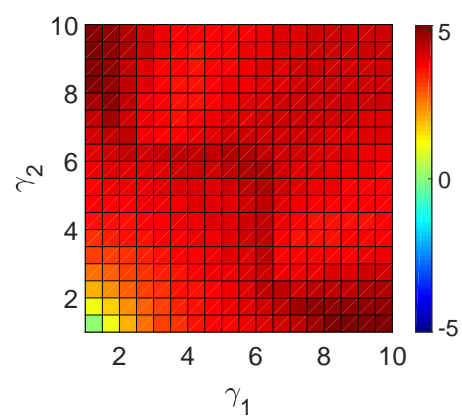

(e) $\%$ error of flow rate for $\mathrm{Dau}_{1}$ in trifurcation case

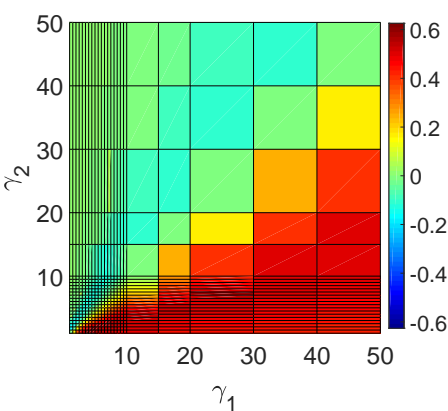

(c) \% error of pressure for $\mathrm{Dau}_{2}$ in trifurcation case

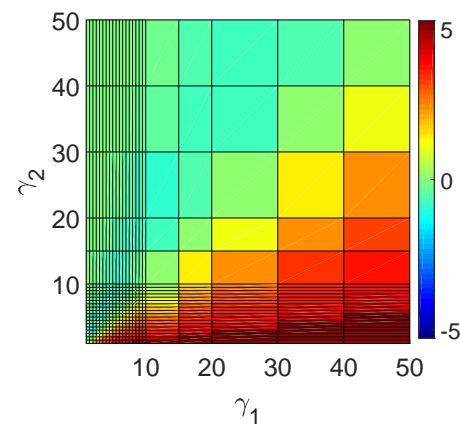

(f) $\%$ error of flow rate for $\mathrm{Dau}_{2}$ in trifurcation case
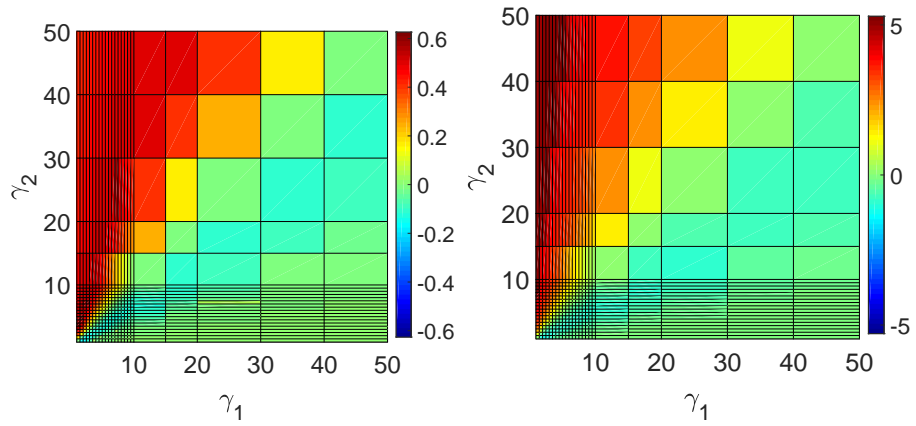

(g) $\%$ error of pressure for (h) $\%$ error of flow rate for $\mathrm{Dau}_{3}$ in trifurcation case $\mathrm{Dau}_{3}$ in trifurcation case

Figure 5.17: Percentage error of static pressure compared with total pressure for trifurcation case for parent (Par) and daughter (Dau) vessels 


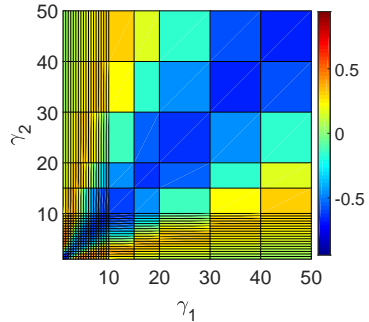

(a) $\%$ error of peak pressure for $\mathrm{Par}_{1}$ in trifurcation case

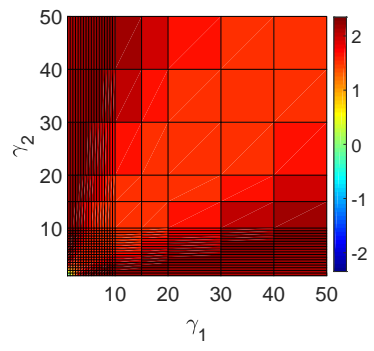

(d) \% error of peak pressure for $D a u_{1}$ in trifurcation case

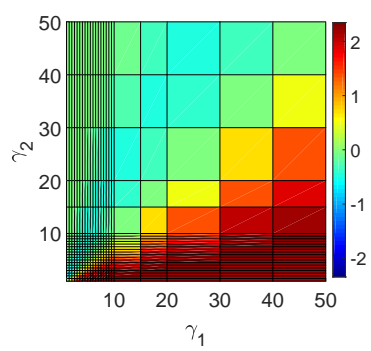

(g) \% error of peak pressure for $\mathrm{Dau}_{2}$ in trifurcation case

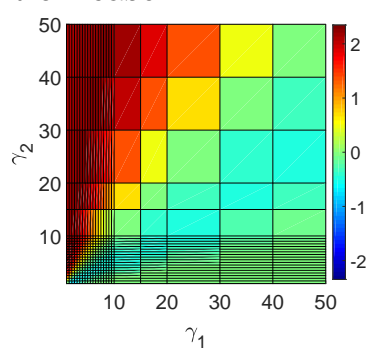

(j) \% error of peak pressure for $\mathrm{Dau}_{3}$ in trifurcation case

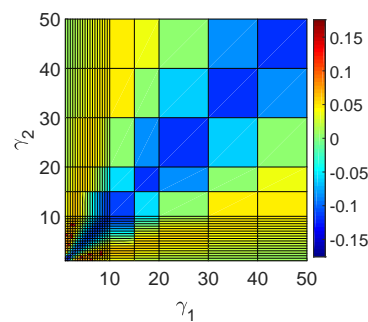

(b) \% error of mean pressure for $\mathrm{Par}_{1}$ in trifurcation case

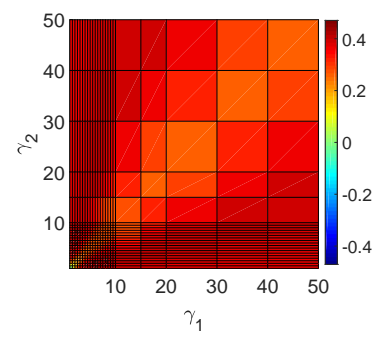

(e) \% error of mean pressure for $D a u_{1}$ in trifurcation case

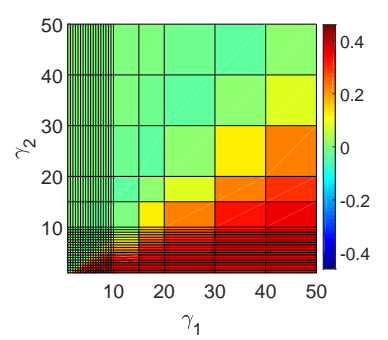

(h) $\%$ error of mean pressure for $\mathrm{Dau}_{2}$ in trifurcation case

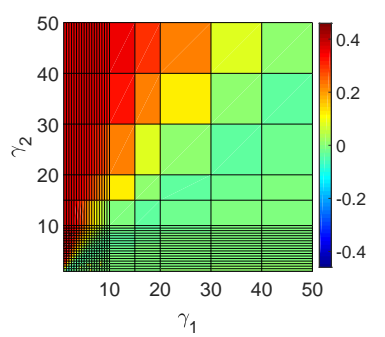

(k) \% error of mean pressure for $\mathrm{Dau}_{3}$ in trifurcation case

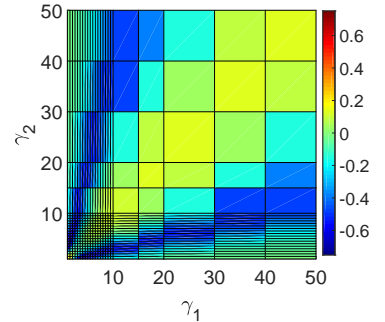

(c) $\%$ error of peak flow rate for $\operatorname{Par}_{1}$ in trifurcation case

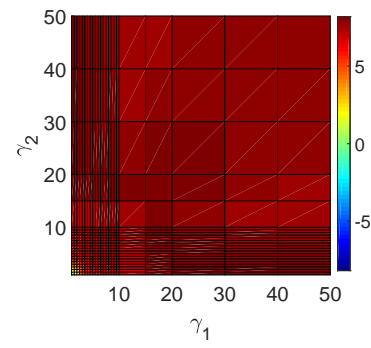

(f) $\%$ error of peak flow rate for $D a u_{1}$ in trifurcation case

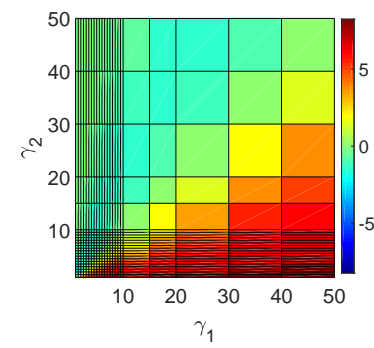

(i) $\%$ error of peak flow rate for $\mathrm{Dau}_{2}$ in trifurcation case

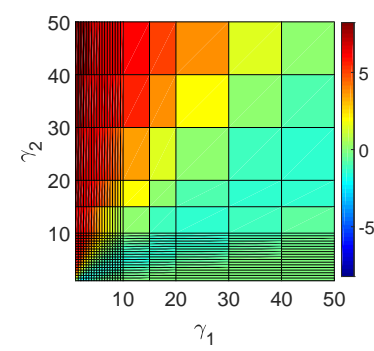

(l) $\%$ error of peak flow rate for $\mathrm{Dau}_{3}$ in trifurcation case

Figure 5.18: Percentage error of static pressure compared with total pressure for the trifurcation case for parent (Par) and daughter (Dau) vessels 


\section{Chapter 6}

\section{Parameter Estimation and Measure- ment Integration}

This chapter introduces the various methodologies used to estimate the parameters of the $1 \mathrm{D}$ and $\mathrm{OD}$ cardiovascular model components. These model components are used to construct the 1D-0D closed-loop cardiovascular model of pregnancy, which is described and implemented in chapter 7. The parameters used in the model are generally estimated by at least one of the following: 1) from literature, and are generally based on either in-vivo or in-vitro measurements; 2) from physiological measurements of height, weight, pressures, heart rate, and cardiac output, which can aid the estimation of model resistances, compliances, and vessel lengths.

Parameter estimation is perhaps the most difficult and most uncertain component of cardiovascular modelling. Many of these reasons relate to the difficulty in obtaining physiological in-vivo data or experimental in-vitro. This could be due to the expense required for performing medical imaging techniques such as CT and MRI, and also the expense of obtaining other equipment of a sufficient quality for accurate measurements. Furthermore, many of these techniques and equipment require sufficient expertise to reliably perform physiological measurements. The requirement to adhere to ethical laws also prevents the use of several potentially useful types of data. For example, during pregnancy an ultrasound device may be used almost indefinitely on the patient, however many more useful types of imaging data such as CT and MRI are restricted due to potential risks to foetal development [74], and are only used if the diagnostic usefulness clearly outweighs any risk. In addition, many blood vessels are inaccessible externally and thus would require invasive measurements, such as catheterisation which can be used for pressure measurements. However such procedures are unethical unless a patient already requires surgical intervention. Many of the measurement devices used in clinics have a potential error of the measured quantity by $\pm 5 \%$ to $\pm 10 \%$ [268], so there is significant uncertainty even with the physiological measurement.

Considering these difficulties, it is important to develop as accurate an estimation of the physiological parameters as possible for a given, and often limited set of 
physiological measurement data. These parameters can often be used either directly into any model, as is the case for flow rate measurements as an inflow boundary condition [41]; or be used to inform the model to aid in choosing parameters, such as the pulse pressure being used to aid estimation of arterial compliance [327, 326].

A particularly important and challenging aspect of implementing a model of such complexity while using physiological measurement data, is:

- the choice of the initial conditions, which includes initial pressure and flow estimations in the 1D and lumped model systems. This is important as a model with poor initial conditions will take significantly longer to converge to a periodic solution;

- parameter estimation within the model, which includes both initial parameter estimation, and iterative adaptation of parameters to improve their estimation. There exists several aspects to parameter estimation as not only does the total effect of parameters need to be accounted for, such as the total peripheral resistance and total compliances in each part of the circulation; but also the distribution of these resistances and compliances throughout the network. Typically, measurements such as pressure and estimations of cardiac output can be used to estimate total resistance and compliance parameters, but do not inform of the distribution of said parameters in the network. There also exists several additional components to consider in a closed-loop model, such as the total blood volume in the system being implicitly defined based on initial pressures and initial parameter values;

- the connectivities of the cardiovascular network, the geometry and material properties of blood vessels, should be different from person to person. Such data on a patient's cardiovascular network is unrealistic to obtain due to expense and ethics. Hence, the same connectivities in the network and the vessel geometry are treated equal among participants. The material properties are estimated from pulse pressure measurements.

Of particular interest in this thesis is the ability to estimate and adapt parameters in the numerical model which enables the simulated solutions of pressure and cardiac output / flow rates, to match measurements performed on participants. Currently no such technique has been published which achieves this for such a complicated 
closed-loop system. A few studies have presented methodologies to estimate resistance and compliance distributions within a small network of the systemic arteries [325, 327, 326, 9, 354, 11, 158], and a study investigated an iterative approach to adapt and improve estimation of these parameters during the simulation [372]. Although iterative schemes have generally only been performed on much smaller networks from the systemic arteries, such as the carotid artery and aortic bifurcation. These iterative schemes were employed on the vascular bed parameters only, with an assumption that the $1 \mathrm{D}$ vessel parameters such as compliance were unchanged for all simulations. This is physiologically unrealistic as there are significant differences between individuals for total arterial compliance, including the compliance of major vessels, and resistances. The vessel with the largest compliance is the aorta, which has a significant impact on the pulse pressure [201, 73, 91, 141, 151, 323]; as pulse pressure is primarily effected by stroke volume, aortic and downstream arterial compliance; although the rate for change of the flow rate also influences the pulse pressure.

This chapter will begin by presenting the model components and parameters which are chosen from literature, which includes details of the 1D vascular networks and all the components required for the closed-loop 1D-0D system. A description of the physiological measurement data will then be given, which leads to the methodologies used in initial and iterative parameter estimation.

\subsection{Model Parameters From Literature}

This section describes the modelling components that remain unchanged from literature. This choice is due to: (1) patient measurement information being insufficient to provide an accurate patient-specific estimation of each parameter, such as in the heart and valve models; or (2) that the modelling component is not of primary interest and is mainly there to complete the model, such as the coronary system being included to drain approximately $5 \%$ of cardiac output from the aorta; or (3) a combination of both such as in the pulmonary system, in which the mean pressures are scaled to achieve healthy pulmonary pressures, as no measurement data was available for the pulmonary system and the primary interest of the model is the systemic circulation. 


\subsubsection{Heart Model}

The heart model parameters used in this thesis are primarily the same as those defined in [226, 230], the description of the heart model and its implementation in this thesis was previously described in chapter 3.2.4, and a lumped parameter diagram of the heart model was shown in figure 2.9. Table 6.1 contains information of the heart parameters used in this thesis.

\begin{tabular}{|l||l|l|l|l|}
\hline & LV & LA & RV & RA \\
\hline$E_{\text {min }},\left(\mathrm{mmHg} \mathrm{ml}^{-1}\right)$ & 0.07 & 0.09 & 0.035 & 0.045 \\
\hline$E_{\text {max }},\left(\mathrm{mmHg} \mathrm{ml}^{-1}\right)$ & 2.8 & 0.13 & 0.45 & 0.09 \\
\hline$\tau_{1},(\mathrm{~s})$ & $0.26875 \mathrm{~T}$ & $0.0525 \mathrm{~T}$ & $0.26875 \mathrm{~T}$ & $0.0525 \mathrm{~T}$ \\
\hline$\tau_{2},(\mathrm{~s})$ & $0.5025 \mathrm{~T}$ & $0.1725 \mathrm{~T}$ & $0.5025 \mathrm{~T}$ & $0.1725 \mathrm{~T}$ \\
\hline$m_{1}$ & 1.32 & 1.99 & 1.32 & 1.99 \\
\hline$m_{2}$ & 21.9 & 11.2 & 21.9 & 11.2 \\
\hline$V_{0},(\mathrm{ml})$ & 10 & 3 & 40 & 7 \\
\hline$V_{t=0},(\mathrm{ml})$ & 136 & 71 & 172 & 67 \\
\hline$K_{s}, 10^{-3}(\mathrm{ml})$ & 0.5 & 0.25 & 1 & 0.5 \\
\hline$\kappa$ & 6 & 2 & 6 & 2 \\
\hline$\mu_{A V},\left(\mathrm{~g} \mathrm{~cm}^{-7} \mathrm{~s}^{-1}\right)$ & 0 & 0.033 & 0 & 0.05 \\
\hline$t_{\text {onset }},(\mathrm{s})$ & 0 & $0.8125 \mathrm{~T}$ & 0 & $0.8125 \mathrm{~T}$ \\
\hline
\end{tabular}

Table 6.1: Heart chamber parameters with units of measurement. Items without a unit of measurement are dimensionless. Abbreviations are as follows: left ventricle/atria -(LV/LA, right ventricle/atria - RV/RA.

\subsubsection{Valve Model}

The valve model and its implementation are described in chapter 3.2.4. The valve model is similar to [226], where venous valve parameters are from [226], while heart valve parameters are from [230]. Table 6.2 contains the parameter values of the heart valve and venous valve models.

\subsubsection{D Vascular Networks}

The majority of the vascular network and vascular bed configurations are the same as [230], however in this thesis additional vessels and vascular beds are included in order to model important physiological adaptations which occur during pregnancy. 


\begin{tabular}{|l||l|l|l|l|l|}
\hline & AV & MV & PV & TV & VV \\
\hline$A_{\text {eff,max }},\left(\mathrm{cm}^{2}\right)$ & 6.9 & 5.1 & 5.7 & 6.0 & $A_{\text {con }}$ \\
\hline$A_{\text {eff,min }},\left(\mathrm{cm}^{2}\right)$ & 0 & 0 & 0 & 0 & 0 \\
\hline$l_{\text {eff }},(\mathrm{cm})$ & 1.5 & 2 & 1.5 & 2 & 1 \\
\hline$K_{v o},\left(\mathrm{~cm}^{2} \mathrm{~s}^{2} \mathrm{~g}^{-1}\right)$ & 0.02 & 0.02 & 0.02 & 0.03 & 0.03 \\
\hline$K_{v c},\left(\mathrm{~cm}^{2} \mathrm{~s}^{2} \mathrm{~g}^{-1}\right)$ & 0.02 & 0.04 & 0.02 & 0.04 & 0.03 \\
\hline
\end{tabular}

Table 6.2: Valve model parameters with units of measurement. Abbreviations are: AV - aortic valve, MV - mitral valve, PV - pulmonary valve, TV - tricuspid valve, $\mathrm{VV}$ - venous valve. The effective maximum area of the venous valve is set to the current area of the connecting vessel $A_{\text {con }}$.

\section{Pulmonary System}

The 1D pulmonary system is identical to that in [230], which used fractal relations from [275] to create the network. Vascular bed compliances and the distribution of pulmonary vascular resistance are also held the same as in [230]. However, the total pulmonary vascular resistance is scaled to achieve physiologically healthy pulmonary pressures. The pulmonary system network is shown in figure 6.1.

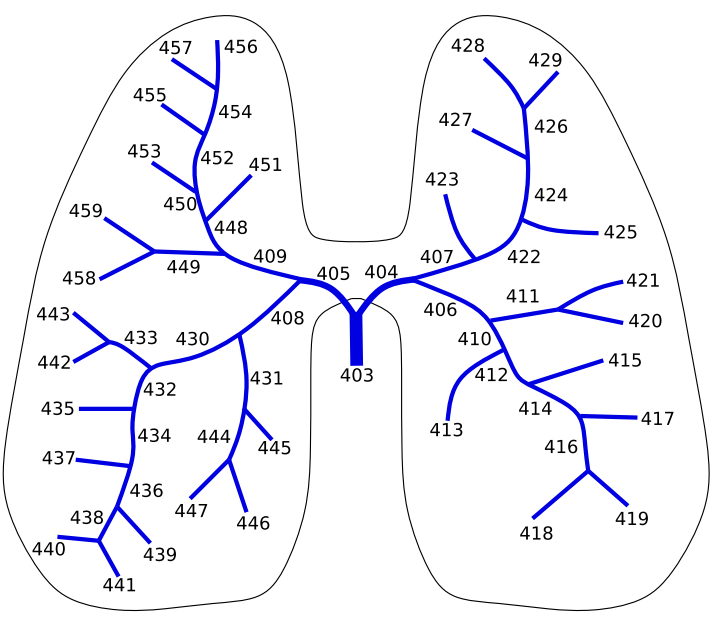

(a) 1D Pulmonary Arteries

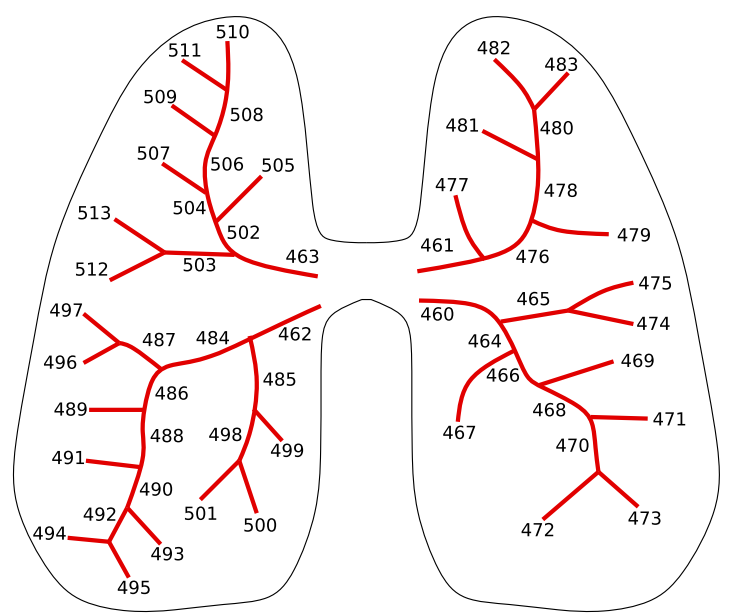

(b) 1D Pulmonary Veins

Figure 6.1: 1D pulmonary artery and venous vascular networks.

\section{Systemic System}

The majority of the 1D network parameters are identical to the model proposed in [230], with the exception of the added vessels for pregnancy modelling, and vessel 
lengths. The vessel lengths are scaled using the height of the patient in the following way

$$
L_{\text {vessels }}=\gamma L_{\text {vessel,base },} \quad \gamma=\frac{\text { Height }}{\text { Base Height }},
$$

where $L_{\text {vessels }}$ is the new length of each vessel, $L_{\text {vessel,base }}$ is the base length as defined in [230], $\gamma$ is a scaling term defined by the fraction of patient height with the models base height of $6 \mathrm{ft} 1 \mathrm{in}$. There exists more complicated relationships such as allometric scaling laws [302, 95], which vary both vessel lengths and diameters. However, in this thesis the simple scaling relationship is used, as there are already significant uncertainties in the vascular network, and thus the choice of scaling law is not likely to have a large impact on the solution, particularly as vessel compliances are adapted based on the patient pressure measurements.

The main systemic vessels of the model, excluding the cerebral arteries and portal system circulation, are shown in figure 6.2, the cerebral vessels are shown in figure 6.3 , and the hepatic portal system circulation is shown in figure 6.4c.

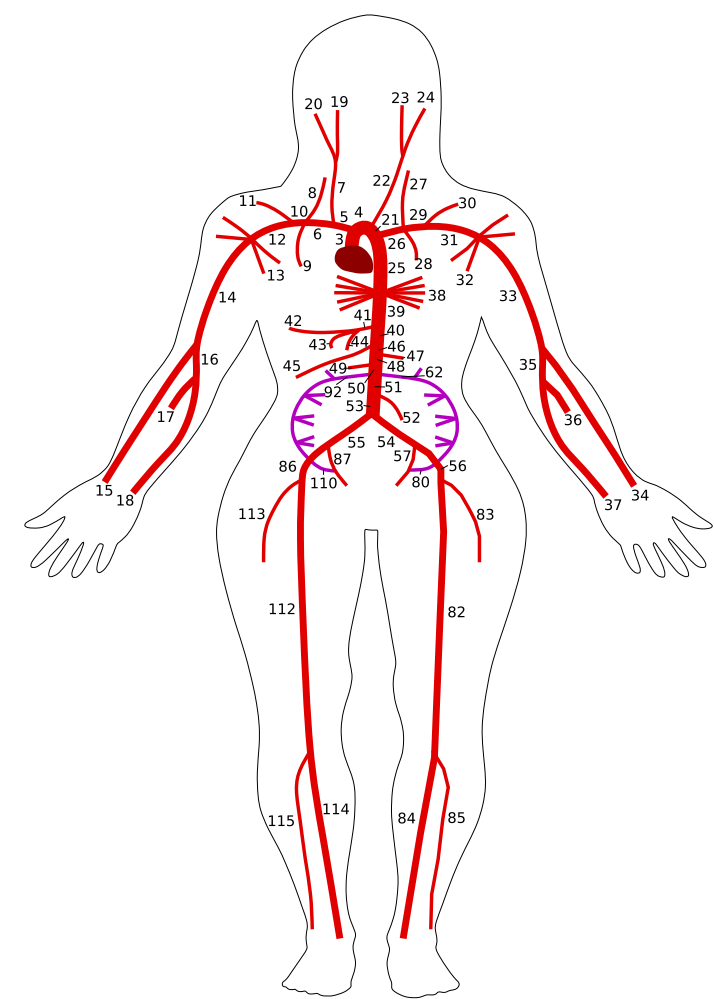

(a) Major arteries in 1D systemic network

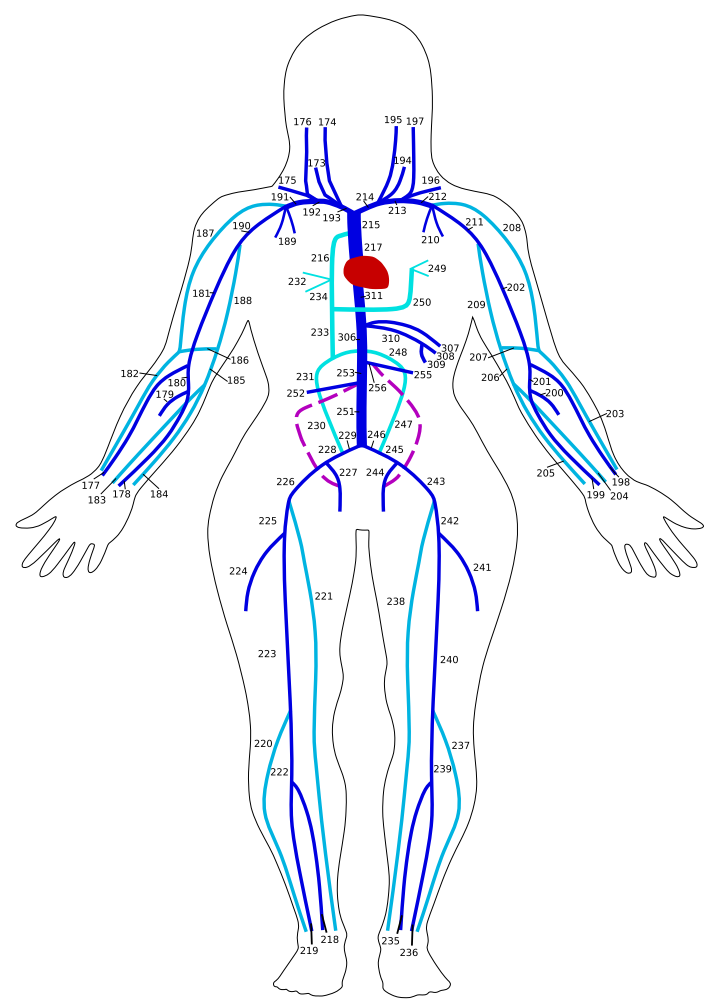

(b) Major veins in 1D systemic network

Figure 6.2: 1D systemic system network 


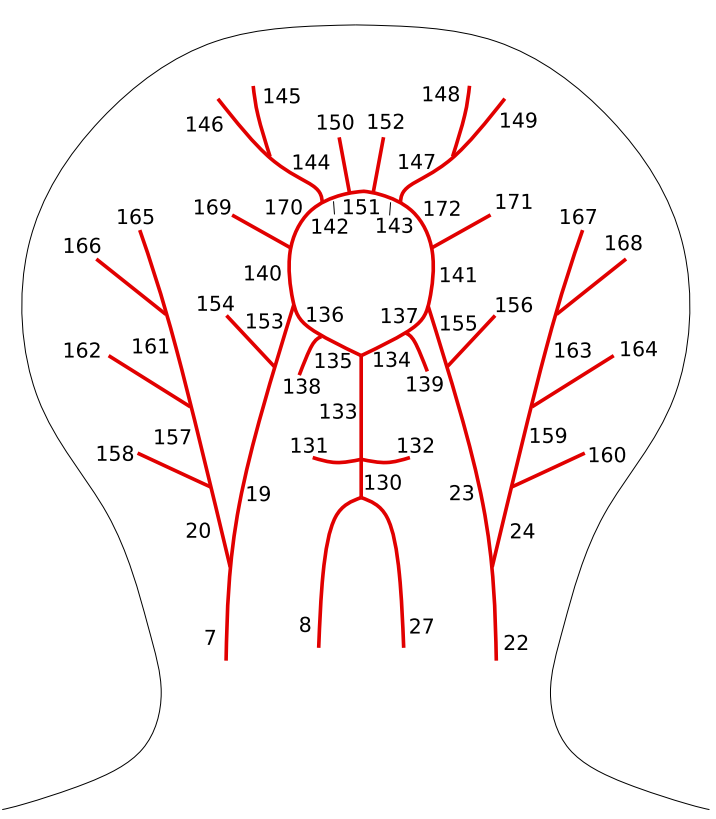

(a) $1 \mathrm{D}$ cerebral arteries

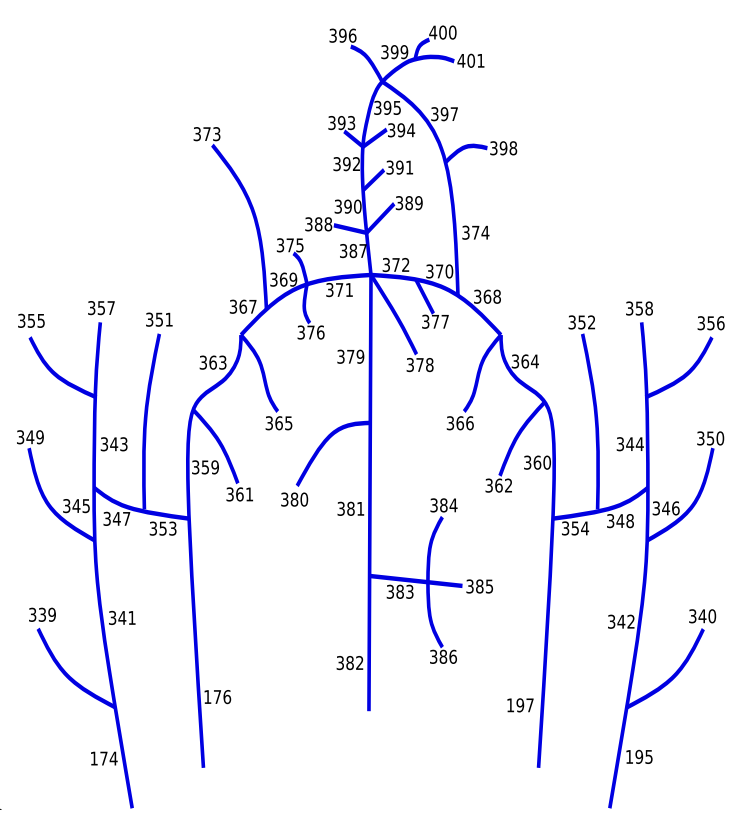

(b) $1 \mathrm{D}$ cerebral veins

Figure 6.3: 1D cerebral artery and venous vascular networks.

\section{Coronary Network and Coronary Vascular Bed Model}

The 1D coronary network and lumped coronary vascular bed parameters are the same as described in [230], but are briefly described here for completeness. The coronary model was previous described in chapter 3.2.4 in this thesis, the vascular bed configuration can be seen in figure 3.3 and the coronary arterial and venous network can be seen in figure 6.4.

The coronary network contains the main arteries and veins in the coronary system. The coronary vascular bed is split into four main compartments: the epicardium, subepicardium, midwall, and subendocardium. In addition the coronary vascular bed experiences external pressure from the left and/or right ventricle, with vascular resistances are non-linear and dependent on the blood volume in each compliance compartment $C_{1}, C_{2}$. The distribution of resistances, compliances, and myocardial volumes, is identical to that presented by Mynard and Smolich [230]. 


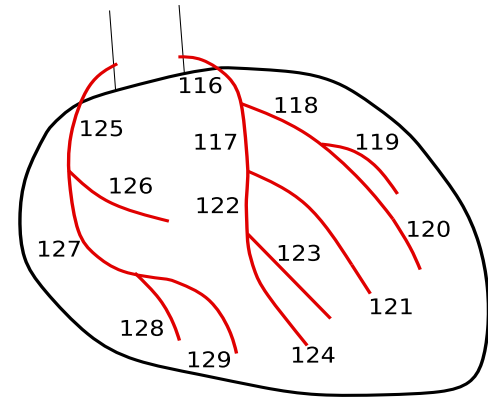

(a) 1D coronary arteries

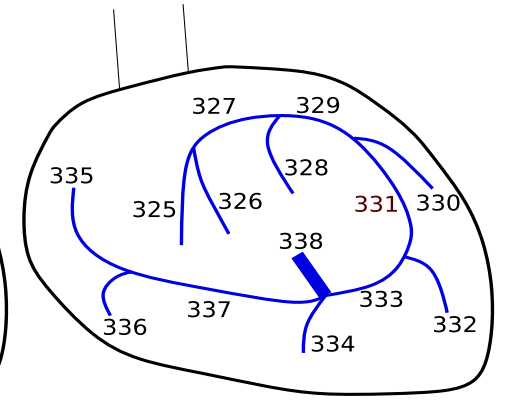

(b) $1 \mathrm{D}$ coronary veins

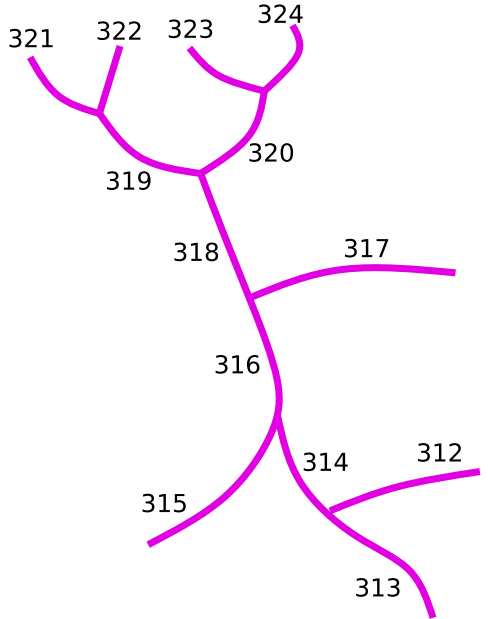

(c) 1D hepatic portal network

Figure 6.4: 1D coronary artery and venous vascular networks, and hepatic portal system.

\subsubsection{Utero-Ovarian Circulation}

The anatomical configuration used in this thesis is the most common. However, several variations of the vessel network in the utero-ovarian system exist [13]. Table 6.3 shows the vessel lumen diameters of arteries and veins in the utero-ovarian circulation, comparing those which are given in literature, and the corresponding diameters used in the model, with both non-pregnant and pregnant values (late pregnancy) given. There are several vessels for which there exists no information on their diameter in literature, and for the majority of values, the lengths are not given, and hence are chosen from anatomical considerations (for lengths), or by assuming the veins are 1.25 times larger in diameter than their arterial counterparts. The utero-ovarian circulation network used in this thesis is shown in figure 6.5, along with vessel numbering which is given in table 6.4 and table 6.5. There are several potential pathways for blood to reach the uterus from the aorta. The circulation is generally symmetric, with left and right pathways containing the same vessels and connectivities. The main pathway is via the uterine arteries, which branches from the anterior branch of the internal iliac artery, before travelling towards the uterus. The uterine artery typically splits into two branches: a descending branch, which connects to the vaginal artery, supplying the vagina and cervix with blood; and the 


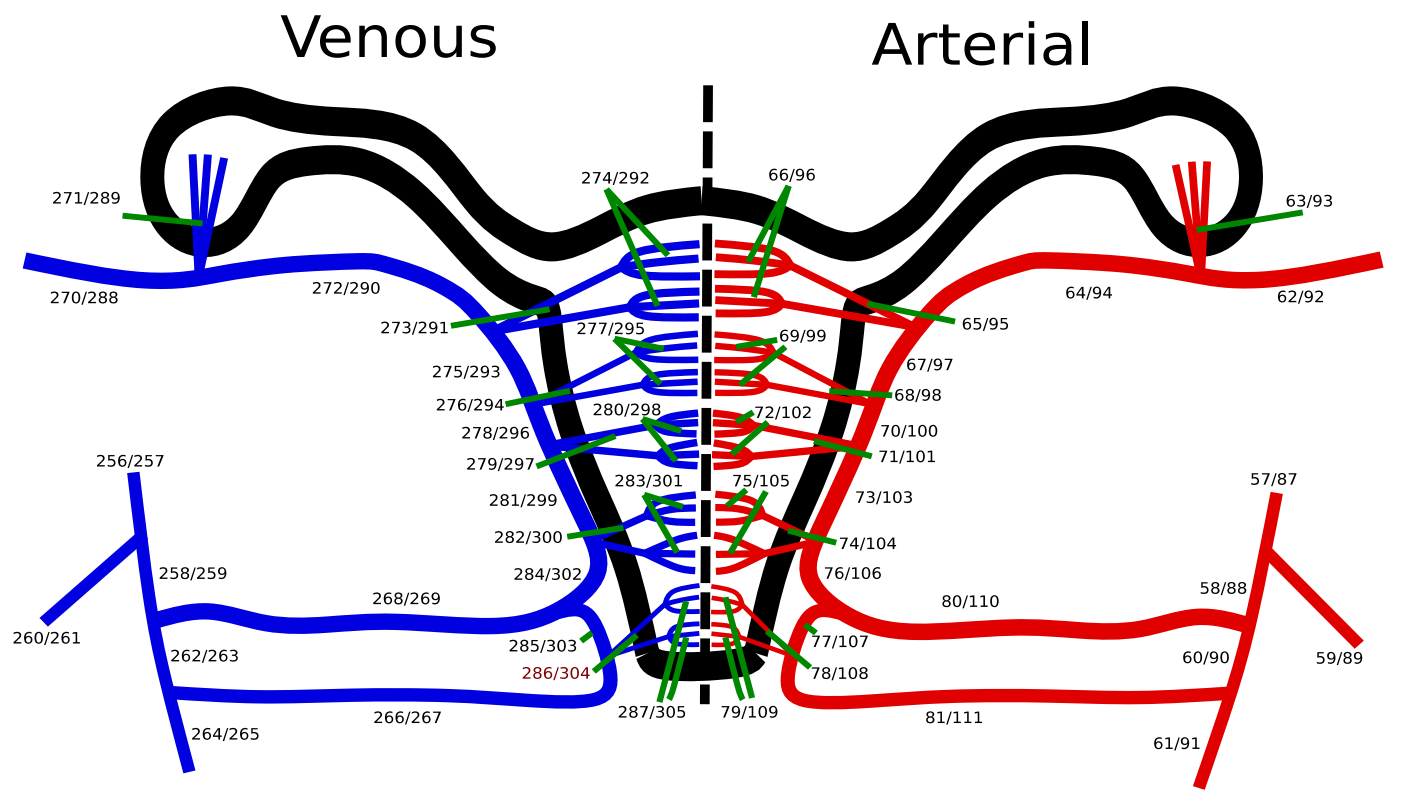

Figure 6.5: Utero-Ovarian System

ascending uterine artery, which travels upwards along the wall of the uterus. Several arcuate arteries branch from the ascending uterine arteries, often anastomosing, creating a connection of the left and right sided blood vessels of the uterus. The radial arteries branch from the arcuate arteries, penetrating the myometrium, containing branches of basal arteries, and finally becoming spiral arteries. The spiral arteries bring blood to the inner most layer of the uterus, and during pregnancy, approximately 200 of these vessels [52] open into the intervillous space of the placenta. The second most common pathway for blood to reach the uterus is via the utero-ovarian communicating arteries, which bifurcate from the ovarian artery and often anastomose with ascending uterine artery near the fundus of the uterus. There exist many smaller pathways, which vary significantly between individuals and hence are not considered in this thesis.

\subsection{Measured Data}

The data utilised in this thesis was collected by Mike Lewis' research group [63, $59,62,60,61,100]$. The equipment used for collecting measurements was the Task Force $^{\circledR}$ monitor by CNSystems (Graz, Austria). There were a total of 58 participants 
in the cohort at the first trimester. However, this decreased to 36 participants in the second trimester, 29 participants in the third trimester, and finally, 24 participants in the post-partum group. An overview of these participants can be found in Table 6.6. The device has the ability to perform:

- impedance cardiography (ICG).

- two-channel electrocardiography (ECG).

- heart rate monitoring (HR)

- non-invasive continuous beat-to-beat blood pressure monitoring via the index and middle fingers.

- oscillometric blood pressure which also acts as a corrector for the continuous blood pressure monitor;

This allows a wide range of measurements and parameters can be extracted. Allowing the following beat-to-beat variables to be measured or estimated:

- the systolic, diastolic, and mean blood pressure in the brachial artery.

- tracking of heart rate and measurements of stroke volume enables the calculation of cardiac output.

- the total peripheral resistance or systemic vascular resistance (TPR) can be estimated from the mean arterial pressure and cardiac output.

- an estimation of the left ventricular ejection time (LVET).

- heart rate and blood pressure variability can be investigated by a power spectral analysis, which is a frequency domain analysis of RR-interval, and the beat-to-beat systolic and/or diastolic pressure signal respectively.

- baroreceptor reflex sensitivity can be investigated by a combination of measured variables such as heart rate and blood pressures.

In addition, participant height and weight were recorded, and body surface area (BSA) was estimated, allowing the calculation of various indices which related cardiovascular parameters with BSA (such as cardiac index and stroke volume index). 
The participants were measured for approximately five minutes in each of the following body positions and conditions:

- Approximately supine, where the patients' torso was approximately at a 45 degree angle to avoid compression of inferior vena cava due to the gravid uterus.

- standing (and hence baro-receptor reflex sensitivity, as measurements from supine to standing were continuous).

- light exercise to increase cardiac output.

- seated recovery.

- mental tasks (mathematical tasks to increase stress levels).

- metronomic breathing (breathing with a constant frequency for the respiratory cycle).

- spontaneous breathing (natural breathing).

Although in this thesis only the data in the supine position has been utilised as gravitational effects and baro-receptor modelling is not the focus of this thesis.

\subsection{Initial Parameter Estimation}

The choice of initial conditions will inevitably effect the convergence behaviour of the iterative parameter estimation scheme (which is presented in section 6.4). The parameter estimation uses the collected data, as discussed in section 6.2. The initial conditions of the model assumes flow rates to be zero everywhere which is typical of a closed-loop cardiovascular model [220, 230]; while initial pressures are treated in the following way:

- The initial pressure in the systemic arterial system is set to the aimed diastolic pressure (diastolic pressure given by measured data).

- Initial pressure in the systemic venous system is set to equal the systemic vein reference pressure $P_{d}(5 \mathrm{mmHg})$. 
- Initial pressure in the hepatic portal system is set to equal the hepatic portal vein reference pressure $P_{d}(8.5 \mathrm{mmHg})$.

- Initial pressure in the pulmonary arterial system is set to equal the pulmonary artery reference pressure $P_{d}(11 \mathrm{mmHg})$.

- Initial pressure in the pulmonary venous system is set to equal the pulmonary vein reference pressure $P_{d}(10 \mathrm{mmHg})$.

- The initial pressure for each heart chamber is defined by $P_{c h}=E_{c h}\left(V_{c h}-V_{c h, 0}\right)$, where the heart model parameters used in the simulations are defined in table 6.1. This often defines a discontinuity in hydrostatic pressure between atria and venous systems, although this assumption has no knock-on effects on the final solution.

- Initially, the venous valves are assumed open, and hence the pressure in the venous valve element is assumed equal in magnitude to its neighbouring $1 \mathrm{D}$ vessel elements.

- Initial pressures in the vascular beds are calculated by ignoring compliance elements. This allows the initial vascular bed pressures to be solved as a steady state system. The pressure at each node can then be easily calculated from the initial pressures in the connecting $1 \mathrm{D}$ vessels.

Furthermore, the reference pressures (i.e. the pressure at which the vessel area is defined) throughout the network is shown in table 6.7

\subsubsection{Total Peripheral Resistance}

The estimation of total peripheral resistance (TPR) is well studied [9, 354, 11, 158]. In this thesis the initial estimation of total peripheral resistance uses the following commonly used formula

$$
T P R \approx \frac{M A P-M C V P}{C O}
$$

where $M A P$ is the mean arterial pressure (from measurements), $M C V P$ is the mean central venous pressure (assumed $5 \mathrm{mmHg}$ ), and $C O$ is the cardiac output (from measurements). The distribution of this resistance is performed from a combination 
of two methodologies. The first involves the use of expected percentage of cardiac output to each region/organ of the body, based on normal distributions found in humans [184].

The second methodology is a variant of Murray's law of bifurcations [223], which involves assuming the flow distribution to a region is dependant on vessel geometry, thus requiring the sum of the radii cubed of parent vessels must equal the sum of the radii cubed of child vessels.

$$
\sum_{i=1: N P} r_{i}^{3}=\sum_{j=1: N D} r_{j}^{3}, \quad \mathrm{NP} \text { - number of parents, ND - number of children. }
$$

Once this distribution is determined the vascular bed parameters can be found by acknowledging the total resistance in the vascular region is determined from the summation of characteristic impedances from both arteries and veins, and a combined arteriole, capillary, venule resistance. The characteristic impedances at the reference wave speed are determined from the geometry and material properties in the $1 \mathrm{D}$ system, thus can be readily calculated.

The initial pressure across the vascular bed models can be found solving the pressure drop from arteries to veins from the initial pressures in the 1D system.

\subsubsection{Estimation of Total Systemic Compliance}

The majority of studies investigating compliance tend to focus on total arterial compliance [325, 327, 326, 73], rather than the total compliance in the systemic system or pulmonary system. In addition the distribution of compliance has not been fully investigated, although the compliance of large vessels against small vessels has been investigated [33]. This serves to show the difference in truly elastic arteries such as the aorta and the more muscular arteries, which have a greater control on blood flow distribution. There have been several studies investigating the compliance in the systemic venous system, linking the compliance to various pathologies such as hypertension $[246,375,363,134]$.

The initial estimation of the total arterial compliance (TAC) is performed via a defined $R C$ constant, which relates to the drop off rate of pressure due to the interaction of the total peripheral resistance $T P R$ and total arterial compliance $C_{T}$. 
The drop of constant is given by

$$
\tau=T P R \times C_{T}
$$

where the time constant is chosen to be $\tau=1.3$ [11], although in literature the time constant has varied significantly from $\tau=0.81$ [325] to $\tau=2.72$ [5]. The total compliance is the sum of $1 \mathrm{D}$ and $0 \mathrm{D}$ models, as the initial compliance of the 1D vessels can be determined via the constitutive law, an estimate of compliance in the vascular bed can be determined from

$$
C_{0 D}=C_{T}-C_{1 D}
$$

While the distribution of compliance is determined using the inverse distribution of peripheral resistance. The total venous compliance is estimated to be thirty times the compliance of the arteries [134], the 1D venous compliance is subtracted from the total venous compliance, then the remaining compliance is distributed to the vascular beds using the same distribution as the arterial side.

\subsection{Iterative Parameter Estimation}

A complication that arises in a closed-loop cardiovascular model is that of judging the initial conditions of the model. Typically flow rates are assumed zero everywhere at the beginning of the simulation. As the vessels within the network change in length based on patient height, it is difficult to predict how the initial blood volume in the system relates to the final periodic solution, making the cardiac output challenging to estimate. This also means the initial resistance/compliance estimations that are calculated using the cardiac output from patient measurements will initially be incorrect. An accurate iterative parameter estimation technique is critical to achieve convergence of the models haemodynamic parameters to the measured participant data. This section presents an automated parameter adaptation technique that: improves the estimation of resistances and compliances within the systemic system to converge the model solutions of diastolic, systolic, and mean arterial pressures to the measured data; and also adds or removes blood volume from the cardiovascular model to achieve the aimed cardiac output. It should be noted 


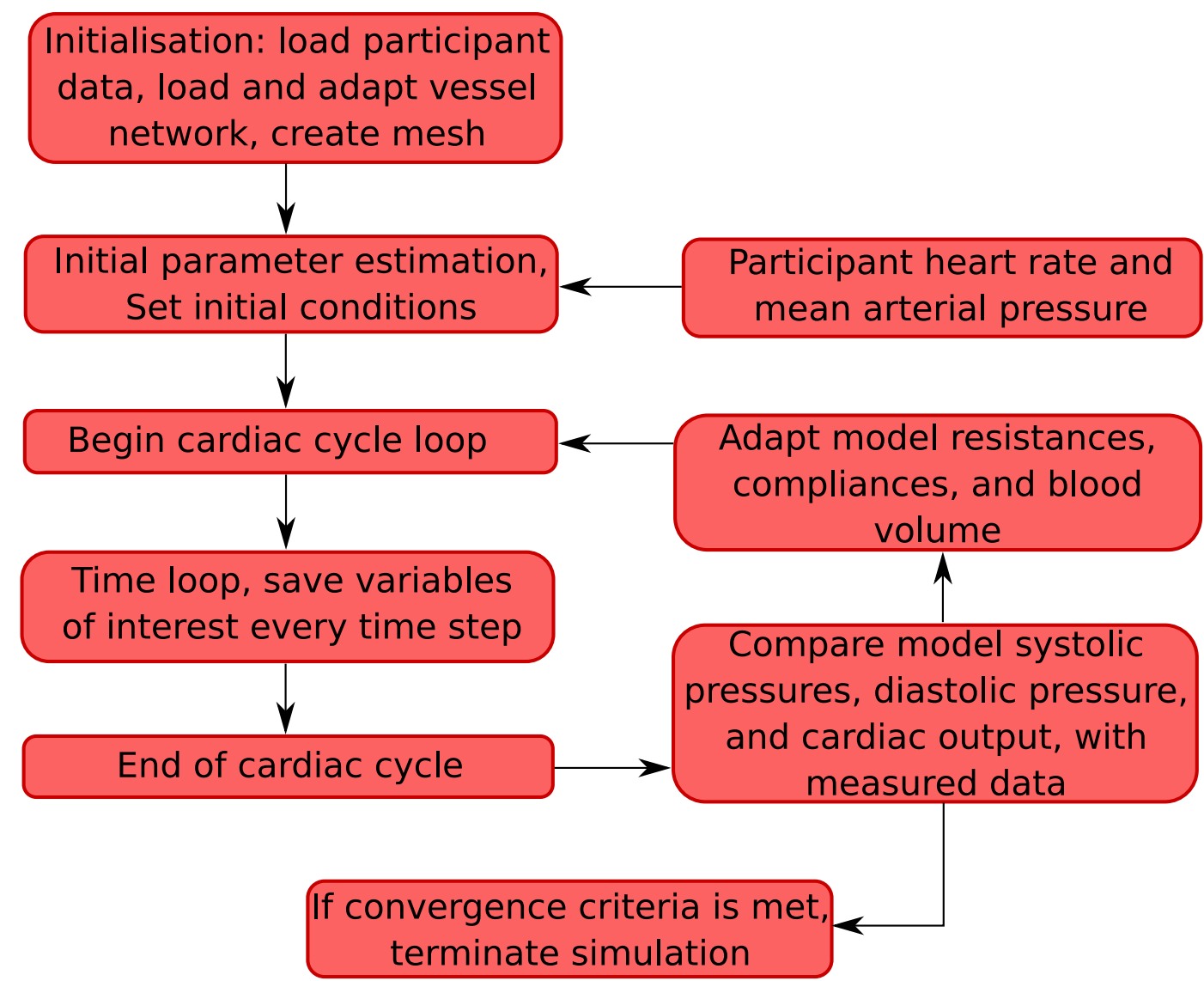

Figure 6.6: Flowchart of the implementation of the initial and iterative parameter estimation techniques

that the model simulation is performed for 8 cardiac cycles before any iterative adaptation occurs, which means the solution is close to periodic with approximately a $2-5 \%$ change in flow and pressure waveforms from the previous cardiac cycle. An overview of the initial and iterative parameter estimation techniques, and their location within the computational algorithm, is shown in Figure 6.6.

\subsubsection{Blood Volume}

Due to the impossible task of accurately estimating the models cardiac output from the initial conditions, it is necessary to add/remove volume into the system. In order to implement this novel technique, the first choice is to decide where the blood will be added or removed from the system. A natural choice to do this is via the venule compliance element in the vascular beds as the 0D compliance element is derived 
from the continuity equation. The $1 \mathrm{D}$ variant of the continuity equation with a source/sink term is given by

$$
C_{1 d} \frac{\partial P_{t m}}{\partial t}+\frac{\partial Q}{\partial x}=\Phi
$$

where $C_{1 d}$ is the vessel compliance and $\Phi$ is the source/sink term. Thus the equations reduction to $0 \mathrm{D}$ becomes

$$
C_{0 d} \frac{\partial P_{t m}}{\partial t}=Q_{\text {in }}-Q_{o u t}+\bar{\Phi}
$$

where $Q_{\text {in }}-Q_{\text {out }}$ is the net change in volumetric flow rate of the compliance element, and $\bar{\Phi}$ is the volumetric flow rate which is added to or taken from to the system.

Only a small amount of blood volume is added to the system at every time step in order to converge to the measured cardiac output in a more controlled manner, which equates to a maximum of a $5 \%$ change in blood volume over a cardiac cycle.

\subsubsection{Total Peripheral Resistance}

The total peripheral resistance is adapted to achieve the measured diastolic pressure in the left brachial artery to match the measurements, although in practice any artery in the system could be used. The iterative update can be performed in a similar fashion to [372]. The iterative update of TPR for the next cardiac cycle $(i+1)$, can be determined from the linearisation of a modified version of the total peripheral resistance equation (6.2) by assuming venous pressure is $0 \mathrm{mmHg}$

$$
T P R^{i+1}=T P R^{i}+\frac{\partial T P R^{i}}{\partial M A P} \delta M A P+\frac{\partial T P R^{i}}{\partial C O} \delta C O
$$

where $\delta M A P$ and $\delta C O$ are the differences of the models mean arterial pressure and cardiac output (defined at the $i^{\text {th }}$ cycle), with the aimed measured data (defined as superscript $m$ ), respectively; and are defined as

$$
\delta M A P=M A P^{m}-M A P^{i}, \quad \text { and } \quad \delta C O=C O^{m}-C O^{i} .
$$


Where the derivatives can be calculated as

$$
\frac{\partial T P R^{i}}{\partial M A P}=\frac{1}{C O^{i}}, \quad \frac{\partial T P R^{i}}{\partial C O}=-\frac{M A P^{i}}{C O^{2, i}}
$$

However, the difference in the current cardiac output to the aimed cardiac output can be quite significant depending on the patient's data. This can cause issues as blood is added or removed from the system slowly and the last term in the righthand side of equation (6.8) can significantly slow down convergence of the total peripheral resistance, as the cardiac output estimation is itself slow to converge. Other linearisations of this term can be performed, such as lagging the cardiac output term behind by setting $\delta C O=C O^{i}-C O^{i-1}$; however this has a relatively small impact on the overall convergence as cardiac output is slow to converge, hence in this thesis the last term in the right-hand side of equation (6.8) is neglected. Once the total peripheral resistance is updated the resistance distribution is kept the same, which essentially means all resistances are scaled by the same amount, which can be written as

$$
\alpha_{R}=T P R^{i+1} \cdot / T P R^{i}
$$

where all resistances in the vascular beds are scaled as $R_{v b}^{i+1}=\alpha_{R} R_{v b}^{i}$, and then the contributions of characteristic impedance (which are updated via the compliance update) are subtracted from this resistance to find the capillary resistance.

\subsubsection{Systemic Arterial Compliance}

The total compliance in the arterial system can be calculated by the summation of $1 \mathrm{D}$ and $0 \mathrm{D}$ compliances. The $0 \mathrm{D}$ compliances are only estimated during the initialisation, and are set during the simulation. However, the 1D compliances are adapted to converge to the pulse pressure from the measurements. This is done by integration of the continuity equation at a reference point(s) in the arterial system over time, between end diastolic pressure and maximum systolic pressure (pulse pressure) in a similar way to [372]. This can be written as

$$
\int_{t_{P, d i a}}^{t_{P, s y s}} C \frac{\partial P}{\partial t}+Q=0
$$


which when assuming $C=C_{d}$, where $C_{d}$ is the compliance at diastolic pressure, can be integrated to

$$
C_{d}\left(P_{s y s}-P_{d i a}\right)+\left(\left.Q\right|_{t=P, s y s}-\left.Q\right|_{t=P, d i a}\right) \Delta t_{p p}=0,
$$

where $\Delta t_{p p}$ is the difference in time between the diastolic $P_{d i a}$ and systolic $P_{s y s}$ pressure, while $\left.Q\right|_{t=P, s y s}$ and $\left.Q\right|_{t=P, d i a}$ is the flow rate at the time of $P_{s y s}$ and $P_{\text {dia }}$, respectively. In order to update the compliance equation (6.13) needs to be linearised, this is performed by first rearranging to solve for compliance in terms of flow and pulse pressure where $P P=P_{\text {sys }}-P_{\text {dia }}$, which gives

$$
C_{d}=\frac{\left(\left.Q\right|_{t=P, s y s}-\left.Q\right|_{t=P, \text { dia }}\right) \Delta t_{p p}}{P P},
$$

and after linearising can be written as

$$
C_{d}^{i+1}=C_{d}^{i}+\frac{\partial C_{d}^{i}}{\partial P P} \delta P P+\frac{\partial C_{d}^{i}}{\partial\left(\left.Q\right|_{t=P, s y s}-\left.Q\right|_{t=P, \text { dia }}\right)} \delta\left(\left.Q\right|_{t=P, \text { sys }}-\left.Q\right|_{t=P, \text { dia }}\right) .
$$

The aimed value and future values of $\left(\left.Q\right|_{t=P, s y s}-\left.Q\right|_{t=P, d i a}\right)$ are not known, however it is reasonable to assume that the difference from previous cardiac cycle is small enough to be neglected (or at least much smaller than the pulse pressure), hence $\delta\left(\left.Q\right|_{t=P, s y s}-\left.Q\right|_{t=P, d i a}\right) \approx 0$.

The compliance change is performed in the $1 \mathrm{D}$ domain via a change in the reference wave speed $c_{0}$. Without knowledge of how the compliance should be distributed, it is assumed to scale by the same amount everywhere in the systemic arteries. Hence the reference wave speed squared $\left(c_{0}^{2}\right)$ is updated by

$$
c_{0}^{2, i+1}=c_{0}^{2, i} \frac{C_{d}^{i}}{C_{d}^{i+1}} .
$$

It should also be noted, that the change in wave speed for the vessels, will also mean the characteristic impedances will need to be recalculated. 


\subsection{Concluding Remarks}

This chapter described the choice of different parameters implemented in the closedloop cardiovascular model. This included parameter and coefficient values from literature, and the initial and iterative parameter estimation techniques utilised in this thesis which use participant measurements to aid the estimation. Details of the measured participant data were described, which includes measurements from the first, second, and third trimesters of pregnancy, as well as a measurement postpartum. The utilisation of the measured data was described via an initial parameter estimation technique, which uses the participant measured heart rate and mean brachial artery pressure as an input. The iterative parameter estimation technique is utilised at the end of every cardiac cycle during the simulation. The iterative technique adapts the models systemic vascular resistances, vessel compliances, and blood volume in the system, in order to converge to the participant measurements of systolic pressure, diastolic pressure, and cardiac output. The parameter estimation techniques are generalised, allowing parts of the techniques to be implemented within both closed-loop and open-loop models. 


\begin{tabular}{|c|c|c|}
\hline Vessel Name & $\begin{array}{l}\text { Literature Diame- } \\
\text { ter (references) }\end{array}$ & Model Diameter \\
\hline Uterine Artery & $\begin{array}{l}0.2-0.5 \mathrm{~cm}(\mathrm{NP})[52] \\
0.2-0.6 \mathrm{~cm}(\mathrm{NP})[331]\end{array}$ & $\begin{array}{llll}0.25 & \mathrm{~cm} & & (\mathrm{NP}) \\
0.4370 & - & 0.5 & \mathrm{~cm} \\
(\mathrm{P}) & & & \end{array}$ \\
\hline $\begin{array}{l}\text { Ascending } \quad \text { Uterine } \\
\text { Artery }\end{array}$ & $\begin{array}{l}0.2 \text { to } 0.5 \mathrm{~cm}(\mathrm{NP}) \\
{[98,52], 0.26 \mathrm{~cm} 2 \mathrm{nd}} \\
\text { trimester and } 0.3 \mathrm{~cm} \\
\text { 3rd trimester }(\mathrm{P})[290]\end{array}$ & $\begin{array}{l}0.25 \mathrm{~cm}(\mathrm{NP}), 0.3-0.4 \\
\mathrm{~cm}(\mathrm{P})\end{array}$ \\
\hline Arcuate Artery & $\begin{array}{l}\text { mid pregnancy about } \\
\text { size of uterine artery, } \\
\text { by term some are dou- } \\
\text { ble diameter }[53]\end{array}$ & $\begin{array}{l}0.125 \mathrm{~cm}(\mathrm{NP}), 0.5046 \\
\mathrm{~cm}(\mathrm{P})\end{array}$ \\
\hline Spiral Artery & $\begin{array}{l}0.02 \text { widening to } \\
0.05-0.1 \mathrm{~cm}[104], \\
0.05 \mathrm{~cm} \text { healthy, } 0.02 \\
\mathrm{~cm} \text { pre-eclampsia }[66], \\
0.1-0.2 \mathrm{~cm}(\mathrm{NP}) \\
{[98], 0.2-0.3 \mathrm{~cm}(\mathrm{P})} \\
{[53]}\end{array}$ & $\begin{array}{ll}0.1 & (\mathrm{NP}), 0.2-0.24 \\
(\mathrm{P}) & \end{array}$ \\
\hline Ovarian Artery & $<0.15 \mathrm{~cm}[52]$ & $\begin{array}{l}0.14 \mathrm{~cm}(\mathrm{NP}), 0.35 \mathrm{~cm} \\
(\mathrm{P})\end{array}$ \\
\hline Ovarian Artery II & No Information & $\begin{array}{l}0.1 \mathrm{~cm}(\mathrm{NP}), 0.2 \mathrm{~cm} \\
(\mathrm{P})\end{array}$ \\
\hline $\begin{array}{l}\text { Utero-Ovarian Com- } \\
\text { municating Artery }\end{array}$ & $\begin{array}{lr}0.05-0.2 \mathrm{~cm} & \text { non- } \\
\text { pregnant (close to } \\
\text { Uterine artery } & \text { when } \\
\text { pregnant) }[52], & 0.05 \\
\mathrm{~cm}(\mathrm{NP})[331] & \end{array}$ & $\begin{array}{l}0.1 \mathrm{~cm}(\mathrm{NP}), 0.3568- \\
0.4 \mathrm{~cm}(\mathrm{P})\end{array}$ \\
\hline Uterine Vein & $0.33 \mathrm{~cm}(\mathrm{NP})[52]$ & $0.31 \mathrm{~cm}$ \\
\hline $\begin{array}{l}\text { Ascending Uterine } \\
\text { Vein }\end{array}$ & $0.33 \mathrm{~cm}(\mathrm{NP})[52]$ & $0.31 \mathrm{~cm}$ \\
\hline Arcuate Vein & No Information & $0.15625 \mathrm{~cm}$ \\
\hline Spiral Vein & No Information & $0.125 \mathrm{~cm}$ \\
\hline Ovarian Vein & $\begin{array}{l}0.32-0.39 \mathrm{~cm}(\mathrm{NP}), \\
1.84[52]\end{array}$ & $0.31 \mathrm{~cm}$ \\
\hline Ovarian Vein II & No Information & $0.125 \mathrm{~cm}$ \\
\hline $\begin{array}{l}\text { Utero-Ovarian Com- } \\
\text { municating Vein }\end{array}$ & No Information & $0.0625 \mathrm{~cm}$ \\
\hline
\end{tabular}

Table 6.3: Diameters of utero-placenta and ovarian circulation. 


\begin{tabular}{|c|c|l|l|l|l|}
\hline No. & Name & $\begin{array}{l}\text { Length } \\
(\mathrm{cm})\end{array}$ & $\begin{array}{l}\text { Area } \\
\text { NP } \\
\left(\mathrm{cm}^{2}\right)\end{array}$ & $\begin{array}{l}\text { Area P } \\
\left(\mathrm{cm}^{2}\right)\end{array}$ & $\begin{array}{l}\text { Vascular } \\
\text { Bed }\end{array}$ \\
\hline 62 & Ovarian Artery I & 3 & 0.0154 & 0.03079 & \\
63 & Ovarian Artery II [x3] & 2 & 0.0079 & 0.015 & Ovary \\
64 & Communicating Artery & 5 & 0.004418 & 0.048105 & \\
65 & Arcuate Arteries I [x4] & 12 & 0.0123 & 0.1257 & \\
66 & Radial/Spiral Arteries I [x50] & 3 & 0.000314 & 0.001963 & Fundus \\
67 & Ascending Uterine Artery I & 3 & 0.02405 & 0.048105 & \\
68 & Arcuate Arteries II [x4] & 12 & 0.0123 & 0.1257 & \\
69 & Radial/Spiral Arteries II [x50] & 3 & 0.000314 & 0.001963 & Uterus \\
70 & Ascending Uterine Artery II & 3 & 0.02405 & 0.048105 & \\
71 & Arcuate Arteries III [x4] & 12 & 0.0123 & 0.1257 & \\
72 & Radial/Spiral Arteries III [x50] & 3 & 0.000314 & 0.001963 & Placenta \\
73 & Ascending Uterine Artery III & 3 & 0.02405 & 0.048105 & \\
74 & Arcuate Arteries IV [x4] & 12 & 0.0123 & 0.1257 & \\
75 & Radial/Spiral Arteries IV [x50] & 3 & 0.000314 & 0.001963 & Uterus \\
76 & Ascending Uterine Artery I & 3 & 0.02405 & 0.048105 & \\
77 & Descending Uterine Artery & 3 & 0.000314 & 0.001963 & \\
78 & Arcuate Arteries V [x4] & 12 & 0.0123 & 0.1257 & \\
79 & Radial/Spiral Arteries V [x50] & 3 & 0.000314 & 0.001963 & Cervix \\
80 & Uterine Artery & 8 & 0.02405 & 0.048105 & \\
81 & Vaginal Artery & 7 & 0.00307 & 0.04909 & \\
\hline
\end{tabular}

Table 6.4: Utero-ovarian artery vessel information. Right and left sides assumed to be equal in length and area. 


\begin{tabular}{|c|c|c|c|c|c|}
\hline No. & Name & $\begin{array}{l}\text { Length } \\
(\mathrm{cm})\end{array}$ & $\begin{array}{l}\text { Area } \\
\mathrm{NP} \\
\left(\mathrm{cm}^{2}\right)\end{array}$ & $\begin{array}{l}\text { Area P } \\
\left(\mathrm{cm}^{2}\right)\end{array}$ & $\begin{array}{l}\text { Vascular } \\
\text { Bed }\end{array}$ \\
\hline 270 & Ovarian Vein I & 3 & 0.0767 & 0.7854 & \\
\hline 271 & Ovarian Vein II [x3] & 2 & 0.0123 & 0.0491 & Ovary \\
\hline 272 & Communicating Vein & 5 & 0.0767 & 0.7854 & \\
\hline 273 & Arcuate Veins I [x4] & 12 & 0.0192 & 0.1963 & \\
\hline 274 & Radial/Spiral Veins I [x50] & 3 & 0.0124 & 0.0491 & Fundus \\
\hline 275 & Ascending Uterine Vein I & 3 & 0.02405 & 0.048105 & \\
\hline 276 & Arcuate Veins II $[\mathrm{x} 4]$ & 12 & 0.0192 & 0.1963 & \\
\hline 277 & Radial/Spiral Veins II [x50] & 3 & 0.0124 & 0.0491 & Uterus \\
\hline 278 & Ascending Uterine Vein II & 3 & 0.02405 & 0.048105 & \\
\hline 279 & Arcuate Veins III [x4] & 12 & 0.0192 & 0.1963 & \\
\hline 280 & Radial/Spiral Veins III [x50] & 3 & 0.0124 & 0.0491 & Placenta \\
\hline 281 & Ascending Uterine Vein III & 3 & 0.02405 & 0.048105 & \\
\hline 282 & Arcuate Veins IV $[\mathrm{x} 4]$ & 12 & 0.0192 & 0.1963 & \\
\hline 283 & Radial/Spiral Veins IV [x50] & 3 & 0.0124 & 0.0491 & Uterus \\
\hline 284 & Ascending Uterine Vein I & 3 & 0.02405 & 0.048105 & \\
\hline 285 & Descending Uterine Vein & 3 & 0.000314 & 0.001963 & \\
\hline 286 & Arcuate Veins V $[\mathrm{x} 4]$ & 12 & 0.0192 & 0.1963 & \\
\hline 287 & Radial/Spiral Veins V [x50] & 3 & 0.0124 & 0.0491 & Cervix \\
\hline 268 & Uterine Vein & 8 & 0.0767 & 0.7854 & \\
\hline 266 & Vaginal Vein & 7 & 0.0098 & 0.02405 & \\
\hline
\end{tabular}

Table 6.5: Utero-ovarian venous vessel information. Right and left sides assumed to be equal in length and area.

\begin{tabular}{|c|c|c|c|c|}
\hline Age Range & Trimester 1 & Trimester 2 & Trimester 3 & $\begin{array}{c}\text { Non-Pregnant } \\
\text { Post-Partum }\end{array}$ \\
\hline Age $<25$ & 17 & 9 & 7 & 2 \\
\hline $25 \leq$ Age $<30$ & 18 & 10 & 6 & 6 \\
\hline $30 \leq$ Age $<35$ & 18 & 13 & 12 & 12 \\
\hline Age $>35$ & 5 & 4 & 4 & 4 \\
\hline \hline Total number of data & 58 & 36 & 29 & 24 \\
\hline
\end{tabular}

Table 6.6: Characteristics of the cohort, showing age range and number of participant data for each trimester. 


\begin{tabular}{|c|c|}
\hline Location & Pressure $(\mathrm{mmHg})$ \\
\hline \hline systemic arteries & 80 \\
\hline systemic veins (excluding hepatic portal system) & 5 \\
\hline hepatic portal veins & 8.5 \\
\hline pulmonary arteries & 11 \\
\hline pulmonary veins & 10 \\
\hline
\end{tabular}

Table 6.7: Defined reference pressures $P_{d}$. 


\section{Chapter 7}

\section{Computational Model of Pregnancy}

This chapter begins by describing the various types of physiological adaptation in the maternal circulation over the course of a human pregnancy. The development of the foetus, placenta, and uterus will be presented and a description of the physiological role of the placenta and umbilical cord will be given. The computational algorithm for the closed-loop pregnancy model will then be described. The computational simulations performed will then be given in separate sections, which includes: the patient-specific model, which integrates in-vivo physiological measurements taken during the first, second, and third trimesters, and for post-partum conditions; an idealised model of pregnancy for all gestational weeks; and two additional cases, which are an asymmetric adaptation of the uterine system, and the effect of changing the diameter of the utero-ovarian communicating artery. Finally, a sensitivity analysis is performed on the model to investigate the effect of the initial conditions, and cardiac parameters on the final model solutions, which tests the effectiveness of the iterative adaptation technique.

\subsection{Physiological Adaptation}

Pregnancy induces many physiological and structural adaptations. Compared to the non-pregnant circulatory systems, pregnant individuals show increases in cardiac output by $30-50 \%$ caused by an increase in both stroke volume and heart rate $[155$, $176,52,300,209,320]$; there is a considerable increase in total blood volume, with an increase in plasma volume by just under $50 \%$, and an increase in red cell mass by approximately $18 \%$, leading to an overall decrease in the density of red blood cells within the plasma $[161,250]$. A study also observed a lower overall blood viscosity in pregnant individuals, while there was no significant change in the viscosity of plasma alone [296]. There is also an increase in interstitial fluid (oedema) during pregnancy, with a further increase in extracellular fluid observed in patients with hypertension [46].

Even with the extreme increase in cardiac output, the pressures in the systemic arterial system remain relatively consistent throughout pregnancy [209], with mean 
arterial pressure observed to fall slightly during the first to second trimesters, and rising to pre-pregnant values towards term. The mean pressures are kept relatively consistent due to the decrease of systemic vascular resistance, as increase in vascular compliance, vascular remodelling, and vaso-dilation of vessels [209, 52].

If sufficient physiological adaptation does not occur, several pathologies can develop. Hypertension may develop if vascular modelling does not occur sufficiently, which means the systemic vascular resistance does not decrease sufficiently to counter the increase in cardiac output; hypotension may result if the vascular remodelling, increase in vascular compliance, and decrease in systemic vascular resistance occur to a greater extent than the relative increase in blood volume. Several clinically serious pathologies are also associated with hypertension, such as preeclampsia, which is a leading cause of maternal mortality, occurring in $3-7 \%$ of all pregnancies [6], and is regarded as a renal complication which tends to develop after week twenty of gestation. Pre-eclampsia causes hypertension, proteinuria (protein in urine), is linked to poor placentation (formation and structure of the placenta), and can lead to an increase in oedema [343, 263, 20, 131]. Placental insufficiency can also develop, and is caused by poor placentation, poor placental development, and poor adaptation of the uterine vasculature, leading to increased placental and uterine resistances, hypertension, and restricted foetal growth [127, 232, 181].

During pregnancy the maternal vasculature undergoes substantial adaptation. These changes include: the remodelling of the heart, which results in an increase of left ventricular mass and wall thickness $[150,145]$; the creation and development of a specialised organ, the placenta, which facilitates nutrient and waste product exchange between maternal and foetal systems; remodelling of the vascular network, including an increase in vessel lumen diameters and compliance, particularly in the uterine system, with significant changes to the micro-structure of the spiral-arteries which penetrate the wall of the uterus [53, 66, 153, 200, 270, 367].

There are also significant changes to the distribution of cardiac output to each body region. In particular with regional increases of blood flow to the uterus, ovaries, kidneys, and skin. Blood flow to the uterus occurs via several pathways. The uterus receives the majority of its blood supply needs from the left and right uterine arteries, while the remaining supply reaches the uterus from: the utero-ovarian communicating artery, which is a small vessel anastomosing the ovarian artery with the ascending uterine artery; and large number of smaller (often unnamed) vessels. 
The uterus has the largest increase in blood flow of all body regions, where the total bi-lateral blood flow through the uterine arteries increases from approximately $3.5 \%$ of cardiac output in early pregnancy, to $12 \%$ of cardiac output by term of pregnancy [340], with an example of the uterine artery waveforms for the non-pregnant case and for the first trimester are shown in 7.2. While the utero-ovarian communicating artery increases its diameter significantly, and in some cases vaso-dilation occurs to the extent that the utero-ovarian communicating vessel can supply the uterus with the majority of its blood supply needs [52].

\subsection{The Uterus and The Placenta}

The vasculature of the uterus undergoes tremendous adaptation over the course of pregnancy to accommodate its role change, from acting as the site of embryo fertilisation pre-pregnancy, to protecting and aiding in the development of the foetus during pregnancy, and even plays a critical role at term by contracting and facilitating childbirth. The uterus has three major layers: a thin outer layer called the perimetrium which mainly acts as an external border; a middle layer called the myometrium, which is a thick muscular layer responsible for uterine contractions during childbirth; and the inner layer called the endometrium, which contains two sub-layers, a basal layer and a functional layer. The functional layer is shed if fertilisation of an embryo does not occur, and is the location where a fertilised embryo attaches to and develops in the uterine wall; while the basal layer remains in place and does not shed during menstruation. Figure 7.1 gives an overview of the uterine wall and its vessels for pregnant and non-pregnant individuals.

The uterine vessels begin to remodel in early pregnancy. The most significant vessel vaso-dilation occurs in the uterine, utero-ovarian, ovarian, arcuate, and spiral vessels, for both arteries and veins [52, 104, 331, 53, 66, 290, 98, 358]. The spiral arteries undergo the most significant changes, dilating up to five times of their non-pregnant values by term. Up to 120-200 of these spiral arteries adapt further, becoming the utero-placental vessels, which drain blood directly to the intervillous space in the placenta $[104,52]$. The compliance of these uterine vessels are affected by an invasion of trophoblast cells from the placenta (originating from the foetus), which progressively travel further into the maternal vessel walls and tissues over 


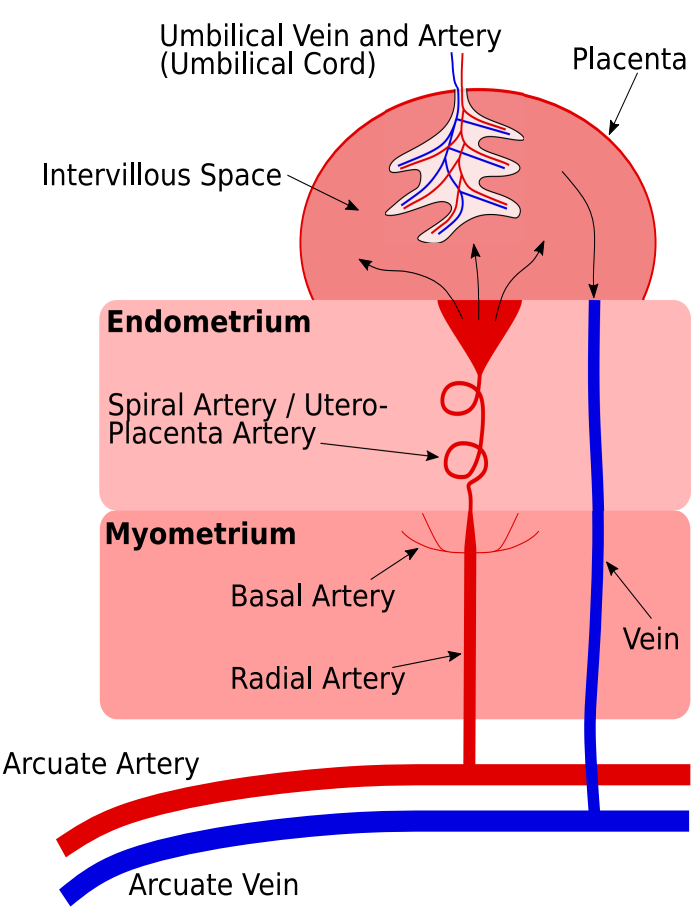

(a) Diagram of the pregnant uterus.

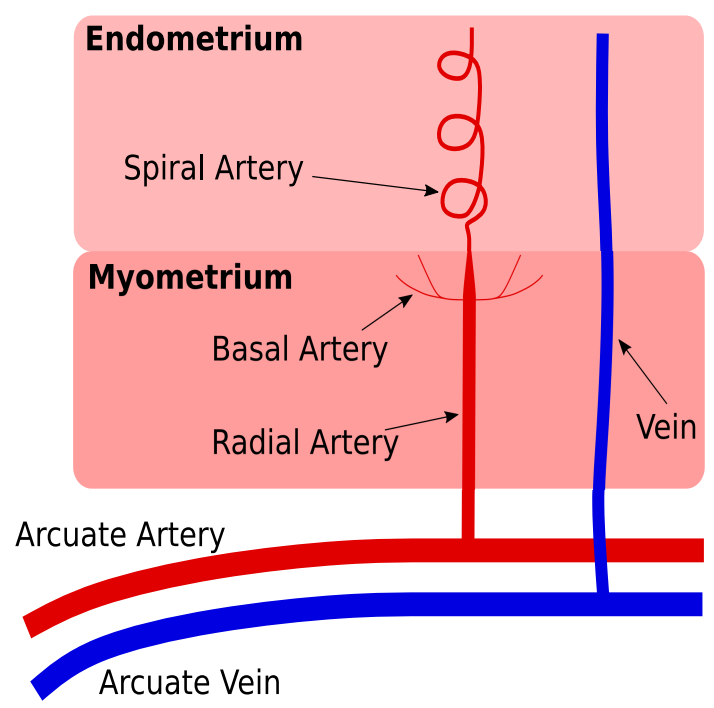

(b) Diagram of the non-pregnant uterus.

Figure 7.1: Comparison of diagram of the non-pregnant and pregnant human uterus, including the development and vasculature of the placenta.

the course of pregnancy, with these trophoblasts even being observed in the uterine arteries in later pregnancy [270, 104, 358, 367, 366].

The placenta plays a critical role during pregnancy. It is a unique organ that: receives blood from both maternal and foetal systems; acts as a barrier that keeps maternal and foetal blood separate; and facilitates nutrient exchange between maternal and foetal systems. An increased weight and size of the placenta has been shown to aid in foetal development, and increase birth weight [292]. Poor placentation may result in a number of pathologies, such as pre-eclampsia, uteroplacental dysfunction (placental insufficiency), and foetal growth restriction [6, 181]. These pathologies often affect the entire uterine system, with poor adaptation of uterine arteries occurring in conjunction with poor placental development. 


\subsection{Doppler Studies of Pregnancy}

The uterine arteries (one on left and one on the right side) are the main vessels which supplies blood to the uterus and placenta, and hence flow in this artery is critical to the development of the foetus, as insufficient nutrient supply may result in foetal growth restriction $[6,181]$. Clinically, the use of Doppler ultrasound on the uterine artery to view blood flow rates, enables an estimation of uterine resistance and compliance. Of particular interest is the presence of a notching in flow waveforms, as this is an indicator for many of the pathologies which may develop during pregnancy, such as pre-eclampsia and placental insufficiency. Figure 7.2 shows a healthy uterine artery waveform from a digitisation of a Doppler ultrasound for a non-pregnant human where a notch is present, and a healthy uterine artery waveform from a digitisation of a Doppler ultrasound in the first trimester. The uterine artery flow waveform has been studied extensively, particularly when investigating risk factors in pregnancy. This led to the development of several measures such as: the pulsatility index, resistance index, types of notching present, and systolic to diastolic flow ratios, that have been used to link uterine waveform shape features to the development of pathologies [98, 137, 109, 297, 173, 307, 22, 136, 193, 306, 138, $256,54,208,43,72,204,255,16,78,19,152,123,83]$.

The uterine artery has received a lot of attention as it is relatively straight forward to access. However, no other vessels in the uterus have been studied to the same extent. For example, due to the complex flow behaviour, attempts to measure the flow in the utero-ovarian communicating artery have been unsuccessful $[262,47]$. The uterine circulation in the venous system is also much less studied, with only the uterine vein waveforms having been studied [344].

\subsection{Computational Modelling Of Pregnancy}

The majority of models of pregnancy have primarily focused on the foetal circulation $[266,130,227,211]$, umbilical cord [273, 311, 188], or the placenta [81, 75]. Only one model of the maternal system has been proposed [89], which is a multi-compartment lumped model. However, lumped models struggle to account for wave-propagation phenomena, and would only have limited use, as the majority of indices used in the

Pg. $179 / 284$ 

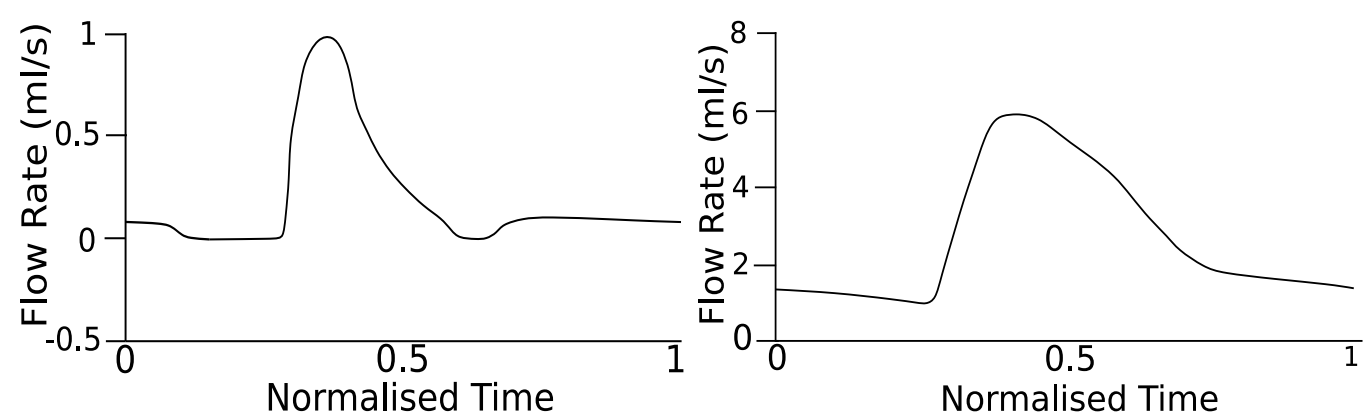

(a) Typical digitised Doppler waveform (b) Typical digitised Doppler wavefor the uterine artery in non-pregnant form for the first trimester in the first women [307] trimester [264]

Figure 7.2: Typical digitised Doppler waveforms for a non-pregnant woman and for the first trimester

detection of pathology, use uterine artery waveforms to determine compliance and resistance estimates of the uterine region. The pressure and flow rate waveforms in the cardiovascular system rely on wave-reflections which lumped models poorly capture [103]. Thus in this thesis, a distributed 1D model is used for the major vessels, which can capture wave-reflection and transmission behaviour.

The maternal system model proposed in this thesis, extends the closed-loop 1D0D model of Mynard and Smolich [230], by adding the utero-ovarian circulation. An automated parameter estimation and adaptation is implemented within the model which allows the integration of measured in-vivo data. The predicted increase of vessel diameters uses the curve presented in [155] for the expected increase in cardiac output over the course of pregnancy, which can be seen in figure 7.3. The description of model parameters and the automated parameter estimation and adaptation is described in Chapter 6; the numerical scheme is described in Chapter 3, and an overview of the computational algorithm for the closed-loop system is described in the next section. In the sections which follow, the closed-loop system is implemented with measured participant data as an input, to inform the model on various parameters. Three of these patients will be included in a small case study and are analysed in more detail, with a comparison of flow and pressure waveforms. The model is then performed for an idealised case in pregnancy, which is performed from a non-pregnant case, and covers all weeks in pregnancy. The results of which are compared with values and indices in literature. The chapter ends with additional 


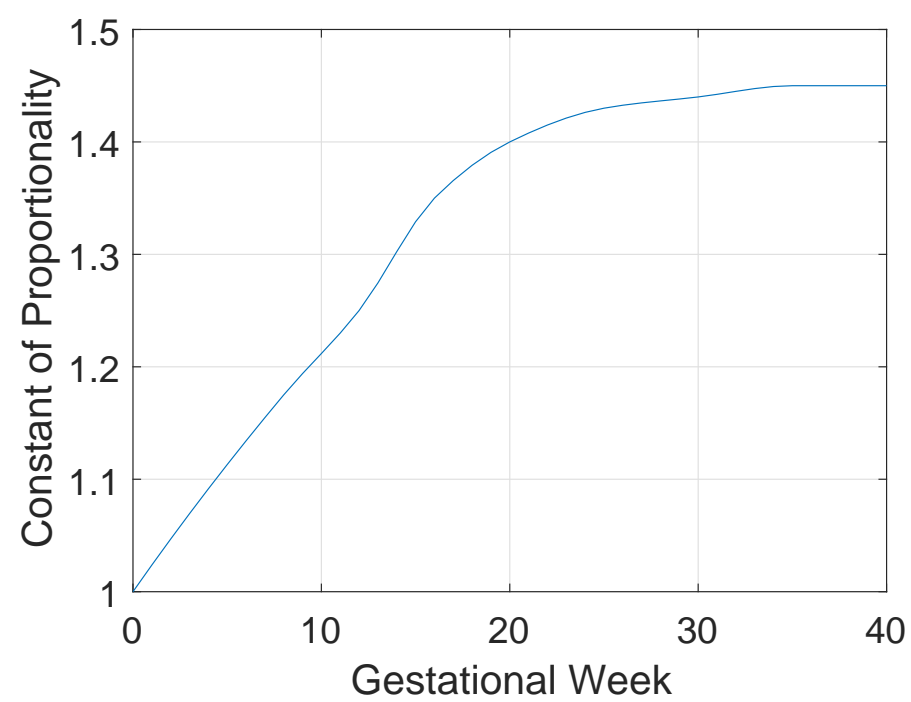

Figure 7.3: Estimated increase of problem parameters.

cases, which includes: an asymmetric adaptation of the left to right side of the uterine circulation; and a sensitivity analysis for several cardiac variables, which is used to test the effectiveness, and consistency of the automated parameter estimation technique.

\subsection{Computational Algorithm}

In order to advance the solution forward in time from $t^{n}$ to $t^{n+1}$ the boundary conditions for the lumped models need to be known. The only true boundary conditions for the closed loop model are external pressures which act on the compliant (and elastance) elements in the vascular bed (and heart) models. These external pressures can either be prescribed such as for vascular beds, or are constrained by certain conditions using Lagrange multipliers such as in the heart model. The general computational algorithm for the ETM scheme is as follows:

- Pre-Processing Step. Involves reading the input file, patient specific measurement data, mesh creation, vectorisation, initial parameter estimation, and pre-allocation memory.

- For loop until periodic convergence and convergence to measured participant data. 
- For loop in time

* Determine if blood needs to be added or removed from the system (only performed after the fifth cardiac cycle, to converge towards periodic solution).

* Within the Newton iteration (if more than one iteration is used):

- Parameters such as vascular resistances, heart elastances, valve opening coefficients are updated to the current time level.

- Calculate and construct elemental matrices for 1D and lumped models.

- Assemble global matrix and source/sink vector, account for boundary conditions, and solve the linear system of equations for pressures and Lagrange multipliers.

- Calculate/update flow rates from the elemental matrices.

- Update volume changes in the heart (and track volume in the system).

- Check convergence of Newton iteration (if more that one iteration is used).

* Update variables and move the the next time step.

- Compare numerical solution with measured participant data to check for convergence.

- If not converged, recalculate total resistance and compliance based on current numerical solution and measured data (pressures and cardiac output).

- If converged to measured data, check convergence to periodicity. If periodic solution detected exit loop.

- Post-processing steps which include saving of solutions to a file and plotting monitoring pressures.

\subsection{Pulse Wave Velocity and Pulsatility Index}

One of the main indicators of pathology development during pregnancy is arterial stiffness [197, 291, 298, 105, 18, 218, 139]. In early pregnancy, an increase in arterial 
stiffness has been linked to an increased risk of pre-eclampsia [192, 164, 352, 303, 167], placental insufficiency [248], and decreased foetal growth [102] later in pregnancy. Several clinical methods have been proposed to estimated arterial stiffness in pregnancy, including the use of pulse pressure [339, 147, 378], pulse wave velocity [172, 236, 251, 121], pulsatility index [22, 109, 137, 144, 146, 337], and resistance index $[43,123,146,148]$.

The current gold standard of estimating aortic stiffness is pulse wave velocity (PWV) [267]. There are several techniques used in the estimation of pulse wave velocity: the femoral-carotid method is recognised as the best method to estimate PWV [267]; the brachial-ankle method [251] is non-invasive, using the brachial artery in the arm and tibial artery in the lower leg, and is used due to the accessibility of these arteries; the carotid-brachial method can also be used [267]. Pulse wave velocity methods estimate the velocity at which a pressure pulse travels in the systemic arteries. The method measures the time difference for a pulse to reach two arteries in different regions of the body, and the distance between the two arteries.

Two different estimations of PWV are implemented in order to compare with literature: the first uses the wave speed in the ascending aorta as calculated from the $1 D$ blood flow equations $P W V_{\text {aorta }}$; the second uses the brachial-ankle estimation $P W V_{b-a}[251]$. There exist two wave speeds, one for a forward-propagating wave and one for a backward-propagating wave, which can be written as

$$
\lambda_{ \pm}=u \pm c
$$

However, this can be estimated from the initial conditions by assuming the initial velocity is $u=u_{0}=0$ and $c=c_{0}$. The aortic PWV can then be estimated using $P W V_{\text {aorta }}=c_{0}$. The brachial-ankle method uses the same technique as described in [251], which estimates PWV using

$$
P W V_{b-a}=\frac{L_{b}-L_{a}}{\Delta t_{b-a}},
$$

where $L_{b}$ is the distance from the aortic valve and brachial artery, $L_{a}$ is the distance from the aortic valve and anterior tibial artery, where both lengths are estimated from patient height. $\Delta t_{b-a}$ is the time difference between the beginning of systole (initial pressure rise) of the brachial artery and anterior tibial artery. 


\subsection{PULSE WAVE VELOCITY AND PULSATILITY INDEX}

The pulsatility index (PI) is a measure of the variability in systolic and diastolic velocities, found from

$$
P I=\frac{u_{\text {max }}-u_{\text {min }}}{u_{\text {mean }}}
$$

where $u_{\text {max }}, u_{\text {min }}$, and $u_{\text {mean }}$, are the maximum systolic, minimum diastolic, and mean velocities respectively. In the model the velocity is determined as $u=\frac{Q}{A}$. 


\subsection{Patient Specific Pregnancy Modelling}

This section will begin by giving an overview of the measured data, and a general comparison of the simulation results with the measured data and data from literature. This will include: the measure of errors from simulated results and measured variables to test the effectiveness of the automated parameter estimation technique; and comparing the simulated results with data from literature for the PI, PWV, and flow distributions through the uterine vessels. The section will end with a comparison of the solutions for three participants, which includes a comparison of Doppler waveforms from literature in the uterine arteries.

The patient-specific closed-loop model is implemented for measured data collected from various studies, led by Prof. Mike Lewis [63, 59, 62, 60, 61, 100]. The number of patients, split into age ranges for each trimester and post-partum, is shown in table 7.1 .

\begin{tabular}{|c|c|c|c|c|}
\hline Age Range & Trimester 1 & Trimester 2 & Trimester 3 & $\begin{array}{c}\text { Non-Pregnant } \\
\text { Post-Partum }\end{array}$ \\
\hline Age $<25$ & 17 & 9 & 7 & 2 \\
\hline $25 \leq$ Age $<30$ & 18 & 10 & 6 & 6 \\
\hline $30 \leq$ Age $<35$ & 18 & 13 & 12 & 12 \\
\hline Age $>35$ & 5 & 4 & 4 & 4 \\
\hline \hline Total number of data & 58 & 36 & 29 & 24 \\
\hline
\end{tabular}

Table 7.1: Characteristics of the cohort, showing age range and number of participant data for each trimester.

\subsubsection{Results Overview}

The results for the non-pregnant cases are designated week zero in all figures, though are from post-partum measurements. There is a discrepancy in literature of studies taken in the first 8 weeks of pregnancy, primarily as many do not realise they are pregnant until the 6 week mark, and it takes time to recruit participants to any study. The simulations are performed until the model cardiac output, diastolic, and systolic blood pressures are within $1 \%$ of the measured data at the end of a cardiac cycle (convergence to measurements), and maximum pressures in the last 
cardiac cycle must be within $0.5 \%$ of the previous cardiac cycle (models periodic convergence).

Figure 7.4a and 7.4b shows the PI for the uterine and utero-ovarian artery. The model estimated PI of the uterine artery is within physiologically realistic values, although the models population mean is slightly lower than the values from [337]. Nevertheless good agreement is observed, with the behaviour of the PI closely following the behaviour of values presented in [337] as pregnancy progresses. The PI of non-pregnant individuals from literature has been measured to be approximately in the range $\mathrm{PI}=2-6$, and varies over the menstrual cycle [144]; this is in agreement with the model prediction $\mathrm{PI} \approx 4$.

No studies have involved the PI in the utero-ovarian communicating artery (UOCA), in fact previous attempts at measuring flow in these arteries using Doppler have been unsuccessful $[262,47]$. The model predicts the PI in the UOCA to follow a similar behaviour to that of the uterine artery, although the model predicts the magnitude of the PI in the UOCA to be lower than in the uterine arteries.

Figures $7.4 \mathrm{c}$ and $7.4 \mathrm{~d}$ shows the percentage of cardiac output to the uterus via the uterine arteries and UOCAs respectively. Table 7.2 shows the estimated total percentage of cardiac output and volumetric flow rates through the uterine arteries from literature, for at various stages in pregnancy and post-partum. The modelpredicted percentage of cardiac output through the uterine arteries is in excellent agreement with literature. The model-predicted population mean percentage of CO through the uterine arteries is: $0.5 \%$ for the non-pregnant simulations, $5.6201 \%$ in the first trimester, $10.5231 \%$ for the second trimester, and $12.9485 \%$ in the third trimester. For the non-pregnant simulations, the flow rate is in agreement with literature, in one study the total measured flow rate in the uterine arteries was found to be $20-50 \mathrm{ml} \mathrm{min}^{-1}$ [249], while the population mean of the model is 27.1 $\mathrm{ml} \mathrm{min}{ }^{-1}$; indicating the flows predicted by the model are physiologically realistic.

The model-predicted percentage of $\mathrm{CO}$ in the utero-ovarian communicating arteries is: $0.0436 \%$ for the non-pregnant case, $3.5552 \%$ in the first trimester, $7.2417 \%$ in the second trimester, and $8.9177 \%$ in the third trimester. These cannot be compared with literature as attempts to use Doppler measurements in these vessels have so far been unsuccessful $[262,47]$, owing this failure to the complex geometry and flow behaviour of this region. However in [52] it is stated that if the UOCA reaches the same diameter as the ascending uterine artery, then it can supply the uterus 


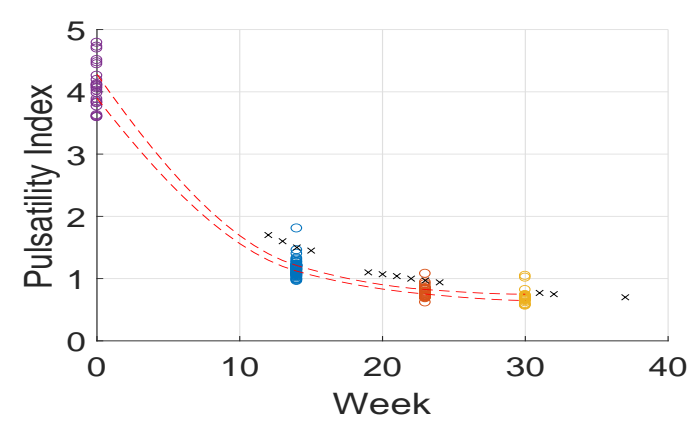

(a) Pulsatility Index in the left uterine artery for patients. The black $\mathrm{x}$ points are the mean pulsatility indices from [337].

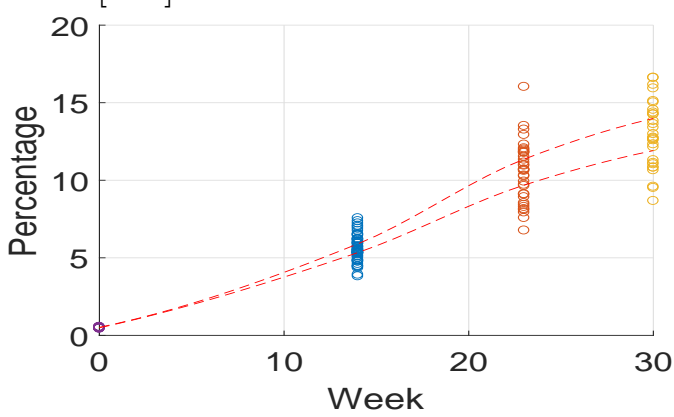

(c) Combined percentage of cardiac output through the left and right uterine arteries.

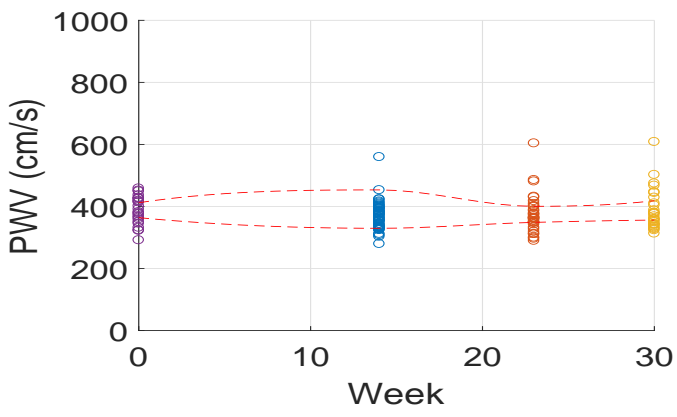

(e) Aortic PWV calculated from the models wave speed $c_{0}$.

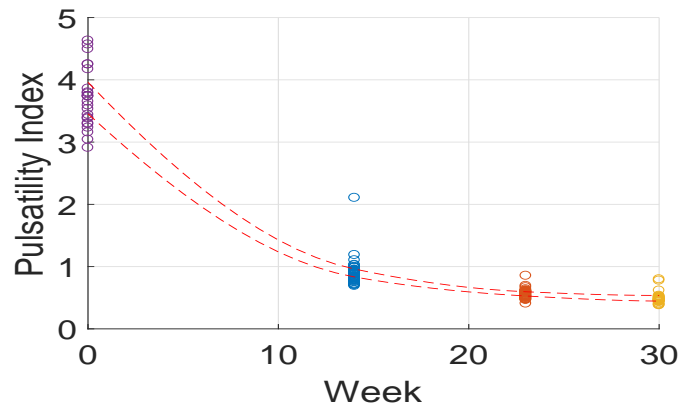

(b) Pulsatility Index in the left uteroovarian communicating artery for patients.

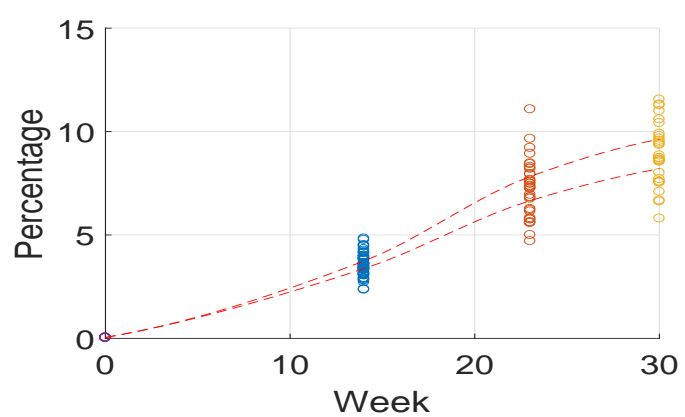

(d) Combined percentage of cardiac output through the left and right uteroovarian communicating artery.

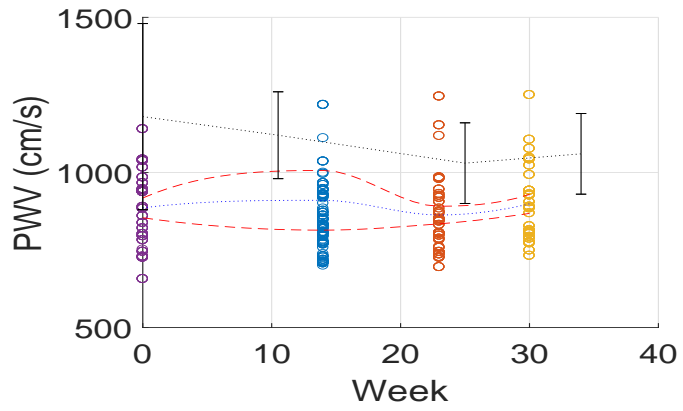

(f) Brachial-ankle PWV, where the black dotted line is the mean and \pm standard deviation of PWV from [251], while the blue dashed line is the mean of simulated values.

Figure 7.4: The pulsatility index and percentage of total cardiac output through the uterine arteries and utero-ovarian communicating arteries; and pulse wave velocity using Aortic PWV from the model and an estimate of brachial-ankle PWV, for all patients. Circles represent patient simulated values, and the dashed red line is the $95 \%$ confidence interval of the simulated data. 
with its full blood supply needs; and as the model assumes the UOCA does in fact reach this diameter, which was assumed as there is little information on the diameter of the UOCA, then the models predicted flow rate in the UOCA being close the that of the uterine artery can be considered quite reasonable.

Figures 7.4e and 7.4f show the aortic PWV, and the PWV calculated from the brachial-ankle method. The aortic PWV is significantly lower that the estimation from the brachial-ankle method, although both show similar trends. This is expected as the PWV in the aorta is generally around $4-5 \mathrm{~m} \mathrm{~s}^{-1}$, increasing to $8-9 \mathrm{~m} \mathrm{~s}^{-1}$ in the abdominal aorta [267]; which means estimation of PWV by methods such as brachial-ankle, and carotid-femoral, will produce higher PWV values than the actual aortic PWV. Figure 7.4f also compares the model-predicted PWV with the mean and standard deviation of the brachial-ankle method from a longitudinal study [251] (from a different cohort). The behaviour of the models PWV estimates are similar to that of [251], with the models population mean showing a decrease from the first trimester to a minimum in the second trimester, before increasing again towards the third trimester and term. Overall the general behaviour of the model is in agreement with literature.

\subsubsection{Three Participant Comparison}

In this section, three of the participant measurements are compared for trimesters one, two, three, and also post-partum. The participant characteristics, which includes participant information and simulation results, are shown in table 7.3.

The uterine artery (UA) flow rate waveforms are shown in figure 7.5, and are compared with digitised Doppler waveforms. For the first trimester waveforms, which are shown in figure $7.5 \mathrm{a}$, the general shape of the uterine artery flow rate waveforms are in good agreement with Doppler waveforms from literature [264], with participant one in particular showing excellent agreement. In the first trimester, it is not uncommon for notching in the waveform to be observed, occurring in 46.3 $\%$ of pregnancies [137]. However, for these patients no notching is observed. The second trimester flow rate waveforms are shown in figure $7.5 \mathrm{c}$, the model gives good agreement with the overall waveform shape from a Doppler study [240], with the shape of participant one's waveform showing excellent agreement with the digitised Doppler waveform. The third trimester flow rate waveforms are again in good agree- 
7.7. PATIENT SPECIFIC PREGNANCY MODELLING

\begin{tabular}{|l||l|l|l|l|}
\hline & Status & $\begin{array}{l}\text { Flow } \\
\text { rate }\end{array}$ & $\begin{array}{l}\text { Percentage } \\
\text { of CO }\end{array}$ & Reference \\
\hline $\begin{array}{l}\text { bi-lateral as- } \\
\text { cending uterine } \\
\text { artery }\end{array}$ & $\begin{array}{l}\text { Early Preg- } \\
\text { nancy }\end{array}$ & -- & $3.5 \%$ & {$[340]$} \\
\hline $\begin{array}{l}\text { bi-lateral as- } \\
\text { cending uterine } \\
\text { artery }\end{array}$ & At Term & -- & $12 \%$ & {$[340]$} \\
\hline $\begin{array}{l}\text { bi-lateral uterine } \\
\text { artery }\end{array}$ & $\begin{array}{l}\text { Non Preg- } \\
\text { nant }\end{array}$ & $\begin{array}{l}20-50 \\
\mathrm{ml} / \mathrm{min}\end{array}$ & -- & {$[249]$} \\
\hline $\begin{array}{l}\text { bi-lateral uterine } \\
\text { artery }\end{array}$ & $\begin{array}{l}\text { Singleton } \\
\text { Pregnancies }\end{array}$ & $\begin{array}{l}450 \\
800 \\
\mathrm{ml} / \mathrm{min}\end{array}$ & -- & {$[249]$} \\
\hline $\begin{array}{l}\text { bi-lateral uterine } \\
\text { artery }\end{array}$ & $\begin{array}{l}\text { Week } \\
\text { (Term, } \\
\text { estimate) }\end{array}$ & $\begin{array}{l}700 \\
900) \\
\mathrm{ml} / \mathrm{min}\end{array}$ & -- & {$[249]$} \\
\hline $\begin{array}{l}\text { bi-lateral uterine } \\
\text { artery }\end{array}$ & Week 20 & $\begin{array}{l}531 \\
\mathrm{ml} / \mathrm{min}\end{array}$ & -- & {$[178]$} \\
\hline $\begin{array}{l}\text { bi-lateral uterine } \\
\text { artery }\end{array}$ & Week 38 & $\begin{array}{l}970 \\
\mathrm{ml} / \mathrm{min}\end{array}$ & -- & {$[178]$} \\
\hline
\end{tabular}

Table 7.2: Flow to uterus from the uterine arteries from literature.

ment with the overall shape from digitised Doppler waveforms [137], which can be seen in figure 7.5e. With the waveform of participant two showing the closest agreement to the digitised Doppler waveform. The post-partum UA flow rate waveform is shown in $7.5 \mathrm{~g}$ also compares favourably with digitised Doppler waveforms [307], where the waveform is quite narrow in comparison with the pregnant waveforms, and notching can be seen at the start of diastole. Overall the uterine artery flow rate waveforms predicted by the model are in good agreement with those of published Doppler studies.

The mean flow rates, which are shown in table 7.3 are also in good agreement with published data, which is shown in table 7.2. With participants mean flow rates for the combined left and right uterine arteries, in the non-pregnant case being within published ranges of $20-50 \mathrm{ml} / \mathrm{min}$ [249], and increasing to $883.1 \mathrm{~mm} \mathrm{~min}^{-1}$ in the third trimester.

The venous system is much less studied compared to the arterial side, and there are few studies on the uterine venous vessels. Two Doppler studies have been pub- 
7.7. PATIENT SPECIFIC PREGNANCY MODELLING

\begin{tabular}{|c|c|c|c|}
\hline Participant & One & Two & Three \\
\hline Age & 27 & 37 & $20 / 21$ \\
\hline HR & $\begin{array}{l}68.1 / 72.9 / \\
71.4 / 58.4\end{array}$ & $\begin{array}{l}102.6 / 114.8 / \\
106.0 / 71.4\end{array}$ & $\begin{array}{l}112.1 / 87.7 / \\
107.3 / 83.9\end{array}$ \\
\hline $\mathrm{CO}\left(\mathrm{lmin}^{-1}\right)$ & $\begin{array}{l}6.43 / 7.30 / \\
7.05 / 5.38\end{array}$ & $\begin{array}{l}9.28 / 8.42 / \\
9.74 / 5.96\end{array}$ & $\begin{array}{l}6.57 / 6.83 / \\
7.07 / 6.21\end{array}$ \\
\hline $\mathrm{SBP}(\mathrm{mmHg})$ & $\begin{array}{l}118.8 / 96.5 / \\
129.5 / 99.8\end{array}$ & $\begin{array}{l}98.2 / 102.5 / \\
109.1 / 93.4\end{array}$ & $\begin{array}{l}120.6 / 118.0 / \\
118.8 / 108.7\end{array}$ \\
\hline $\begin{array}{l}\text { DBP } \\
(\mathrm{mmHg})\end{array}$ & $\begin{array}{l}69.4 / 54.4 / \\
67.7 / 66.5\end{array}$ & $\begin{array}{l}61.4 / 64.7 / \\
72.5 / 57.6\end{array}$ & $\begin{array}{l}79.0 / 69.2 / \\
55.2 / 64.9\end{array}$ \\
\hline $\begin{array}{l}\text { PI (uterine } \\
\text { artery) }\end{array}$ & $\begin{array}{l}1.40 / 0.94 / \\
1.05 / 3.98\end{array}$ & $\begin{array}{l}1.07 / 0.73 / \\
0.69 / 4.10\end{array}$ & $\begin{array}{l}1.06 / 0.87 / \\
1.02 / 4.08\end{array}$ \\
\hline $\begin{array}{l}\text { Uterine Flow } \\
\left(\mathrm{ml} \mathrm{min}^{-1}\right)\end{array}$ & $\begin{array}{l}389.2 / 567.8 / \\
855.1 / 25.7\end{array}$ & $\begin{array}{l}351.2 / 678.7 / \\
836.2 / 26.5\end{array}$ & $\begin{array}{l}427.2 / 829.9 / \\
883.3 / 30.1\end{array}$ \\
\hline $\begin{array}{l}\text { Utero- } \\
\text { Ovarian Flow } \\
\left(\mathrm{ml} \mathrm{min}^{-1}\right)\end{array}$ & $\begin{array}{l}248.9 / 400.4 / \\
670.7 / 2.2\end{array}$ & $\begin{array}{l}217.5 / 468.8 / \\
559.6 / 2.3\end{array}$ & $\begin{array}{l}273.5 / 568.7 / \\
608.6 / 2.6\end{array}$ \\
\hline
\end{tabular}

Table 7.3: Characteristics of the three participant comparison. Values are shown for each trimester, and for post-partum measurements, and are written in the form first trimester / second trimester / third trimester / post-partum. The following abbreviations are used: HR - heart rate, CO - cardiac output, SBP - systolic blood pressure, DPB - diastolic blood pressure, and PI - pulsatility index.

lished $[344,162]$. The mean uni-lateral volumetric flow rate in the uterine vein was found to be $274 \mathrm{ml} \mathrm{min}^{-1}$ in the third trimester [162]. This compares very favourably with the model-predicted values of $314 \mathrm{ml} \mathrm{min}^{-1}$ for participant one, $270 \mathrm{ml} \mathrm{min}^{-1}$ for participant two, and $280 \mathrm{ml} \mathrm{min}^{-1}$ for participant three. The model-predicted volumetric flow rate waveforms are shown in figure 7.6 for all three trimesters, and the non-pregnant (post-partum) case. From literature the velocity (and flow) waveforms in the third trimester are generally continuous with low pulsatility [344], which can be observed in the model waveforms in Figure 7.6c. Unfortunately, no information was found in the literature on uterine venous flows for non-pregnant individuals or in early pregnancy. However, the model shows the general observed shape for flow rate waveforms, and the expected mean flow rates in the third trimester.

The model-predicted volumetric flow rate waveforms in the ascending aorta for all participants, in all trimesters and post-partum, are shown in figure 7.7. The cardiac output is much larger for participant two during pregnancy, especially during the 
first and third trimesters, and can be observed in the ascending aorta flow rate waveforms in figures $7.7 \mathrm{a}$ and $7.7 \mathrm{c}$. The pressures in participant two are generally lower than participants one and three, particularly in the first trimester. Considering that the cardiac output is significantly higher and the pulse pressure is lower for participant two, this requires a significant increase in compliance, and decrease in total peripheral resistance for participant two compared with the other participants. The effect of this increased compliance can be observed from the low pulsatility index in the uterine artery shown in table 7.3, and in figure 7.8 for the brachial artery pressure waveforms. Participant two's waveforms are absent of notches which are observed for participants one and three in all three trimesters, although the notch is observed in the post-partum waveform. 

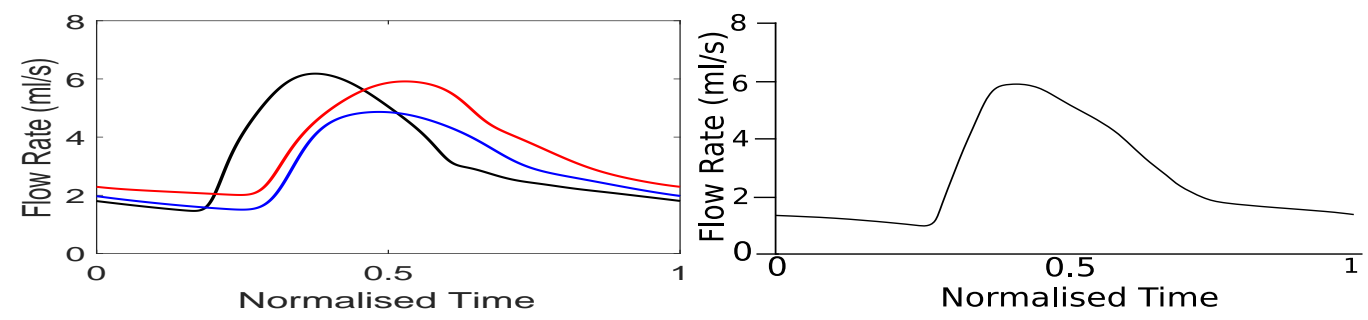

(a) First trimester

(b) Digitised Doppler first trimester [264]
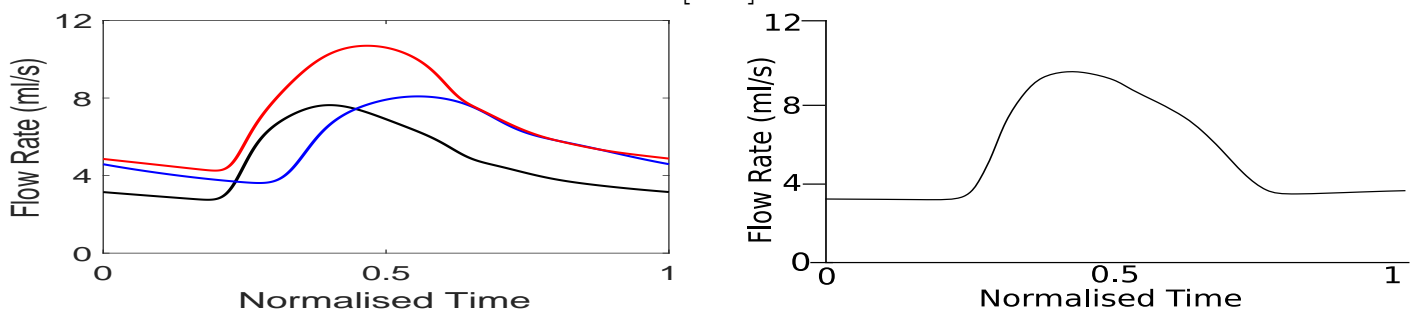

(c) Second trimester

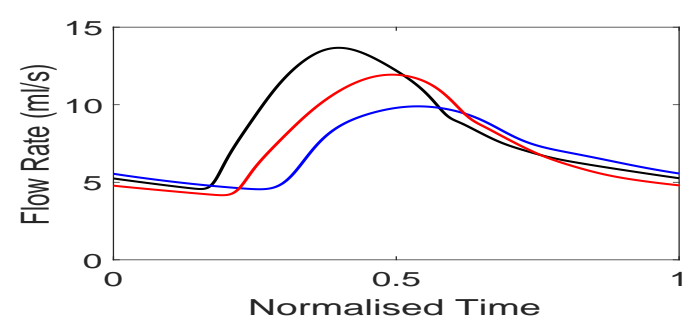

(d) Digitised Doppler second trimester [240]

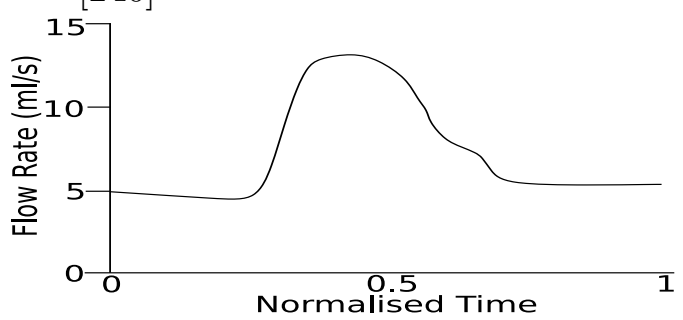

(e) Third trimester

(f) Digitised Doppler third trimester [137]

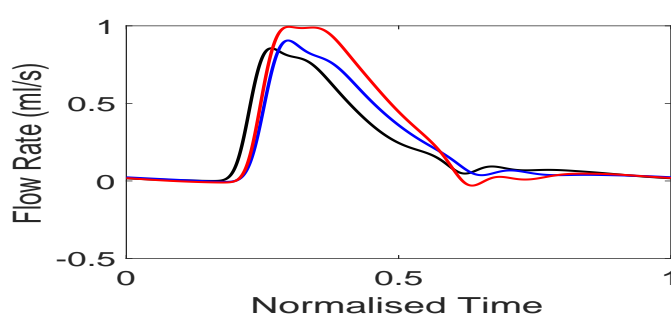

(g) Post-partum

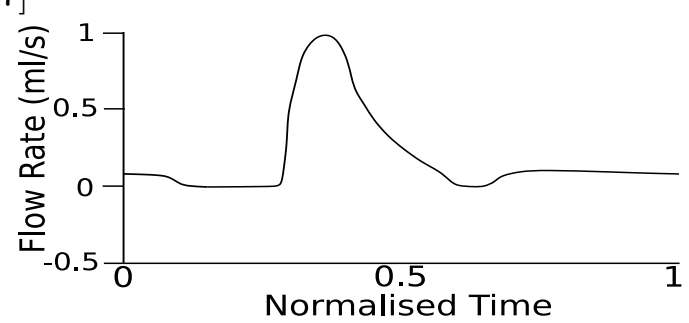

(h) Digitised Doppler non-pregnant [307]

Figure 7.5: Right uterine artery flow rate waveforms for three patients over the course of pregnancy, and post-partum, compared with in-vivo waveforms from literature. The black line is for participant one, the blue line is participant two, and the red line is participant three. Waveforms are normalised in time for comparison purposes. Digitised waveforms are scaled by physiologically realistic areas, as they are originally velocity waveforms for Doppler studies. 


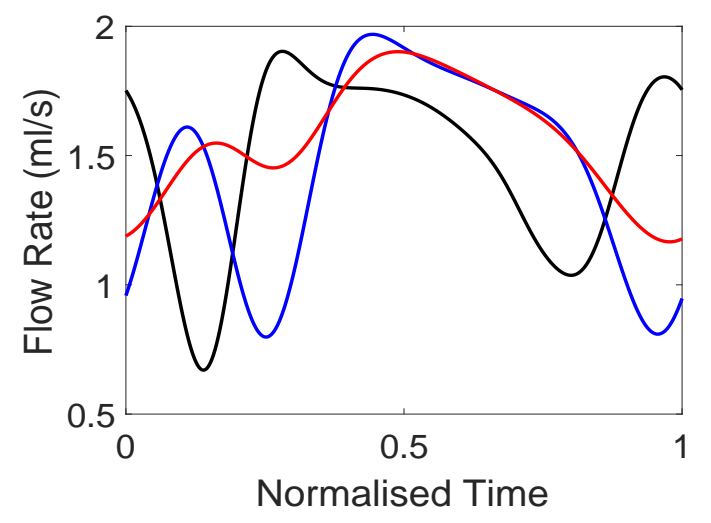

(a) First trimester

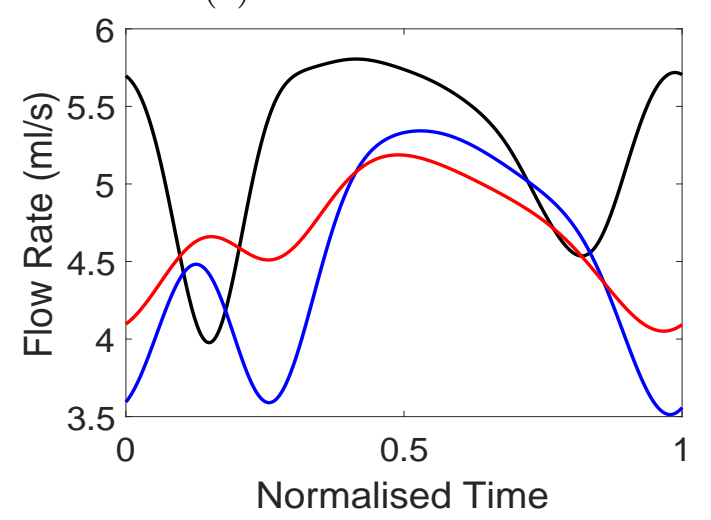

(c) Third trimester

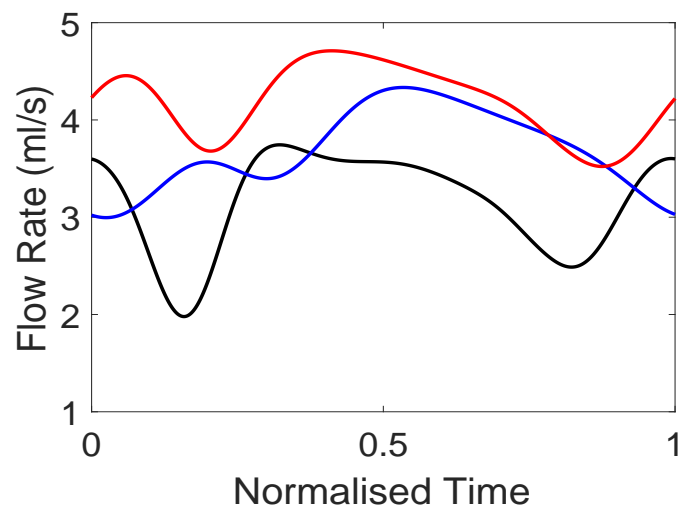

(b) Second trimester

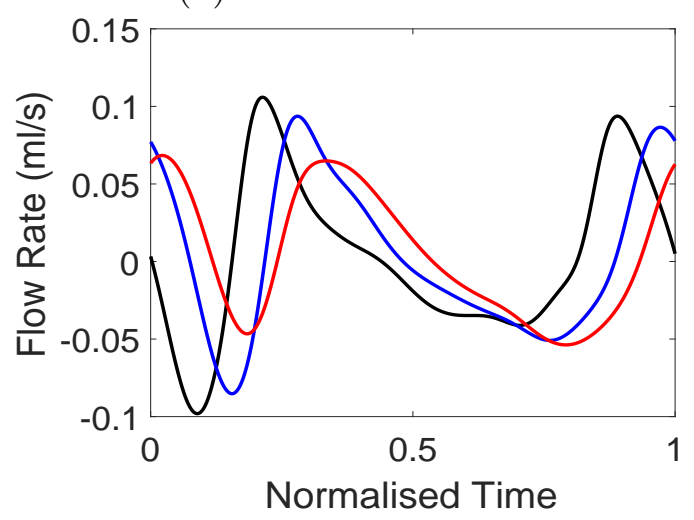

(d) Post-partum

Figure 7.6: Right uterine vein flow rate waveforms for three patients over the course of pregnancy, and post-partum. The black line is for participant one, the blue line is participant two, and the red line is participant three. 


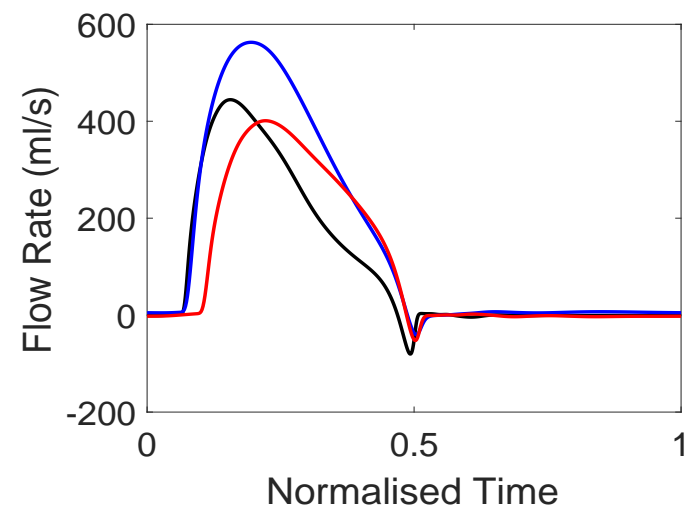

(a) First trimester

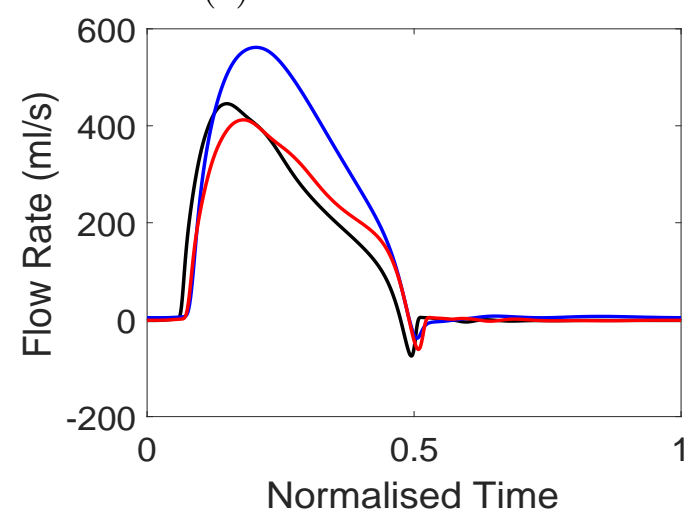

(c) Third trimester

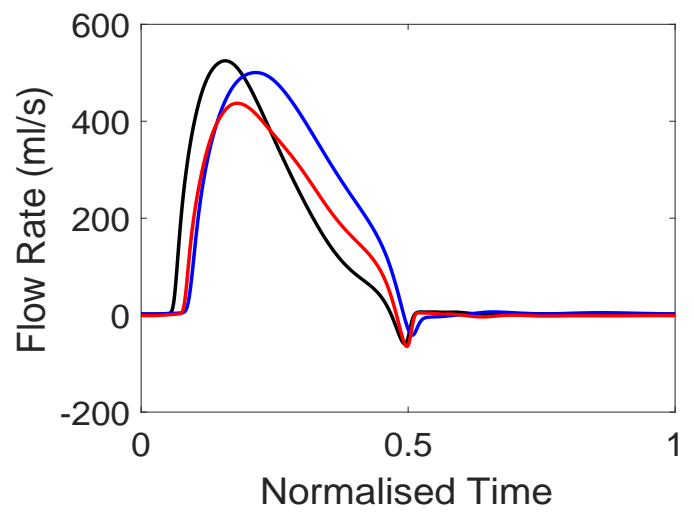

(b) Second trimester

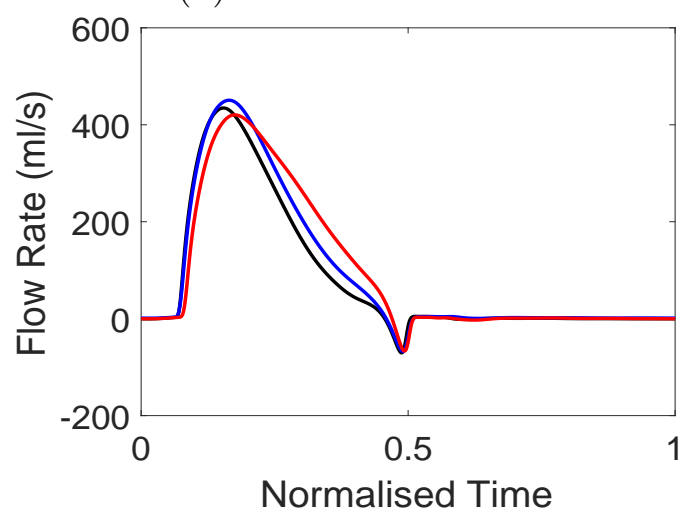

(d) Post-partum

Figure 7.7: Ascending aorta flow rate waveforms for three patients over the course of pregnancy, and post-partum. The black line is for participant one, the blue line is participant two, and the red line is participant three. 


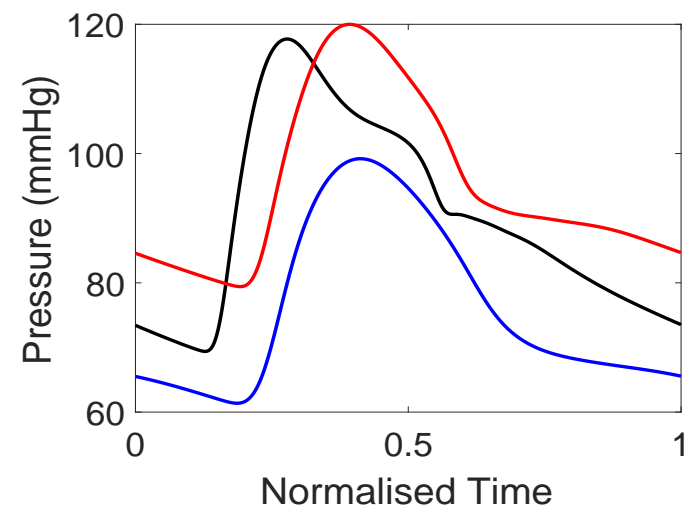

(a) First trimester

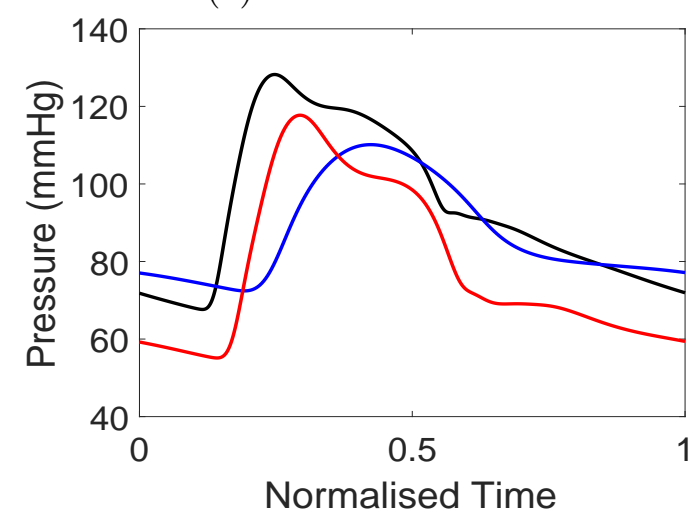

(c) Third trimester

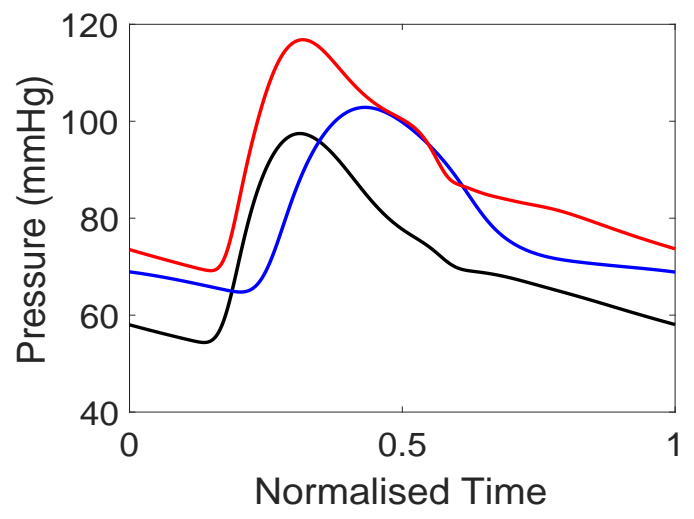

(b) Second trimester

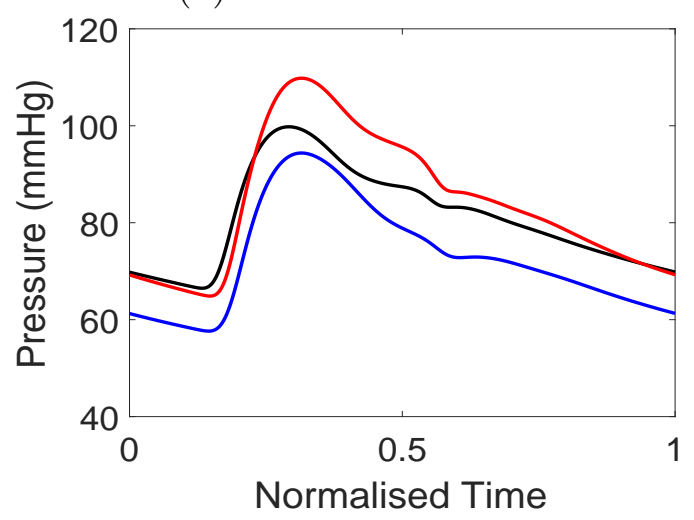

(d) Post-partum

Figure 7.8: Left brachial artery pressure waveforms for three patients over the course of pregnancy, and post-partum. The black line is for participant one, the blue line is participant two, and the red line is participant three. 


\subsection{An Idealised Pregnancy Model For All Weeks}

Although a patient specific model is the ultimate aim of this thesis, it is important to remember that measured patient data which has been collected retrospectively, rarely contains data which is perfect. Therefore, it is necessary to simulate an idealised model, for which the model can be run for all weeks in pregnancy, while patient measurements are only performed at three points in time over the course of their pregnancy. Hence, using published data from various studies, it is possible to construct a model which captures the general behaviour of a healthy pregnancy.

A difficulty in developing a full closed loop model is that the majority of studies contain measurements only in the systemic arterial system [25, 26, 58, 1, 124]. Although a few studies have investigated pulmonary hypertension in pregnancy [269, 174, 241]. The systemic venous system has been under-investigated, particularly as many health issues related to pregnancy are due in part to poor adaptation in the systemic venous system. Although other factors can result in pathology, such as in supine hypotensive syndrome, which is a phenomena seen in a minority of pregnant patients in the late second to third trimesters, where the uterus and foetus exerts pressure on the descending aorta and inferior vena cava, lowering venous return (and hence cardiac output), which has a knock-on effect of reducing mean arterial pressures.

In this section an idealised model will be implemented, with expected changes of the maternal system being imposed on the model. This allows an investigation of the adaptation over all weeks of pregnancy. The model is tested for a different rates of adaptation in the UOCA, which will give an indication to how sensitive flow rate through this vessel is on the vessel lumen size, particularly as attempts at measuring blood flow through this additional pathway to the uterus have been unsuccessful $[262,47]$. Finally a sensitivity analysis is performed for several model parameters.

The model primarily uses an expected increase of blood volume as an estimation of how much the maternal cardiovascular system is required to adapt between nonpregnant conditions and term of pregnancy, which is considered to be week 40 . The predicted increase in parameters such as stroke volume and heart rate, and the rate of adaptation of vessel diameter sizes, follows the curve presented in [155], 


\begin{tabular}{|c|c|c|c|}
\hline Cardiac Output & HR & Diastolic Pressure & Systolic Pressure \\
\hline $5.9-8.55501 \mathrm{~min}^{-1}$ & $75-90 \mathrm{BPM}$ & $70 \mathrm{mmHg}$ & $115 \mathrm{mmHg}$ \\
\hline
\end{tabular}

Table 7.4: Initial parameter values for the non-pregnant case to week 40 of pregnancy.

which is shown in figure 7.3. The problem parameters are described in table 7.4. The parameters are chosen via the mean of the measured data, and are also in agreement with published values $[281,312,150]$. The simulations are performed until the difference between model parameters and the aimed input parameters (systolic and diastolic blood pressure, and cardiac output) is less than $1 \%$.

\subsubsection{Simulation Results}

The overview of results for the simulation of non-pregnant conditions and all weeks of pregnancy is shown in figure 7.9. The effectiveness of the parameter estimation technique can be observed in figures 7.9a and 7.9c.

The models predicted total peripheral resistance decreases by approximately 32 $\%$ from non-pregnant conditions to term of pregnancy, which is in agreement with literature estimations [150] which gives a range between $25-35 \%$ during a healthy pregnancy.

Due to an increase in cardiac output during pregnancy, the body needs to adapt its compliances in order to keep pulse pressures relatively consistent over the course of pregnancy. The total arterial compliance (TAC) predicted by the model for all weeks of pregnancy is shown in figure 7.10c. The TAC percentage increase predicted by the model from the non-pregnant case to term is approximately $39 \%$, which is in agreement with the estimate from [150] of between $30-45 \%$.

The PI is compared with published data from [137] and shows excellent agreement with the behaviour from week 11 through to week 40, however, the study did not include data from before week 11 . Another study showed reference ranges for healthy non-pregnant women of $\mathrm{PI}=2-6$ [144], though this still means there is missing data for early pregnancy. The aortic pulse wave velocity predicted by the model shows a similar behaviour to that shown in literature [251], although has significantly different magnitudes compared with the estimation in literature, as the clinical estimation used a brachial-femoral method. The differences in the brachial- 


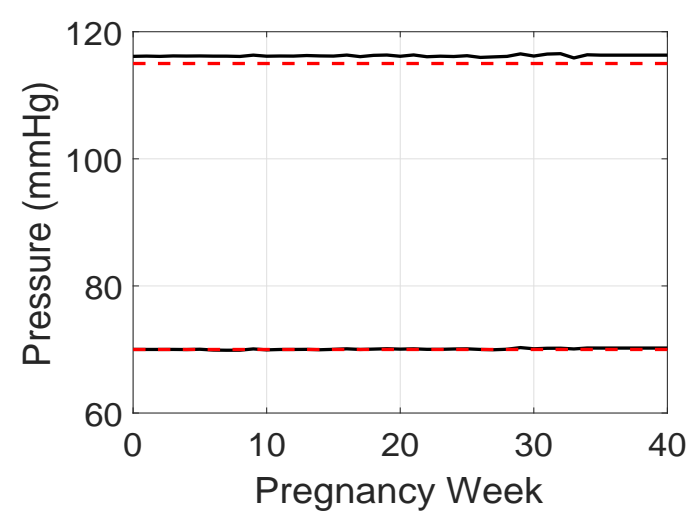

(a) Comparing systolic and diastolic pressures in left brachial artery of the model output against idealised data

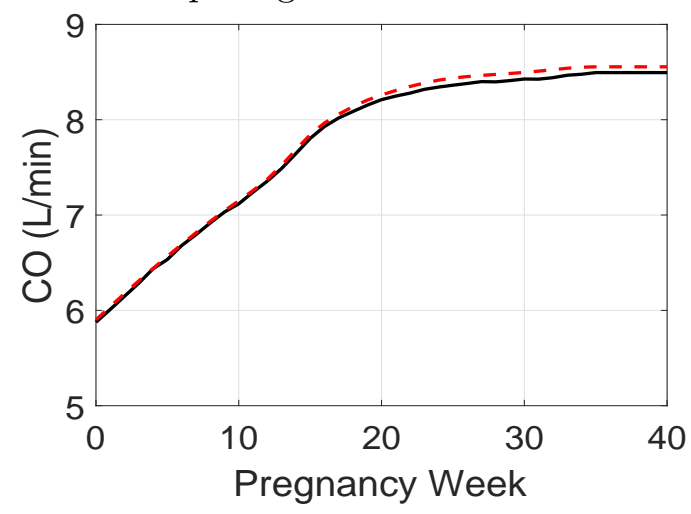

(c) Comparing modelled cardiac output against idealised data

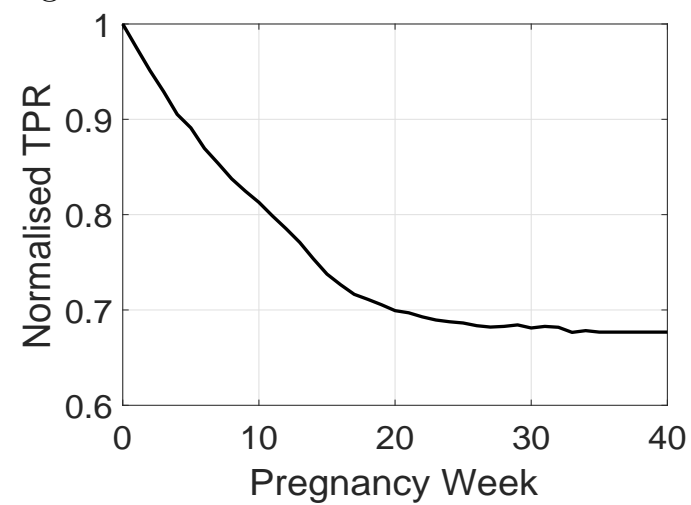

(e) Total peripheral resistance

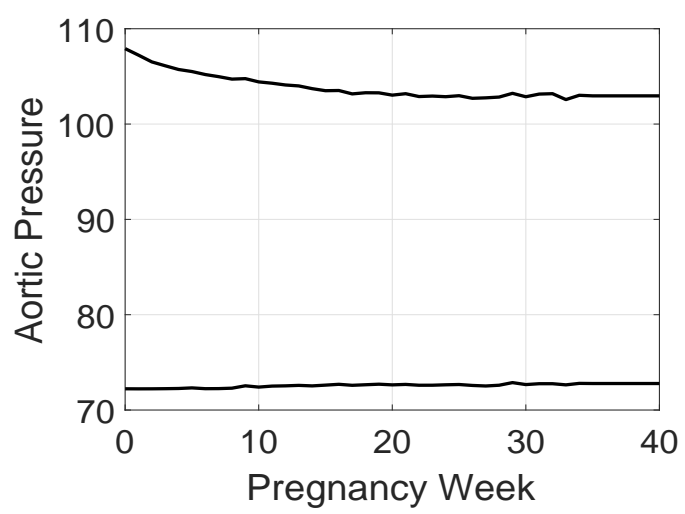

(b) Comparing systolic and diastolic pressures in the ascending aorta of the model

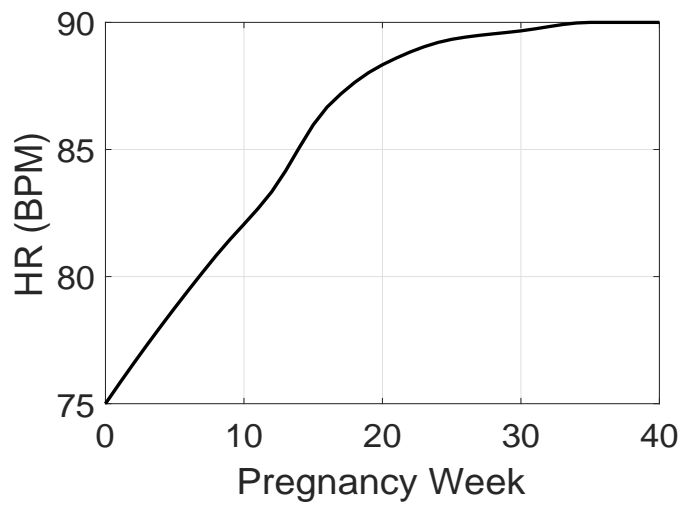

(d) Idealised heart rate change over the course of pregnancy

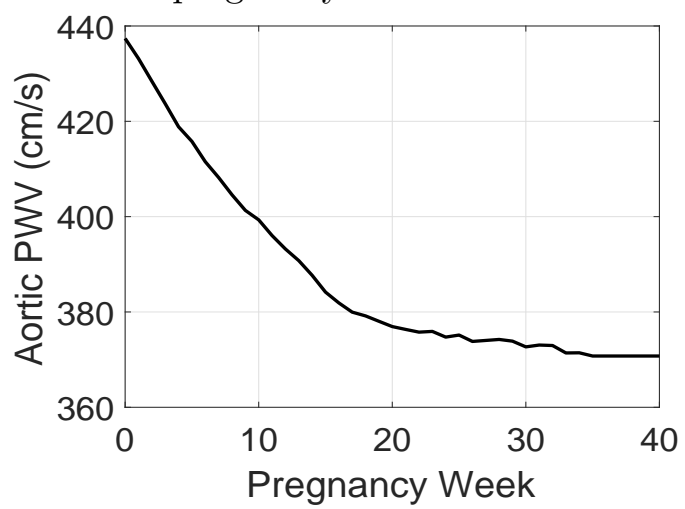

(f) Aortic pulse wave velocity

Figure 7.9: Comparison of model outputs with idealised data. The aimed parameters which the parameter estimation technique converges towards are shown by the red dashed line, where applicable. 


\subsection{AN IDEALISED PREGNANCY MODEL FOR ALL WEEKS}

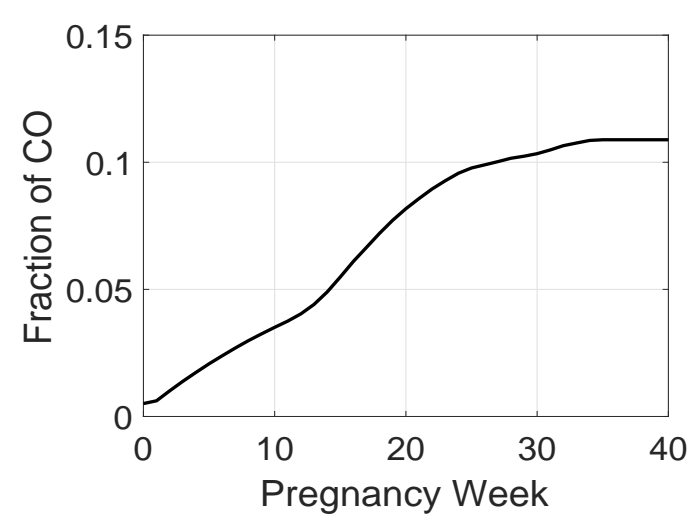

(a) Percentage of cardiac output to uterus via the uterine arteries (bilateral)

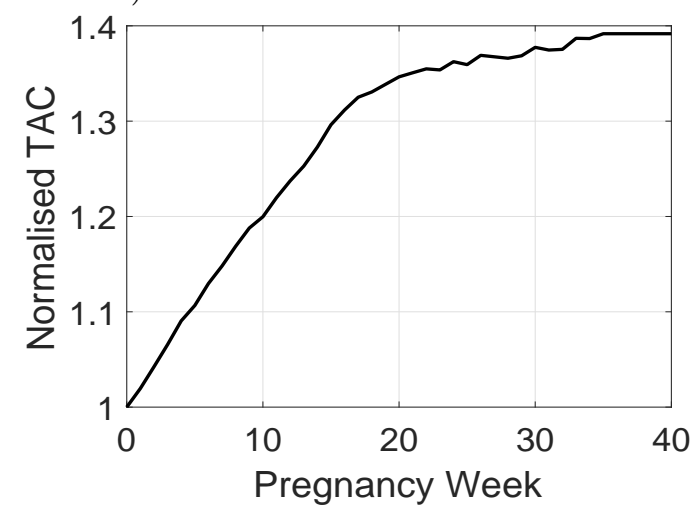

(c) Total arterial compliance over the course of pregnancy.

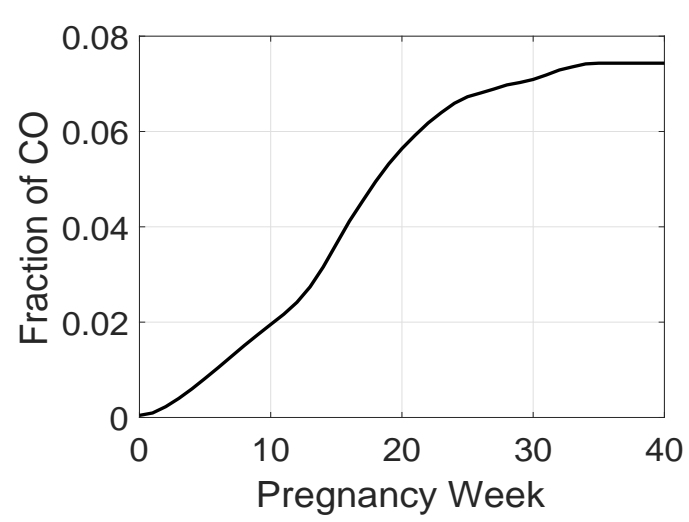

(b) Percentage of cardiac output to uterus via the ovarian arteries (bilateral)

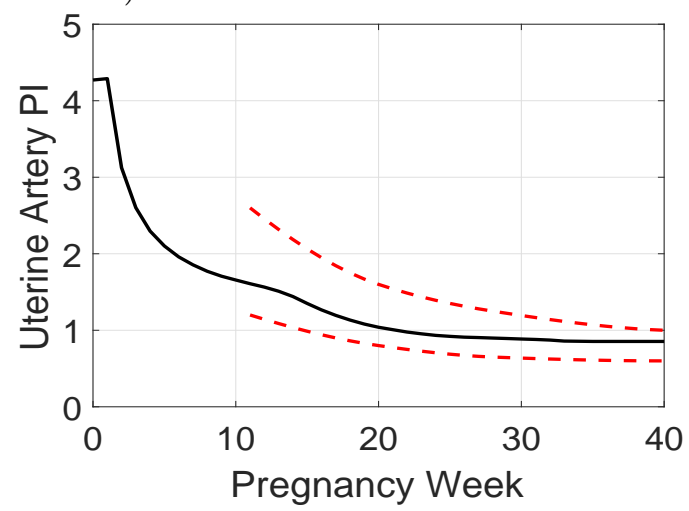

(d) Uterine artery pulsatility index, red dashed line is the $5^{\text {th }}$ and $95^{\text {th }}$ centile from $[137]$

Figure 7.10: Comparison of model outputs with idealised data, and convergence behaviour.

femoral estimation of the model, and the aortic PWV is discussed in section 7.7.1 for the measured data cases. Furthermore, in [251] the PWV increases from the second to third trimester, while the model predicts a small decrease. This could be attributed to the models assumption that the pressure remains consistent during pregnancy, while in reality there is often a decrease in mean arterial blood pressure during the first and second trimesters (where PWV will decrease), followed by an increase in mean arterial blood pressure towards term (where PWV will increase).

The model prediction for the combined percentage of cardiac output reaching the uterus from the two uterine arteries, shown in figure 7.10a, is close to the expected 


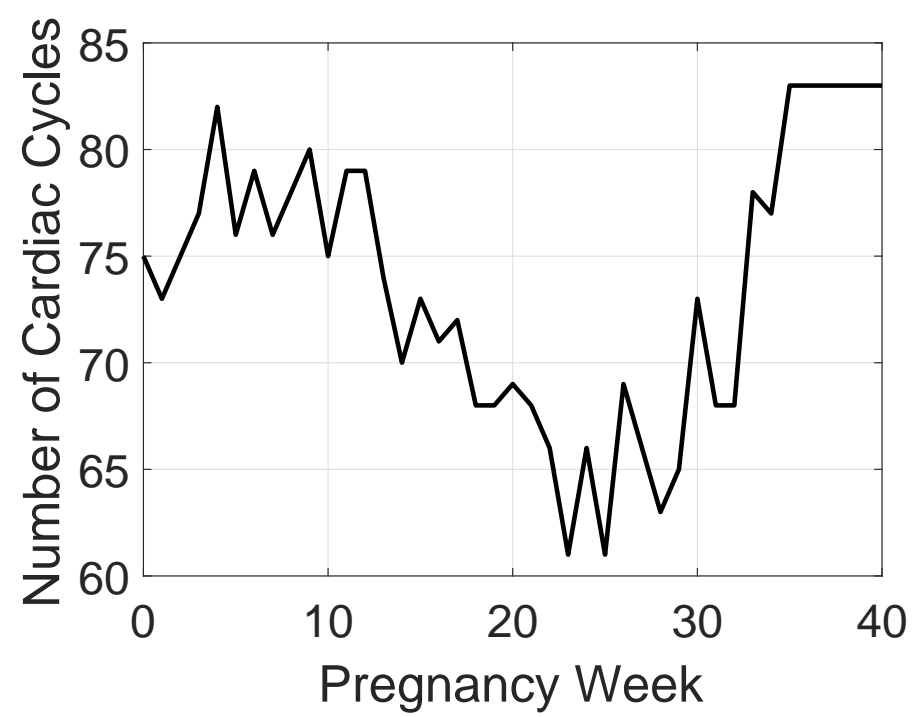

Figure 7.11: Number of cardiac cycles required to achieve convergence to the idealised data input and periodic convergence

range given in literature, from $0.5 \%$ [249] in the pre-pregnant simulation, to $11 \%$ at week $40(\approx 12 \%$ in [340]). The model-predicted mean flow rates through the uterine arteries reaches $917 \mathrm{ml} \mathrm{min}^{-1}$, which is in the physiologically expected range $[249,178]$. The model-predicted percentage of cardiac output which travels via the ovarian arteries and UOCA reaches an additional 7.6 \%, and its change over the 40 weeks of pregnancy is shown in figure $7.10 \mathrm{~b}$. It is unknown exactly how much blood should travel via this pathway as previous attempts to measure it have failed [262, 47]. However, it has been mention in [52] that these vessels are capable of supplying the uterus with its full blood supply needs if the diameter increases to match the diameter of the ascending uterine artery (as assumed in the model). The estimated blood supply which the uterus needs is $450-800 \mathrm{ml} / \mathrm{min}$ [249], while the combined left and right UOCA flow rate predicted by the model is $626.3286 \mathrm{ml} \mathrm{min}^{-1}$, and hence is in the correct range.

The number of cardiac cycles needed to achieve convergence to the aimed input variables via the automated parameter estimation algorithm is shown in figure 7.11. The model generally takes approximately 75-80 cardiac cycles to converge, however each cardiac cycle only takes approximately $12-18$ seconds (depending on heart rate). It may be possible to improve the convergence behaviour, but this is not the focus of the thesis and is left for potential future work. 


\subsubsection{Changes in Vessel Adaptation}

In this section two additional cases are considered. The first case involves the adaptation of the utero-ovarian communicating artery. Information on this artery is generally quite scarce, as it is very small before pregnancy, with a diameter of approximately $0.5 \mathrm{~mm}$. However, the vessel can increase its diameter by approximately 5 times its pre-pregnancy size by the third trimester, which means its diameter can be approximately the same as the ascending uterine artery. When this occurs it is expected to be capable of supplying the uterus with its full blood supply needs [52]. In the previous section, the model-predicted flow rates through the utero-ovarian communicating arteries almost reached the values seen in the uterine arteries, which are normally the primary vessels of blood transport to the uterus. Hence, it would be useful to understand how the flow to the utero-ovarian vessel changes if it does not adapt to its maximum potential lumen size. The cases considered are from week 8 and week 40 in the idealised pregnancy model. The first case considered a symmetric arterial network.

Figure 7.12 shows the flow rates in the left utero-ovarian communicating artery and the left uterine artery. The three cases considered are: the baseline case, where the area of the utero-ovarian artery is the same as the ascending uterine artery; a case where the utero-ovarian artery has only undergone half of the adaptation so its diameter is half its baseline value; the utero-ovarian artery area only reaches a quarter of its baseline value.

The combined left and right volumetric flow rate which travels through the uteroovarian arteries is reduced significantly when the vessel lumen area is reduced. The percentage of cardiac output which travels via these utero-ovarian arteries is reduced from the baseline value of $7.43 \%$, to $4.31 \%$ when the area is reduced by half, and finally to $2.01 \%$ when the area is a quarter of the baseline value. The flow rate in the uterine artery does not change significantly between the three cases considered, with only a slight increase in flow rate when the utero-ovarian artery is smaller. The percentage of cardiac output through the uterine arteries varies from $10.89 \%$ for the baseline, through to $11.24 \%$ for the most restricted case $(A / 4$ in the figures). Indicating that only a small proportion of the extra flow, created by a reduced volume through the utero-ovarian vessels, is redistributed to the uterine arteries.

For the asymmetric cases, the vessels in the left side of the network have been 


\subsection{AN IDEALISED PREGNANCY MODEL FOR ALL WEEKS}

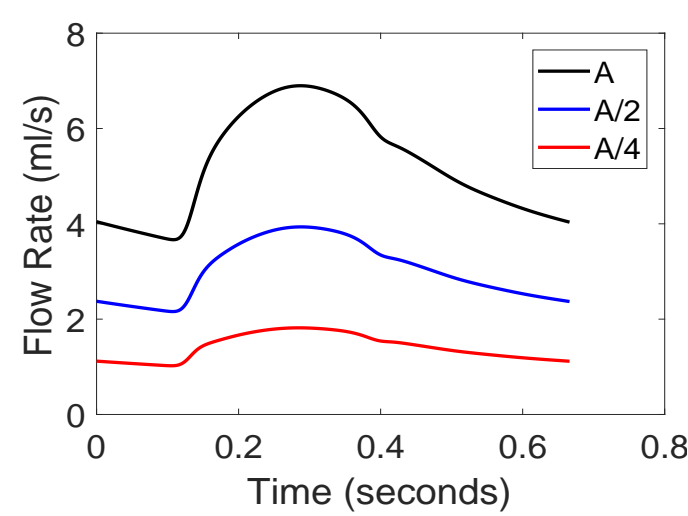

(a) Flow rate in the utero-ovarian communicating artery.

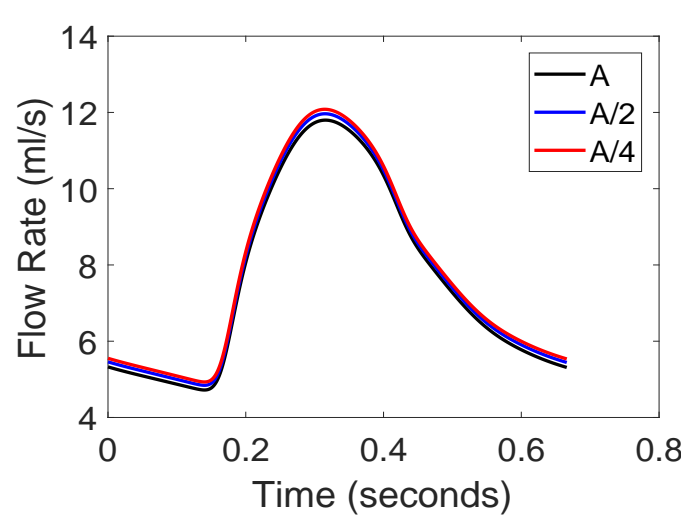

(b) Flow rate in the uterine artery

Figure 7.12: Volumetric flow rates in vessels toward the uterus for the following cases: the utero-ovarian communicating artery has the same area as the ascending uterine - $\mathrm{A}$, has half the original area $-\mathrm{A} / 2$, and has a quarter of the original area $\mathrm{A} / 4$.

assumed to adapt half as much as the right sided vessels. Figure 7.13 show results for a symmetric and asymmetric adaptation of the uterine vessels. The normalised flow rate waveforms are quite similar, with the solution for the asymmetric case showing slightly more definition with notching in the waveform shown in $7.13 \mathrm{a}$, however the majority of these differences are only seen in the week 8 simulation. For the week 40 case, the increased $1 \mathrm{D}$ vessel stiffness affects the flow rate waveforms to a much less extent, which indicates the downstream compliance (compliance of the placenta and uterus vascular beds) has a dominant effect on the solution.

For the asymmetric case the percentage of cardiac output through the left and right uterine vessels varies significantly. In the week 8 simulations the left uterine artery receives $0.59 \%$ with a $P I=2.0469$, while the right uterine artery receives $1.56 \%$ of cardiac output with a $P I=1.7131$. For week 40 the pulsatility index is similar for both left and right sides with 0.8372 in the right side and 0.8271 in the left side, once again indicating that the downstream compliance is dominant; nevertheless, significant differences are observed in the percentage of cardiac output through each vessel, with $5.7 \%$ travelling through the right side, and only $2.74 \%$ travelling through the left uterine artery. 


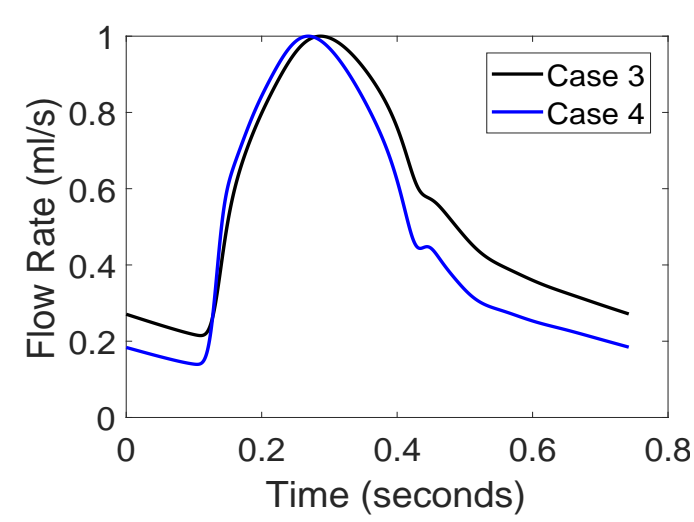

(a) Normalised flow rate in the left uteroovarian communicating artery in gestational week 8 .

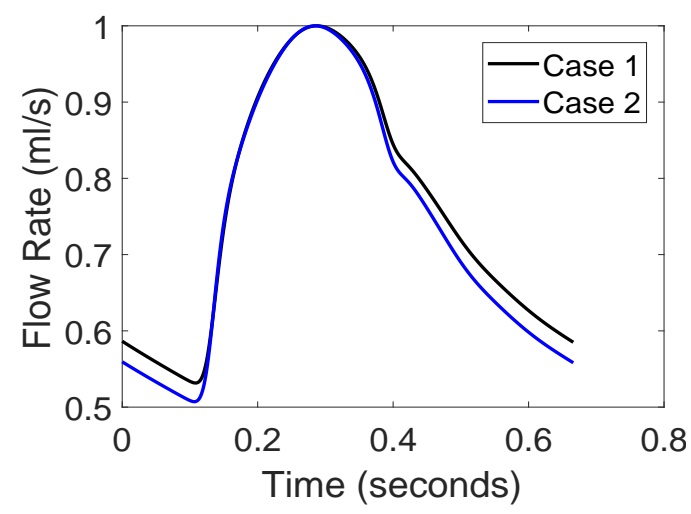

(c) Normalised flow rate in the left uteroovarian communicating artery in gestational week 40 .

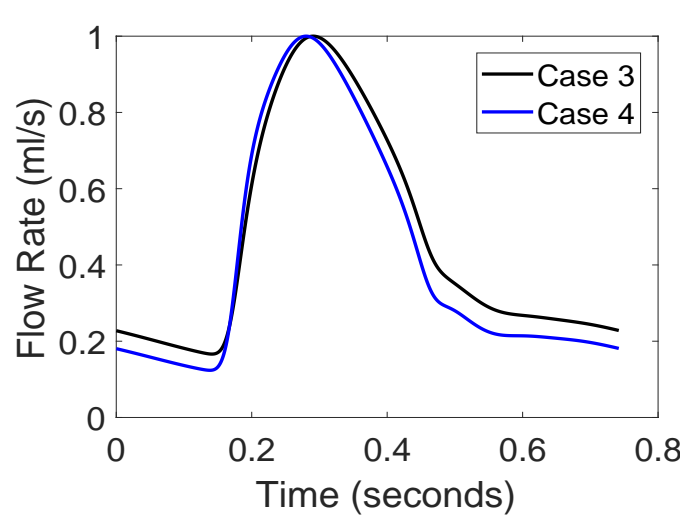

(b) Normalised flow rate in the left uterine artery in gestational week 8 .

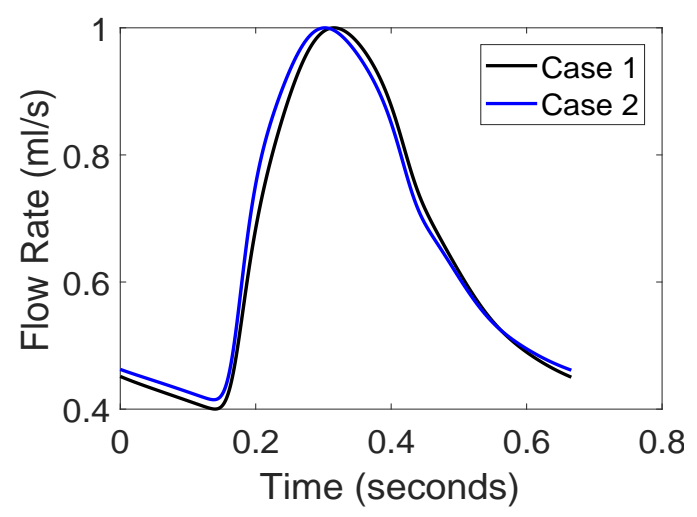

(d) Normalised flow rate in the left uterine artery in gestational week 40.

Figure 7.13: Comparison of flow rate waveforms in vessels carrying blood to the uterus for symmetric (case 1 and 3) and asymmetric (case 2 and 4)adaptation

\subsubsection{Sensitivity of Parameters}

In this section several cardiac and haemodynamic parameters are perturbed in order to test the models sensitivity to various estimated parameters and initial conditions. As the primary region of interest is in the quantification of solutions in the uterine circulation, the uterine artery and utero-ovarian communicating artery flow rates are investigated, along with pressure in the brachial artery, which is where the measured data pressure readings were taken. The sensitivity test is performed by increasing or reducing the parameter by $\pm 5 \%$ of their baseline values. The baseline values are the simulations at week 40 for the idealised model given in table 7.4. For the 
sensitivity analysis only solutions in the systemic arteries are considered, as this is where the measurements were performed for the participants. The following are perturbed:

- the aimed cardiac output, diastolic blood pressure and systolic blood pressure;

- the cardiac parameters of the left ventricle: $E_{\max }, \tau_{1}, \tau_{2}, m_{1}$, and $m_{2}$, which affect the contractility behaviour.

- the initial compliance estimation, which will affect the models compliance distribution;

- and the initial pressures in the entire system, which will change the initial blood volume in the system, and in turn will effect the vascular bed resistance calculation, which depends on both the initial and current pressures in the system.

Figure 7.14 shows the mean absolute percentage errors for flow rates in the UOCA and UA, and pressures in the brachial artery. The aimed parameters, which are the parameters for which the automated parameter estimation converges towards, are shown in figure 7.14a. Changing the aimed cardiac output has little impact on the flow rates in the uterine vessels, or pressure in the brachial artery. Changing the aimed systolic and diastolic pressures in the brachial artery has a much greater impact on the flows in the uterine vessels, and of course the mean pressure in the brachial artery itself. The results indicate that the input parameters of systolic and diastolic blood pressures have a greater impact on the solution than changing the cardiac output. This is due to the treatment of vessel compliance in the parameter estimation technique, which estimates the change needed in the total arterial compliance from the pulse pressure at the end of cardiac cycle, and modifies the compliances of all vessels equally to achieve the measured pulse pressure.

In the remainder of the results the aimed cardiac output, systolic, and diastolic blood pressures are unchanged. Thus it is expected that the mean pressures in the brachial artery will vary by only a small amount. The effect of changing various cardiac parameters is shown in figures $7.14 \mathrm{~b}$ and $7.14 \mathrm{c}$. The results deviated from the baseline solution by less that $1 \%$ for all cardiac parameters tested. This indicates that changing the various cardiac parameters has a minor effect on the solution of flow rates in the uterine vessels, and pressure in the brachial artery. 


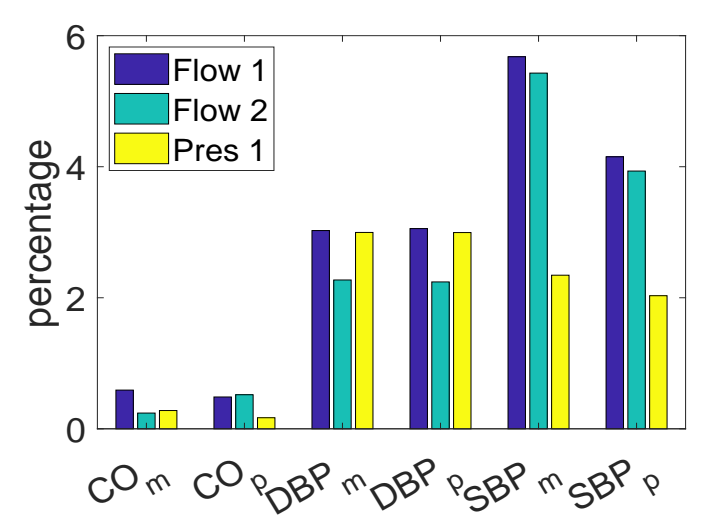

(a) Aimed input parameters

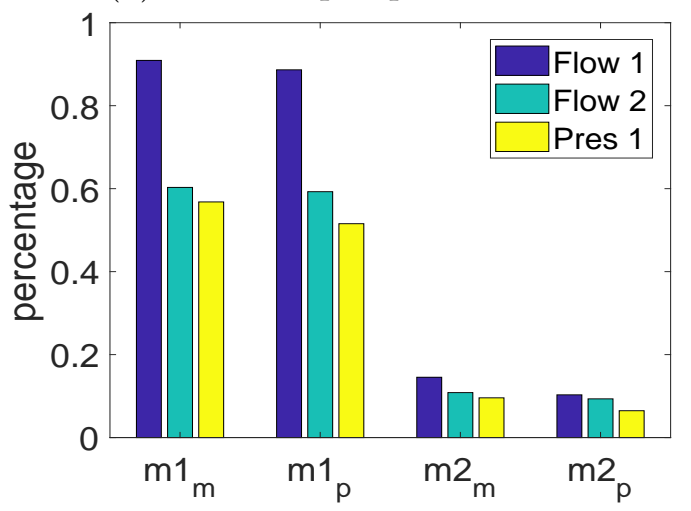

(c) Cardiac parameters

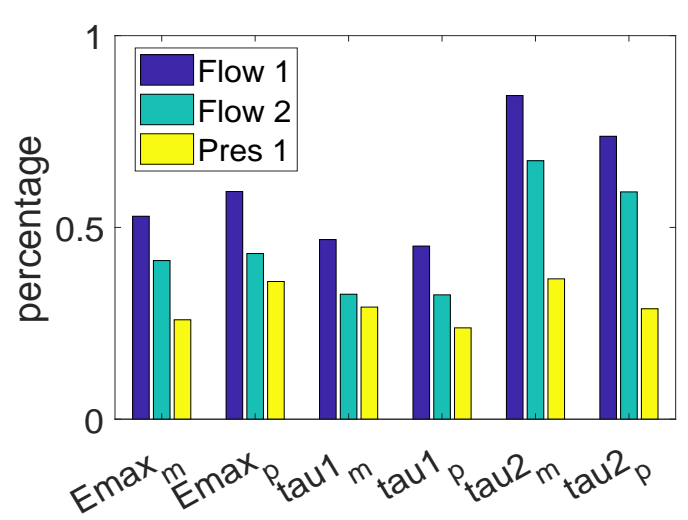

(b) Cardiac parameters

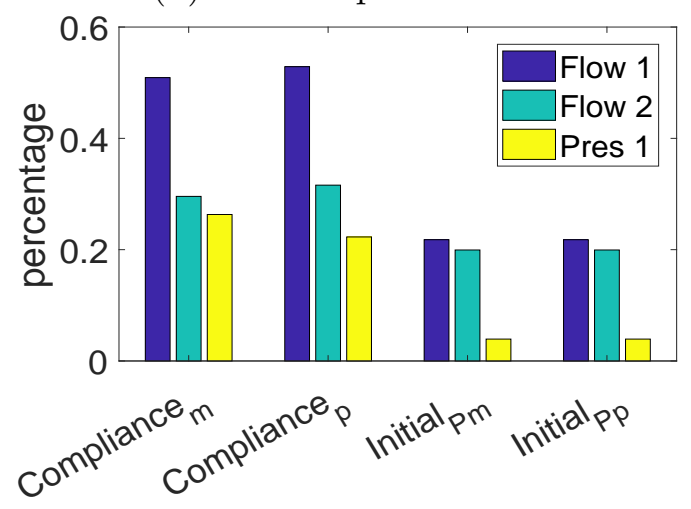

(d) Initial compliance and pressures

Figure 7.14: Mean absolute percentage error in flow rates through the utero-ovarian communicating and uterine arteries, and pressures in the brachial artery. Subscripts $p$ and $m$ in the figures indicate whether the parameter is perturbed by an increase, or decrease respectively.

Figure 7.14d shows the impact of the total arterial compliance estimation, and the estimation of initial pressures in the system. Changing the initial estimation of compliance will also change the distribution of compliance in the arterial system. The results indicate that the model is relatively insensitive to the changes in the estimation of total arterial compliance, and of the defined initial blood pressures.

The volumetric flow rate waveforms in the UOCA and UA are shown in figures $7.15 \mathrm{a}$ and $7.15 \mathrm{~b}$, while the pressure waveforms in the brachial artery is shown in 7.15c. The overall shape of all waveforms are very similar, and for the majority of cases the magnitudes of the waveforms are in good agreement. More significant differences in the magnitudes of the waveforms are observed for the perturbations of the input parameters for diastolic and systolic blood pressures, which will impact 


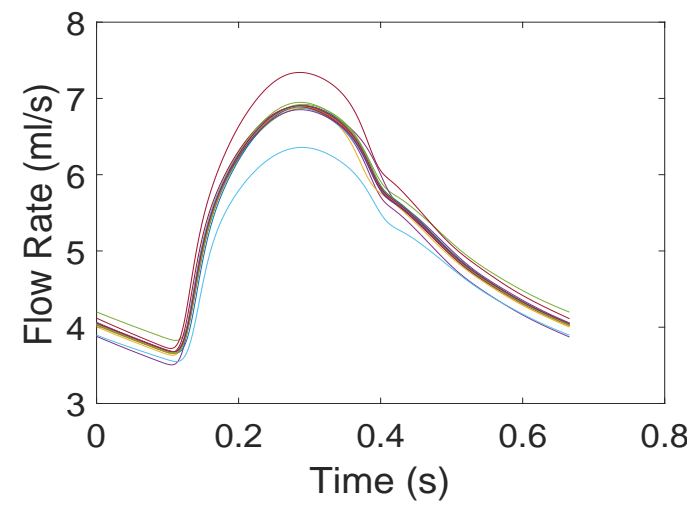

(a) Flow rate in UOCA

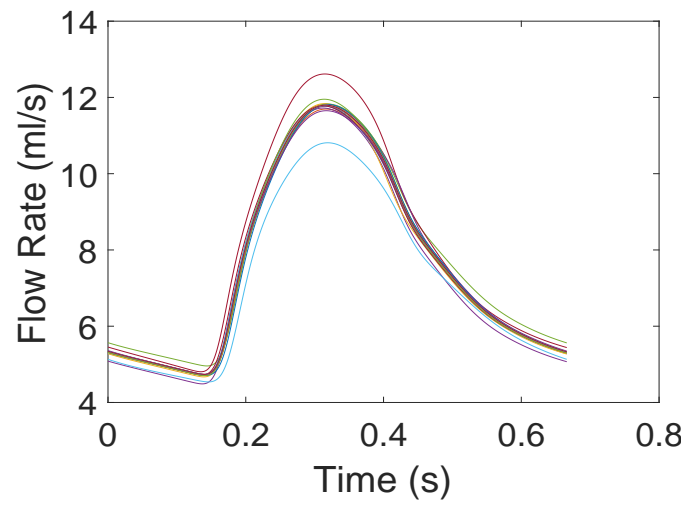

(b) Flow rate in UA

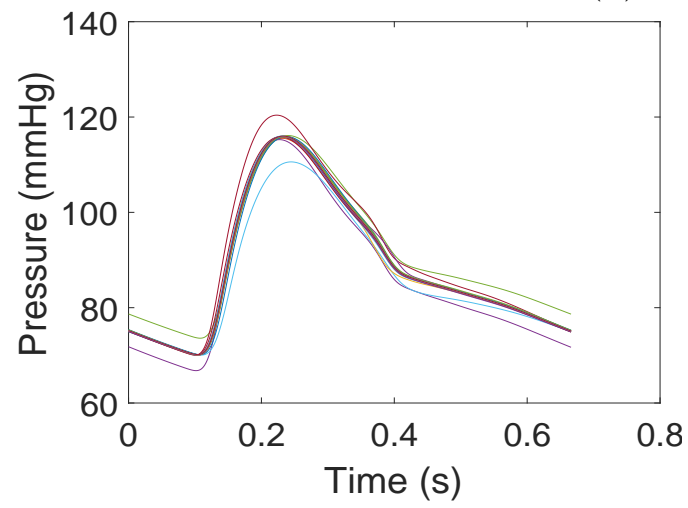

(c) Brachial artery pressures

Figure 7.15: Comparison of flow rate waveforms in the utero-ovarian communicating arteries and uterine arteries, and pressure waveforms in the brachial artery

the estimation of compliance in the entire circulation.

\subsection{Limitations}

The sensitivity analysis performed in this chapter was used to test the effectiveness of the initial and adaptive parameter estimation techniques. This is used to investigate uncertain cardiac parameters within the model that are likely to affect the pressure and flow rate solutions. However, in order to fully appreciate the model, a sensitivity analysis involving mutual variations of parameters must be performed, as this could lead to different results. In addition, a more comprehensive sensitivity analysis could help indicate which parameters are essential for clinicians to measure, which would also provide a greater confidence in the model-predicted solutions. 
In this study a general network was implemented for all patient cases as no vessel network information was available. A sensitivity analysis on the vessel lengths and sizes within the network could also be performed to show whether the assumption of a general network is acceptable.

In this study, no uterine artery Doppler waveforms were available for the participants. In order to have predictive value and to detect pathologies, a further study involving patient uterine artery Doppler waveform data, must be performed.

\subsection{Conclusions}

The automated parameter estimation technique employed was found to be very effective at converging to the in-vivo measurement data, taking between 62 and 83 cardiac cycles to converge to within $1 \%$ of the measured cardiac output, systolic pressure, and diastolic pressure. After the model's convergence to this measured data was achieved, the model solutions of pulsatility index, pulse wave velocity, and expected percentage of cardiac output in the uterine vessels, showed good agreement with the values and observed behaviour from literature.

The model was able to give an estimate for the volume of blood that reaches the uterus via the utero-ovarian communicating arteries. This could have a clinical significance, as currently it has not been possible to measure flow in these arteries due to the complex anatomical configuration of that region. Therefore the total blood reaching the uterus from the major vessels can be found via the model, but not via any current clinical measurements. The model indicated that approximately $7.43 \%$ of the cardiac output would reach the uterus from these vessels, if the diameter of the utero-ovarian communicating arteries increases to equal the diameter of the uterine arteries. This percentage decreases to $4.31 \%$ if the diameter of the utero-ovarian communicating arteries increases to be half the diameter of the uterine arteries, and $2.01 \%$ when the diameter of the utero-ovarian communicating arteries reaches a quarter of the uterine arteries diameter. The volumetric flow rate to the uterus via the uterine artery was insensitive to the change of diameter of the utero-ovarian communicating arteries, varying between $10.89-11.24 \%$ of cardiac output over the three different diameters of the utero-ovarian vessels. This suggests that if the uteroovarian arteries reach the diameter of those in the uterine arteries, approximately 
$20 \%$ of cardiac output reaches the uterus.

The model will need to be further validated through a comparison of patient specific waveforms, however, the waveforms are similar to those seen in published Doppler studies. In the future the model may be able to aid patient treatment planning by determining which pregnant woman are at a high risk of developing pathologies, such as pre-eclampsia and placental insufficiency; and be able to determine whether a sufficient volume of blood is reaching the placenta and foetus to ensure a healthy level of growth. If this can be achieved in the future, it would be a ground-breaking development, as currently age and patient history are the main clinical methods to determine patients with a high-risk of developing a pathology. The patient history utilised involves any pre-existing cardiovascular disease was present, or if the pregnant woman developed pathology in previous pregnancies. There are no current methods of predicting the development of a pathology in later pregnancy for patients with no previous history of being pregnant, or no previous pathology that could affect the outcome of the pregnancy.

The results of the sensitivity analysis indicate that the model is most sensitive to the input parameters provided by the measured data, specifically the blood pressure measurements, and is much less sensitive to the model's initial conditions. This is a direct result of the model's parameter estimation technique, which assumes the distribution of compliance in the 1D network does not change during a simulation, so when the compliance is adapted to achieve the measured pulse pressure, the compliance is changed everywhere in the systemic arteries. Overall the model's performance in modelling the various aspects of pregnancy was very satisfactory.

The work in this chapter can provide a foundation for future development and improve understanding of the mechanisms involved in the adaptation of the maternal cardiovascular system during pregnancy. The model developed successfully utilised a parameter adaptation algorithm to converge to participant measurements. This iterative scheme has the potential to be used in many areas of cardiovascular modelling, not just for pregnancy. Such a parameter estimation technique could also be utilised for open-loop systems, thus if only the arterial system is of interest, then the remainder of the network could be neglected, while the adaptive parameter estimation technique could be utilised to predict participant resistances and compliances within the network. The parameter estimation technique could also be used to aid the modelling of several clinical procedures and diagnostic tools, such as: 
fractional flow research (FFR), which is a measurement of the pressure ratio across a coronary stenosis that estimates how severe the vessel narrowing is on coronary function; haemodialysis, where a machine extracts and cleans the blood of patients whose kidneys are not functioning effectively; baro receptor reflex sensitivity, which indicates the body's ability to adapt to posture change and exercise; and to investigate the precurser to haemodialysis, by modelling the kidneys regulation of fluid volume, and in predicting fluid change in the body due to a decrease in kidney function. 


\section{Chapter 8}

\section{The Lymphatic System}

\subsection{Introduction}

The lymphatic system is one of the lesser known and under-appreciated systems in the human body. However, as the various functions of the lymphatic system are being learned and understood, the importance of this still relatively unknown system is steadily growing $[79,280]$. The lymphatic system is often considered to be constructed from two semi-independent components: a network of lymphatic vessels; and lymph tissues and organs, such as lymph nodes, the spleen, tonsils. Unlike the cardiovascular system, the lymphatic network is: an open-looped system, as the initial lymphatics drain fluid from body tissues, while the outlet of the lymphatic system drains directly into the systemic venous system (mainly into the subclavian veins in the neck); and is a uni-directional system as valves present in the large lymphatic vessels prevent back flow [202] .

Fluid from the arterial side of the capillary beds in the cardiovascular system is forced out of the vessels due to hydrostatic pressure, while oncotic pressures, which are pressures primarily exerted by proteins, cause most of this fluid to be re-absorbed into the venous side of the capillary beds; however some fluid remains within the interstitia [360]. The primary role of the lymphatic system is to drain this interstitial fluid and return the fluid back to the cardiovascular system. Although the amount of fluid the lymphatic system carries varies significantly with estimates between 1 litre per day [308] and 12 litres per day [305].

In addition to returning leaked fluid to the cardiovascular system, the lymphatic system plays an important role in protein and ion transport [79] and has a critical role in supporting the immune system [280], both in fighting infection and disease.

If the lymphatic system is unable to provide sufficient fluid drainage of the interstitia then swelling will occur. This pathology is known as oedema, although there are many different types depending on the location or cause of the swelling. For example, pulmonary oedema occurs if there is excess fluid retention in body tissue around the lungs, while peripheral oedema refers to excess fluid in the legs or arms. The term lymphedema is used to describe oedema which has occurred as a result 
of damage to the lymph vessels or lymph nodes. Lymphedema can occur in cancer patients who have undergone either surgical treatment to remove a lymph node, or radiation therapy [168].

During pregnancy approximately $80 \%$ of woman develop peripheral oedema [94], and also have a greater risk of developing pulmonary oedema. It has been proposed that the lymphatic system may play an important role at the maternal-foetal interface as significant lymphangiogenesis (creation of new lymphatic vessels) occurs in the region near the placenta. The role of the lymphatic system near the placenta is still unknown, but may be to prevent excess fluid build up in the nearby body tissues, which could add pressure to the growing foetus [282].

\subsubsection{Lymph}

The fluid carried by the lymphatic is called lymph, which is translated into English as 'clear water'. Compositionally, lymph is similar to interstitial fluid and blood plasma, containing water, ions, and protein. Interstitial fluid is primarily formed through the leaking of substances from the cardiovascular capillaries by Starling forces (a balance of hydrostatic and oncotic pressures), whereby substances leak into the interstitia via hydrostatic pressure in the capillaries, and substances being pushed back into the blood capillaries via colloid osmotic pressure (oncotic). Not all of the leaked fluid returns to the blood capillaries, and instead remains in the interstitia until being taken up by the lymphatic capillary vessels.

\subsubsection{Lymph Vessels}

The lymphatic vessels intra-connect to form a complex network which begins in the lymphatic capillaries, shown in figure 8.1, and weave between various tissues and cardiovascular capillaries. The lymphatic network contains vessel types with significantly different properties, while the vessels can be broadly split into lymph capillaries (initial lymphatics), pre-collecting vessels, collecting vessels, trunks, and ducts [202]. Figure 8.1 shows the position of lymph capillaries in relation to blood capillaries.

The lymph capillaries [44], sometimes referred to as initial lymphatics, are responsible for uptake of lymph from the interstitial fluid. The vessel walls are made

Pg. $211 / 284$ 
8.1. INTRODUCTION

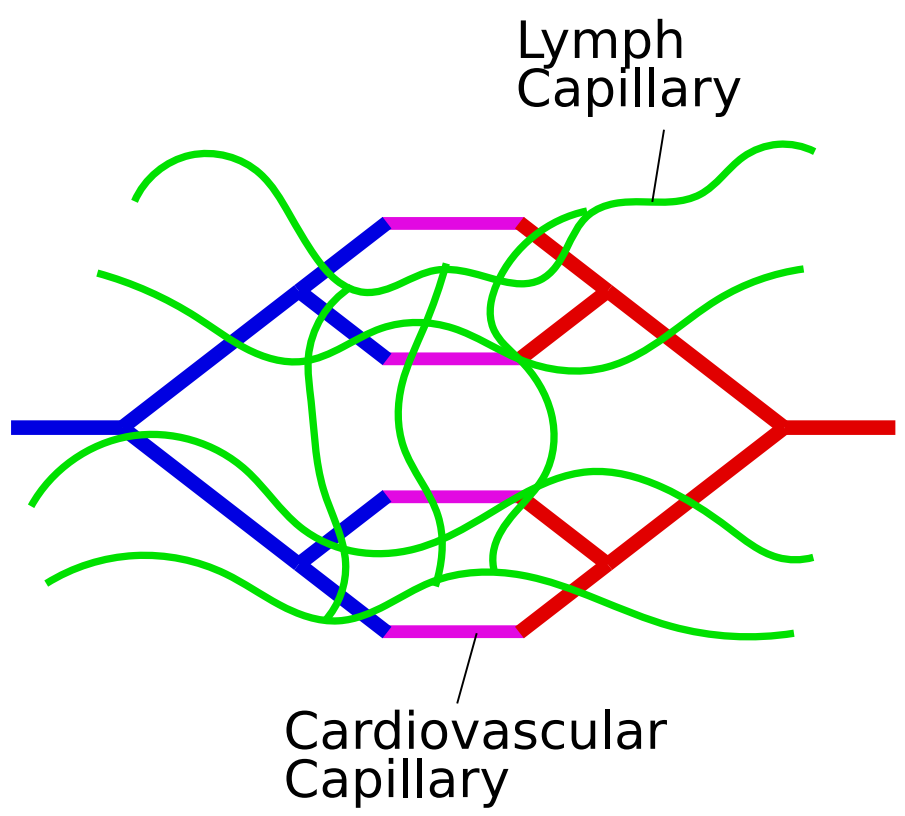

Figure 8.1: Overview of lymphatic system in relation to the cardiovascular system

up of single endothelial cells, while the size and network structure of these vessels can vary widely depending on the organ or tissue at which they are located, and vary significantly between species. Initial lymphatics in the human skin are approximately $35-70 \mu \mathrm{m}$ [111] in diameter, although estimates for the size of initial lymphatics have also been suggested as $10-60 \mu \mathrm{m}[202,289]$. Due to their unique structure, which includes irregular walls with an incomplete basement membrane [44], the initial lymphatics function similarly to one way valves, allowing fluid to leak into the vessel from a positive pressure gradient, while preventing the fluid from leaking back into the interstitial. Lymphangiogenesis, which is the term used to describe any growth of the lymphatic network, can be induced by events such as tissue damage, repair, and tumor growth [90]. The capillaries eventually meet at confluences to form the pre-collecting lymphatics.

Pre-collecting lymphatics have irregular network and structure patterns with discontinuous, irregular smooth muscle cells which function to propagate fluid through one-wave bi-cuspid valves, often called primary valves, which are located at irregular intervals [294]; and contains parts without smooth muscle cells, with an endothelium discontinuous basal lamina similar to the lymphatic capillaries. The structure of the pre-collecting vessels start to become more regular further from the capillary, and

Pg. $212 / 284$ 
eventually connect to become the collecting vessels.

The collecting lymphatic vessels are much more regular in structure than the precollecting vessels. They contain a complete basal lamina, valves which are normally bi-cuspid at regular intervals which are often called secondary valves, and a cellular structure more similar to blood vessels with three layers which are composed of endothelial cells, smooth muscle cells, and collagen fibres. The vessel segments inbetween valves are called lymphangions. The contracting smooth muscle cells in these lymphangions act as the pumping mechanism for the lymphatic system, and propel fluid forward through the one-way valves.

There are controversial arguments describing the behaviour of individual lymphangions [202], and of neighbouring lymphangions including across vessel junctions. There have been claims which suggest chains of lymphangions contract in a peristaltic manner $[113,376]$ and is currently the widely accepted view, although these studies were not on human lymphatics. However others have observed unperistaltic like behaviour, or a mix of peristaltic and un-peristaltic behaviour [237] where contractions were observed to be peristaltic $84 \%$ of the time, or the whole lymph vessel contracting approximately at the same time [93]. This indicates that although there may be interaction between neighbouring lymphangions, there is also likely an in-built mechanism for each lymphangion. The contradictions on lymphangion contraction behaviour in literature indicate that the exact mechanisms behind lymphangion contraction are still not fully understood. Figure 8.2 shows the general configuration of a series of lymphangions which are separated by bi-cuspid valves.

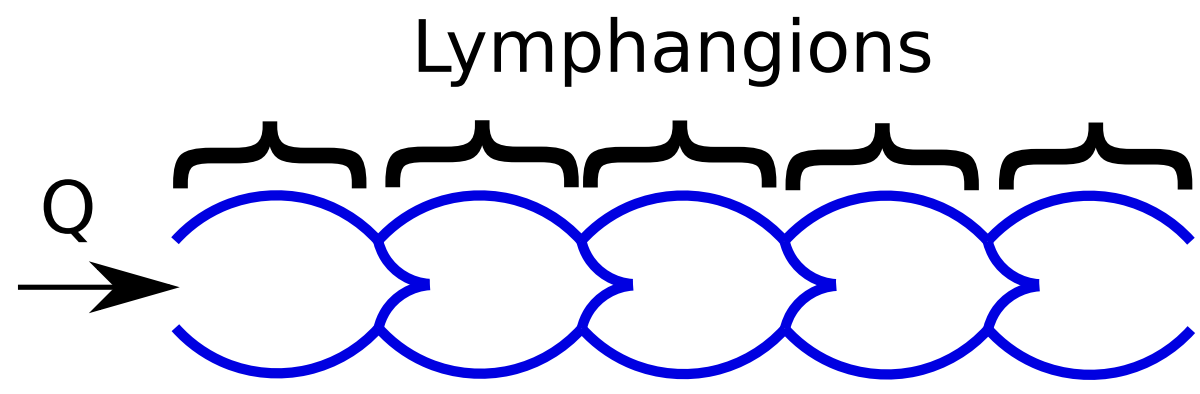

Figure 8.2: A lymph vessel containing a chain of 5 lymphangions

The collecting vessels confluence to form the lymph trunks, which are larger vessels whose primary role is to transport lymph to lymph ducts which finally return the fluid to the systemic venous system. The two largest ducts are the thoracic duct 
and the right lymphatic duct, which usually drain into the left and right subclavian veins respectively. The largest of these is the thoracic duct which has a diameter of approximately $2.5 \mathrm{~mm}$ in healthy human volunteers [309].

There exists several organs related to the lymphatic system which are split into primary lymph organs and secondary organs. The primary organs, which includes bone marrow and thymus gland, are where lymphocytes are created and mature. The secondary organs, such as lymph nodes, tonsils, spleen, and Peyer's patches, act as filters and fluid monitors and are the site of lymphocyte activation and are critical in immune response [202]. Each of these secondary organs have tissue arranged in different ways. The appendix is also considered a secondary lymphatic organ, although has little function in humans.

The lymph nodes are distributed throughout the lymphatic network, primarily acting to filter and monitor lymph in the network of collecting lymphatics. Lymph nodes have a highly complex structure [238], have a high resistance to flow [48, 257], and contain smooth muscle cells on the exterior walls which contract [206, 342, 159] which facilitates lymph transport. However, the study of lymph nodes in human subjects is limited, with the structure of a lymph node studied in a rat [238], and [206, 159] observing the phenomena of contraction in bovine and [342] observing contraction in ovine lymph nodes.

In this thesis the primary components of interest are the collecting vessels. To this end, a mathematical model based on reduced order lumped parameter modelling is presented. The model is simulated for idealised cases to test the various components used to build the model, including a comparison with the published model from [27].

\subsection{Mathematical Model of Lymphatics}

In this section the various model components which are used to construct a model of lymphatics is presented. The section describes briefly, the 1D system of equations and their reduction to a lumped representation of lymphatics; and proposes the use of automated contraction frequency based on lymphangion pressure.

The majority of current lymphatic system models use reduced-order methodologies (1D or 0D) and tend to only include the collecting lymphatics as they consider 
lymphangions at regular intervals $[283,285,284,196,274,28,29,27,165,88]$. Generally lymph capillaries and lymph nodes are not included in such models.

Reddy [283] is attributed with producing the first model of the entire circulation which includes some of the major pathways, a lumped model for initial lymphatics, and the thoracic duct; however due to the lack of data that currently exists, it is unknown how physiologically accurate the lymphatic network that was presented actually is as there is currently no recent model of the entire circulation using modern imaging techniques. The model uses a simplification of the 1D Navier-Stokes equations by assuming inertia effects are negligible, leading to a system of ordinary differential equations; although the model is claimed to be one-dimensional, the model spatially averages the pressure for each lymphangion and due to the assumed simplifications, essentially becomes a multi-compartment lumped model.

The model was extended by [196] to include passive and active terms in the constitutive law, with contraction magnitude and phase of each lymphangion being pre-defined. The model used a first order in time and space discretisation, choosing an explicit upwind finite difference approach, which is well known to be highly diffusive, an additional damping term and tension term were added to the system as oscillations were originally seen in the solution space. The extended model was only implemented for a small chain of lymphangions.

A lumped model of small chains of lymphangions was presented in [28, 29, 27, 165], which focused primarily on the contracting regimes. Each contraction was predefined and the phase difference between contractions of neighbouring lymphangions was also pre-defined.

A recent study has implemented a one-dimensional model using equations in the same form as the $1 \mathrm{D}$ blood flow equations with a modified systemic venous system constitutive law [88]. The model uses an electro-fluid-mechanical contraction (EFMC) model to initiate and control both the phase and magnitude of lymphangion contractions. The EMFC model is based on a modified FitzHugh-Nagumo model and has the advantage of not needing the contraction behaviour to be pre-defined, albeit with a significant increase in the number of user-defined parameters for the EMFC model in each lymphangion. The contraction model uses four additional ordinary differential equations per lymphangion, which includes calcium dynamics and nitric oxide to provide a stimulus and refraction period. Although calcium dynamics is likely to be involved in lymphangion contraction [362], as it is involved 
in vascular smooth muscle contraction [15], the exact mechanism behind lymphatic contraction and its regulation is still unknown.

\subsubsection{Modelling Lymph Vessels, Lymphangions}

The lymphatic system is essentially a fluid travelling in a tube-like structure which are under internal and external forces. The governing equations can be derived in the same way as the cardiovascular system as shown in chapter 2.2.1 and can be written in the same form as given in equation (2.15). For completeness the governing equations are repeated here and are given by the continuity equation

$$
\frac{\partial A}{\partial t}+\frac{\partial Q}{\partial x}=0
$$

the momentum equation

$$
\frac{\rho}{A} \frac{\partial Q}{\partial t}+\frac{\rho}{A} \frac{\left(\frac{Q^{2}}{A}\right)}{\partial x}+\frac{\partial P}{\partial x}+\frac{\xi \pi \mu Q}{A^{2}}=0
$$

and a constitutive law linking pressure and area

$$
P(A, x, t)=P_{e x t}+f_{p}(A, x, t)+f_{a}(A, x, t)
$$

where $f_{p}$ represents passive vessel wall properties, and $f_{a}$ represents the active contraction of smooth muscles in the lymphangion wall.

In the lymphatic system the flow rates and Reynold numbers are very small, particularly in the lymph capillaries and collecting lymphatics, with the only exception being the larger lymph vessels close to where lymph is drained back into the systemic venous system, although an average flow of $1-2$ litres per day in the largest vessel, the thoracic duct, would generally be considered small under normal circumstances, particularly when compared to cardiovascular flows; although these estimates are based on the assumption of total lymph flow is around 2 litres per day, while others estimate up to 12 litres per day [305]. Due to the small flows, several studies have neglected either non-linear convection effects only [283, 285, $284,196,274]$, or both inertial and convective effects [28, 29, 27, 165]. Recently a 1D-0D model of collecting lymphatic pumping was proposed, which includes both 
convective an inertial terms in the momentum equation [88]. In this thesis fluid inertia effects are considered, however the non-linear convection effects are neglected as the small Reynolds number in the region of interest is small, and viscous effects would be expected to dominate. Furthermore each lymphangion is connected to two valves, one at the inlet and one at the outlet, which means that fluid tends to only travel the length of two neighbouring lymphangions at most, which is approximately $6-10 \mathrm{~mm}$ before being blocked by a valve, further limiting the effect of the non-linear convection term.

The model proposed here is a multi-compartmental similar to that of $[29,27]$ with the addition of fluid inertia and a different valve model, which is described in section 8.2.3. The one-dimensional equations (8.1), (8.2), and (8.3) are simplified by assuming an average diameter for the lymphangion, which is a reasonable assumption due to observations that the entire wall of the lymph vessel contracts at the same time [93]; however, the method can be easily and readily extended to include additional spatial variation in a lymphangion if desired, which may be necessary for the larger lymph vessels, such as the trunks and ducts.

Each lymphangion is represented by a volume (capacitance-like) element, two resistance elements, and two inertial elements. This configuration can be seen in figure 8.3. The lumped equations being solved are written in terms of diameter, flow

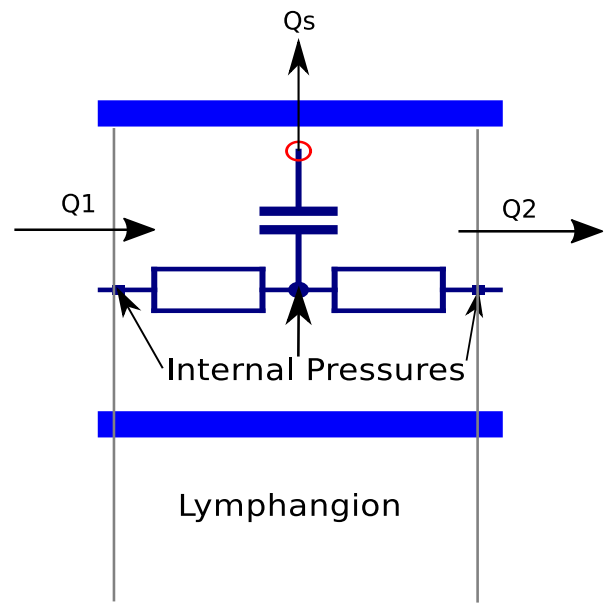

Figure 8.3: Lumped representation of a lymphangion

rate, and hydrostatic pressure. Each half of a lymphangion contains a resistance and 
inertance element which can be expressed as

$$
\Delta x \frac{\rho}{\pi(D / 2)^{2}} \frac{\partial Q}{\partial t}+\Delta P+\Delta x \frac{128 \mu Q}{\pi D^{4}}=0
$$

where $\Delta x=L / 2$ is half the length of the lymphangion, while the compliant element is the continuity equation

$$
\frac{\partial A}{\partial D} \frac{\partial D}{\partial t}+\frac{\Delta Q}{\Delta x}=0, \quad \frac{\partial A}{\partial D}=\frac{\pi D}{2}
$$

with $\Delta Q=Q_{2}-Q_{1}=-Q_{s}$, where $Q_{s}$ is the net flow as shown in figure 8.3.

\subsubsection{Constitutive Law and Contraction Model}

The main constitutive laws which have been proposed are: a thin-walled tube model [283, 285, 284], a thick-walled tube model [196], two mathematical relations by Bertram [28, 29, 27, 165], and a re-purposing of the cardiovascular venous constitutive law by [88]. In addition there are several contraction models which have been proposed, including a pre-defined elastance curve model similar to a heart model [274]; a pre-defined sine wave contraction curve which affects an active term in the constitutive law [28, 29, 27, 165], or changes the elastic modulus [196]; and an EFMC model utilised by [88] which uses calcium and nitric oxide dynamics to determine the contraction function on a time-step per time-step basis by changing the elastic modulus.

The constitutive model utilised in this thesis is similar to [27]. The constitutive law is split into passive and active (contraction) terms. However, unlike in [27] which pre-defines the contraction function and relaxation period, which takes into account that a lymphangion rests before contracting again, in this thesis the contraction state is determined via an ordinary differential equation, similar to the valve model [226], which changes its contraction frequency based on the average pressure in the lymphangion during the previous contraction cycle. This allows a network to be created without needing to define a contraction cycle for every single lymphangion, while the pumping regimes are automatically determined numerically.

The constitutive law links the spatially averaged diameter in a lymphangion with its mean pressure using a passive term and active term in the form of equation (8.3). 
For comparison purposes with [29], the passive term is given by

$$
\begin{aligned}
f_{p}(A, x, t) & =P_{d}\left[c_{1}\left(\frac{D}{c_{9}}-c_{2}\right)^{2}+c_{3} \exp \left(c_{4}\left(\frac{D}{c_{9}}-c_{5}\right)\right)+c_{6}\right. \\
& \left.+c_{7}\left(\frac{D}{c_{9}}-c_{8}\right)+c_{10}\left(\frac{c_{9}}{D}\right)^{3}\right],
\end{aligned}
$$

where $c_{1}=-2.34457751, c_{2}=1.1262924, c_{3}=3.76013762, c_{4}=79.991135, c_{5}=$ $1.0028029, c_{6}=1.59133174, c_{7}=3.69692633, c_{8}=0.20699868, c_{9}=D_{d} / c_{11}, c_{10}=$ $-0.0180867408, c_{11}=0.32538081, D_{d}=0.025$, and $P_{d}$ is a scaling term. The passive constitutive term can be seen in figure $8.4 \mathrm{a}$. The active term is

$$
f_{a}(A, x, t)=\frac{2 M_{0} M_{t}}{D}
$$

where $M_{0}$ is a scaling factor for the maximum force of contraction, and $M_{t}$ is the contraction state which is updated by

$$
\left\{\begin{array}{lr}
\frac{d M_{t}}{d t}=K_{c o n} \omega\left(1-M_{t}\right), \quad \text { Contraction } \\
\frac{d M_{t}}{d t}=-K_{r e l} \omega\left(M_{t}\right), \quad \text { Relaxation }
\end{array}\right.
$$

where $K_{c o n}, K_{\text {rel }}$ are opening and closing coefficients, and $\omega$ is a frequency term which depends on the pressure (created by Daniel Watson, Swansea University, PhD student), which was constructed from data in [207].

$$
\omega=\frac{1}{1+(-0.71 P+0.56)}
$$

produces a relation between contraction frequency and period and pressure as shown in figure 8.4c and 8.4d. In comparison [29] pre-defines the contraction cycle using a sine wave and a time delay between contractions; however the model proposed here in equation (8.8) does not require defining a time delay, moreover the contraction and relaxation behaviour is more physiological, as the decrease in force during the relaxation phase is normally slower than the increase in force during the contraction phase. Figure 8.4b compares the contraction model of [29], with equation (8.8). 


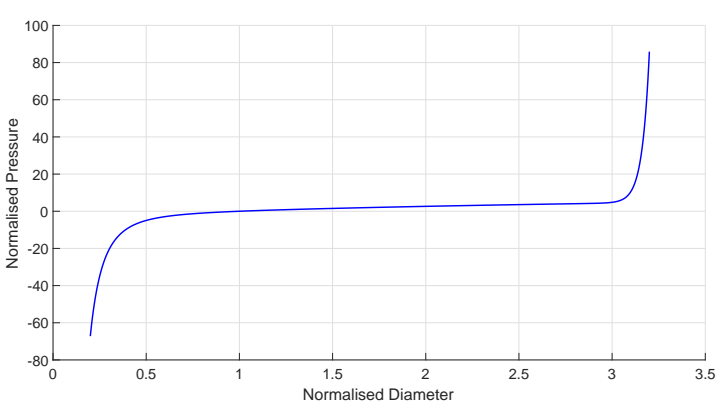

(a) The passive constitutive relationship given in equation (8.6), pressure and diameter are normalised by $P_{d}$ and $D_{d}$ respectively

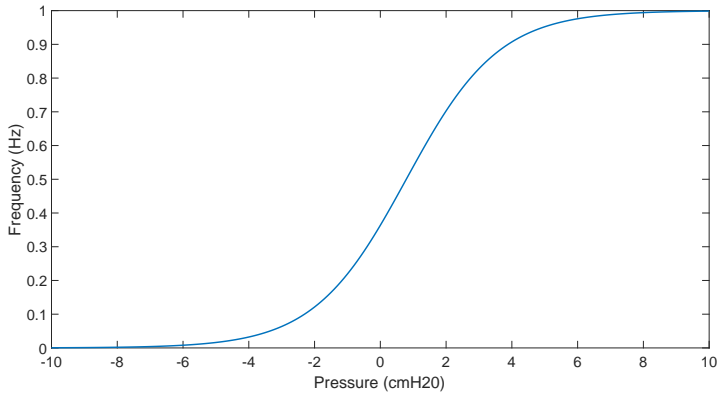

(c) Relationship between frequency of contraction and pressure

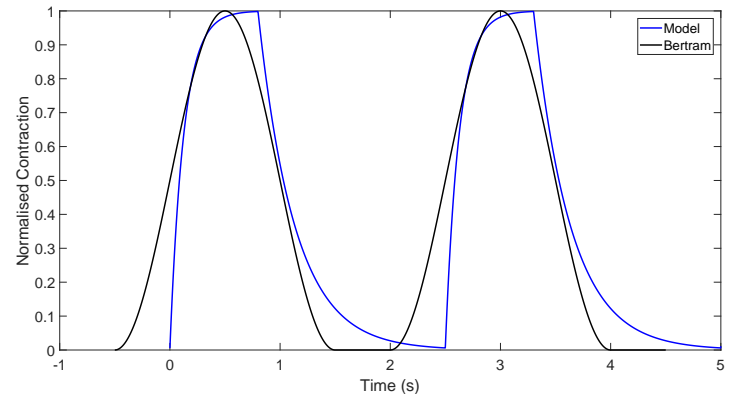

(b) Comparison of the contraction models from [29], and the model used in this thesis. Contaction force is normalised by $M_{0}$

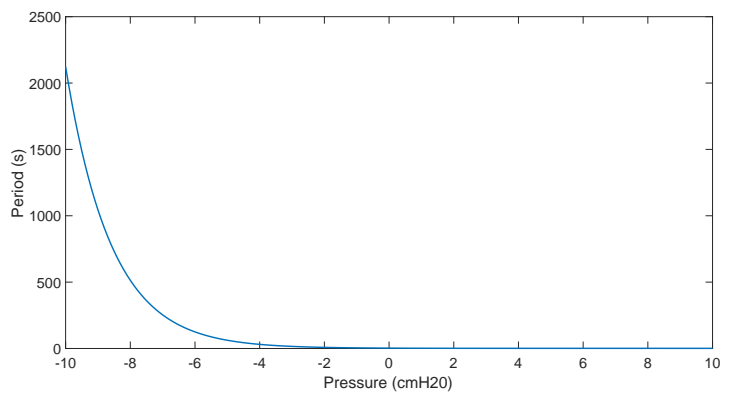

(d) Relationship between period of contraction and pressure

Figure 8.4: Comparison of passive constitutive relationships and contraction functions

\subsubsection{Valve Model}

The valve model implemented in this thesis is described in chapter 2.3.3 and is the same as the cardiovascular model, hence is not repeated in detail here. The model allows a valve to be anything between open and closed, can be set up to be biased towards opening or closing, and its opening state is updated based on the pressure differential across the valve [226]. The equation that governs flow through a valve is given by

$$
\Delta P=B|Q| Q+R Q+L \frac{d Q}{d t} .
$$

However, in the majority of test cases implemented here, only the viscous effect is considered for comparison purposes. In the lymphatic system viscous effects are considered dominant over inertial and convective terms, which are normally 
neglected. The remainder of the valve model is described in chapter 2.3.3. The lymphangion and valve model configuration in a collecting lymph vessel is shown in figure 8.5.

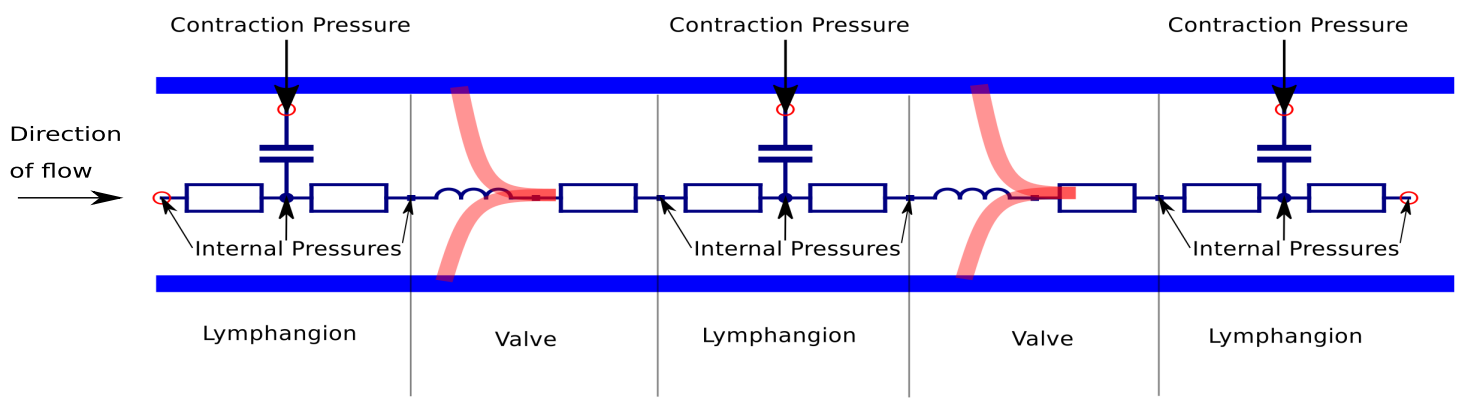

Figure 8.5: Lumped representation of lymphangion chain

The valve model is entirely different to that of [29]. Due to the majority of the lymphatic model implemented here being modified from [29], it is useful to compare the two valve models. Figure 8.5 shows the model representation of a lymphangion chain. The valve model from [29] uses an empirical formula to determine resistance of the valve, based on the pressure difference across it. The model equations are

$$
\Delta P=R Q,
$$

where the resistance is determined as

$$
R=R_{\min }+\frac{R_{v}}{1+\exp \left(-s_{o}\left(\Delta P-\Delta P_{0}\right)\right)}
$$

where $R_{\text {min }}$ is the minimum resistance, and $R_{v}, s_{o}$, and $\Delta P_{0}$ are tuning parameters. A comparison of the two valve models can be seen if figure 8.6.

\subsubsection{Linearisation and Methodology}

The linearisation of the equations is performed in a similar way to the cardiovascular equations. The time derivatives are discretised using second order backward differences

$$
\frac{\partial Q}{\partial t}=\frac{2}{3 \Delta t} Q^{n+1}-\frac{2}{\Delta t} Q^{n}+\frac{1}{2 \Delta t} Q^{n-1},
$$




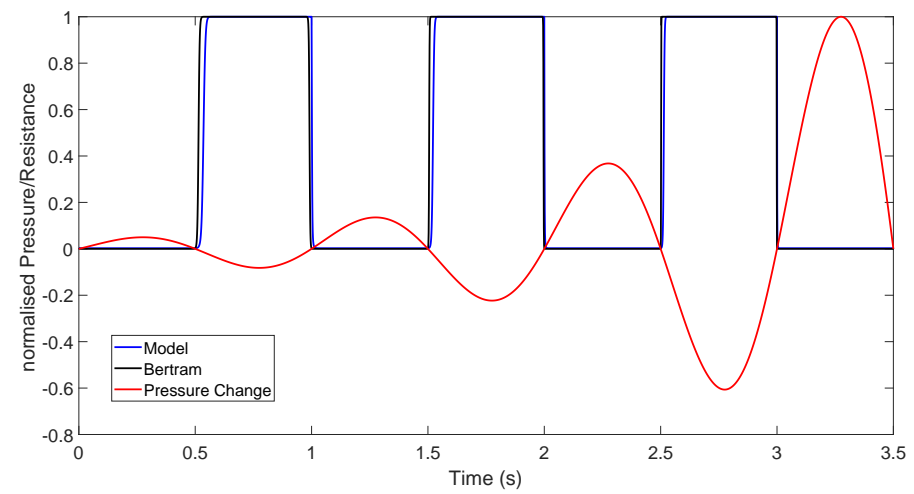

Figure 8.6: Comparison of lymph valve models. Resistance and pressure are normalised by maximum resistance and maximum pressure respectively

and

$$
\frac{\partial D}{\partial t}=\frac{2}{3 \Delta t} D^{n+1}-\frac{2}{\Delta t} D^{n}+\frac{1}{2 \Delta t} D^{n-1},
$$

The terms of the momentum equation are linearised as follows:

$$
\begin{gathered}
\frac{\rho}{\pi(D / 2)^{2}}{ }^{n+1} \approx{\frac{\rho}{\pi(D / 2)^{2}}}^{n+1, j}, \quad \Delta P^{n+1} \approx \Delta P^{n+1, j+1} . \\
\Delta x{\frac{128 \mu Q^{n+1}}{\pi D^{4}}}^{n} \approx \Delta x{\frac{128 \mu^{n+1, j}}{\pi D^{4}}}^{n+1, j+1},
\end{gathered}
$$

while for the continuity equation is

$$
\frac{\pi D^{n+1}}{2} \approx \frac{\pi D^{n+1, j}}{2}, \quad \Delta Q^{n+1}=-Q_{s}^{n+1} \approx-Q_{s}^{n, j+1}
$$

The constitutive law terms are linearised in the following way

$$
\begin{gathered}
P^{n+1} \approx P^{n, j+1}, \quad P_{e x t}^{n+1} \approx P_{e x t}^{n+1, j} \\
f_{p}^{n+1} \approx f_{p}^{n+1, j}+\frac{\partial f_{p}^{n+1, j}}{\partial D} \delta D, \quad f_{a}^{n+1} \approx f_{a}^{n+1, j}+\frac{\partial f_{a}^{n+1, j}}{\partial D} \delta D,
\end{gathered}
$$

where $\delta D=D^{n+1, j+1}-D^{n+1, j}$. This allows the system of equations to be written in the form

$$
F_{e} P_{e}+G_{e} Q_{e}=f_{e}
$$

which allows the same treatment of flux terms as in the cardiovascular system. 


\subsubsection{Example of an Assembled Matrix for a Lymphangion}

In order to construct the elemental system matrix it is advantageous to initially consider only the momentum equation 8.4. Writing this for each half of the lymphangion leads to the system

$$
\left[\begin{array}{ccc}
K_{11}^{1} & K_{12}^{1} & 0 \\
K_{21}^{1} & K_{22}^{2}+K_{11}^{2} & K_{12}^{2} \\
0 & K_{21}^{2} & K_{22}^{2}
\end{array}\right]^{n+1, j}\left[\begin{array}{c}
P_{1} \\
P_{2} \\
P_{3}
\end{array}\right]^{n+1, j+1}=\left[\begin{array}{c}
Q_{1} \\
-Q_{2}+Q_{3} \\
Q_{4}
\end{array}\right]^{n+1, j+1}+\left[\begin{array}{c}
f_{1} \\
-f_{2}+f_{3} \\
f_{4}
\end{array}\right]^{n+1, j}
$$

where the superscripts on $K$ denotes the element number. However, this system ignores any change in diameter, contracting pressures, and the fact that the flow leaving element one is now not necessarily the same as the flow entering element two (i.e. $-Q_{2}+Q_{3} \neq 0$ ). Which means that some fluid storage can occur at this position. Therefore, a net flow can be defined for mass conservation $-Q_{2}+Q_{3}-Q_{s}=0$. Bringing this net flow to the left side of the system, treating it as a variable, and adding the linearised equations (8.5) and (8.3) adds diameter as another variable. Thus, the full system can be written as

$$
\left[\begin{array}{ccccc}
K_{11}^{1} & K_{12}^{1} & 0 & 0 & 0 \\
K_{21}^{1} & K_{22}^{2}+K_{11}^{2} & K_{12}^{2} & 0 & 1 \\
0 & K_{21}^{2} & K_{22}^{2} & 0 & 0 \\
0 & 0 & 0 & K_{1}^{m} & K_{2}^{m} \\
0 & K_{1}^{c} & 0 & K_{2}^{c} & 0
\end{array}\right]\left[\begin{array}{c}
P_{1} \\
P_{2} \\
P_{3} \\
D \\
Q_{s}
\end{array}\right]^{n+1}=\left[\begin{array}{c}
Q_{1} \\
0 \\
Q_{4} \\
f^{m} \\
f^{c}
\end{array}\right]^{n+1}
$$

where superscript $m$ and $c$ are the continuity and constitutive equations. Note that this matrix can be reduced in size by rearranging the fourth row as

$$
Q_{s}=\left(f_{m}-K_{1}^{m} D\right) / K_{2}^{m}
$$

and substituting the right hand side into row 2 of the system of equations (8.22).

The lymphangion model and all other lumped models can be coupled by sharing a pressure node, while at junctions conservation of mass and conservation of hydrostatic pressure are held as system constraints using Lagrange multipliers. 


\subsection{Lymphatic Simulation Results}

In this section several computational tests are performed using the newly proposed methodology which includes a valve model with a variable opening state, and the frequency of contraction which is dependant on the pressure in the lymphangion.

\subsubsection{Single Lymphangion}

The first test shows a comparison of the model in [29] for both the original valve model in equation (8.12), the proposed valve model in equation (8.10) with a viscous resistance only, and also shows the solution of the problem using the proposed contraction model. The problem parameters are shown in table 8.1.

Figure 8.7 compares the results for the three simulation cases. First the effect of using a different valve model is investigated. The pressure in the lymphangion is shown in figure 8.7a and shows excellent agreement between the two valve models; the flow through both valves is shown in $8.7 \mathrm{c}$, the diameter of the lymphangion is shown in figure 8.7e, and the contraction state is shown in figure 8.7g. The results indicate that the new valve model can give solutions in excellent agreement with the valve model of [29].

The waveforms on the right hand side of figure 8.7 shows the solution of the proposed contraction and valve model, tuned to have similar contraction behaviour as the left hand side waveforms. Slight differences in the shape and magnitudes of pressure, diameter, flow rates and contraction state are observed. The main reasons for these differences is that the newly proposed contraction function has a slower rate of relaxation, which means fluid filling of the lymphangion is slightly lowered, reducing pressures and flow rates when compared with the model of [29]. It would not be expected that two different models would give the exact same solution, and the overall behaviour is very similar, indicating the new model gives a satisfactory solution to this problem.

\subsubsection{Chain of Four Lymphangions}

In this section a chain of four lymphangions is considered. Each of the lymphangions have the same diameter and length, while the boundary conditions and pumping 


\begin{tabular}{|c|c|}
\hline Parameters & Values \\
\hline$P_{d}$ & 35 \\
\hline$P_{\text {ext }}, \mathrm{cmH}_{2} \mathrm{O}$ & 2.1407 \\
\hline$P_{\text {in }}, \mathrm{cm} \mathrm{H}_{2} \mathrm{O}$ & 2.1916 \\
\hline$P_{\text {out }}, \mathrm{cm} \mathrm{H}_{2} \mathrm{O}$ & 2.4975 \\
\hline $\mathrm{M}_{0}$ & 10 \\
\hline$L, \mathrm{~cm}$ & 0.3 \\
\hline
\end{tabular}

Table 8.1: Input parameters for single lymphangion model, where $P_{\text {ext }}$ is the external pressure, $P_{\text {in }}$ is the inlet boundary pressure, $P_{\text {out }}$ is the outlet boundary pressure, $L$ is the length of a lymphangion.

parameters are chosen to be the same as the single lymphangion case for the baseline case. The problem parameters are shown in table 8.1. Four cases are simulated, three with different initial conditions but all other parameters kept the same, and an additional case with an increased pressure differential from inlet to outlet. Figure 8.8 shows the pressure and diameter results of all simulations.

The first comparison is between three configurations with the same problem parameters, but with different initial conditions. The baseline problem defines the initial pressure everywhere to be $\mathrm{P}=2.4975 \mathrm{~cm} \mathrm{H}_{2} \mathrm{O}$, meaning the initial transmural pressure is zero, furthermore all valves are initially in a closed position. The second case uses an initial condition of $P=2 P_{\text {ext }}$ with all valves initially open, while the third uses $P=P_{\text {ext }} / 2$ with all valves initially open. The results are compared for the last 160 seconds of the simulation. The mean solutions between these cases are within $0.25 \%$ for all parameters, where the percentage is calculated using the mean absolute percentage error. The parameters compared were: the flow rates through each valve, mean, maximum, and minimum pressures in each lymphangion, the contraction frequency for each lymphangion, and the diameter of each lymphangion. Furthermore, the differences between solutions decreases when a longer simulation time is used. The results are in excellent agreement with each other, and it can be concluded that the model is insensitive to the initial condition, and will converge towards a periodic solution.

The fourth test case performed uses a larger pressure differential from inlet to outlet with an increase in outlet pressure to $P_{\text {out }}=3.4975 \mathrm{~cm} \mathrm{H}_{2} \mathrm{O}$, and an increase in the contraction parameter $M_{0}=50$ to help overcome this un-physiological pressure differential. The main objective of this example is to showcase the effect of frequency 
changes for neighbouring lymphangions. The effective pumping of lymph is still drastically reduced even with the increase in pumping force, with a $95 \%$ reduction mean flow through each valve in the system. This will create un-physiologically high pressures in the lymphangion during pumping. The increased pressure differential has a significant effect on the contraction frequency term $\omega$, decreasing from a mean of $0.72 \mathrm{~s}^{-1}$ to $0.43 \mathrm{~s}^{-1}$.

The models contraction behaviour has periods of peristaltic-like behaviour shown in figure $8.8 \mathrm{~g}$ where each lymphangion contracts in sequence; and periods of unperistaltic behaviour shown in figure $8.8 \mathrm{~h}$, this phenomena is observed physiologically [237], and would not be possible to model if the contraction behaviour is pre-defined.

As mentioned the pressure differential is un-physiological for such a short chain of lymphangions, resulting in low flow rates and high pressures. In order to overcome this an increased in the rate of contraction and a change in the force of contraction could be introduced, which will limit the maximum force applied via contraction, avoiding the un-physiologically high pressures, and better control flow rate and contraction behaviour; however this is outside the focus of this thesis, which mainly aims to present a numerical scheme for lymphatics.

\subsection{Limitations}

The development of the lymphatic model components are subject to the following major limitations: 1) The mechanisms involved in the lymphatic system are not fully understood, which includes the mechanical, electrical, and chemical processes responsible for the contraction of a lymphangion wall. This means that the contraction function in current models generally try to match some observed behaviour, such as the contraction behaviour of a chain of lymphangions [29]. This means that the model may be representative of an ex-vivo experiment with a specific set up, but may not be generalisable to in-vivo conditions, lymphangions from other locations in the body, or lymphatics in other animals. In order to develop an accurate model of the full lymphatic system, a much greater understanding of these physiological mechanisms is required; 2) No accurate vessel network of the full lymphatic system currently exists, this means that the majority of models implement either a lumped 
model [283, 285, 284], which ignores spatial variability, or considers a very small network of less than ten lymphatic vessels [196, 28, 29, 27, 165, 88]. In order to model fluid movement within the lymphatic system, a full network of the lymphatic system needs to be developed.

\subsection{Conclusions}

This chapter presents a new numerical scheme for reduced-order modelling of the collecting lymphatic vessels. The model was compared to the another reduced-order model for a single lymphangion case from [29], and showed good agreement with the overall behaviour. A particular strength of the model, is the ability for the lymphangion contraction frequency to be determined automatically, and does not need to be pre-defined as in the majority of currently published works [196, 28, 29, $27,165]$. The proposed numerical scheme for the lymphatic system could form a basis for the future development of lymphatic system modelling. Before this occurs, a greater understanding is needed of how lymphangion contraction occurs. Once this is achieved, more clinically relevant research can be investigated.

One particular avenue to pursue, is in the understanding of how fluid transport in the lymphatic system changes if a lymph node is removed from the network. This is a problem which can occur in cancer patients, where after a lymph node is removed, the fluid drainage from other body regions is affected, causing oedema, which is when swelling of a body part occurs as a result of fluid build up within the interstitia. Being able to model this may eventually help clinicians choose a treatment for the patient which lowers the likelihood of oedema developing.

Eventually the cardiovascular system and lymphatic system models could be coupled, which occurs in physiology. This coupling within the model could allow the investigation of fluid transport within the entire circulation, and lead to improved understanding of what happens during haemodialysis, which causes significant fluid shifts in the body. This could aid clinicians in developing a more efficient and effective treatment of haemodialysis patients, who suffer from excess fluid build up in body tissues as a result of poor renal function, or renal failure.

During pregnancy, oedema is very common, occurring in approximately $80 \%$ of woman [94], particularly affecting the lower legs and arms. A coupled cardiovascu- 
lar and lymphatic system model, could indicate why peripheral oedema occurs in pregnancy, and eventually provide an opportunity to investigate how oedema can be managed or prevented during pregnancy. 


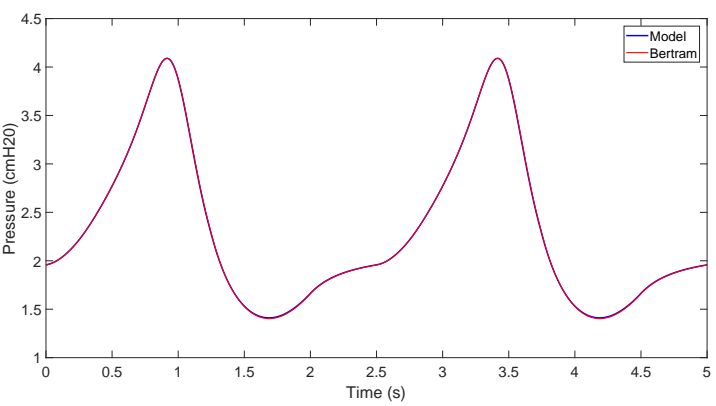

(a) Pressure at centre of lymphangion

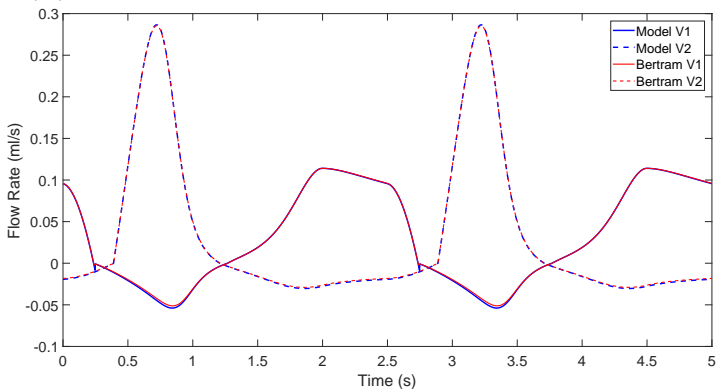

(c) Flow rate through valves $V_{1}$ and $V_{2}$

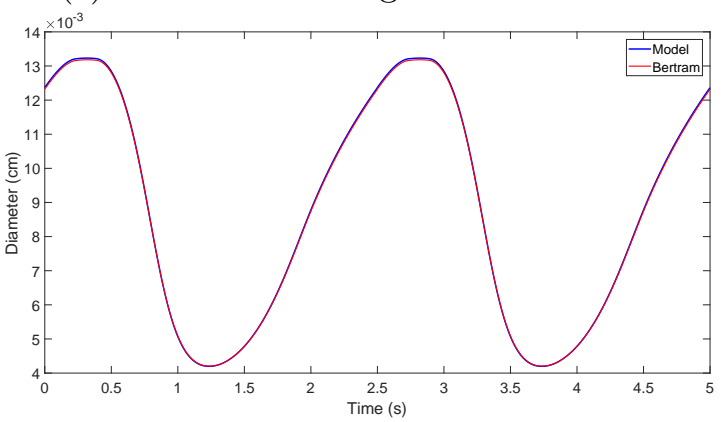

(e) Diameter at centre of lymphangion

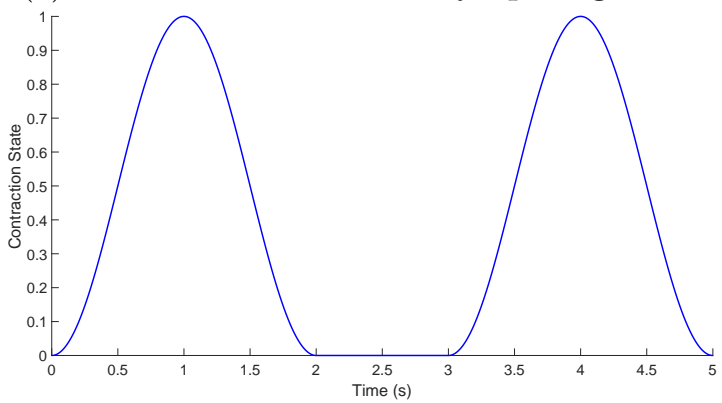

(g) Contraction state in lymphangion

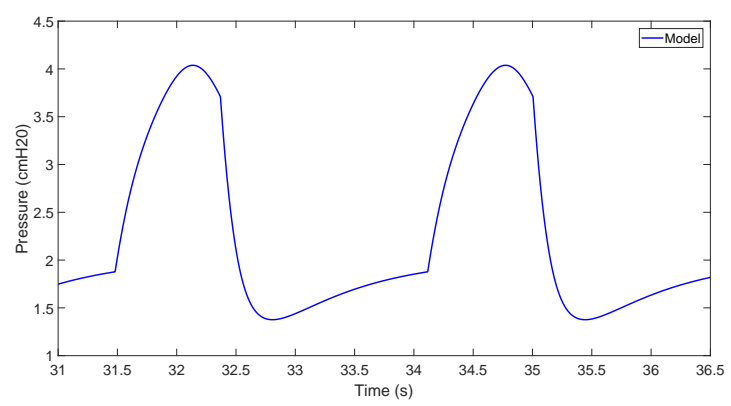

(b) Pressure at centre of lymphangion

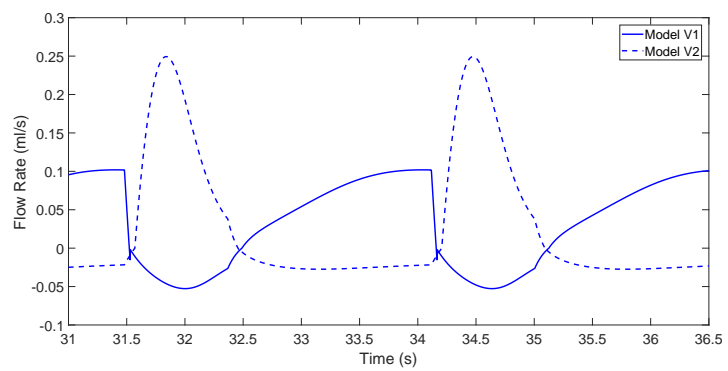

(d) Flow rate through valves $V_{1}$ and $V_{2}$

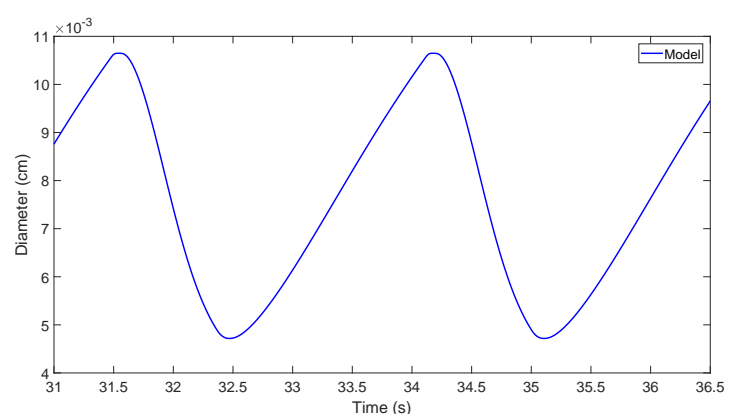

(f) Diameter at centre of lymphangion

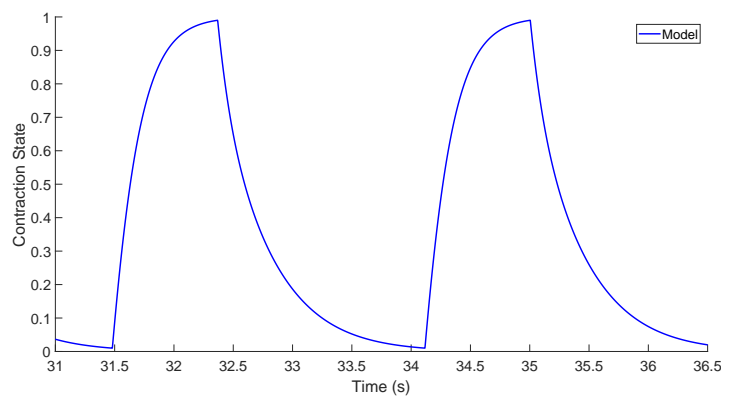

(h) Contraction state in lymphangion

Figure 8.7: Results of a single lymphangion test comparing the valve model of Bertram [29], the proposed valve model modified from [226], and the new contraction model. 


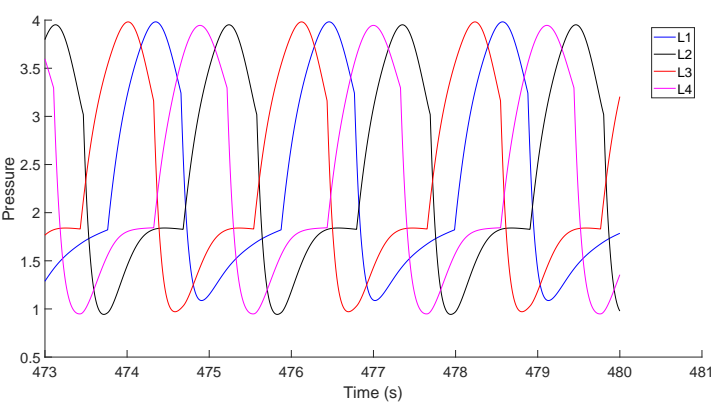

(a) Pressure at centre of lymphangion, Case 1

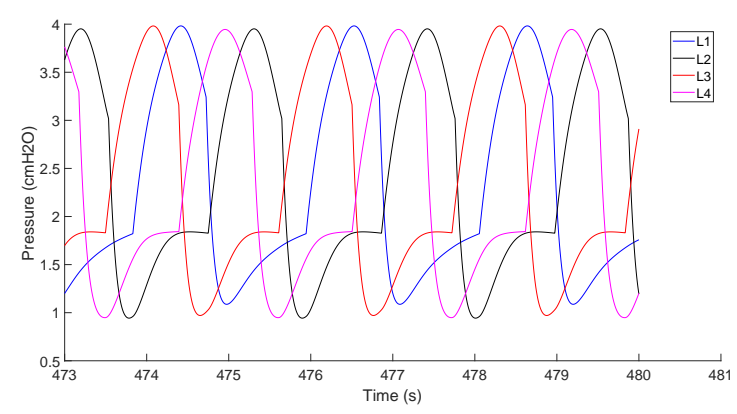

(c) Pressure at centre of lymphangion, Case 2

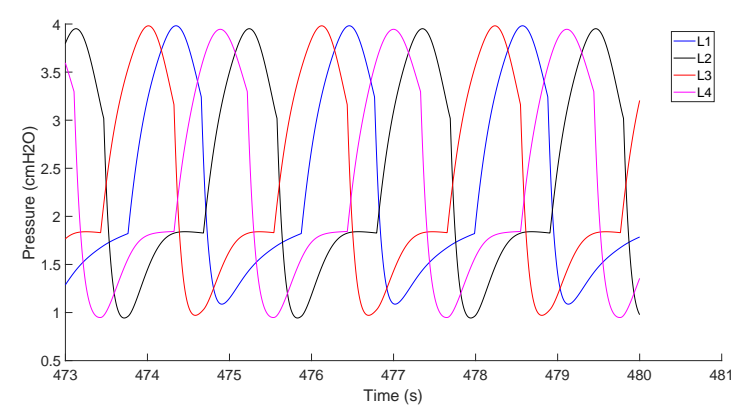

(e) Pressure at centre of lymphangion, Case 3

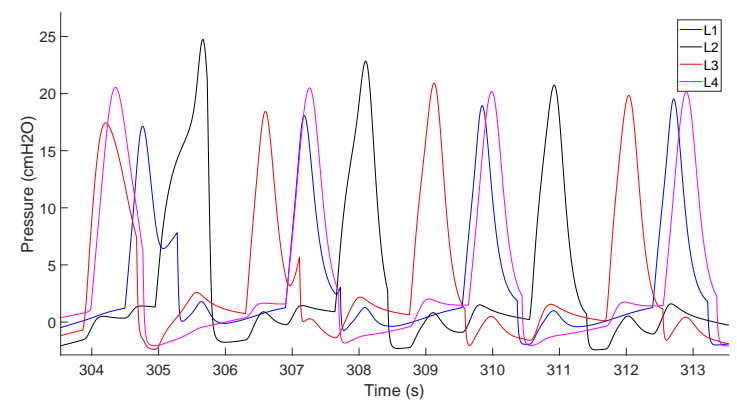

(g) Pressure at centre of lymphangion, Case 4 , peristaltic behaviour

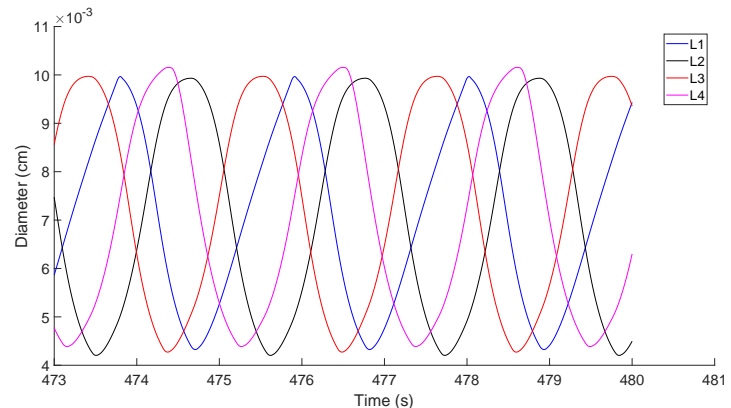

(b) Diameter at centre of lymphangion, Case 1

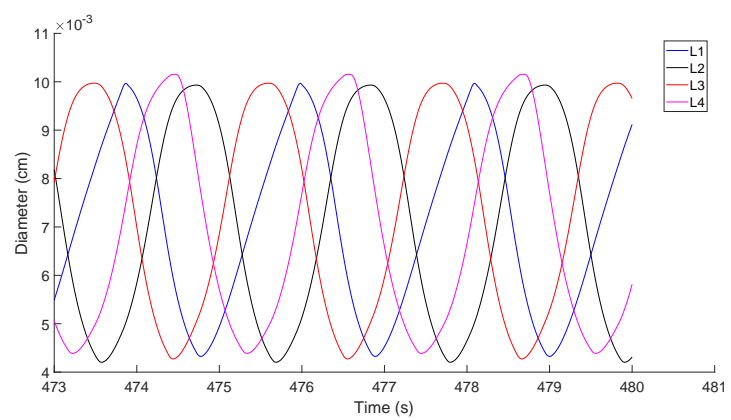

(d) Diameter at centre of lymphangion, Case 2

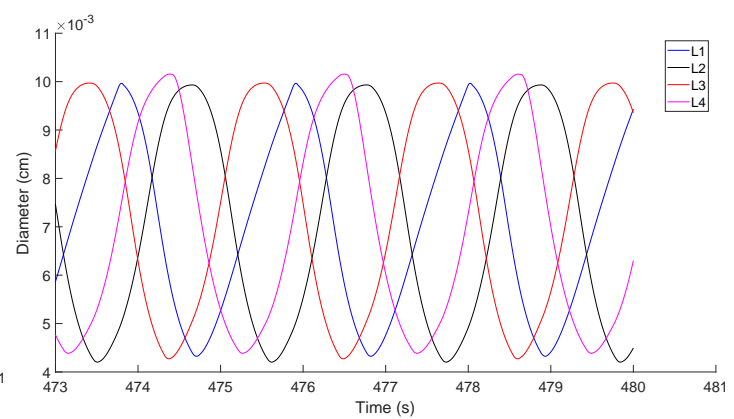

(f) Diameter at centre of lymphangion, Case 3

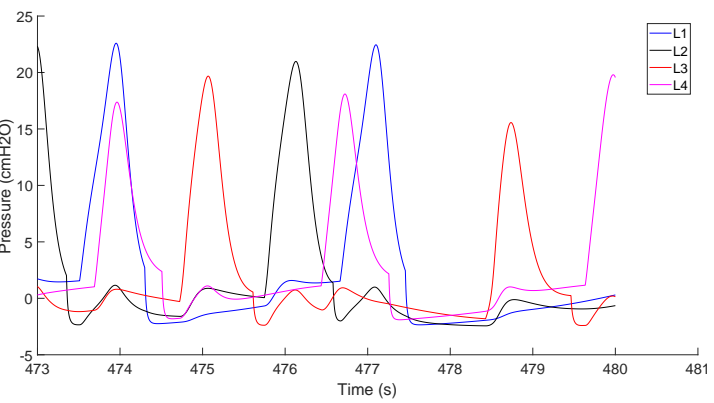

(h) Pressure at centre of lymphangion, Case 4 , un-peristaltic behaviour

Figure 8.8: Results for a chain of four lymphangions 


\section{Chapter 9}

\section{Conclusions}

In this chapter a general discussion is provided that outlines: the main achievements of this thesis, including the use of techniques developed in this thesis that are being used in other research areas; and the possible applications, extensions, and views on potential future clinical impact of the model for pregnancy, and for other areas of cardiovascular and lymphatic system research.

\subsection{Summary of Thesis Achievements}

In this thesis a comprehensive closed-loop 1D-0D cardiovascular model of human pregnancy was developed. The model successfully used an automated parameter adaptation algorithm to integrate in-vivo measurements from pregnant woman at three stages during pregnancy, and post-partum. The model was further extended to include all weeks of gestation by using published data for an idealised cardiovascular adaptation model of pregnancy.

An implicit numerical scheme called the enhanced trapezoidal rule method (ETM), was developed and presented in Chapter 3. The scheme was a modification of the pre-existing simplified trapezoidal rule (STM). The main modification was for the treatment of vessel junctions, where the ETM used Lagrange multipliers to constrain a conservation of pressure, allowing a straightforward change between the use of: static pressure conservation, total pressure conservation, and can easily allow pressure loss methods into the formulation.

The ETM scheme was rigorously tested in Chapter 4 by comparing with: published benchmark problems, which included in-silico and in-vitro data, and included problem from a single pulse in a reflection free vessel, small networks which were compared with 3D model solutions, a 37-vessel arterial network which had an equivalent experimental set-up with in-vitro measurements, and an arterial network with 56 arteries for which one-dimensional schemes were compared; and carefully chosen cases with analytical solutions, including numerically challenging problems with discontinuities in material properties, geometries, and a shock-wave. The scheme gave excellent agreement with the published solutions to the benchmark problems, 
and showed excellent accuracy when compared with the problems which have an analytical solution. A convergence test revealed that the ETM scheme is superlinearly convergent in both temporal and spatial domains. Furthermore, the scheme showed excellent mass conservation properties, which is an important criteria for a closed-loop cardiovascular model, as the volume of blood must be conserved to achieve sensible solutions.

An automated parameter estimation technique was presented in Chapter 6, with the purpose of integrating into the model measured in-vivo data. The parameter estimation technique determines the difference from the model solutions and measurement data at the end of a cardiac cycle, and iteratively adapts resistances, compliances, and blood volumes in the system to converge to the measured data. The effectiveness of the parameter estimation technique was shown in Section 7.7 within the closed-loop 1D-0D cardiovascular model of pregnancy.

The solutions of the pregnancy model were compared with published clinical indices which are used to aid detection of pathologies, such as hypertension, preeclampsia, and placental insufficiency. The model solutions of the patient specific simulations for the pulsatility index in the uterine arteries closely followed the expected behaviour from literature $[137,144]$ for pregnancy week 11 , where the range predicted by the model for all patient specific simulations was in the expected range of $P I \in[1.1,2.6]$, through to week 40 , where the range predicted by the model was in the expected range of $P I \in[0.55,1.00]$. The model predicted that the pulsatility index in the utero-ovarian communicating arteries would generally be lower in magnitude than the PI in the uterine arteries, although it follows the same general behaviour over the course of pregnancy by continually decreasing until term. Currently the measurement of the PI in these communicating vessels within the clinical setting have been unsuccessful. Thus the model gives an estimate of this previously unknown parameter. In addition, the brachial-ankle pulse wave velocity predicted by the model follows the expected behaviour described by [251], showing a decrease in PWV from the first trimester to the second trimester, before increasing again towards the third trimester, and a further increase to term. The model showed that the aortic PWV is much lower in magnitude than the brachial-ankle PWV, but that it shows the same general behaviour over the course of pregnancy.

The model-predicted percentage of cardiac output that reaches the uterus via the pathway of the uterine arteries was $0.5 \%$ in the non-pregnant case (averaged 
for the cohort), rising to $5.6 \%$ in the first trimester, $10.5 \%$ in the second trimester, and eventually reaching $12.9 \%$ late in the third trimester, which agree with values measured in literature [249, 340]. Additionally the model gave a first estimation of the percentage of cardiac output which reaches the uterus via the utero-ovarian communicating arteries. The volumetric flow rate through the utero-ovarian communicating arteries is currently unmeasured, as previous attempts to measure these flows have been unsuccessful due to the complex geometry and flow behaviour in the pelvic region $[262,47]$. If the utero-ovarian communicating arteries reached a lumen size equal to that of the uterine arteries, then they supplied an additional $9 \%$ of cardiac output to the uterus at term for the patient specific simulations.

Three of the participant measurements were investigated in more detail, for all three trimesters and post-partum. The uterine artery flow rate waveforms showed good agreement with published Doppler studies for all three trimesters and post pregnancy. The model-predicted flow rates in the uterine arteries were in excellent agreement with the value of $20-50 \mathrm{ml} \mathrm{min}^{-1}$ from literature [249], with 25.7 $\mathrm{ml} \mathrm{min}-1,26.5 \mathrm{ml} \mathrm{min}^{-1}$, and $30.1 \mathrm{ml} \mathrm{min}^{-1}$ for participant one, two, and three respectively. The total volumetric flow rate in the uterine arteries at term is 855.1 $\mathrm{ml} \mathrm{min}{ }^{-1}, 855.1 \mathrm{ml} \mathrm{min}^{-1}$, and $855.1 \mathrm{ml} \mathrm{min}^{-1}$ for participant one, two, and three respectively, while the range given in literature is $600-900 \mathrm{ml} \mathrm{min}^{-1}$ [249]. The flow in the uterine veins was also compared to values from literature for the third trimester (no data from earlier trimesters was available). The model-predicted values were in close agreement to that of $[344,162]$, where the volumetric flow rate in the third trimester was measured to be $274 \mathrm{ml} \mathrm{min}^{-1}$, while the model-predicted values were $314 \mathrm{ml} \mathrm{min}^{-1}$ for participant one, $270 \mathrm{ml} \mathrm{min}^{-1}$ for participant two, and $280 \mathrm{ml} \mathrm{min}^{-1}$ for participant three.

Through the use of published data, the pregnancy model was extended to become an idealised model of human pregnancy in Section 7.7, covering the 40 weeks of gestation. This made it possible to fill in the gaps and model every week of pregnancy, as the patient-specific model only had measurement data from four different time points. The idealised model showed excellent agreement with the published data of cardiovascular adaptation in pregnancy, which included the predicted changes to total peripheral resistance, total arterial compliance, the pulsatility index, and the fraction of cardiac output reaching the uterus via the uterine artery throughout pregnancy. The iterative parameter estimation technique took between 62 and 
83 cardiac cycles to converge to within $1 \%$ of the aimed cardiac output, systolic pressure, and diastolic pressure.

A sensitivity analysis was performed on cardiac parameters, model initial conditions, and the haemodynamic parameters which the iterative parameter estimation algorithm utilises. The solutions in the utero-ovarian circulation were shown to be insensitive to the initial conditions, and the cardiac model parameters. However, the solutions were more sensitive to the aimed haemodynamic parameters of systolic and diastolic blood pressures, indicating the importance of accurate measurement techniques to supply the model with reliable data.

In addition the model was simulated for different lumen diameter estimates of the utero-ovarian communicating artery and indicated that the blood volume supplied to the uterus from these communicating arteries was highly depended on the vessel lumen diameter. If the communicating arteries reached the same diameter as the uterine arteries, then they provided an additional $7.43 \%$ of cardiac output to the uterus. This reduced to $4.31 \%$ if their diameter was halved, and $2.01 \%$ if their diameter was quartered.

Another contribution made in the thesis, is a new numerical scheme for collecting lymphatics. The scheme is constructed in such a way as to be easily compatible with the cardiovascular models numerical scheme, which will allow the cardiovascular model and lymphatic models to be coupled in a straight forward manner in the future, with the potential of creating a combined framework for the full human circulatory system. However, before this is achieved, significant development needs to occur for lymphatic system modelling, as current models of the lymphatic system are mainly trying to match observed behaviour, but the exact mechanisms behind the behaviour are not understood.

In conclusion, this thesis' main contribution was the development of a closedloop 1D-0D cardiovascular model of human pregnancy, which can incorporate in-vivo measurement data. The model can provide important insights in the cardiovascular adaptation processes which occur during pregnancy, with the potential to be used for improving understanding of the development of various pathologies, which may eventually assist in diagnosis of cardiovascular disease as a results of poor adaptation in pregnancy. 


\subsection{Additional Achievements}

The previous section focused on the main outcomes of the work developed and investigated in this thesis. The techniques developed in this thesis are also currently being utilised in other cardiovascular research areas. In this section an overview of these other areas is described. The numerical scheme and model implemented in this work has been utilised in a paper investigating blood flow induced vibrations of the head in an attempt to detect and predict the severity of a carotid stenosis [71].

In addition the initial and iterative estimation technique is currently being utilised in the development of a reduced-order fractional flow reserve software. Fractional flow reserve is a measurement used in the clinic to estimate the severity of a stenosis located in the coronary arteries. The parameter estimation technique is used to estimate the inflow conditions of the patient-specific coronary system, as extracted from the segmentation of CT scans.

\subsection{Main Limitations}

A sensitivity analysis for the cardiovascular model of pregnancy was performed on the variation of a single parameter at a time. This enabled a test of the iterative parameter estimation technique for uncertain parameters. However, to fully understand the affect of all model parameters, and their interaction, a sensitivity analysis involving mutual variation of parameters must be performed, as this could lead to different results. This could also aid clinicians in determining which parameters are most important to measure.

In this work there were no patient-specific Doppler waveforms available. This mean that the model predicted waveforms, and various indices such as the pulsatility index, had to be compared to measurements found in literature, which may vary significantly with the patient data in this thesis. Therefore, in the future it is imperative to compare the model to patient-specific data which includes waveforms, and ideally pulse wave velocity measurements, in order to fully validate the model.

A generic cardiovascular network was utilised, as no information of the vessel network, such as vessel lengths and diameters, was available for the patient-specific simulations. It would aid the model prediction if some vessel information was avail- 
able, especially the geometry of the uterine vessels.

The lymphatic system is much more difficult to model as the mechanisms involves in lymphatic contraction, which is responsible for the propulsion of lymph fluid in the network, are currently not known. Moreover, the majority of models only consider very small networks that are not based on physiological networks (due to lack of available data). Hence, before clinical questions can be answered, the understanding of the mechanisms involved in lymphangion contraction and the geometry of the lymphatic network must be improved.

\subsection{Future Work}

This section describes the possible further applications and development of the modelling framework described in this thesis. The section is separated into two main parts: the first part describes the possible extension and application of the model with regards to pregnancy; while the second part describes other areas where the modelling methodologies developed in this thesis could also be utilised.

\subsubsection{Pregnancy Modelling and Clinical Applications}

The natural and ideal future work for the pregnancy model requires access to additional patient data. This data must have the uterine artery Doppler waveforms, along with the parameters that are already utilised in this thesis for the parameter estimation technique, which includes heart rate, stroke volume, systolic pressure and diastolic pressure. It would also be useful to obtain a pulse wave velocity measurement for these patients. If this type of data can be obtained, then the model can be fully validated, and confidence can then be assured when attempting to model the development of various pathologies that can occur during pregnancy.

After validating for a 'healthy' pregnancy, the model could be used to investigate all cardiovascular related pathologies that can develop over the course of pregnancy, which includes pre-eclampsia, placental insufficiency, hypertension, hypotension, supine hypotensive syndrome. In the future, this could provide a better understanding of how these pathologies develop, and may even lead to a model that is capable of predicting whether a pathology is likely to develop later in a pregnancy. This could lead to a significant impact on healthcare systems by providing an ear- 
lier diagnosis of a developing pathology, which will improve the management and treatment of patients during pregnancy.

An even more comprehensive model, which involves the coupling of the maternalfoetal systems, could be developed. A model of the foetal cardiovascular system has already been developed by [227], thus only a more complex model of the maternalfoetal interface, i.e. the placenta, needs to be created in order to achieve this coupled system. This could allow a number of interesting aspects to be investigated, such as: the effect of placental placement within the uterus on blood and nutrient supply to the foetus; and in understanding the impact of the placental micro-structure on the resistance in the uterine system, and how it affects nutrient exchange between the maternal and foetal systems.

Peripheral oedema occurs in around $80 \%$ of pregnancies [94], and pregnant woman have a greater risk of developing other types of oedema, such as pulmonary oedema [320]. When lymphatic system modelling is at a stage where the lymphatic vessel network is known and the mechanisms involved in lymphangion contraction are better understood, a coupled cardiovascular and lymphatic system model could be implemented for pregnancy to provide insight into why oedema occurs in a large majority of pregnancies, and why extensive lymphangiogenesis occurs near the placenta [282]. This could provide a treatment plan and aid in the management of oedema during pregnancy.

\subsubsection{Other Modelling and Clinical Applications}

The 1D-0D framework developed in this thesis used an automated parameter estimation technique to converge model solutions towards patient-specific measurement data. Although the current model focused on pregnancy, and thus contains female specific vessels, and vascular beds, the model can be easily extended for males, or for a general case by ignoring reproductive organs. This could make for an interesting comparison between female and male specific cardiovascular network models.

The model presented in this thesis primarily focused on and utilised in-vivo data from the systemic arterial system. However, the model could be easily extended to integrate measured data from the systemic veins or from the pulmonary system, within the same framework. This could lead to investigations for pulmonary hypertension, where the pressure in the pulmonary arteries increases above $25 \mathrm{mmHg}$. 
Pulmonary hypertension is a serious pathology that is difficult to diagnose as symptoms vary widely [157], thus a model of this disease could help understand how the pathology develops, which may lead to an improved diagnosis system. With the inclusion of gravity and the auto-regulation mechanisms involved in the cardiovascular system, there are a number of other interesting areas of research that could be perused. For example, venous insufficiency is a disease where venous valves do not function correctly, which means blood collects in the lower legs and causes swelling. Chronic venous insufficiency commonly occurs in patients who have suffered a blood clot in a deep vein (called deep vein thrombosis). In addition, the body's reaction to exercise and the baroreceptor reflex sensitivity could be investigated, which could lead to a model to investigate the effect of a haemorrhage or blunt force trauma on the cardiovascular system response.

There are many pathologies which can develop in the cardiovascular system. The parameter estimation technique proposed in this thesis can be used to aid the modelling of many of these pathologies. For instance it could be used to:

- estimate inlet and outlet boundary conditions of the coronary system for the calculation of fractional flow reserve, which is a clinical procedure involving the measurement of the pressure drop across a coronary artery stenosis, which estimates the severity and functional significance of the lesion.

- utilise of patient measurements to investigate the severity of stenoses that are located in other regions of the body, such as a carotid or cerebral stenosis.

- utilise of patient data to determine the effect of an aneurysm in various regions of the body, such as the abdominal aortic aneurysm, aortic aneurysm, or cerebral aneurysm.

- model the functional impact of the formation of a thrombosis (blood clot) in various regions of the body to improve the treatment pathway, such as: in the cerebral arteries which can lead to a cerebral embolism and a stroke; in the coronary arteries which can lead to a coronary embolism and an acute myocardial infarction; or in the pulmonary arteries, which can lead to a pulmonary embolism.

The thesis also proposed a numerical scheme for the collecting lymphatic vessels, although current lymphatic networks are small and do not include full body lymph 
networks, the model can be extended when sufficient information is available in the literature on the lymphatic network, and when the mechanisms involved in lymphangion contraction are understood. The model could be utilised to investigate the causes and potential treatments of various types of oedema. When adequate development of lymphatic system modelling has occurred, it will also open the possibility of coupling cardiovascular and lymphatic system models. This will be particularly useful for investigating pathologies which are related to fluid retention and excretion from the body. These pathologies include:

- the treatment of cancer patients, where lymph nodes are surgically removed or damaged from radiation therapy which leads to the development of lymphedema, particularly in limbs [168]. A coupled cardiovascular and lymphatic model could aid in the understanding of why oedema sometimes occurs in areas that are located far away from the damaged or removed lymph node.

- the treatment of renal failure patients, where fluid gathers in the interstitia of the lower limbs as a result of poor or no kidney function. These patients undergo haemodialysis, which takes approximately four hours at a time, and is required three days a week. A coupled cardiovascular and lymphatic model could help in developing a more effective and efficient treatment for these patients.

\subsection{Concluding Remarks}

It is currently an exciting time for cardiovascular research, primarily due to the recent surge of activity trackers, such as watches that can track heart rate throughout the day, it is expected that future studies could have improved longitudinal data, enabling very interesting research to be performed. As the understanding of physiology improves, and as computational resources become easier and cheaper to obtain, larger cardiovascular and lymphatic networks could be simulated, allowing an investigation of physiological mechanisms at a local and global level. In recent years, the introduction of computed fractional flow reserve into the patient treatment pathway, could provide an opportunity for other such software to gain recognition for capabilities in diagnosing and predicting pathology. This could lead to a monumental change in healthcare systems around the world in treating various 
types of cardiovascular pathology, ranging from: stenosis and aneurysm diagnosis and treatment; improved treatment for cancer patients; more effective and efficient treatment for haemodialysis patients; and improving the management of patients during pregnancy. 


\section{References}

[1] Amr E. Abbas, Steven J. Lester, and Heidi Connolly. "Pregnancy and the cardiovascular system". In: International Journal of Cardiology 98.2 (2005), pp. 179-189. DOI: 10.1016/j.ijcard.2003.10.028.

[2] Mohsen Abdi et al. "A lumped parameter mathematical model to analyze the effects of tachycardia and bradycardia on the cardiovascular system". In: International Journal of Numerical Modelling: Electronic Networks, Devices and Fields 28.3 (2014), pp. 346-357. DOI: 10.1002/jnm. 2010.

[3] G. L. Acacio. "Uterine artery Doppler patterns in abdominal pregnancy". In: Ultrasound in Obstetrics and Gynecology 20.2 (2002), pp. 194-196. DOI: 10.1046/j.1469-0705.2002.00769.x.

[4] S. Lee Adamson et al. "Effect of placental resistance, arterial diameter, and blood pressure on the uterine arterial velocity waveform: A computer modeling approach". In: Ultrasound in Medicine $6 \mathcal{G}$ Biology 15.5 (1989), pp. 437442. DOI: $10.1016 / 0301-5629$ (89) 90096-3.

[5] J Aguado-Sierra et al. "Separation of the reservoir and wave pressure and velocity from measurements at an arbitrary location in arteries". In: Proceedings of the Institution of Mechanical Engineers, Part H: Journal of Engineering in Medicine 222.4 (2008), pp. 403-416. DOI: 10.1243/09544119jeim315.

[6] Al-Jameil. "A Brief Overview of Preeclampsia". In: Journal of Clinical Medicine Research (2013). DOI: 10.4021/jocmr1682w.

[7] J. Alastruey. "Numerical modelling of pulse wave propagation in the cardiovascular system : development, validation and clinical applications". Imperial College London, 2006.

[8] J. Alastruey et al. "Analysing the pattern of pulse waves in arterial networks: a time-domain study". In: Journal of Engineering Mathematics 64.4 (2009), pp. 331-351. DOI: 10.1007/s10665-009-9275-1.

[9] J. Alastruey et al. "Lumped Parameter Outflow Models for 1-D Blood Flow Simulations: Effect on Pulse Waves and Parameter Estimation". In: Communications in Computational Physics 4 (2 2008). 
[10] J. Alastruey et al. "Reduced modelling of blood flow in the cerebral circulation: Coupling 1-D, 0-D and cerebral auto-regulation models". In: International Journal for Numerical Methods in Fluids 56.8 (2008), pp. 1061-1067. DOI: $10.1002 / \mathrm{fld} .1606$.

[11] Jordi Alastruey et al. "Physical determining factors of the arterial pulse waveform: theoretical analysis and calculation using the 1-D formulation". In: Journal of Engineering Mathematics 77.1 (2012), pp. 19-37. DOI: 10.1007/ s10665-012-9555-z.

[12] Jordi Alastruey et al. "Pulse wave propagation in a model human arterial network: Assessment of 1-D visco-elastic simulations against in vitro measurements". In: Journal of Biomechanics 44.12 (2011), pp. 2250-2258. ISSN: 0021-9290. DOI: $10.1016 / \mathrm{j} \cdot \mathrm{jbiomech} .2011 .05 .041$.

[13] Dana Albulescu, C. Constantin, and Gabriela Constantin. "Uterine artery emerging variants - angiographic aspects". In: Current Health Sciences Journal 3 (2014), pp. 214-216. DOI: 10.12865/CHSJ .40.03.11.

[14] Lori L. Altshuler, Victoria Hendrick, and Lee S. Cohen. "An Update on Mood and Anxiety Disorders During Pregnancy and the Postpartum Period." In: Primary care companion to the Journal of clinical psychiatry 2 (6 Dec. 2000), pp. 217-222. ISSN: 1523-5998.

[15] Gregory C. Amberg and Manuel F. Navedo. "Calcium Dynamics in Vascular Smooth Muscle". In: Microcirculation 20.4 (2013), pp. 281-289. DOI: 10 . 1111/micc. 12046.

[16] A. Antsaklis et al. "The effect of gestational age and placental location on the prediction of pre-eclampsia by uterine artery Doppler velocimetry in lowrisk nulliparous women". In: Ultrasound in Obstetrics and Gynecology 16.7 (2000), pp. 635-639. DOI: 10.1046/j.1469-0705.2000.00288.x.

[17] Andrea Arnold et al. "Uncertainty Quantification in a Patient-Specific OneDimensional Arterial Network Model: EnKF-Based Inflow Estimator". In: Journal of Verification, Validation and Uncertainty Quantification 2.1 (2017), p. 011002. DOI: $10.1115 / 1.4035918$.

[18] B. Avni et al. "Aortic stiffness in normal and hypertensive pregnancy". In: Blood Pressure 19.1 (2009), pp. 11-15. DOI: 10.3109/08037050903464535. 
[19] R. Axt-Fliedner et al. "The value of uterine artery Doppler ultrasound in the prediction of severe complications in a risk population". In: Archives of Gynecology and Obstetrics 271.1 (2004), pp. 53-58. DOI: 10.1007/s00404004-0648-4.

[20] Ayoubi. "Pre-eclampsia: pathophysiology, diagnosis, and management". In: Vascular Health and Risk Management (2011), p. 467. DOI: 10.2147/vhrm. s20181.

[21] Guha Balakrishnan, Fredo Durand, and John Guttag. "Detecting Pulse from Head Motions in Video". In: 2013 IEEE Conference on Computer Vision and Pattern Recognition. IEEE, 2013. DOI: 10.1109/cvpr.2013.440.

[22] Mojgan Barati et al. "Diagnostic evaluation of uterine artery Doppler sonography for the prediction of adverse pregnancy outcomes." In: Journal of research in medical sciences : the official journal of Isfahan University of Medical Sciences 19 (6 2014), pp. 515-519. ISSN: 1735-1995.

[23] A. C. L. Barnard et al. "A Theory of Fluid Flow in Compliant Tubes". In: Biophysical Journal 6.6 (1966), pp. 717-724. DOI: 10.1016/s0006-3495(66) 86690-0.

[24] Stefan Baumann et al. "Coronary CT angiography-derived fractional flow reserve correlated with invasive fractional flow reserve measurements - initial experience with a novel physician-driven algorithm". In: European Radiology 25.4 (2014), pp. 1201-1207. DOI: 10.1007/s00330-014-3482-5.

[25] Riccarda Bene et al. "Cardiovascular function in pregnancy: effects of posture". In: BJOG: An International Journal of Obstetrics and Gynaecology 108.4 (2001), pp. 344-352. DOI: 10.1111/j.1471-0528.2001.00099.x.

[26] Ira M. Bernstein et al. "The Influence of Pregnancy on Arterial Compliance". In: Obstetrics \& Gynecology 105.3 (2005), pp. 621-625. DOI: 10.1097/01. aog.0000152346.45920.45.

[27] C. D. Bertram, C. Macaskill, and J. E. Moore Jr. "An improved model of an actively contracting lymphatic vessel composed of several lymphangions: pumping characteristics". In: (Dec. 3, 2015). arXiv: 1512.01269v1 [physics.flu-dyn]. 
[28] C. D. Bertram, C. Macaskill, and J. E. Moore. "Simulation of a Chain of Collapsible Contracting Lymphangions With Progressive Valve Closure". In: Journal of Biomechanical Engineering 133.1 (2011), p. 011008. DOI: 10 . 1115/1.4002799.

[29] C. D. Bertram et al. "Development of a model of a multi-lymphangion lymphatic vessel incorporating realistic and measured parameter values". In: Biomechanics and Modeling in Mechanobiology 13.2 (2013), pp. 401-416. DOI: 10.1007/s10237-013-0505-0.

[30] D. Bessems, M. Rutten, and F. van de Vosse. "A wave propagation model of blood flow in large vessels using an approximate velocity profile function". In: Journal of Fluid Mechanics 580 (2007), pp. 145-168. DOI: 10.1017 / S0022112007005344. URL: https: //www . cambridge .org/core/article/ div-class-title-a-wave-propagation-model-of-blood-flow-inlarge-vessels-using-an-approximate-velocity-profile-functiondiv/5F7BA2D61342495601306523186D2E23.

[31] David Bessems et al. "Experimental validation of a time-domain-based wave propagation model of blood flow in viscoelastic vessels". In: Journal of Biomechanics 41.2 (2008), pp. 284-291. DOI: 10.1016/j.jbiomech.2007.09.014.

[32] N. Bessonov et al. "Methods of Blood Flow Modelling". In: Mathematical Modelling of Natural Phenomena 11.1 (2015). Ed. by V. Volpert, pp. 1-25. DOI: $10.1051 / \mathrm{mmnp} / 201611101$.

[33] A. Bhuiyan et al. "Distribution and Correlates of Arterial Compliance Measures in Asymptomatic Young Adults: The Bogalusa Heart Study". In: American Journal of Hypertension 18.5 (2005), pp. 684-691. DOI: 10 .1016/ j . amjhyper.2004.11.039.

[34] P. J. Blanco and R. A. Feijóo. "A dimensionally-heterogeneous closed-loop model for the cardiovascular system and its applications". In: Medical Engineering $\mathscr{6}$ Physics 35.5 (2013), pp. 652 -667. ISSN: 1350-4533. DOI: 10.1016/ j . medengphy . 2012 .07 .011. URL: //www . sciencedirect. com/science/ article/pii/S135045331200197X. 
[35] P. J. Blanco, R. A. Feijóo, and S. A. Urquiza. "A unified variational approach for coupling 3D-1D models and its blood flow applications". In: Computer Methods in Applied Mechanics and Engineering 196.41-44 (2007), pp. 43914410. DOI: $10.1016 /$ j.cma.2007.05.008.

[36] Pablo J. Blanco et al. "An Anatomically Detailed Arterial Network Model for One-Dimensional Computational Hemodynamics". In: IEEE Transactions on Biomedical Engineering 62.2 (2015), pp. 736-753. DOI: 10.1109/tbme. 2014. 2364522 .

[37] Pablo J. Blanco et al. "Blood flow distribution in an anatomically detailed arterial network model: criteria and algorithms". In: Biomechanics and Modeling in Mechanobiology 13.6 (2014), pp. 1303-1330. DOI: 10.1007/s10237014-0574-8.

[38] W. E. Bodley. "The non-linearities of arterial blood flow". In: Physics in Medicine and Biology 16.4 (1971).

[39] R P Boggon and A J Palfrey. "The microscopic anatomy of human lymphatic trunks." In: Journal of anatomy 114 (Pt 3 1973), pp. 389-405. ISSN: 00218782 .

[40] Etienne Boileau and Perumal Nithiarasu. "One-Dimensional Modelling of the Coronary Circulation. Application to Noninvasive Quantification of Fractional Flow Reserve (FFR)". In: Lecture Notes in Computational Vision and Biomechanics. Springer International Publishing, 2015, pp. 137-155. DOI: 10.1007/978-3-319-15799-3_11.

[41] Etienne Boileau et al. "A benchmark study of numerical schemes for onedimensional arterial blood flow modelling". In: International Journal for Numerical Methods in Biomedical Engineering 31.10 (2015), n/a-n/a. ISSN: 2040-7947. DOI: 10.1002/cnm.2732. URL: http://dx . doi .org/10.1002/ cnm. 2732 .

[42] Etienne Boileau et al. "Estimating the accuracy of a reduced-order model for the calculation of fractional flow reserve (FFR)". In: International Journal for Numerical Methods in Biomedical Engineering 34.1 (2017), e2908. DOI: $10.1002 / \mathrm{cnm} .2908$. 
[43] S Bower, S Bewley, and S Campbell. "Improved prediction of preeclampsia by two-stage screening of uterine arteries using the early diastolic notch and color Doppler imaging." In: Obstetrics and gynecology 82 (1 1993), pp. 78-83. ISSN: 0029-7844.

[44] Jerome W. Breslin. "Mechanical forces and lymphatic transport". In: $M i$ crovascular Research 96 (2014), pp. 46-54. DOI: 10.1016/j.mvr.2014.07. 013.

[45] B. S. Brook, S. A. E. G. Falle, and T. J. Pedley. "Numerical solutions for unsteady gravity-driven flows in collapsible tubes: evolution and roll-wave instability of a steady state". In: Journal of Fluid Mechanics 396 (1999), pp. 223-256. DOI: 10.1017/s0022112099006084.

[46] M A Brown, V C Zammit, and D M Mitar. "Extracellular fluid volumes in pregnancy-induced hypertension." In: Journal of hypertension 10 (1 1992), pp. 61-68. ISSN: 0263-6352.

[47] V. A. Browne et al. "Uterine artery blood flow, fetal hypoxia and fetal growth". In: Philosophical Transactions of the Royal Society B: Biological Sciences 370.1663 (2015), pp. 20140068-20140068. DOI: 10 . 1098 / rstb. 2014.0068.

[48] N. L. Browse, R. L. Doig, and D. Sizeland. "The resistance of a lymph node to lymph flow". In: British Journal of Surgery 71.3 (1984), pp. 192-196. DOI: 10.1002/bjs. 1800710308 .

[49] A. Ya. Bunicheva et al. "Studying the influence of gravitational overloads on the parameters of blood flow in vessels of greater circulation". In: Mathematical Models and Computer Simulations 5.1 (2013), pp. 81-91. DOI: 10.1134/ S207004821301002X.

[50] R. Burattini and G. Gnudi. "Computer identification of models for the arterial tree input impedance: Comparison between two new simple models and first experimental results". In: Medical \& Biological Engineering $\&$ Computing 20.2 (1982), pp. 134-144. DOI: 10.1007/bf02441348. 
[51] Roberto Burattini and Paola Oriana Di Salvia. "Development of systemic arterial mechanical properties from infancy to adulthood interpreted by fourelement windkessel models". In: Journal of Applied Physiology 103.1 (2007), pp. 66-79. DOI: 10.1152/japplphysiol.00664.2006.

[52] Fred Burbank. Fibroids, Menstruation, Childbirth, and Evolution: The Fascinating Story of Uterine Blood Vessels. WHEATMARK INC, 2009. 296 pp. ISBN: 1604941707.

[53] G.J. Burton et al. "Rheological and Physiological Consequences of Conversion of the Maternal Spiral Arteries for Uteroplacental Blood Flow during Human Pregnancy". In: Placenta 30.6 (2009), pp. 473-482. DOI: 10.1016/ j.placenta.2009.02.009.

[54] S Campbell, S Bewley, and T Cohen-Overbeek. "Investigation of the uteroplacental circulation by Doppler ultrasound." In: Seminars in perinatology 11 (4 1987), pp. 362-368. ISSN: 0146-0005.

[55] Sunčica Čanić. "Blood flow through compliant vessels after endovascular repair: wall deformations induced by the discontinuous wall properties". In: Computing and Visualization in Science 4.3 (2002), pp. 147-155. DOI: 10 . $1007 / \mathrm{s} 007910100066$.

[56] Mary M. Canobbio et al. "Management of Pregnancy in Patients With Complex Congenital Heart Disease: A Scientific Statement for Healthcare Professionals From the American Heart Association". In: Circulation 135.8 (2017), e50-e87. DOI: 10.1161/CIR.0000000000000458.

[57] B. A. Carabello. "Introduction to Aortic Stenosis". In: Circulation Research 113.2 (2013), pp. 179-185. DOI: 10.1161/circresaha.113.300156.

[58] Andrew Carlin and Zarko Alfirevic. "Physiological changes of pregnancy and monitoring". In: Best Practice 85 Research Clinical Obstetrics 85 Gynaecology 22.5 (2008), pp. 801-823. DOI: 10.1016/j . bpobgyn.2008.06.005.

[59] R E Carpenter et al. "Changes in heart rate variability and QT variability during the first trimester of pregnancy". In: Physiological Measurement 36.3 (2015), pp. 531-545. DOI: 10.1088/0967-3334/36/3/531. 
[60] R. E. Carpenter et al. "Influence of antenatal physical exercise on heart rate variability and QT variability". In: The Journal of Maternal-Fetal \& Neonatal Medicine 30.1 (2016), pp. 79-84. DOI: 10.3109/14767058.2016.1163541.

[61] R. E. Carpenter et al. "Influence of physical exercise on baroreceptor sensitivity during pregnancy". In: The Journal of Maternal-Fetal $\&$ Neonatal Medicine 30.5 (2016), pp. 514-519. DOI: 10.1080/14767058.2016.1179275.

[62] R. E. Carpenter et al. "Recruitment of pregnant women to an exerciseintervention study". In: Journal of Obstetrics and Gynaecology 36.2 (2015), pp. 200-207. DOI: 10.3109/01443615.2015.1049988.

[63] Rhiannon Emma Carpenter et al. "Influence of antenatal physical exercise on haemodynamics in pregnant women: a flexible randomisation approach". In: BMC Pregnancy and Childbirth 15.1 (2015). DOI: 10.1186/s12884-0150620-2.

[64] Jason Carson and Raoul Van Loon. "An implicit solver for 1D arterial network models". In: International Journal for Numerical Methods in Biomedical Engineering 33.7 (2016), e2837. ISSN: 2040-7947. DOI: 10.1002/cnm. 2837. URL: http://dx.doi.org/10.1002/cnm. 2837.

[65] Jason Carson, Raoul Van Loon, and Perumal Nithiarasu. "Mathematical Techniques for Circulatory Systems". In: Reference Module in Biomedical Sciences. Elsevier, 2018. DOI: 10.1016/b978-0-12-801238-3.99982-3.

[66] J. E. Cartwright et al. "Remodelling at the maternal-fetal interface: relevance to human pregnancy disorders". In: Reproduction 140.6 (2010), pp. 803-813. DOI: $10.1530 / R E P-10-0294$.

[67] Yasmin Casmod, Barbara Van Dyk, and E. Nicolaou. "Uterine artery Doppler screening as a predictor of pre-eclampsia". In: Health SA Gesondheid 21 (2016), pp. 391-396. DOI: 10.1016/j.hsag.2016.06.004.

[68] Marcelo A. Castro, Christopher M. Putman, and Juan R. Cebral. "PatientSpecific Computational Modeling of Cerebral Aneurysms With Multiple Avenues of Flow From 3D Rotational Angiography Images". In: Academic Radiology 13.7 (2006), pp. 811-821. DOI: 10.1016/j.acra.2006.03.011. 
[69] Vincenzo Casulli, Michael Dumbser, and Eleuterio F. Toro. "Semi-implicit numerical modeling of axially symmetric flows in compliant arterial systems". In: International Journal for Numerical Methods in Biomedical Engineering 28.2 (2011), pp. 257-272. DOI: 10.1002/cnm. 1464.

[70] H Celepci and E Brenner. "Position of valves within the subclavian and axillary veins". In: Journal of Vascular Surgery 54.6 (2011), 70S76S. DOI: $10.1016 / j \cdot j v s .2011 .06 .083$.

[71] Neeraj Kavan Chakshu et al. "A semi-active human digital twin model for detecting severity of carotid stenoses from head vibration - a coupled computational mechanics and computer vision method". In: International Journal for Numerical Methods in Biomedical Engineering (Jan. 2019), e3180. DOI: 10.1002/cnm. 3180 .

[72] F. Y. Chan et al. "Pregnancy Screening by Uterine Artery Doppler Velocimetry - Which Criterion Performs Best?" In: Obstetrics \& Gynecology 85.4 (1995), pp. 596-602. DOI: 10.1016/0029-7844(95)00006-d.

[73] Denis Chemla et al. "Total arterial compliance estimated by stroke volume-toaortic pulse pressure ratio in humans". In: American Journal of PhysiologyHeart and Circulatory Physiology 274.2 (1998), H500-H505. DOI: 10.1152/ ajpheart.1998.274.2.h500.

[74] Morie M. Chen et al. "Guidelines for Computed Tomography and Magnetic Resonance Imaging Use During Pregnancy and Lactation". In: Obstetrics $\&$ Gynecology 112.2, Part 1 (2008), pp. 333-340. DOI: 10 . 1097 /aog . Ob013e318180a505.

[75] I.L. Chernyavsky, O.E. Jensen, and L. Leach. "A Mathematical Model of Intervillous Blood Flow in the Human Placentone". In: Placenta 31.1 (2010), pp. 44-52. DOI: $10.1016 / \mathrm{j} \cdot \mathrm{placenta} .2009 .11 .003$.

[76] Katharine L. Cheung and Richard A. Lafayette. "Renal Physiology of Pregnancy". In: Advances in Chronic Kidney Disease 20.3 (2013), pp. 209-214. DOI: $10.1053 / \mathrm{j}$. ackd.2013.01.012. 
[77] Claudio Chiastra et al. "On the necessity of modelling fluid-structure interaction for stented coronary arteries". In: Journal of the Mechanical Behavior of Biomedical Materials 34 (2014), pp. 217-230. DOI: 10.1016/j .jmbbm. 2014.02 .009 .

[78] P F Chien et al. "How useful is uterine artery Doppler flow velocimetry in the prediction of pre-eclampsia, intrauterine growth retardation and perinatal death? An overview." In: BJOG : an international journal of obstetrics and gynaecology 107 (2 2000), pp. 196-208. ISSN: 1470-0328.

[79] I. Choi, S. Lee, and Y.-K. Hong. "The New Era of the Lymphatic System: No Longer Secondary to the Blood Vascular System". In: Cold Spring Harbor Perspectives in Medicine 2.4 (2012), a006445-a006445. DOI: 10.1101 / cshperspect.a006445.

[80] Jin Wook Chung et al. "Reference Values for the Augmentation Index and Pulse Pressure in Apparently Healthy Korean Subjects". In: Korean Circulation Journal 40.4 (2010), p. 165. DOI: 10.4070/kcj.2010.40.4.165.

[81] A. R. Clark et al. "Multiscale modelling of the feto-placental vasculature". In: Interface Focus 5.2 (2015), p. 20140078. DOI: 10.1098/rsfs.2014.0078.

[82] Alys R. Clark et al. "Understanding abnormal uterine artery Doppler waveforms: A novel computational model to explore potential causes within the utero-placental vasculature". In: Placenta 66 (2018), pp. 74-81. DOI: 10 . 1016/j.placenta.2018.05.001.

[83] J. S. Cnossen et al. "Use of uterine artery Doppler ultrasonography to predict pre-eclampsia and intrauterine growth restriction: a systematic review and bivariable meta-analysis". In: Canadian Medical Association Journal 178.6 (2008), pp. 701-711. DOI: 10.1503/cmaj.070430.

[84] Alberto Coccarelli and Perumal Nithiarasu. "A Robust Finite Element Modeling Approach to Conjugate Heat Transfer in Flexible Elastic Tubes and Tube Networks". In: Numerical Heat Transfer, Part A: Applications 67.5 (2014), pp. 513-530. DOI: 10.1080/10407782.2014.937284. 
[85] Alberto Coccarelli et al. "An advanced computational bioheat transfer model for a human body with an embedded systemic circulation". In: Biomechanics and Modeling in Mechanobiology 15.5 (2015), pp. 1173-1190. DOI: 10.1007/ s10237-015-0751-4.

[86] Adriaan Coenen et al. "Fractional Flow Reserve Computed from Noninvasive CT Angiography Data: Diagnostic Performance of an On-Site Clinicianoperated Computational Fluid Dynamics Algorithm". In: Radiology 274.3 (2015), pp. 674-683. DOI: 10.1148/radiol.14140992.

[87] Kirk P. Conrad. "Maternal vasodilation in pregnancy: the emerging role of relaxin". In: American Journal of Physiology-Regulatory, Integrative and Comparative Physiology 301.2 (2011), R267-R275. DOI: 10.1152/ajpregu . 00156.2011.

[88] Christian Contarino and Eleuterio F. Toro. "A one-dimensional mathematical model of collecting lymphatics coupled with an electro-fluid-mechanical contraction model and valve dynamics". In: Biomechanics and Modeling in Mechanobiology (2018). DOI: 10.1007/s10237-018-1050-7.

[89] Chiara Corsini et al. "Mathematical modelling of the maternal cardiovascular system in the three stages of pregnancy". In: Medical Engineering $\&$ Physics 47 (2017), pp. 55-63. DOI: 10.1016/j .medengphy.2017.06.025.

[90] Leah N. Cueni and Michael Detmar. "The Lymphatic System in Health and Disease". In: Lymphatic Research and Biology 6.3-4 (2008), pp. 109-122. DOI: 10.1089/lrb.2008.1008.

[91] Anthony M Dart and Bronwyn A Kingwell. "Pulse pressure - a review of mechanisms and clinical relevance". In: Journal of the American College of Cardiology 37.4 (2001), pp. 975-984. DOI: 10.1016/s0735-1097(01)011081.

[92] Sanjay Datta. Obstetric Anesthesia Handbook. Springer New York, 2006. DoI: 10.1007/978-0-387-88602-2. URL: http://www. ebook.de/de/product/ 11430341 /sanjay_datta_obstetric_anesthesia_handbook.html. 
[93] Michael J. Davis et al. "Determinants of valve gating in collecting lymphatic vessels from rat mesentery". In: American Journal of Physiology-Heart and Circulatory Physiology 301.1 (2011), H48-H60. DOI: 10 . 1152 / ajpheart . 00133.2011.

[94] J. M. Davison. "Edema in pregnancy." In: Kidney international. Supplement 59 (June 1997), S90-S96. ISSN: 0098-6577.

[95] Thomas Dawson. "Allometric Relations and Scaling Laws for the Cardiovascular System of Mammals". In: Systems 2.2 (2014), pp. 168-185. DOI: $10.3390 /$ systems 2020168

[96] Kenna Degner, Ronald R. Magness, and Dinesh M. Shah. "Establishment of the Human Uteroplacental Circulation: A Historical Perspective". In: Reproductive Sciences 24.5 (2016), pp. 753-761. DOI: 10.1177/1933719116669056.

[97] O. Delestre and P.-Y. Lagrée. "A 'well-balanced' finite volume scheme for blood flow simulation". In: International Journal for Numerical Methods in Fluids 72.2 (2012), pp. 177-205. DOI: 10.1002/fld.3736.

[98] R. Dickey. "Doppler ultrasound investigation of uterine and ovarian blood flow in infertility and early pregnancy". In: Human Reproduction Update 3.5 (1997), pp. 467-503. DOI: 10.1093/humupd/3.5.467.

[99] Jingliang Dong et al. "Fluid-structure interaction analysis of the left coronary artery with variable angulation". In: Computer Methods in Biomechanics and Biomedical Engineering 18.14 (2014), pp. 1500-1508. DOI: 10.1080 / 10255842.2014.921682.

[100] Lindsay A D'Silva et al. "Influence of somatic state on cardiovascular measurements in pregnancy". In: Physiological Measurement 35.1 (2013), pp. 1529. DOI: $10.1088 / 0967-3334 / 35 / 1 / 15$.

[101] M. G. Elliot and B. J. Crespi. "Genetic recapitulation of human pre-eclampsia risk during convergent evolution of reduced placental invasiveness in eutherian mammals". In: Philosophical Transactions of the Royal Society B: Biological Sciences 370.1663 (2015), pp. 20140069-20140069. DOI: 10.1098 / rstb.2014.0069. 
[102] A. Elvantaspinar et al. "Arterial stiffness and fetal growth in normotensive pregnancy". In: American Journal of Hypertension 18.3 (2005), pp. 337-341. DOI: $10.1016 / j . a m j$ hyper.2004.10.020.

[103] Sally Epstein et al. "Reducing the number of parameters in 1D arterial blood flow modeling: less is more for patient-specific simulations". In: American Journal of Physiology - Heart and Circulatory Physiology 309.1 (2015), H222H234. DOI: 10.1152/ajpheart.00857.2014.

[104] Jimmy Espinoza et al. "Normal and abnormal transformation of the spiral arteries during pregnancy". In: Journal of Perinatal Medicine 34.6 (2006). DOI: $10.1515 /$ JPM. 2006.089.

[105] M.-E. Estensen et al. "Increased Arterial Stiffness in Pre-eclamptic Pregnancy at Term and Early and Late Postpartum: A Combined Echocardiographic and Tonometric Study". In: American Journal of Hypertension 26.4 (2013), pp. 549-556. DOI: 10.1093/ajh/hps067.

[106] Pythoud F., Stergiopulos N., and Meister J.-J. "Forward and backward waves in the arterial system: nonlinear separation using Riemann invariants". In: Technology and Health Care 3.3 (1995), pp. 201-207. ISSN: 0928-7329. DOI: 10.3233/THC-1995-3305.

[107] F. Fantin et al. "Is augmentation index a good measure of vascular stiffness in the elderly?" In: Age and Ageing 36.1 (2006), pp. 43-48. DOI: 10.1093/ ageing/afl115.

[108] I Fernstrom. "Arteriography of the uterine artery: its value in the diagnosis of uterine fibromyoma, tubal pregnancy, adnexal tumour, and placental site localization in cases of intra-uterine pregnancy." In: Acta radiologica. Supplementum 122 (1955), pp. 1-128. ISSN: 0365-5954.

[109] E. Ferrazzi et al. "Uterine artery blood flow volume in pregnant women with an abnormal pulsatility index of the uterine arteries delivering normal or intrauterine growth restricted newborns". In: Placenta 32.7 (2011), pp. 487492. DOI: $10.1016 / \mathrm{j} \cdot \mathrm{placenta} .2011 .04 .004$. 
[110] C. A. Figueroa et al. "A coupled momentum method for modeling blood flow in three-dimensional deformable arteries". In: Computer Methods in Applied Mechanics and Engineering 195.41-43 (2006), pp. 5685-5706. DOI: 10.1016/ j.cma.2005.11.011.

[111] M. Fischer et al. "Flow velocity of single lymphatic capillaries in human skin". In: American Journal of Physiology-Heart and Circulatory Physiology 270.1 (1996), H358-H363. DOI: 10.1152/ajpheart.1996.270.1.h358.

[112] K. Flo, T. Wilsgaard, and G. Acharya. "A new non-invasive method for measuring uterine vascular resistance and its relationship to uterine artery Doppler indices: a longitudinal study". In: Ultrasound in Obstetrics $\&$ Gynecology 37.5 (2011), pp. 538-542. DOI: 10.1002/uog. 8907.

[113] H Florey. "Observations on the contractility of lacteals: Part I." In: The Journal of physiology 62 (3 Jan. 1927), pp. 267-272. ISSN: 0022-3751.

[114] R. Fogliardi, M. Di Donfrancesco, and R. Burattini. "Comparison of linear and nonlinear formulations of the three-element windkessel model". In: American Journal of Physiology-Heart and Circulatory Physiology 271.6 (Dec. 1996), H2661-H2668. DOI: 10.1152/ajpheart.1996.271.6.h2661.

[115] M. R. Foley. "Maternal Cardiovascular and hemodynamic adaptations to pregnancy". In: uptodate (2017). URL: https : //www . uptodate.com/contents/ maternal-cardiovascular-and-hemodynamic-adaptations-to-pregnancy.

[116] L. Formaggia et al. "On the coupling of 3D and 1D Navier-Stokes equations for problems in compliant vessels". In: Computer Methods in Applied Mechanics and Engineering 191.6-7 (2001), pp. 561-582. DOI: 10.1016/S00457825 (01) 00302-4.

[117] Luca Formaggia, Daniele Lamponi, and Alfio Quarteroni. "One-dimensional models for blood flow in arteries". In: Journal of Engineering Mathematics 47.3 (2003), pp. 251-276. ISSN: 1573-2703. DOI: 10 . 1023 / B : ENGI . 0000007980 . 01347 . 29. URL: http : / / dx . doi .org/10 . 1023/B : ENGI . 0000007980.01347 .29 .

[118] Luca Formaggia et al. "Multiscale modelling of the circulatory system: a preliminary analysis". In: Computing and Visualization in Science 2.2-3 (Dec. 1999), pp. 75-83. DOI: 10.1007/s007910050030. 
[119] Luca Formaggia et al. "Numerical modeling of 1D arterial networks coupled with a lumped parameters description of the heart". In: Computer Methods in Biomechanics and Biomedical Engineering 9.5 (Oct. 2006), pp. 273-288. DOI: $10.1080 / 10255840600857767$.

[120] O. Frank. "Die Grundform des arteriellen Pulses". In: Zeitschrift fr Biologie 37 (1899), pp. 483-526.

[121] Maximilian B. Franz et al. "Augmentation index and pulse wave velocity in normotensive and pre-eclamptic pregnancies". In: Acta Obstetricia et Gynecologica Scandinavica 92.8 (May 2013), pp. 960-966. DOI: 10.1111/aogs . 12145.

[122] S.V. Frolov et al. "Experimental and CFD flow studies in an intracranial aneurysm model with Newtonian and non-Newtonian fluids". In: Technology and Health Care 24.3 (May 2016), pp. 317-333. ISSN: 0928-7329. DOI: 10. 3233/THC-161132.

[123] T Frusca et al. "Role of uterine artery Doppler investigation in pregnant women with chronic hypertension." In: European journal of obstetrics, gynecology, and reproductive biology 79 (1 July 1998), pp. 47-50. ISSN: 0301-2115.

[124] Mika Fujime et al. "Central aortic blood pressure and augmentation index during normal pregnancy". In: Hypertension Research 35.6 (Feb. 2012), pp. 633-638. DOI: 10.1038/hr.2012.1.

[125] Jose-Maria Fullana and S. Zaleski. "A branched one-dimensional model of vessel networks". In: Journal of Fluid Mechanics 621 (Feb. 2009), p. 183. DOI: $10.1017 / \mathrm{s} 0022112008004771$.

[126] A Gaggiati. "The venous valves of the lower limbs". In: Phlebolymphology 20.2 (2013), pp. 87 -95. ISSN: 1286-0107.

[127] Robert Gagnon. "Placental insufficiency and its consequences". In: European Journal of Obstetrics \& Gynecology and Reproductive Biology 110 (Sept. 2003), S99-S107. DOI: 10.1016/s0301-2115(03)00179-9.

[128] R. Gaillard et al. "Second- and Third-Trimester Placental Hemodynamics and the Risks of Pregnancy Complications: The Generation R Study". In: American Journal of Epidemiology 177.8 (Mar. 2013), pp. 743-754. DOI: 10. 1093/aje/kws296. 
[129] Timur M. Gamilov et al. "Virtual fractional flow reserve assessment in patientspecific coronary networks by 1D hemodynamic model". In: Russian Journal of Numerical Analysis and Mathematical Modelling 30.5 (Jan. 2015). DOI: 10.1515/rnam-2015-0024.

[130] Patricia Garcia-Canadilla et al. "A Computational Model of the Fetal Circulation to Quantify Blood Redistribution in Intrauterine Growth Restriction". In: PLoS Computational Biology 10.6 (June 2014). Ed. by Amit Gefen, e1003667. DOI: 10.1371/journal.pcbi.1003667.

[131] P Gathiram and J Moodley. "Pre-eclampsia: its pathogenesis and pathophysiolgy". In: Cardiovascular Journal of Africa 27.2 (May 2016), pp. 71-78. DOI: $10.5830 / c v j a-2016-009$.

[132] Juliana Gebb and Pe'er Dar. "Colour Doppler ultrasound of spiral artery blood flow in the prediction of pre-eclampsia and intrauterine growth restriction". In: Best Practice \& Research Clinical Obstetrics \& Gynaecology 25.3 (June 2011), pp. 355-366. DOI: 10.1016/j. bpobgyn.2011.01.008.

[133] Rob J. van der Geest and Pankaj Garg. "Advanced Analysis Techniques for Intra-cardiac Flow Evaluation from 4D Flow MRI". In: Current Radiology Reports 4.7 (May 2016). DOI: 10.1007/s40134-016-0167-7.

[134] Simon Gelman. "Venous Function and Central Venous Pressure". In: Anesthesiology 108.4 (Apr. 2008), pp. 735-748. DOI: 10.1097/aln.0b013e3181672607.

[135] Arthur Ghigo et al. "Linear and nonlinear viscoelastic arterial wall models: application on animals". In: Journal of Biomechanical Engineering 139.1 (Nov. 2016), p. 011003. DOI: 10.1115/1.4034832. arXiv: 1607.07973v1 [physics.bio-ph].

[136] Rosalba Giordano et al. "Uterine artery Doppler flow studies in obstetric practice." In: Journal of prenatal medicine 4 (4 Oct. 2010), pp. 59-62. ISSN: 1971-3290.

[137] O. Gómez et al. "Reference ranges for uterine artery mean pulsatility index at 11-41 weeks of gestation". In: Ultrasound in Obstetrics and Gynecology 32.2 (Aug. 2008), pp. 128-132. DOI: 10.1002/uog. 5315. 
[138] O. Gómez et al. "Uterine artery Doppler at 11-14 weeks of gestation to screen for hypertensive disorders and associated complications in an unselected population". In: Ultrasound in Obstetrics and Gynecology 26.5 (Sept. 2005), pp. 490-494. DOI: 10.1002/uog. 1976.

[139] YH Gomez et al. "Pulse Pressure Amplification and Arterial Stiffness in LowRisk, Uncomplicated Pregnancies". In: Angiology 67.4 (Aug. 2015), pp. 375383. DOI: $10.1177 / 0003319715590056$.

[140] J. Gonzalez-Alonso et al. "Dehydration reduces cardiac output and increases systemic and cutaneous vascular resistance during exercise". In: Journal of Applied Physiology 79.5 (Nov. 1995), pp. 1487-1496. DOI: 10.1152/jappl. 1995.79 .5 .1487$.

[141] S.E. Greenwald. "Pulse pressure and arterial elasticity". In: QJM 95.2 (Feb. 2002), pp. 107-112. DOI: 10.1093/qjmed/95.2.107.

[142] L. Grinberg et al. "Modeling Blood Flow Circulation in Intracranial Arterial Networks: A Comparative 3D/1D Simulation Study". In: Annals of Biomedical Engineering 39.1 (July 2010), pp. 297-309. DOI: 10.1007/s10439-0100132-1.

[143] L. Guedes-Martins et al. "Internal iliac and uterine arteries Doppler ultrasound in the assessment of normotensive and chronic hypertensive pregnant women". In: Scientific Reports 4.1 (Jan. 2014). DOI: 10.1038/srep03785.

[144] Luís Guedes-Martins et al. "Reference Ranges for Uterine Artery Pulsatility Index during the Menstrual Cycle: A Cross-Sectional Study". In: PLOS ONE 10.3 (Mar. 2015). Ed. by Fatima Crispi-Brillas, e0119103. DOI: 10 . 1371 / journal.pone.0119103.

[145] S. De Haas et al. "Cardiac remodeling in normotensive pregnancy and in pregnancy complicated by hypertension: systematic review and meta-analysis". In: Ultrasound in Obstetrics $\&$ Gynecology 50.6 (Dec. 2017), pp. 683-696. DOI: $10.1002 /$ uog. 17410 .

[146] T. Habara et al. "Elevated blood flow resistance in uterine arteries of women with unexplained recurrent pregnancy loss." In: Human reproduction (Oxford, England) 17 (1 Jan. 2002), pp. 190-194. ISSN: 0268-1161. 
[147] Sarah Hale et al. "Pulse Pressure and Arterial Compliance Prior to Pregnancy and the Development of Complicated Hypertension During Pregnancy". In: Reproductive Sciences 17.9 (July 2010), pp. 871-877. DOI: 10.1177/1933719110376545.

[148] Sarah A. Hale et al. "Relationship Between Prepregnancy and Early Pregnancy Uterine Blood Flow and Resistance Index". In: Reproductive Sciences 16.11 (Aug. 2009), pp. 1091-1096. DOI: 10.1177/1933719109341843.

[149] John E. Hall. Guyton and Hall Textbook of Medical Physiology. Elsevier LTD, Oxford, 2015. ISBN: 1455770051. URL: https://www.ebook.de/de/product/ 23640278 / john _ e _ hall_ guyton_ and _ hall_ textbook _ of _ medical _ physiology.html.

[150] Michael E. Hall, Eric M. George, and Joey P. Granger. "The Heart During Pregnancy". In: Revista Española de Cardiología (English Edition) 64.11 (Nov. 2011), pp. 1045-1050. DOI: 10.1016/j.rec.2011.07.008.

[151] B. A. Haluska et al. "Pulse pressure vs. total arterial compliance as a marker of arterial health". In: European Journal of Clinical Investigation 35.7 (July 2005), pp. 438-443. DOI: 10.1111/j.1365-2362.2005.01513.x.

[152] K. Harrington et al. "The value of uterine artery Doppler in the prediction of uteroplacental complications in multiparous women". In: Ultrasound in Obstetrics and Gynecology 23.1 (Dec. 2003), pp. 50-55. DOI: 10.1002/uog. 932.

[153] L. K. Harris. "Review: Trophoblast-Vascular Cell Interactions in Early Pregnancy: How to Remodel a Vessel". In: Placenta 31 (Mar. 2010), S93-S98. DOI: $10.1016 / \mathrm{j} \cdot$ placenta.2009.12.012.

[154] Kamran Hassani, Mahdi Navidbakhsh, and Mostafa Rostami. "Modeling of the aorta artery aneurysms and renal artery stenosis using cardiovascular electronic system". In: BioMedical Engineering OnLine 6.1 (2007), p. 22. DOI: $10.1186 / 1475-925 \mathrm{X}-6-22$.

[155] B. H. Heidemann and J. H. McClure. "Changes in maternal physiology during pregnancy". In: BJA CEPD Reviews 3.3 (June 2003), pp. 65-68. DOI: 10 . 1093/bjacepd/mkg065. 
[156] Leif Rune Hellevik et al. "An assessment of ductus venosus tapering and wave transmission from the fetal heart". In: Biomechanics and Modeling in Mechanobiology 8.6 (2009), p. 509. ISSN: 1617-7940. DOI: 10.1007/s10237009-0155-4. URL: http://dx.doi.org/10 .1007/s10237-009-0155-4.

[157] Marius M Hoeper et al. "Pulmonary Hypertension." In: Deutsches Arzteblatt international 114 (5 Feb. 2017), pp. 73-84. ISSN: 1866-0452. DOI: 10.3238/ arztebl.2017.0073.

[158] W. Huberts et al. "A pulse wave propagation model to support decisionmaking in vascular access planning in the clinic". In: Medical Engineering $\&$ Physics 34.2 (Mar. 2012), pp. 233-248. DOI: 10.1016/j .medengphy. 2011. 07.015.

[159] G. A. Hughes and J. M. Allen. "Neural modulation of bovine mesenteric lymph node contraction". In: Experimental Physiology 78.5 (Sept. 1993), pp. 663-674. DOI: 10.1113/expphysiol.1993.sp003714.

[160] Thomas J. R. Hughes and J. Lubliner. "On the one-dimensional theory of blood flow in the larger vessels". In: Mathematical Biosciences 18.1 (1973), pp. 161 -170. ISSN: 0025-5564. DOI: 10.1016/0025-5564(73)90027-8. URL: http://www. sciencedirect.com/science/article/pii/0025556473900278.

[161] F. Hytten. "Blood volume changes in normal pregnancy." In: Clinics in haematology 14 (3 Oct. 1985), pp. 601-612. ISSN: 0308-2261.

[162] Bashar Issa et al. "Quantification of blood velocity and flow rates in the uterine vessels using echo planar imaging at 0.5 Tesla". In: Journal of Magnetic Resonance Imaging 31.4 (Apr. 2010), pp. 921-927. DOI: 10 . 1002 / jmri . 22119.

[163] Lucian Itu et al. "A machine-learning approach for computation of fractional flow reserve from coronary computed tomography". In: Journal of Applied Physiology 121.1 (Apr. 2016), pp. 42-52. DOI: 10 . 1152 / japplphysiol . 00752.2015 .

[164] S. JAIN et al. "Changes in arterial stiffness in pregnancy induced hypertension following delivery". In: International Journal of Cardiology 137 (Oct. 2009), S96. DOI: 10.1016/j.ijcard.2009.09.324. 
[165] Samira Jamalian et al. "Network Scale Modeling of Lymph Transport and Its Effective Pumping Parameters". In: PLOS ONE 11.2 (Feb. 2016). Ed. by Jerome W Breslin, e0148384. DOI: 10.1371/journal.pone.0148384.

[166] Bo-nan Jiang. Least-Squares Finite Element Method. 2013. URL: https: // www . ebook. de/de/product/33542861/bo_nan_jiang_least_squares_ finite_element_method.html.

[167] Christina Kaihura et al. "Maternal arterial stiffness in pregnancies affected by preeclampsia". In: American Journal of Physiology-Heart and Circulatory Physiology 297.2 (Aug. 2009), H759-H764. DOI: 10.1152/ajpheart.01106. 2008.

[168] Oguz Kayiran et al. "Lymphedema: From diagnosis to treatment". In: Turkish Journal of Surgery 33.2 (June 2017), pp. 51-57. DOI: 10.5152/turkjsurg. 2017.3870 .

[169] J. M. T. Keijsers et al. "A 1D pulse wave propagation model of the hemodynamics of calf muscle pump function". In: International Journal for $\mathrm{Nu}$ merical Methods in Biomedical Engineering 31.7 (Apr. 2015), n/a-n/a. ISSN: 2040-7947. DOI: 10 .1002/cnm.2714. URL: http://dx .doi .org/10 .1002/ cnm. 2714.

[170] Zahra Keshavarz-Motamed et al. "Non-Invasive Determination of Left Ventricular Workload in Patients with Aortic Stenosis Using Magnetic Resonance Imaging and Doppler Echocardiography". In: PLoS One 9.1 (2014). Ed. by Vincenzo Lionetti, e86793. DOI: 10.1371/journal.pone.0086793.

[171] A. Khalil et al. "Longitudinal changes in maternal hemodynamics in a population at risk for pre-eclampsia". In: Ultrasound in Obstetrics \& Gynecology 44.2 (June 2014), pp. 197-204. DOI: 10.1002/uog. 13367.

[172] Asma Khalil et al. "Pulse Wave Analysis in Normal Pregnancy: A Prospective Longitudinal Study". In: PLoS ONE 4.7 (July 2009). Ed. by Fernando Althabe, e6134. DOI: 10.1371/journal pone.0006134.

[173] Su Lynn Khong et al. "First-Trimester Uterine Artery Doppler Analysis in the Prediction of Later Pregnancy Complications". In: Disease Markers 2015 (2015), pp. 1-10. DOI: 10.1155/2015/679730. 
[174] David G Kiely et al. "Pregnancy and pulmonary hypertension: a practical approach to management". In: Obstetric Medicine 6.4 (Aug. 2013), pp. 144154. DOI: $10.1177 / 1753495 \times 13495193$.

[175] H. J. Kim et al. "On Coupling a Lumped Parameter Heart Model and a Three-Dimensional Finite Element Aorta Model". In: Annals of Biomedical Engineering 37.11 (July 2009), pp. 2153-2169. DOI: 10.1007/s10439-0099760-8.

[176] Hermann H. Klein and Sibylle Pich. "Physiologische Änderungen des HerzKreislauf-Systems in der Schwangerschaft". In: Herz 28.3 (May 2003), pp. 173174. DOI: $10.1007 / \mathrm{s} 00059-003-2455-2$.

[177] Brian S. Ko et al. "Noninvasive CT-Derived FFR Based on Structural and Fluid Analysis". In: JACC: Cardiovascular Imaging (Oct. 2016). DOI: 10.1016/j . jcmg. 2016.07.005.

[178] Justin C. Konje et al. "A longitudinal study of quantitative uterine blood flow with the use of color power angiography in appropriate for gestational age pregnancies". In: American Journal of Obstetrics and Gynecology 185.3 (Sept. 2001), pp. 608-613. DOI: 10.1067/mob.2001.117187.

[179] Bon-Kwon Koo et al. "Diagnosis of Ischemia-Causing Coronary Stenoses by Noninvasive Fractional Flow Reserve Computed From Coronary Computed Tomographic Angiograms". In: Journal of the American College of Cardiology 58.19 (Nov. 2011), pp. 1989-1997. DOI: 10.1016/j.jacc.2011.06.066.

[180] Theodosios Korakianitis and Yubing Shi. "Numerical simulation of cardiovascular dynamics with healthy and diseased heart valves". In: Journal of Biomechanics 39.11 (Jan. 2006), pp. 1964-1982. DOI: 10.1016/j . jbiomech. 2005.06 .016 .

[181] Usha Krishna and Sarita Bhalerao. "Placental Insufficiency and Fetal Growth Restriction". In: The Journal of Obstetrics and Gynecology of India 61.5 (Oct. 2011), pp. 505-511. DOI: 10.1007/s13224-011-0092-x.

[182] W. Kroon et al. "A Numerical Method of Reduced Complexity for Simulating Vascular Hemodynamics Using Coupled 0D Lumped and 1D Wave Propagation Models". In: Computational and Mathematical Methods in Medicine 
2012 (2012), pp. 1-10. DOI: 10.1155/2012/156094. URL: http://doi.org/ $10.1155 / 2012 / 156094$.

[183] Jonathan Lai et al. "Uterine Artery Doppler at 30-33 Weeks' Gestation in the Prediction of Preeclampsia". In: Fetal Diagnosis and Therapy 33.3 (2013), pp. 156-163. DOI: 10.1159/000343665.

[184] BM Lantz et al. "Regional distribution of cardiac output: normal values in man determined by video dilution technique". In: American Journal of Roentgenology 137.5 (Nov. 1981), pp. 903-907. DOI: 10.2214/ajr.137.5. 903.

[185] Kevin D. Lau and C. Alberto Figueroa. "Simulation of short-term pressure regulation during the tilt test in a coupled 3D-0D closed-loop model of the circulation". In: Biomechanics and Modeling in Mechanobiology 14.4 (Jan. 2015), pp. 915-929. DOI: 10.1007/s10237-014-0645-x.

[186] Merzaka Lazdam et al. "Prevention of Vascular Dysfunction after Preeclampsia: A Potential Long-Term Outcome Measure and an Emerging Goal for Treatment". In: Journal of Pregnancy 2012 (2012), pp. 1-8. DOI: 10.1155/ 2012/704146.

[187] Hae-Young Lee and Byung-Hee Oh. "Aging and Arterial Stiffness". In: Circulation Journal 74.11 (2010), pp. 2257-2262. DOI: 10.1253/circj.cj-100910.

[188] Paul. Roger. Leinan. "Biomechanical modeling of fetal veins: The umbilical vein and ductus venosus bifurcation". phd. Norwegian University of Science and Technology, NTNU Trondheim, Norway., 2012.

[189] R. P. Lewis et al. "The mitral valve prolapse epidemic: fact or fiction." In: Transactions of the American Clinical and Climatological Association 98 (1987), pp. 222-236. ISSN: 0065-7778.

[190] H. Li et al. "Increased uterine artery vascular impedance is related to adverse outcome of pregnancy but is present in only one-third of late thirdtrimester pre-eclamptic women". In: Ultrasound in Obstetrics and Gynecology 25.5 (2005), pp. 459-463. DOI: 10.1002/uog. 1895. 
[191] Fuyou LIANG and Hao LIU. "A Closed-Loop Lumped Parameter Computational Model for Human Cardiovascular System". In: JSME International Journal Series C 48.4 (2005), pp. 484-493. DOI: 10.1299/jsmec.48.484.

[192] Wai Yee Lim et al. "A cohort evaluation on arterial stiffness and hypertensive disorders in pregnancy". In: BMC Pregnancy and Childbirth 12.1 (Dec. 2012). DOI: $10.1186 / 1471-2393-12-160$.

[193] S Lin et al. "Uterine artery Doppler velocimetry in relation to trophoblast migration into the myometrium of the placental bed." In: Obstetrics and gynecology 85 (5 Pt 1 May 1995), pp. 760-765. ISSN: 0029-7844.

[194] K. Low et al. "An improved baseline model for a human arterial network to study the impact of aneurysms on pressure-flow waveforms". In: International Journal for Numerical Methods in Biomedical Engineering 28.12 (Dec. 2012), pp. 1224-1246. DOI: 10.1002/cnm. 2533.

[195] Jianwen Luo and Elisa E. Konofagou. "Imaging of Wall Motion Coupled With Blood Flow Velocity in the Heart and Vessels in Vivo: A Feasibility Study". In: Ultrasound in Medicine $\mathscr{G}$ Biology 37.6 (June 2011), pp. 980-995. DOI: 10.1016/j.ultrasmedbio.2011.03.004.

[196] A. J. Macdonald et al. "Modeling flow in collecting lymphatic vessels: onedimensional flow through a series of contractile elements". In: American Journal of Physiology-Heart and Circulatory Physiology 295.1 (July 2008), H305H313. DOI: 10.1152/ajpheart.00004.2008.

[197] M. L. Macedo et al. "Maternal Wave Reflections and Arterial Stiffness in Normal Pregnancy as Assessed by Applanation Tonometry". In: Hypertension 51.4 (Feb. 2008), pp. 1047-1051. DOI: 10 . 1161 / HYPERTENSIONAHA . 107 . 106062.

[198] Adam Mahdi et al. "Modeling the Afferent Dynamics of the Baroreflex Control System". In: PLoS Computational Biology 9.12 (Dec. 2013). Ed. by Julia C. Arciero, e1003384. DOI: 10.1371/journal.pcbi.1003384.

[199] A. Cristiano I. Malossi et al. "Implicit Coupling of One-Dimensional and Three-Dimensional Blood Flow Models with Compliant Vessels". In: Multiscale Modeling \& Simulation 11.2 (Jan. 2013), pp. 474-506. DOI: 10.1137/ 120867408. 
[200] Maurizio Mandala and George Osol. "Physiological Remodelling of the Maternal Uterine Circulation during Pregnancy". In: Basic \& Clinical Pharmacology \& Toxicology 110.1 (Oct. 2011), pp. 12-18. DOI: $10.1111 /$ j.17427843.2011.00793.x.

[201] S J Marchais et al. "Arterial compliance and blood pressure." In: Drugs 46 Suppl 2 (1993), pp. 82-87. ISSN: 0012-6667.

[202] K. N. Margaris and R. A. Black. "Modelling the lymphatic system: challenges and opportunities". In: Journal of The Royal Society Interface 9.69 (Jan. 2012), pp. 601-612. DOI: 10.1098/rsif.2011.0751.

[203] Elaine Nicpon Marieb. Human Anatomy $\&$ Physiology (5th Edition). BenjaminCummings Pub Co, 2000. ISBN: 0-8053-4989-8. URL: https://www . amazon . com / Anatomy - Physiology - Elaine - Nicpon - Marieb / dp / $0805349898 ?$ SubscriptionId = 0JYN1NVW651KCA56C102 \& tag = techkie -20 \& linkCode $=$ xm2\&camp $=2025 \&$ creative $=165953 \&$ creativeASIN $=0805349898$.

[204] R Matijevic and T Johnston. "In vivo assessment of failed trophoblastic invasion of the spiral arteries in pre-eclampsia." In: British journal of obstetrics and gynaecology 106 (1 Jan. 1999), pp. 78-82. ISSN: 0306-5456.

[205] Koen S. Matthys et al. "Pulse wave propagation in a model human arterial network: Assessment of 1-D numerical simulations against in vitro measurements". In: Journal of Biomechanics 40.15 (2007), pp. 3476-3486. ISSN: 0021-9290. DOI: 10.1016/j.jbiomech.2007.05.027.

[206] J G McGeown and M J Gallagher. "The effects of field stimulation on bovine mesenteric lymph node contractility." In: Pflugers Archiv : European journal of physiology 416 (6 Aug. 1990), pp. 667-672. ISSN: 0031-6768.

[207] N G McHale and I C Roddie. "The effect of transmural pressure on pumping activity in isolated bovine lymphatic vessels." In: The Journal of physiology 261 (2 Oct. 1976), pp. 255-269. ISSN: 0022-3751.

[208] A. McKelvey et al. "Total uterine artery blood volume flow rate in nulliparous women is associated with birth weight and gestational age at delivery". In: Ultrasound in Obstetrics \& Gynecology 49.1 (Jan. 2017), pp. 54-60. DOI: 10.1002/uog. 15917. 
[209] Victoria L Meah et al. "Cardiac output and related haemodynamics during pregnancy: a series of meta-analyses". In: Heart 102.7 (Jan. 2016), pp. 518526. DOI: $10.1136 /$ heartjnl-2015-308476.

[210] M.H Meissner. "Lower Extremity Venous Anatomy". In: Seminars in Interventional Radiology 22.3 (2005), pp. 147-158. DOI: 10.1055/s-2005-921948.

[211] E Ménigault et al. "Feto-maternal circulation: mathematical model and comparison with Doppler measurements". In: European Journal of Ultrasound 7.2 (Apr. 1998), pp. 129-143. DOI: 10.1016/s0929-8266 (98)00018-4.

[212] Vuk Milišić and Alfio Quarteroni. "Analysis of lumped parameter models for blood flow simulations and their relation with 1D models". In: ESAIM: Mathematical Modelling and Numerical Analysis 38.4 (July 2004), pp. 613632. DOI: 10 . 1051/m2an : 2004036. URL: https : / / www . cambridge . org / core / article / div-class - title - analysis - of - lumped - parameter models-for-blood-flow-simulations-and-their-relation-with-1dmodels-div/DC38700CCA82BAC1865609A4DE2107D1.

[213] James K. Min et al. "Diagnostic Accuracy of Fractional Flow Reserve From Anatomic CT Angiography". In: JAMA 308.12 (Sept. 2012), p. 1237. DOI: 10.1001/2012.jama.11274.

[214] G. F. Mitchell et al. "Changes in Aortic Stiffness and Augmentation Index After Acute Converting Enzyme or Vasopeptidase Inhibition". In: Hypertension 46.5 (Oct. 2005), pp. 1111-1117. DOI: 10.1161/01. HYP. 0000186331. 47557.ae.

[215] Shohei Miyazaki et al. "Validation of numerical simulation methods in aortic arch using 4D Flow MRI". In: Heart and Vessels 32.8 (Apr. 2017), pp. 10321044. DOI: $10.1007 / \mathrm{s} 00380-017-0979-2$.

[216] Lucas O. Mller and Eleuterio F. Toro. "Well-balanced high-order solver for blood flow in networks of vessels with variable properties". In: International Journal for Numerical Methods in Biomedical Engineering 29.12 (July 2013), pp. 1388-1411. DOI: $10.1002 / \mathrm{cnm} .2580$.

[217] Larry Y. L. Mo et al. "A transmission line modelling approach to the interpretation of uterine doppler waveforms". In: Ultrasound in Medicine 86 Biology 14.5 (1988), pp. 365-376. DOI: 10.1016/0301-5629(88)90072-5. 
[218] Erin A. Morris et al. "Pregnancy induces persistent changes in vascular compliance in primiparous women". In: American Journal of Obstetrics and Gynecology 212.5 (May 2015), 633.e1-633.e6. DOI: 10.1016/j.ajog.2015.01. 005.

[219] Paul D. Morris et al. "Virtual Fractional Flow Reserve From Coronary Angiography: Modeling the Significance of Coronary Lesions". In: JACC: Cardiovascular Interventions 6.2 (Feb. 2013), pp. 149-157. DOI: $10.1016 / \mathrm{j}$. jcin.2012.08.024.

[220] Lucas O. Müller and Eleuterio F. Toro. "A global multiscale mathematical model for the human circulation with emphasis on the venous system". In: International Journal for Numerical Methods in Biomedical Engineering 30.7 (2014), pp. 681-725. ISSN: 2040-7947. DOI: 10.1002/cnm. 2622. URL: http: //dx.doi.org/10.1002/cnm. 2622.

[221] Lucas O. Müller and Eleuterio F. Toro. "Enhanced global mathematical model for studying cerebral venous blood flow". In: Journal of Biomechanics 47.13 (2014), pp. 3361 -3372. ISSN: 0021-9290. DOI: 10.1016/j.jbiomech. 2014.08.005. URL: http://www.sciencedirect.com/science/article/ pii/S0021929014004291.

[222] Lucas O. Müller et al. "A high-order local time stepping finite volume solver for one-dimensional blood flow simulations: application to the ADAN model". In: International Journal for Numerical Methods in Biomedical Engineering 32.10 (Jan. 2016), e02761. DOI: 10.1002/cnm.2761.

[223] C D Murray. "The Physiological Principle of Minimum Work: I. The Vascular System and the Cost of Blood Volume". In: Proceedings of the National Academy of Sciences of the United States of America 12.3 (1926), pp. 207214.

[224] J. P. Mynard and P. Nithiarasu. "A 1D arterial blood flow model incorporating ventricular pressure, aortic valve and regional coronary flow using the locally conservative Galerkin (LCG) method". In: Communications in Numerical Methods in Engineering 24.5 (Mar. 2008), pp. 367-417. ISSN: 1099-0887. DOI: 10.1002/cnm.1117. URL: http://dx.doi.org/10.1002/cnm.1117. 
[225] J. P. Mynard et al. "A numerical model of neonatal pulmonary atresia with intact ventricular septum and RV-dependent coronary flow". In: International Journal for Numerical Methods in Biomedical Engineering (2010), n/a-n/a. DOI: $10.1002 / \mathrm{cnm} .1384$.

[226] J. P. Mynard et al. "A simple, versatile valve model for use in lumped parameter and one-dimensional cardiovascular models". In: International Journal for Numerical Methods in Biomedical Engineering 28.6-7 (Sept. 2012), pp. 626-641. ISSN: 2040-7947. DOI: 10 . 1002/cnm. 1466. URL: http://dx . doi.org/10.1002/cnm.1466.

[227] Jonathan Mynard. "Computer modelling and wave intensity analysis of perinatal cardiovascular function and dysfunction". PhD Thesis. 2011.

[228] Jonathan P. Mynard, Daniel J. Penny, and Joseph J. Smolich. "Scalability and in vivo validation of a multiscale numerical model of the left coronary circulation". In: American Journal of Physiology - Heart and Circulatory Physiology 306.4 (2014), H517-H528. ISSN: 0363-6135. DOI: 10.1152/ajpheart. 00603 . 2013. eprint: http://ajpheart . physiology .org/content/306/4/ H517 . full . pdf. URL: http: //ajpheart . physiology . org/content/306/ $4 / \mathrm{H} 517$.

[229] Jonathan P. Mynard and Joseph J. Smolich. "Influence of anatomical dominance and hypertension on coronary conduit arterial and microcirculatory flow patterns: a multiscale modeling study". In: American Journal of PhysiologyHeart and Circulatory Physiology 311.1 (July 2016), H11-H23. DOI: 10.1152/ ajpheart. 00997.2015.

[230] Jonathan P. Mynard and Joseph J. Smolich. "One-Dimensional Haemodynamic Modeling and Wave Dynamics in the Entire Adult Circulation". In: Annals of Biomedical Engineering 43.6 (Apr. 2015), pp. 1443-1460. ISSN: 1573-9686. DOI: 10.1007/s10439-015-1313-8. URL: http://dx.doi.org/ $10.1007 / \mathrm{s} 10439-015-1313-8$.

[231] Jonathan P. Mynard and Kristian Valen-Sendstad. "A unified method for estimating pressure losses at vascular junctions". In: International Journal for Numerical Methods in Biomedical Engineering 31.7 (2015), n/a-n/a. ISSN: 
2040-7947. DOI: 10.1002/cnm.2717. URL: http://dx .doi .org/10.1002/ $\mathrm{cnm} .2717$.

[232] Mark G. Neerhof and Larry G. Thaete. "The Fetal Response to Chronic Placental Insufficiency". In: Seminars in Perinatology 32.3 (June 2008), pp. 201205. DOI: $10.1053 /$ j.semperi.2007.11.002.

[233] B Newman, C Derrington, and C Dore. "Cardiac output and the recumbent position in late pregnancy." In: Anaesthesia 38 (4 Apr. 1983), pp. 332-335. ISSN: 0003-2409.

[234] F. Nobile. "Coupling strategies for the numerical simulation of blood flow in deformable arteries by 3D and 1D models". In: Mathematical and Computer Modelling 49.11-12 (2009), pp. 2152-2160. DOI: 10.1016/j .mcm. 2008 .07. 019.

[235] Bjarne L. Nørgaard et al. "Diagnostic Performance of Noninvasive Fractional Flow Reserve Derived From Coronary Computed Tomography Angiography in Suspected Coronary Artery Disease". In: Journal of the American College of Cardiology 63.12 (Apr. 2014), pp. 1145-1155. DOI: $10.1016 / \mathrm{j} \cdot$ jacc . 2013.11 .043$.

[236] Hasan Obeid et al. "Numerical assessment and comparison of pulse wave velocity methods aiming at measuring aortic stiffness". In: Physiological Measurement 38.11 (Oct. 2017), pp. 1953-1967. DOI: 10 . 1088/1361-6579 / aa905a.

[237] T. Ohhashi, T. Azuma, and M. Sakaguchi. "Active and passive mechanical characteristics of bovine mesenteric lymphatics". In: American Journal of Physiology-Heart and Circulatory Physiology 239.1 (July 1980), H88-H95. DOI: 10.1152/ajpheart.1980.239.1.h88.

[238] Osamu Ohtani and Yuko Ohtani. "Structure and function of rat lymph nodes". In: Archives of Histology and Cytology 71.2 (2008), pp. 69-76. DOI: 10.1679/aohc. 71.69 .

[239] J.B. Olansen et al. "A Closed-Loop Model of the Canine Cardiovascular System That Includes Ventricular Interaction". In: Computers and Biomedical Research 33.4 (Aug. 2000), pp. 260-295. DOI: 10.1006/cbmr.2000.1543. 
[240] Olufemi Adebari Oloyede and Faye Iketubosin. "Uterine artery Doppler study in second trimester of pregnancy". In: Pan African Medical Journal 15 (2013). DOI: $10.11604 / p a m j .2013 .15 .87 .2321$.

[241] Karen M. Olsson and Richard Channick. "Pregnancy in pulmonary arterial hypertension". In: European Respiratory Review 25.142 (Nov. 2016), pp. 431437. DOI: $10.1183 / 16000617.0079-2016$.

[242] M. S. Olufsen. "Blood pressure and blood flow variation during postural change from sitting to standing: model development and validation". In: Journal of Applied Physiology 99.4 (June 2005), pp. 1523-1537. DOI: 10 . 1152/japplphysiol.00177.2005.

[243] M S Olufsen. "Structured tree outflow condition for blood flow in larger systemic arteries." In: The American journal of physiology 276 (1 Pt 2 Jan. 1999), H257-H268. ISSN: 0002-9513.

[244] Mette S. Olufsen et al. "Numerical Simulation and Experimental Validation of Blood Flow in Arteries with Structured-Tree Outflow Conditions". In: Annals of Biomedical Engineering 28.11 (2000), pp. 1281-1299. ISSN: 15739686. DOI: 10 .1114/1.1326031. URL: http://dx .doi .org/10 .1114/1. 1326031.

[245] R. Orabona et al. "Elastic properties of ascending aorta in women with previous pregnancy complicated by early- or late-onset pre-eclampsia". In: Ultrasound in Obstetrics $\&$ Gynecology 47.3 (Feb. 2016), pp. 316-323. DOI: 10.1002/uog. 14838.

[246] Shmuel Oren, Ehud Grossman, and Edward D. Frohlich. "Arterial and venous compliance in obese and nonobese subjects". In: The American Journal of Cardiology 77.8 (Mar. 1996), pp. 665-667. DOI: 10.1016/s0002-9149(97) 89331-9.

[247] World Health Organisation. Cardiovascular disease fact sheet. May 17, 2017. URL: http://www . who . int/news-room/fact-sheets/detail/cardiovasculardiseases-(cvds). 
[248] Mohamed Waseem Osman et al. "Association between arterial stiffness and wave reflection with subsequent development of placental-mediated diseases during pregnancy". In: Journal of Hypertension 36.5 (May 2018), pp. 10051014. DOI: $10.1097 / \mathrm{hjh} .0000000000001664$.

[249] G. Osol and M. Mandala. "Maternal Uterine Vascular Remodeling During Pregnancy". In: Physiology 24.1 (Feb. 2009), pp. 58-71. DOI: 10 . 1152 / physiol.00033.2008.

[250] Joseph G. Ouzounian and Uri Elkayam. "Physiologic Changes During Normal Pregnancy and Delivery". In: Cardiology Clinics 30.3 (Aug. 2012), pp. 317329. DOI: $10.1016 /$ j.ccl.2012.05.004.

[251] Mizuho Oyama-Kato et al. "Change in pulse wave velocity throughout normal pregnancy and its value in predicting pregnancy-induced hypertension: A longitudinal study". In: American Journal of Obstetrics and Gynecology 195.2 (Aug. 2006), pp. 464-469. DOI: 10.1016/j.ajog.2006.01.104.

[252] Sanjay Pant et al. "Data assimilation and modelling of patient-specific singleventricle physiology with and without valve regurgitation". In: Journal of Biomechanics 49.11 (July 2016), pp. 2162-2173. DOI: 10.1016/j . jbiomech . 2015.11.030.

[253] George Papadakis. "Coupling 3D and 1D fluid-structure-interaction models for wave propagation in flexible vessels using a finite volume pressurecorrection scheme". In: Communications in Numerical Methods in Engineering 25.5 (May 2009), pp. 533-551. DOI: 10.1002/cnm. 1212.

[254] Michail I. Papafaklis et al. "Fast virtual functional assessment of intermediate coronary lesions using routine angiographic data and blood flow simulation in humans: comparison with pressure wire - fractional flow reserve". In: EuroIntervention 10.5 (Sept. 2014), pp. 574-583. DOI: 10.4244/eijy14m07_01.

[255] Aris T Papageorghiou, Christina K.H Yu, and Kypros H Nicolaides. "The role of uterine artery Doppler in predicting adverse pregnancy outcome". In: Best Practice \&5 Research Clinical Obstetrics $\&$ Gynaecology 18.3 (June 2004), pp. 383-396. DOI: 10.1016/j. bpobgyn.2004.02.003. 
[256] Aris T. Papageorghiou et al. "Assessment of risk for the development of pre-eclampsia by maternal characteristics and uterine artery Doppler". In: BJOG: An International Journal of Obstetrics and Gynaecology 112.6 (June 2005), pp. 703-709. DOI: 10.1111/j.1471-0528.2005.00519.x.

[257] M Papp, G B Makara, and B Hajtman. "The resistance of in situ perfused lymph trunks and lymph nodes to flow." In: Experientia 27 (4 Apr. 1971), pp. 391-392. ISSN: 0014-4754.

[258] K. H. Parker and C. J. H. Jones. "Forward and Backward Running Waves in the Arteries: Analysis Using the Method of Characteristics". In: Journal of Biomechanical Engineering 112.3 (1990), p. 322. DOI: 10.1115/1.2891191.

[259] Kim H. Parker. "An introduction to wave intensity analysis". In: Medical 8 Biological Engineering \& Computing 47.2 (Feb. 2009), pp. 175-188. DOI: 10.1007/s11517-009-0439-y.

[260] Kim H. Parker et al. "What stops the flow of blood from the heart?" In: Heart and Vessels 4.4 (Dec. 1988), pp. 241-245. DOI: 10.1007/bf02058593.

[261] T.A. Parlikar et al. "Model-based estimation of cardiac output and total peripheral resistance". In: Computers in Cardiology (Sept. 2007). DOI: 10 . $1109 /$ cic.2007.4745501.

[262] Jason A. Pates et al. "Determining uterine blood flow in pregnancy with magnetic resonance imaging". In: Magnetic Resonance Imaging 28.4 (May 2010), pp. 507-510. DOI: 10.1016/j.mri.2009.12.009.

[263] Beth Payne et al. "PIERS Proteinuria: Relationship With Adverse Maternal and Perinatal Outcome". In: Journal of Obstetrics and Gynaecology Canada 33.6 (June 2011), pp. 588-597. DOI: 10.1016/s1701-2163(16)34907-6.

[264] Alberto Borges Peixoto et al. "Reference ranges for the uterine arteries Doppler and cervical length measurement at 11-13(+6) weeks of gestation in a Brazilian population". In: The Journal of Maternal-Fetal $\&$ Neonatal Medicine (Nov. 2015), pp. 1-6. DOI: 10.3109/14767058.2015.1111334.

[265] P. Pellizzari et al. "Assessment of uterine artery blood flow in normal firsttrimester pregnancies and in those complicated by uterine bleeding". In: Ultrasound in Obstetrics and Gynecology 19.4 (Apr. 2002), pp. 366-370. DOI: 10.1046/j.1469-0705.2002.00667.x. 
[266] G. Pennati, M. Bellotti, and R. Fumero. "Mathematical modelling of the human foetal cardiovascular system based on Doppler ultrasound data". In: Medical Engineering \& Physics 19.4 (June 1997), pp. 327-335. DOI: 10.1016/ s1350-4533(97) 84634-6.

[267] Tânia Pereira, Carlos Correia, and João Cardoso. "Novel Methods for Pulse Wave Velocity Measurement". In: Journal of Medical and Biological Engineering 35.5 (Oct. 2015), pp. 555-565. DOI: 10.1007/s40846-015-0086-8.

[268] Dean S. Picone et al. "Accuracy of Cuff-Measured Blood Pressure". In: Journal of the American College of Cardiology 70.5 (Aug. 2017), pp. 572-586. DOI: 10.1016/j.jacc. 2017.05 .064 .

[269] P. G. Pieper and E. S. Hoendermis. "Pregnancy in women with pulmonary hypertension". In: Netherlands Heart Journal 19.12 (Nov. 2011), pp. 504508. DOI: $10.1007 / \mathrm{s} 12471-011-0219-9$.

[270] R. Pijnenborg, L. Vercruysse, and M. Hanssens. "The Uterine Spiral Arteries In Human Pregnancy: Facts and Controversies". In: Placenta 27.9-10 (Sept. 2006), pp. 939-958. DOI: 10.1016/j.placenta.2005.12.006.

[271] Antoine Pironet et al. "Parameter Identification Methods in a Model of the Cardiovascular System**This work was supported by the French Community of Belgium, the Belgian Funds for Scienti_c Research (F.R.S.-FNRS) and EU Marie Curie Actions (FP7-PEOPLE-2012-IRSES)." In: IFAC-PapersOnLine 48.20 (2015), pp. 366-371. DOI: 10.1016/j.ifacol.2015.10.167.

[272] Alfio Quarteroni and Luca Formaggia. "Mathematical Modelling and Numerical Simulation of the Cardiovascular System". In: Handbook of Numerical Analysis. Elsevier, 2004, pp. 3-127. DOI: 10.1016/s1570-8659(03)12001-7.

[273] Abdullah Bin Queyam et al. "Simulation and Analysis of Umbilical Blood Flow using Markov-based Mathematical Model". In: International Journal of Intelligent Systems and Applications 9.3 (Mar. 2017), pp. 41-50. DOI: 10. 5815/ijisa.2017.03.06.

[274] Christopher M. Quick et al. "First-order approximation for the pressureflow relationship of spontaneously contracting lymphangions". In: American Journal of Physiology-Heart and Circulatory Physiology 294.5 (May 2008), H2144-H2149. DOI: 10.1152/ajpheart.00781.2007. 
[275] M. Umar Qureshi et al. "Numerical simulation of blood flow and pressure drop in the pulmonary arterial and venous circulation". In: Biomechanics and Modeling in Mechanobiology 13.5 (Mar. 2014), pp. 1137-1154. DOI: 10. 1007/s10237-014-0563-y.

[276] R. Raghu et al. "Comparative Study of Viscoelastic Arterial Wall Models in Nonlinear One-Dimensional Finite Element Simulations of Blood Flow". In: Journal of Biomechanical Engineering 133.8 (Sept. 2011), p. 081003. DOI: 10.1115/1.4004532.

[277] Jeffrey K. Raines, Michel Y. Jaffrin, and Ascher H. Shapiro. "A computer simulation of arterial dynamics in the human leg". In: Journal of Biomechanics 7.1 (Jan. 1974), pp. 77-91. DOI: 10.1016/0021-9290 (74)90072-4.

[278] K S Rammos et al. "A computer model for the prediction of left epicardial coronary blood flow in normal, stenotic and bypassed coronary arteries, by single or sequential grafting." In: Cardiovascular surgery (London, England) 6 (6 Dec. 1998), 635-48; discussion 649-51. ISSN: 0967-2109.

[279] M. W. Ramsey and M. Sugawara. "Arterial wave intensity and ventriculoarterial interaction." In: Heart and vessels Suppl 12 (1997), pp. 128-134. ISSN: 0910-8327.

[280] Gwendalyn J. Randolph et al. "The Lymphatic System: Integral Roles in Immunity". In: Annual Review of Immunology 35.1 (Apr. 2017), pp. 31-52. DOI: 10.1146/annurev-immunol-041015-055354.

[281] Fernanda Rebelo et al. "Blood Pressure Variation Throughout Pregnancy According to Early Gestational BMI: A Brazilian Cohort". In: Arquivos Brasileiros de Cardiologia (2015). DOI: 10.5935/abc. 20150007.

[282] K. Red-Horse. "Lymphatic Vessel Dynamics in the Uterine Wall". In: Placenta 29 (Mar. 2008), pp. 55-59. DOI: 10.1016/j.placenta.2007.11.011.

[283] N P Reddy, T A Krouskop, and P H Newell. "Biomechanics of a lymphatic vessel." In: Blood vessels 12 (5 1975), pp. 261-278. ISSN: 0303-6847.

[284] Narender P. Reddy. "A note on the lymphatic vessel network design". In: Journal of Biomechanics 13.6 (Jan. 1980), pp. 529-531. DOI: 10.1016/0021$9290(80) 90346-2$. 
[285] Narender P. Reddy, Thomas A. Krouskop, and Paul H. Newell. "A computer model of the lymphatic system". In: Computers in Biology and Medicine 7.3 (July 1977), pp. 181-197. DOI: 10.1016/0010-4825 (77)90023-3.

[286] Matthias Renker et al. "Comparison of Diagnostic Value of a Novel Noninvasive Coronary Computed Tomography Angiography Method Versus Standard Coronary Angiography for Assessing Fractional Flow Reserve". In: The American Journal of Cardiology 114.9 (Nov. 2014), pp. 1303-1308. DOI: 10. 1016/j.amjcard.2014.07.064.

[287] P. Reymond et al. "Validation of a one-dimensional model of the systemic arterial tree". In: AJP: Heart and Circulatory Physiology 297.1 (May 2009), H208-H222. DOI: 10.1152/ajpheart.00037. 2009.

[288] Philippe Reymond et al. "Validation of a patient-specific one-dimensional model of the systemic arterial tree". In: American Journal of PhysiologyHeart and Circulatory Physiology 301.3 (Sept. 2011), H1173-H1182. DOI: 10.1152/ajpheart.00821.2010.

[289] Domenico Ribatti. "The Origins of Lymphatic Vessels". In: Milestones in Immunology. Elsevier, 2017, pp. 129-162. DOI: 10.1016/b978-0-12-8113134.00010-3.

[290] S. Rigano et al. "Blood flow volume of uterine arteries in human pregnancies determined using 3D and bi-dimensional imaging, angio-Doppler, and fluiddynamic modeling". In: Placenta 31.1 (Jan. 2010), pp. 37-43. DOI: 10.1016/ j.placenta.2009.10.010.

[291] A. O. Robb et al. "Influence of the Menstrual Cycle, Pregnancy, and Preeclampsia on Arterial Stiffness". In: Hypertension 53.6 (Apr. 2009), pp. 952-958. DOI: 10.1161 /HYPERTENSIONAHA . 109.130898.

[292] Marie Cecilie Paasche Roland et al. "Fetal Growth versus Birthweight: The Role of Placenta versus Other Determinants". In: PLoS ONE 7.6 (June 2012). Ed. by Nick Harvey, e39324. DOI: 10.1371/journal.pone.0039324.

[293] E. Rooz, D. F. Young, and T. R. Rogge. "A finite-element simulation of pulsatile flow in flexible obstructed tubes." In: Journal of biomechanical engineering 104 (2 May 1982), pp. 119-124. ISSN: 0148-0731. 
[294] G Sacchi et al. "The structure of superficial lymphatics in the human thigh: precollectors." In: The Anatomical record 247 (1 Jan. 1997), pp. 53-62. ISSN: 0003-276X.

[295] Masashi Saito et al. "One-Dimensional Model for Propagation of a Pressure Wave in a Model of the Human Arterial Network: Comparison of Theoretical and Experimental Results". In: Journal of Biomechanical Engineering 133.12 (2011), p. 121005. DOI: 10.1115/1.4005472.

[296] K Sakai et al. "How blood viscosity influences changes in circulation during pregnancy?" In: Fukuoka igaku zasshi = Hukuoka acta medica 83 (8 Aug. 1992), pp. 328-332. ISSN: 0016-254X.

[297] N. Salavati et al. "The relationship between human placental morphometry and ultrasonic measurements of utero-placental blood flow and fetal growth". In: Placenta 38 (Feb. 2016), pp. 41-48. DOI: 10.1016/j . placenta. 2015. 12.003.

[298] A. A. Salmi et al. "Older age group in pregnancy is associated with increased arterial stiffness". In: Medical Journal Of Malaysia 65.2 (2010). DOI: 23756794.

[299] A A Salmi et al. "Older Age Group in Pregnancy is Associated with Increased Arterial Stiffness." In: The Medical journal of Malaysia 65 (2 June 2010), pp. 115-118. ISSN: 0300-5283.

[300] M. Sanghavi and J. D. Rutherford. "Cardiovascular Physiology of Pregnancy". In: Circulation 130.12 (Sept. 2014), pp. 1003-1008. DOI: 10.1161/ CIRCULATIONAHA . 114.009029.

[301] Sethuraman Sankaran et al. "Uncertainty quantification in coronary blood flow simulations: Impact of geometry, boundary conditions and blood viscosity". In: Journal of Biomechanics 49.12 (Aug. 2016), pp. 2540-2547. DOI: 10.1016/j.jbiomech.2016.01.002.

[302] Van M. Savage, Eric J. Deeds, and Walter Fontana. "Sizing Up Allometric Scaling Theory". In: PLoS Computational Biology 4.9 (Sept. 2008). Ed. by Brian J. Enquist, e1000171. DOI: 10.1371/journal .pcbi.1000171. 
[303] Makrina D. Savvidou et al. "Maternal Arterial Stiffness in Women Who Subsequently Develop Pre-Eclampsia". In: PLoS ONE 6.5 (May 2011). Ed. by Jeffrey S. Berger, e18703. DOI: 10.1371/journal.pone.0018703.

[304] Igor Sazonov et al. "A novel method for non-invasively detecting the severity and location of aortic aneurysms". In: Biomechanics and Modeling in Mechanobiology (Feb. 2017). DOI: 10.1007/s10237-017-0884-8.

[305] Joshua P. Scallan et al. "Lymphatic pumping: mechanics, mechanisms and malfunction". In: The Journal of Physiology 594.20 (Aug. 2016), pp. 57495768. DOI: $10.1113 /$ jp272088.

[306] H Schulman et al. "Development of uterine artery compliance in pregnancy as detected by Doppler ultrasound." In: American journal of obstetrics and gynecology 155 (5 Nov. 1986), pp. 1031-1036. ISSN: 0002-9378.

[307] Anthony C. Sciscione and Edward J. Hayes. "Uterine artery Doppler flow studies in obstetric practice". In: American Journal of Obstetrics and Gynecology 201.2 (Aug. 2009), pp. 121-126. DOI: 10.1016/j.ajog.2009.03.027.

[308] H. Seeger, M. Bonani, and S. Segerer. "The role of lymphatics in renal inflammation". In: Nephrology Dialysis Transplantation 27.7 (May 2012), pp. 26342641. DOI: $10.1093 / \mathrm{ndt} / \mathrm{gfs} 140$.

[309] Marcus Seeger et al. "Terminal Part of Thoracic Duct: High-Resolution US Imaging". In: Radiology 252.3 (Sept. 2009), pp. 897-904. DOI: 10 . 1148 / radiol. 2531082036.

[310] P. Segers et al. "Peripheral "Oscillatory" Compliance Is Associated With Aortic Augmentation Index". In: Hypertension 37.6 (June 2001), pp. 14341439. DOI: $10.1161 / 01$. HYP.37.6.1434.

[311] Ruchit G. Shah et al. "Hemodynamic analysis of blood flow in umbilical artery using computational modeling". In: Placenta 57 (Sept. 2017), pp. 912. DOI: $10.1016 / \mathrm{j} \cdot \mathrm{placenta} .2017 .05 .017$.

[312] Minxue Shen et al. "Trajectory of blood pressure change during pregnancy and the role of pre-gravid blood pressure: a functional data analysis approach". In: Scientific Reports 7.1 (July 2017). DOI: 10.1038/s41598-01706606-0. 
[313] S. J. Sherwin et al. "Computational modelling of 1D blood flow with variable mechanical properties and its application to the simulation of wave propagation in the human arterial system". In: International Journal for $\mathrm{Nu}$ merical Methods in Fluids 43.6-7 (2003), pp. 673-700. ISSN: 1097-0363. DOI: 10.1002/fld.543. URL: http://dx.doi.org/10.1002/fld.543.

[314] S.J. Sherwin et al. "One-dimensional modelling of a vascular network in space-time variables". In: Journal of Engineering Mathematics 47.3/4 (Dec. 2003), pp. 217-250. DOI: 10.1023/b:engi.0000007979.32871.e2.

[315] Changzheng Shi et al. "A study of noninvasive fractional flow reserve derived from a simplified method based on coronary computed tomography angiography in suspected coronary artery disease". In: BioMedical Engineering OnLine 16.1 (Apr. 2017). DOI: 10.1186/s12938-017-0330-2.

[316] Yubing Shi, Patricia Lawford, and Rodney Hose. "Review of Zero-D and 1-D Models of Blood Flow in the Cardiovascular System". In: BioMedical Engineering OnLine 10.1 (2011), p. 33. DOI: 10.1186/1475-925X-10-33.

[317] Fabrício da Silva Costa, Sérgio Pereira da Cunha, and Aderson Tadeu Berezowski. "Qual o melhor período para a realização do doppler das artérias uterinas na predição de complicações da gestação?" In: Radiologia Brasileira 39.2 (Apr. 2006), pp. 97-102. DOI: 10.1590/S0100-39842006000200005.

[318] N. P. Smith, A. J. Pullan, and P. J. Hunter. "An Anatomically Based Model of Transient Coronary Blood Flow in the Heart". In: SIAM Journal on Applied Mathematics 62.3 (Jan. 2002), pp. 990-1018. DOI: 10.1137/s0036139999355199.

[319] Eric R. Sokol. "Clinical Anatomy of the Uterus, Fallopian Tubes, \& Ovaries". In: The Global Library of Women's Medicine (2009). DOI: 10.3843/GLOWM. 10001.

[320] P Soma-Pillay et al. "Physiological changes in pregnancy". In: Cardiovascular Journal of Africa 27.2 (May 2016), pp. 89-94. DOI: 10.5830/CVJA-2016-021.

[321] Benjamin J. Sprague et al. "The effects of the ovarian cycle and pregnancy on uterine vascular impedance and uterine artery mechanics". In: European Journal of Obstetrics $\&$ Gynecology and Reproductive Biology 144 (May 2009), S170-S178. DOI: 10.1016/j.ejogrb.2009.02.041. 
[322] B.N. Steele et al. "In vivo validation of a one-dimensional finite-element method for predicting blood flow in cardiovascular bypass grafts". In: IEEE Transactions on Biomedical Engineering 50.6 (June 2003), pp. 649-656. DOI: 10.1109/tbme.2003.812201.

[323] Jochen Steppan et al. "Vascular Stiffness and Increased Pulse Pressure in the Aging Cardiovascular System". In: Cardiology Research and Practice 2011 (2011), pp. 1-8. DOI: 10.4061/2011/263585.

[324] N Stergiopoulos. "Computer simulation of arterial blood flow". PhD thesis. Iowa State University, 1990.

[325] N. Stergiopoulos, D. Young, and T. Rogge. "Computer simulation of arterial flow with applications to arterial and aortic stenoses". In: Journal of Biomechanics 25.12 (1992), pp. $1477-1488$.

[326] N. Stergiopulos, J. J. Meister, and N. Westerhof. "Evaluation of methods for estimation of total arterial compliance." In: The American journal of physiology 268 (4 Pt 2 Apr. 1995), H1540-H1548. ISSN: 0002-9513.

[327] N. Stergiopulos, J. J. Meister, and N. Westerhof. "Simple and accurate way for estimating total and segmental arterial compliance: The pulse pressure method". In: Annals of Biomedical Engineering 22.4 (July 1994), pp. 392397. DOI: $10.1007 / \mathrm{bf} 02368245$.

[328] N. Stergiopulos, Y. Tardy, and J.-J. Meister. "Nonlinear separation of forward and backward running waves in elastic conduits". In: Journal of Biomechanics 26.2 (Feb. 1993), pp. 201-209. DOI: 10.1016/0021-9290 (93)90049-k.

[329] N. Stergiopulos, B. E. Westerhof, and N. Westerhof. "Total arterial inertance as the fourth element of the windkessel model." In: The American journal of physiology 276 (1 Pt 2 Jan. 1999), H81-H88. ISSN: 0002-9513.

[330] N. Stergiopulos et al. "On the wave transmission and reflection properties of stenoses". In: Journal of Biomechanics 29.1 (Jan. 1996), pp. 31-38. DOI: 10.1016/0021-9290(95)00023-2.

[331] Meenakshi Sundaram et al. "Laparoscopic myomectomy with uterine artery ligation: Review article and comparative analysis". In: Journal of Gynecological Endoscopy and Surgery 2.1 (2011), p. 3. DOI: 10.4103/0974-1216.85272. 
[332] T. E. Sweeney et al. "Intravascular pressure and diameter profile of the uteroovarian resistance artery network: estrous cycle-dependent modulation of resistance artery tone". In: AJP: Heart and Circulatory Physiology 293.5 (Sept. 2007), H2937-H2944. DOI: 10.1152/ajpheart.01019. 2006.

[333] Michał Szpinda and Anna Szpinda. "Normative Growth Data for the External Diameters of the External and Internal Iliac Arteries in Human Fetuses An Anatomical, Digital and Statistical Study". In: Advances in Clinical and Experimental Medicine 21.2 (2012). URL: http : / / www . advances . umed. wroc.pl/pdf/2012/21/2/143.pdf.

[334] Michał Szpinda et al. "The normal growth of the common iliac arteries in human fetuses - an anatomical, digital and statistical study". In: Medical Science Monitor 18.3 (2012), BR109-BR116. DOI: 10.12659/msm. 882516.

[335] Kenji Takizawa and Tayfun E. Tezduyar. "Fluid-Structure Interaction Modeling of Patient-Specific Cerebral Aneurysms". In: Visualization and Simulation of Complex Flows in Biomedical Engineering. Springer Netherlands, Nov. 2013, pp. 25-45. DOI: 10.1007/978-94-007-7769-9_2.

[336] D. G. Talbert. "Uterine flow velocity waveform shape as an indicator of maternal and placental development failure mechanisms: a model-based synthesizing approach". In: Ultrasound in Obstetrics and Gynecology 6.4 (1995), pp. 261-271. DOI: 10.1046/j.1469-0705.1995.06040261.x.

[337] A. Tayyar et al. "Uterine artery pulsatility index in the three trimesters of pregnancy: effects of maternal characteristics and medical history". In: Ultrasound in Obstetrics $\mathscr{G}$ Gynecology 45.6 (Apr. 2015), pp. 689-697. DOI: 10.1002/uog. 14789.

[338] Shivendra G. Tewari et al. "Analysis of cardiovascular dynamics in pulmonary hypertensive C57BL6/J mice". In: Frontiers in Physiology 4 (2013). DOI: $10.3389 /$ fphys . 2013.00355.

[339] R Thadhani et al. "Pulse pressure and risk of preeclampsia: a prospective study." In: Obstetrics and gynecology 97 (4 Apr. 2001), pp. 515-520. ISSN: 0029-7844. 
[340] Israel Thaler et al. "Changes in uterine blood flow during human pregnancy". In: American Journal of Obstetrics and Gynecology 162.1 (Jan. 1990), pp. 121125. DOI: 10.1016/0002-9378(90)90834-t.

[341] Thenappan Thenappan et al. "Pulmonary arterial hypertension: pathogenesis and clinical management". In: BMJ (Mar. 2018), j5492. DOI: 10.1136/bmj . j5492.

[342] K D Thornbury et al. "Nerve-mediated contractions of sheep mesenteric lymph node capsules." In: The Journal of physiology 422 (Mar. 1990), pp. 513522. ISSN: 0022-3751.

[343] Charlene E Thornton et al. "Role of proteinuria in defining pre-eclampsia: Clinical outcomes for women and babies". In: Clinical and Experimental Pharmacology and Physiology 37.4 (Apr. 2010), pp. 466-470. DOI: 10.1111/ j.1440-1681.2009.05334.x.

[344] A. Thuring, R. Laurini, and K. Maršál. "Uterine venous blood flow in normal and complicated pregnancies: a methodological study". In: Ultrasound in Obstetrics and Gynecology 35.4 (Apr. 2010), pp. 462-467. DOI: 10.1002 / $\operatorname{uog} .7572$.

[345] Ryo Torii et al. "Fluid-structure interaction analysis of a patient-specific right coronary artery with physiological velocity and pressure waveforms". In: Communications in Numerical Methods in Engineering 25.5 (May 2009), pp. 565-580. DOI: $10.1002 / \mathrm{cnm} .1231$.

[346] Ryo Torii et al. "Fluid--structure interaction modeling of blood flow and cerebral aneurysm: Significance of artery and aneurysm shapes". In: Computer Methods in Applied Mechanics and Engineering 198.45-46 (Sept. 2009), pp. 3613-3621. DOI: $10.1016 /$ j.cma.2008.08.020.

[347] Monique Trbs et al. "Comparison of Fractional Flow Reserve Based on Computational Fluid Dynamics Modeling Using Coronary Angiographic Vessel Morphology Versus Invasively Measured Fractional Flow Reserve". In: The American Journal of Cardiology 117.1 (Jan. 2016), pp. 29-35. DOI: 10.1016/ j. amj card. 2015.10.008. 
[348] Shengxian Tu et al. "Fractional Flow Reserve Calculation From 3-Dimensional Quantitative Coronary Angiography and TIMI Frame Count". In: JACC: Cardiovascular Interventions 7.7 (July 2014), pp. 768-777. DOI: 10.1016/j . jcin.2014.03.004.

[349] S.A. Urquiza et al. "Multidimensional modelling for the carotid artery blood flow". In: Computer Methods in Applied Mechanics and Engineering 195.3336 (July 2006), pp. 4002-4017. DOI: 10.1016/j.cma.2005.07.014.

[350] Kristian Valen-Sendstad et al. "Direct numerical simulation of transitional flow in a patient-specific intracranial aneurysm". In: Journal of Biomechanics 44.16 (Nov. 2011), pp. 2826-2832. DOI: 10.1016/j · jbiomech.2011.08.015.

[351] Alvaro Valencia et al. "Fluid Structural Analysis of Human Cerebral Aneurysm Using Their Own Wall Mechanical Properties". In: Computational and Mathematical Methods in Medicine 2013 (2013), pp. 1-18. DOI: 10.1155/2013/ 293128.

[352] Karlijn Vollebregt et al. "712: Arterial stiffness is higher in early pregnancy in women, who will develop preeclampsia". In: American Journal of Obstetrics and Gynecology 199.6 (Dec. 2008), S203. DOI: 10.1016/j . ajog. 2008.09 . 743.

[353] Sharona Vonck et al. "Why non-invasive maternal hemodynamics assessment is clinically relevant in early pregnancy: a literature review". In: BMC Pregnancy and Childbirth 16.1 (Oct. 2016). DOI: 10.1186/s12884-016-1091-9.

[354] Frans N. van de Vosse and Nikos Stergiopulos. "Pulse Wave Propagation in the Arterial Tree". In: Annual Review of Fluid Mechanics 43.1 (Jan. 2011), pp. 467-499. DOI: 10.1146/annurev-fluid-122109-160730.

[355] William A.W. Walters. "Chapter 10 Maternal adaptation to pregnancy". In: Reproductive Endocrinology and Biology. Elsevier, 1998, pp. 183-209. DOI: 10.1016/s1569-2582(98)80102-5.

[356] Rui Wang et al. "Diagnostic value of quantitative stenosis predictors with coronary CT angiography compared to invasive fractional flow reserve". In: European Journal of Radiology 84.8 (Aug. 2015), pp. 1509-1515. DOI: 10 . 1016/j.ejrad.2015.05.010. 
[357] Xiaofei Wang, Jose-Maria Fullana, and Pierre-Yves Lagrée. "Verification and comparison of four numerical schemes for a 1D viscoelastic blood flow model". In: Computer Methods in Biomechanics and Biomedical Engineering 18.15 (Aug. 2014), pp. 1704-1725. DOI: 10.1080/10255842.2014.948428.

[358] Yuping Wang and Shuang Zhao. "Vascular Biology of the Placenta". In: (2010). DOI: 10.4199/C00016ED1V01Y201008ISP009.

[359] Sansuke M. Watanabe, Pablo J. Blanco, and Raúl A. Feijóo. "Mathematical Model of Blood Flow in an Anatomically Detailed Arterial Network of the Arm". In: ESAIM: Mathematical Modelling and Numerical Analysis 47.4 (June 2013), pp. 961-985. DOI: 10.1051/m2an/2012053.

[360] Jim Waterhouse, Marina Sawdon, and Emrys Kirkman. "Capillary dynamics and the interstitial fluid-lymphatic system". In: Anaesthesia $\mathscr{G}$ Intensive Care Medicine 14.2 (Feb. 2013), pp. 72-78. DOI: 10.1016/j.mpaic. 2012. 11.016.

[361] R.L. Watrous. A Computational Model of Cardiovascular Physiology and Heart Sound Generation. IEEE, Sept. 2009. DOI: 10 . 1109/IEMBS . 2009 . 5332548.

[362] P Y von der Weid. "Review article: lymphatic vessel pumping and inflammationthe role of spontaneous constrictions and underlying electrical pacemaker potentials." In: Alimentary pharmacology 85 therapeutics 15 (8 Aug. 2001), pp. 1115-1129. ISSN: 0269-2813.

[363] Toby N. Weingarten, Juraj Sprung, and James R. Munis. "Peripheral Venous Pressure as a Measure of Venous Compliance During Pheochromocytoma Resection". In: Anesthesia \& Analgesia 99.4 (Oct. 2004), pp. 1035-1037. DOI: $10.1213 / 01$. ane.0000130853.58560.5d.

[364] Nico Westerhof, Jan-Willem Lankhaar, and Berend E. Westerhof. "The arterial Windkessel". In: Medical \& Biological Engineering $\&$ Computing 47.2 (June 2008), pp. 131-141. DOI: 10.1007/s11517-008-0359-2.

[365] Nicolaas Westerhof et al. "Analog studies of the human systemic arterial tree". In: Journal of Biomechanics 2.2 (May 1969), pp. 121-143. DOI: 10 . 1016/0021-9290(69)90024-4. 
[366] G.St.J. Whitley and J.E. Cartwright. "Cellular and Molecular Regulation of Spiral Artery Remodelling: Lessons from the Cardiovascular Field". In: Placenta 31.6 (June 2010), pp. 465-474. DOI: 10.1016/j.placenta. 2010. 03.002.

[367] Guy St J. Whitley and Judith E. Cartwright. "Trophoblast-mediated spiral artery remodelling: a role for apoptosis". In: Journal of Anatomy 215.1 (July 2009), pp. 21-26. DOI: 10.1111/j.1469-7580.2008.01039.x.

[368] Ian B Wilkinson et al. "Increased central pulse pressure and augmentation index in subjects with hypercholesterolemia". In: Journal of the American College of Cardiology 39.6 (Mar. 2002), pp. 1005-1011. DOI: 10.1016/S07351097 (02) 01723-0.

[369] Marie Willemet and Jordi Alastruey. "Arterial Pressure and Flow Wave Analysis Using Time-Domain 1-D Hemodynamics". In: Annals of Biomedical Engineering 43.1 (Aug. 2014), pp. 190-206. DOI: 10.1007/s10439-014-1087-4.

[370] B.J.B.M. Wolters et al. "A patient-specific computational model of fluid-structure interaction in abdominal aortic aneurysms". In: Medical Engineering $\&$ Physics 27.10 (Dec. 2005), pp. 871-883. DOI: 10.1016/j .medengphy . 2005.06.008.

[371] B.J.B.M. Wolters et al. "Assessment of endoleak significance after endovascular repair of abdominal aortic aneurysms: A lumped parameter model". In: Medical Engineering 6 Physics 29.10 (Dec. 2007), pp. 1106-1118. DOI: 10.1016/j . medengphy . 2006.11.008.

[372] N. Xiao, J. Alastruey, and C. A. Figueroa. "A systematic comparison between 1-D and 3-D hemodynamics in compliant arterial models". In: International Journal for Numerical Methods in Biomedical Engineering 30.2 (Sept. 2013), pp. 204-231. DOI: 10.1002/cnm. 2598.

[373] Dongbin Xiu and Spencer J. Sherwin. "Parametric uncertainty analysis of pulse wave propagation in a model of a human arterial network". In: Journal of Computational Physics 226.2 (Oct. 2007), pp. 1385-1407. DOI: 10.1016/ j.jcp.2007.05.020. 
[374] C. K. H. Yu et al. "Prediction of pre-eclampsia by uterine artery Doppler imaging: relationship to gestational age at delivery and small-for-gestational age". In: Ultrasound in Obstetrics and Gynecology 31.3 (2008), pp. 310-313. DOI: $10.1002 / \mathrm{uog} .5252$.

[375] Paolo Zamboni et al. "Venous compliance and clinical implications". In: Veins and Lymphatics 7.2 (May 2018). DOI: 10.4081/v1.2018.7367.

[376] D. C. Zawieja et al. "Distribution, propagation, and coordination of contractile activity in lymphatics". In: American Journal of Physiology-Heart and Circulatory Physiology 264.4 (Apr. 1993), H1283-H1291. DOI: 10.1152/ ajpheart.1993.264.4.h1283.

[377] Constantinos Zervides et al. "The role of venous valves in pressure shielding". In: BioMedical Engineering OnLine 7.1 (2008), p. 8. DOI: $10.1186 / 1475-$ $925 \mathrm{x}-7-8$.

[378] Jun Zhang et al. "Blood pressure dynamics during pregnancy and spontaneous preterm birth". In: American Journal of Obstetrics and Gynecology 197.2 (Aug. 2007), 162.e1-162.e6. DOI: 10.1016/j.ajog.2007.03.053.

[379] Jun-Mei Zhang et al. "Simplified Models of Non-Invasive Fractional Flow Reserve Based on CT Images". In: PLOS ONE 11.5 (May 2016). Ed. by Salvatore De Rosa, e0153070. DOI: 10.1371/journal pone.0153070.

[380] J. Zhou and Y. C. Fung. "The degree of nonlinearity and anisotropy of blood vessel elasticity". In: Proceedings of the National Academy of Sciences 94.26 (Dec. 1997), pp. 14255-14260. DOI: 10.1073/pnas.94.26.14255.

[381] Olek C. Zienkiewicz, Robert L. Taylor, and J. Z. Zhu. The Finite Element Method: Its Basis and Fundamentals. Elsevier LTD, Oxford, 2013. 768 pp. ISBN: 1856176339. URL: https://www . ebook.de/de/product/20688519/ olek_c_zienkiewicz_robert_l_taylor_j_z_zhu_the_finite_element_ method_its_basis_and_fundamentals.html. 